\title{
AN UPDATED CHECKLIST OF THE ORCHIDACEAE OF PANAMA
}

\author{
Diego Bogarín ${ }^{1,2,4}$, Zuleika Serracín ${ }^{2}$, Zabdy Samudio ${ }^{2}$, Rafael Rincón ${ }^{2}$ \\ \& Franco PuPULIN ${ }^{1,3}$
}

\author{
${ }^{1}$ Jardín Botánico Lankester, Universidad de Costa Rica. P.O. Box 302-7050 Cartago, Costa Rica, A.C. \\ ${ }^{2}$ Herbario UCH, Universidad Autónoma de Chiriquí, 0427, David, Chiriquí, Panama \\ ${ }^{3}$ Harvard University Herbaria, 22 Divinity Avenue, Cambridge, Massachusetts, U.S.A.; \\ Marie Selby Botanical Gardens, Sarasota, FL, U.S.A. \\ ${ }^{4}$ Author for correspondence: diego.bogarin@ucr.ac.cr
}

AbSTRACT. The Orchidaceae is one of the most diverse vascular plant families in the Neotropics and the most diverse in Panama. The number of species is triple that of other well-represented families of angiosperms such as Rubiaceae, Fabaceae and Poaceae. Despite its importance in terms of diversity, the latest checklist was published ten years ago and the latest in-depth taxonomic treatments were published in 1949 and 1993. The accumulation of information over the years and the need to update the nomenclature and to clarify taxonomic concepts made necessary the publication of an up-dated checklist of the Orchidaceae of Panama. This checklist was completed by studying specimens strictly collected in Panama and vouchered in herbaria. Species are presented alphabetically with their synonyms and herbarium vouchers. The data were analyzed to explain the patterns of geographic distribution, most diverse taxa, endemism, exotic species and relationships with other nearby floras. The checklist contains 1365 species (including two natural hybrids and three subspecies) in four subfamilies, 16 tribes, 27 subtribes and 187 genera. Four exotic species were recorded. A total of 296 (21.7\%) species are endemic. Epidendroideae is the most diverse group housing more than $90 \%$ of species. The most diverse subtribes are Pleurothallidinae (30 genera, 405 spp.), Laeliinae (16 genera, 292 spp.), Oncidiinae (29 genera, 157 spp.) and Maxillariinae (18 genera, 132 spp.). The most diverse genera are: Epidendrum (206 spp.), Stelis (88 spp.), Lepanthes (66 spp.) and Pleurothallis (54 spp.). Nomenclatural changes are proposed in Maxillariella, Pleurothallis, Specklinia, Stelis and Trichocentrum. Many areas remain unexplored for orchids, and we estimate that much work remains to complete a floristic treatment that reveals more realistic data on the orchid flora that Panama harbors. This checklist is an important initial step toward the development of an illustrated treatment of the Orchidaceae of Panama.

RESUMEN. Orchidaceae es una de las familias de plantas vasculares más diversas del Neotrópico y la más diversa en Panamá. Su número de especies triplica a las otras familias más diversas de angiospermas como Rubiaceae, Fabaceae y Poaceae. A pesar de su importancia en terminos de diversidad, el último listado de especies se publicó hace diez años y los últimos tratamientos taxonómicos profundos fueron publicados en 1949 y 1993 . La acumulación de información a través de esos años así como la necesidad de actualizar la nomenclatura y aclarar conceptos taxonómicos dudosos hace necesaria la publicación de un listado actualizado de las Orchidaceae de Panamá. El presente listado se completó mediante el estudio de especímenes estrictamente recolectados en Panamá y con un testigo en herbario. Las especies se presentan alfabéticamente con sus respectivos sinónimos y se citan los testigos de herbario. Se analizaron los datos para explicar los patrones de distribución, taxa más diversos, endemismos, especies exóticas y relación con floras cercanas. El listado contiene 1365 especies (dos híbridos naturales y tres subespecies) en cuatro subfamilias, 16 tribus, 27 subtribus y 187 géneros. Se registraron 4 especies exóticas. Un total de 296 (21.7\%) son especies endémicas. Epidendroideae es el grupo más diverso, concentrando más del $90 \%$ de las especies. Las sutribus más diversas son: Pleurothallidinae (30 géneros, 405 spp.), Laeliinae (16 géneros, 292 spp.), Oncidiinae (29, 157 spp.) y Maxillariinae (18 géneros, 132 spp.). Los géneros más diversos son: Epidendrum (206 spp.), Stelis (88 spp.), Lepanthes (66 spp.) y Pleurothallis (54 spp.). Se proponen cambios nomenclatoriales en Maxillariella, Pleurothallis, Specklinia, Stelis y Trichocentrum. Muchas zonas permanecen sin explorar desde el punto de vista orquideológico y estimamos que todavía se necesita mucho trabajo para completar un tratamiento que revele datos más realistas sobre la flora de orquídeas que alberga Panamá. Este listado es un importante paso inicial hacia el desarrollo de un tratamiento ilustrado de las Orchidaceae de Panamá.

KEY WORDS: floristics, taxonomy, systematics, biogeography, species distribution 
Introduction. The orchid flora of Panama is one of the most diverse in the Neotropics. The early efforts to understand this family were ephemeral and made by European explorers or expeditions passing through the isthmus on the route to North or South America. James Wallace in 1700 was perhaps the first plant collector in Panama. Wallace collected in the settlement of New Caledonia, on the Atlantic coast of the Kuna Yala (now Punta Escocés and Isla de Oro), in a failed attempt of Scotland to colonize lands in the New World. However, these specimens were not studied by the botanists at the time and no orchid species based on those collections are recorded or mentioned in literature (Wallace 17001701). The first documented record of an orchid in Panama was an illustration of Catasetum sp., made by Joseph Guío, illustrator of the expedition led by Alessandro Malaspina between 1789 and 1794. The illustration was based on a collection by Louis Neé on Taboga Island in December 1790 (Fig. 1). One of the earliest mentions of an orchid collected in Panama in a floristic treatment was made by Beurling (1800-1866), in the "Primitiae florae Portobellensis" who cited a specimen of Brassavola nodosa Lindl., a plant collected "in rupibus extimis ad introitum portus" by J.E. Billberg in 1826 (Fig. 2). However, the first descriptions of new species based on collecting expeditions in Panama were published subsequent to the exploration of Hugh Cuming (Fig. 3A) for the nursery firm of Messrs Loddiges of Hackney. Around 1829-1831 he collected in some areas of the Pacific coast such as Taboga Island, the Las Perlas Archipelago (Pearl Islands), Bay of Montijo and Chiriquí River. Although several of his collections were made at these sites and others on the Pacific coast of Colombia, almost all herbarium specimens were labeled "Panama et Columbia occidentalis". Lindley (1830-1840) described some of these collections: Aspasia epidendroides, Dichaea panamensis, Hexisea bidentata and Oncidium ampliatum (Fig. 4). Another
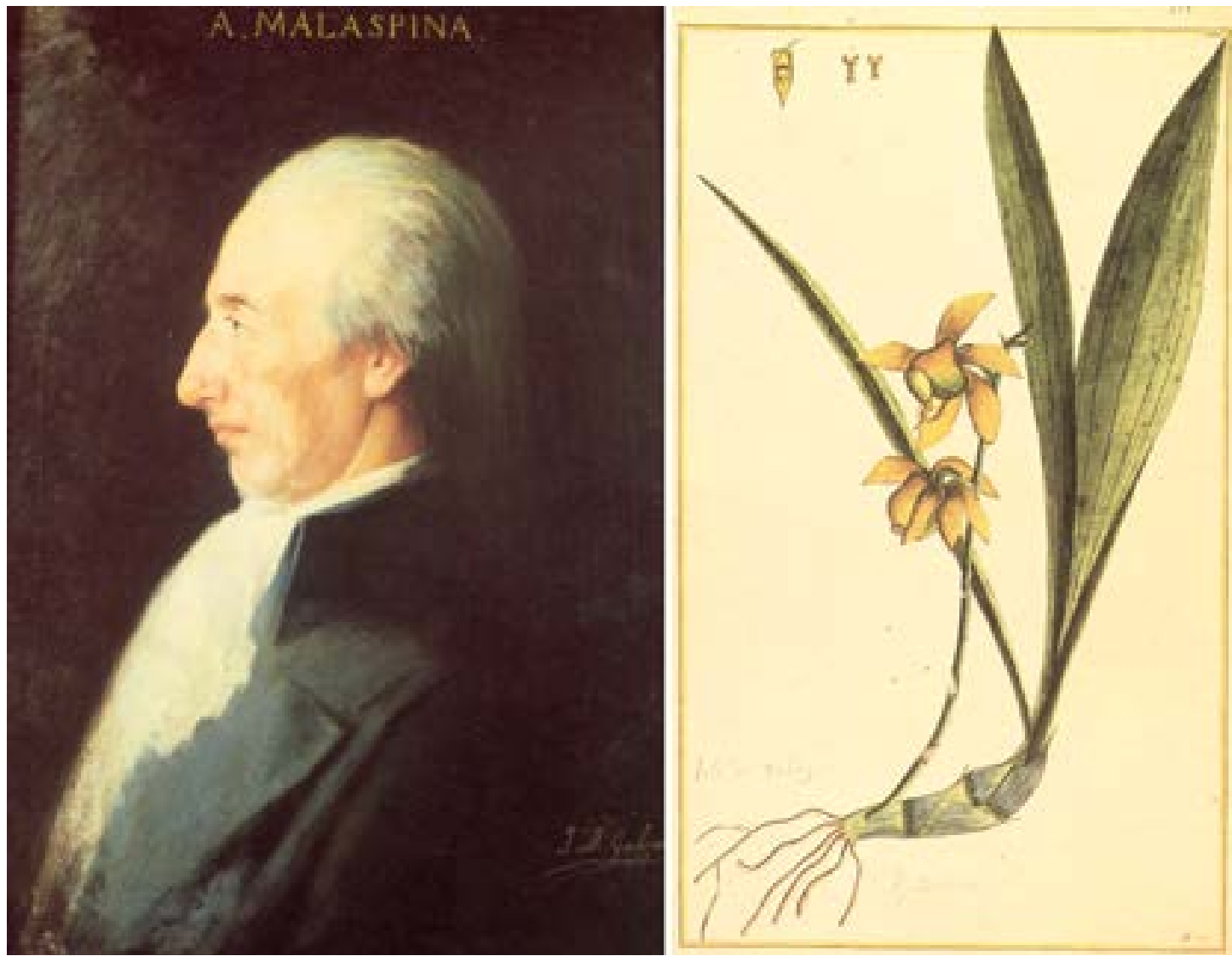

Figure 1. Left, Alessandro Malaspina (1754-1810). Navy Museum, Madrid. Right, The first documented record of an orchid in Panama: Catasetum sp. Illustration by José Guío in Real Jardín Botánico de Madrid, 1989: plate 137. 


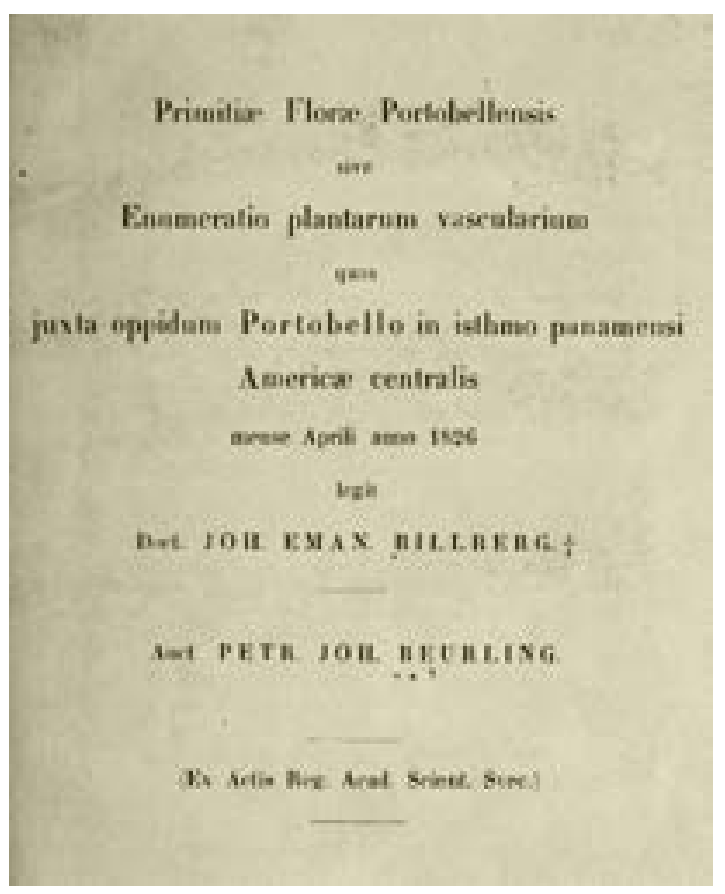

Figure 2. Cover of "Primitiae florae Portobellensis" by Beurling (1800-1866)

expedition that reached Panama and Taboga Island was The Voyage of the HMS Sulphur between 1836-1842. The botanical collections were led by Andrew Sinclair, George Barclay and Richard B. Hinds (1812-1847). A few collections of orchids, including Lockhartia micrantha Rchb.f., Oncidium stipitatum Lindl. ex Benth. and Ornithocephalus bicornis Lindl. ex Benth., were reported (Hinds 1844, Reichenbach 1852). They also correspond to the first orchid species described from Central America (Fig. 5). These species are typical of coastal areas, indicating that explorations did not penetrate deep into Panama.

Berthold Seemann was another of the pioneer explorers and the author of the work entitled Flora of the Isthmus of Panama published in 1854. In 1846 he undertook his first exploration to Panama under The Voyage of the H.M.S Herald during the years 18451851 (Seemann 1852-1857). He explored Chagres, Panama City, Coiba Island, Chiriquí and Darién (Fig. 3B). Seemann (1854) managed to accumulate a total of 104 species of Panamanian orchids.

However, the first plant collector to undertake a serious expedition into the interior was Josef Ritter von Rawicz Warscewicz (Fig. 3C). In 1848, he crossed the intricate Cordillera de Talamanca, starting in the Pacific at David, Chiriquí, ascending towards Dolega to Boquete and Volcán Barú (the highest elevation of Panama) and finishing at Laguna de Chiriquí Grande on the Caribbean (Savage 1970, Fig. 6). Warscewicz (1850) recorded 18 species mainly from Chiriquí and Veraguas. Later, Reichenbach (1866) recorded 63 species that were collected by Warscewicz in Panama. Of them, 34 species and one variety were described
A

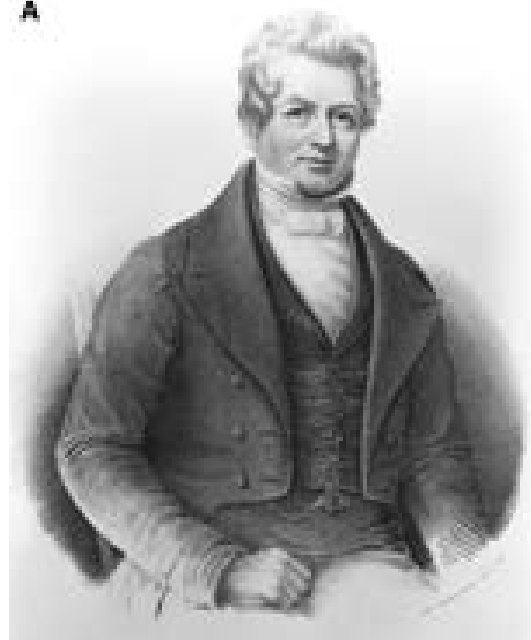

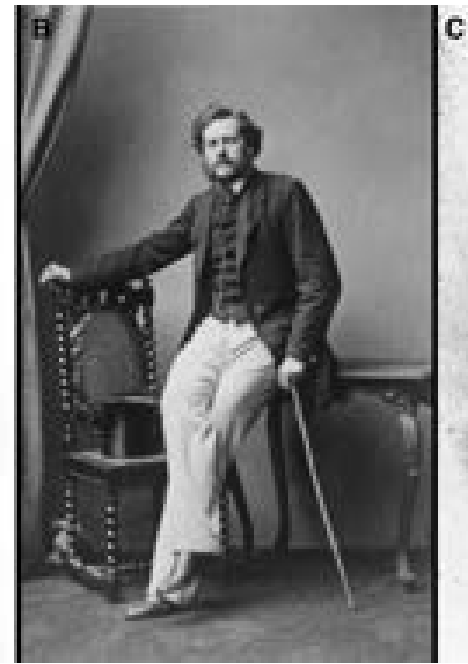

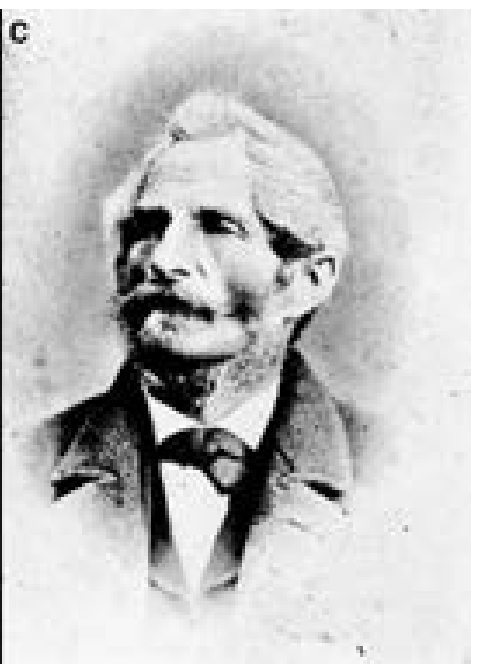

Figure 3. Hugh Cuming (1791-1865). From a lithograph by Hawkins, 1850 1850. Courtesy of Rudolf Jenny. B Carl Berthold Seemann (1825-1871). From Gardeners' Chron., 1871. Courtesy of the Hunt Institute for Botanical Documentation C - Josef Ritter von Rawicz Warscewicz (1812-1866). Courtesy of the Hunt Institute for Botanical 


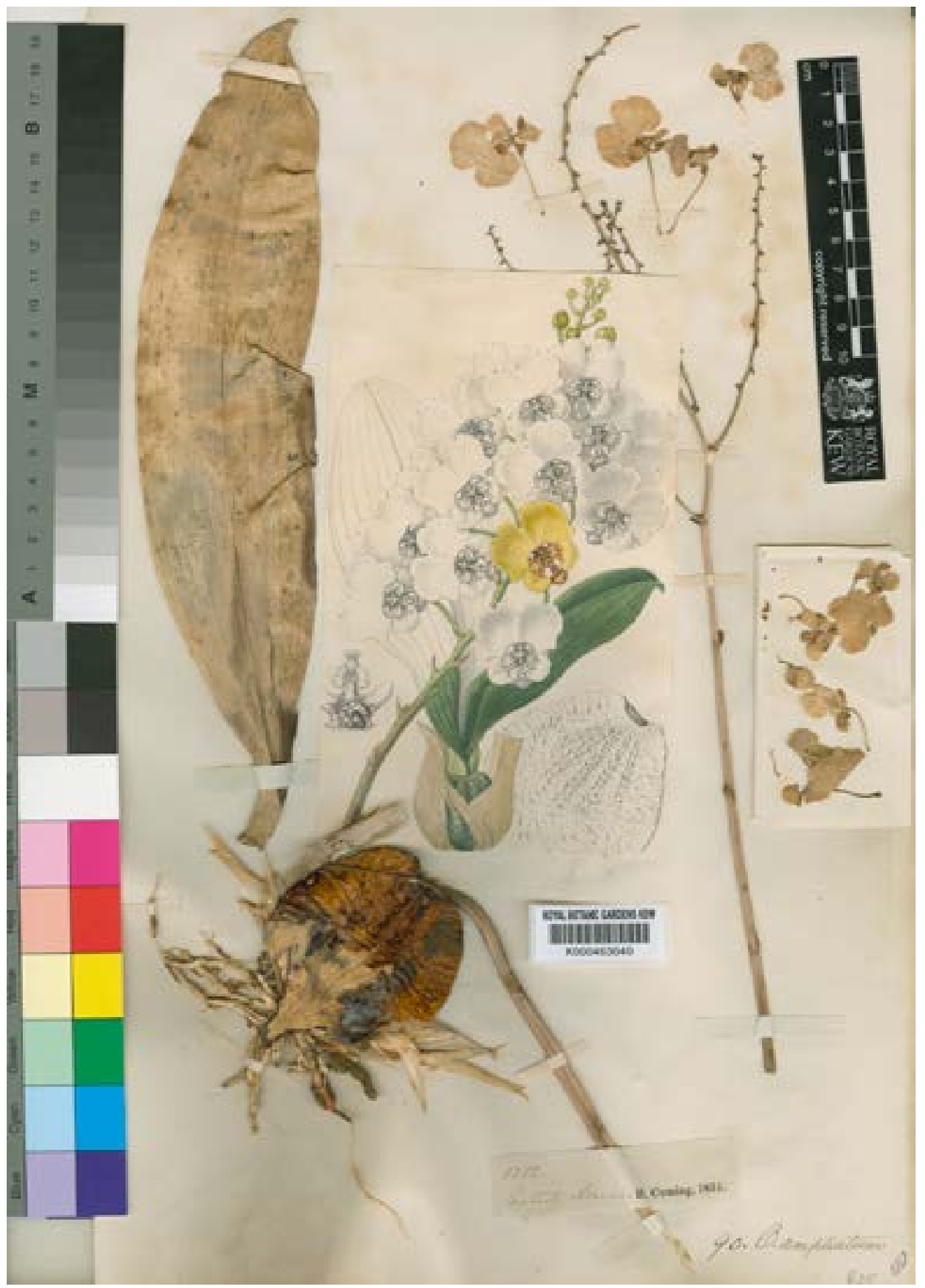

FiguRE 4. Type specimen of Oncidium ampliatum Lindl., collected in "Panama et Columbia occidentali" by H. Cuming in 1831. Reproduced with permission by the Board of Trustees, Royal Botanic Gardens, Kew. 


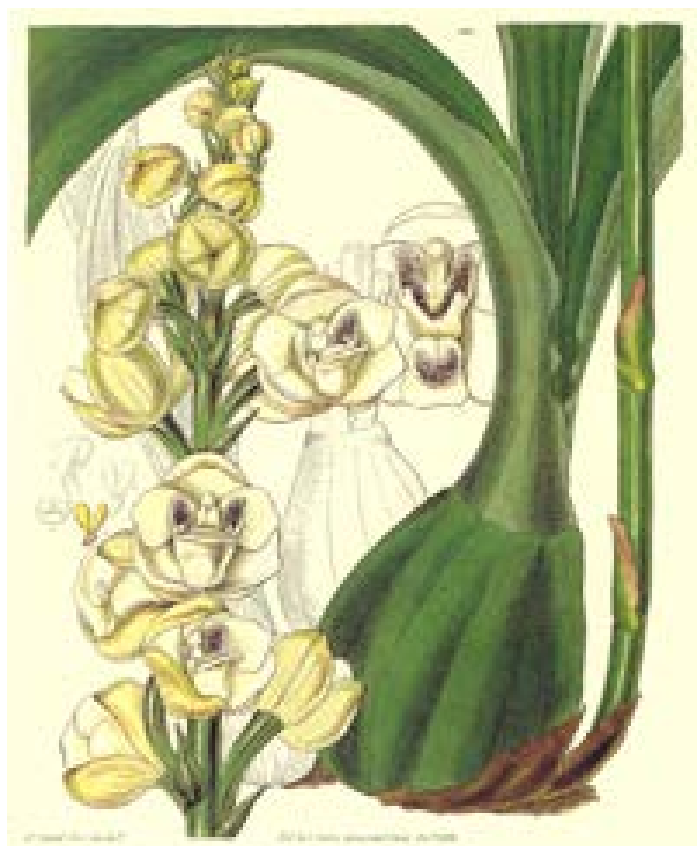

Figure 5. Peristeria elata Hook., the National Flower of Panama described in 1831 by Sir William Jackson Hooker. Taken from the Curtis's Botanical Magazine, t. 3116.

as new to science (Reichenbach 1852, 1854, 1866). Warscewicz returned to the country in 1851 and was the first to introduce living orchid plants from Panama to Europe. Then, William Hemsley (1884) published an annotated checklist to the orchids of Central America in the third volume of the botany series of Godman and Salvin's Biologia Centrali-Americana, listing 54 species mainly based on the collections of Behr, Cuming, E.P. Duchassaing, S. Hayes, P. Henderson, A. F. Fendler, Seemann, A. Sinclair, F.F. Stange, Stanger, G. Wallis, Warscewicz and G. Zahn. In the fourth volume, Hemsley (1884) listed 104 species and 41 genera for the flora of Panama (Fig. 7).

After sporadic expeditions by European travelers, floristic studies received a major boost with the construction of the Panama Canal between 1904 and 1914 (Fig. 8). From 1910 until 1912 Henry Pittier collected plants in the isthmus as part of the Biological Exploration of Panama by the Smithsonian Institution (Fig. 9). He visited places such as Colón, Darién, San Blas, Puerto Obaldía and the province of Chiriquí. Along with Robert W. Maxon, he explored the region of Boquete in the Chiriquí highlands (the Eldorado of the orchid-lovers in Panama according to Rudolf

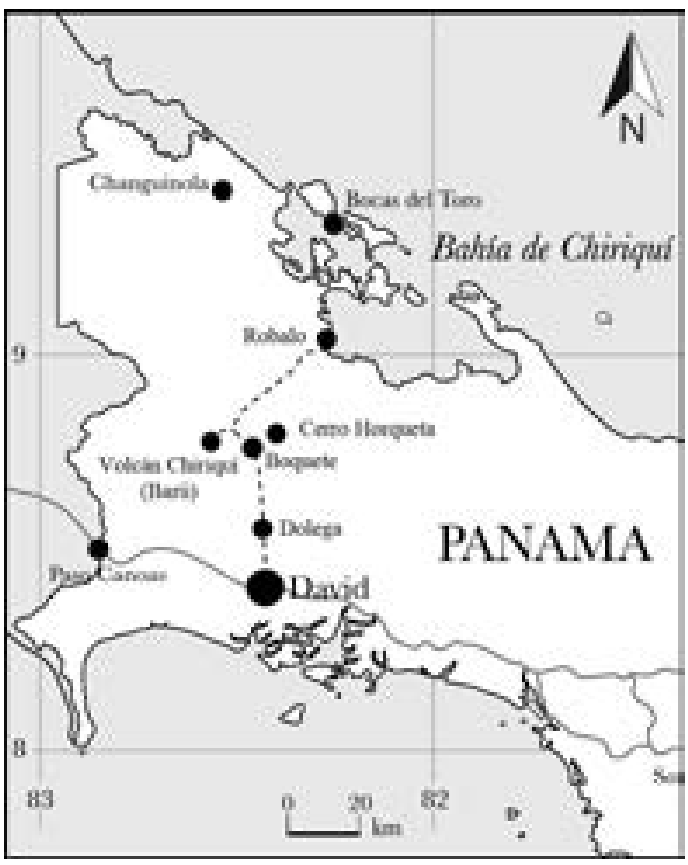

Figure 6. The route of Josef von Warscewicz in Panama (Savage 1970).

Schlechter), Volcán Barú, Alto de Las Palmas and the Cerro de La Horqueta (Fig. 10). Some 18 species of orchids from these expeditions were described by Schlechter (1913a,1913b, 1918), including Lepanthes eciliata Schltr. and L. maxonii Schltr. Schlechter (1918), who listed a total of 117 species for the country. Subsequently, Schlechter (1920a, 1920b, 1921) received a few specimens from Panama mainly by W. Joseph, Pittier and M. Wagner. He described nine species from those collections.

The first major effort to cultivate and study orchids in Panama was undertaken by Charles Wesley Powell. He kept a large collection of orchids near Balboa in Panama City and become the most prolific discoverer of Panamanian orchids (Fig. 11). With Abel A. Hunter he explored the country. Initially, he established contact with Robert Rolfe in Kew. However, after Rolfe's death in 1921, the identification of Powell' specimens ceased (Fig. 12B). Through a correspondent in Costa Rica, Schlechter contacted Powell who later sent his first set of samples to Berlin (Fig. 12C). Based on this material Schlechter (1922) published Orchidaceae Powellianae Panamensis, a treatment of collections made by Powell in Panama. Although this work was 

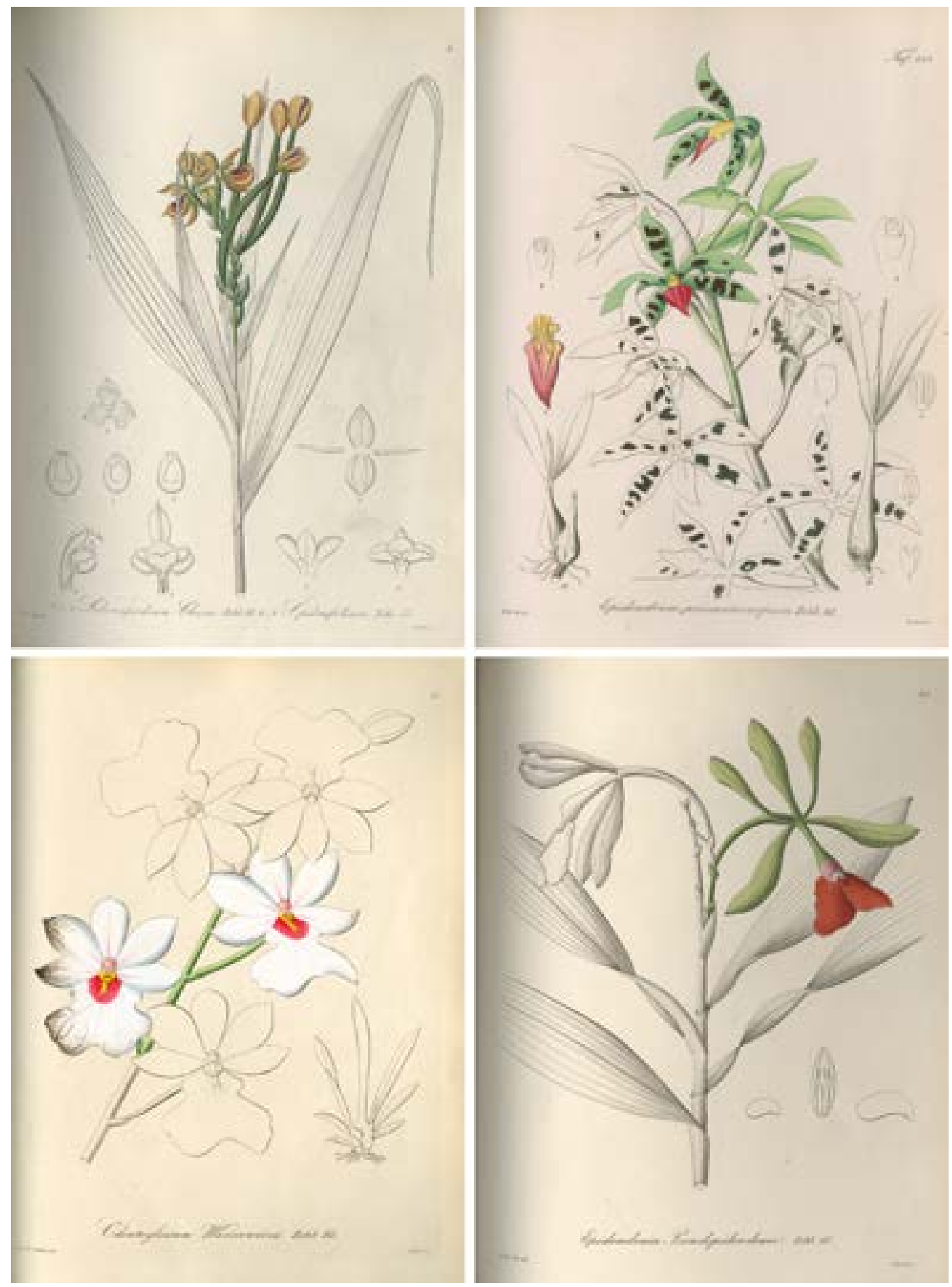

FIGURE 7. Orchid species collected in Panama and illustrated in Xenia Orchidacea I,II,III. A — Selenipedium chica Rchb.f. B — Epidendrum prismatocarpum Rchb.f. C — Odontoglossum warszewiczii Rchb.f. D — Pseudepidendrum spectabile Rchb.f. 


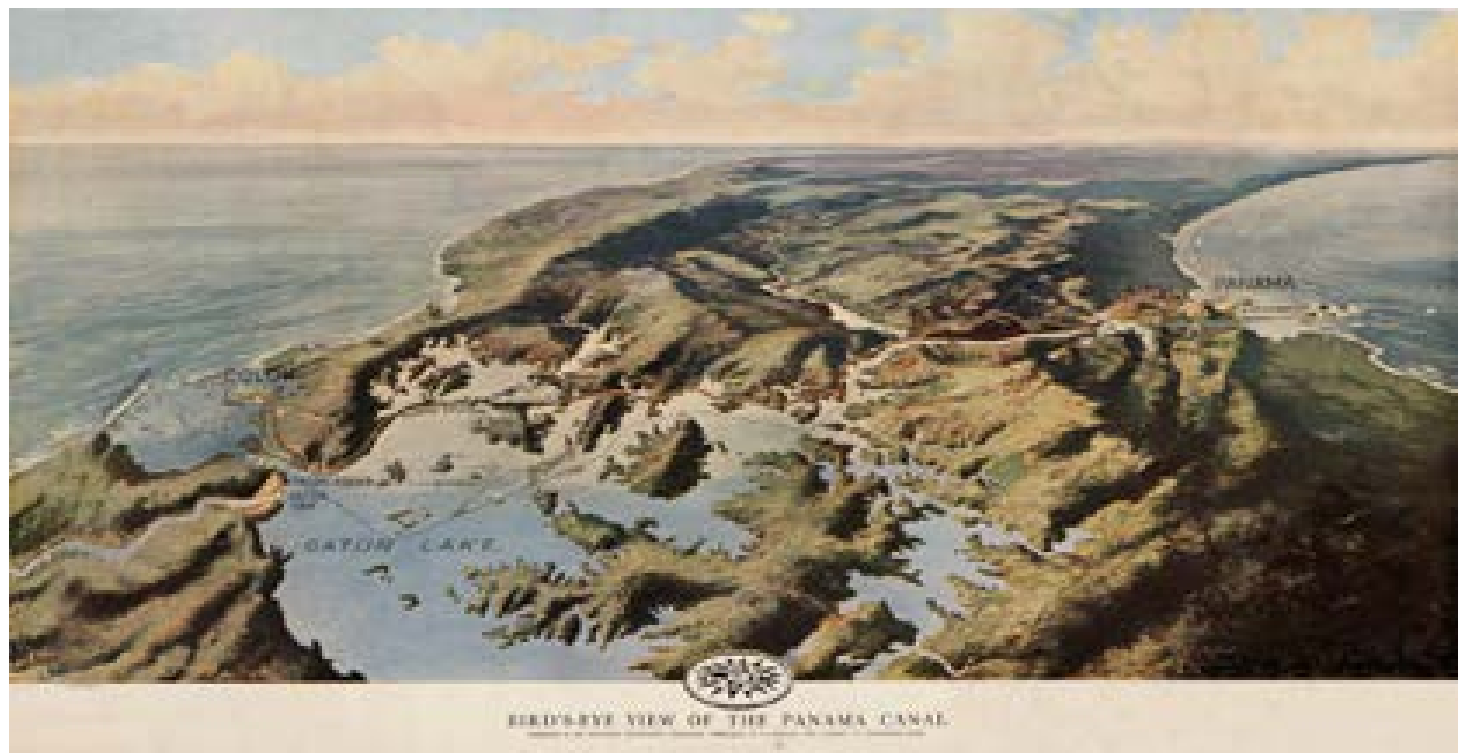

Figure 8. Panama Canal. National Geographic Society (U.S), 1912.

not a formal treatment of the Orchidaceae, at the time it constituted the largest published compilation on the family (including 260 collections) in the country. From that material, Schlechter identified 184 species in 60 genera, with 75 species described for the first time (Fig. 13). With this contribution, the number of genera in Panama increased to 72 and the number of species to 192. Unfortunately, these were the last of Schlechter's contributions, as he died in 1925 (Ossenbach 2009).

After a short visit to Panama where he met Powell, the American orchid specialist Oakes Ames received some of Powell's specimens. Of these, 29 were new to science. Ames (1921, 1922a, 1922b), Ames \& Schweinfurth $(1925,1930,1937)$ published the latest orchid species from Panama based on collections by A.M. Bouché, S. Hayes, E.P. Killip, W.R. Lindsay, W.R. Maxon, Pittier and R.S.Williams (Fig. 12A, 12D). Ames and collaborators added a total of 49 species new to science. However, no compilation of all of the orchids of Panama had yet been published. During this period only a few floristic works for specific regions were published, most significantly, the Flora of Barro Colorado Island (Standley 1927) covering 15 species and Flora of the Panama Canal Zone (Ames 1928) with 134 species in 57 genera. Also, 25 species were listed in The Botany of San Jose Island (Gulf of Panama) by Johnston (1949) (where the Lycaste campbellii C. Schweinf. was described). Subsequently, the Flora of Barro Colorado

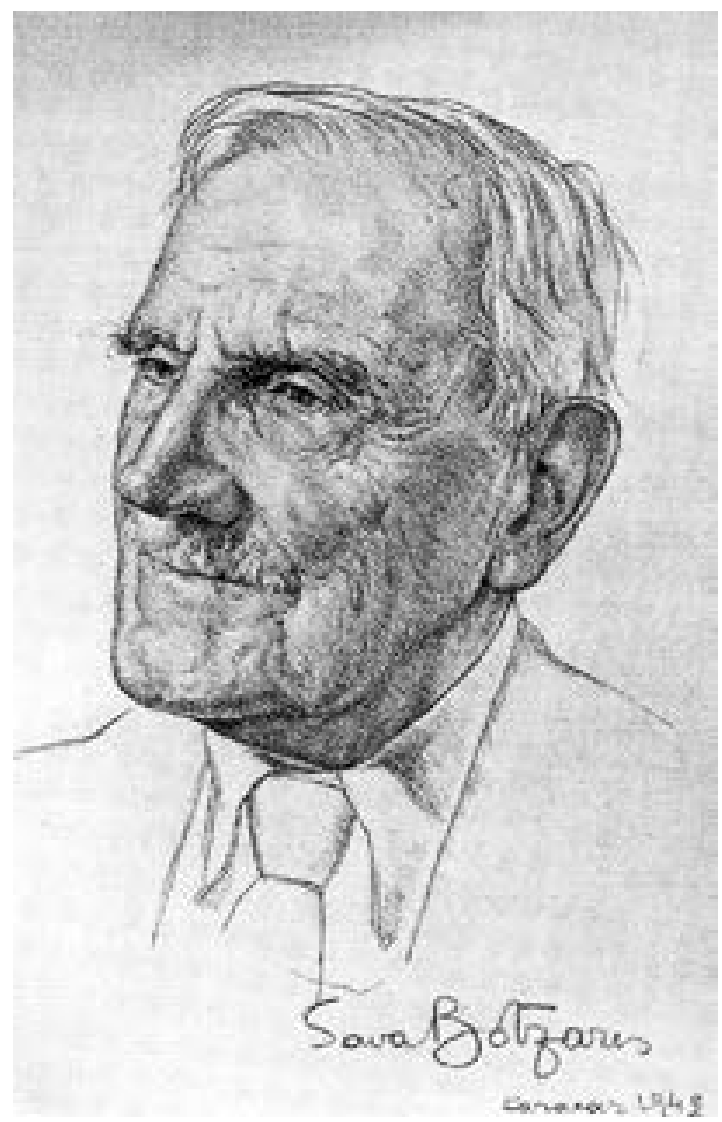

Figure 9. Henri Francois Pittier (1857-1950) by Sava Botzaris in the frontispiece of Häsler \& Baumann, 2000. 


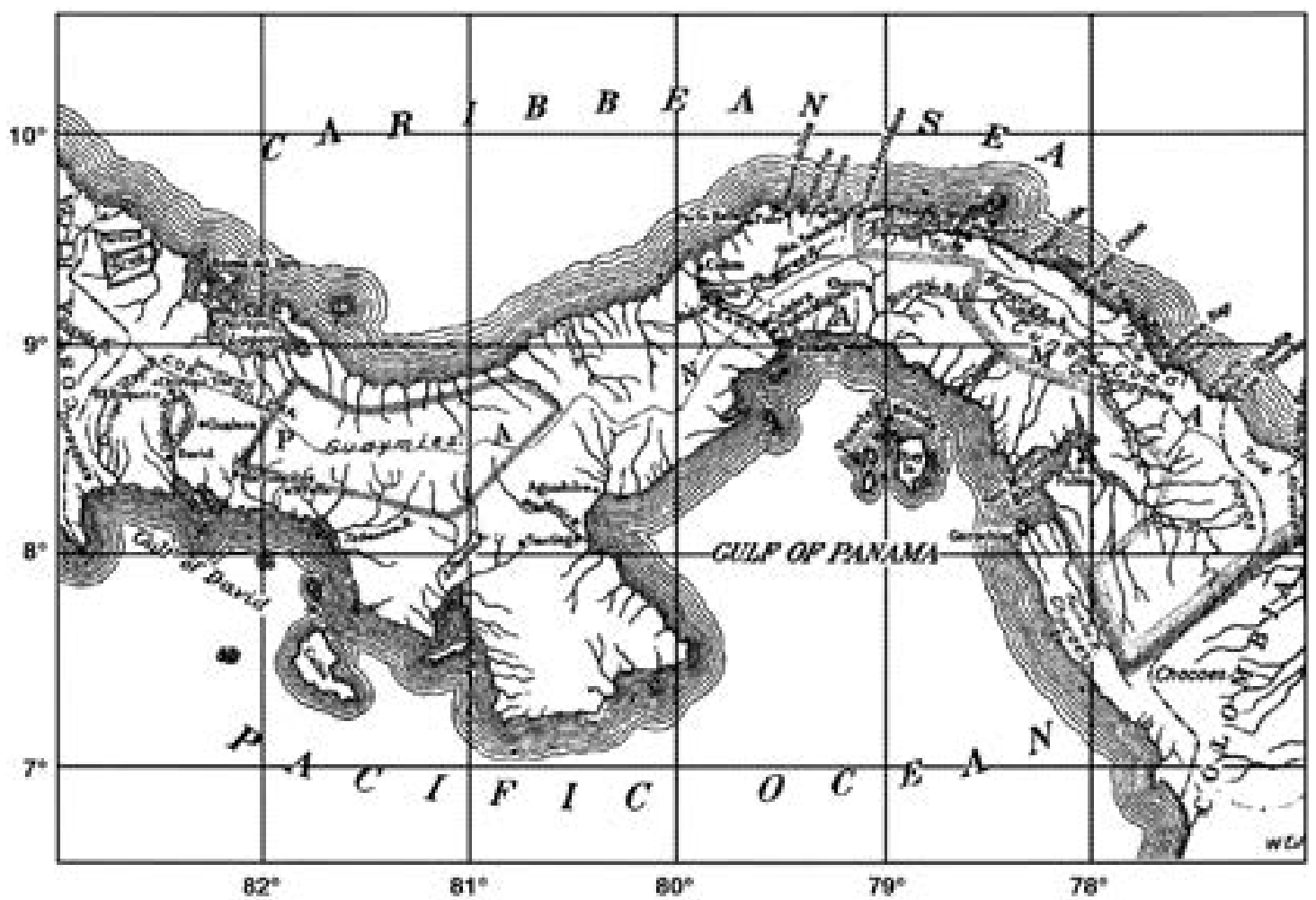

Figure 10. Map of Panama by H. Pittier. In Heckadon-Moreno, 1998: 113.
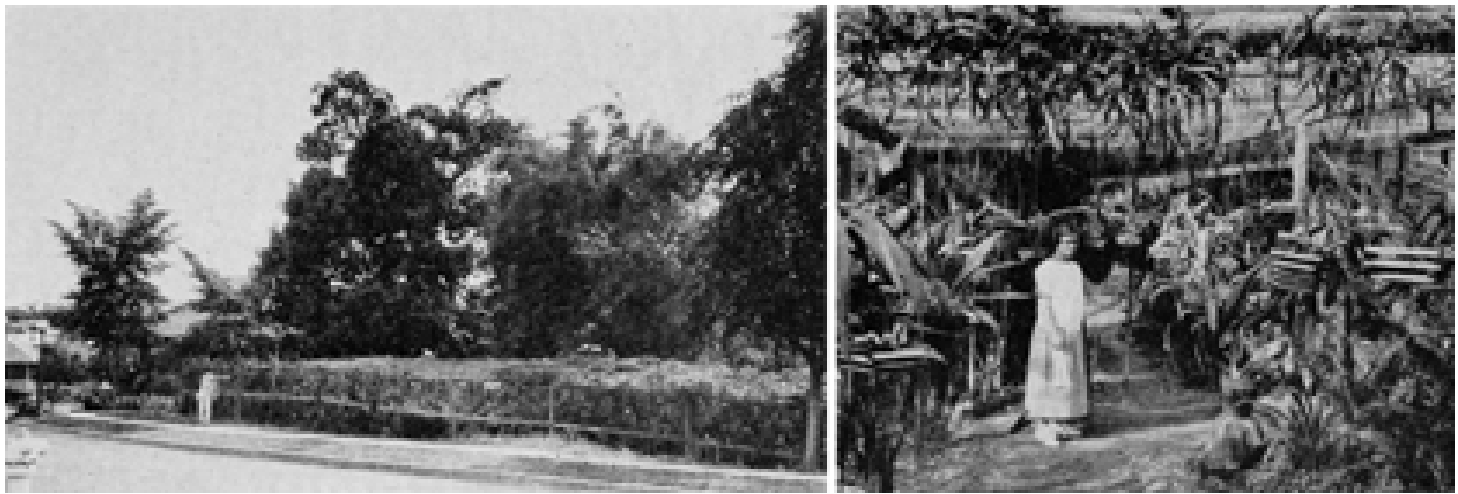

FIGURE 11. Left: exterior side of the orchid garden of Charles W. Powell in Balboa, Canal Zone, Panamá. Right: view of the Powell's living collection of over 7000 specimens of Panamanian orchids. Taken from page 376 of the 1924 Annual Report of the Board of Regents of the Smithsonian Institution.

Island by Croat (1978) treated 89 species.

Woodson \& Schery (1943-1981) edited the first formal floristic treatment for the Flora of Panama, based on the accumulated data from herbarium collections. Orchid collections increased from 1930 through the efforts of collectors such as: P.H. Allen, H.P. Butcher, H. A. Dunn, F. H. von Hagen, I. M. Johnston, E.M.
Kieswetter, W.R. Lindsay, R.J. Seibert, O. Shattuck, M.E. Spence Davidson, E. D. Starry, W.R. Taylor, R.H. Woodworth, P.A. Vestal, H. von Wedel, G. and P. White, V. Wolfgang and R.E. Woodson. The orchid contribution for Flora of Panama, prepared by Louis O. Williams and Paul H. Allen (Fig. 14), comprised a total of 469 species (Williams 1940, 1946a, 1946b, Allen 

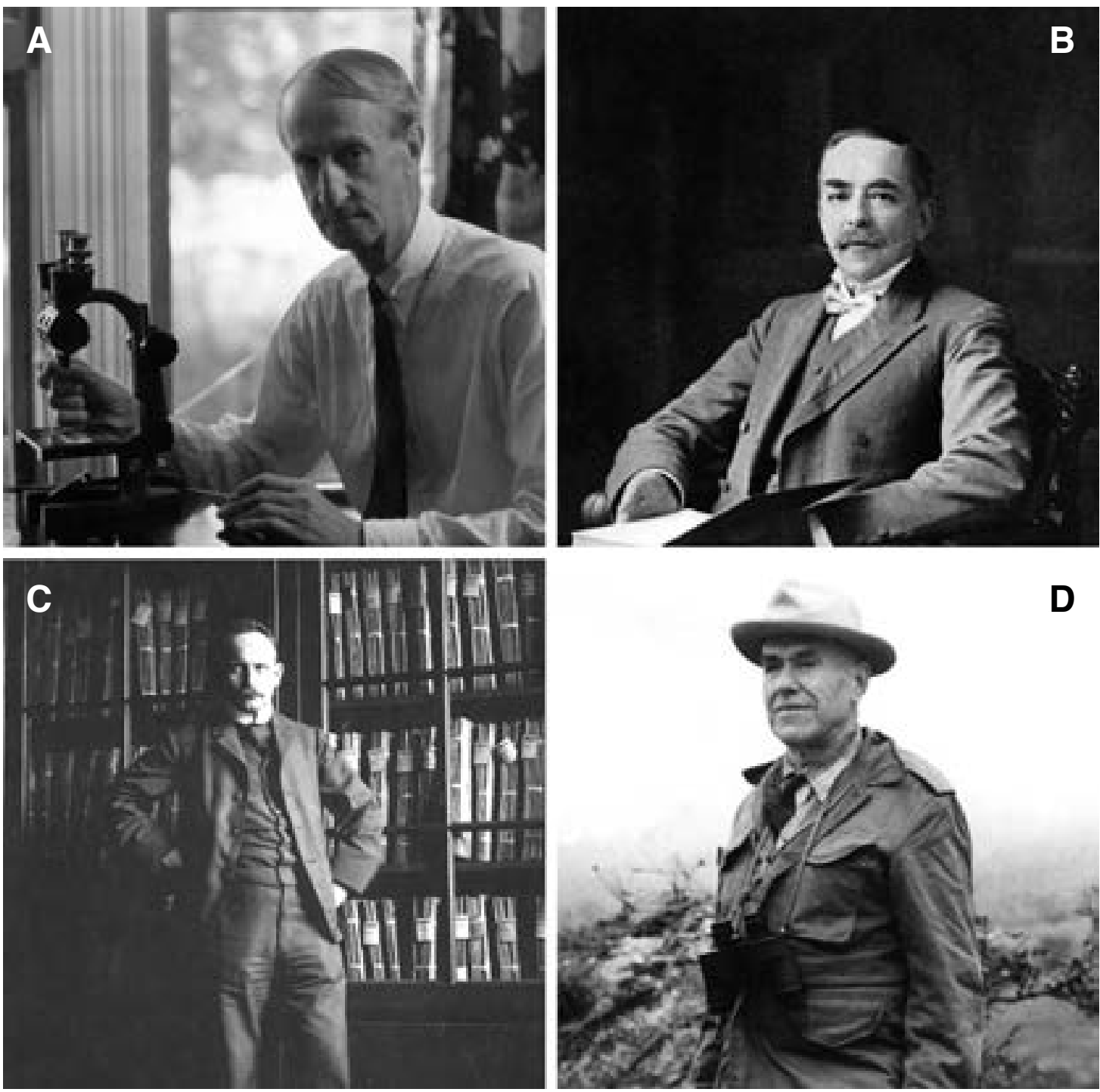

Figure 12. A - Oakes Ames (1874-1950). Courtesy of the Oakes Ames Herbarium, Harvard University. B - Robert Allen Rolfe (1855-1921). Courtesy of the Oakes Ames Herbarium, Harvard University. C - Rudolf Schlechter (1872-1925), Courtesy of Dr. N. Kilian, Archives BGBM Berlin-Dahlem. D — Charles C. Schweinfurth (1890-1970). Courtesy of the Oakes Ames Herbarium, Harvard University.

1949a, 1949b, 1953, Williams \& Allen 1980). A further five species were added to the Flora of Panama in an update of the Orchidaceae of Mesoamerica, by Williams (1956), producing a total of 474 species in 88 genera.

Robert L. Dressler who worked at the Smithsonian Tropical Research Institute in Panama (STRI) was the most important orchid specialist based in Panama (Fig. 15). In over 20 years of work, he managed to add 328 new records to Williams \& Allen (1980) list. D'Arcy
(1987) continued to update the checklist generated during the Flora of Panama project, recording 893 orchid species.

Subsequently, the floristic and taxonomic work of Dressler gave a new impetus to the study of the orchids on the Isthmus. He also encouraged other orchid specialists such as John T. Atwood (Maxillarinae), Eric Hágsater (Epidendrum), Carlyle A. Luer (Pleurothallidinae), Norris H. Williams and Mark Whitten (Oncidiinae, 

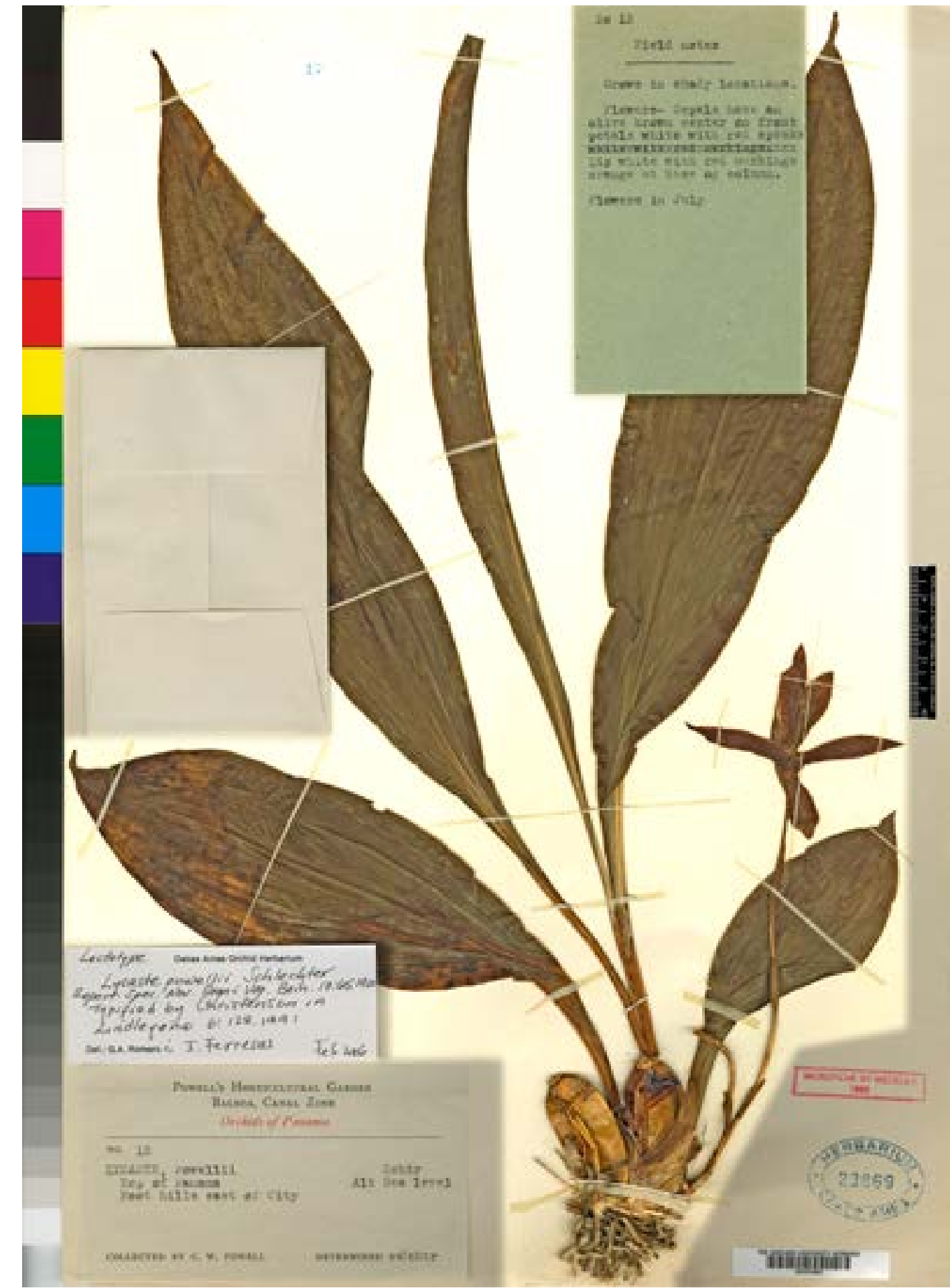

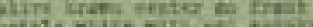

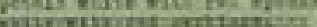

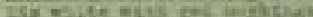

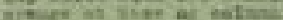

aretre in thit?

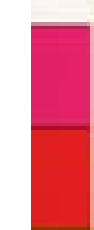

FigURE 13. Lectotype of Lycaste powellii Schltr., collected by C.W. Powell on hills near Panama City. Courtesy of the Oakes Ames Herbarium, Harvard University. 

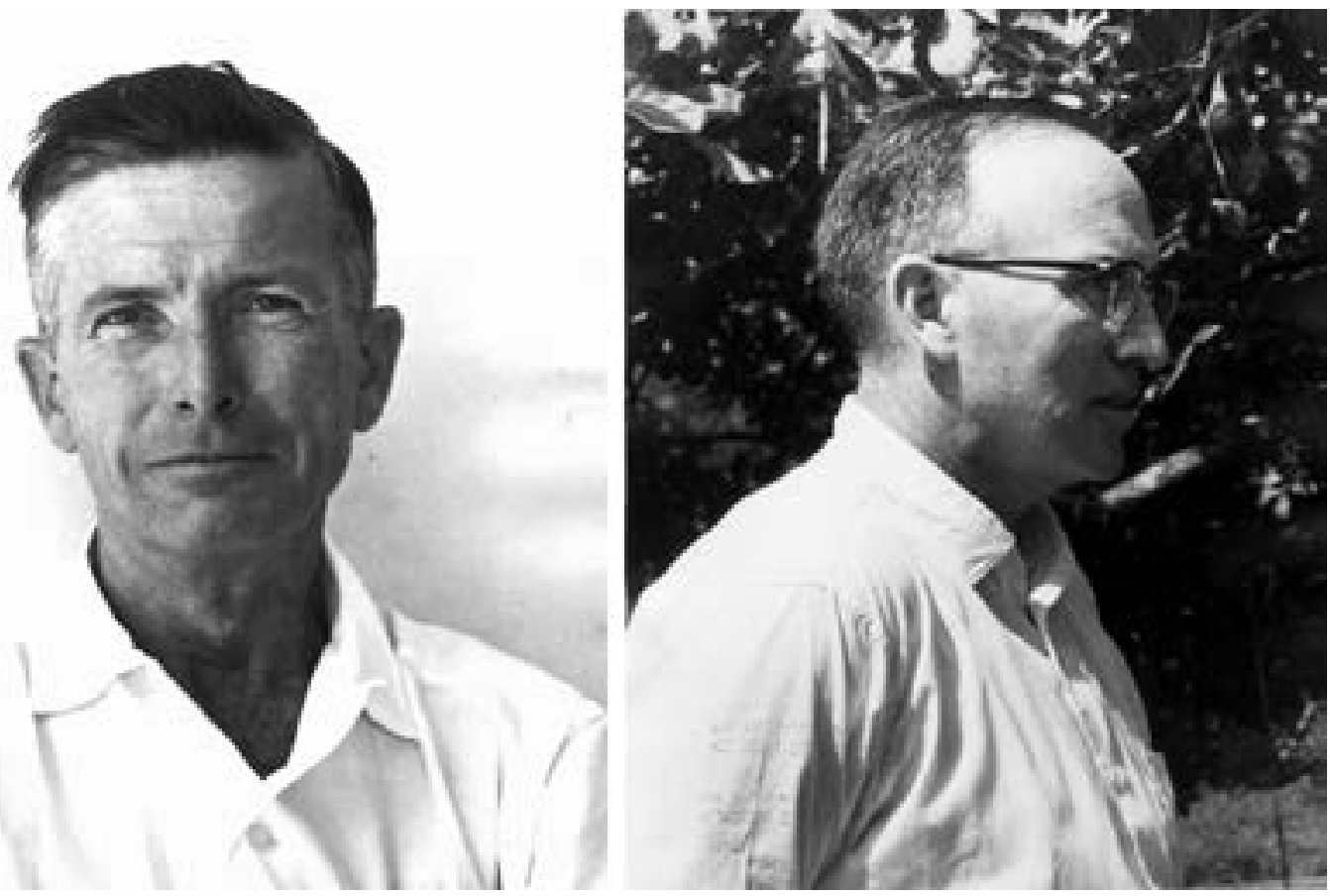

Figure 14. A - Paul H. Allen (111-1963). B — Louis O. Williams (1908-1991). Courtesy of L.D. Gómez.

Stanhopeinae) who all making outstanding floristic contributions (Fig. 16). In 1993, Dressler published his Field Guide to the Orchids of Costa Rica and Panama, where some 950 species of orchids are recorded. In the following years Dressler added many new species (Dressler 1997, 2000, 2002, 2003a, 2003b, 2003d, 2003e, 2004a, 2004b, 2004d, 2005a, 2005b, 2006a, 2006b, 2006c, 2007, Dressler \& Pupulin 2003, Pupulin \& Dressler 2006, Dressler \& Bogarín 2007a, 2007b, 2010). From 1960 other new species and new records for Panama were made by: T. Antonio, W. G. D’Arcy, H. Butcher, D. Cáceres, M.D. Correa, T.B. Croat, R.L. Dressler, J. Duke, J. D. Dwyer, J. Folsom, C. Galdames, A. Gentry, Hágsater, B.E. Hammel, H. Kennedy, L.A. Kenoyer, J. Kirkbride, S. Knapp, W. Lewis, Luer, A. Maduro, G. McPherson, S. Mori, M. Nee, E. Olmos, I. Oviedo, D. Porter, R. Rincón, P.C. Standley, K. Sytsma, Whitten, and Norris Williams among others.

Correa et al. (2004), in the Catalogue of Vascular Plants of Panama, listed 1150 orchid species. Although herbarium vouchers (exsiccata) were not cited in most cases, but there is a reference citation for each species. In this treatment, Orchidaceae was the most diverse family of plants in Panama (12.08\% of the 9520 species listed), surpassing any other angiosperm family, and also the family with most endemic species with an impressive 173 species. Ossenbach et al. (2007) listed a total 1385 species and 267 endemics, adding 235 species and 94 endemic species but no herbarium vouchers were cited.

Species lists are useful to present an overview of the diversity of a specific area, providing basic information on species distribution, biogeography, conservation, standardization and updating of nomenclature that are starting points for more detailed floristic and ecological studies (Funk et al. 2007, Brundu \& Camarda 2013).

The aim of this paper is to present an updated checklist of all orchid species found so far in Panama and supporting herbarium vouchers. The data presented here are analyzed to explain the patterns of geographic distribution of species, most diverse taxa, endemism, and relationships with nearby floras. This checklist is a basis for a detailed and updated floristic treatment "Flora Panamensis: Orchidaceae" currently developed within the framework of the project "Towards a modern orchid flora of Panama" (Bogarín et al. 2013b) by the Jardín Botánico Lankester, Universidad de Costa Rica and the UCH Herbarium of the Universidad Autónoma de Chiriquí, David, Panama. 

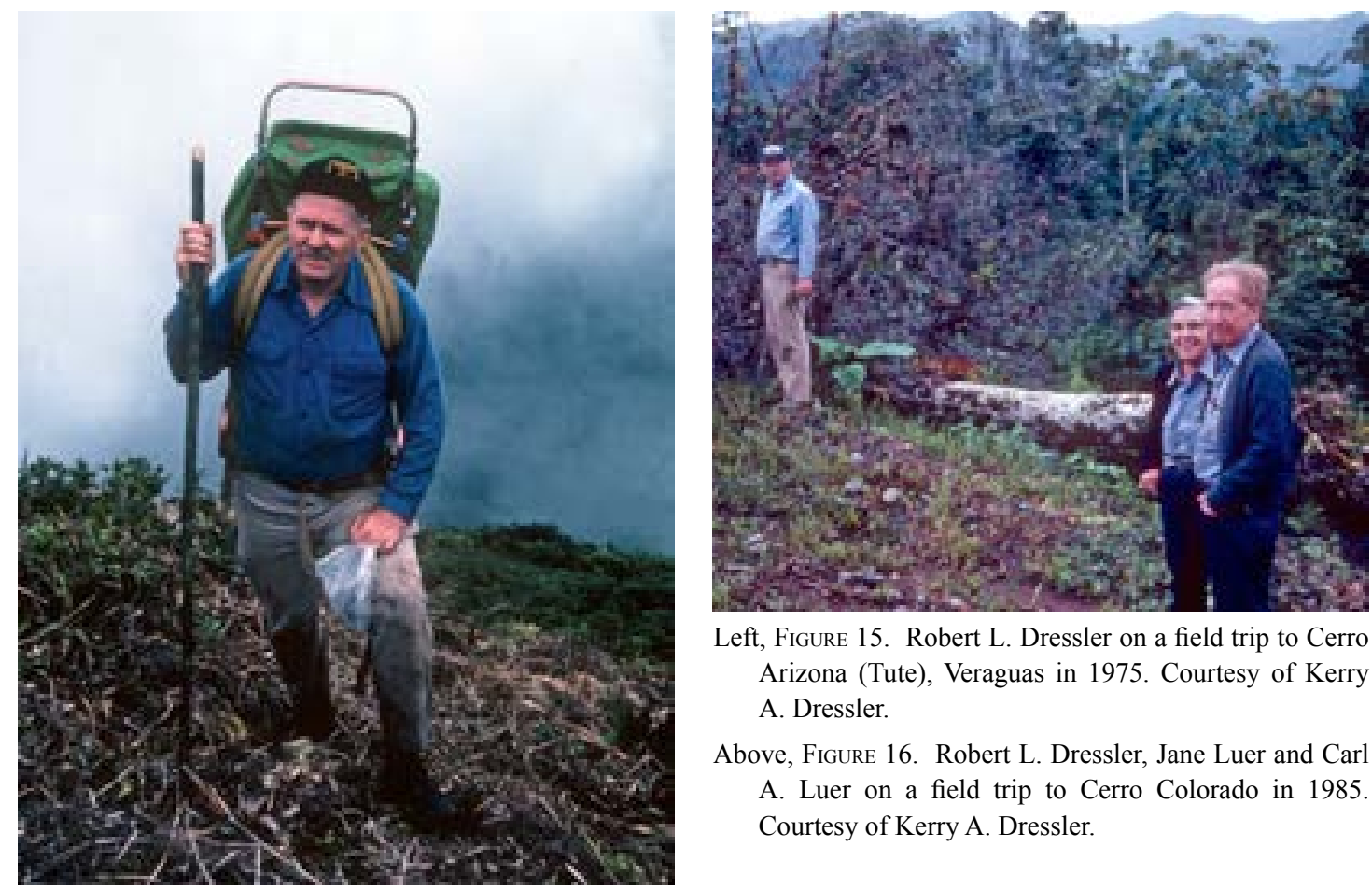

Left, Figure 15. Robert L. Dressler on a field trip to Cerro Arizona (Tute), Veraguas in 1975. Courtesy of Kerry A. Dressler.

Above, Figure 16. Robert L. Dressler, Jane Luer and Carl A. Luer on a field trip to Cerro Colorado in 1985. Courtesy of Kerry A. Dressler.

\section{Materials and methods}

Study area. Geography-. Panama lies in the south-east of Central America $\left(9^{\circ} 38^{\prime} \mathrm{N}-7^{\circ} 12^{\prime} \mathrm{N}, 8^{\circ} 03^{\prime} \mathrm{N}-77^{\circ} 09^{\prime} \mathrm{W}\right)$ and comprises the narrowest and lowest land strip of America separating the Atlantic from the Pacific oceans (Figs. 17, 18, 19). The country is well known because of the Panama Canal, which allows the trade between the two oceans and is placed along the narrowest point of Panama that is less than $50 \mathrm{~km}$ wide. Panama runs from west to east and comprises $75,517 \mathrm{~km}^{2}$ of land and $2,210 \mathrm{~km}^{2}$ of territorial waters. Politically, it is divided in ten provinces: Bocas del Toro, Coclé, Chiriquí, Colón, Darién, Herrera, Los Santos, Panamá Oeste, Panamá, Veraguas and five indigenous "comarcas" or regions: Emberá, Guna Yala, Kuna de Madugandí, Kuna de Wargandí and Ngöbe-Buglé. Panama is usually divided in western, central and eastern Panama. Western Panama is the continuation of the Cordillera de Talamanca, which spans much of Costa Rica and extends to Chiriquí and Veraguas. This mountain range has the highest elevations of the isthmus, usually above $3000 \mathrm{~m}$. No active volcanoes are found in this range. It is mostly protected by the La Amistad International Park. The highest peaks are found there close to the border with Costa Rica: Volcán Barú (3475 m), Cerro Fábrega (3335 m), Itamut (3293 m) and Echandi (3162 $\mathrm{m})$. The Cordillera de Talamanca crosses the provinces of Bocas del Toro, Chiriquí, Veraguas and Comarca Ngöbe-Buglé. It loses elevation towards central Panama in the provinces of Veraguas and Coclé where it is also known as Serranía del Tabasará. Its main slope is: Cerro Santiago $(2127 \mathrm{~m})$. The provinces of Herrera, Los Santos and part of Veraguas are called central provinces and comprise mostly the Península de Azuero. Here the highest point is Cerro Hoya (1559 m), which is isolated from the Cordillera de Talamanca and Tabasará by the dry plains of Veraguas. Colón, Panama and Panama Oeste are in Central Panama, on the narrowest stretch of the country where elevations are relatively low, usually less than $1000 \mathrm{~m}$. The area includes the slopes of Cerro Azul (571 m), Brewester (899 m), Campana (1030 m), Gaital (1185 m), Jefe (1007 m) and Nombre de Dios (587 $\mathrm{m})$. Eastern Panama comprises the provinces of Darién (the easternmost province and adjacent to Colombia) and Panama towards the boundary with Colombia and the comarcas Emberá, Guna Yala, Kuna de Madugandí and Kuna de Wargandí. The Pan-American highway 


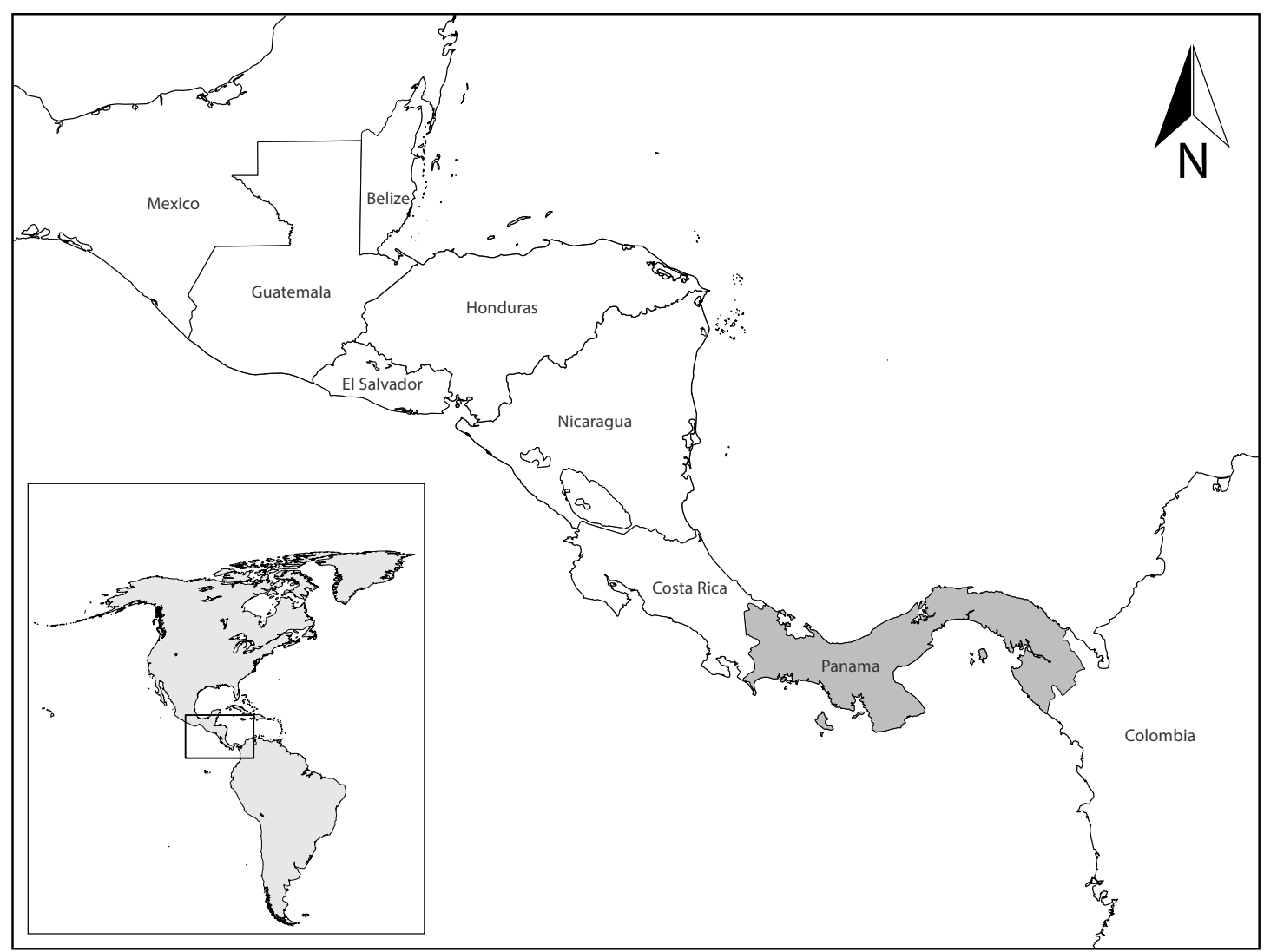

Figure 17. Panama lies in the southeastern part of Central America.

terminates at Yaviza in Darién. There is no connection by road with Colombia after this point. The ridge of Darien which extends along the Atlantic has the highest peaks of eastern Panama: Sasardi (610 m), Bell (1046 m), Chucurtí (1695 m), Gandi (1160 m), Armila (1421 $\mathrm{m})$, Tanela (1421 m), Tacarcuna $(1875 \mathrm{~m})$ and Nique $(1550 \mathrm{~m})$. Serranía de San Blas along the Kuna Yala extends along the coast from central to the southeastern point, with Cerro Habú (747 m), Ebitan (736 m) and Diablo $(518 \mathrm{~m})$, among the highest points. Along the Pacific side, the main ranges are Majé with its highest points Cerro Chucantí $(1430 \mathrm{~m})$ and Pechito Parado (591 m), which is isolated from Serranía del Sapo with Cerro La Piña (1580 m); Jurado with Cocalita (1052 m), Bagre and Pirre with Cerro Pirre (1569 m). This area of Darién is perhaps the richest in plant species but the least known botanically. Panama has over 480 rivers. The most important are Changuinola, Cricamola and Teribe in Bocas del Toro; Caldera, Chiriquí, Chiriquí Viejo and Piedra in Chiriquí; Tabasará in Comarca Ngöbe-Buglé;
Santa María in Coclé and Herrera; Balsas, Chagres, Chucunaque (the longest with $231 \mathrm{~km}$ ) and Tuira in central-eastern Panama. There are three main lakes: Alajuela (also known as Madden), Bayano and Gatún (artificial). The main islands are all off the Pacific coast: Cébaco $\left(80 \mathrm{~km}^{2}\right)$ in Montijo Bay, Coiba $\left(493 \mathrm{~km}^{2}\right)$ and Isla del Rey $\left(234 \mathrm{~km}^{2}\right)$ in the Archipiélago de Las Perlas. Coiba is the largest island in the Pacific of Central America (IGTG 2007).

Geology -. Panama lies on the Costa Rica-Panama microplate, a complex area of interaction of three tectonic plates: Nazca, Cocos and Caribe. Panama emerged as an archipelago of islands about 15 Mya ago in the middle Miocene. However, the complex evolution leading to the formation of the Isthmus started during the Late Cretaceous and ended about 3.5 Mya in the Pliocene, resulting in the complete closing of the isthmus separating the Atlantic and Pacific oceans. The ranges of Costa Rica and Panama together with those of western Colombia are among the youngest in 


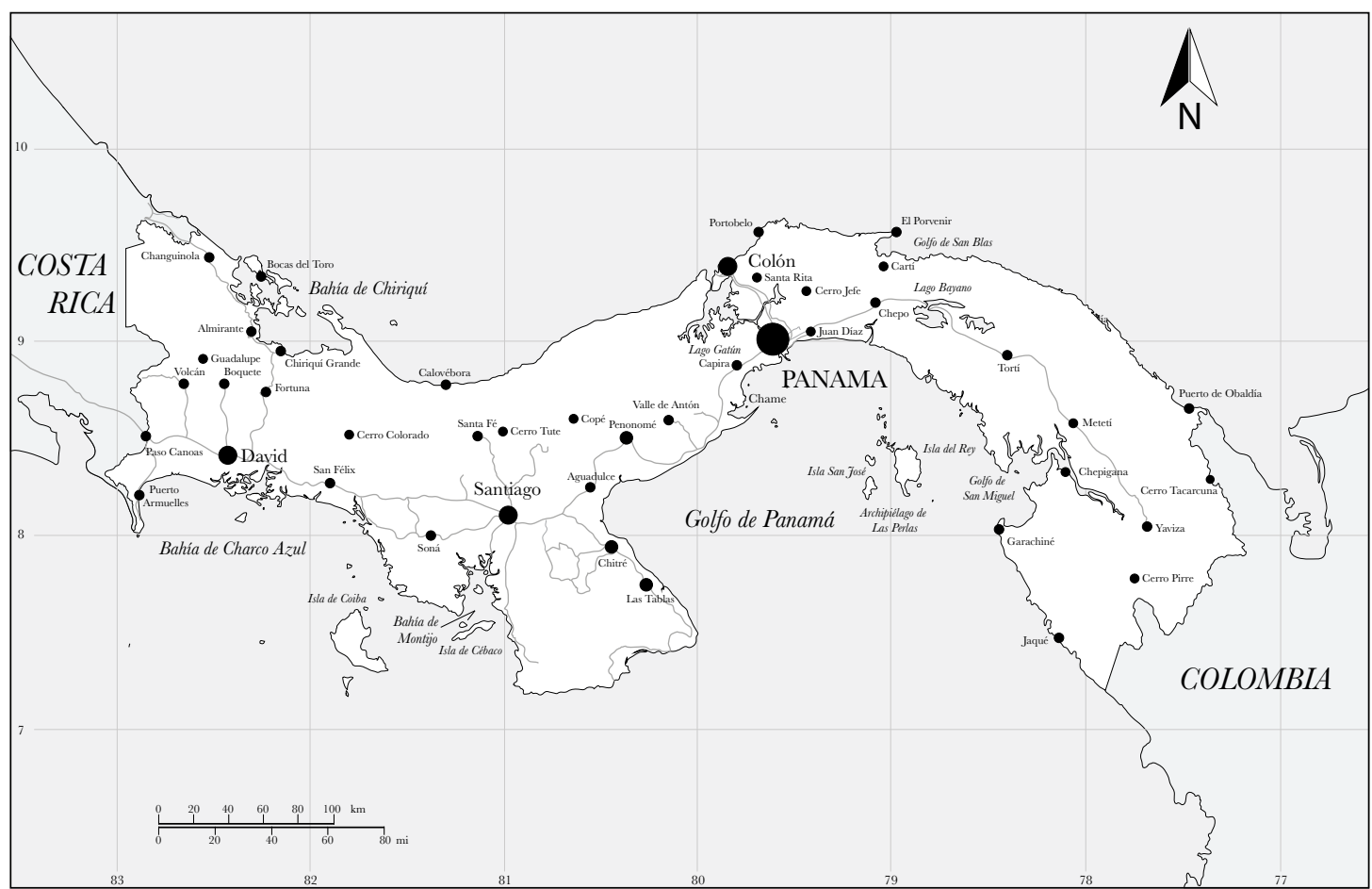

FIGURE 18. Map of Panama showing some of the most important cities and places visited by botanists. Map by D. Bogarín.

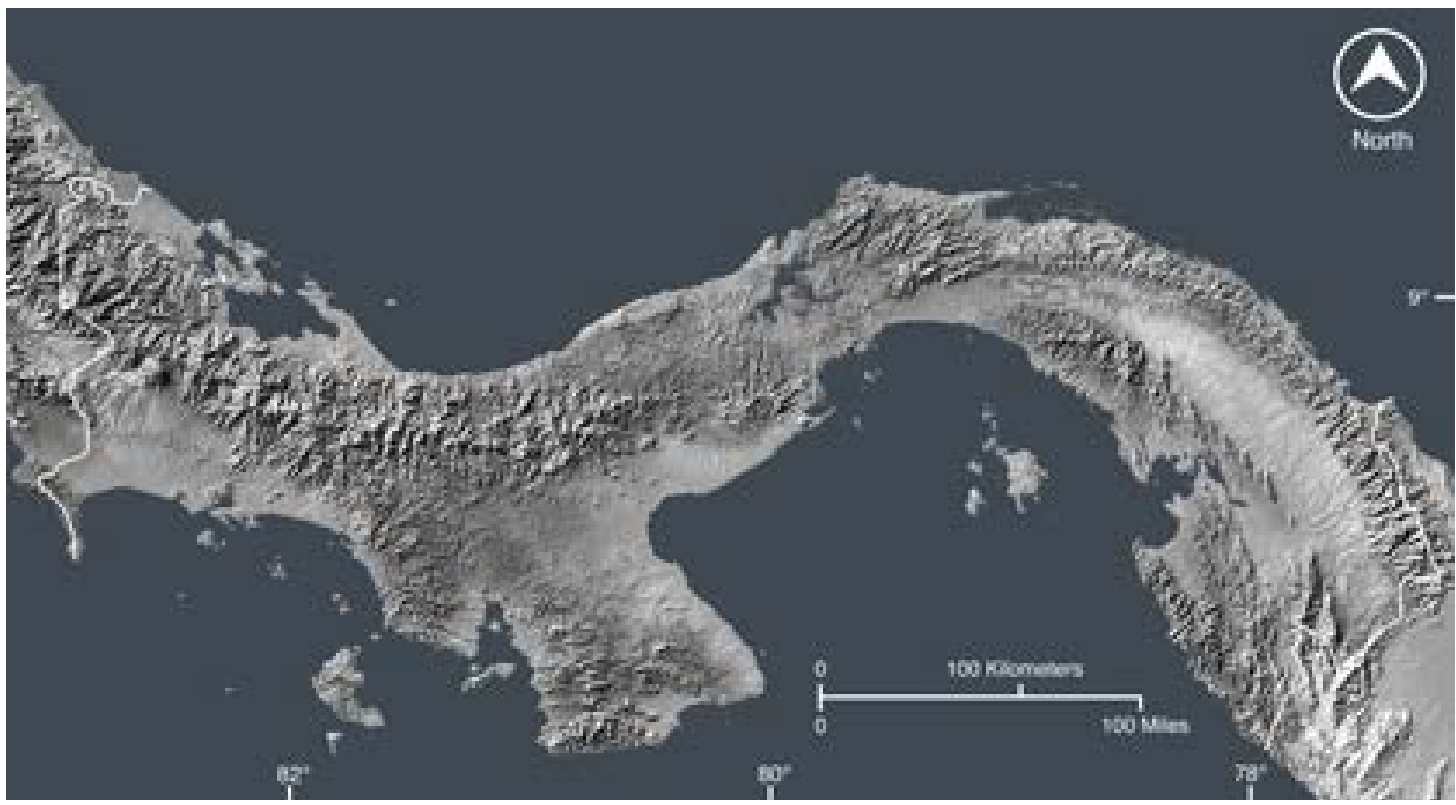

FIgURE 19. Geomorphology of Panama. Modified from false-color image of Panama by the Shuttle Radar Topography Mission, NASA/JPL/NIMA/SRTM Team.

the Neotropics (Kirby 2011). Some exotic lands that were volcanic islands in the ancient archipielago now make up Azuero, Burica and Coiba. These islands were pushed by the Cocos Plate toward the subduction zone with Caribbean Plate along the Pacific coast of Costa Rica and Panama. According to Coates et al. (1992) 
and Coates \& Obando (1996) three major events led the tectonic evolution of the southern Central American isthmus. First, the volcanic activity caused by the convergent tectonics of the eastern Pacific subduction zone, one of the primary forces that created the volcanic arc extending from North America to the south. Another tectonic effect was the subduction of the Cocos Ridge on the Pacific. This submarine range led to the rise of the isthmus from the Arenal Volcano in Costa Rica to nearby Cerro Campana and Gaital at El Valle, Panama. From this region, the Cordillera de Talamanca (which contains the highest points of the isthmus) emerged and reduced its marine connections. The southern region of the isthmus was affected by the collision of the volcanic arc with north-western South America and led to the uplift of eastern Panama (San Blas, and Darién ranges) and northern Andes of Colombia and Venezuela in the late Neogene (Coates et al. 2004).

Climate -. The climate of Panama is affected by its geographical position close to the Equator, the narrow strip of land surrounded by two oceans, the mountain chains with different elevation ranges and the prevailing wet winds of the Caribbean. Usually the Caribbean lowlands are moist and warm. Precipitation originates mostly by the trade winds from the Caribbean colliding with the mountain ranges, primarily on the Cordillera de Talamanca and the action of the Inter-tropical Convergence Zone (Kohlmann et al. 2002, ANAM 2010). This climate is present from Bocas del Toro, to the Caribbean of Veraguas, Coclé and Colón. Average temperature varies from $25-27^{\circ} \mathrm{C}$ and precipitation from $2500-4500 \mathrm{~mm} / \mathrm{y}$. The Cordillera de Talamanca has broad elevation ranges and the wind system is quite complex. The western Pacific slope of Talamanca, and mid-elevation areas of Coclé, Panamá, San Blas and Darién are wet due to the interaction of local winds with the moist air from the Pacific Ocean. Average temperature varies from $26-28^{\circ} \mathrm{C}$ and precipitation from 2500-3500 mm/y. The Peninsula de Azuero is warm and dry with marked seasonality and an extended dry season. Average temperature varies from $27-28^{\circ} \mathrm{C}$ and precipitation is less than $2500 \mathrm{~mm} / \mathrm{y}$. In central Panama, there are low hills such as Campana and Gaital and Hoya in Azuero, where montane forests predominate from the interaction of the wet winds. In eastern Panama the climate is warm and rainy, (similar to Chocó in
Colombia), and is one of the wettest regions on Earth (Dressler 1993). In general, there are two seasons in Panama: the dry season extending from the late of November to March-April and the wet season from May to November. The wet and dry seasons are better defined on the Pacific slope, while along the Caribbean slope, rains are seasonally variable, with no pronounced dry season (ANAM 2010).

Vegetation - The flora of Panama is greatly influenced by the biotic exchange between North and South America, resulting in one of the richest countries in terms of plant diversity in the Neotropics. About 3 Mya ago, the vegetation of Panama consisted of open savannas. Some elements of the Laurasian flora colonized the Isthmus: Alfaroa Standl., Alnus Hill., Berberis L., Billia Peyr., Caryocar L., Castilleja Mutis ex L. f., Magnolia Juss., Papaver L., Prunus L., Quercus L., Rubus L., Saurauia Willd., Ulmus L. and Vaccinium L. At the same time, other southern groups moved northward including, Drymis Juss., Gunnera L. and Weinmannia L. Panama has 12 life zones (ANAM 2010). The most complete account of the flora of Panama by Correa et al. (2004) recognizes about $10,444(91.2 \%)$ species of vascular plants and some 924 (8.8\%) species are nonvascular. About $80 \%$ of the vascular plants are angiosperms. Of all angiosperms, $29.77 \%$ are monocotyledons and $60.15 \%$ are dicotyledons. Ferns comprise $9.85 \%$ but gymnosperms are poorly represented with less than $0.23 \%$ of species. The largest families in terms of species number in relation to the total vascular species in Panama are: Orchidaceae (13\%), Rubiaceae (5.2\%), Fabaceae (5.1\%), Poaceae (4.4\%), Araceae $(3.6 \%)$, Melastomataceae (3.2\%) and Asteraceae (3.1\%). The families with most epiphytic species are Orchidaceae, Araceae, Bromeliaceae (1.9\%) and Gesneriaceae (1.8\%). Families containing greatest endemism are the Orchidaceae (23.6\%), Rubiaceae (8.9\%), Araceae (8.6\%), Myrsinaceae (6.1\%) and Melastomataceae (4.9\%). According to the Vegetation Map of Panama (ANAM 2000) there are 24 vegetation types. An estimated of $46 \%$ of the territory is covered by forest, where the tropical ombrophile evergreen, latifoliated lowland forest occupies the largest area (24.48\%). Regarding the state of conservation of the flora, ANAM (2000) recorded 1,305 endemic 
species, 3,615 vulnerable, 1,041 endangered and 37 critically endangered. The mountains of Darién, Cerro Tacarcuna, the Cordillera de Talamanca, Cerro Azul and Cerro Jefe are considered the main centers of endemism (Correa et al. 2004).

Methods. This checklist was completed from studying specimens strictly collected in Panama and deposited in different herbaria mainly: AMES, CR, F, JBL, K, MO, NY, PMA, SEL, UCH, US and USJ. We included vouchers cited in: several taxonomic treatments (Williams \& Allen 1980, Hágsater \& Salazar 1993, Hágsater 1999, 2001, 2010, 2013, Dressler 2003c, Hágsater \& Sánchez 2004, 2006, 2007, 2008, 2009, Hills 2012, Luer 1986, 1987, 1997a, 1997b, 1998a, 1998b, 1999, 2001a, 2001b, 2004, 2005, 2006, 2007, 2009, 2010, 2011, Ormerod 2004, 2005, 2007, 2008, 2009, 2013, Szlachetko et al. 2005, Pupulin 2010, among others), vouchers directly studied in herbaria, other historical collections and specimens collected by the authors in the field.

For the species described on the basis of material collected in Panama preference was given to the type collections as vouchers and the information was obtained directly from protologues (see Literature cited). The main taxonomic and floristic works studied are: Williams (1946a, 1946b), Allen (1949a, 1949b), Williams \& Allen (1980) D’Arcy (1987) and Correa et al. (2004). Ossenbach et al. (2007) was used as reference, though the absence of herbarium vouchers did not allow us to confirm or compare some taxonomic concepts adopted there. Therefore, these examples were not discussed in the excluded species section (see format of the checklist). Some other cases of questionable or misapplied species for the flora of Panama in D'Arcy (1987) and Dressler (1993) were clarified by Correa et al. (2004) (see excluded species). Specimen citations, synonyms and information localities cited in the literature were also obtained from the following online electronic databases: EPIDENDRA (The Botanical Databases by Lankester Botanical Garden, http://www.epidendra.org), IPNI (International Plants name Index, http://www.ipni. org) WCSP (World Checklist of Selected Plant Families: Royal Botanic Gardens, Kew, http://apps. kew.org/wcsp/home.do) and TROPICOS (Missouri Botanical Garden Databases, http://www.tropicos. org). Information on publications and protologues was obtained from EPIDENDRA, BHL (Biodiversity Heritage Library, http://www.biodiversitylibrary. org) and the Library of Royal Botanic Gardens, Kew, Lankester Botanical Garden and Herbarium UCH. We added new records collected and documented between January 2012 and August 2014 as part of the activities and preliminary results of the project "Towards a modern orchid flora of Panama" (see introduction). These new records were documented by photographs and by drawings using the techniques described by Pupulin et al. (2012) and Bogarín et al. (2014). Georeferencing for specimens were obtained using a Garmin eTrex Vista GPS and Google Earth 6.1.0 (C) and herbarium vouchers were deposited at JBL, UCH and PMA herbaria.

Format of the checklist. Species are presented as an alphabetical list of accepted names with their respective homotypic and heterotypic synonyms and herbarium vouchers. Accepted names are indicated in bold and italics. A herbarium voucher for each accepted name is given. Homotypic and heterotypic synonyms are listed in italics but without bold face and are arranged chronologically. Some species are known to occur in Panama or have been cited in formal taxonomic treatments elsewhere but we have not been able to locate a voucher. The voucher is cited as "not seen" and we refer to the taxonomic work that cited it. In some cases we added a short note warning of a particular case such as species with disjunct distributions with a voucher from a cultivated plant or where we suspect a taxonomic problem.

Several names have been misapplied to the flora of Panama or they have changed since previous taxonomic works. For example species currently accepted under a broad concept that have been redefined or clarified, infraspecific taxa that are now accepted in the range of species or some errors or omissions. To clarify these records, we present a list of excluded species (Appendix 3), in which we discuss the exclusion of doubtful species or the taxonomic clarification of certain concepts that no longer apply to the Panamanian material previously cited in the literature. The endemic species are listed separately. We included only the accepted names for the endemic species list. 
A basionym list is presented with the respective accepted name in bold. This list is useful as reference for the species whose type locality is within Panama and for future taxonomic and nomenclatural treatments. Locality data and citations for this list were obtained directly from protologues (see Literature cited).

Nomenclatural considerations. In all cases where it is intended to make a list of the orchid species for a particular region, there are always issues regarding to the classification system followed, mainly from the generic viewpoint. The nomenclature of Orchidaceae has undergone many changes after the publication of several phylogenetic studies (Chase \& Palmer 1989, 1992, van den Berg et al. 2000, Cameron et al. 1999, Cameron 2001, Pridgeon et al. 2001a, Chase et al. 2003, Salazar et al. 2003, Whitten et al. 2005 and Williams et al. 2005, among others). This list relies largely on the generic concepts proposed in Genera Orchidacearum (Pridgeon et al. 1999, 2001a, 2001b, 2003, 2005a, 2005b, 2005c). In a few cases we adopted redefined concepts proposed after Genera Orchidacearum (Chase \& Whitten 2011, Karremans 2014).
To facilitate the understanding of nomenclature issues, we present three tables summarizing the main nomenclatural changes that affected the orchid flora of Panama. Table 1 provides a guide to the genera that have been lumped into other genera and accepted for this work. Table 2 presents the genera that have been segregated into various other genera. As a guide for the reader, Table 3 presents the general classification followed in this checklist with the genera and their respective number of species accepted for Panama including the total of endemic species. The list also provides an overview of the genera that are represented in the country.

This catalog is not intended to discuss the different generic classifications but to serve as a study guide for future taxonomic and floristic works in Panama. Clearly, there are different trends in orchid classification and acceptance of generic boundaries varies from one author to another. Nevertheless, citation of homotypic synonyms will help the reader to determine which names have been proposed by different studies and to easily find the latest changes affecting Panamanian species even if the reader is not familiar with those changes.

\section{Results and discussion}

This checklist contains 1,365 taxa (1,360 species, two natural hybrids and three subspecies) in four subfamilies, 16 tribes, 27 subtribes, 187 genera (Figs. 20-26, Table 3). Since the last checklist published in the Catalogue of Vascular Plants of Panama by Correa et al. (2004) this is an increase of 210 species published since the last decade. We were able to add 32 records in 17 genera that have not been mentioned so far in the literature. With regard to Ossenbach et al. (2007) there is a reduction of 25 species although it was not possible to corroborate the voucher specimens in that treatment. Though we do not yet know the exact number of orchid species that exist in Panama, the country has one of the richest floras of Mesoamerica. The orchid diversity index (calculated as the number of species $/ \mathrm{km}^{2}$ ) shows 0.018 $\mathrm{spp} . / \mathrm{km}^{2}$. The number of species and diversity index are among the highest in the Mesoamerican region together with Costa Rica and southeastern Mexico (Ossenbach et al. 2007). The area between Costa Rica and Panama treated as Lower Central America (LCA) is widely recognized for its high number of endemic species, and the number for Orchidaceae is not an exception
(Myers et al. 2000). Now that we have more accurate numbers, both countries contain a total of 2,030 species of orchids (between $6.5-8 \%$ of the global biodiversity of the family) in $130,000 \mathrm{~km}^{2}$ (about $0.09 \%$ of Earth's land area). Some factors explaining this extraordinary diversity are: 1) a natural land bridge uniting three of the 25 recognized hotspots world-wide (Mesoamerica, Choco/Darién/Western Ecuador and Tropical Andes) that.are centers of diversity of many groups of orchids 2) the climatic influence of the Pacific and Atlantic oceans. 3) the orographic and tectonic factors such as the lifting of the Cordillera de Talamanca between Costa Rica and Panama and the formation of foothills of Majé, Darién and San Blas from Panama and western Colombia.

Epidendroideae, with more than $90 \%$ of species and over $85 \%$ of the genera, is the most diverse group in Panama, significantly outnumbering the other three recorded subfamilies. Orchidoideae contains less than $13 \%$ and Vanilloideae and Cypripedioideae less than $1 \%$ of the species (Table 4). The most diverse tribes of the 16 recorded are Epidendreae, Cymbidieae, Cranichideae and Sobralieae. These groups make up $25 \%$ of tribes, 
TABLE 1. List of orchid genera which have been lumped into other genera by recent authors affecting the nomenclature of the orchids of Panama.

\begin{tabular}{|c|c|c|}
\hline Genus & Reduced under & Reference \\
\hline Acostaea Schltr. & Specklinia Lindl. & Pridgeon \& Chase (2001) \\
\hline Ada Lindl. & Brassia R.Br. & Chase \& Whitten (2011) \\
\hline Amparoa Schltr. & Rhynchostele Rchb.f. & Hágsater \& Soto (2003) \\
\hline Areldia Luer & Specklinia & This paper \\
\hline Chelyorchis Dressler \& N.H.Williams & Rossioglossum (Schltr.) Garay \& G.C.Kenn. & Chase et al. (2008) \\
\hline Cohniella Pfitzer & Trichocentrum Poepp. \& Endl. & Williams et al. (2001) \\
\hline Cucumeria Luer & Specklinia & This paper \\
\hline Goniochilus M.W.Chase & Leochilus Knowles \& Westc. & Chase et al. (2008) \\
\hline Hexisea Lindl. & Scaphyglottis Poepp. \& Endl. & Dressler et al. (2004a) \\
\hline Hybochilus Schltr. & Leochilus & Chase et al. (2008) \\
\hline Leucohyle Klotzsch & Trichopilia Lindl. & Dressler (2004c) \\
\hline Lophiaris Raf. & Trichocentrum & Williams et al. (2005) \\
\hline Mesospinidium Rchb.f. & Brassia & Chase \& Whitten (2011) \\
\hline Oerstedella Rchb.f. & Epidendrum $\mathrm{L}$. & Hágsater \& Soto (2005) \\
\hline Osmoglossum (Schltr.) Schltr. & Cuitlauzina La Llave \& Lex. & Dressler \& Williams (2003) \\
\hline Pachystele Schltr. & Scaphyglottis & Dressler et al. (2004a) \\
\hline Platyglottis L.O. Williams & Scaphyglottis & Dressler et al. (2004a) \\
\hline Pachyphyllum Kunth & Fernandezia Ruiz \& Pav. & Chase \& Whitten (2011) \\
\hline Pleurothallis R.Br. & Stelis Sw. & Pridgeon \& Chase (2001) \\
\hline Psygmorchis Dodson \& Dressler & Erycina Lindl. & Williams et al. (2001) \\
\hline Restrepiopsis Luer & Pleurothallopsis Porto \& Brade & Pridgeon \& Chase (2001) \\
\hline Salpistele Dressler & Stelis & Pridgeon \& Chase (2001) \\
\hline Scelochilus Klotzsch & Comparettia Poepp. \& Endl. & Chase et al. (2008) \\
\hline Sigmatostalix Rchb.f. & Oncidium Sw. & Chase et al. (2008) \\
\hline Stellilabium Schltr. & Telipogon Kunth & Williams et al. (2005) \\
\hline Ticoglossum R.L.Rodr. ex Halb. & Rossioglossum & Chase et al. (2008) \\
\hline
\end{tabular}

TABLE 2. List of orchid genera segregated by recent authors affecting the nomenclature of the orchids of Panama.

\begin{tabular}{l|l|l}
\hline Genus & Segregate Genera & Reference \\
\hline Chondrorhyncha Lindl. & $\begin{array}{l}\text { Benzingia Dodson } \\
\text { Daiotyla Dressler } \\
\text { Stenotyla Dressler }\end{array}$ & $\begin{array}{l}\text { Romero-González \& Dodson (2010) } \\
\text { Whitten et al. (2005) } \\
\text { Whitten et al. (2005) }\end{array}$ \\
\hline Elleanthus C.Presl & $\begin{array}{l}\text { Adeneleuterophora Barb. Rodr. } \\
\text { Evelyna Poepp. \& Endl. }\end{array}$ & $\begin{array}{l}\text { Dudek \& Szlachetko (2010) } \\
\text { Dudek \& Szlachetko (2010) }\end{array}$ \\
\hline Eltroplectris Raf. & Callistanthos Szlach. & Szlachetko \& Rutkowski (2008) \\
\hline Erythrodes Blume & Coilostylis Raf. & Whitner \& Harding (2004) \\
\hline Aspidogyne Garay & Ormerod (2007), Ormerod (2009) \\
& Kreodanthus Garay & Ormerod (2008)
\end{tabular}


TABle 2. Continues.

\begin{tabular}{|c|c|c|}
\hline Genus & Segregate Genera & Reference \\
\hline Erythrodes Blume & $\begin{array}{l}\text { Microchilus C.Presl } \\
\text { Platythelys Garay }\end{array}$ & $\begin{array}{l}\text { Ormerod (2002) } \\
\text { Ormerod (2007) }\end{array}$ \\
\hline Habenaria Willd. & $\begin{array}{l}\text { Bertauxia Szlach } \\
\text { Habenella Small } \\
\text { Platantheroides Szlach. }\end{array}$ & $\begin{array}{l}\text { Szlachetko (2004a) } \\
\text { Szlachetko \& Kras (2006) } \\
\text { Szlachetko (2004b) }\end{array}$ \\
\hline Kefersteinia Rchb.f. & Senghasia Szlach. & Szlachetko (2003) \\
\hline Ligeophila Garay & Aspidogyne Garay & Ormerod (2007) Meneguzzo (2012) \\
\hline Lycaste Lindl. & Selbyana Archila & Archila (2010) \\
\hline Malaxis Sol. ex Sw. & Microstylis (Nutt.) Eaton & Szlachetko \& Margońska (2006) \\
\hline Masdevallia Ruiz \& Pav. & $\begin{array}{l}\text { Acinopetala Luer } \\
\text { Alaticaulia Luer } \\
\text { Buccella Luer } \\
\text { Diodonopsis Pridgeon \& M.W.Chase } \\
\text { Fissia (Luer) Luer } \\
\text { Reichantha Luer } \\
\text { Spilotantha Luer } \\
\text { Zahleria Luer }\end{array}$ & $\begin{array}{l}\text { Luer (2006) } \\
\text { Luer (2006) } \\
\text { Luer (2006) } \\
\text { Pridgeon \& Chase (2001) } \\
\text { Luer (2006) } \\
\text { Luer (2006) } \\
\text { Luer (2006) } \\
\text { Luer (2006) }\end{array}$ \\
\hline Maxillaria Ruiz \& Pav. & $\begin{array}{l}\text { Adamanthus Szlach. } \\
\text { Calawaya Szlach. \& Sitko } \\
\text { Camaridium Lindl. } \\
\text { Chaseopsis Szlach. \& Sitko } \\
\text { Chelyella Szlach. \& Sitko } \\
\text { Christensonella Szlach., Mytnik, Górniak \& Smiszek } \\
\text { Heterotaxis Lindl. } \\
\text { Inti M.A.Blanco } \\
\text { Laricorchis Szlach. } \\
\text { Mapinguari Carnevali \& R.B.Singer } \\
\text { Maxillariella M.A.Blanco \& Carnevali } \\
\text { Mormolyca Fenzl } \\
\text { Nitidobulbon Ojeda, Carnevali \& G.A.Romero } \\
\text { Ornithidium Salisb. ex R. Br. } \\
\text { Pseudocymbidium Szlach. \& Sitko } \\
\text { Pseudomaxillaria Hoehne } \\
\text { Psittacoglossum La Llave \& Lex. } \\
\text { Rhetinantha M.A.Blanco } \\
\text { Sauvetrea Szlach. } \\
\text { Viracocha Szlach. \& Sitko } \\
\text { Xanthoxerampellia Szlach. \& Sitko }\end{array}$ & $\begin{array}{l}\text { Szlachetko \& Śmiszek 2006[2007] } \\
\text { Szlachetko \& Sitko (2012) } \\
\text { Blanco et al. (2007) } \\
\text { Szlachetko \& Sitko (2012) } \\
\text { Szlachetko \& Sitko (2012) } \\
\text { Szlachetko, Mytnik, Górniak \& } \\
\text { Ojeda, Carnevali \& Romero (2005) } \\
\text { Blanco et al. (2007) } \\
\text { Szlachetko \& Sitko (2012) } \\
\text { Blanco et al. (2007) } \\
\text { Blanco et al. (2007) } \\
\text { Blanco et al. (2007) } \\
\text { Ojeda, Carnevali \& Romero (2009) } \\
\text { Blanco et al. (2007) } \\
\text { Szlachetko \& Sitko (2012) } \\
\text { Szlachetko \& Sitko (2012) } \\
\text { Szlachetko \& Sitko (2012) } \\
\text { Blanco et al. (2007) } \\
\text { Blanco et al. (2007) } \\
\text { Szlachetko \& Sitko (2012) } \\
\text { Szlachetko \& Sitko (2012) }\end{array}$ \\
\hline Oncidium Sw. & Brevilongium Christenson & Christenson (2006b) \\
\hline
\end{tabular}


TABLE 2. Continues.

\begin{tabular}{|c|c|c|}
\hline Genus & Segregate Genera & Reference \\
\hline Oncidium Sw. & $\begin{array}{l}\text { Chelyorchis Dressler \& N.H.Williams } \\
\text { Heteranthocidium Szlach., Mytnik \& Romowicz } \\
\text { Otoglossum (Schltr.) Garay \& Dunst. } \\
\text { Rossioglossum (Schltr.) Garay \& G.C.Kenn. } \\
\text { Stacyella Szlach. } \\
\text { Trichocentrum Poepp. \& Endl. } \\
\text { Vitekorchis Romowicz \& Szlach. }\end{array}$ & $\begin{array}{l}\text { Dressler \& Williams (2000), Carnevali et al. } \\
\text { Szlachetko, Mytnik \& Romowicz (2006) } \\
\text { Williams et al. (2001) } \\
\text { Chase et al. (2008) } \\
\text { Szlachetko (2006) } \\
\text { Williams et al. (2001) } \\
\text { Romowicz \& Szlachetko (2006) }\end{array}$ \\
\hline Pleurothallis R.Br. & $\begin{array}{l}\text { Aberrantia Luer } \\
\text { Acronia C.Presl } \\
\text { Acianthera Scheidw. } \\
\text { Anathallis Barb.Rod. } \\
\text { Ancipitia (Luer) Luer } \\
\text { Apoda-prorepentia (Luer) Luer } \\
\text { Areldia Luer } \\
\text { Crocodeilanthe Rchb.f. \& Warsz. } \\
\text { Cucumeria Luer } \\
\text { Didactylus Luer } \\
\text { Dracontia (Luer) Luer } \\
\text { Echinella Pridgeon \& M.W.Chase } \\
\text { Echinosepala Pridgeon \& M.W.Chase } \\
\text { Effusiella Luer } \\
\text { Elongatia (Luer) Luer } \\
\text { Empusella (Luer) Luer } \\
\text { Gerardoa Luer } \\
\text { Kraenzlinella Kuntze } \\
\text { Lankesteriana Karremans } \\
\text { Lalexia Luer } \\
\text { Loddigesia Luer } \\
\text { Lomax Luer } \\
\text { Muscarella Luer } \\
\text { Niphantha Luer } \\
\text { Pabstiella Brieger \& Senghas } \\
\text { Panmorphia Luer } \\
\text { Phloeophila Hoehne \& Schltr. } \\
\text { Rhynchopera Klotzsch } \\
\text { Ronaldella Luer } \\
\text { Sarcinula Luer } \\
\text { Specklinia Lindl. } \\
\text { Stelis Sw. }\end{array}$ & $\begin{array}{l}\text { Luer (2005) } \\
\text { Luer (2005) } \\
\text { Pridgeon \& Chase (2001) } \\
\text { Pridgeon \& Chase (2001), Hágsater \& Soto } \\
\text { Luer (2004) } \\
\text { Luer (2004) } \\
\text { Luer (2004) } \\
\text { Luer (2004) } \\
\text { Luer (2004) } \\
\text { Luer (2005) } \\
\text { Luer (2004) } \\
\text { Pridgeon \& Chase (2001) } \\
\text { Pridgeon \& Chase (2002) } \\
\text { Luer (2007) } \\
\text { Luer (2004) } \\
\text { Luer (2004) } \\
\text { Luer (2006) } \\
\text { Luer (2004), Hágsater \& Soto (2003) } \\
\text { Karremans (2014) } \\
\text { Luer (2011) } \\
\text { Luer (2006) } \\
\text { Luer (2006) } \\
\text { Luer (2006) } \\
\text { Luer (2010) } \\
\text { Luer (2007) } \\
\text { Luer (2006) } \\
\text { Pridgeon \& Chase (2001), Luer (2006) } \\
\text { Szlachetko \& Margońska (2001) } \\
\text { Luer (2006) } \\
\text { Luer (2006) } \\
\text { Pridgeon \& Chase (2001), Hágsater \& Soto } \\
\text { Pridgeon \& Chase (2001) }\end{array}$ \\
\hline
\end{tabular}


TABle 2. Continues.

\begin{tabular}{l|l|l}
\hline Genus & Segregate Genera & Reference \\
\hline Pleurothallis R.Br. & Tribulago Luer & Luer 2004, Luer (2006) \\
& $\begin{array}{l}\text { Sylphia Luer } \\
\text { Unciferia (Luer) Luer } \\
\text { Unguella (Luer) Luer }\end{array}$ & Luer (2004) \\
& Luer (2005) & Szlachetko \& Margońska (2001), Szlachetko \& \\
& Kulak (2006) \\
\hline Prosthechea Knowles \& Westc. & Anacheilium Rchb.f. ex Hoffmanns. & Withner \& Harding (2004) \\
& Hormidium (Lindl.) Heynh. & Withner \& Harding (2004) \\
& Panarica Withner \& P. A. Harding & Withner \& Harding (2004) \\
& Pollardia Withner \& P. A. Harding & Withner \& Harding (2004) \\
& Pseudencyclia Chiron \& V.P. Castro & Chiron \& Castro-Neto (2003) \\
\hline Stanhopea Frost ex Hook. & Stanhopeastrum Rchb.f & Szlachetko (2007) \\
\hline Trichosalpinx Luer & Tubella (Luer) Archila & Archila (2000) \\
\hline
\end{tabular}

$87.7 \%$ of the genera and more than $90 \%$ of the species. Ten tribes (62.5\%) contain less than three genera and ten species (Table 5). These groups also encompass the most diverse subtribes. Of the 27 recorded, Pleurothallidinae has 30 genera and $405 \mathrm{spp}$. (by far the most diverse), Laeliinae 16 genera and 292 spp., Oncidiinae 29 genera and 157 spp. and Maxillariinae with 18 genera and 132 spp. are the most numerous in terms of species richness and endemism. All these subtribes together surpass 100 species and account for $50 \%$ of the genera and $72 \%$ of species. Seven subtribes (26\%) have more than ten species while ten $(37 \%)$ contain less than ten species. A comparison among the most diverse subtribes is shown in Table 6. Recent estimates of the age of the Orchidaceae based on molecular clock calculations by Ramírez et al. (2007) and subsequent recalibration by Gustafsson et al. (2010) and Guo et al. (2012) show that the common ancestor of Orchidaceae existed in the Late Cretaceous 80-90 Mya. From this perspective, current subfamilies diverged before the mass extinction of the Cretaceous/ Paleogene (K-Pg). Most notably, after this period, Epidendroideae and also Orchidoideae diversified in the numbers that we know today (Ackerman 2014). The diversification of the Pleurothallidinae, Laeliinae, Oncidiinae and Maxillariinae, the largest subtribes within Epidendroideae, may have occurred about 15-20 Mya in the Early Miocene. This diversification coincided with geological events that shaped the tropical Andes and the Isthmus of Panama and were perhaps key geological events in the evolutionary history of these groups. In fact, they are the two best represented subfamilies in the tropical Andes and Costa Rica-Panama. The uplift of the Cordillera de Talamanca, resulting in the final closure of the isthmus about 3.5 Mya in the Pliocene as well as the abrupt appearance of the Andes between 6-10 Mya created new environments, isolation of regions and local climate changes (appearance of cold and humid regions, where the highest number of species and endemism are found) thus promoting rapid diversification by allopatric speciation. Further work is required to study the biological processes that could boost radiations of diverse groups (see discussion of endemic species). Other groups did not diversify and contain few species in the region. For example, Cypripedioideae, has only three representatives on the isthmus. Selenipedium chica Rchb.f. is the sole member of the subfamily with plicate leaves in Central America and is found only from central Panama to the southeast (Fig. 7A). Guo et al. (2010) indicate that the isthmus served as a corridor for species that managed to diversify in South America but not in Costa Rica and Panama. Selenipedium Rchb.f. evolved during the Paleocene, while representatives with conduplicate leaves (Phragmipedium Rolfe) originated around the Eocene. Vanilloideae is a pantropical group with few representatives in Panama, but the isthmus could also be used as a bridge for future dispersion between the two land masses. Other representatives of Old World diverse 
TABLE 3. Classification of the Orchidaceae of Panama, species number and endemism.

\begin{tabular}{l|c|c}
\hline $\begin{array}{l}\text { Tribe Vanilleae } \\
\text { Subtribe Pogoniinae } \\
\text { Cleistes }\end{array}$ & 1 & \\
$\begin{array}{l}\text { Subtribe Vanillinae } \\
\text { Vanilla }\end{array}$ & 8 & \\
\hline
\end{tabular}

Subtribe Coeliopsidinae Coeliopsis Peristeria

Subtribe Cyrtopodiinae Cyrtopodium

Subtribe Eriopsidinae Eriopsis

\begin{tabular}{l|c|c}
\multicolumn{3}{c}{ Subfamily CYPRIPEDIOIDEAE } \\
\hline $\begin{array}{l}\text { Phragmipedium } \\
\text { Selenipedium }\end{array}$ & 2 & \\
\hline \multicolumn{2}{c}{ Subfamily ORCHIDOIDEAE } \\
\hline
\end{tabular}

Subtribe Eulophiinae Eulophia Oeceoclades

\begin{tabular}{|c|c|c|}
\hline \multicolumn{3}{|c|}{ Subfamily ORCHIDOIDEAE } \\
\hline Tribe Cranichideae & & \\
\hline $\begin{array}{l}\text { Subtribe Cranichidinae } \\
\text { Gomphichis }\end{array}$ & 1 & \\
\hline Pseudocentrum & 1 & \\
\hline Pterichis & 1 & \\
\hline Solenocentrum & 1 & \\
\hline Baskervilla & 2 & 1 \\
\hline Prescottia & 2 & \\
\hline Ponthieva & 4 & \\
\hline Cranichis & 6 & \\
\hline \multicolumn{3}{|l|}{ Subtribe Goodyerinae } \\
\hline Aspidogyne & 5 & 1 \\
\hline Goodyera & 2 & \\
\hline Kreodanthus & 3 & 2 \\
\hline Microchilus & 12 & 6 \\
\hline Platythelys & 3 & \\
\hline \multicolumn{3}{|l|}{ Subtribe Spiranthinae } \\
\hline Beloglottis & 2 & \\
\hline Brachystele & 1 & \\
\hline Coccineorchis & 7 & \\
\hline Cyclopogon & 9 & 1 \\
\hline Discyphus & 1 & \\
\hline Eurystyles & 3 & \\
\hline Pelexia & 4 & 1 \\
\hline Sacoila & 1 & \\
\hline Sarcoglottis & 4 & 1 \\
\hline Stenorrhynchos & 1 & \\
\hline \multicolumn{3}{|l|}{ Tribe Orchideae } \\
\hline Subtribe Orchidinae & & \\
\hline Habenaria & 20 & 4 \\
\hline
\end{tabular}

Subtribe Maxillariinae Camaridium Christensonella Cryptocentrum Heterotaxis

Inti

Lycaste

Mapinguari

Maxillaria

Maxillariella

Mormolyca

Neomoorea

Nitidobulbon

Ornithidium

Rhetinantha

Rudolfiella

Teuscheria

Trigonidium

Xylobium

Subtribe Oncidiinae

Aspasia

Brassia

Cischweinfia

Comparettia

Cuitlauzina

Cyrtochiloides

Eloyella

Erycina

Fernandezia

Ionopsis

Leochilus

Lockhartia

Macradenia

Macroclinium

Miltoniopsis

Notylia

Oncidium

Ornithocephalus

Otoglossum

Plectrophora

Psychopsis

Rhynchostele

Rodriguezia

Rossioglossum

Systeloglossum

Telipogon

Trichocentrum

Trichopilia

Trizeuxis

Cycnoches

Dressleria

Galeandra

Mormodes 
TABle 3. Continues.

\begin{tabular}{|c|c|c|c|c|c|}
\hline Taxa & Species number & Endemism & Taxa & Species number & Endemism \\
\hline Coryanthes & 4 & 2 & Dresslerella & 4 & 3 \\
\hline Gongora & 10 & 1 & Dryadella & 6 & 2 \\
\hline Horichia & 1 & & Echinosepala & 6 & 1 \\
\hline Houlletia & 2 & & Kraenzlinella & 1 & \\
\hline Kegeliella & 2 & & Lalexia & 1 & \\
\hline Lacaena & 1 & & Lankesteriana & 4 & \\
\hline Paphinia & 2 & 1 & Lepanthes & 66 & 21 \\
\hline Polycycnis & 5 & 1 & Lepanthopsis & 2 & 1 \\
\hline Sievekingia & 3 & 1 & Masdevallia & 26 & 9 \\
\hline Stanhopea & 10 & 2 & Muscarella & 3 & \\
\hline Subtribe Zygopetalinae & & & Myoxanthus & 7 & \\
\hline Benzingia & 1 & & Octomeria & 5 & \\
\hline Chaubardiella & 1 & & Pabstiella & 1 & \\
\hline Chondroscaphe & 3 & 1 & Phloeophila & 2 & 1 \\
\hline Cochleanthes & 1 & & Platystele & 20 & 3 \\
\hline Cryptarrhena & 2 & & Pleurothallis & 54 & 23 \\
\hline Daiotyla & 2 & & Pleurothallopsis & 2 & \\
\hline Dichaea & 26 & 3 & Restrepia & 3 & \\
\hline Euryblema & 1 & 1 & Scaphosepalum & 4 & 1 \\
\hline Galeottia & 1 & & Specklinia & 34 & 7 \\
\hline Huntleya & 2 & & Stelis & 88 & 23 \\
\hline Kefersteinia & 11 & 4 & Trichosalpinx & 18 & 4 \\
\hline Koellensteinia & 1 & & Trisetella & 4 & 1 \\
\hline Pescatoria & 2 & & Zootrophion & 3 & \\
\hline Stenotyla & 3 & 1 & Subtribe Ponerinae & & \\
\hline Warczewiczella & 2 & & Helleriella & 1 & \\
\hline Warrea & 1 & & Isochilus & 3 & \\
\hline Warreopsis & 1 & & Nemaconia & 1 & \\
\hline Tribe Epidendreae & & & Tribe Gastrodieae & & \\
\hline Subtribe Bletiinae & & & Uleiorchis & 1 & \\
\hline Bletia & 2 & & Tribe Malaxideae & & \\
\hline Subtribe Chysinae & & & Crossoglossa & 5 & 2 \\
\hline Chysis & 3 & 2 & Liparis & 3 & \\
\hline Subtribe Coeliinae & & & Malaxis & 15 & 2 \\
\hline Coelia & 1 & & Tribe Neottieae & & \\
\hline Subtribe Laeliinae & & & Palmorchis & 4 & \\
\hline Acrorchis & 1 & & Tribe Dendrobieae & & \\
\hline Barkeria & 1 & & Subtribe Bulbophyllinae & & \\
\hline Brassavola & 2 & & Bulbophyllum & 2 & \\
\hline Cattleya & 1 & & Tribe Sobralieae & & \\
\hline Caularthron & 1 & & Elleanthus & 23 & 2 \\
\hline Dimerandra & 1 & & Sobralia & 39 & 16 \\
\hline Dinema & 1 & & Tribe Triphoreae & & \\
\hline Encyclia & 7 & 1 & Subtribe Triphorinae & & \\
\hline Epidendrum & 206 & 53 & Monophyllorchis & 1 & \\
\hline Guarianthe & 1 & & Psilochilus & 3 & 1 \\
\hline Homalopetalum & 1 & & Triphora & 4 & \\
\hline Jacquiniella & 6 & 1 & Tribe Tropidieae & & \\
\hline Laelia & 1 & & Corymborkis & 1 & \\
\hline Nidema & 2 & & Tribe Vandeae & & \\
\hline Prosthechea & 21 & 2 & Subtribe Aeridinae & & \\
\hline Scaphyglottis & 39 & 5 & Phalaenopsis & 1 & \\
\hline Subtribe Pleurothallidinae & & & Subtribe Angraecinae & & \\
\hline Acianthera & 13 & 1 & Campylocentrum & 9 & \\
\hline Anathallis & 4 & & Subtribe Polystachyinae & & \\
\hline Barbosella & 5 & 1 & Polystachya & 2 & \\
\hline Brachionidium & 12 & 5 & & & \\
\hline Diodonopsis & 1 & & & & \\
\hline Dracula & 6 & 3 & TOTAL & 1365 & 296 \\
\hline
\end{tabular}


TABLE 4. Number of taxa, percentage of total (\%) and endemism in each subfamily recorded in Panama.

\begin{tabular}{lccccc}
\hline Subfamily & Tribes & Subtribes & Genera & Species & Endemism \\
\hline Epidendroideae & $13(81.3)$ & $21(77.8)$ & $159(85.5)$ & $1257(92.1)$ & $278(94.3)$ \\
Orchidoideae & $2(12.5)$ & $4(14.8)$ & $24(12.8)$ & $96(7.0)$ & $17(5.8)$ \\
Vanilloideae & $1(6.3)$ & $2(7.4)$ & $2(1.1)$ & $9(0.7)$ & $0(0)$ \\
Cypripedioideae & $0(0)$ & $0(0)$ & $2(1.1)$ & $3(0.2)$ & $1(0)$ \\
Total & 16 & 27 & 187 & 1365 & 296 \\
\hline
\end{tabular}

TABLE 5. Genera, species, endemism and percentage of total $(\%)$ in the 16 tribes present in the orchid flora of Panama.

\begin{tabular}{lccc}
\hline Tribe & Genera & Species & Endemism \\
\hline Epidendreae & $52(27.8)$ & $708(51.9)$ & $174(58.8)$ \\
Cymbidieae & $87(46.5)$ & $427(31.3)$ & $81(27.4)$ \\
Cranichideae & $23(12.3)$ & $76(5.6)$ & $13(4.4)$ \\
Sobralieae & $2(1.1)$ & $62(4.5)$ & $18(6.1)$ \\
Malaxideae & $3(1.6)$ & $23(1.7)$ & $4(1.4)$ \\
Orchideae & $1(0.5)$ & $20(1.5)$ & $4(1.4)$ \\
Vandeae & $3(1.6)$ & $12(0.9)$ & $0(0.0)$ \\
Vanilleae & $2(1.1)$ & $9(0.7)$ & $1(0.0)$ \\
Triphoreae & $3(1.6)$ & $8(0.6)$ & $1(0.3)$ \\
Calypsoeae & $2(1.1)$ & $6(0.4)$ & $1(0.3)$ \\
Neottieae & $1(0.5)$ & $4(0.3)$ & $0(0.0)$ \\
Collabieae & $2(1.1)$ & $2(0.1)$ & $0(0.0)$ \\
Dendrobieae & $1(0.5)$ & $1(0.1)$ & $0(0.0)$ \\
Arethuseae & $1(0.5)$ & $1(0.1)$ & $0(0.0)$ \\
Gastrodieae & $1(0.5)$ & $1(0.1)$ & $0(0.0)$ \\
Tropidieae & $1(0.5)$ & $1(0.1)$ & $0(0.0)$ \\
\hline & & &
\end{tabular}

subtribes (Angraecinae and Bulbophyllinae) have their centers of diversity in other regions such as Brazil and not on the Isthmus of Panama. On the other hand, groups such as Eriopsidinae, Gastrodieae, Ponerinae, and Triphorinae, are not diverse in the Neotropics.

The genera with the most species are shown in Tables 3 and 7. The most diverse are: Epidendrum, 206 spp. (70\% of Laeliinae and alone exceeding 100 species), Stelis (88 spp.), Lepanthes Sw. (66 spp.) and Pleurothallis (54 spp.) (together accounting for $50 \%$ of Pleurothallidinae), Camaridium 48 spp., Scaphyglottis and Sobralia Ruiz \& Pav. with 39 spp. Of the 187 genera recorded, 159 (85\%) have fewer than ten species
TABLE 6. Genera, species, endemism and percentage of total (\%) in many different subtribes in the orchid flora of Panama.

\begin{tabular}{lccc}
\hline Subtribe/Tribe $^{*}$ & Genera & Species & Endemism \\
\hline Pleurothallidinae & $30(16)$ & $405(29.6)$ & $110(37.2)$ \\
Laeliinae & $16(8.6)$ & $292(21.4)$ & $62(21.0)$ \\
Oncidiinae & $29(15.5)$ & $157(11.5)$ & $39(13.2)$ \\
Maxillariinae & $18(9.6)$ & $132(9.7)$ & $15(5.1)$ \\
Sobralieae* & $2(1.1)$ & $62(4.5)$ & $18(6.1)$ \\
Zygopetalinae & $17(9.1)$ & $61(4.5)$ & $10(3.4)$ \\
Stanhopeinae & $11(5.9)$ & $44(3.2)$ & $9(3.1)$ \\
Spiranthinae & $10(5.3)$ & $33(2.4)$ & $3(1.0)$ \\
Goodyerinae & $5(2.7)$ & $25(1.8)$ & $9(3.1)$ \\
Catasetinae & $6(3.2)$ & $25(1.8)$ & $8(2.7)$ \\
Malaxideae* & $3(1.6)$ & $23(1.7)$ & $4(1.4)$ \\
Orchidinae & $1(0.5)$ & $20(1.5)$ & $4(1.4)$ \\
\hline
\end{tabular}

and represent $52 \%$ of the flora, while the remaining $15 \%$ of the genera contains $48 \%$ of the species (Fig. 20-26). Similar to its geological history, Panama and Costa Rica show a strong floristic relationship with the Tropical Andes. When analyzing the most numerous genera of the Pleurothallidinae (the most diverse Neotropical subtribe) that reach the highest number of species in the Andes such as Lepanthes, Pleurothallis s.l., Stelis s.l. as well as Brachionidium Lindl. and Platystele Schltr., we found a clear trend. These genera are more diverse in Costa Rica and Panama than in other regions of Central America. When comparing the floristic data of Panama with the flora of Ecuador, one of the most intensively studied Andean countries (Dodson 2003), we found that there is proportionately the same trend. The genera with the most species are Epidendrum, Pleurothallis (> 400 spp.), and Stelis s.s., Lepanthes (> $300 \mathrm{spp}$.). Other groups with high number of species in 
TABle 7. The most diverse orchid genera in Panama.

\begin{tabular}{lcc}
\hline Genera & $\begin{array}{c}\text { Species } \\
\text { number }\end{array}$ & $\begin{array}{c}\text { Percentage } \\
\text { of the flora }\end{array}$ \\
\hline Epidendrum & 206 & 15.09 \\
Stelis & 88 & 6.45 \\
Lepanthes & 66 & 4.84 \\
Camaridium & 48 & 3.96 \\
Pleurothallis & 48 & 3.52 \\
Scaphyglottis & 39 & 2.86 \\
Sobralia & 39 & 2.86 \\
Specklinia & 34 & 2.49 \\
Oncidium & 32 & 2.34 \\
Dichaea & 26 & 1.90 \\
Masdevallia & 26 & 1.90 \\
Telipogon & 23 & 1.68 \\
Elleanthus & 23 & 1.68 \\
Maxillaria & 21 & 1.54 \\
Prosthechea & 21 & 1.54 \\
Habenaria & 20 & 1.47 \\
Platystele & 18 & 1.47 \\
Trichosalpinx & 20 & 1.10 \\
Malaxis & & \\
\hline & & (5) \\
\hline
\end{tabular}

the Andes include Fernandezia Lindl., Telipogon Kunth and terrestrial genera such as $A a$ Rchb.f. and Petrichis Lindl. are other examples of Andean influence (Dodson 2003, García-Castro et al. 1993). The results found are similar to that of Costa Rica in terms of number of taxa (Dressler 2003c). The definition of LCA treating Costa Rica and Panama as two areas with high biological similarity is supported by our data. Unpublished results on the floristic affinities of LCA with other areas of the Neotropics show that Panama shares at least 70\% of its orchid flora with Costa Rica (Bogarín in prep.). Floristic inventories in Costa Rica have also revealed a strong component of Panamanian species mainly in the region of the Cordillera de Talamanca, strengthening a close biological relationship (Bogarín et al. 2008, Karremans et al. 2012, Fernández et al. 2014). Most species of Epidendrum, Lepanthes, Pleurothallis, and Stelis found in both countries are in the region of Talamanca. However, the whole country does not have close relations with Costa Rica. The central regions

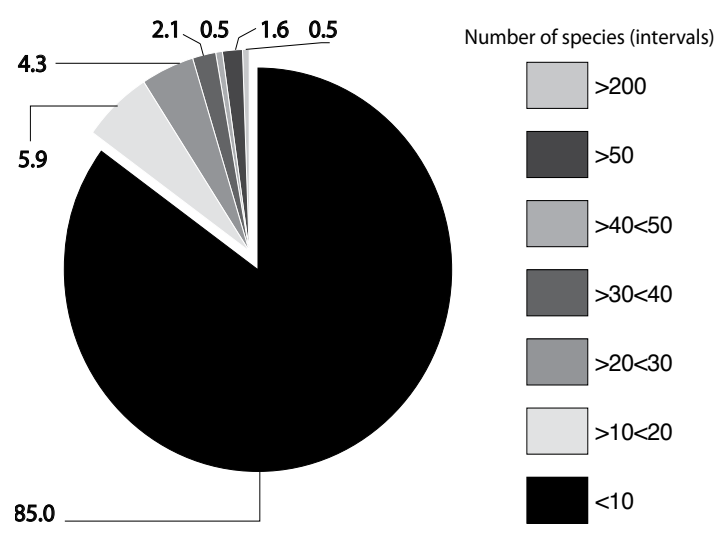

FiguRE 20. Percentage of the number of genera with their respective number of species.

(Coclé), and southeastern Panama (Panama, Darién and comarcas Emberá, Guna Yala, Kuna de Madugandí and Kuna de Wargandí) show more affinities to South American taxa, which are not distributed in Costa Rica or even in the western provinces (Chiriquí and Bocas del Toro and part of Veraguas). Among them we can mention Houlletia odoratissima Linden ex Lindl. \& Paxton, Huntleya fasciata Fowlie, Kefersteinia mystacina Rchb.f., Lycaste campbellii, Lycaste powellii Schltr., Miltoniopsis roezlii (Rchb.f.) God.-Leb., Selenipedium chica and genera such as Eloyella P. Ortiz, Euryblema Dressler, Koellensteinia Rchb.f., Neomoorea Rolfe and Rudolfiella Hoehne, absent in northern Central America (Dressler 2003c).

The Southern Pacific region of Panama is geologically and biologically more related to the Department of Chocó in Colombia. Recent studies in the Serranía del Baudó support this floristic relationship. Misas (2005) documented from this region with several species described from Panamanian material. Of the 357 species recorded, $167(48 \%)$ are also found in Panama, mostly in the central and southeast. Among the species recorded are: Epidendrum flexuosissimum C.Schweinf., Lockhartia obtusata L.O. Williams, Lycaste campbellii, L. powellii, Scaphyglottis coriacea (L.O. Williams) Dressler and Specklinia areldii (Luer) Pridgeon \& M.W. Chase among others. Additionally, we determined a specimen of Cryptocentrum misasii $\mathrm{P}$. Ortiz \& Carnevali, recently described from Serranía del Baudó by Carnevali et al. (2012) that was collected in 
the Darién National Park. These data demonstrate that the increasing collecting efforts and the publication of floristic treatments in the border provinces of Panama and Colombia will help to better understand its narrow phytogeographical relationships. There are few groups with greater diversity in northern Mesoamerica than is found in Panama. Barkeria Knowles \& Westc. with about 20 species in Mesoamerica and Rhynchostele Rchb.f. with a center of diversity in Mexico and Guatemala, among others, contain a single species in Panama. LCA can be seen as a biotic unit, but it could in turn be subdivided on its orchid diversity into biogeographic provinces proposed for other groups such as fish, insects and birds (Myers et al. 2000).

Although at genus level there is a similarity with neighboring areas of the isthmus, at the species level the picture may be different for some groups. By analyzing the most diverse genera we noted that few species (mainly in the Pleurothallidinae) are shared between the tropical Andes and LCA. For example, of the nearly 150 species of Lepanthes of Costa Rica and Panama, only two species are shared with Colombia and Ecuador, countries whose floras each reported over 300 species (Luer 2006, Luer \& Thoerle 2012). Again floristic work intensification in the southeastern region of Panama may change this scenario. However, it is clear that there are certain trends towards more specific biogeographic characterization for LCA. The other diverse groups such as Maxillaria, Pleurothallis and Stelis have been subject to nomenclatural changes and generic recircumscriptions (Pridgeon \& Chase 2001, Luer 2002a, Blanco et al. 2007). Furthermore, Epidendrum (206 spp.), the largest genus in Panama, is accepted as a broadly defined genus. However, because of their heterogeneity, about 35 genera proposed so far are currently reduced in synonymy (Hágsater \& Salazar 1993, Hágsater 1999, 2001, 2010, 2013, Hágsater \& Sánchez 2004, 2006, 2007, 2008, 2009, Hágsater \& Soto Arenas 2005). All these nomenclatural issues suggest that the phytogeographic conclusions should be interpreted in context.

A distribution analysis of species recorded in this study reveals that Panama shares the majority of taxa with: Costa Rica (90.6\%), Colombia (15.6\%), Ecuador $(12.5 \%)$ and northern Mesoamerica (9.3\%). In the past decade 108 species, belonging to 38 genera, were described based on Panamanian material. Some species described prior 2004 were not included by Correa et al. (2004). About $44.9 \%$ of these records are still endemic to Panama, $47.3 \%$ are shared with Costa Rica, $6.7 \%$ with Colombia and $2.4 \%$ with other regions of the Neotropics. These data support the narrow floristic relations between Costa Rica and Panama formerly discussed. However, we must also consider that there is some bias regarding botanical exploration, with most expeditions undertaken of greater intensity in the provinces bordering Costa Rica and less intense toward the provinces bordering Colombia. Despite the low percentage of species that Panama shares with Colombia and Ecuador in terms of new floristic contributions of the last decade, it is expected that an increase in botanical exploration in the province of Darién and surrounding areas will reveal more species in common. Unfortunately, the access to this area is restricted and problematic, hampering research efforts. It is likely that the number of records now regarded as endemic will decrease with the increasing botanical explorations in regions neighboring Costa Rica and Colombia.

Ecologically, the family is more diverse at elevations from 1500 to $2800 \mathrm{~m}$ where most of the species are found. Regions such as the dry lowland areas of Azuero at sea level are poor in orchid species. The highlands of Volcán Barú, Cerro Echandi, Cerro Fábrega and Cerro Itamut are less diverse as they are located above $3000 \mathrm{~m}$ elevation; however, they are the only places in Panama where it is possible to observe some genera such as Pterichis or some species of Telipogon. About 173 $(12,7 \%)$ of the species recorded are terrestrials and 1193 $(87,3 \%)$ are epiphytes. Some species (Eriopsis Lindl., or some species of Epidendrum, Sobralia and Elleanthus) exhibit both habits, existing as either terrestrials and / or epiphytes. Other species such as Vanilla Mill. with eight species can be considered climbing or scandent vines. Some species of Pleurothallidinae, Laeliinae, Maxillariinae can be seen in rocky, humid, mossy areas but they cannot be considered strictly terrestrials.

So far we have analyzed some of the biological, evolutionary and geological factors behind the numbers found in the flora of Panama. However, the knowledge of a region involves an analysis of the level of effort that the scientific community has undertaken in order to understand the diversity of a particular site.

A total of 686 taxa described (658 species, 3 subspecies and 25 varieties) in 131 genera were based 
TABLE 8. Authors who published most of the basionyms of Panama.

\begin{tabular}{llc}
\hline Author & $\begin{array}{l}\text { Standard } \\
\text { form }\end{array}$ & $\begin{array}{c}\text { Number of } \\
\text { species }\end{array}$ \\
\hline Carlyle A. Luer & Luer & 148 \\
Rudolf Schlechter & Schltr. & 117 \\
Robert L. Dressler & Dressler & 112 \\
Heinrich G. Reichenbach & Rchb.f. & 64 \\
Eric Hágsater & Hágsater & 55 \\
Oakes Ames & Ames & 51 \\
Louis Williams & L.O.Williams & 43 \\
Charles Schweinfurth & C.Schweinf. & 34 \\
John T. Atwood & J.T.Atwood & 13 \\
John Lindley & Lindley & 9 \\
\hline
\end{tabular}

on type material collected in Panama. Of the total, 392 $(57.2 \%)$ names are accepted as names lacking synonyms, $158(23.0 \%)$ are accepted as homotypic synonyms and $136(19.8 \%)$ have been treated as heterotypic synonyms. The genera with most species described are: Epidendrum (112 spp.), Pleurothallis (86 spp.), Lepanthes (45 spp.), Sobralia (33 spp.), Maxillaria (31 spp.), Oncidium and Stelis (30 spp.). The authors that published most species of orchids in the flora of Panama are shown in Table 8. Luer, Schlechter and Dressler are the major contributors to the taxonomy of Orchidaceae of Panama (Figs. 12C, 15, 16). Schlechter through C.W. Powell managed to describe several species in the early stages of orchid research in Panama (Figs. 11, 12C). Dressler's major contributions were made during his more than 20 years at the STRI in Balboa, Canal Zone and Luer contributed with the taxonomy of Pleurothallidinae, the largest subtribe in Panama. The most important herbaria for the orchid flora of Panama in terms of type specimens are summarized in Table 9. Most of the type specimens of the species described by Schlechter were lost in Berlin (Christenson 1991a, 1991b, 1991c). We recorded 97 names that have been lectotypified. Most of the specimens that were lost at Berlin correspond to Powell's collections described by Schlechter. Duplicates are mostly found at AMES, BM, K, MO, S and W (Christenson 1991b). AMES is an important herbarium because it houses copies of Schlechter's original drawings of types made under his supervision (Ames 1944) as well as the majority of
TABLE 9. Number of type specimens deposited in the most important herbaria for Panama.

\begin{tabular}{cccc}
\hline $\begin{array}{c}\text { Herbaria } \\
\text { (Acronym) }\end{array}$ & Holotypes & Isotypes & Lectotypes \\
\hline MO & 157 & 53 & 4 \\
B & 115 & 0 & 0 \\
SEL & 104 & 10 & 0 \\
AMES & 97 & 37 & 81 \\
W & 54 & 0 & 6 \\
US & 37 & 7 & 0 \\
AMO & 33 & 6 & 0 \\
PMA & 20 & 47 & 0 \\
K & 14 & 13 & 5 \\
F & 11 & 10 & 0 \\
FLAS & 9 & 6 & 0 \\
CR & 5 & 5 & 0 \\
NY & 4 & 6 & 0 \\
\hline
\end{tabular}

lectotypes (Table 9). Syntypes are cited for ten names, all described by Schlechter or Reichenbach. Seven herbaria in the United States keep more than $60 \%$ of the holotypes, $10 \%$ is kept in Europe (about $17 \%$ were lost in B), 5\% in Mexico at AMO (mostly Epidendrum) and only 3\% are kept in Panama (all at PMA herbarium). The MO herbarium is the most important in terms of type specimens for the orchid flora of Panama (Table 9). Figure 17 shows some locations where type specimens have been collected in Panama.

Some genera have traditionally been studied more intensively than others. In addition to the recognized contributions of Schlechter, Ames and L.O. Williams to the Panamanian orchids, Dressler promoted the study of Orchidaceae of the Isthmus in a broad sense. He made major contributions in Sobralia and Elleanthus, Chysis Lindl., Oncidium, Trichopilia and Telipogon among others. Hágsater and colleagues have led to a better understanding of Epidendrum, Atwood in Maxillaria s.l. and Luer in Pleurothallidinae. The decreasing activity of these scientists in the flora of Panama, has led to a slowdown in terms of scientific publications in comparison with neighboring countries such as Costa Rica (Bogarín et al. 2013b). Furthermore, many areas remain unexplored for orchids. The most urgent areas to be explored are the Cordillera de Talamanca, on both Pacific and Caribbean slopes, indigenous comarcas 
TABLE 10. Twenty genera with the most endemic species in the orchid flora of Panama.

\begin{tabular}{lcc}
\hline Genus & Endemic species & Total species \\
\hline Epidendrum & $53(18.0)$ & 206 \\
Pleurothallis & $23(7.8)$ & 54 \\
Stelis & $23(7.8)$ & 88 \\
Lepanthes & $21(7.1)$ & 66 \\
Telipogon & $17(5.8)$ & 23 \\
Sobralia & $16(5.4)$ & 39 \\
Masdevallia & $9(3.1)$ & 26 \\
Camaridium & $8(2.7)$ & 48 \\
Specklinia & $7(2.4)$ & 34 \\
Oncidium & $6(2.0)$ & 32 \\
Microchilus & $6(2.0)$ & 12 \\
Scaphyglottis & $5(1.7)$ & 39 \\
Brachionidium & $5(1.7)$ & 12 \\
Trichopilia & $5(1.7)$ & 12 \\
Habenaria & $4(1.4)$ & 20 \\
Trichosalpinx & $4(1.4)$ & 18 \\
Kefersteinia & $4(1.4)$ & 11 \\
Dichaea & $3(1.0)$ & 26 \\
Maxillaria & $3(1.0)$ & 21 \\
Platystele & $3(1.0)$ & 20 \\
\hline & &
\end{tabular}

(albeit with inherent problems obtaining collection permits), the Caribbean side of Veraguas, Colón, Panama and the foothills of Majé, San Blas, Sapo and Darién. Panama is a privileged country biologically; however we estimate that much work remains to complete a floristic treatment that reveals more realistic data on the orchid flora that the country hosts. This checklist is a starting point in this direction and we hope that this contribution will encourage and boost orchid research on the Isthmus of Panama and the surrounding regions. We are still working to develop a taxonomic illustrated treatment covering all the Orchidaceae of Panama (Bogarín et al. 2013b).

Endemism. A total of 296 (21.7\%) endemic species in $69(36.9 \%)$ genera were recorded (Table 3). This percentage of endemism is superior to other Central American countries except Costa Rica and southern
TABLE 11. Relationship between the number of endemic species and the total of species of the genera with most endemic species.

\begin{tabular}{|c|c|}
\hline Genus & Endemics/total \\
\hline Telipogon & 73.9 \\
\hline Microchilus & 50.0 \\
\hline Pleurothallis & 42.6 \\
\hline Brachionidium & 41.7 \\
\hline Trichopilia & 41.7 \\
\hline Sobralia & 41.0 \\
\hline Kefersteinia & 36.4 \\
\hline Masdevallia & 34.6 \\
\hline Lepanthes & 31.8 \\
\hline Stelis & 26.1 \\
\hline Epidendrum & 25.7 \\
\hline Trichosalpinx & 22.2 \\
\hline Specklinia & 20.6 \\
\hline Habenaria & 20.0 \\
\hline Oncidium & 18.8 \\
\hline Camaridium & 16.7 \\
\hline Platystele & 15.0 \\
\hline Maxillaria & 14.3 \\
\hline Scaphyglottis & 12.8 \\
\hline Dichaea & 11.5 \\
\hline
\end{tabular}

Mexico (Ossenbach et al. 2007). The high number of species in the most diverse taxa of the flora of Panama is proportionally reflected in the number of endemic species. For example, Epidendroideae concentrates $94.3 \%$ of the endemic species, the tribes Epidendreae (58.8\%) and Cymbidieae (27.5\%) and subtribes Pleurothallidinae (37.2\%), Laeliinae $(20.9 \%)$ and Oncidiinae (13.2\%) are the groups with the most endemic species in the country (Tables 4,5,6). The genera with the highest number of endemic species are Epidendrum (53 spp., 18\%), Pleurothallis (23 spp., 7.8\%), Stelis (23 spp., 7.8\%), Lepanthes (21 spp., 7.1\%), Telipogon (17 spp., 5.8\%) and Sobralia (16 spp., 5.4\%). These six genera account for $50 \%$ of endemic species (Table 10). The remaining genera have less than ten endemic species and 31 (45\%) of the genera have only one endemic species. The percentages of endemism within Pleurothallidinae are 
high. It is likely that the complex pollination systems propitiated narrow distributions in some genera. According to Ackerman (2014), the exploitation of highly diverse groups of pollinators such as Diptera (Pleurothallidinae), Lepidoptera (Epidendrum) and Hymenoptera (Sobralia) is one of the possible reasons for the diversification of Neotropical orchids. Clear examples are the genera pollinated by sexual deception such as Lepanthes and Telipogon that "exploit" highly diverse groups of insects belonging to Sciaridae and Tachinidae respectively (Dodson 2003, Blanco \& Barboza 2005). Both groups are quite diverse in the Andes and the highlands of Costa Rica and Panama where the levels of endemism are also high.

Within the system of classification followed in this paper we did not document endemic genera for the flora of Panama. However, this conclusion should be viewed with caution. The arbitrary political boundaries of countries and different nomenclatural trends could alter or skew biogeographic interpretations. For example the group of species allied to Salpistele Dressler (= Stelis s.l.) is not endemic to Panama as there is one species in Costa Rica and if considered a member of Stelis s.l. it would not be endemic to Panama. But nomenclatural issues and political boundaries aside, clearly the Salpistele group is endemic to Costa Rica and Panama. Other groups that are more diverse on the isthmus are Dracontia Luer and Unciferia (Luer) Luer (= Stelis s.l.), Certain clades, including Specklinia, Epidanthus L.O. Williams, Neowilliamsia Garay and Oerstedella, members of Epidendrum; and Panarica Withner \& P.A.Harding (=Prosthechea Knowles \& Westc.) among others, can be considered endemic or more diverse in Costa Rica and Panama than in neighboring areas (Dressler 2003c). Other genera that have suffered nomenclatural changes, such as Camaridium and Scaphyglottis, also show the tendency to have a greater number of endemic species in Costa Rica and Panama (Dressler et al. 2004, Kirby 2011). Despite being a geologically young region, the evolution of the orchids on the isthmus is complex. There are several theories that attempt to explain this phenomenon. Some are related to the ancient volcanic arc of islands that created favorable sites for speciation that joined and mixed their floras with the existing continental populations. This is a probable hypothesis to explain the presence of certain lineages on the isthmus but has yet to be tested by integrating molecular and biogeographical data (Burger 1980).

By comparing the ratio of the number of endemic species with the total number of species in the flora of Panama for each genus, the highest percentage of endemism is found in Telipogon (73.9\%) followed by Microchilus (50\%), Pleurothallis, Brachionidium, Trichopilia and Sobralia (between 41-43\%), Kefersteinia, Masdevallia and Lepanthes (between 31$36 \%$ ), Epidendrum and Stelis (25-26\%), Trichosalpinx $(22.2 \%)$ and the remaining genera have relationships between 7 and $20 \%$ (Table 11). Two genera have all their species endemic: Dressleria Dodson (3 spp.) and Euryblema (1 sp.). Dresslerella Luer has four species recorded of which three (75\%) are endemic. The high percentage of endemism in Telipogon (Dodson \& Escobar 1993) should not be surprising considering its complex pollination syndrome (Dodson 2003). However, if we consider that those species were collected very close to Costa Rica, it is likely that some of them will also be found there. On the other hand, the high number of endemic species in Telipogon, Sobralia, Epidendrum and other groups within Pleurothallidinae is also attributable to a greater effort in the study of those genera. The above groups were the preferential study subjects of the taxonomists who made the major contributions to the orchid flora of Panama. It is still necessary to make more comprehensive taxonomic studies, especially in subtribes Laeliinae, Pleurothallidinae, Goodyerinae and Spiranthinae both in Panama and in its neighboring countries, in order to have a more precise conclusion regarding endemism.

Interpretations of endemism evaluated in a context of political boundaries are also biased and lose sense in biological terms. For example, Panama "lost" 11 endemic species of Lepanthes because they were recorded in Costa Rica (Fernández et al. 2014). However, these collections of Lepanthes in Costa Rica come from biogeographical regions related to Panama, such as the Cordillera de Talamanca. Despite the caution required when making interpretations of endemism at the country level, it is nevertheless necessary to record these data because the political decisions on conservation and the sustainable use of natural resources vary from one border to another.

Exotic flora. In Panama, four $(0.3 \%)$ species are currently recorded as non-native wild populations. 
The percentage is low compared to the West Indies but is similar to Costa Rica (Dressler 2003c, Ackerman 2014). At least Arundina graminifolia (D. Don) Hochr., Spathoglottis plicata Blume from Asia and Oeceoclades maculata (Lindl.) Lindl. from Africa and Madagascar are widely dispersed in the Neotropics. Arundina graminifolia and $S$. plicata plants are often grown as ornamentals in gardens and naturalization was recorded in Costa Rica, Cuba and Hawaii (Pupulin 2005b, Ackerman 2007). In the case of A. graminifolia, the flowers with a trumpet-like shaped lip (similar to Sobralia) apparently attract bees that pollinate them. Spathoglottis plicata plants are often self-pollinated and do not require native pollinators for reproduction. Both species are terrestrial and grow in places with direct sunlight such as rocky roadsides (Cohen \& Ackerman 2009).

Oeceoclades maculata is a fast growing orchid and was classified as an invasive species (Ackerman 2007). It is perhaps the most common introduced orchid in
Panama. It is found throughout the Neotropics from Florida (USA) to Brazil. Populations of O. maculata are common along both the Caribbean and Pacific lowlands were they are often found among understory vegetation. The adaptation to grow in a wide variety of environments contributes to its invasive prowess. Reproductive success is due to self-pollination (it does not need native pollinators) and high rates of fruit production (Cohen \& Ackerman 2009).

The most curious case is perhaps the naturalization of Phalaenopsis stuartiana Rchb.f., an endemic orchid from the island of Mindanao in the Philippines (Fig. 26B). It has only been reported from Isla Colón in Bocas del Toro Archipielago where it probably encountered climatic conditions and coastal environments like those on Mindanao. It is the only epiphytic orchid naturalized in Panama. Its introduction, population status and pollination have yet to be investigated. To date, the few herbarium records that exist are all known from this site.

AcKnowledgments. We are grateful to Clotilde Arrocha, Bolívar González (UCH), and Jorge Warner (JBL) for making possible the development of this project and for believing in us with their unconditional support. A special thanks to Ivonne del Carmen Oviedo Espinoza and Ricardo Blas for their kind hospitality, friendship and support. To the staff and colleagues of UCH: Tina Hofmann, Idalmi Martínez, Eyvar Rodríguez-Quiel, Rosemary Ríos and Rosa Villarreal and JBL: Jaime Aguilar, Karen Barquero, Maricruz Bonilla, Jorge Brenes, Melania Fernández, Adam Karremans and Giovanni Meza for their help with fieldwork and logistics. Esteban Jiménez and Melissa Varela, research assistants at JBL helped with data analysis. To our dear friends Jerry and Linda Harrison of Altos de Cerro Azul for always supporting us unconditionally in the study of the flora of Panama particularly in the region of Cerro Jefe and for their useful comments on the manuscript. Bill Adsett (Cerro Azul), Carlos Espinosa (STRI-Fortuna), the late Andrés Maduro and Erick Olmos (Finca Drácula), Bonarge Rodríguez (Panama) and Steve and Marjorie Sarner of Boquete for providing plants, information and useful discussions on Panamanian species. The staff and curators of PMA especially Mireya D. Correa, Rodolfo Flores and Vielka Murillo. We want to acknowledge the scientific services of Autoridad Nacional del Ambiente (ANAM) of Panama for issuing the scientific permits under which part of this study was performed. The Rector of UNACHI, Etelvina de Bonagas, Héctor Renquena (formerly Rector of UNACHI), Henning Jensen Rector of UCR and Alice Pérez, Vice-President of Research of UCR for supporting our visits and activities at UCH and JBL. This research was enabled by the Project 814-B2-161 "Hacia una moderna flora de orquídeas de Panamá" by JBL and UCH, supported by the Vice-Presidency of Research, Universidad de Costa Rica and Universidad Autónoma de Chiriquí, Panama. 


\section{CHECKLIST OF THE ORCHIDACEAE OF PANAMA}

\section{ACianthera Scheidw.}

Acianthera aberrans (Luer) Pupulin \& Bogarín, Lankesteriana 8(2): 53. 2008.

Pleurothallis aberrans Luer, Selbyana 2: 382. 1978.

Aberrantia aberrans (Luer) Luer, Monogr. Syst. Bot. Missouri Bot. Gard. 103: 310. 2005.

Voucher: C. Luer \& R.L. Dressler 1628 (SEL).

Acianthera butcheri (L.O.Williams) Pridgeon \& M.W.Chase, Lindleyana 16: 242. 2001.

Pleurothallis butcheri L.O.Williams, Fieldiana, Bot. 29(6): 346. 1961.

Didactylus butcheri (L.O.Williams) Luer, Monogr.

Syst. Bot. Missouri Bot. Gard. 95: 257. 2004.

VOUCHER: H. P. Butcher 651 (F).

Acianthera cogniauxiana (Schltr.) Pridgeon \& M.W.Chase, Lindleyana 16: 243. 2001.

Pleurothallis cogniauxiana Schltr., Repert. Spec.

Nov. Regni Veg 3(42-43): 246. 1907.

Pleurothallis congruens Luer, Selbyana 2(4): 391. 1978.

Acianthera congruens (Luer) Pridgeon \&

M.W.Chase, Lindleyana 16(4): 243. 2001.

VOucher: C.A. Luer et al. 987 (SEL).

Acianthera decipiens (Ames \& C.Schweinf.) Pridgeon \& M.W.Chase, Lindleyana 16: 243. 2001.

Pleurothallis decipiens Ames \& C.Schweinf., Schedul. Orchid. 8: 26-27. 1925.

Pleurothallis pterocaulis L.O.Williams, Ann. Missouri Bot. Gard. 27(3): 280-281, t. 32, f. 1-7. 1940.

Voucher: P.H Allen 1239 (AMES), C. A. Luer \& H. P. Butcher 1124 (SEL).

Acianthera ellipsophylla (L.O.Williams) Pridgeon \& M.W.Chase, Lindleyana 16: 243. 2001.

Pleurothallis ellipsophylla L.O.Williams, Ann. Missouri Bot. Gard. 29(4): 344. 1942.

Voucher: H. von Wedel 560 (AMES).

Acianthera johnsonii (Ames) Pridgeon \& M.W.Chase, Lindleyana 16: 244. 2001.

Pleurothallis johnsonii Ames, Schedul. Orchid. 2: 21. 1923.

Brenesia johnsonii (Ames) Luer, Monogr. Syst.

Bot. Missouri Bot. Gard. 95: 255. 2004.
Brenesia costaricensis Schltr., Repeeert. Spec. Nov. Regni Veg. Beih. 19: 200. 1923.

Pleurothallis lateralis L.O.Williams, Ceiba 5: 84. 1956.

VOUCHER: not seen, cited by D'Arcy (1987) and Luer (2003).

Acianthera juxtaposita (Luer) Luer, Monogr. Syst. Bot. Missouri Bot. Gard. 95: 253. 2004.

Pleurothallis juxtaposita Luer, Lindleyana 6(2): 103-105. 1991.

VOUCHER: C. Luer et al. 10585 (SEL).

Acianthera lepidota (L.O.Williams) Pridgeon \& M.W.Chase, Lindleyana 16: 244. 2001.

Pleurothallis lepidota L.O.Williams, Ann. Missouri Bot. Gard. 27(3): 279., t. 32, f. 81-2. 1940.

VOUCHER: P.H. Allen 1552 (AMES).

Acianthera lojae (Schltr.) Luer, Monogr. Syst. Bot. Missouri Bot. Gard. 95: 254. 2004.

Pleurothallis citrophila Luer, Selbyana 3(3-4): 266-267, f. 234. 1977.

Pleurothallis lojae Schltr., Repert. Spec. Nov. Regni Veg. Beih. 8: 60. 1921.

Acianthera citrophila (Luer) Pridgeon \& M.W.Chase, Lindleyana 16(4): 243. 2001.

Voucher: C. A. Luer et al. 10619 (MO).

Acianthera oscitans (Ames) Pridgeon \& M.W.Chase, Lindleyana 16: 245. 2001.

Pleurothallis oscitans Ames, Bot. Mus. Leafl. 2: 25. 1934.

Didactylus oscitans (Ames) Luer, Monogr. Syst. Bot. Missouri Bot. Gard. 103: 310. 2005.

Voucher: C. A. Luer et al. 681 (MO).

Acianthera pubescens (Lindl.) Pridgeon \& M.W.Chase, Lindleyana 16: 245. 2001.

Pleurothallis pubescens Lindl., Compan. Bot. Mag. 2: 355. 1836.

Humboldtia pubescens (Lindl.) Kuntze, Revis. Gen. Pl. 2: 668. 1891.

Pleurothallis vittata Lindl., Edwards's Bot. Reg. 24(Misc.): 73. 1838.

Pleurothallis smithiana Lindl., Edwards's Bot. Reg. 29(Misc.): 57. 1843.

Pleurothallis polystachya A.Rich. \& Galeotti, Ann. Sci. Nat., Bot., sér. 3, 3: 16. 1845. 
Pleurothallis bufonis Klotzsch, Allg. Gartenzeitung 22: 225.1854.

Pleurothallis truxillensis Rchb.f., Bonplandia (Hannover) 2: 25. 1854.

Pleurothallis janeirensis Barb.Rodr., Gen. Spec. Orchid. 2: 29. 1881.

Pleurothallis riograndensis Barb.Rodr., Gen. Spec. Orchid. 2: 28. 1881.

Pleurothallis riograndensis var. viridicata Barb. Rodr., Gen. Spec. Orchid. 2: 30. 1881.

Pleurothallis janeirensis var. viridicata Barb.Rodr., Gen. Spec. Orchid. 2: 30. 1882.

Pleurothallis coriacea Bello, Anales Soc. Esp. Hist. Nat. 12: 116. 1883.

Humboldtia bufonis (Klotzsch) Kuntze, Revis. Gen. P1. 2: 667. 1891.

Humboldtia polystachya (A.Rich. \& Galeotti) Kuntze, Revis. Gen. P1. 2: 668. 1891., nom. illeg. Humboldtia smithiana (Lindl.) Kuntze, Revis. Gen. Pl. 2: 668. 1891.

Humboldtia truxillensis (Rchb.f.) Kuntze, Revis. Gen. P1. 2: 668. 1891.

Humboldtia vittata (Lind1.) Kuntze, Revis. Gen. P1. 2: 668. 1891.

Pleurothallis riograndensis var. longicaulis Cogn. in C.F.P.von Martius \& auct. suc. (eds.), Fl. Bras. 3(4): 542. 1896.

Pleurothallis smithiana var. viridicata Cogn. in C.F.P.von Martius \& auct. suc. (eds.), Fl. Bras. 3(4): 533. 1896.

Pleurothallis mandibularis Kraenzl., Vidensk. Meddel. Naturhist. Foren. Kjøbenhavn 71: 169. 1920.

Pleurothallis bourgeaui Kraenzl., Ark. Bot. 16(8): 15. 1921.

Pleurothallis porphyrantha Kraenzl., Ark. Bot. 16(8): 10. 1921.

Voucher: T.B. Croat 14764 (MO).

Acianthera sicaria (Lindl.) Pridgeon \& M.W.Chase, Lindleyana 16: 246. 2001.

Pleurothallis sicaria Lindl., Edwards's Bot. Reg. 27: Misc. 91. 1841.

Humboldtia sicaria (Lindl.) Kuntze, Revis. Gen. Pl 2: 668. 1891.

Pleurothallis alpina Ames, Schedul. Orchid. 5: 1417, f. 3. 1923.

Acianthera alpina (Ames) Pridgeon \& M.W.Chase,
Lindleyana 16(4): 242. 2001.

Voucher: C. A. Luer \& et al. 1355 (SEL).

Acianthera verecunda (Schltr.) Pridgeon \& M.W.Chase, Lindleyana 16: 246. 2001.

Pleurothallis verecunda Schltr., Repert. Spec. Nov. Regni Veg. Beih. 17: 24. 1922.

VOUCHER: C.W. Powell 91 (AMES, MO).

\section{Acineta Lindl.}

Acineta chrysantha (C.Morren) Lindl. \& Paxton, Paxt. Fl. Gard. 1: 31-32. 1850.

Neippergia chrysantha C.Morren, Ann. Soc. Roy. Agric. Gand 5: 375. 1849.

Acineta warscewiczii Klotzsch, Allg. Gartenzeitung 20(19): 145-146. 1852.

Voucher: J. T. Witherspoon \& F. Witherspoon 8452 (MO).

Acineta densa Lindl., Paxton's Fl. Gard. 1: 91. 1851.

VOuCHer: A. Maduro 00/3424 (M).

Acineta mireyae G.Gerlach \& M.H.Weber, J. Orchideenfr. 10: 230. 2003.

Voucher: A. Maduro 330 (PMA).

Acineta sella-turcica Rchb. f., Bot. Zeitung (Berlin) 10: 705.1852.

VOUCHER: J. Warscewicz s.n. (W).

\section{ACrorchis Dressler}

Acrorchis roseola Dressler, Orquídea (Mexico City), n.s., 12: 14. 1990.

VOUCHER: R.L. Dressler 6103 (MO).

Anathallis Barb.Rodr.

Anathallis funerea (Barb.Rodr.) Luer, Monogr. Syst. Bot. Missouri Bot. Gard. 115: 258. 2009.

Lepanthes funerea Barb.Rodr., Vellosia, ed. 2, 1: 118. 1891.

Pleurothallis funerea (Barb.Rodr.) Cogn. in C.F.P.von Martius \& auct. suc. (eds.), Fl. Bras. 3(4): 567. 1896.

Specklinia funerea (Barb.Rodr.) Luer, Monogr. Syst. Bot. Missouri Bot. Gard. 95: 260. 2004.

Panmorphia funerea (Barb.Rodr.) Luer, Monogr. Syst. Bot. Missouri Bot. Gard. 105: 156. 2006. Pleurothallis breviscapa C.Schweinf., Bot. Mus. Leafl. 3: 79. 1935.

Pleurothallis praemorsa Luer, Selbyana 2: 388. 1978. 
Anathallis breviscapa (C.Schweinf.) Pridgeon \& M.W.Chase, Lindleyana 16: 248. 2001.

Specklinia breviscapa (C.Schweinf.) Luer, Monogr. Syst. Bot. Missouri Bot. Gard. 95: 259. 2004.

Specklinia praemorsa (Luer) Luer, Monogr. Syst.

Bot. Missouri Bot. Gard. 95: 263. 2004.

VOucher: S. Laube et al. 521 (PMA).

Anathallis grayumii (Luer) Luer, Monogr. Syst. Bot. Missouri Bot. Gard. 115: 258. 2009.

Pleurothallis grayumii Luer, Lindleyana 11: 78. 1996. Specklinia grayumii (Luer) Luer, Monogr. Syst.

Bot. Missouri Bot. Gard. 95: 260. 2004.

Panmorphia grayumii (Luer) Luer, Monogr. Syst.

Bot. Missouri Bot. Gard. 105: 157. 2006.

VOUCHER: R.L. Dressler 5692 (PMA).

Anathallis lewisiae (Ames) Solano \& Soto Arenas, Icon. Orchid. 5-6: x. 2003.

Pleurothallis lewisiae Ames, Proc. Biol. Soc. Wash. 44: 42. 1931.

Specklinia lewisiae (Ames) Luer, Monogr. Syst.

Bot. Missouri Bot. Gard. 95: 261. 2004.

Panmorphia lewisiae (Ames) Luer, Monogr. Syst.

Bot. Missouri Bot. Gard. 105: 164-165. 2006.

Voucher: R.L. Dressler 2918 (SEL).

Anathallis sertularioides (Sw.) Pridgeon \&

M.W.Chase, Lindleyana 16: 250. 2001.

Epidendrum sertularioides Sw., Prodr. 122. 1788.

Dendrobium sertularioides (Sw.) Sw., Nova Acta

Regiae Soc. Sci. Upsal. 6: 83. 1799.

Specklinia sertularioides (Sw.) Lindl., Gen. Sp.

Orchid. P1. 8. 1830.

Pleurothallis sertularioides (Sw.) Spreng., Syst.

Veg. 3: 731. 1826.

Humboldtia sertularioides (Sw.) Kuntze, Revis.

Gen. P1. 2: 668. 1891.

Specklinia sertularioides (Sw.) Luer, Monogr. Syst.

Bot. Missouri Bot. Gard. 95: 263. 2004. nom. superfl.

Panmorphia sertularioides (Sw.) Luer, Monogr.

Syst. Bot. Missouri Bot. Gard. 105: 174. 2006.

VOUCHER: C. Luer \& H. Butcher 1253 (SEL).

\section{Arundina Blume}

Arundina graminifolia (D.Don) Hochr., Bull. New York Bot. Gard. 6: 270. 1910.

Bletia graminifolia D.Don, Prodr. Fl. Nepal.: 29. 1825.
Arundina bambusifolia Lindl., Gen. Sp. Orchid. P1.: 125. 1831, nom. illeg.

Cymbidium bambusifolium Roxb., Fl. Ind. ed. 1832, 3: 460. 1832.

VOUCHER: J.D. Dwyer 9511 (PMA).

\section{Aspasia Lindl.}

Aspasia epidendroides Lindl., J. Bot. (Hooker) 1: 6. 1834.

Aspasia fragrans Klotzsch, Index Seminum (B) 1853: 12.1853.

Aspasia barclayi Rolfe, Bull. Misc. Inform. Kew 1892: 210. 1892.

Odontoglossum aspasia Rchb.f. in W.G.Walpers, Ann. Bot. Syst. 6: 851. 1864.

Voucher: H. Cuming 1297 (K).

Aspasia principissa Rchb.f., Bot. Zeitung (Berlin) 10: 637. 1852.

Aspasia biberiana Rchb.f., Bonplandia (Hannover) 2: 90.1854.

Odontoglossum principissa (Rchb.f.) Rchb.f. in W.G.Walpers, Ann. Bot. Syst. 6: 852. 1864.

Odontoglossum biberianum (Rchb.f.) Rchb.f. in

W.G.Walpers, Ann. Bot. Syst. 6: 851. 1864.

Aspasia epidendroides var. principissa (Rchb.f.)

P.H.Allen, Ann. Missouri Bot. Gard. 36: 165. 1949.

Aspasia rousseauae Schltr., Gartenflora 71: 76. 1922.

VOUCHER: J. Warscewicz s.n. (W).

\section{Aspidogyne Garay}

Aspidogyne clavigera (Rchb.f.) Meneguzzo, Orquidario 26, 3: 89. 2012.

Ligeophila clavigera (Rchb.f.) Garay, Bradea 2: 195. 1977.

Physurus claviger Rchb.f., Bonplandia (Hannover) 4: 211.1856.

Erythrodes clavigera (Rchb.f.) Ames, Orchidaceae 7: 70. 1922.

VOUCHER: not seen, cited by Dressler (2003c).

Ormerod (2013) did not cite specimens from Panama. However, he cited two specimens from Costa Rica very close to the boundary with Panama in the Sixaola river basin. Dressler (2003c) stated that this species is present in Panama. 
Aspidogyne grandis (Ormerod) Ormerod, Harvard Pap. Bot. 11(2): 147. 2007.

Ligeophila grandis Ormerod, Harvard Pap. Bot. 9(2): 395-397, f. 6. 2005.

VOUCHER: P. H. Allen 4857 (MO).

Aspidogyne roseoalba (Dressler) Ormerod, Harvard Pap. Bot. 11: 150. 2007.

Erythrodes roseoalba Dressler, Orquídea (Mexico City), n.s., 13(1-2): 257, 260. 1993.

Platythelys roseoalba (Dressler) Szlach., Fragm.

Florist. Geobot., Suppl. 3: 115. 1995.

VOUCHER: R.L. Dressler 5959 (PMA).

Aspidogyne stictophylla (Schltr.) Garay, Bradea 2(28): 204. 1977.

Physurus stictophyllus Schltr., Repert. Spec. Nov.

Regni Veg. 10(248-250): 249. 1911.

Erythrodes stictophylla (Schltr.) Ames,

Orchidaceae 7: 76. 1922.

Voucher: J.P. Folsom, R.L. Dressler \& K. Dressler 5401 (MO).

Aspidogyne tuerckheimii (Schltr.) Garay, Bradea 2: 204. 1977.

Physurus tuerckheimii Schltr., Repert. Spec. Nov. Regni Veg. 2(22): 132. 1906.

Erythrodes tuerckheimii (Schltr.) Ames,

Orchidaceae 7: 77. 1922.

VOUCHER: J. P. Folsom et al. 5761 (MO).

\section{Barbosella Schltr.}

Barbosella circinata Luer, Selbyana 3: 204. 1977.

Voucher: C. Luer et al. 1575 (SEL).

Barbosella dolichorhiza Schltr., Repert. Spec. Nov. Regni Veg. Beih. 7: 117. 1920.

Masdevallia anaristella Kraenzl., Repert. Spec. Nov. Regni Veg. 17: 417. 1921.

Barbosella bradeorum Schltr., Repert. Spec. Nov. Regni Veg. Beih. 19: 111. 1923.

Pleurothallis bradeorum (Schltr.) Ames, F.T.Hubb. \& C.Schweinf., Bot. Mus. Leafl. 3: 39. 1934.

Pleurothallis anaristella (Kraenzl.) L.O.Williams, Ceiba 1: 186. 1950.

Barbosella fuscata Garay, Orquideología 4: 153. 1969.

Barbosella anaristella (Kraenzl.) Garay, Orquideología 9: 114. 1974.

Triaristellina anaristella (Kraenzl.) Rauschert,
Feddes Repert. 94: 469. 1983.

VOUCHER: R.L. Dressler 6058 (MO).

Barbosella geminata Luer, Phytologia 46(6): 345. 1980.

Voucher: T. B. Croat \& D. M. Porter 16083 (MO).

Barbosella orbicularis Luer, Selbyana 3: 10. 1976.

Voucher: R.L. Dressler 3285 (SEL).

Barbosella prorepens (Rchb.f.) Schltr., Repert. Spec. Nov. Regni Veg. 15: 263. 1918.

Restrepia prorepens Rchb. f., Gard. Chron., n.s., 7(183): 810. 1877.

Pleurothallis prorepens (Rchb. f.) Ames, F.T.Hubb. \& C.Schweinf., Bot. Mus. Leafl. 3(2): 39-40. 1934.

Pleurothallis monstrabilis Ames, Schedul. Orchid. 6: 63. 1923.

VOUCHER: R. L. Dressler 5727 (MO).

BARKeria Knowles \& Westc.

Barkeria obovata (C.Presl) Christenson, Lindleyana 3: 221.1988 [1989].

Oncidium obovatum C.Presl., Reliq. Haenk. 99. 1827.

Voucher: G.White \& P. White 100 (MO).

\section{BASKERVILLA Lindl.}

Baskervilla colombiana Garay, Svensk Bot. Tidskr. 47: 196. 1953.

Baskervilla nicaraguensis Hamer \& Garay, Icon. Pl. Trop. 7: t. 610. 1982.

VOUCHER: W.D. Stevens 18314 (PMA).

Baskervilla stenopetala Dressler, Bol. Inst. Bot. (Guadalajara) 5: 70. 1998.

VOUCHER: J. Folsom s.n. (MO).

\section{Beloglottis Schltr.}

Beloglottis costaricensis (Rchb.f.) Schltr., Beih. Bot. Centralbl. 37(2): 365. 1920.

Spiranthes costaricensis Rchb. f., Bonplandia 3(15/16): 214. 1855.

Gyrostachys costaricensis (Rchb. f.) Kuntze, Revis. Gen. P1. 2: 664. 1891.

Voucher: W. L. Stern et al. 1910 (MO).

Beloglottis subpandurata (Ames \& C.Schweinf.) Garay, Bot. Mus. Leafl. 28: 302. 1980[1982]. 
Spiranthes subpandurata Ames \& C.Schweinf.,

Schedul. Orchid. 8: 4-6, f. 1.1925.

Voucher: $C W$. Powell 377 (AMES).

\section{Benzingia Dodson}

Benzingia reichenbachiana (Schltr.) Dressler, Lankesteriana 9: 527. 2010.

Chondrorhyncha reichenbachiana Schltr., Repert. Spec. Nov. Regni Veg. 17: 15. 1921.

Chondrorhyncha lamellata Rchb.f., Ms. ined., W-Rchb Orch.

Zygopetalum lamellatum Rchb.f., Ms. ined., W-Rchb Orch.

VOUCHER: R.L. Dressler 5977 (MO).

\section{Bletia Ruiz \& Pav.}

Bletia campanulata Lex. in P.de La Llave \& J.M.de Lexarza, Nov. Veg. Descr. 2: 17. 1825.

Limodorum campanulatum (Lex.) Ames \& C.Schweinf., Schedul. Orchid. 10: 79. 1930.

Bletia anomala A.Rich. \& Galeotti, Ann. Sci. Nat., Bot., III, 3: 23. 1845.

Bletia wageneri Rchb.f., Linnaea 26: 143. 1854.

Bletia landsbergii Rchb.f., Ned. Kruidk. Arch. 4: 317. 1859.

Serapias diphylla Sessé \& Moc., Pl. Nov. Hisp.: 153. 1890.

Bletia mandonii Schltr., Repert. Spec. Nov. Regni Veg. Beih. 10: 48. 1922.

Limodorum lankesteri Ames \& C.Schweinf., Schedul. Orchid. 10: 78. 1930.

Bletia edwardsii Ames, Proc. Biol. Soc. Wash. 45: 1. 1932.

Bletia lankesteri (Ames \& C.Schweinf.) Ames, F.T.Hubb. \& C.Schweinf., Bot. Mus. Leafl. 3: 41. 1934.

Bletia altilamellata Garay, Svensk Bot. Tidskr. 47: 222. 1953.

Bletia wageneri var. cobra Garay \& Dunst., Venez.

Orchids Ill. 6: 60. 1976.

VOUCHER: I. de Blas et al. 498 (UCH).

Bletia purpurea (Lam.) A.DC., Rapp. [Not.] Pl. Rar. Genève 8: 23. 1840.

Limodorum purpureum Lam., Encycl. 3: 515. 1792.

Serapias purpurea (Lam.) Poir. in J.B.A.M.de Lamarck, Tabl. Encycl. 3: 306. 1823.
Limodorum tuberosum Jacq., Collectanea 4: 108. 1790., nom. illeg.

Limodorum verecundum Salisb., Prodr. Stirp. Chap. Allerton: 9. 1796.

Cymbidium verecundum (Salisb.) Sw., Nova Acta Regiae Soc. Sci. Upsal. 6: 75. 1799.

Limodorum trifidum Michx., Fl. Bor.-Amer. 2: 159. 1803.

Cymbidium altum Willd., Sp. P1. 4: 105. 1805.

Cymbidium trifidum (Michx.) Sw., Neues J. Bot. 1: 76. 1805.

Epidendrum altum (Willd.) Poir. in J.B.A.M.de Lamarck, Encycl., Suppl. 1: 374. 1810.

Gyas verecunda (Salisb.) Salisb., Trans. Hort. Soc. London 1: 299. 1812.

Bletia verecunda (Salisb.) R.Br. in W.T.Aiton, Hortus Kew. 5: 206. 1813.

Helleborine americana Steud., Nomencl. Bot.: 398. 1821.

Bletia pallida Lodd., Bot. Cab. 7: t. 629. 1822.

Bletia acutipetala Hook., Bot. Mag. 60: t. 3217. 1833.

Bletia havannensis Lindl., Edwards's Bot. Reg. 24: t. 28.1838.

Bletia pulchella auct., Gard. Chron. 1850: 168. 1850.

Bletia pottsii S.Watson, Proc. Amer. Acad. Arts 22: 478. 1887.

Bletia purpurea var. pittieri Schltr., Repert. Spec. Nov. Regni Veg. Beih. 17: 50. 1922.

Bletia purpurea var. alba Ariza-Julia \& J.Jiménez Alm., Rhodora 62: 236. 1960.

Voucher: D. Cáceres et al. 649 (UCH).

\section{BRACHIONIDIUM Lindl.}

Brachionidium calypso Luer, Monogr. Syst. Bot. Missouri Bot. Gard. 57: 24. 1995.

VOUCHER: R.L. Dressler 5838 (MO).

Brachionidium dentatum Luer \& Dressler, Monogr. Syst. Bot. Missouri Bot. Gard. 57: 34. 1995.

Voucher: R.L. Dressler s.n. (MO).

Brachionidium dressleri Luer, Monogr. Syst. Bot. Missouri Bot. Gard. 57: 40. 1995.

Voucher: R.L. Dressler 5837 (MO).

Brachionidium filamentosum Luer \& Hirtz, Monogr. Syst. Bot. Missouri Bot. Gard. 57: 50. 1995.

VOuCHer: C. Luer et al. 15563 (MO). 
Brachionidium folsomii Dressler, Orquideología 15: 154. 1982.

VOUCHER: R.L. Dressler 6032 (MO).

Brachionidium kuhniarum Dressler, Orquideología 15: 157. 1982.

VOUCHER: R.L. Dressler 5858 (MO).

Brachionidium lucanoideum Luer, Monogr. Syst. Bot. Missouri Bot. Gard. 57: 78. 1995.

Voucher: S. Knapp \& R. Schmalzel 4829 (MO).

Brachionidium peltarion Luer, Monogr. Syst. Bot. Missouri Bot. Gard. 57: 92. 1995.

Voucher: R.L. Dressler \& E. Hágsater 6005 (MO).

Brachionidium polypodium Luer, Monogr. Syst. Bot. Missouri Bot. Gard. 57: 98. 1995.

VOUCHER: T. Antonio 1413 (MO).

Brachionidium pusillum Ames \& C.Schweinf., Schedul. Orchid. 10: 52. 1930.

VOUCHER: C.A. Luer \& et al. 10584 (MO).

Brachionidium satyreum Luer, Monogr. Syst. Bot. Missouri Bot. Gard. 57: 112. 1995.

VOucher: R.L. Dressler, K. Dressler \& N. H. Williams 5809 (MO).

Brachionidium valerioi Ames \& C.Schweinf.,

Schedul. Orchid. 10: 53-55. 1930.

VOUCHER: C.A. Luer 19009 (MO).

\section{Brachystele Schltr.}

Brachystele guayanensis (Lindl.) Schltr., Beih. Bot. Centralbl., Abt. 2 37(2, Heft 3): 373-374. 1920.

Goodyera guayanensis Lindl., Gen. Sp. Orchid. P1. 494. 1840.

Spiranthes guayanensis (Lindl.) Cogn., Fl. Bras. 3(4): 209. 1895.

Spiranthes aguacatensis Rchb.f., Bonplandia (Hannover) 3: 214. 1855.

Spiranthes hostmannii Rchb.f. ex Griseb., Fl. Brit.

W. I.: 640. 1864.

Gyrostachys aguacatensis Kuntze, Revis. Gen. Pl. 2: 664. 1891.

Gyrostachys hostmannii (Rchb.f. ex Griseb.)

Kuntze, Revis. Gen. P1. 2: 664. 1891.

Spiranthes brenesii Schltr., Repert. Spec. Nov.

Regni Veg. 10: 481. 1912.

Brachystele aguacatensis (Rchb.f.) Schltr., Beih.

Bot. Centralbl. 37(2): 371. 1920.

Brachystele brenesii (Schltr.) Schltr., Beih. Bot.

Centralbl. 37(2): 372. 1920.

Voucher: M. E. Davidson 680 (F).
Brassavola R.Br.

Brassavola acaulis Lindl. \& Paxton, Paxton's Fl. Gard. 2: 152. 1851.

Brassavola lineata Hook. f., Bot. Mag. 79: t. 4734. 1853.

Voucher: C. W. Powell 102 (MO).

Brassavola nodosa (L.) Lindl., Gen. Sp. Orchid. Pl.: 114. 1831.

Epidendrum nodosum L., Sp. Pl.: 953. 1753.

Cymbidium nodosum (L.) Sw., Nova Acta Regiae Soc. Sci. Upsal. 6: 73. 1799.

Bletia nodosa (L.) Rchb.f. in W.G.Walpers, Ann. Bot. Syst. 6: 437. 1862.

Brassavola rhopalorrhachis Rchb.f., Bot. Zeitung (Berlin) 10: 855. 1852.

Bletia rhopalorrhachis (Rchb.f.) Rchb.f. in

W.G.Walpers, Ann. Bot. Syst. 6: 437. 1862.

Brassavola nodosa var. rhopalorrhachis Schltr., Orchis 13: 77. 1919.

Brassavola scaposa Schltr., Orchis 13: 77. 1919.

Voucher: I. de Blas et al. 507 (UCH).

Brassia R.Br.

Brassia allenii L.O.Williams ex C.Schweinf., Bot. Mus. Leafl. 13: 145. 1948.

Ada allenii (L.O.Williams ex C.Schweinf.) N.H.Williams, Brittonia 24(1): 104-105. 1972.

VOUCHer: P.H. Allen 5150 (AMES).

Brassia arcuigera Rchb.f., Gard. Chron. 1869: 388. 1869.

Voucher: P.H. Allen 2752 (AMES).

Brassia caudata (L.) Lindl., Bot. Reg. 10: t. 832. 1825. Epidendrum caudatum L., Syst. Nat. (ed. 10) 2: 1246. 1759.

Voucher: C. W. Powell 3231 (AMES).

Brassia chlorops Endrés \& Rchb.f., Gard. Chron. 1873: 542. 1873.

Ada chlorops (Endrés \& Rchb.f.) N.H.Williams, Brittonia 24: 104. 1972.

Brassia parviflora Ames \& C.Schweinf., Schedul. Orchid. 8: 74: 1925.

VOUCHER: R.E. Woodson et al. 874 (MO).

Brassia filiana M.W.Chase, Phytotaxa 20: 27. 2011.

Mesospinidium warscewiczii Rchb.f., Bot. Zeitung (Berlin) 10: 929. 1852.

Mesospinidium endresii (Kraenzl.) Garay ex H.R.Sweet, Orquideología 8: 92. 1973. 
Solenidium endresii Kraenzl. in H.G.A.Engler (ed.), Pflanzenr., IV, 50(80): 317. 1922.

VOuCher: S.A. Mori 3919 (MO).

Brassia gireoudiana Rchb.f. \& Warsz., Allg. Gartenzeitung 22: 273. 1854.

VOUCHER: D. Cáceres et al. 619 (UCH).

Brassia horichii (I.Bock) M.W.Chase, Phytotaxa 20: 28. 2011.

Mesospinidium horichii I.Bock, Orchidee (Hamburg) 42: 232. 1991.

VOUCHER: R.L. Dressler 5350 (PMA).

Brassia panamensis (Garay) M.W.Chase, Phytotaxa 20: 28. 2011.

Mesospinidium panamense Garay, Orchidee

(Hamburg) 24: 185. 1973.

VOUCHER: P.H. Allen 2785 (AMES).

\section{Bulbophyllum Thouars}

Bulbophyllum aristatum (Rchb.f.) Hemsl., Biol. Cent.-Amer., Bot. 3: 213. 1884.

Bolbophyllaria aristata Rchb.f., Beitr. Orchid.-K. C. Amer.: 60. 1866.

Phyllorkis aristata (Rchb.f.) Kuntze, Revis. Gen. P1. 2: 677. 1891.

Voucher: A.A. Hunter \& P.H. Allen 375 (AMES, $\mathrm{MO})$.

Bulbophyllum pachyrachis (A.Rich.) Griseb., Fl. Brit. W. I.: 613. 1864.

Pleurothallis pachyrachis A.Rich. in R.de la Sagra, Hist. Fis. Cuba, Bot. 11: 234. 1850.

Bolbophyllaria pachyrachis (A.Rich.) Rchb.f. in W.G.Walpers, Ann. Bot. Syst. 6: 241. 1861.

Phyllorkis pachyrachis (A.Rich.) Kuntze, Revis. Gen. Pl. 2: 677. 1891.

Pachyrhachis pineliana A.Rich., Herb. Gén. Amateur, II, 3: 23. 1845.

Bolbophyllaria oerstedii Rchb.f., Bonplandia (Hannover) 3: 223. 1855.

Bulbophyllum oerstedii (Rchb.f.) Hemsl., Biol. Cent.-Amer., Bot. 3: 213. 1884.

Phyllorkis oerstedii (Rchb.f.) Kuntze, Revis. Gen. P1. 2: 677. 1891.

Bulbophyllum vinosum Schltr., Beih. Bot. Centralbl. 36(2): 411. 1918.

Bulbophyllum wagneri Schltr., Repert. Spec. Nov. Regni Veg. 17: 142. 1921.

Voucher: M. Wagner s.n. (M).

\section{Calanthe R.Br.}

Calanthe calanthoides (A.Rich. \& Galeotti) Hamer \& Garay in F.Hamer, Orquid. El Salvador 1: 90. 1974.

Ghiesbreghtia calanthoides A.Rich. \& Galeotti, Ann. Sci. Nat., Bot., III, 3: 28. 1845.

Calanthe mexicana Rchb.f., Linnaea 18: 406. 1845.

Calanthe cubensis Linden \& Rchb.f., Bonplandia (Hannover) 4: 322. 1856.

Calanthe granatensis Rchb.f., Bonplandia (Hannover) 4: 322. 1856.

Alismorkis cubensis (Linden \& Rchb.f.) Kuntze, Revis. Gen. Pl. 2: 650. 1891.

Alismorkis granatensis (Rchb.f.) Kuntze, Revis. Gen. P1. 2: 650. 1891.

Alismorkis mexicana (Rchb.f.) Kuntze, Revis. Gen. P1. 2: 650. 1891.

Ghiesbreghtia mexicana (Rchb.f.) Schltr., Repert. Spec. Nov. Regni Veg. Beih. 19: 135. 1923.

Calanthe mexicana var. lanceolata Correll, Lloydia 10: 214. 1947[1948].

Calanthe mexicana var. retusa Correll, Lloydia 10: 214. 1947[1948].

VOUCHER: R. Villarreal et al. 391 (UCH).

Camaridium Lindl.

Camaridium adolphi Schltr., Repert. Spec. Nov. Regni Veg. Beih. 19: 141. 1923.

Maxillaria adolphi (Schltr.) Ames \& Correll, Bot. Mus. Leafl. 11: 18. 1943.

Ornithidium tonduzii Schltr., Repert. Spec. Nov. Regni Veg. 3: 250. 1907.

Voucher: B. Stein 1297 (PMA).

Camaridium allenii (L.O.Williams) M.A.Blanco, Lankesteriana 7: 519. 2007.

Maxillaria allenii L.O.Williams, Ann. Missouri Bot. Gard. 27: 282. 1940.

Adamanthus allenii (L.O.Williams) Szlach. \& Sitko, Biodivers. Res. Conservation 25: 21. 2012.

VOUCHER: P.H. Allen 1650 (MO).

Camaridium ampliflorum (C.Schweinf.) M.A.Blanco, Lankesteriana 7: 519. 2007.

Camaridium grandiflorum Ames, Proc. Biol. Soc. Wash. 34: 149. 1921., nom. illeg.

Maxillaria ampliflora C.Schweinf., Bot. Mus. Leafl. 8: 188. 1940. 
Camaridium ampliflorum (C.Schweinf.) Szlach. \& Sitko, Biodivers. Res. Conservation 25: 24. 2012.

VOUCHER: P. Killip 3565 (AMES).

Camaridium anceps (Rchb.f.) M.A.Blanco, Lankesteriana 7: 519. 2007.

Ornithidium anceps Rchb.f., Beitr. Orchid.-K. C. Amer.: 75. 1866.

Maxillaria pseudoneglecta J.T.Atwood, Lindleyana 8: 30. 1993.

Pseudomaxillaria anceps (Rchb. f.) Szlach. \& Sitko, Biodivers. Res. Conservation 25: 34. 2012.

Voucher: G. McPherson 9916 (MO).

Camaridium aurantiacum (Schltr.) M.A.Blanco, Lankesteriana 7: 519. 2007.

Ornithidium aurantiacum Schltr., Repert. Spec. Nov. Regni Veg. Beih. 19: 241. 1923.

Maxillaria bilobulata Senghas in F.R.R.Schlechter, Orchideen Beschreib. Kult. Zücht., ed. 3, I/B(28): 1751. 1993.

Maxillaria lankesteri Ames, Schedul. Orchid. 7: 11. 1924.

Maxillaria jugata Garay, Orquideología 4: 159. 1969.

Chaseopsis aurantiaca (Schltr.) Szlach. \& Sitko, Biodivers. Res. Conservation 25: 25. 2012.

Voucher: M.A. Blanco \& D. Penneys 2903 (MO).

Camaridium biolleyi (Schltr.) Schltr., Beih. Bot. Centralbl. 36(2): 498. 1918.

Ornithidium biolleyi Schltr., Repert. Spec. Nov. Regni Veg. 9: 29. 1910.

Maxillaria biolleyi (Schltr.) L.O.Williams, Ann.

Missouri Bot. Gard. 28: 425. 1941.

Adamanthus biolleyi (Schltr.) Szlach. \& Sitko, Biodivers. Res. Conservation 25: 21. 2012.

Voucher: G. McPherson 13582 (PMA).

Camaridium bracteatum (Schltr.) Schltr., Repert. Spec. Nov. Regni Veg. Beih. 19: 57. 1923.

Ornithidium bracteatum Schltr., Repert. Spec. Nov. Regni Veg. 9: 217. 1911.

Maxillaria bracteata (Schltr.) Ames \& Correll, Bot. Mus. Leafl. 11: 14. 1943.

Maxillaria vagans Ames \& C.Schweinf., Schedul. Orchid. 8: 65. 1925.

Maxillaria admonens I.Bock, Orchidee (Hamburg) 48: 105. 1997.

Chelyella bracteata (Schltr.) Szlach. \& Sitko,
Biodivers. Res. Conservation 25: 26. 2012.

VOUCHER: R. Foster et al. s.n. (PMA).

Camaridium bradeorum Schltr., Repert. Spec. Nov. Regni Veg. Beih. 19: 141. 1923.

Maxillaria bradeorum (Schltr.) L.O.Williams, Ann. Missouri Bot. Gard. 28: 425. 1941.

Maxillaria semiorbicularis Ames \& C.Schweinf., Schedul. Orchid. 8: 64. 1925.

Voucher: M.A. Blanco \& D. Penneys 2941 (PMA).

Camaridium brenesii Schltr., Repert. Spec. Nov. Regni Veg. Beih. 19: 237. 1923.

Maxillaria trilobata Ames \& Correll, Bot. Mus. Leafl. 11: 17. 1943.

VOUCHER: G. McPherson 13544 (PMA).

Camaridium brevilabium (Ames \& Correll)

M.A.Blanco, Lankesteriana 7: 5192007.

Maxillaria brevilabia Ames \& Correll, Bot. Mus. Leafl. 11: 15. 1943.

Ornithidium alfaroi Ames \& C.Schweinf., Schedul. Orchid. 10: 98. 1930.

Pseudomaxillaria brevilabia (Ames \& Correll) Szlach. \& Sitko, Biodivers. Res. Conservation 25: 34. 2012.

Voucher: G. Davidse \& W.G. D'Arcy 10189 (MO).

Camaridium costaricense Schltr., Repert. Spec. Nov. Regni Veg. 3: 250. 1907.

Maxillaria heidelbergensis Senghas in F.R.R.Schlechter, Orchideen Beschreib. Kult. Zücht., ed. 3, I/B(28): 1751. 1993.

Camaridium tonduzii Schltr., Repert. Spec. Nov. Regni Veg. 8: 571. 1910.

Maxillaria tonduzii (Schltr.) Ames \& Correll, Bot. Mus. Leafl. 11: 17. 1943.

VOUCHER: R.L. Dressler 4841 (PMA).

Camaridium ctenostachys (Rchb.f.) Schltr., Repert. Spec. Nov. Regni Veg. Beih. 19: 238. 1923.

Maxillaria ctenostachys Rchb.f., Gard. Chron. 1870: 39. 1870.

Camaridium arachnites Schltr., Repert. Spec. Nov. Regni Veg. Beih. 17: 73. 1922.

Voucher: C.W. Powell 210 (MO).

Camaridium cucullatum (Lindl.) M.A.Blanco,

Lankesteriana 7: 520. 2007.

Maxillaria cucullata Lindl., Edwards's Bot. Reg. 26: t. 12. 1840.

Maxillaria atrata Rchb.f., Beitr. Orchid.-K. C. Amer.: 31. 1866. 
Psittacoglossum cucullatum (Lindl.) Szlach. \& Sitko, Biodivers. Res. Conservation 25: 33. 2012.

Maxillaria hematoglossa A.Rich. \& Galeotti, Ann. Sci. Nat., Bot., sér. 3, 3: 24. 1845.

Maxillaria punctostriata Rchb.f., Linnaea 41: 28. 1876.

Camaridium punctostriatum (Rchb.f.) Soto Arenas, Icon. Orchid. 10: xx. 2008.

Maxillaria rubrilabia Schltr., Repert. Spec. Nov. Regni Veg. Beih. 19: 236. 1923.

VOUCHER: not seen, cited by Atwood (2003).

Camaridium dendrobioides Schltr., Beih. Bot. Centralbl. 36(2): 415. 1918.

Maxillaria dendrobioides (Schltr.) L.O.Williams, Ann. Missouri Bot. Gard. 27: 283. 1940.

Adamanthus dendrobioides (Schltr.) Szlach., Richardiana 7: 30. 2007[2006].

Camaridium jimenezii Schltr., Beih. Bot. Centralbl. 36(2): 416. 1918.

Camaridium simile Schltr., Repert. Spec. Nov. Regni Veg. Beih. 19: 239. 1923.

VouCHER: R.L. Dressler 5949 (PMA).

Camaridium dichotomum Schltr., Repert. Spec. Nov. Regni Veg. Beih. 8: 98. 1921.

Maxillaria dichotoma (Schltr.) L.O.Williams, Bot. Mus. Leafl. 9: 15. 1940.

Viracocha dichotoma (Schltr.) Szlach. \& Sitko, Biodivers. Res. Conservation 25: 36. 2012.

Voucher: T.B. Croat 27754 (MO).

Camaridium exaltatum Kraenzl., Bot. Jahrb. Syst. 37: 386. 1906.

Maxillaria exaltata (Kraenzl.) C.Schweinf., Bot. Mus. Leafl. 11: 272. 1945.

Pleuranthium exaltatum (Kraenzl.) Brieger, Die Orchideen 9(33-36) 514. 1977., nom. inval.

Adamanthus exaltatus (Kraenzl.) Szlach., Richardiana 7: 31. 2007[2006].

Calawaya exaltata (Kraenzl.) Szlach. \& Sitko, Biodivers. Res. Conservation 25: 24. 2012. nom. inval.

Voucher: M. Blanco \& D. Penneys 2917 (PMA).

Camaridium falcatum (Ames \& Correll) M.A.Blanco, Lankesteriana 7: 520. 2007.

Maxillaria falcata Ames \& Correll, Bot. Mus. Leafl. 11: 15. 1943.

Ornithidium costaricense Schltr., Repert. Spec.
Nov. Regni Veg. 8: 456. 1910.

Adamanthus costaricensis (Schltr.) Szlach. \& Sitko, Biodivers. Res. Conservation 25: 21. 2012.

Voucher: T.B. Croat 13760 (MO).

Camaridium fragrans (J.T.Atwood) M.A.Blanco, Lankesteriana 7: 520. 2007.

Maxillaria fragrans J.T.Atwood, Selbyana 22: 131. 2001.

Adamanthus fragrans (J.T. Atwood) Szlach. \& Sitko, Biodivers. Res. Conservation 25: 21. 2012.

Voucher: S. Mori \& J. Kallunki 3770 (SEL).

Camaridium gomezianum (J.T.Atwood) M.A.Blanco, Lankesteriana 7: 520. 2007.

Maxillaria gomeziana J.T.Atwood, Lindleyana 11: 202. 1996.

Chelyella gomeziana (J.T. Atwood) Szlach. \& Sitko, Biodivers. Res. Conservation 25: 26. 2012 .

VOUCHER: W.D. Stevens 18135 (MO).

Camaridium horichii (Senghas) M.A.Blanco, Lankesteriana 7: 520. 2007.

Maxillaria horichii Senghas, Orchidee (Hamburg) 28: 13.1977.

Sepalosaccus horichii (Senghas) Senghas in F.R.R.Schlechter, Orchideen Beschreib. Kult. Zücht., ed. 3, I/B(28): 1745. 1993.

Pseudomaxillaria horichii (Senghas) Szlach. \& Sitko, Biodivers. Res. Conservation 25: 34. 2012.

Voucher: R.L. Dressler 3127 (PMA).

Camaridium imbricatum Schltr., Beih. Bot. Centralbl. 36(2): 415.1918.

Ornithidium schlechterianum C.Schweinf., Bot. Mus. Leafl. 4: 94. 1937., nom. superfl.

Maxillaria wrightii var. imbricata (Schltr.) Ames \& Correll, Bot. Mus. Leafl. 11: 18. 1943.

Maxillaria schlechteriana J.T.Atwood, Lindleyana 9: 231.1994.

Viracocha imbricata (Schltr.) Szlach. \& Sitko, Biodivers. Res. Conservation 25: 36. 2012.

Voucher: M.A. Blanco \& D. Penneys 2963 (PMA).

Camaridium inauditum (Rchb.f.) M.A.Blanco, Lankesteriana 7: 520. 2007.

Maxillaria inaudita Rchb.f., Beitr. Orchid.-K. C. Amer.: 76. 1866. 
Maxillaria inaudita var. minor Ames \& C.Schweinf., Schedul. Orchid. 10: 94. 1930.

VOUCHER: G. McPherson 13655 (PMA).

Camaridium insolitum (Dressler) M.A.Blanco, Lankesteriana 7: 520. 2007.

Maxillaria insolita Dressler, Orquideología 14: 204. 1981.

Calawaya insolita (Dressler) Szlach. \& Sitko, Biodivers. Res. Conservation 25: 24. 2012.

Voucher: R.L. Dressler 4064 (US).

Camaridium lankesteri (Ames) M.A.Blanco,

Lankesteriana 7: 520. 2007.

Maxillaria quadrata Ames \& Correll, Bot. Mus. Leafl. 11: 16. 1943.

Ornithidium lankesteri Ames, Schedul. Orchid. 4: 52. 1923.

VOUCHER: B.E. Hammel 7138 (MO).

Camaridium latifolium Schltr., Repert. Spec. Nov. Regni Veg. Beih. 17: 74. 1922.

Maxillaria planicola C.Schweinf., Bot. Mus. Leafl. 8: 188.1940.

Sauvetrea planicola (C.Schweinf.) Szlach., Richardiana 7: 29. 2007.

Mapinguari planicola (C.Schweinf.) Szlach. \& Sitko, Biodivers. Res. Conservation 25: 30. 2012.

Voucher: C.W. Powell 8 (AMES).

\section{Camaridium longicolumna (J.T.Atwood)}

M.A.Blanco, Lankesteriana 7: 520. 2007.

Maxillaria longicolumna J.T.Atwood, Selbyana 22: 132. 2001.

Chelyella longicolumna (J.T. Atwood) Szlach. \& Sitko, Biodivers. Res. Conservation 25: 26. 2012.

VOUCHER: J.P. Folsom 1574 (MO).

Camaridium lutheri (J.T.Atwood) M.A.Blanco, Lankesteriana 7: 5202007.

Maxillaria lutheri J.T.Atwood, Selbyana 19: 257. 1998[1999].

Viracocha lutheri (J.T. Atwood) Szlach. \& Sitko, Biodivers. Res. Conservation 25: 36. 2012.

VOUCHER: H. Luther et al. 1068 (SEL).

Camaridium microphyton (Schltr.) M.A.Blanco, Lankesteriana 7: 520. 2007.

Maxillaria microphyton Schltr., Repert. Spec. Nov. Regni Veg. 8: 457. 1910.

Ornithidium parvulum Schltr., Repert. Spec. Nov.
Regni Veg. 9: 292. 1911.

Ornithidium pallidiflavum Schltr., Repert. Spec.

Nov. Regni Veg. Beih. 19: 143. 1923.

Maxillaria pallidiflava (Schltr.) Senghas in F.R.R.Schlechter, Orchideen Beschreib. Kult. Zücht., ed. 3, I/B(28): 1751. 1993.

Chaseopsis microphyton (Schltr.) Szlach. \& Sitko, Biodivers. Res. Conservation 25: 25. 2012.

VOuCHER: K. Systma et al. 4825 (PMA).

Camaridium minus Schltr., Beih. Bot. Centralbl. 36(2): 417. 1918.

Maxillaria minor (Schltr.) L.O.Williams, Amer. Orchid Soc. Bull. 10: 273. 1942.

Viracocha minor (Schltr.) Szlach. \& Sitko, Biodivers. Res. Conservation 25: 36. 2012.

Voucher: M.Blanco \& D. Penneys 2940 (PMA).

Camaridium monteverdense (J.T.Atwood \& Barboza) M.A.Blanco, Lankesteriana 7: 521. 2007.

Maxillaria monteverdensis J.T.Atwood \& Barboza, Lindleyana 9: 241. 1994.

Adamanthus monteverdensis (J.T. Atwood \& Barboza) Szlach. \& Sitko, Biodivers. Res. Conservation 25: 21. 2012.

VOUCHER: T.B. Croat 66483 (PMA).

Camaridium neglectum (Schltr.) M.A.Blanco, Lankesteriana 7: 521. 2007.

Ornithidium neglectum Schltr., Repert. Spec. Nov. Regni Veg. Beih. 19: 242. 1923.

Maxillaria neglecta (Schltr.) L.O.Williams, Ann. Missouri Bot. Gard. 29: 348. 1942.

Pseudomaxillaria neglecta (Schltr.) Szlach. \& Sitko, Biodivers. Res. Conservation 25: 34. 2012.

Voucher: R.L. Dressler 5153 (PMA).

Camaridium nutantiflorum Schltr., Beih. Bot. Centralbl. 36(2): 417. 1918.

Maxillaria umbratilis L.O.Williams, Ann. Missouri Bot. Gard. 28: 425. 1941

Camaridium vinosum Schltr., Repert. Spec. Nov. Regni Veg. Beih. 19: 240 (1923), nom. illeg.

Maxillaria vinosa Senghas in F.R.R.Schlechter, Orchideen Beschreib. Kult. Zücht., ed. 3, I/B(28): 1751. 1993.

Voucher: R.L. Dressler 5861 (PMA).

Camaridium obscurum (Linden \& Rchb.f.) M.A.Blanco, Lankesteriana 7: 521. 2007.

Maxillaria obscura Linden \& Rchb.f., Beitr. 
Orchid.-K. C. Amer.: 31. 1866.

Maxillaria atrata var. brachyantha Rchb.f., Beitr. Orchid.-K. C. Amer.: 78. 1866.

Psittacoglossum obscurum (Linden \& Rchb. f.)

Szlach. \& Sitko, Biodivers. Res. Conservation 25: 33. 2012.

VOUCHER: J. Linden S.n. (W).

Camaridium ochroleucum Lindl., Bot. Reg. 10: 844. 1824.

Cymbidium ochroleucum (Lindl.) Lindl., Gen. Sp. Orchid. P1.: 168. 1833.

Maxillaria camaridii Rchb.f., Hamburger GartenBlumenzeitung 19: 547. 1863.

Ornithidium album Hook., Bot. Mag. 61: t. 3306. 1834.

Maxillaria lutescens Scheidw., Allg. Gartenzeitung 7: 145.1839.

Camaridium lutescens (Scheidw.) Rchb.f. in W.G.Walpers, Ann. Bot. Syst. 6: 541. 1863.

Camaridium cyrtopodanthum Barb.Rodr., Contr. Jard. Bot. Rio de Janeiro 4: 101. 1907.

Camaridium affine Schltr., Repert. Spec. Nov. Regni Veg. Beih. 17: 72. 1922.

Camaridium amazonicum Schltr., Beih. Bot. Centralbl. 42(2): 135. 1925.

Maxillaria hoehneana P.F.Hunt, Bot. Mag. 175: t. 472. 1965.

Voucher: A. Ibáñez et al. 569 (PMA).

Camaridium paleatum (Rchb.f.) M.A.Blanco, Lankesteriana 7: 521. 2007.

Ornithidium paleatum Rchb.f., Linnaea 41: 36. 1876.

Maxillaria paleata (Rchb.f.) Ames \& Correll, Bot. Mus. Leafl. 11: 15. 1943.

Camaridium wrightii Schltr., Repert. Spec. Nov. Regni Veg. 16: 448. 1920.

Ornithidium wrightii (Schltr.) C.Schweinf., Bot. Mus. Leafl. 4: 95.1937.

Maxillaria wrightii (Schltr.) Ames \& Correll, Bot. Mus. Leafl. 11: 18. 1943.

Viracocha paleata (Rchb. f.) Szlach. \& Sitko, Biodivers. Res. Conservation 25: 36. 2012.

Voucher: B.E. Hammel 5795 (MO).

Camaridium pygmaeum M.A.Blanco, Lankesteriana 7: 521. 2007.

Ornithidium wercklei Schltr., Repert. Spec. Nov. Regni Veg. Beih. 19: 60. 1923.
Maxillaria wercklei (Schltr.) L.O.Williams, Ann. Missouri Bot. Gard. 27: 284. 1940.

Chaseopsis wercklei (Schltr.) Szlach. \& Sitko, Biodivers. Res. Conservation 25: 25. 2012.

VOUCHER: C.A. Luer 9226 (MO).

Camaridium ramonense (Schltr.) M.A.Blanco, Lankesteriana 7: 521. 2007.

Ornithidium ramonense Schltr., Repert. Spec. Nov. Regni Veg. Beih. 19: 243. 1923.

Maxillaria flava Ames, F.T.Hubb. \& C.Schweinf., Bot. Mus. Leafl. 3: 41. 1934.

Chaseopsis ramonensis (Schltr.) Szlach. \& Sitko, Biodivers. Res. Conservation 25: 25. 2012.

VOUCHER: R.L. Dressler 5441 (PMA).

Camaridium scalariforme (J.T.Atwood) M.A.Blanco, Lankesteriana 7: 521. 2007.

Maxillaria scalariformis J.T.Atwood, Selbyana 19: 257. 1998[1999].

Adamanthus scalariformis (J.T. Atwood) Szlach. \& Sitko, Biodivers. Res. Conservation 25: 21. 2012.

VOUCHER: R.L. Dressler 6050 (SEL).

Camaridium sigmoideum (C.Schweinf.) M.A.Blanco, Lankesteriana 7: 521. 2007.

Ornithidium sigmoideum C.Schweinf., Bot. Mus. Leafl. 4: 121. 1937.

Maxillaria sigmoidea (C.Schweinf.) Ames \& Correll, Bot. Mus. Leafl. 11: 16. 1943.

Viracocha sigmoidea (C.Schweinf.) Szlach. \& Sitko, Biodivers. Res. Conservation 25: 37. 2012.

Voucher: R.L. Dressler \& J. T. Atwood 6240 (MO).

Camaridium standleyi M.A.Blanco, Lankesteriana 7: 521. 2007.

Maxillaria parvilabia Ames \& C.Schweinf., Schedul. Orchid. 8: 62. 1925.

Voucher: M.E. Davidson 126 (MO).

Camaridium strumatum (Endrés \& Rchb.f.)

M.A.Blanco, Lankesteriana 7: 521. 2007.

Ornithidium strumatum Endrés \& Rchb.f., Gard. Chron., n.s., 2: 772.1874.

Maxillaria strumata (Endrés \& Rchb.f.) Ames \& Correll, Bot. Mus. Leafl. 11: 17. 1943.

Sepalosaccus strumatus (Endrés \& Rchb.f.) Garay, Caldasia 8: 525. 1962.

Sepalosaccus humilis Schltr., Repert. Spec. Nov. Regni Veg. Beih. 19: 245. 1923. 
Pseudomaxillaria strumata (Endrés \& Rchb. f.) Szlach. \& Sitko, Biodivers. Res. Conservation 25: 34. 2012.

Voucher: H.P. Butcher s.n. (SEL).

Camaridium synsepalum (J.T.Atwood) M.A.Blanco, Lankesteriana 7: 521. 2007.

Maxillaria synsepala J.T.Atwood, Selbyana 19: 260. 1998[1999].

Adamanthus synsepalus (J.T. Atwood) Szlach. \& Sitko, Biodivers. Res. Conservation 25: 22. 2012.

VOUCHER: H. van der Werff \& J. Herrera 6435 (SEL).

Camaridium tigrinum (C.Schweinf.) M.A.Blanco, Lankesteriana 7: 521. 2007.

Maxillaria tigrina C.Schweinf., Amer. Orchid Soc. Bull. 37: 409. 1968.

Chrysocycnis tigrinum (C.Schweinf.) J.T.Atwood, Icon. Pl. Trop. 14: t. 1306. 1989.

VOUCHER: A. Gentry 5881 (PMA).

Camaridium tricarinatum (J.T.Atwood) M.A.Blanco, Lankesteriana 7: 522. 2007.

Maxillaria tricarinata J.T.Atwood, Selbyana 19: 260. 1998[1999].

Adamanthus tricarinatus (J.T. Atwood) Szlach. \& Sitko, Biodivers. Res. Conservation 25: 22. 2012.

Voucher: R.L. Dressler 5810 (SEL).

Camaridium tuberculare (J.T.Atwood) M.A.Blanco, Lankesteriana 7: 522. 2007.

Maxillaria tubercularis J.T.Atwood, Lindleyana 9: 229. 1994.

Chelyella tubercularis (J.T. Atwood) Szlach. \& Sitko, Biodivers. Res. Conservation 25: 26. 2012.

VOUCHER: G. McPherson 13567 (MO).

Camaridium tutae (J.T.Atwood) M.A.Blanco, Lankesteriana 7: 522. 2007.

Maxillaria tutae J.T.Atwood, Selbyana 19: 262. 1998[1999].

Adamanthus tutae (J.T. Atwood) Szlach. \& Sitko, Biodivers. Res. Conservation 25: 22. 2012.

Voucher: S. Knapp \& K. Sytsma 2641 (MO).

Camaridium vaginale (Rchb.f.) M.A.Blanco, Lankesteriana 7: 522. 2007.

Maxillaria vaginalis Rchb.f., Beitr. Orchid.-K. C. Amer.: 77. 1866.

Camaridium wercklei Schltr., Repert. Spec. Nov.
Regni Veg. Beih. 19: 58. 1923.

Viracocha vaginalis (Rchb. f.) Szlach. \& Sitko, Biodivers. Res. Conservation 25: 37. 2012.

Voucher: C. Galdames et al. 3340 (PMA).

Camaridium valerioi (Ames \& C.Schweinf.) M.A.Blanco, Lankesteriana 7: 522. 2007.

Maxillaria valerioi Ames \& C.Schweinf., Schedul. Orchid. 10: 96. 1930.

Adamanthus valerioi (Ames \& C.Schweinf.) Szlach. \& Sitko, Biodivers. Res. Conservation 25: 22. 2012

Voucher: M. Correa et al. 2136 (PMA).

\section{Campylocentrum Benth}

Campylocentrum brenesii Schltr., Repert. Spec. Nov. Regni Veg. Beih. 19: 268. 1923.

Campylocentrum longicalcaratum Ames \& C.Schweinf., Schedul. Orchid. 10: 111-112. 1930.

Campylocentrum parvulum Schltr., Repert. Spec. Nov. Regni Veg. Beih. 19: 157.1923.

Voucher: R.E. Woodson, Jr. \& R.W. Schery 579 (MO).

Campylocentrum fasciola (Lindl.) Cogn. in C.F.P.von Martius \& auct. suc. (eds.), Fl. Bras. 3(6): 520. 1906.

Angraecum fasciola Lindl., Edward's Bot. Reg. 26: sub t. 68. 1840.

Aeranthus fasciola (Lindl.) Rchb.f., Walp. Ann. Bot. Syst. 6: 902. 1864.

Campylocentrum sullivanii Fawc. \& Rendle, J. Bot. 47: 128. 1909.

Campylocentrum lankesteri Ames, Schedul. Orchid. 4: 57. 1923.

Voucher: S. Laube et al. 376 (PMA).

Campylocentrum micranthum (Lindl.) Maury, J. Bot.

(M. Louis Morot) 3: 273. 1889.

Angraecum micranthum Lindl., Edward's Bot. Reg. 21: t. 1772. 1835.

Aeranthus micranthus (Lindley) Rchb.f., in W.G.Walpers, Ann. Bot. Syst. 6: 901.1984.

Epidorchis micrantha (Lindl.) Kuntze, Revis. Gen. P1. 2: 660. 1891.

Mystacidium micranthum (Lind1.) T. Durand \& Schinz, Consp. Fl. Afr. 5: 54. 1895.

Campylocentrum micranthum (Lindl.) Rolfe, Orchid Rev. 11(128): 245. 1903. nom. superfl.

Campylocentrum peniculus Schltr., Repert. Spec. Nov. Regni Veg. Beih. 17: 91. 1922. 
Campylocentrum tuerckheimii Schltr., Repert. Spec. Nov. Regni Veg. 10: 363. 1912.

VOUCHER: R.L. Dressler 5523 (PMA).

Campylocentrum pachyrrhizum (Rchb.f.) Rolfe, Orchid Rev. 11: 246. 1903.

Aeranthus pachyrrhizus Rchb. f., Flora 48: 279. 1865.1865.

Voucher: O. Shattuck 844 (F).

Campylocentrum panamense Ames, Orchidaceae 7: 88. 1922.

VOucher: S. Hayes 988 (NY).

Campylocentrum poeppigii (Rchb.f.) Rolfe, Orchid Rev. 11: 246. 1903.

Angraecum poeppigii Rchb.f., Linnaea 22: 858. 1850.

VOUCHER: R.L. Dressler 3774 (MO).

Campylocentrum schiedei (Rchb.f.) Benth. ex Hemsl., Biol. Cent.-Amer., Bot. 3: 292. 1884.

Angraecum schiedei Rchb.f., Linnaea 22: 857. 1850.

Aeranthes schiedei (Rchb.f.) Rchb.f. in

W.G.Walpers, Ann. Bot. Syst. 6: 901.1864.

Todaroa micrantha A. Rich \& Galeotti, Ann. Sci.

Nat., Bot., II, 3: 28. 1845. nom. illeg.

Campylocentrum acutum Schltr., Repert. Spec.

Nov. Regni Veg. Beih. 19: 268. 1923.

Voucher: R.L. Dressler 4859 (MO).

Campylocentrum tenellum Todzia, Ann. Missouri Bot. Gard. 72: 877. 1985.

VOUCHER: R.L. Dressler 3758 (CR).

Campylocentrum tyrridion Garay \& Dunst., Venez. Orchid. Ill. 2: 54-55. 1961.

Campylocentrum dressleri H.Dietr. \& M.A.Díaz, Orchidee (Hamburg) 35(1): 28-30, f. 1984.

VOucher: J. Bisse et al. s.n. (HAJB).

\section{Catasetum Rich. ex Kunth}

Catasetum bicolor Klotzsch, Allg. Gartenzeitung 22: 337. 1854.

Catasetum gongoroides Kraenzl., Repert. Spec.

Nov. Regni Veg. 27: 254. 1930.

Voucher: J.P. Folsom 3782 (MO).

Catasetum maculatum Kunth, Syn. P1. 1: 331. 1822.

Catasetum oerstedii Rchb.f., Bonplandia

(Hannover) 3: 218. 1855.

Catasetum rostratum Klinge, Trudy Imp. S.-

Peterburgsk. Bot. Sada 17(1): 134. 1898.
Catasetum brenesii Schltr., Repert. Spec. Nov. Regni Veg. Beih. 19: 136, 225. 1923.

VOUCHER: P.H. Allen 3512 (MO).

Catasetum viridiflavum Hook., Bot. Mag. 69: t. 4017. 1843.

Catasetum serratum Lindl., Edwards's Bot. Reg. 33: t. 24.1847.

VOUCHER: K. J. Sytsma 1277 (MO).

\section{Cattleya Lindl.}

Cattleya dowiana Bateman \& Rchb.f., Gard. Chron. 1866: 922.1866.

Cattleya labiata var. dowiana (Bateman \& Rchb.f.) A.H.Kent in H.J.Veitch, Man. Orchid. P1. 2: 16. 1887.

Voucher: A.G.B. Fairchild s.n. (MO).

\section{Caularthron Raf.}

Caularthron bilamellatum (Rchb.f.) R.E.Schult., Bot. Mus. Leafl. 18: 92. 1958.

Epidendrum bilamellatum Rchb.f. in W.G.Walpers, Ann. Bot. Syst. 6: 345. 1862.

Diacrium bilamellatum (Rchb.f.) Hemsl., Biol. Cent.-Amer., Bot. 3: 222. 1884.

Epidendrum bigibberosum Rchb.f. in W.G.Walpers, Ann. Bot. Syst. 6: 346. 1862.

Epidendrum indivisum Bradford ex Griseb., Fl. Brit. W. I.: 614. 1864.

Diacrium bigibberosum (Rchb.f.) Hemsl., Biol. Cent.-Amer., Bot. 3: 222. 1884.

Diacrium bicornutum var. indivisum (Bradford ex Griseb.) Cogn. in C.F.P.von Martius \& auct. suc. (eds.), Fl. Bras. 3(5): 188. 1898.

Diacrium venezuelanum Schltr., Repert. Spec. Nov. Regni Veg. Beih. 6: 41. 1919.

Diacrium bilamellatum var. reichenbachianum Schltr., Repert. Spec. Nov. Regni Veg. Beih. 17: 47. 1922.

Diacrium bivalvatulum Schltr., Repert. Spec. Nov. Regni Veg. Beih. 19: 132. 1923.

Caularthron bivalvatulum (Schltr.) H.G.Jones, Adansonia, n.s., 14: 300. 1974.

Caularthron indivisum (Bradford ex Griseb.) Garay \& Dunst., Orchids Venezuela: 107. 1979.

Caularthron bilamellatum f. indivisum (Bradford ex Griseb.) Carnevali \& I.Ramírez in 
J.A.Steyermark \& al. (eds.), Fl. Venez. Guayana

7: 273. 2003.

VOUCHER: H.A. Behr s.n. (W).

\section{Chaubardiella Garay}

Chaubardiella subquadrata (Schltr.) Garay, Orquideología 4: 149. 1969.

Kefersteinia subquadrata Schltr., Repert. Spec.

Nov. Regni Veg. Beih. 19: 300. 1923.

Chondrorhyncha subquadrata (Schltr.)

L.O.Williams, Ceiba 5: 195. 1956.

Stenia chasmatochila Fowlie, Orchid Digest 29: 347. 1965.

Chaubardiella chasmatochila (Fowlie) Garay, Orquideología 4: 148. 1969.

VOUCHER: not seen, cited by Pupulin (2010).

Chondroscaphe (Dressler) Senghas \& G.Gerlach

Chondroscaphe atrilinguis Dressler, Orquideología 22: 16. 2001.

VOUCHER: R.L. Dressler 6289 (MO).

Chondroscaphe bicolor (Rolfe) Dressler, Orquideología 22: 22. 2001.

Chondrorhyncha bicolor Rolfe, Bull. Misc. Inform. Kew 1894: 393. 1894.

Chondrorhyncha endresii Schltr., Repert. Spec. Nov. Regni Veg. 17: 14. 1921.

Chondroscaphe laevis Dressler, Orquideología 22: 20. 2001.

Chondroscaphe endresii (Schltr.) Dressler, Lankesteriana 3: 28. 2002.

Voucher: R.L. Dressler 6098 (MO).

Chondroscaphe eburnea (Dressler) Dressler, Orquideología 22: 22. 2001.

Chondrorhyncha eburnea Dressler, Orchidee

(Hamburg) 34: 224. 1983.

VOUCHER: R.L. Dressler 6070 (US).

\section{Christensonella Szlach.}

Christensonella subulifolia (Schltr.) S.Koehler, Bot.

J. Linn. Soc. 168: 467. 2012.

Maxillaria subulifolia Schltr., Repert. Spec. Nov. Regni Veg. Beih. 7: 172. 1920.

Voucher: K.P. Reading s.n. (MO).

Christensonella uncata (Lindl.) Szlach., Mytnik, Górniak \& Smiszek, Polish Bot. J. 51: 59. 2006.
Maxillaria uncata Lindl., Edwards's Bot. Reg. 23: t. 1986.1837.

Camaridium uncatum (Lindl.) Hoehne, Ind. Bibl. Pl. Col. Com. Rondon: 166. 1951.

Maxillaria nana Hook., Hooker's Icon. Pl. 4: t. 315. 1841.

Maxillaria squamata Barb.Rodr., Gen. Spec. Orchid. 1: 118. 1877.

Ornithidium squamatum (Barb.Rodr.) Barb.Rodr., Gen. Spec. Orchid. 2: 209. 1882.

Ornithidium nanum Rolfe, Bull. Misc. Inform. Kew 1894: 395. 1894.

Maxillaria stenostele Schltr., Beih. Bot. Centralbl. 36(2): 414. 1918.

Maxillaria striatella Kraenzl., Repert. Spec. Nov. Regni Veg. 24: 359. 1928.

Camaridium squamatum (Barb.Rodr.) Hoehne, Arq. Bot. Estado São Paulo, n.s., f.m., 2: 72. 1947.

Christensonella squamata (Barb.Rodr.) S.Koehler, Lankesteriana 7: 523. 2007.

VOUCHER: G. McPherson 20065 (PMA).

Chysis Lindl.

Chysis bruennowiana Rchb.f. \& Warsz., Bot. Zeitung (Berlin) 15: 157. 1857.

Chysis aurea var. maculata Hook., Bot. Mag. 77: t. 4576.1851.

Chysis costaricensis Schltr., Repert. Spec. Nov. Regni Veg. Beih. 19: 297. 1923.

Chysis maculata (Hook.) Fowlie, Orchid Digest 35: 86. 1971.

VOUCher: C.W. Powell 309 (AMES).

Chysis pluricostata Dressler, Orquideología 24(2): 142-151. 2006.

VOUCHER: M. Whitten 2968 (PMA).

Chysis violacea Dressler, Orquideología 22: 237. 2003.

VOUCHER: R.L. Dressler 6340 (MO).

Cischweinfia Dressler \& N.H.Williams

Cischweinfia dasyandra (Rchb.f.) Dressler \& N.H.Williams, Amer. Orchid Soc. Bull. 39: 991. 1970.

Trichopilia dasyandra Rchb.f., Xenia Orchid. 3: 64, t. 230.1883.

VOUCHER: N.H. Williams s.n. (MO). 
Cischweinfia nana Dressler, Selbyana 22: 9. 2001. VOUCHER: R.L. Dressler 5700 (MO).

Cischweinfia pusilla (C.Schweinf.) Dressler \& N.H.Williams, Amer. Orchid Soc. Bull. 39: 992. 1970. Aspasia pusilla C.Schweinf., Bot. Mus. Leafl. 10: 21. 1941.

Trichopilia pusilla (C.Schweinf.) Garay, Orquideología 5: 20. 1970.

Cischweinfia sheehaniae Christenson, Orchids (West Palm Beach) 72: 126. 2003.

Voucher: M.E. \& R.A. Terry 1502 (AMES).

Cischweinfia pusilla subsp. furcata Dressler \& Dalström, Selbyana 25(1): 5, 9, f. 12. 2004.

VOuCher: R.L. Dressler $6396 A$ (MO).

\section{Cheistes Rich. ex Lindl.}

Cleistes rosea Lindl., Gen. Sp. Orchid. P1.: 410. 1840. Pogonia rosea (Lindl.) Rchb.f., Xenia Orchid. 2: 89. 1865.

Epistephium monanthum Poepp. \& Endl., Nov. Gen. Sp. P1. 1: 53. 1836.

Pogonia rosea var. augusta Hoehne, Relat. Commiss. Linhas Telegr. Estratég. Matto Grosso Amazonas 5(4): 9. 1912.

VOUCHER: R.L. Dressler 2922 (PMA).

\section{Clowesia Lindl.}

Clowesia warczewitzii (Lindl. \& Paxton) Dodson, Selbyana 1: 136. 1975.

Catasetum warczewitzii Lindl. \& Paxton, Paxton's Fl. Gard. 1: 45. 1850.

Myanthus warczewitzii (Lindl. \& Paxton) Beer, Prakt. Stud. Orchid.: 272. 1854.

Catasetum scurra Rchb.f., Gard. Chron. 1872: 1003. 1872.

Voucher: G. Skinner ex J. Warscewicz s.n. (K).

\section{CoccineorChis Schltr.}

Coccineorchis bracteosa (Ames \& C.Schweinf.) Garay, Bot. Mus. Leafl. 28: 306. 1980[1982].

Stenorrhynchos bracteosum Ames \& C.Schweinf., Schedul. Orchid. 8: 6. 1925.

Spiranthes bracteosa (Ames \& C.Schweinf.) L.O.Williams, Ceiba 5: 33. 1956., nom. illeg. VOUCHER: R.L. Dressler 5395 (FLAS).
Coccineorchis cernua (Lindl.) Garay, Fl. Ecuador 9(225: 1): 237. 1978.

Stenorrhynchos cernuum Lindl., Ann. Mag. Nat. Hist. 15: 386. 1845.

Spiranthes coccinea Garay, Bot. Mus. Leafl. 21: 250. 1967.

Spiranthes corymbosa Kraenzl., Beibl. Bot. Jahrb. Syst. 117: 17. 1916.

Coccineorchis corymbosa (Kraenzl.) Schltr., Beih. Bot. Centralbl. 37(2): 434. 1920.

VOUCHER: H. van der Werff \& J. Herrera 6443 (MO).

Coccineorchis cristata Szlach., Rutk. \& Mytnik, Ann.

Bot. Fenn. 41: 482. 2004.

VOUCher: R.L. Dressler 4233 (FLAS).

Coccineorchis dressleri Szlach., Rutk. \& Mytnik, Ann. Bot. Fenn. 41: 481. 2004.

Voucher: C.A. Luer et al. 1322 (SEL).

Coccineorchis navarrensis (Ames) Garay, Bot. Mus. Leafl. 28: 306. 1980[1982].

Stenorrhynchos navarrense Ames, Schedul. Orchid. 9: 13. 1925.

Spiranthes navarrensis (Ames) L.O.Williams, Ann. Missouri Bot. Gard. 29: 337. 1942.

VOUCHER: T.M. Antonio 1972 (MO).

Coccineorchis standleyi (Ames) Garay, Bot. Mus. Leafl. 28: 306. 1980[1982].

Stenorrhynchos standleyi Ames, Schedul. Orchid. 9: 14. 1925.

Spiranthes standleyi (Ames) L.O.Williams, Ceiba 1: 128. 1950.

Voucher: G. McPherson 12854 (MO).

Coccineorchis warszewicziana Szlach., Rutk. \& Mytnik, Ann. Bot. Fenn. 41: 480. 2004.

Voucher: S.A. Mori \& J.A. Kallunki 3753 (MO).

Cochleanthes Raf.

Cochleanthes aromatica (Rchb.f.) R.E.Schult. \& Garay, Bot. Mus. Leafl. 18: 323. 1959.

Zygopetalum aromaticum Rchb.f., Bot. Zeitung (Berlin) 10: 668. 1852.

Warczewiczella aromatica (Rchb.f.) Rchb.f. in W.G.Walpers, Ann. Bot. Syst. 6: 654. 1863.

Chondrorhyncha aromatica (Rchb.f.) P.H.Allen, Ann. Missouri Bot. Gard. 36: 85. 1949.

Zygopetalum wendlandii Rchb.f., Beitr. Orchid.-K. C. Amer.: 74. 1866.

Warczewiczella wendlandii (Rchb.f.) Rchb.f. ex 
B.S.Williams, Orchid Album 3: t. 126. 1884.

Warczewiczella wendlandii var. discolor

B.S.Williams, Orchid Album 3: t. 126. 1884. Zygopetalum wendlandii discolor (B.S.Williams)

G.Nicholson, Ill. Dict. Gard. 4: 248. 1887.

Bollea wendlandii (Rchb.f.) auct., Gard. \& Forest 1: 315.1888.

Voucher: D. Solano et al. 5569 (PMA).

\section{Coelia Lindl.}

Coelia macrostachya Lindl. in G.Bentham, Pl. Hartw.: 92. 1842.

Coelia macrostachya var. genuina Rchb.f., Beitr.

Orchid.-K. C. Amer.: 41. 1866., nom. inval.

Bothriochilus macrostachyus (Lindl.)

L.O.Williams, Bot. Mus. Leafl. 8: 148. 1940.

Coelia macrostachya var. integrilabia Rchb.f.,

Beitr. Orchid.-K. C. Amer.: 41. 1866.

VOUCHER: J. Warscewicz s.n. (W).

\section{Coeliopsis Rchb.f.}

Coeliopsis hyacinthosma Rchb.f., Gard. Chron. 1872:

9, 477. 1872.

Voucher: W. Wilson Saunders s.n. (W).

Comparettia Poepp. \& Endl.

Comparettia aurea (Schltr.) M.W.Chase \& N.H.Williams, Lindleyana 21(3): 27. 2008.

Scelochilus aureus Schltr., Repert. Spec. Nov. Regni Veg. Beih. 19: 144. 1923.

Voucher: not seen, cited by Dressler (2003c).

Comparettia falcata Poepp. \& Endl., Nov. Gen. Sp. Pl. 1: 42.1836.

VOUCHER: W.G. D'Arcy \& J.J. D'Arcy 6664 (MO).

\section{Coryanthes Hook.}

Coryanthes hunteriana Schltr., Repert. Spec. Nov. Regni Veg. Beih. 17: 63. 1922.

Coryanthes powellii Schltr., Repert. Spec. Nov.

Regni Veg. Beih. 17: 641922.

Coryanthes horichiana Jenny, Orchidee (Hamburg) 37: 126. 1986.

VOUCHER: C.W. Powell 19 (MO).

Coryanthes kaiseriana G.Gerlach, Lankesteriana 8: 23. 2003.
VOUCHER: E. Olmos et al. s.n. sub BGM-N 02/2958 (M).

Coryanthes maduroana G.Gerlach, Lankesteriana 4 : 70. 2004.

Voucher: A. Maduro \& E. Olmos 512 (PMA).

Coryanthes panamensis G.Gerlach, Trop. Subtrop. Pflanzenwelt 83: 141. 1993.

VOUCHER: H.G. Seeger O-18594 (HEID).

\section{Corymborkis Thouars}

Corymborkis flava (Sw.) Kuntze, Revis. Gen. Pl. 2: 658. 1891.

Serapias flava Sw., Prodr. Veg. Ind. Occ.: 119. 1788.

Neottia flava (Sw.) Sw., Fl. Ind. Occid. 3: 1417. 1806.

Stenorrhynchos flavum (Sw.) Spreng., Syst. Veg. 3: 710. 1826.

Tomotris flava (Sw.) Raf., Fl. Tellur. 2: 89. 1837.

Chloidia flava (Sw.) Rchb.f. in W.G.Walpers, Ann. Bot. Syst. 6: 644. 1863.

Corymbis flava (Sw.) Hemsl., Biol. Cent.-Amer., Bot. 3: 297. 1884

Chloidia decumbens Lindl., Gen. Sp. Orchid. Pl.: 484. 1840.

Macrostylis decumbens (Lindl.) Rchb.f., Bonplandia (Hannover) 2: 11. 1854.

Corymbis decumbens (Lindl.) Cogn. in C.F.P.von Martius \& auct. suc. (eds.), Fl. Bras. 3(6): 276. 1895.

Tropidia decumbens (Lindl.) Schltr., Bot. Jahrb. Syst. 45: 395. 1911.

Corymborkis decumbens (Lindl.) L.O.Williams, Lilloa 5: 9. 1939.

Voucher: P.H. Allen 1009 (MO).

\section{Cranichis Sw.}

Cranichis ciliata (Kunth.) Kunth, Syn. Pl. 1: 324. 1822

Ophrys ciliata Kunth in F.W.H.von Humboldt, A.J.A.Bonpland \& C.S.Kunth, Nov. Gen. Sp. 1: 334. 1816., nom. illeg.

Cranichis schlimii Rchb.f., Linnaea 41: 19. 1876.

Cranichis atrata Schltr., Repert. Spec. Nov. Regni Veg. Beih. 7: 581920.

Cranichis pleioneura Schltr., Repert. Spec. Nov. Regni Veg. Beih. 7: 60. 1920. 
Cranichis polyblephara Schltr., Repert. Spec. Nov. Regni Veg. Beih. 7: 61. 1920.

Cranichis sororia Schltr., Repert. Spec. Nov. Regni Veg. Beih. 8: 43. 1921.

Cranichis mandonii Schltr., Repert. Spec. Nov. Regni Veg. Beih. 10: 38. 1922.

Cranichis pachnodes Løjtnant, Bot. Not. 130: 164. 1977.

Voucher: G. Davidse \& W. G. D'Arcy 10317 (MO).

Cranichis diphylla Sw., Prodr. Veg. Ind. Occ.: 120. 1788.

Cranichis guatemalensis Schltr., Repert. Spec. Nov. Regni Veg. 2: 129. 1906.

Cranichis nigrescens Schltr., Repert. Spec. Nov. Regni Veg. 10: 482. 1912.

Cranichis pittieri Schltr., Repert. Spec. Nov. Regni Veg. 11: 41. 1912.

Cranichis ovatilabia Schltr., Repert. Spec. Nov. Regni Veg. Beih. 7: 59. 1920.

Cranichis stictophylla Schltr., Repert. Spec. Nov.

Regni Veg. Beih. 7: 62. 1920.

Cranichis alfredii Schltr., Repert. Spec. Nov. Regni

Veg. Beih. 19: 82. 1923.

Voucher: D. Bogarín et al. 10748 (JBL).

Cranichis lankesteri Ames, Schedul. Orchid. 4: 5. 1923.

Voucher: S. Knapp 1500 (MO).

Cranichis muscosa Sw., Prodr. Veg. Ind. Occ.: 120. 1788.

Cranichis bradei Schltr., Anexos Mem. Inst. Butantan, Secç. Bot. 1(4): 32. 1922.

Voucher: J.P. Folsom 1585 (MO).

Cranichis polyantha Schltr., Repert. Spec. Nov. Regni Veg. Beih. 7: 61. 1920.

Cranichis polyantha var. caquetaensis Renz, Candollea 11: 262. 1948.

VOUCHER: R.L. Dressler 6225 (MO).

Cranichis wageneri Rchb.f., Linnaea 41: 19. 1876.

Cranichis subcordata Schltr., Repert. Spec. Nov. Regni Veg. 2: 130. 1906.

Cranichis pilosa Fawc. \& Rendle, J. Bot. 47: 265. 1909.

Cranichis costaricensis Schltr., Repert. Spec. Nov. Regni Veg. Beih. 19: 12. 1923.

Cranichis viereckii Ames, Schedul. Orchid. 7: 1. 1924.

VOUCHER: W. H. Lewis 418 (MO).
Crossoglossa Dressler \& Dodson

Crossoglossa bifida Dressler, Orquideología 20: 255. 1997.

VOUCHER: R.L. Dressler 3890 (MO).

Crossoglossa blephariglottis (Schltr.) Dressler ex Dodson, Native Ecuadorian Orchids 1: 148. 1993.

Microstylis blephariglottis Schltr., Repert. Spec.

Nov. Regni Veg. 12(317-321): 202. 1913.

Malaxis blephariglottis (Schltr.) Ames, Proc. Biol.

Soc. Wash. 35: 84. 1922.

VOUCHER: W.R. Maxon 5667 (AMES).

Crossoglossa elliptica Dressler, Orquideología 20: 256. 1997.

VOUCHER: J.P. Folsom s.n. (MO).

Crossoglossa fratrum (Schltr.) Dressler ex Dodson, Native Ecuadorian Orchids 1: 149. 1993.

Liparis fratrum Schltr., Repert. Spec. Nov. Regni Veg. Beih. 19: 92. 1923.

VOUCHER: P.H. Allen 1528 (MO).

Crossoglossa tipuloides (Lindl.) Dodson, Native Ecuadorian Orchids 1: 149. 1993.

Microstylis tipuloides Lindl., Ann. Mag. Nat. Hist. 15: 256. 1845.

Malaxis tipuloides (Lindl.) Kuntze, Revis. Gen. Pl. 2: 673. 1891.

Liparis tipuloides (Lindl.) Schltr., Repert. Spec. Nov. Regni Veg. Beih. 19: 91. 1923.

VOUCHER: R.L. Dressler 4830 (MO).

\section{Cryptarrhena R.Br.}

Cryptarrhena guatemalensis Schltr., Repert. Spec. Nov. Regni Veg. 10: 253. 1911.

VOUCHER: S. Laube et al. 436 (PMA).

Cryptarrhena lunata R.Br., Bot. Reg. 2: t. 153. 1816.

VOUCHER: B.E. Hammel et al. 16585 (MO).

\section{Cryptocentrum Benth.}

Cryptocentrum caespitosum Carnevali, Harvard Pap. Bot. 5: 472. 2001.

Voucher: P.H. Allen 1911 (US).

Cryptocentrum calcaratum (Schltr.) Schltr., Orchideen: 449. 1914.

Pittierella calcarata Schltr., Repert. Spec. Nov. Regni Veg. 3: 81. 1906.

Cryptocentrum costaricense Schltr., Repert. Spec. Nov. Regni Veg. 10: 389. 1912. 
VOUCHER: T.B. Croat 49826 (MO).

Cryptocentrum latifolium Schltr., Repert. Spec. Nov. Regni Veg. Beih. 19: 247. 1923.

Cryptocentrum latifolium var. brachypetalum Garay, Svensk Bot. Tidskr. 47: 228. 1953.

Voucher: J.P. Folsom et al. 5563 (MO).

Cryptocentrum lehmannii (Rchb.f.) Garay, Bot. Mus. Leafl. 18: 209. 1958.

Aeranthes lehmannii Rchb.f., Otia Bot. Hamburg.: 10. 1878.

Epidorkis lehmannii (Rchb.f.) Kuntze, Revis. Gen. P1. 2: 660. 1891.

Campylocentrum lehmannii (Rchb.f.) Schltr., Repert. Spec. Nov. Regni Veg. Beih. 8: 164. 1921.

Cryptocentrum jamesonii Benth. in G.Bentham \& J.D.Hooker, Gen. P1. 3: 557. 1883.

Cryptocentrum gracilipes Schltr., Repert. Spec. Nov. Regni Veg. Beih. 19: 246. 1923.

VOUCHER: R.L. Dressler 5245 (MO).

Cryptocentrum misasii P.Ortiz \& Carnevali, Phytotaxa 56: 2. 2012.

Voucher: J. Polanco 2926 (PMA).

Cryptocentrum roseans (Schltr.) A.D.Hawkes, Orchid J. 2: 379. 1953.

Anthosiphon roseans Schltr., Repert. Spec. Nov.

Regni Veg. Beih. 7: 183-184. 1920.

VOUCHER: M. Whitten 2554 (FLAS).

The Voucher of this species is from a cultivated plant at Finca Dracula ostensibly collected in Panama.

Cryptocentrum standleyi Ames, Schedul. Orchid. 9: 55. 1925.

Cryptocentrum longiscapum Brieger, Bot. Jahrb. Syst. 97: 568. 1977.

VOUCHER: M. Blanco 2827 (PMA).

\section{Cuitlauzina Lex.}

Cuitlauzina convallarioides (Schltr.) Dressler \& N.H.Williams, Selbyana 24: 44. 2003.

Osmoglossum convallarioides Schltr., Repert. Spec. Nov. Regni Veg. Beih. 19: 148. 1923. Odontoglossum convallarioides (Schltr.) Ames \& Correll, Bot. Mus. Leafl. 11: 19. 1943.

VOUCHER: S. Knapp 1482 (MO).

Cuitlauzina egertonii (Lindl.) Dressler \& N.H.Williams, Selbyana 24: 44. 2003.

Odontoglossum egertonii Lindl., Edwards's Bot.
Reg. 31(Misc.): 50. 1845.

Oncidium egertonii (Lindl.) Beer, Prakt. Stud. Orchid.: 285. 1854.

Osmoglossum egertonii (Lindl.) Schltr., Repert. Spec. Nov. Regni Veg. Beih. 17: 79. 1922.

Odontoglossum candidum Linden \& André, Ill. Hort. 22: 58. 1875.

Osmoglossum acuminatum Schltr., Repert. Spec. Nov. Regni Veg. Beih. 17: 97. 1922.

Osmoglossum anceps Schltr., Repert. Spec. Nov.

Regni Veg. Beih. 19: 147. 1923.

Osmoglossum candidum (Linden \& André) Garay, Bot. Mus. Leafl. 26: 28. 1978.

VOUCHER: C.W. Powell 255 (AMES).

Cyclopogon C.Presl.

Cyclopogon comosus (Rchb.f.) Burns-Bal. \& E.W.Greenw., Orquídea (Mexico City), n.s., 10: 92. 1986.

Spiranthes comosa Rchb.f., Linnaea 18: 408. 1845. Gyrostachys comosa (Rchb.f.) Kuntze, Revis. Gen. P1. 2: 664. 1891.

Beadlea comosa (Rchb.f.) Hamer \& Garay in F.Hamer, Orquid. El Salvador 3: 48. 1981.

Voucher: H. Butcher s.n. (FLAS).

Cyclopogon dressleri Szlach., Novon 4: 172. 1994.

Cocleorchis dressleri (Szlach.) Szlach., Fragm.

Florist. Geobot. 39: 558. 1994.

Cocleorchis sarcoglottidis Szlach., Fragm. Florist. Geobot. 39: 559. 1994.

VOUCHER: R.L. Dressler 5986 (FLAS).

Cyclopogon elatus (Sw.) Schltr., Repert. Spec. Nov. Regni Veg. Beih. 6: 53. 1919.

Satyrium elatum Sw., Prodr. Veg. Ind. Occ.: 119. 1788.

Neottia elata (Sw.) Sw., Neues J. Bot. 1: 51. 1805.

Ibidium elatum (Sw.) Salisb., Trans. Hort. Soc. London 1: 291. 1812.

Spiranthes elata (Sw.) Rich., De Orchid. Eur.: 37. 1817.

Sauroglossum richardii Ames, Orchidaceae 1: 44. 1905.

Beadlea elata (Sw.) Small ex Britton, Brooklyn Bot. Gard. Mem. 1: 38. 1918.

Neottia minor Jacq., Collectanea 3: 172. 1791.

Spiranthes elata var. humilis Rchb.f., Linnaea 25: 235. 1852. 
Gyrostachys haenkeana Kuntze, Revis. Gen. P1. 2: 663. 1891.

Gyrostachys minor (Jacq.) Kuntze, Revis. Gen. Pl. 2: 663. 1891.

Spiranthes elata var. foliosa Cogn. in C.F.P.von Martius \& auct. suc. (eds.), Fl. Bras. 3(4): 192. 1895.

Spiranthes elata var. longipetiolata Cogn. in C.F.P.von Martius \& auct. suc. (eds.), Fl. Bras. 3(4): 192. 1895.

Spiranthes elata var. minutiflora Cogn. in C.F.P.von Martius \& auct. suc. (eds.), Fl. Bras. 3(4): 192. 1895.

Spiranthes elata var. ovata Cogn. in C.F.P.von Martius \& auct. suc. (eds.), Fl. Bras. 3(4): 192. 1895.

Spiranthes elata var. parvifolia Cogn. in C.F.P.von Martius \& auct. suc. (eds.), Fl. Bras. 3(4): 192. 1895.

Spiranthes variegata Kraenzl., Bot. Jahrb. Syst. 37: 392. 1906., nom. illeg.

Cyclopogon densiflorus Schltr., Repert. Spec. Nov. Regni Veg. Beih. 9: 59. 1921.

Voucher: C. Calderón et al. 827 (PMA).

Cyclopogon laxiflorus Ekman \& Mansf., Ark. Bot. 22A(8): 11. 1929.

Spiranthes laxiflora (Ekman \& Mansf.) Jiménez, Phytologia 8: 326. 1962.

Beadlea laxiflora (Ekman \& Mansf.) Garay, Bot. Mus. Leafl. 28: 300. 1980[1982].

Voucher: M.E. Davidson 1365 (F).

Cyclopogon miradorensis Schltr., Repert. Spec. Nov. Regni Veg. 21: 332. 1925.

Beadlea miradorensis (Schltr.) Garay \& Dunst., Orchids Venezuela: 26. 1979.

VOUCHER: B.E. Hammel 922 (MO).

Cyclopogon plantagineus Schltr., Beih. Bot. Centralbl. 37(2): 393. 1920.

Spiranthes plantaginea Lindl., Gen. Sp. Orchid. P1.: 468. 1840, nom. illeg.

Beadlea plantaginea (Schltr.) Garay, Fl. Ecuador 9(225: 1): 272. 1978.

VOUCHER: G. McPherson 10575 (MO).

Cyclopogon prasophylloides (Garay) Mora-Ret. \& J.B. García, Brenesia 37: 80-81.1992.

Cyclopogon prasophylloides (Garay) Szlach., Candollea 48: 435. 1993.
Beadlea prasophylloides Garay, Bot. Mus. Leafl. 28: 301. 1980[1982].

Spiranthes prasophylla var. cleistogama Ames \& Correll, Bot. Mus. Leafl. 10: 65. 1942.

VOUCHER: G. McPherson 7966 (MO).

Cyclopogon prasophyllum (Rchb.f.) Schltr., Beih. Bot. Centralbl. 37(2): 393. 1920.

Spiranthes prasophylla Rchb.f., Beitr. Orchid.-K. C. Amer.: 65. 1866.

Gyrostachys prasophylla (Rchb.f.) Kuntze, Revis. Gen. P1. 2: 664. 1891.

Beadlea prasophylla (Rchb.f.) Hamer \& Garay in F.Hamer, Orquid. El Salvador 3: 27. 1981.

Spiranthes epiphytica Schltr., Repert. Spec. Nov. Regni Veg. 2: 130. 1906.

VOUCHER: R.L. Dressler 3257 (FLAS).

Cyclopogon storeri (Chapm.) Szlach., Mytnik \& Rutk., Genus, Suppl.: 58. 2003.

Spiranthes storeri Chapm., Fl. South. U.S., ed. 2, Suppl. 2: 696. 1892.

Beadlea storeri (Chapm.) Small, Fl. S.E. U.S.: 319. 1903.

Sauroglossum nigricans Schltr., Beih. Bot. Centralbl. 36(2): 379. 1918.

VOUCHER: R.L. Dressler 3330 (FLAS).

\section{Cycnoches Lindl.}

Cycnoches aureum Lindl. \& Paxton, Paxton's Fl. Gard. 3: t. 75. 1852.

Cycnoches egertonianum var. aureum (Lindl. \& Paxton) P.H.Allen, Orchid J. 1: 351. 1952.

Voucher: P.H. Allen 3628 (MO).

Cycnoches chlorochilon Klotzsch, Allg. Gartenzeitung 6: 225.1838.

Cycnoches ventricosum var. chlorochilon (Klotzsch) P.H.Allen, Orchid J. 1: 401.1952.

VOUCHER: P.H. Allen 65 (AMES).

Cycnoches dianae Rchb.f., Bot. Zeitung (Berlin) 10: 636. 1852.

VOUCHER: J. Warszewicz s.n. (W).

Cycnoches egertonianum Bateman, Orchid. Mexico Guatemala: t. 40. 1842.

Voucher: C. Galdames et al. 4034 (PMA).

Cycnoches guttulatum Schltr., Repert. Spec. Nov. Regni Veg. Beih. 17: 56. 1922.

Voucher: C.W. Powell 14 (MO).

Cycnoches pachydactylon Schltr., Repert. Spec. Nov. 
Regni Veg. Beih. 17: 57. 1922.

Voucher: C.W. Powell 159 (AMES).

Cycnoches powellii Schltr., Repert. Spec. Nov. Regni

Veg. Beih. 17: 58. 1922.

Voucher: C.W. Powell 111 (AMES).

Cycnoches stenodactylon Schltr., Repert. Spec. Nov. Regni Veg. Beih. 17: 59. 1922.

Voucher: C.W. Powell 173 (AMES).

Cycnoches warszewiczii Rchb.f., Bot. Zeitung (Berlin) 10: 734.1852.

Cycnoches ventricosum var. warszewiczii (Rchb.f.)

P.H.Allen, Orchid J. 1: 401. 1952.

Cycnoches tonduzii Schltr., Repert. Spec. Nov.

Regni Veg. Beih. 19: 298. 1923.

VOUCHER: J. Warszewicz s.n. (W).

Cyrtochiloides N.H.Williams \& M.W.Chase

Cyrtochiloides ochmatochila (Rchb.f.) N.H.Williams \& M.W.Chase, Lindleyana 16: 285. 2001.

Oncidium ochmatochilum Rchb.f., Bot. Zeitung (Berlin) 10: 698. 1852.

Oncidium cardiochilum Lindl., Fol. Orchid. 6: 27. 1855.

Odontoglossum cardiochilum (Lindl.) Kraenzl. in H.G.A.Engler (ed.), Pflanzenr., IV, 50(80): 288. 1922.

Oncidium chelidon Kraenzl. in H.G.A.Engler (ed.), Pflanzenr., IV, 50(80): 216. 1922.

Oncidium chelidonizon Kraenzl. in H.G.A.Engler (ed.), Pflanzenr., IV, 50(80): 217. 1922.

Cyrtochiloides cardiochila (Lindl.) N.H.Williams

\& M.W.Chase, Lindleyana 16: 285. 2001.

VOUCHER: R.L. Dressler 5466 (MO).

Cyrtochiloides panduriformis (Ames \& C.Schweinf.) N.H.Williams \& M.W.Chase, Lindleyana 16: 285. 2001.

Oncidium panduriforme Ames \& C.Schweinf., Schedul. Orchid. 8: 77. 1925.

VOUCHER: R.E. Woodson \& R.W. Schery 704 (AMES).

\section{Cyrtopodium R.Br.}

Cyrtopodium macrobulbon (Lex.) G.A.Romero \& Carnevali, Harvard Pap. Bot. 4: 331. 1999.

Epidendrum macrobulbon Lex. in P.de La Llave \& J.M.de Lexarza, Nov. Veg. Descr. 2(Orchid. Opusc.): 42. 1825.
Cyrtopodium punctatum var. salvadorense Hamer \& Garay in F.Hamer, Orquid. El Salvador 3: 84. 1981.

Cyrtopodium macrobulbon $\mathrm{f}$. salvadorense (Hamer \& Garay) G.A.Romero \& Carnevali, Harvard Pap. Bot. 4: 336. 1999.

VOUCHER: Mr. Walker s.n. sub P.H. Allen 3542 (AMES).

\section{Daiotyla Dressler}

Daiotyla albicans (Rolfe) Dressler, Lankesteriana 5: 92. 2005.

Chondrorhyncha albicans Rolfe, Bull. Misc. Inform. Kew 40: 195. 1898.

Voucher: R.L. Dressler s.n. (MO).

Daiotyla crassa (Dressler) Dressler, Lankesteriana 5: 92. 2005.

Chondrorhyncha crassa Dressler, Orchidee (Hamb.) 34: 222. 1983.

VOUCHER: R.L. Dressler 6055 (US).

\section{Dichaea Lindl.}

Dichaea costaricensis Schltr., Repert. Spec. Nov. Regni Veg. Beih. 19: 73. 1923.

VOUCHER: R.L. Dressler 5365 (PMA).

Dichaea cryptarrhena Rchb.f. ex Kraenzl., Pflanzenr. IV. 50(Heft 83): 36. 1923.

Dichaea verrucosa Ames \& C.Schweinf., Schedul. Orchid. 8: 83. 1925.

Dichaea ovatipetala Schltr., Repert. Spec. Nov. Regni Veg. Beih. 19: 266. 1923.

VOUCHER: N. Williams s.n. (MO).

Dichaea dammeriana Kraenzl., Pflanzenr. IV. 50(Heft 83): 41.1923.

VOUCHER: N. Williams s.n. (MO).

Dichaea dressleri Folsom, Novon 16: 336. 2006.

Voucher: J.P. Folsom 11278A (PMA).

Dichaea eligulata Folsom, Orchid Digest 58(4): 185186. 1994.

VOUCHER: J.P. Folsom 10261 (MO).

Note: Type collected in Costa Rica or adjacent Panama (Folsom 1994).

Dichaea elliptica Dressler \& Folsom, Lankesteriana 3(2): 25-26. 2002.

Voucher: R.L. Dressler 5641 (MO).

Dichaea filiarum Pupulin, Vanishing Beauty, Native Costa Rican Orchids 1: 206. 2005. 
VOUCHER: D. Bogarín 10971 (UCH).

Dichaea fragrantissima Folsom, Orchid Digest 58: 99. 1994.

VOUCHER: J.P. Folsom 11283 NN (PMA).

Dichaea fragrantissima subsp. eburnea Dressler \& Pupulin, Novon 16(3): 340, f. 3. 2006.

Voucher: R.L. Dressler 4838 (MO).

Dichaea glauca (Sw.) Lindl., Gen. Sp. Orchid. P1. 209. 1833.

Epidendrum glaucum Sw., Prodr. Veg. Ind. Occ.: 124. 1788.

Cymbidium glaucum (Sw.) Sw., Nova Acta Regiae Soc. Sci. Upsal. 6: 71. 1799.

Dichaeopsis glauca (Sw.) Schltr., Beih. Bot. Centralbl. 36(2): 519. 1918.

Dichaea oerstedii Rchb.f., Bonplandia (Hannover) 3: 219. 1855.

Epithecia oerstedii (Rchb.f.) Schltr., Orchis 8: 101. 1914.

Dichaeopsis oerstedii (Rchb.f.) Schltr., Beih. Bot. Centralbl. 36(2): 519. 1918.

Dichaea willdenowiana Kraenzl. in H.G.A.Engler (ed.), Pflanzenr., IV, 50(83): 46. 1923.

Voucher: A. Maduro \& E. Olmos 302 (MO).

Dichaea globosa Dressler \& Pupulin, Novon 16(3): 340-343. 2006.

VOuCHER: A. Maduro \& J. Nuñez 220 (MO).

Dichaea hystricina Rchb. f., Flora 48(18): 279. 1865.

Dichaea ciliolata Rolfe, Bull. Misc. Inform. Kew 1917: 83. 1917.

Dichaea selaginella Schltr., Repert. Spec. Nov. Regni Veg. Beih. 7: 202. 1920.

Dichaea lycopodioides Rchb.f. ex Kraenzl. in H.G.A.Engler (ed.), Pflanzenr., IV, 50(83): 35. 1923.

Voucher: R.L. Dressler 5150 (MO).

Dichaea lankesteri Ames, Sched. Orch. 4: 56-57. 1923.

VOUCHER: R.L. Dressler 5103 (MO).

Dichaea morrisii Fawc. \& Rendle, J. Bot. 48(568): 107-108. 1910.

Epithecia morrisii (Fawc. \& Rendle) Schltr., Orchis 9: 26. 1915.

Dichaeopsis morrisii (Fawc. \& Rendle) Schltr. in I.Urban, Symb. Antill. 8: 146. 1920.

Cymbidium muricatum Sw., Nova Acta Regiae Soc. Sci. Upsal. 6: 71. 1799., nom. utique rej.
Epidendrum muricatum (Sw.) Poir. in J.B.A.M.de Lamarck, Encycl., Suppl. 1: 368. 1810.

Dichaea muricata (Sw.) Lindl., Gen. Sp. Orchid. P1.: 209. 1833.

Dichaea muricata var. moritzii (Rchb.f.) Cogn. in C.F.P.von Martius \& auct. suc. (eds.), Fl. Bras. 3(6): 488. 1906.

Dichaea bradeorum Schltr., Repert. Spec. Nov. Regni Veg. Beih. 19: 154. 1923.

Voucher: R.L. Dressler 5338 (MO).

Dichaea muyuyacensis Dodson, Orquideología 19: 109. 1993.

Dichaea tuberculilabris Folsom, Orchid Digest 58: 95. 1994.

VOUCHER: J.P. Folsom 11301J (TEX).

Dichaea obovatipetala Folsom, Orchid Digest 58(4): 186-187, f.. 1994.

Voucher: J.P. Folsom 11302M (PMA).

Dichaea oxyglossa Schltr., Repert. Spec. Nov. Regni Veg. Beih. 19: 267. 1923.

VOUCHER: R.L. Dressler 5478 (MO).

Dichaea panamensis Lindl., Gen. Sp. Orchid. Pl. 209. 1833.

Epithecia panamensis (Lindl.) Schltr., Orchis 9: 25. 1915.

Dichaeopsis panamensis (Lindl.) Schltr., Beih. Bot. Centralbl., Abt. 2 36(2): 519. 1918.

Dichaea brachypoda Rchb.f., Beitr. Orchid.-K. C. Amer.: 78. 1866.

Epithecia brachypoda (Rchb. f.) Schltr., Orchis 9: 25. 1915.

Dichaeopsis brachypoda (Rchb. f.) Schltr., Beih.

Bot. Centralbl. 36(2): 519. 1918.

VOuCHer: H. Cuming 1292 (K).

Dichaea pendula (Aubl.) Cogn., Symb. Antill. 4: 182183. 1903.

Limodorum pendulum Aubl., Hist. Pl. Guiane 2: 819. 1775.

Epidendrum echinocarpon Sw., Prodr. Veg. Ind. Occ.: 124. 1788.

Cymbidium echinocarpon (Sw.) Sw., Nova Acta Regiae Soc. Sci. Upsal. 6: 71. 1799.

Pachyphyllum echinocarpon (Sw.) Spreng., Syst. Veg. 3: 731. 1826.

Dichaea echinocarpa (Sw.) Lindl., Gen. Sp. Orchid. P1.: 208. 1833., nom. illeg.

Dichaea pendula var. ciliata Cogn. in C.F.P.von 
Martius \& auct. suc. (eds.), Fl. Bras. 3(6): 487. 1906.

Dichaea echinocarpa var. lobata Ames \& Correll, Bot. Mus. Leafl. 11: 71. 1943.

Dichaea lobata (Ames \& Correll) L.O.Williams, Ceiba 1: 189. 1950.

Dichaea pendula var. swartzii C.Schweinf., Bot. Mus. Leafl. 77: 62. 1955.

Dichaea swartzii (C.Schweinf.) Garay \&

H.R.Sweet, J. Arnold Arbor. 53: 397. 1972.

Voucher: R.L. Dressler 3625 (MO).

Dichaea poicillantha Schltr., Repert. Spec. Nov. Regni Veg. Beih. 19: 73. 1923.

VOUCHER: N. Williams 463 (MO).

Dichaea retroflexiligula Folsom, Orchid Digest 58: 187. 1994.

VOUCHER: J.P. Folsom 11301K (PMA).

Dichaea squarrosa Lindl., Ann. Nat. Hist. 4: 384. 1840.

Dichaea suaveolens Kraenzl. in H.G.A.Engler

(ed.), Pflanzenr., IV, 50(83): 39. 1923.

Voucher: R.E. Woodson et al. 834 (K).

Dichaea trichocarpa (Sw.) Lindl., Gen. Sp. Orchid. P1.: 209. 1833.

Epidendrum trichocarpum Sw., Prodr. Veg. Ind. Occ.: 124. 1788.

Cymbidium trichocarpon (Sw.) Sw., Nova Acta Regiae Soc. Sci. Upsal. 6: 71. 1799.

Voucher: William L. Stern 1109 (MO).

Dichaea trulla Rchb. f., Beitr. Orchid.-K. C. Amer. 104. 1866.

Epithecia trulla (Rchb.f.) Schltr., Orchis 9: 26. 1915.

Dichaeopsis trulla (Rchb.f.) Schltr., Beih. Bot. Centralbl. 36(2): 519. 1918.

Dichaea coriacea Barb.Rodr., Gen. Spec. Orchid. 2: 181.1882.

Epithecia coriacea (Barb.Rodr.) Schltr., Orchis 9: 25. 1915.

Dichaea powellii Schltr., Repert. Spec. Nov. Regni Veg. Beih. 17: 90. 1922.

Dichaea brenesii Schltr., Repert. Spec. Nov. Regni Veg. Beih. 19: 264. 1923.

Voucher: J.P. Folsom 2869 (MO).

Dichaea tuerckheimii Schltr., Orchis 10(8): 188-190, t. 44, 17-24. 1916.

Dichaea guatemalensis Schltr., Orchis 10: 190. 1916.
Dichaea glabrescens Kraenzl. in H.G.A.Engler (ed.), Pflanzenr., IV, 50(83): 44. 1923.

Dichaea wercklei Schltr., Repert. Spec. Nov. Regni Veg. Beih. 19: 74. 1923.

Voucher: S. Laube et al. SL520 (PMA).

Dichaea violacea Folsom, Orchid Digest 58: 190. 1994.

VOUCHER: J.P. Folsom 11283MM (PMA).

\section{Dimerandra Schltr.}

Dimerandra emarginata (G.Mey.) Hoehne, Bol. Agric. (São Paulo) 34: 618. 1933[1934].

Oncidium emarginatum G.Mey., Prim. Fl. Esseq.: 259. 1818.

Epidendrum lamellatum Westc. ex Lindl., Edwards's Bot. Reg. 29(Misc.): 46. 1843.

Dimerandra lamellata (Westc. ex Lindl.) Siegerist ex C.W.Ham., Icon. P1. Trop. 13: 1213. 1985.

Epidendrum stenopetalum Hook., Bot. Mag. 62: t. 3410. 1835.

Caularthron umbellatum Raf., Fl. Tellur. 2: 41. 1837., nom. superfl.

Dimerandra stenopetala (Hook.) Schltr., Repert. Spec. Nov. Regni Veg. Beih. 17: 44. 1922.

Isochilus elegans Focke, Tijdschr. Natuurk. Wetensch. Kunsten 4: 68. 1851.

Dimerandra elegans (Focke) Siegerist, Bot. Mus. Leafl. 30: 207. 1986.

Dimerandra isthmii Schltr., Repert. Spec. Nov. Regni Veg. Beih. 17: 44. 1922.

Dimerandra major Schltr., Repert. Spec. Nov. Regni Veg. Beih. 27: 136. 1924.

Dimerandra latipetala Siegerist, Bot. Mus. Leafl. 30: 211. 1986.

Dimerandra emarginata var. alba L.C.Menezes, Bol. CAOB 33: 69. 1998.

Dimerandra emarginata f. alba (L.C.Menezes) F.Barros \& J.A.N.Bat., Orquidologia SulAmer.: 102. 2004.

Voucher: C.W. Powell 17 (MO).

\section{Dinema Lindl.}

Dinema polybulbon (Sw.) Lindl., Gen. Sp. Orchid. P1.: 111. 1831

Epidendrum polybulbon Sw., Prodr. Veg. Ind. Occ.: 
124. 1788.

Encyclia polybulbon (Sw.) Dressler, Brittonia 13: 265. 1961.

Bulbophyllum occidentale Spreng., Syst. Veg. 3: 732. 1826.

Epidendrum polybulbon var. luteoalbum Miethe, Orchis 8: 33. 1914.

Epidendrum cubincola Borhidi, Acta Bot. Acad. Sci. Hung. 22: 295. 1976. [1977].

Dinema cubincola (Borhidi) H.Dietr., Wiss. Z. Friedrich-Schiller-Univ. Jena, Math.Naturwiss. Reihe 29: 524. 1980.

VOUCHER: R.L. Dressler 6218 (PMA).

This species is known from Mexico to Nicaragua, Antilles and Panama. The voucher cited here was prepared from a cultivated plant at Finca Dracula ostensibly collected in Panama.

\section{Diodonopsis Pridgeon \& M.W.Chase}

Diodonopsis erinacea (Rchb.f.) Pridgeon \& M.W.Chase, Lindleyana 16: 253. 2001.

Masdevallia erinacea Rchb.f., Linnaea 41: 11. 1876.

Scaphosepalum erinaceum (Rchb.f.) Schltr., Repert. Spec. Nov. Regni Veg. Beih. 7: 219. 1920.

Masdevallia echinocarpa Schltr., Repert. Spec. Nov. Regni Veg. Beih. 7: 78. 1920.

Masdevallia horrida Teusch. \& Garay, Amer.

Orchid Soc. Bull. 29: 23. 1960.

Voucher: R.L. Dressler 5377 (PMA).

\section{Discyphus Schltr.}

Discyphus scopulariae (Rchb.f.) Schltr., Repert. Spec. Nov. Regni Veg. 15: 417. 1919.

Spiranthes scopulariae Rchb.f., Bonplandia (Hannover) 2: 11. 1854.

Gyrostachys scopulariae (Rchb.f.) Kuntze, Revis.

Gen. P1. 2: 664. 1891.

VOUCHER: R.J. Seibert 528 (MO).

\section{Dracula Luer}

Dracula agnosia A.Doucette, Phytotaxa 56: 23. 2012. Voucher: A. Doucette 4011 (BH).

Dracula erythrochaete (Rchb.f.) Luer, Selbyana 2: 195. 1978.

Masdevallia erythrochaete Rchb.f., Gard. Chron., n.s., 18: 392. 1882.

Masdevallia gaskelliana Rchb.f., Gard. Chron., n.s., 20: 294. 1883.

Masdevallia erythrochaete var. gaskelliana (Rchb.f.) Woolward, Masdevallia: 11. 1886.

Dracula gaskelliana (Rchb.f.) Luer, Selbyana 2: 195. 1978.

Voucher: C.A. Luer 1383 (SEL).

Dracula immunda A.Doucette, Phytotaxa 16: 38. 2011.

Voucher: A. Doucette 3389 (BH).

Dracula maduroi Luer, Monogr. Syst. Bot. Missouri Bot. Gard. 95: 234. 2004.

Voucher: A. Maduro \& E. Olmos 410 (MO).

Dracula olmosii Luer \& Maduro, Monogr. Syst. Bot. Missouri Bot. Gard. 76: 136. 1999.

VOUCHer: C.A. Luer 18990 (MO).

Dracula pusilla (Rolfe) Luer, Selbyana 2: 196. 1978.

Masdevallia pusilla Rolfe, Bull. Misc. Inform. Kew 1893: 335. 1893.

Masdevallia johannis Schltr., Repert. Spec. Nov. Regni Veg. 10: 359. 1912.

Dracula vagabunda Luer \& R.Escobar, Orquideología 15: 35. 1981.

Voucher: C.A. Luer 1601 (SEL).

\section{Dresslerella Luer}

Dresslerella elvallensis Luer, Selbyana 3: 2. 1976. Voucher: C.A. Luer et al. 1079 (SEL).

Dresslerella hispida (L.O.Williams) Luer, Selbyana 3: 4. 1976.

Pleurothallis hispida L.O.Williams, Ann. Missouri Bot. Gard. 27: 277. 1940.

Voucher: P.H. Allen 782 (AMES).

Dresslerella pertusa (Dressler) Luer, Selbyana 3: 6. 1976.

Pleurothallis pertusa Dressler, Orquideología 5: 76. 1970.

VOUCHER: R.L. Dressler 3152 (US).

Dresslerella powellii (Ames) Luer, Selbyana 3: 8. 1976.

Cryptophoranthus powellii Ames, Schedul. Orchid. 4: 8. 1923.

Pleurothallis deceptrix Dressler, Orquideología 5: 78. 1970.

Voucher: C.W. Powell 278 (MO). 


\section{Dressleria Dodson}

Dressleria allenii H.G.Hills, Lindleyana 15: 171. 2000.

Voucher: J. Ackerman 1336 (SEL).

Dressleria kerryae H.G.Hills, Lindleyana 15: 173. 2000.

VOUCHER: R.L. Dressler 3515 (FLAS).

Dressleria severiniana H.G.Hills, Amer. Orchid Soc. Bull. 62: 616. 1993.

VOUCHER: H.G. Hills 90015 (AMES).

\section{Dryadella Luer}

Dryadella butcheri Luer, Monogr. Syst. Bot. Missouri Bot. Gard. 76: 160. 1999.

VOUCHER: C. Luer 13804A (MO).

Dryadella dressleri Luer, Monogr. Syst. Bot. Missouri

Bot. Gard. 76: 162. 1999.

VOUCHER: C. Luer 10627 (MO).

Dryadella gnoma (Luer) Luer, Selbyana 2: 208. 1978. Pleurothallis gnoma Luer, Selbyana 3: 110. 1976.

Voucher: C. Luer 6438 (SEL).

Dryadella guatemalensis (Schltr.) Luer, (Schltr.) Luer, Selbyana 2: 208. 1978.

Masdevallia guatemalensis Schltr., Repert. Spec.

Nov. Regni Veg. 15: 201. 1918.

Masdevallia simula var. guatemalensis (Schltr.)

Kraenzl., Repert. Spec. Nov. Regni Veg. Beih.

34: 191. 1925.

VOUCHER: C. Luer 6423 (SEL).

Dryadella odontostele Luer, Lindleyana 11: 54. 1996.

Voucher: A. Gentry 6178 (MO).

Dryadella sororcula Luer, Lindleyana 11: 56. 1996.

VOUCHER: C. Luer et al. 10606 (MO).

Echinosepala Pridgeon \& M.W.Chase

Echinosepala aspasicensis (Rchb.f.) Pridgeon \&

M.W.Chase, Lindleyana 17: 101. 2002.

Pleurothallis aspasicensis Rchb.f., Bonplandia

(Hannover) 3: 73. 1855.

Humboldtia aspasicensis (Rchb.f.) Kuntze, Revis. Gen. P1. 2: 667. 1891.

Myoxanthus aspasicensis (Rchb.f.) Luer, Monogr.

Syst. Bot. Missouri Bot. Gard. 15: 38. 1986

Echinella aspasicensis (Rchb.f.) Pridgeon \&

M.W.Chase, Lindleyana 16: 253. 2001.

Brenesia aspasicensis (Rchb.f.) Luer, Monogr.

Syst. Bot. Missouri Bot. Gard. 95: 255. 2004.
Pleurothallis pastacensis Luer, Selbyana 3: 156. 1976.

Myoxanthus pastacensis (Luer) Luer, Monogr. Syst. Bot. Missouri Bot. Gard. 15: 38. 1986.

Voucher: A. Gentry et al. 28639 (MO).

Echinosepala balaeniceps (Luer \& Dressler) Pridgeon \& M.W.Chase, Lindleyana 17: 101. 2002.

Pleurothallis balaeniceps Luer \& Dressler, Orquideología 16: 47. 1986.

Myoxanthus balaeniceps (Luer \& Dressler) Luer, Monogr. Syst. Bot. Missouri Bot. Gard. 44: 94. 1992.

Echinella balaeniceps (Luer \& Dressler) Pridgeon \& M.W.Chase, Lindleyana 16: 253. 2001.

Brenesia balaeniceps (Luer \& Dressler) Luer, Monogr. Syst. Bot. Missouri Bot. Gard. 95: 255. 2004.

VOUCHER: R.L. Dressler 6063 (MO).

Echinosepala lappiformis (A.H.Heller \& L.O.Williams) Pridgeon \& M.W.Chase, Lindleyana 17: 101. 2002.

Pleurothallis lappiformis A.H.Heller \& L.O.Williams, Fieldiana, Bot. 31: 42.1964.

Myoxanthus lappiformis (A.H.Heller \& L.O.Williams) Luer, Monogr. Syst. Bot. Missouri Bot. Gard. 15: 38. 1986.

Echinella lappiformis (A.H.Heller \& L.O.Williams) Pridgeon \& M.W.Chase, Lindleyana 16: 253. 2001.

Brenesia lappiformis (A.H.Heller \& L.O.Williams) Luer, Monogr. Syst. Bot. Missouri Bot. Gard. 95: 255. 2004.

VOUCHER: R.L. Dressler 1034 (MO).

Echinosepala pan (Luer) Pridgeon \& M.W.Chase, Lindleyana 17: 101. 2002.

Pleurothallis pan Luer, Selbyana 3: 360. 1977.

Myoxanthus pan (Luer) Luer, Monogr. Syst. Bot. Missouri Bot. Gard. 15: 38. 1986.

Echinella pan (Luer) Pridgeon \& M.W.Chase, Lindleyana 16: 253. 2001.

Brenesia pan (Luer) Luer, Monogr. Syst. Bot. Missouri Bot. Gard. 95: 255. 2004.

VOUCHER: R.L. Dressler s.n. (SEL).

Echinosepala sempergemmata (Luer) Pridgeon \& M.W.Chase, Lindleyana 17: 101. 2002.

Pleurothallis sempergemmata Luer, Selbyana 3: 386. 1977. 
Myoxanthus sempergemmatus (Luer) Luer, Monogr. Syst. Bot. Missouri Bot. Gard. 15: 38. 1986.

Echinella sempergemmata (Luer) Pridgeon \& M.W.Chase, Lindleyana 16: 253. 2001.

Brenesia sempergemmata (Luer) Luer, Monogr. Syst. Bot. Missouri Bot. Gard. 95: 255. 2004.

Voucher: C.A. Luer 1360 (MO).

Echinosepala uncinata (Fawc.) Pridgeon \& M.W.Chase, Lindleyana 17: 101. 2002.

Pleurothallis uncinata Fawc., J. Bot. 33: 12. 1895. Myoxanthus uncinatus (Fawc.) Luer, Monogr. Syst.

Bot. Missouri Bot. Gard. 15: 38. 1986.

Echinella uncinata (Fawc.) Pridgeon \&

M.W.Chase, Lindleyana 16: 253. 2001.

Brenesia uncinata (Fawc.) Luer, Monogr. Syst.

Bot. Missouri Bot. Gard. 95: 255. 2004.

VOUCHER: G. McPherson 13634 (MO).

\section{Elleanthus C.Presl.}

Elleanthus ampliflorus Schltr., Repert. Spec. Nov. Regni Veg. Beih. 27: 13. 1924.

Voucher: B.E. Hammel 7260 (PMA).

Elleanthus aurantiacus (Lindl.) Rchb.f. in W.G.Walpers, Ann. Bot. Syst. 6: 482. 1863.

Evelyna aurantiaca Lindl. in G.Bentham, P1. Hartw.: 149. 1845.

Evelyna bractescens Lindl., Orchid. Linden.: 10. 1846.

Evelyna hallii Rchb.f., Bot. Zeitung (Berlin) 10: 711. 1852.

Evelyna lindenii Rchb.f., Bot. Zeitung (Berlin) 10: 709. 1852.

Evelyna lindenii var. cymbibractea Linden \& Rchb.f., Bonplandia (Hannover) 2: 282. 1854.

Elleanthus bractescens (Lindl.) Rchb.f. in W.G.Walpers, Ann. Bot. Syst. 6: 475. 1862.

Elleanthus lindenii (Rchb.f. ex Linden) Rchb.f. in W.G.Walpers, Ann. Bot. Syst. 6: 477. 1862.

Elleanthus lindenii var. cymbibracteus (Linden \& Rchb.f.) Rchb.f. in W.G.Walpers, Ann. Bot. Syst. 6: 477. 1862.

Elleanthus hallii (Rchb.f.) Rchb.f. in W.G.Walpers, Ann. Bot. Syst. 6: 482. 1863.

Elleanthus gallipanensis Schltr., Repert. Spec. Nov. Regni Veg. Beih. 6: 28. 1919.

Elleanthus cajamarcae Schltr., Repert. Spec. Nov.
Regni Veg. Beih. 9: 44. 1921.

Elleanthus pallidiflavus Schltr., Repert. Spec. Nov. Regni Veg. Beih. 9: 48. 1921.

Elleanthus hoppii Schltr., Repert. Spec. Nov. Regni

Veg. Beih. 27: 15. 1924.

VOUCHER: S. Knapp 1606 (MO).

Elleanthus bradeorum Schltr., Repert. Spec. Nov. Regni Veg. Beih. 19: 78. 1923.

Voucher: S.A. Mori 6061 (PMA).

Elleanthus capitatellus Dressler, J. Orchideenfr. 11: 143. 2004.

VOUCHER: R.L. Dressler 6387 (MO).

Elleanthus caricoides Nash, Bull. Torrey Bot. Club 34(3): 119-120, t. 7. 1907.

Voucher: T.B. Croat 22306 (MO).

Elleanthus cynarocephalus (Rchb.f.) Rchb.f. in W.G.Walpers, Ann. Bot. Syst. 6: 476. 1862.

Evelyna cynarocephala Rchb.f., Bonplandia (Hannover) 4: 216. 1856.

Voucher: R.L. Dressler \& J.T. Atwood 6237 (PMA).

Elleanthus decipiens Dressler, J. Orchideenfr. 11: 146. 2004.

Voucher: T.F. Daniel et al. 5574 (CAS).

Elleanthus fractiflexus Schltr., Repert. Spec. Nov. Regni Veg. Beih. 8: 35. 1921.

Adeneleuterophora fractiflexa (Schltr.) Dudek \& Szlach., Richardiana 11: 4. 2010.

Elleanthus tenellus L.O.Williams, Lilloa 6: 241. 1941.

VOUCHER: R.L. Dressler 4774 (PMA).

Elleanthus glaucophyllus Schltr., Repert. Spec. Nov. Regni Veg. 8: 567. 1910.

Voucher: G. McPherson 12494 (PMA).

Elleanthus graminifolius (Barb.Rodr.) Løjtnant, Bot. Not. 129: 447. 1977.

Adeneleuterophora graminifolia Barb.Rodr., Gen. Spec. Orchid. 2: 171. 1882.

Elleanthus pusillus Schltr., Notizbl. Bot. Gart. Berlin-Dahlem 8: 117. 1922.

Elleanthus linifolius C.Presl, Reliq. Haenk. 1: 97. 1827.

VOUCHER: R.L. Dressler 2975 (MO).

Elleanthus hymenophorus (Rchb.f.) Rchb.f. in W.G.Walpers, Ann. Bot. Syst. 6: 480. 1862.

Evelyna hymenophora Rchb.f., Bot. Zeitung (Berlin) 10: 710. 1852.

Elleanthus alberti Schltr., Repert. Spec. Nov. Regni 
Veg. Beih. 19: 162. 1923.

Elleanthus curtii Schltr., Repert. Spec. Nov. Regni

Veg. Beih. 19: 79. 1923.

VOUCHER: J. Warscewicz s.n. (W).

Elleanthus jimenezii (Schltr.) C.Schweinf., Bot. Mus. Leafl. 5: 38. 1937.

Epilyna jimenezii Schltr., Beih. Bot. Centralbl. 36(2): 375. 1918.

Voucher: H.W. Churchill 4557 (MO).

Elleanthus lancifolius C.Pres1, Reliq. Haenk. 1: 97. 1827.

Elleanthus laxus Schltr., Repert. Spec. Nov. Regni Veg. 12: 213. 1913.

Elleanthus macer Schltr., Repert. Spec. Nov. Regni

Veg. 14: 388. 1916.

Voucher: R.L. Hartman 12413 (PMA).

Elleanthus lentii Barringer, Brittonia 37: 286. 1985.

VOUCHER: R.L. Dressler 5398 (PMA).

Elleanthus ligularis Dressler \& Bogarín, Lankesteriana 7: 539. 2007.

Adeneleuterophora ligularis (Dressler \& Bogarín)

Dudek \& Szlach., Richardiana 11: 4. 2010.

Voucher: T.B. Croat 27756 (MO).

Elleanthus longibracteatus (Lindl. ex Griseb.) Fawc., Prov. List Pl. Jamaica: 38. 1893.

Evelyna longibracteata Lindl. ex Griseb., Fl. Brit. W. I.: 623.1864.

Elleanthus xanthocomus Rchb.f., Bot. Mag. 99: t. 6016. 1873.

Evelyna xanthocoma (Rchb.f.) Rollisson, Nursery Cat. (Rollisson) 1875-1876: 22. 1875.

Elleanthus longibracteatus var. latifolius Cogn. in I.Urban, Symb. Antill. 6: 564. 1910.

Elleanthus brenesii Schltr., Repert. Spec. Nov. Regni Veg. 11: 44. 1912.

Elleanthus trilobatus Ames \& C.Schweinf., Schedul. Orchid. 8: 53. 1925.

Voucher: C.W. Powell 3267 (AMES).

Elleanthus muscicola Schltr., Repert. Spec. Nov. Regni Veg. Beih. 19: 10. 1923.

VOUCHER: P.H. Allen 2159 (MO).

Elleanthus poiformis Schltr., Repert. Spec. Nov. Regni Veg. Beih. 19: 164. 1923.

Adeneleuterophora poiformis (Schltr.) Dudek \& Szlach., Richardiana 11: 5. 2010.

VOuCHer: M.D. Correa \& R.L. Dressler 821 (PMA).
Elleanthus stolonifer Barringer, Brittonia 37: 287. 1985. Adeneleuterophora stolonifera (Barringer) Dudek \& Szlach., Richardiana 11: 5. 2010.

Voucher: M.A. Blanco \& D. Penneys 2934 (PMA).

Elleanthus tillandsioides Barringer, Brittonia 37: 288. 1985.

Adeneleuterophora tillandsioides (Barringer)

Dudek \& Szlach., Richardiana 11: 5. 2010.

VOUCHER: R.L. Dressler 3892 (PMA).

Elleanthus tonduzii Schltr., Repert. Spec. Nov. Regni Veg. 8: 567. 1910.

Voucher: S. Knapp 5325 (PMA).

Elleanthus tricallosus Ames \& C.Schweinf., Schedul.

Orchid. 8: 51. 1925.

VOucher: M.D. Correa et al. 2188 (PMA).

Elleanthus wercklei Schltr., Repert. Spec. Nov. Regni

Veg. Beih. 19: 11. 1923.

Voucher: R.L. Dressler \& J.T. Atwood 6280 (PMA).

\section{Eloyella P.Ortiz}

Eloyella panamensis (Dressler) Dodson, Icon. Pl. Trop., II, 5: t. 455. 1989.

Phymatidium panamense Dressler, Orquideología 6: 143.1971.

VOUCHER: R.L. Dressler 3570 (US)

ENCYCLIA Hook.

Encyclia ceratistes (Lind1.) Schltr., Repert. Spec. Nov. Regni Veg. Beih. 6: 74. 1919.

Epidendrum ceratistes Lindl., Edwards's Bot. Reg. 30(Misc.): 91. 1844.

Epidendrum ramonense Rchb.f., Beitr. Orchid.-K. C. Amer.: 81. 1866.

Encyclia ramonensis (Rchb.f.) Schltr., Beih. Bot. Centralbl. 36(2): 473. 1918.

Encyclia powellii Schltr., Repert. Spec. Nov. Regni Veg. Beih. 17: 46. 1922.

Encyclia oncidioides var. ramonensis (Rchb.f.) Ames, F.T.Hubb. \& C.Schweinf., Bot. Mus. Leafl. 3: 103. 1935.

Epidendrum oncidioides var. ramonense (Rchb.f.) Ames, F.T.Hubb. \& C.Schweinf., Bot. Mus. Leafl. 3: 103. 1935.

Voucher: C.W. Powell 83 (AMES).

Encyclia chloroleuca (Hook.) Neumann, Rev. Hort. (Paris), sér. 2, 4: 138. 1846. 
Epidendrum chloroleucum Hook., Bot. Mag. 64: t. 3557. 1837.

Epidendrum chloranthum Lindl., Edwards's Bot. Reg. 24(Misc.): 25. 1838.

Epidendrum chloroleucum var. fuscoluteum Regel, Index Seminum (LE) 1857: 29. 1858.

Epidendrum amandum Ames, Schedul. Orchid. 4: 36. 1923.

Encyclia acuta Schltr., Beih. Bot. Centralbl. 42(2): 104. 1925.

Epidendrum acutum (Schltr.) A.D.Hawkes, Orquídea (Rio de Janeiro) 18: 168. 1957.

Encyclia amanda (Ames) Dressler, Phytologia 21: 440. 1971.

Encyclia viridiflava L.C.Menezes, Orchid Digest 55: 21. 1991.

Voucher: C.W. Powell 271 (AMES).

Encyclia cordigera (Kunth) Dressler, Taxon 13: 247. 1964.

Cymbidium cordigerum Kunth in F.W.H.von Humboldt, A.J.A.Bonpland \& C.S.Kunth, Nov. Gen. Sp. 1: 341. 1816.

Epidendrum cordigerum (Kunth) Foldats, Bol. Soc. Venez. Ci. Nat. 28: 234. 1969.

Epidendrum macrochilum Hook., Bot. Mag. 63: t. 3534. 1836.

Epidendrum macrochilum var. roseum Bateman, Orchid. Mexico Guatemala: t. 17. 1840.

Limodorum purpureum Lour. ex Steud., Nomencl. Bot., ed. 2, 2: 46.1841.

Encyclia macrochila (Hook.) Neumann, Rev. Hort. (Paris), sér. 2, 4: 137. 1846.

Epidendrum macrochilum var. albopurpurea C.Morren, Ann. Soc. Roy. Agric. Gand 2: 365, t. 86.1846.

Epidendrum atropurpureum var. roseum (Bateman) Rchb.f., Bonplandia (Hannover) 2: 19. 1854.

Epidendrum atropurpureum var. B.S.Williams, Orch.-Grow. Man., ed. 2: 101. 1862.

Epidendrum longipetalum God.-Leb., Orchidophile (Argenteuil) 12: 257. 1892., nom. illeg.

Encyclia atropurpurea var. leucantha Schltr., Repert. Spec. Nov. Regni Veg. Beih. 17: 45. 1922.

Encyclia atropurpurea var. rhodoglossa Schltr., Repert. Spec. Nov. Regni Veg. Beih. 17: 45. 1922.
Epidendrum atropurpureum var. laciniatum Ames, F.T.Hubb. \& C.Schweinf., Bot. Mus. Leafl. 3: 63. 1935.

Encyclia doeringii Hoehne, Arq. Bot. Estado São Paulo, n.s., f.m., 2: 24. 1946.

Encyclia atropurpurea var. rosea (Bateman) Summerh., Bot. Mag. 171: t. 290. 1957.

Epidendrum doeringii (Hoehne) A.D.Hawkes, Orquídea (Rio de Janeiro) 18: 170. 1957.

Encyclia cordigera var. rosea (Bateman) H.G.Jones, Darwiniana 15: 23. 1969.

Encyclia cordigera f. leucantha (Schltr.) Withner, Cattleyas \& Relatives 5: 104. 1998.

Voucher: D. Cáceres et al. 615 (UCH).

Encyclia elegantula Dressler, Orchid Digest 68: 244. 2004.

Voucher: A. Maduro \& E. Olmos 303 (MO).

Encyclia mooreana (Rolfe) Schltr., Orchideen: 210. 1914.

Epidendrum mooreanum Rolfe, Bull. Misc. Inform. Kew 1891: 190. 1891.

Epidendrum oncidioides var. mooreanum (Rolfe) Ames, F.T.Hubb. \& C.Schweinf., Bot. Mus. Leafl. 3: 106. 1935.

Encyclia oncidioides var. mooreana (Rolfe) Hoehne, Arq. Bot. Estado São Paulo, n.s., f.m., 2(6): 154. 1952.

Encyclia brenesii Schltr., Repert. Spec. Nov. Regni Veg. Beih. 19: 221. 1923.

Encyclia tonduziana Schltr., Repert. Spec. Nov. Regni Veg. Beih. 19: 132. 1923.

Epidendrum tonduzianum Schltr., Repert. Spec.

Nov. Regni Veg. Beih. 19: 132. 1923.

Voucher: D.Bogarin et al. 8627 (JBL).

Encyclia silverarum Leopardi \& Carnevali, Phytotaxa 183 (3): 161. 2014.

Voucher: G. \& K. Silvera s.n. (PMA).

Encyclia stellata (Lind1.) Schltr., Orchideen: 211. 1914. Epidendrum stellatum Lindl., Fol. Orchid. 3: 21. 1853.

Encyclia hunteriana Schltr., Repert. Spec. Nov. Regni Veg. Beih. 17: 46. 1922.

Epidendrum alanjense Ames, Schedul. Orchid. 1: 13. 1922.

Encyclia alanjensis (Ames) Carnevali \& G.A.Romero ex Pupulin, Lankesteriana 4: 18. 2002.

VOUCHER: H. Pittier s.n. (US). 


\section{EPIDENDRUM L.}

Epidendrum aberrans Schltr., Repert. Spec. Nov. Regni Veg. 15: 206. 1918.

Oerstedella aberrans (Schltr.) Hamer, Icon. P1. Trop. 9: t. 891. 1983.

VOUCHER: G. McPherson 12684 (MO).

Epidendrum acunae Dressler, Amer. Orchid Soc. Bull. 28: 358. 1959.

VOUCHER: R.E. Woodson 1898 (MO).

Epidendrum adnatum Ames \& C.Schweinf., Schedul. Orchid. 8: 41. 1925.

Voucher: T.B. Croat 60034 (MO).

Epidendrum alberti Schltr., Repert. Spec. Nov. Regni Veg. Beih. 19: 208. 1923.

VOUCHER: T.B. Croat 66623 (MO).

Epidendrum alfaroi Ames \& C.Schweinf., Schedul. Orchid. 10: 55. 1930.

Epidanthus alfaroi (Ames \& C.Schweinf.) Garay, Orchid Digest 41: 22. 1977.

Neowilliamsia alfaroi (Ames \& C.Schweinf.)

Dressler, Orquídea (Mexico City), n.s., 8: 28. 1981.

VOUCHER: H.W. Churchill 4604 (MO).

Epidendrum allenii L.O.Williams, Ann. Missouri Bot. Gard. 28: 418. 1941.

VOUCHer: P.H. Allen 2203 (AMES).

Epidendrum allochronum Hágsater, Icon. Orchid. 2: t. 104.1993.

Epidendrum coriifolium var. purpurascens Schltr., Repert. Spec. Nov. Regni Veg. Beih. 17: 31. 1922.

Voucher: C.W. Powell 119 (AMES).

Epidendrum anceps Jacq., Select. Stirp. Amer. Hist.: 244. 1763.

Amphiglottis anceps (Jacq.) Britton, Sci. Surv.

Porto Rico \& Virgin Islands 5: 200. 1924.

Epidendrum anceps var. typicum Stehlé, Bull.

Agric. Martinique 8: 221. 1939., nom. inval.

Epidendrum fuscatum Sm., Spic. Bot.: 21. 1792.

Amphiglottis lurida Salisb., Trans. Hort. Soc. London 1: 294. 1812.

Tritelandra fuscata (Sm.) Raf., Fl. Tellur. 2: 86. 1837.

Epidendrum fuscatum var. virescens Lodd. ex Lindl., Fol. Orchid. 3: 65. 1853.

Epidendrum cearense Barb.Rodr., Gen. Spec. Orchid. 2: 141. 1882.
Epidendrum schreineri Barb.Rodr., Gen. Spec. Orchid. 2: 142. 1882.

Epidendrum anceps var. virescens (Lodd. ex Lindl.)

Hemsl., Biol. Cent.-Amer., Bot. 3: 255. 1883.

VOUCHER: H. von Wedel 2921 (MO).

Epidendrum angustilobum Fawc. \& Rendle, J. Bot. 47: 124. 1909.

Epidendrum nocturnum var. latifolium Lindl., Edwards's Bot. Reg. 23: t. 1961. 1837.

Amphiglottis nocturna var. latifolia (Lindl.) Acuña,

Bol. Estaçión Exp. Agron. Santiago de las Vegas 60: 100. 1938.

Epidendrum latifolium (Lindl.) Garay \&

H.R.Sweet, J. Arnold Arbor. 53: 392. 1972.

VOUCHER: P.H. Allen 3924 (MO).

Epidendrum angustisegmentum (L.O.Williams) Hágsater, Icon. Orchid. 3: t. 308. 1999.

Epidendrum rigidum var. angustisegmentum L.O.Williams, Ann. Missouri Bot. Gard. 33: 371. 1946.

VOUCHER: H. von Wedel 1708 (AMES).

Epidendrum anoglossoides Ames \& C.Schweinf., Schedul. Orchid. 10: 56. 1930.

Voucher: L.D. Gómez P. 22373 (CR).

Epidendrum anoglossum Schltr., Repert. Spec. Nov. Regni Veg. 9: 214. 1911.

VOUCHER: G. McPherson 11835 (MO).

Epidendrum antonense Hágsater, Icon. Orchid. 2: t. 108. 1993.

VOUCHER: R.L. Dressler 4071 (AMO).

Epidendrum astroselaginella Hágsater \& E.Santiago, Icon. Orchid. 9: t. 910. 2007.

VOUCHER: S.A. Mori 3158 (MO).

Epidendrum atwoodii Hágsater \& L.Sánchez, Icon. Orchid. 3: t. 311. 1999.

VOUCHER: Z. Samudio 85 (UCH).

Epidendrum barbae Rchb.f., Beitr. Orchid.-K. C. Amer.: 83. 1866.

VOUCHER: G. McPherson 11929 (MO).

Epidendrum barbeyanum Kraenzl., Bull. Herb. Boissier 3: 607. 1895.

Neolehmannia barbeyana (Kraenzl.) Garay \& Dunst., Venez. Orchids Ill. 6: 38. 1976.

Epidendrum amparoanum Schltr., Repert. Spec.

Nov. Regni Veg. Beih. 19: 34. 1923.

VOUCHER: J.P. Folsom 6000 (MO).

Epidendrum baumannianum Schltr., Repert. Spec. 
Nov. Regni Veg. Beih. 7: 126. 1920.

VOUCHER: G.C. de Nevers 4867 (MO).

Epidendrum belloi Hágsater, Icon. Orchid. 3: t. 313. 1999.

VOUCHER: A. Gentry et al. 3678 (MO).

Epidendrum bisulcatum Ames, Schedul. Orchid. 5:

24. 1923.

VOUCHER: C.W. Powell 280 (AMES).

Epidendrum brachybotrys Ackerman \& Montalvo,

Selbyana 9: 129. 1986.

VOUCHER: A.M. Montalvo 383 (SEL).

Epidendrum brachyrepens Hágsater, Icon. Orchid. 3: t. 315.1999.

VOUCHER: P.H. Allen 1530 (AMES).

Epidendrum buenaventurae F.Lehm. \& Kraenzl., Bot. Jahrb. Syst. 26: 470. 1899.

Epidendrum nocturnum var. panamense Schltr., Repert. Spec. Nov. Regni Veg. Beih. 17: 36. 1922.

VOUCHER: C.W. Powell 35 (AMES).

Epidendrum bugabense Hágsater, Icon. Orchid. 2: t. 115. 1993.

VOUCHER: H. van der Werff \& J. Herrera 6427 (AMO). Epidendrum caligarium Rchb.f., Gard. Chron. 1869: 1110. 1869.

Oerstedella caligaria (Rchb.f.) Hágsater, Orquídea

(Mexico City), n.s., 8: 21. 1981.

VOUCHER: W. W. Buller s.n. (W).

Epidendrum caluerorum Hágsater, Icon. Orchid. 2: t. 116. 1993.

VOUCHER: R.L. Dressler sub E. Hagsater 6299 (AMO).

Epidendrum cancanae (P.Ortiz) Hágsater, Lankesteriana 5: 73. 2005.

Oerstedella cancanae P.Ortiz, Orquideología 22: 4. 2001.

Epidendrum gymnopodum Hágsater, Icon. Orchid. 7: t. 741.2004.

VOUCHER: B.E. Hammel et al. 16342 (MO).

Epidendrum candelabrum Hágsater, Orquídea

(Mexico City), n.s., 11: 23. 1988.

Voucher: J.P. Folsom 4448 (MO).

Epidendrum cardiophorum Schltr., Repert. Spec.

Nov. Regni Veg. 9: 214. 1911.

Voucher: W.H. Lewis 2004 (AMES).

Epidendrum caroli Schltr., Repert. Spec. Nov. Regni

Veg. Beih. 19: 35. 1923.

VOUCHER: Roviralta 20 (F).
Epidendrum centropetalum Rchb.f., Bot. Zeitung (Berlin) 10: 732. 1852.

Oerstedella centropetala (Rchb.f.) Rchb.f., Bot. Zeitung (Berlin) 10: 932. 1852.

Oerstedella centradenia Rchb.f., Bot. Zeitung (Berlin) 10: 932. 1852.

Epidendrum centradenia (Rchb.f.) Rchb.f. in W.G.Walpers, Ann. Bot. Syst. 6: 1163. 1865.

Epidendrum tenuiflorum Schltr., Repert. Spec.

Nov. Regni Veg. 3: 49. 1906., nom. illeg.

Epidendrum leprosum Schltr., Repert. Spec. Nov.

Regni Veg. Beih. 19: 38. 1923.

Oerstedella tenuiflora Hágsater, Orquídea (Mexico City), n.s., 8: 24. 1981.

VOUCHER: J. Warszewicz s.n. (W).

Epidendrum chirripoense Hágsater, Icon. Orchid. 2: t. 122.1993.

Voucher: G. Davidse 23912 (MO).

Epidendrum chlorocorymbos Schltr., Repert. Spec. Nov. Regni Veg. Beih. 17: 30-31. 1922.

Voucher: C.W. Powell 82 (K).

Epidendrum ciliare L., Syst. Nat. ed. 10, 2: 1246. 1759.

Auliza ciliaris (L.) Salisb., Trans. Hort. Soc. London 1: 294. 1812.

Epidendrum ciliare var. typicum Stehlé, Bull. Agric. Martinique 8: 216. 1939., nom. inval.

Encyclia ciliaris (L.) A.Lemée, Fl. Guyane Franç. 1: 417. 1955.

Coilostylis ciliaris (L.) Withner \& P.A.Harding, Cattleyas \& Relatives: Debatable Epidendrums: 139. 2004.

Epidendrum cuspidatum G.Lodd., Bot. Cab. 1: t. 10. 1817.

Coilostylis emarginata Raf., Fl. Tellur. 4: 37. 1838.

Epidendrum viscidum Lindl., Edwards's Bot. Reg. 26(Misc.): 81. 1840.

Epidendrum cuspidatum var. brachysepalum Rchb.f., Linnaea 19: 372. 1846.

Epidendrum ciliare var. cuspidatum (G.Lodd.) Lind1., Fol. Orchid. 3: 30. 1853.

Epidendrum ciliare var. viscidum (Lindl.) Lindl., Fol. Orchid. 3: 30. 1853.

Epidendrum ciliare var. minor Stein, Orchid.Buch: 226. 1892.

Epidendrum sanctalucianum H.G.Jones, Novosti Sist. Vyssh. Rast. 12: 140. 1975. 
Coilostylis cuspidata (G.Lodd.) Withner \& P.A.Harding, Cattleyas \& Relatives: Debatable Epidendrums: 142. 2004.

Voucher: T.B. Croat 48822 (MO).

Epidendrum circinatum Ames, Schedul. Orchid. 7: 4. 1924.

Voucher: S. Laube 590 (PMA).

Epidendrum cirrhochiloides Hágsater \& E.Santiago, Icon. Orchid. 11: t. 1112. 2008.

VOuCHER: G. de Nevers et al. 6396 (MO).

Epidendrum confertum Ames \& C.Schweinf., Schedul. Orchid. 10: 61. 1930.

Epidendrum prostratum Schltr., Beih. Bot. Centralbl. 36(2): 407. 1918., nom. illeg.

VOuCHer: E. Hágsater 6530 (AMO).

Epidendrum cordiforme C.Schweinf., Bot. Mus. Leafl. 8: 47. 1940.

VOUCHER: H. Butcher s.n. (AMO).

Epidendrum coriifolium Lindl., J. Hort. Soc. London 6: 218.1851.

Epidendrum fuscopurpureum Schltr., Repert. Spec.

Nov. Regni Veg. Beih. 17: 32. 1922.

Epidendrum subviolascens Schltr., Repert. Spec.

Nov. Regni Veg. Beih. 19: 219. 1923.

Voucher: C.W. Powell 257 (AMES).

Epidendrum cornanthera F.Lehm. \& Kraenzl., Bot. Jahrb. Syst. 26: 463. 1899.

VOUCHER: R.L. Dressler 4868 (AMO).

Epidendrum coronatum Ruiz \& Pav., Syst. Veg. Fl. Peruv. Chil.: 242. 1798.

Epidendrum compositum Vell., Fl. Flumin. 9: t. 39. 1831.

Epidendrum sulphuroleucum Barb.Rodr., Gen.

Spec. Orchid. 1: 56. 1877.

Epidendrum moyobambae Kraenzl., Repert. Spec.

Nov. Regni Veg. 1: 185. 1905.

Epidendrum subpatens Schltr., Repert. Spec. Nov.

Regni Veg. Beih. 17: 40. 1922.

Epidendrum benignum Ames, Schedul. Orchid. 2: 26. 1923.

Epidendrum amazonicum Schltr., Beih. Bot. Centralbl. 42(2): 78. 1925.

VOucher: C.W. Powell 86 (AMES).

Epidendrum criniferum Rchb.f., Gard. Chron. 1871: 1291. 1871.

Voucher: J.P. Folsom 5231 (MO).

Epidendrum cristatum Ruiz \& Pav., Syst. Veg. Fl. Peruv. Chil.: 243. 1798.
Epidendrum raniferum Lindl., Gen. Sp. Orchid. P1.: 109. 1831.

Epidendrum calliferum Lem., Jard. Fleur. 4(Misc.): 65. 1845.

Epidendrum raniferum var. luteum Lindl., Fol. Orchid. 3: 53. 1853.

Epidendrum hexadactylum Barb.Rodr., Gen. Spec. Orchid. 1: 56. 1877.

Epidendrum longovarium Barb.Rodr., Gen. Spec. Orchid. 1: 57. 1877.

Epidendrum tigrinum Sessé \& Moc., Fl. Mexic., ed. 2: 204. 1894., nom. illeg.

Epidendrum raniferum var. hexadactylum (Barb. Rodr.) Cogn. in C.F.P.von Martius \& auct. suc. (eds.), Fl. Bras. 3(5): 99. 1898.

Epidendrum raniferum var. lofgrenii Cogn. in C.F.P.von Martius \& auct. suc. (eds.), Fl. Bras. 3(5): 100. 1898.

Epidendrum raniferum var. obtusilobum Cogn. in C.F.P.von Martius \& auct. suc. (eds.), Fl. Bras. 3(5): 99. 1898.

Epidendrum bathyschistum Schltr., Repert. Spec. Nov. Regni Veg. Beih. 6: 34. 1919.

Epidendrum validum Schltr., Repert. Spec. Nov.

Regni Veg. Beih. 9: 95. 1921.

Epidendrum alexandri Schltr., Anexos Mem. Inst. Butantan, Secç. Bot. 55: 60. 1922.

Epidendrum raniferum var. lutescens Lindl. ex Broadway, Orchid Rev. 34: 199. 1926.

VOUCHER: H. E. Stork 115 (MO).

Epidendrum croatii Hágsater, Icon. Orchid. 3: t. 328. 1999.

Voucher: T.B. Croat 23240 (MO).

Epidendrum cryptanthum L.O.Williams, Amer. Orchid Soc. Bull. 11: 249. 1942.

VOUCHER: Voucher: P.H. Allen 2262 (AMES).

Epidendrum cuneatoides Dodson ex Hágsater, Orquídea (Mexico City), n.s., 13: 217. 1993.

Neowilliamsia cuneata Dressler, Orquídea (Mexico City), n.s., 9: 24. 1983.

VOUCHER: R.L. Dressler 6066 (US).

Epidendrum curvisepalum Hágsater \& Dressler, Icon. Orchid. 2: t. 125. 1993.

VOUCHER: R.L. Dressler 5087 (AMO).

Epidendrum cymbiglossum Hágsater, Icon. Orchid. 2: t. 126.1993.

Voucher: H. van der Werff \& J. Herrera 7186 (SEL). 
Epidendrum dariense Hágsater, Kolan. \& L.Sánchez, Icon. Orchid. 14(10): t. 1421. 2013.

VOUCHER: M. Kolanowska 410 (AMO).

Epidendrum dentiferum Ames \& C.Schweinf.,

Schedul. Orchid. 8: 42. 1925.

Voucher: B.E. Hammel 2063 (MO).

Epidendrum dentilobum Ames, F.T.Hubb. \&

C.Schweinf., Bot. Mus. Leafl. 3: 69. 1935.

VOUCHER: C.W. Powell 342 (AMES).

Epidendrum dosbocasense Hágsater, Orquídea

(Mexico City), n.s., 12: 282. 1992.

VOUCHER: R.L. Dressler 4814 (AMO).

Epidendrum dwyeri Hágsater, Icon. Orchid. 2: t. 130. 1993.

VOUCHER: J.D. Dwyer \& S.M.V. Hayden 7733 (MO).

Epidendrum dwyerioides Hágsater \& E.Santiago, Icon. Orchid. 9: t. 929. 2007.

Voucher: E. Olmos \& J. Nuñez 202 (AMO).

Epidendrum eburneum Rchb.f., Gard. Chron. 1867:

404. 1867.

Epidendrum leucocardium Schltr., Repert. Spec.

Nov. Regni Veg. 12: 206. 1913.

VOUCHER: H. Pittier 4266 (US).

Epidendrum elephantinum Hágsater, Icon. Orchid. 7: t. 734.2004.

Voucher: B. E. Hammel et al. 16421 (MO).

Epidendrum ellipsophyllum L.O.Williams, Ann. Missouri Bot. Gard. 28: 419. 1941.

VOUCHER: P.H. Allen 2178 (AMES).

Epidendrum endresii Rchb.f., Gard. Chron., n.s., 19: 432. 1883.

Oerstedella endresii (Rchb.f.) Hágsater, Orquídea

(Mexico City), n.s., 8: 21. 1981.

Epidendrum adolphii Schltr., Repert. Spec. Nov.

Regni Veg. 3: 108. 1906.

Oerstedella adolphii (Schltr.) Brieger in

F.R.R.Schlechter, Orchideen Beschreib. Kult.

Zücht., ed. 3, 9(33-36): 516. 1977.

VOUCHER: G. McPherson 15917 (MO).

Epidendrum erythrostigma Hágsater, Icon. Orchid. 3: t. 331.1999.

VOUCHER: M. Richardson sub G. McPherson 15905 (AMO).

Epidendrum estrellense Ames, Schedul. Orchid. 4: 39. 1923.

Voucher: T.B. Croat 67942 (MO).

Epidendrum exasperatum Rchb.f., Beitr. Orchid.-K. C. Amer.: 87. 1866.
Oerstedella exasperata (Rchb.f.) Hágsater, Orquídea (Mexico City), n.s., 8: 21. 1981.

Epidendrum chondranthum Kraenzl., Vierteljahrsschr. Naturf. Ges. Zürich 74: 136. 1929.

Voucher: S. Knapp \& M.R. Vodicka 5635 (MO).

Epidendrum exiguum Ames \& C.Schweinf., Schedul.

Orchid. 10: 63. 1930.

Voucher: not seen, cited by Hágsater et al. (2003).

Epidendrum firmum Rchb.f., Beitr. Orchid.-K. C. Amer.: 87. 1866.

Epidendrum difforme var. firmum (Rchb.f.) Ames, F.T.Hubb. \& C.Schweinf., Bot. Mus. Leafl. 2: 55. 1934.

Epidendrum majale Schltr., Beih. Bot. Centralbl. 36(2): 406.1918.

VOUCHER: E. Hágsater 6448 (AMO).

Epidendrum flexuosissimum C.Schweinf., Bot. Mus. Leafl. 14: 55. 1949.

Epidendropsis flexuosissima (C.Schweinf.) Garay \& Dunst., Venez. Orchids Ill. 6: 39. 1976.

Voucher: P.H. Allen 3780 (AMES).

Epidendrum flexuosum G.Mey., Prim. Fl. Esseq.: 260. 1818.

Epidendrum imantophyllum Lindl., Gen. Sp. Orchid. P1.: 106. 1831.

Epidendrum palpigerum Rchb.f., Gard. Chron., n.s., 12: 40. 1879.

Epidendrum buenavistae Kraenzl., Repert. Spec. Nov. Regni Veg. 6: 19. 1908.

Epidendrum lorifolium Schltr., Repert. Spec. Nov. Regni Veg. Beih. 17: 35. 1922.

Voucher: C.W. Powell 138 (AMES).

Epidendrum folsomii Hágsater \& E.Santiago, Icon. Orchid. 8: 832. 2006.

VOUCHER: T. Antonio 1123 (AMO).

Epidendrum fortunae Hágsater \& Dressler, Icon. Orchid. 8: 833. 2006.

VOuCHER: H. van der Werff \& Cor van Hardeveld 6855 (AMO).

Epidendrum fuscinum (Dressler) Hágsater,

Lankesteriana 5: 73. 2005.

Oerstedella fuscina Dressler, Orquídea (Mexico City), n.s., 8: 347. 1982.

VOUCHER: R.L. Dressler 6010 (US).

Epidendrum galeochilum Hágsater \& Dressler, Icon.

Orchid. 7: t. 738. 2004.

VOUCHeR: R.L. Dressler s.n. (AMO). 
Epidendrum gibbosum L.O.Williams, Ann. Missouri Bot. Gard. 28: 420. 1941.

Epidendrum acrostigma Hágsater \& García-Cruz, Icon. Orchid. 3: t. 301. 1999.

VOUCHER: R.E. Woodson \& R.W. Schery 367 (MO).

Epidendrum gregorii Hágsater, Icon. Orchid. 2: t. 138. 1993.

Voucher: E. Hágsater 6410 (AMO).

Epidendrum guanacastense Ames \& C.Schweinf., Schedul. Orchid. 10: 64. 1930.

Epidendrum cocleense Ames, F.T.Hubb. \&

C.Schweinf., Bot. Mus. Leafl. 4: 6. 1936.

VOUCHER: A.A. Hunter \& P.H. Allen 389 (AMES).

Epidendrum gymnochlamys Hágsater \& E.Santiago, Icon. Orchid. 8: 839. 2006.

VOUCHER: G. McPherson 12323 (MO).

Epidendrum hammelii Hágsater \& L.Sánchez, Icon. Orchid. 2: t. 143. 1993.

VOUCHER: B.E. Hammel 1809 (MO).

Epidendrum hawkesii A.H. Heller, Phytologia 14: 2. 1966.

VOUCHER: K. Sytsma 3107 (MO).

Epidendrum hellerianum A.D.Hawkes, Orchid Digest 30: 258. 1966.

VOUCHER: R.L. Dressler 3879 (MO).

Epidendrum hornitense Hágsater \& L.Sánchez, Icon. Orchid. 8: 841. 2006.

Voucher: R.L. Dressler 5326 (AMO).

Epidendrum hunterianum Schltr., Repert. Spec. Nov. Regni Veg. Beih. 17: 33. 1922.

Voucher: C.W. Powell 29 (AMES).

Epidendrum incomptum Rchb.f., Bot. Zeitung (Berlin) 10: 733. 1852.

VOUCHER: J. Warscewicz s.n. (W).

Epidendrum infundibulum Hágsater \& E.Santiago, Icon. Orchid. 9: t. 946. 2007.

VOucher: G. McPherson 11986 (MO).

Epidendrum insolatum Barringer, Brittonia 43: 245. 1991[1992].

Epidanthus crassus Dressler, Orquídea (Mexico City), n.s., 9: 15. 1983.

Epidendrum crassum (Dressler) Mora-Ret. \& García Castro, Brenesia 33: 124. 1990 [1991]., nom. illeg. hom.

Voucher: R.L. Dressler 5686 (US).

Epidendrum intermixtum Ames \& C.Schweinf., Schedul. Orchid. 10: 65 (1930).
Oerstedella intermixta (Ames \& C.Schweinf.) Hágsater, Orquídea (Mexico City), n.s., 8: 21. 1981.

Voucher: Z. Serracin 771 (UCH).

Epidendrum isomerum Schltr., Repert. Spec. Nov. Regni Veg. 2: 132. 1906.

VOUCHER: R.E. Woodson 1886 (MO).

Epidendrum isthmi Schltr., Repert. Spec. Nov. Regni Veg. Beih. 17: 34. 1922.

Voucher: C.W. Powell 104 (AMES).

Epidendrum jefeallenii Hágsater \& García-Cruz, Icon. Orchid. 3: t. 339. 1999.

Voucher: C. Luer \& J. Luer 1400 (SEL).

Epidendrum jefestigma Hágsater \& García-Cruz, Icon. Orchid. 3: t. 340. 1999.

Voucher: W.G. D'Arcy 11379 (MO).

Epidendrum kerichilum Hágsater, Orquídea (Mexico City), n.s., 11: 26. 1988.

Voucher: E. Hágsater \& R.L. Dressler 6519 (AMO).

Epidendrum lacteum Dressler, Orquideología 11: 108. 1978.

Oerstedella lactea (Dressler) Hágsater, Orquídea (Mexico City), n.s., 8: 21. 1981.

VOUCHER: R.L. Dressler 5030 (US).

Epidendrum lacustre Lindl., Fol. Orchid. 3: 50. 1853.

Epidendrum leucochilum Lindl., Orchid. Linden.: 8. 1846., nom. illeg.

Epidendrum obesum Ames, Schedul. Orchid. 2: 31. 1923.

Voucher: D. Cáceres et al. 687 (UCH).

Epidendrum lagenocolumna Hágsater \& L.Sánchez, Icon. Orchid. 2: t. 150. 1993.

Voucher: Greg C. de Nevers 6004 (MO).

Epidendrum lancilabium Schltr., Repert. Spec. Nov. Regni Veg. Beih. 19: 38. 1923.

VOUCHER: R.L. Dressler 5723 (AMO).

Epidendrum laterale Rolfe, Orchid Rev. 20: 280. 1912.

Voucher: S. Laube 572 (PMA).

Epidendrum laucheanum Bonhof ex Rolfe, Bull. Misc. Inform. Kew 1893: 63. 1893.

Epidendrum dolichostachyum Schltr., Repert. Spec. Nov. Regni Veg. 3: 79. 1906.

Epidendrum urostachyum Schltr., Beih. Bot. Centralbl. 36(2): 409. 1918.

Epidendrum cristobalense Ames, Schedul. Orchid. 4: 38. 1923.

VOUCHER: R.L. Dressler 5704 (AMO). 
Epidendrum lockhartioides Schltr., Repert. Spec. Nov. Regni Veg. Beih. 19: 39. 1923.

Epidendrum aporum Garay, Orquideología 4: 78. 1969.

Voucher: G.Davidse \& C.W. Hamilton 23655 (MO).

Epidendrum longibracteatum Hágsater, Icon. Orchid. 3: t. 347. 1999.

Voucher: E. Hágsater, R.L. Dressler \& C. Castro 6526 (MO).

Epidendrum luckei I. Bock, Orchidee (Hamburg) 35: 100. 1984.

VOUCHER: B.E. Hammel 7322 (MO).

Epidendrum lutheri Hágsater, Icon. Orchid. 2: t. 154. 1993.

VOUCHER: H. Luther et al. 1059 (AMES).

Epidendrum macroclinium Hágsater, Orquídea (Mexico City), n.s., 10: 319. 1987.

VOUCHER: H. von Wedel 1126 (AMES).

Epidendrum macroophorum Hágsater \& Dodson, Icon. Orchid. 3: t. 350. 1999.

VOuCHER: S.A. Mori 3988 (MO).

Epidendrum maduroi Hágsater \& García-Cruz, Icon. Orchid. 3: t. 352. 1999.

VOUCHER: E. Hágsater \& R.L. Dressler 6468 (AMO).

Epidendrum magnibracteatum Ames, Schedul. Orchid. 1: 16. 1922.

Epidendrum palmense Ames, Schedul. Orchid. 2:

33. 1923., nom. illeg.

Voucher: R.L. Dressler 5491 (AMO).

Epidendrum mantis-religiosae Hágsater, Orquídea

(Mexico City), n.s., 11: 27. 1988.

VOuCher: R.L Dressler sub Hágsater 6304 (AMO).

Epidendrum microanoglossum Hágsater \&

Karremans, Icon. Orchid. 14(10): t. 1457. 2013.

Voucher: A.P. Karremans et al. 4010 (JBL).

Epidendrum microdendron Rchb.f., Beitr. Orchid.-K.

C. Amer.: 84. 1866.

Epidendrum fundii Ames, Schedul. Orchid. 9: 50. 1925.

VOUCHER: B.E. Hammel 7107 (AMO).

Epidendrum microrigidiflorum Hágsater, Icon. Orchid. 8: 853. 2006.

VOUCHER: R.L. Dressler 6259 (AMO).

Epidendrum miserrimum Rchb.f., Bonplandia (Hannover) 3: 220. 1855.

Jacquiniella miserrima (Rchb.f.) Stehlé, Bull. Soc. Bot. France 84: 425. 1937.[1938].
Epidendrum poiforme Schltr., Repert. Spec. Nov. Regni Veg. Beih. 19: 43. 1923.

Voucher: J.P. Folsom 5649 (MO).

Epidendrum montis-narae Pupulin \& L.Sánchez, Lankesteriana 1: 7. 2001.

Voucher: Z. Serracin \& Z. Samudio 99 (UCH).

Epidendrum mora-retanae Hágsater, Icon. Orchid. 2: t. 160.1993.

VOuCHER: E. Hágsater \& R.L. Dressler 6499 (AMO).

Epidendrum muscicola Schltr., Repert. Spec. Nov. Regni Veg. Beih. 19: 214. 1923.

Epidanthus muscicola (Schltr.) L.O.Williams, Bot. Mus. Leafl. 8: 151. 1940.

Epidendrum linifolium Ames, Schedul. Orchid. 7: 7. 1924.

VOUCHER: B.E. Hammel 2143 (MO).

Epidendrum musciferum Lindl., J. Bot. (Hooker) 1: 6. 1834.

VOUCHER: R.L. Liesner 190 (AMES).

Epidendrum myodes Rchb.f., Beitr. Orchid.-K. C. Amer.: 86. 1866.

Epidendrum polyanthum var. myodes (Rchb.f.) Ames, F.T.Hubb. \& C.Schweinf., Bot. Mus. Leafl. 3: 75. 1935.

VOUCHER: C.W. Hamilton 3825 (MO).

Epidendrum niveocaligarium Hágsater, Icon. Orchid. 8: 860. 2006.

Voucher: W.G. D'Arcy \& K. Sytsma 14547 (MO).

Epidendrum $\times$ nocteburneum Hágsater \& L.Sánchez, Icon. Orchid. 11: t. 1148. 2008.

Voucher: R.L. Dressler sub E. Hágsater 6285 (AMO).

Epidendrum nocturnum Jacq., Enum. Syst. P1.: 29. 1760.

Epidendrum nocturnum var. angustifolium Stehlé, Fl. Descr. Antilles 1: 143. 1939.

Epidendrum nocturnum var. minor Schltr., Repert. Spec. Nov. Regni Veg. Beih. 27: 69. 1924.

Epidendrum nocturnum var. minus Cogn., Bull.

Soc. Roy. Bot. Belgique 43: 323. 1906.

Epidendrum nocturnum var. taguatingense Brieger \& Bicalho, Relat. Ci. Dept. Inst. Genet. Esc. Super. Agric. Luiz Queiroz 11: 26. 1977.

Epidendrum nocturnum var. tridens (Poepp. \& Endl.) Cogn. in C.F.P.von Martius \& auct. suc. (eds.), Fl. Bras. 3(5): 136. 1898.

Epidendrum nocturnum var. tumuc-humaciense Veyret, Bull. Mus. Natl. Hist. Nat., B, 
Adansonia 1982: 286. 1983.

VOUCHER: T.B. Croat 4348 (MO).

Epidendrum notabile Schltr., Repert. Spec. Nov. Regni Veg. Beih. 19: 121. 1923.

Voucher: S. Knapp 2053 (MO).

Epidendrum nutantirhachis Ames \& C.Schweinf., Schedul. Orchid. 10: 69. 1930.

VOUCHER: E. Hágsater 6469 (AMO).

Epidendrum octomerioides Schltr., Repert. Spec. Nov. Regni Veg. 3: 248. 1907.

Voucher: E. Hágsater 7808 (AMO).

Epidendrum odontochilum Hágsater, Orquídea (Mexico City), n.s., 11: 30. 1988.

VOuCHER: G. McPherson 9914 (MO).

Epidendrum oerstedii Rchb.f., Bot. Zeitung (Berlin) 10: 937.1852.

Epidendrum ciliare var. oerstedii (Rchb.f.)

L.O.Williams, Ann. Missouri Bot. Gard. 33: 327. 1946.

Coilostylis oerstedii (Rchb.f.) Withner \& P.A.Harding, Cattleyas \& Relatives: Debatable Epidendrums: 145. 2004.

Epidendrum costaricense Rchb.f., Bot. Zeitung (Berlin) 10: 937. 1852.

Epidendrum umlaufti Zahlbr., Wiener Ill. Gart.Zeitung 1893: t. 2. 1893.

VOUCHER: H. Pittier 3048 (US).

Epidendrum orthodontum Hágsater \& L.Sánchez, Icon. Orchid. 3: t. 361. 1999.

VOUCHER: H. Pittier 3220 (US).

Epidendrum oxyglossum Schltr., Repert. Spec. Nov. Regni Veg. Beih. 19: 40. 1923.

VOUCHER: R.L. Dressler 5228 (AMO).

Epidendrum pachyceras Hágsater \& L.Sánchez, Icon. Orchid. 2: t. 170. 1993.

VOUCHER: J.P. Folsom 4783 (MO).

Epidendrum pachyrachis Ames, Schedul. Orchid. 2:

32. 1923.

VOUCHer: R.L. Dressler 3473 (AMO).

Epidendrum pachytepalum Hágsater \& E.Santiago, Icon. Orchid. 8: 865. 2006.

Voucher: B.E. Hammel 1429 (MO).

Epidendrum pajitense C.Schweinf., Bot. Mus. Leafl. 14: 56.1949.

Oerstedella pajitense (C.Schweinf.) Hágsater, Orquídea (Mexico City), n.s., 8: 21. 1981.

Voucher: Paul H. Allen 3784 (AMES).
Epidendrum pallens Rchb.f., Beitr. Orchid.-K. C. Amer.: 82. 1866.

VOUCHER: B.E. Hammel 7130 (MO).

Epidendrum panamense Schltr., Repert. Spec. Nov. Regni Veg. 12: 212. 1913.

VOUCHER: H. Pittier 5635 (US).

Epidendrum pansamalae Schltr., Repert. Spec. Nov. Regni Veg. 10: 485. 1912.

Oerstedella pansamalae (Schltr.) Hágsater, Orquídea (Mexico City), n.s., 8: 24. 1981.

Epidendrum dunnii A.D.Hawkes, Orchid J. 1: 39. 1952.

VOUCHER: A.D. Hawkes 2392 (UC).

Epidendrum paraguastigmum Hágsater \& GarcíaCruz, Icon. Orchid. 3: t. 367. 1999.

Voucher: R.L. Dressler 3100 (MO).

Epidendrum paranthicum Rchb.f., Bot. Zeitung (Berlin) 10: 732. 1852.

Epidanthus paranthicus (Rchb.f.) L.O.Williams, Bot. Mus. Leafl. 8: 150. 1940.

Epidendrum sancti-ramoni Kraenzl., Vierteljahrsschr. Naturf. Ges. Zürich 74: 137. 1929.

VOUCHER: B.E. Hammel 2298 (MO).

Epidendrum parkinsonianum Hook., Bot. Mag. 66: t. 3778.1840.

Coilostylis parkinsoniana (Hook.) Withner \& P.A.Harding, Cattleyas \& Relatives: Debatable Epidendrums: 145. 2004.

Epidendrum aloifolium Bateman, Orchid. Mexico Guatemala: t. 25. 1841., nom. illeg.

Brassavola pescatorii Rchb.f., Gard. Chron., n.s., 9: 724.1878.

Epidendrum pugioniforme Regel, Trudy Imp. S.Peterburgsk. Bot. Sada 11: 305. 1890[1891].

Epidendrum falcatum var. zeledoniae Schltr., Repert. Spec. Nov. Regni Veg. Beih. 19: 37. 1923.

Coilostylis pugioniformis (Regel) D.P.Banks, Austral. Orchid Rev. 69(4): 18. 2004.

Voucher: P.H. Allen 3515 (K).

Epidendrum parviexasperatum (Hágsater) Hágsater, Lankesteriana 5: 74. 2005.

Oerstedella parviexasperata Hágsater, Orquídea (Mexico City), n.s., 13: 219. 1993.

VOUCHER: B.E. Hammel 6694 (MO).

Epidendrum paucifolium Schltr., Repert. Spec. Nov. Regni Veg. 3: 248. 1907. 
VOUCHER: W.G. D'Arcy 12524 (MO).

Epidendrum pendens L.O.Williams, Ann. Missouri Bot. Gard. 28: 421. 1941.

Voucher: P.H. Allen 2570 (AMES).

Epidendrum pergameneum Rchb.f., Beitr. Orchid.-K.

C. Amer.: 86. 1866.

VOUCHER: M.E. Davidson 457 (MO).

Epidendrum philowercklei Hágsater \& E.Santiago, Icon. Orchid. 8: 870. 2006.

Voucher: A. Maduro \& E. Olmos 200 (AMO).

Epidendrum phragmites A.H.Heller \& L.O.Williams, Fieldiana, Bot. 32: 7. 1968.

Voucher: M.A. Blanco \& D. Penneys 2844 (PMA).

Epidendrum phyllocharis Rchb.f., Xenia Orchid. 3:

11. 1878 .

VOUCHER: R.L. Dressler 4360 (AMO).

Epidendrum physodes Rchb. f., Gard. Chron. 289. 1873.

Physinga physodes (Rchb. f.) Brieger \& Bicalho, Bradea 2(34): 233, f. b. 1978.

Physinga physodes (Rchb. f.) Brieger, Die Orchideen 9(3336): 515. 1977. nom. inval.

VouCHER: D. Bogarin et al. 11552 (UCH).

Epidendrum piliferum Rchb.f., Linnaea 41: 83. 1876.

VOUCHER: J. Warscewicz s.n (W).

Epidendrum pinniferum C.Schweinf., Bot. Mus. Leafl. 5: 92 (1938).

Oerstedella pinnifera (C.Schweinf.) Hágsater, Orquídea (Mexico City), n.s., 8: 24. 1981.

VOUCHER: R. Rincón 963 (UCH).

Epidendrum pirrense Hágsater, Icon. Orchid. 4: t. 471. 2001.

Voucher: J.P. Folsom et al. 6276 (MO).

Epidendrum plagiophyllum Hágsater, Icon. Orchid. 3: t. 370.1999.

Voucher: E. Hágsater et al. 6481 (AMO).

According to Hágsater (1999), the Voucher Dressler 5535 (PMA) determined as E. obliquifolium Ames, F.T.Hubb. \& C.Schweinf. is E. plagiophyllum.

Epidendrum platyphyllostigma Hágsater \& GarcíaCruz, Icon. Orchid. 3: t. 372. 1999.

Voucher: R.L. Dressler 5713 (AMO).

Epidendrum platystigmum Rchb.f., Beitr. Orchid.-K. C. Amer.: 83. 1866.

Voucher: T.B. Croat 49959 (MO).

Epidendrum platystomum Hágsater \& L.Sánchez, Icon. Orchid. 8: 873. 2006.
VOUCHER: A. Gentry 5948 (MO).

Epidendrum pleurothalloides Hágsater, Icon. Orchid. 2: t. 174. 1993.

Voucher: E. Hágsater \& R.L. Dressler 6524 (AMO).

Epidendrum polychlamys Schltr., Repert. Spec. Nov. Regni Veg. 3: 109. 1906.

Voucher: R.L. Dressler 6227 (PMA).

Epidendrum porpax Rchb.f., Bonplandia (Hannover) 3: 220.1855.

Neolehmannia porpax (Rchb.f.) Garay \& Dunst., Venez. Orchids Ill. 6: 37. 1976.

Nanodes porpax (Rchb.f.) Brieger \& Lückel, Orchidee (Hamburg) 29: 179. 1978.

Epidendrum porphyrophyllum Schltr., Repert.

Spec. Nov. Regni Veg. Beih. 17: 37. 1922.

Voucher: C.W. Powell 183 (MO).

Epidendrum powellii Schltr., Repert. Spec. Nov. Regni Veg. Beih. 17: 38. 1922.

Epidendrum arcuiflorum Ames \& C.Schweinf., Schedul. Orchid. 10: 58. 1930.

Voucher: C.W. Powell 228 (AMES).

Epidendrum probiflorum Schltr., Repert. Spec. Nov. Regni Veg. Beih. 17: 39. 1922.

Voucher: C.W. Powell 250 (AMES).

Epidendrum pseudepidendrum Rchb.f., Xenia Orchid. 1: 160. 1856.

Pseudepidendrum spectabile Rchb.f., Bot. Zeitung (Berlin) 10: 733. 1852.

Voucher: J. Warscewicz $16(\mathrm{~W})$.

Epidendrum pseudoramosum Schltr., Repert. Spec.

Nov. Regni Veg. 10: 361. 1912.

Voucher: E. Hágsater 6513 (AMO).

Epidendrum pseudoschumannianum Fowlie, Orchid Digest 33: 255. 1969.

Oerstedella pseudoschumanniana (Fowlie) Hágsater, Orquídea (Mexico City), n.s., 8: 24. 1981.

VOUCHER: C.K. Horich s.n. (UCLA).

Epidendrum pudicum Ames, Schedul. Orchid. 6: 71. 1923.

Voucher: C.W. Powell 319 (AMES).

Epidendrum pumilum Rolfe, Bull. Misc. Inform. Kew 1893: 171. 1893.

Oerstedella pumila (Rolfe) Hágsater, Orquídea (Mexico City), n.s., 8: 24. 1981.

Epidendrum acrochordonium Schltr., Beih. Bot. Centralbl. 36(2): 400. 1918. 
Oerstedella acrochordonia (Schltr.) Hágsater, Orquídea (Mexico City), n.s., 8: 21. 1981.

Voucher: T. S. Cochrane 6261 (F).

Epidendrum radicans Pav. ex Lindl., Gen. Sp. Orchid. P1.: 104. 1831.

Epidendrum rhizophorum Bateman ex Lindl.,

Edwards's Bot. Reg. 24(Misc.): 8. 1838.

Epidendrum pratense Rchb.f., Beitr. Orchid.-K. C.

Amer.: 84. 1866.

Epidendrum radicans var. fuscatum Rchb.f., Gard.

Chron., ser. 3, 5: 5. 1889.

Epidendrum radicans var. chiriquense Schltr.,

Repert. Spec. Nov. Regni Veg. Beih. 17: 39. 1922.

Voucher: C.W. Powell 61 (MO).

Epidendrum rafael-lucasii Hágsater, Icon. Orchid. 2: t. 179.1993.

Voucher: R.L. Dressler sub E. Hágsater 6293 (AMO).

Epidendrum ramosum Jacq., Enum. Syst. Pl.: 29. 1760.

Isochilus ramosus (Jacq.) Spreng., Syst. Veg. 3: 734. 1826.

Spathiger ramosus (Jacq.) Britton, Sci. Surv. Porto Rico \& Virgin Islands 5: 202. 1924.

Epidendrum rigidum G.Lodd., Bot. Cab. 16: t. 1600. 1829., nom. illeg.

Epidendrum sellowii Rchb.f., Linnaea 22: 839. 1850.

Epidendrum imbricatum var. angustifolium Cogn.

in C.F.P.von Martius \& auct. suc. (eds.), Fl. Bras. 3(5): 171. 1898.

Epidendrum modestiflorum Schltr., Repert. Spec.

Nov. Regni Veg. Beih. 19: 213. 1923.

Epidendrum ramosum var. lineare Stehlé, Bull.

Agric. Martinique 8: 249. 1939.

Epidendrum ramosum var. angustifolium (Cogn.)

L.O.Williams, Ann. Missouri Bot. Gard. 28:

422. 1941.

VOUCHER: G. C. de Nevers 8412 (MO).

Epidendrum resectum Rchb.f., Linnaea 41: 83. 1876.

Epidendrum reflexum Ames \& C.Schweinf.,

Schedul. Orchid. 8: 49. 1925.

Voucher: G. Zahn ex J. Veitch 189 (W).

Epidendrum rigidiflorum Schltr., Repert. Spec. Nov. Regni Veg. Beih. 19: 127. 1923.

Voucher: B.E. Hammel 6211 (MO).

Epidendrum rigidum Jacq., Enum. Syst. P1.: 29. 1760.
Spathiger rigidus (Jacq.) Small, Fl. Miami: 55. 1913.

Epidendrum pium Rchb.f. \& Warm. in H.G.Reichenbach, Otia Bot. Hamburg.: 92. 1881.

Voucher: P.M. Peterson 8551 (MO).

Epidendrum romero-castannedae Hágsater \& L.Sánchez, Icon. Orchid. 8: 882. 2006.

VOUCHER: R.L. Dressler 6416 (AMO).

Epidendrum rousseauae Schltr., Beih. Bot. Centralbl., Abt. 2 36(2): 407-408. 1918.

Voucher: Mrs. Rousseau s.n. (B, destroyed).

Epidendrum rugosum Ames, Schedul. Orchid. 6: 74. 1923.

Voucher: S.A. Mori \& J.A. Kallunki 5948 (MO).

Epidendrum sanchoi Ames, Schedul. Orchid. 4: 48. 1923.

Voucher: M.E. Davidson 114 (AMES).

Epidendrum santaclarense Ames, Schedul. Orchid. 4: 49. 1923.

VOUCHER: H. van der Werff 6245 (MO).

Epidendrum schistostemum Hágsater, Laube \& L.Sánchez, Icon. Orchid. 11: t. 1177. 2008.

Voucher: S. Laube 563 (PMA).

Epidendrum schlechterianum Ames, Schedul. Orchid. 7: 9. 1924.

Epidendrum brevicaule Schltr., Repert. Spec. Nov. Regni Veg. Beih. 17: 30. 1922., nom. illeg.

Nanodes schlechterianum (Ames) Brieger, Proc.

World Orchid Conf. 3: 334. 1960.

Nanodes discolor Lindl., Edwards's Bot. Reg. 18: t. 1541.1832.

Epidendrum discolor (Lindl.) Benth., Kew Hand-

List Orch.: 105. 1896., nom. illeg.

VOUCHER: C.W. Powell 18 (AMES).

Epidendrum sculptum Rchb.f., Bonplandia (Hannover) 2: 89. 1854.

Epidendrum florijugum Barb.Rodr., Gen. Spec. Orchid. 1: 57. 1877.

Epidendrum sculptum var. linearifolium Rchb.f., Linnaea 41: 131. 1877.

Epidendrum biflorum Cogn., Bull. Herb. Boissier, sér. 2, 2: 337. 1902., nom. illeg.

Epidendrum boissierianum Schltr., Beih. Bot. Centralbl. 36(2): 459. 1918.

Epidendrum colonense Ames, Schedul. Orchid. 1: 4. 1922. 
Epidendrum sculptum var. arevaloi Schltr., Repert.

Spec. Nov. Regni Veg. Beih. 27: 74. 1924.

Voucher: F.C. Lehmann s.n. (W).

Epidendrum selaginella Schltr., Repert. Spec. Nov. Regni Veg. 3: 48. 1906.

VOUCHER: E. Hágsater 6537 (AMO).

Epidendrum serruliferum Schltr., Repert. Spec. Nov. Regni Veg. Beih. 19: 44. 1923.

Voucher: P.H. Allen 1247 (AMES).

Epidendrum simulacrum Ames, Schedul. Orchid. 6:

75. 1923.

Epidendrum curvicolumna Ames, F.T.Hubb. \& C.Schweinf., Bot. Mus. Leafl. 3: 67. 1935.

VOuCher: C.W. Powell 298 (AMES).

Epidendrum sotoanum Karremans \& Hágsater, Lankesteriana 9(3): 405. 2010.

VOUCHER: D. Bogarin et al. 10367 (UCH-photo).

Epidendrum spathulipetalum Hágsater \& Dressler, Icon. Orchid. 4: t. 486. 2001.

VOUCHER: R.L. Dressler 6230 (AMO).

Epidendrum sphenostele Hágsater \& E.Santiago, Icon. Orchid. 9: t. 989. 2007.

VOUCHER: E. Hágsater 9361 (AMO).

Epidendrum stamfordianum Bateman, Orchid. Mexico Guatemala: t. 11. 1838.

Epidendrum basilare Klotzsch, Allg. Gartenzeitung 11: 193.1843.

Epidendrum cycnostalix Rchb.f., Bot. Zeitung (Berlin) 10: 731. 1852.

Epidendrum stamfordianum var. pictum Lem., Jard. Fleur. 3: t. 251. 1853.

Epidendrum stamfordianum var. parviflorum Regel, Ann. Sci. Nat., Bot., sér. 4, 6: 374. 1856. Epidendrum cycnostachys Benth., J. Linn. Soc., Bot. 18: 314. 1881.

Epidendrum stamfordianum var. wallacei Rchb.f.,

Gard. Chron., ser. 3, 1: 543. 1887.

Epidendrum stamfordianum var. lawrenceanum

Stein, Orchid.-Buch: 240. 1892.

Epidendrum stamfordianum var. pictum Ram.

Goyena, Fl. Nicarag. 2: 759. 1911., nom. illeg.

VOUCHER: J. Warscewicz s.n. (W).

Epidendrum stangeanum Rchb.f., Gard. Chron., n.s., 15: 462.1881.

Epidendrum glandulosum Ames, Schedul. Orchid.

7: 5.1924

VOUCHER: F.F. Stange 602 (AMES).
Epidendrum stenoselaginellum Hágsater \& E.Santiago, Icon. Orchid. 9: t. 991. 2007.

VOUCHER: S. Knapp 5035 (MO).

Epidendrum stolidium Hágsater, Lankesteriana 5: 74. 2005.

Oerstedella ornata Dressler, Orquídea (Mexico City), n.s., 8: 345. 1982.

VOUCHER: R.L. Dressler 5930 (US).

Epidendrum storkii Ames, Schedul. Orchid. 7: 10. 1924.

Voucher: M.E. Davidson 885 (AMES).

Epidendrum strobiliferum Rchb.f., Ned. Kruidk.

Arch. 4: 333. 1859.

Isochilus ramosus Focke, Tijdschr. Natuurk. Wetensch. Kunsten 4: 69. 1851., nom. illeg.

Epidendrum ramosum House, Muhlenbergia 1: 129. 1906., nom. illeg.

Spathiger strobiliferus (Rchb.f.) Small, Man. S.E. Fl.: 390. 1933.

Epidendrum mosenii Barb.Rodr., Gen. Spec. Orchid. 2: 144. 1882., nom. illeg.

Epidendrum rodriguesii Cogn. in C.F.P.von Martius $\&$ auct. suc. (eds.), Fl. Bras. 3(5): 176. 1898.

Epidendrum verecundum Schltr., Repert. Spec. Nov. Regni Veg. Beih. 17: 42. 1922.

Voucher: C.W. Powell 209 (AMES).

Epidendrum subnutans Ames \& C.Schweinf., Schedul. Orchid. 10: 73. 1930.

Voucher: M.E. Davidson 43 (AMES).

Epidendrum summerhayesii Hágsater, Icon. Orchid. 2: t. 189. 1993.

VOUCHer: T. Antonio 5009 (AMO).

Epidendrum suturatum Hágsater \& Dressler, Icon. Orchid. 2: t. 190. 1993.

VOUCHER: R.L. Dressler 5187 (AMO).

Epidendrum tacarcunense Hágsater, Icon. Orchid. 3: t. 384.1999.

Voucher: A. Gentry \& S. Mori 14043 (MO).

Epidendrum talamancanum (J.T.Atwood) Mora-Ret. \& García Castro, Brenesia 33: 124. 1990[1991].

Voucher: G. McPherson 13593 (MO).

Epidendrum tenuisulcatum (Dressler) Hágsater, Orquídea (Mexico City), n.s., 13: 217. 1993.

Neowilliamsia tenuisulcata Dressler, Orquídea (Mexico City), n.s., 8: 29. 1981.

VOUCHER: R.L. Dressler 5673 (US).

Epidendrum tetraceros Rchb.f., Bot. Zeitung (Berlin) 10: 733.1852. 
Oerstedella tetraceros (Rchb.f.) Hágsater, Orquídea (Mexico City), n.s., 8: 24. 1981.

Voucher: J. Warscewicz 2721 (W).

Epidendrum trachythece Schltr., Repert. Spec. Nov. Regni Veg. 3: 249. 1907.

Voucher: M.E. Davidson 124 (AMES).

Epidendrum transversellipticum Hágsater, Icon. Orchid. 4: t. 492. 2001.

Voucher: J. P. Folsom 6101 (MO).

Epidendrum trialatum Hágsater, Orquídea (Mexico City), n.s., 9: 356. 1984.

Voucher: R.L. Dressler sub Hágsater 4674 (AMO).

Epidendrum triangulabium Ames \& C.Schweinf.,

Schedul. Orchid. 10: 75. 1930.

Voucher: P.H. Allen 1245 (MO).

Epidendrum tritropianthum Hágsater \& E.Santiago, Icon. Orchid. 9: t. 998. 2007.

Voucher: R.L. Dressler s.n. (picture sub Epidanthus parathicus, Dressler 1993).

Epidendrum turialvae Rchb.f., Gard. Chron. 1871: 1678. 1871.

VOUCHER: A.H. Gentry 3026 (MO).

Epidendrum unicallosum Hágsater \& E.Santiago, Icon. Orchid. 8: 895. 2006.

Voucher: A. Maduro \& E. Olmos 179 (AMO).

Epidendrum veraguasense Hágsater, Orquídea (Mexico City), n.s., 12: 286. 1992.

Voucher: K. Sytsma \& T. Antonio 3046 (AMO).

Epidendrum vexillium Hágsater, Icon. Orchid. 3: t. 395. 1999.

VOUCHER: R.L. Dressler 5522 (AMO).

Epidendrum villotae Hágsater \& Dodson, Icon. Orchid. 3: t. 398. 1999.

Voucher: R.L. Liesner 1215 (MO).

Epidendrum vincentinum Lindl., J. Bot. (Hooker) 3: 88. 1841.

Epidendropsis vincentina (Lindl.) Garay \& Dunst., Venez. Orchids Ill. 6: 114. 1976.

Epidendrum serricardium Schltr., Repert. Spec.

Nov. Regni Veg. Beih. 19: 218. 1923.

VOUCHER: R.L. Dressler 5661 (AMO).

Epidendrum volutum Lindl. \& Paxton, Paxton's Fl. Gard. 2: 151. 1852.

Epidendrum radlkoferianum Schltr., Repert. Spec.

Nov. Regni Veg. 17: 142. 1921.

VOUCHER: M. Wagner s.n. (M).

Epidendrum vulgoamparoanum Hágsater \&
L.Sánchez, Icon. Orchid. 8: 898. 2006.

VOUCher: T. M. Antonio 2218 (MO).

Epidendrum wallisii Rchb.f., Gard. Chron. 1875(2): 66. 1875.

Oerstedella wallisii (Rchb.f.) Hágsater, Orquídea (Mexico City), n.s., 8: 24. 1981.

Epidendrum pseudowallisii Schltr., Repert. Spec.

Nov. Regni Veg. Beih. 19: 124. 1923.

Oerstedella pseudowallisii (Schltr.) Hágsater,

Orquídea (Mexico City), n.s., 8: 24. 1981.

VOUCHER: A. Gentry 13440 (MO).

Epidendrum warszewiczii Rchb.f., Bot. Zeitung (Berlin) 10: 742. 1852.

Voucher: J. Warszewicz s.n. (W).

Epidendrum wercklei Schltr., Repert. Spec. Nov. Regni Veg. 3: 48. 1906.

Epidanthus wercklei (Schltr.) Garay, Orchid Digest 41: 22. 1977.

Neowilliamsia wercklei (Schltr.) Dressler, Orquídea

(Mexico City), n.s., 8: 29. 1981.

VOUCHER: A. Maduro 277 (MO).

Epidendrum witherspooniorum Hágsater \& Dressler, Icon. Orchid. 7: t. 798. 2004.

VOUCHER: J.T. Witherspoon \& F. Witherspoon 8489 (MO).

Eriopsis Lindl.

Eriopsis biloba Lindl., Edwards's Bot. Reg. 33: t. 9. 1847.

Pseuderiopsis schomburgkii Rchb.f., Linnaea 22: 853. 1850

Eriopsis schomburgkii (Rchb.f.) Rchb.f., Bonplandia (Hannover) 3: 67. 1855.

Eriopsis fuerstenbergii Kraenzl., Orchis 2: 62. 1908.

VOUCHER: T. B. Croat 14424 (MO).

\section{Erycina Lindl.}

Erycina crista-galli (Rchb.f.) N.H.Williams \& M.W.Chase, Lindleyana 16: 136. 2001.

Oncidium crista-galli Rchb.f., Bot. Zeitung (Berlin) 10: 697. 1852.

Psygmorchis crista-galli (Rchb.f.) Dodson, Native Ecuadorian Orchids 4: 883. 2003.

Stacyella crista-galli (Rchb.f.) Szlach., Polish Bot. J. 51: 41 (2006), nom. inval. 
Oncidium iridifolium Lindl., Edwards's Bot. Reg. 22: t. 1911 (1836), nom. illeg.

Oncidium decipiens Lindl., Fol. Orchid. 6: 22 (1855), nom. illeg.

Voucher: M.A. Blanco \& D. Penneys 2825 (PMA).

Erycina pumilio (Rchb.f.) N.H.Williams \& M.W.Chase, Lindleyana 16: 136. 2001.

Oncidium pumilio Rchb.f., Bot. Zeitung (Berlin) 10: 697. 1852.

Tolumnia pumilio (Rchb.f.) Hoehne, Ic. Orch. Bras.: 231. 1949.

Psygmorchis pumilio (Rchb.f.) Dodson \& Dressler, Phytologia 24: 288. 1972.

Oncidium gnomus Kraenzl. in H.G.A.Engler (ed.), Pflanzenr., IV, 50(80): 98. 1922.

Oncidium titania Schltr., Repert. Spec. Nov. Regni Veg. Beih. 19: 67. 1923.

Oncidium oberonia Schltr., Repert. Spec. Nov. Regni Veg. Beih. 27: 113. 1924.

Oncidium hondurense Ames, Bot. Mus. Leafl. 1(5): 1. 1933.

Psygmorchis gnomus (Kraenzl.) Dodson \& Dressler, Phytologia 24: 289. 1972.

Psygmorchis hondurensis (Ames) Garay \& Stacy, Bradea 1: 408. 1974.

Erycina gnomus (Kraenzl.) N.H.Williams \& M.W.Chase, Lindleyana 16: 136. 2001.

Erycina hondurensis (Ames) N.H.Williams \& M.W.Chase, Lindleyana 16: 136. 2001.

VOUCHeR: S. Laube et al. 310 (PMA).

Erycina pusilla (L.) N.H.Williams \& M.W.Chase, Lindleyana 16: 136. 2001.

Epidendrum pusillum L., Sp. Pl. ed. 2: 1352. 1763.

Cymbidium pusillum (L.) Sw., Nova Acta Regiae Soc. Sci. Upsal. 6: 74. 1799.

Oncidium pusillum (L.) Rchb.f. in W.G.Walpers, Ann. Bot. Syst. 6: 714. 1863.

Tolumnia pusilla (L.) Hoehne, Ic. Orch. Bras.: 231. 1949.

Psygmorchis pusilla (L.) Dodson \& Dressler, Phytologia 24: 288. 1972.

Oncidium iridifolium Kunth in F.W.H.von Humboldt, A.J.A.Bonpland \& C.S.Kunth, Nov. Gen. Sp. 1: 344. 1816.

Epidendrum ventilabrum Vell., Fl. Flumin. 9: t. 32. 1831.

Oncidium allemanii Barb.Rodr., Gen. Spec.
Orchid. 2: 185. 1882.

Oncidium pusillum var. megalanthum Schltr., Repert. Spec. Nov. Regni Veg. Beih. 27: 115. 1924.

Psygmorchis allemanii (Barb.Rodr.) Garay \& Stacy, Bradea 1: 408. 1974.

Erycina allemanii (Barb.Rodr.) N.H.Williams \& M.W.Chase, Lindleyana 16: 136. 2001.

VOUCHER: M.D. Correa \& R.L. Dressler 1756 (PMA).

Eulophia R.Br. ex Lindl.

Eulophia alta (L.) Fawc. \& Rendle, Fl. Jamaica 1: 112. 1910.

Cyrtopodium woodfordii Sims, Bot. Mag. 43: t. 1814. 1816.

Dendrobium longifolium Kunth in F.W.H.von Humboldt, A.J.A.Bonpland \& C.S.Kunth, Nov. Gen. Sp. 1: 360. 1816.

Xylobium longifolium (Kunth) Lindl. ex Spreng., Syst. Veg. 3: 733. 1826.

Cypripedium epidendricum Vell., Fl. Flumin. 9: t. 64. 1831.

Maxillaria longifolia (Kunth) Lindl., Gen. Sp. Orchid. Pl.: 150. 1832.

Cyrtopera woodfordii (Sims) Lindl., Gen. Sp. Orchid. P1.: 189. 1833.

Govenia barbata Poepp. \& Endl., Nov. Gen. Sp. P1. 2: 5. 1836.

Cyrtopera longifolia var. pachystelidia Rchb.f., Bot. Zeitung (Berlin) 10: 734. 1852.

Cyrtopera woodfordii var. pachystelidia Rchb.f., Bot. Zeitung (Berlin) 10: 734. 1852.

Cyrtopera longifolia (Kunth) Rchb.f. in W.G.Walpers, Ann. Bot. Syst. 6: 668. 1863.

Lissochilus amazonicus Barb.Rodr., Gen. Spec. Orchid. 1: 89. 1877.

Cyrtopera amazonica (Barb.Rodr.) Barb.Rodr., Gen. Spec. Orchid. 2: 180. 1882.

Cyrtopera vellosiana Barb.Rodr., Gen. Spec. Orchid. 2: 179. 1882., nom. superfl.

Paphiopedilum epidendricum (Vell.) Pfitzer, Bot. Jahrb. Syst. 19: 42. 1894.

Eulophia woodfordii (Sims) Rolfe in D.Oliver \& auct. suc. (eds.), Fl. Trop. Afr. 7: 68. 1897.

Cyrtopera longifolia var. amazonica (Barb.Rodr.) Cogn. in C.F.P.von Martius \& auct. suc. (eds.), Fl. Bras. 3(5): 355. 1901. 
Platypus papilliferus Small \& Nash in J.K.Small, Fl. S.E. U.S.: 329. 1903.

Eulophia longifolia (Kunth) Schltr., Orchideen Beschreib. Kult. Zücht.: 347. 1914.

Eulophia longifolia var. flavescens Schltr., Anexos Mem. Inst. Butantan, Secç. Bot. 1(4): 62. 1922. Eulophia longifolia var. amazonica (Barb.Rodr.) Cogn. in F.C.Hoehne, Fl. Brasílica 12(6): 4. 1942.

Eulophia alta f. pallida P.M.Br., N. Amer. Native Orchid J. 1: 132. 1995.

Eulophia alta var. alba L.C.Menezes, Bol. CAOB 33: 70. 1998.

Eulophia alta f. pelchatii P.M.Br., N. Amer. Native Orchid J. 4: 46. 1998.

Eulophia alta f. Alavescens (Schltr.) F.Barros, Orchid Memories: 20. 2004.

Eulophia alta var. pachystelidia (Rchb.f.)

G.A.Romero, Vanishing Beauty, Native Costa Rican Orchids 1: 318. 2005.

VOUCHER: J. von R. Warszewicz s.n. (W).

\section{Euryblema Dressler}

Euryblema anatonum (Dressler) Dressler, Lankesteriana 5: 94. 2005.

Cochleanthes anatona Dressler, Orchidee (Hamburg) 34: 160. 1983.

Chondrorhyncha anatona (Dressler) Senghas, Orchidee (Hamburg) 41: 91. 1990.

Voucher: R.L. Dressler 5690 (US).

\section{Eurystyles Wawra}

Eurystyles auriculata Schltr., Repert. Spec. Nov. Regni Veg. Beih. 19: 86. 1923.

VOUCHER: R.L. Dressler 4911 (FLAS).

Eurystyles cornu-bovis Szlach., Fragm. Florist. Geobot. 37: 16. 1992.

VOUCHER: R.L. Dressler 5077 (FLAS).

Eurystyles standleyi Ames, Schedul. Orchid. 9: 9. 1925.

VOUCHER: H.P. Butcher 651 (SEL).

Fernandezia Ruiz \& Pav.

Fernandezia crystallina (Lindl.) M.W.Chase, Phytotaxa 20: 29. 2011.

Pachyphyllum crystallinum Lindl., Orchid. Linden.: 18. 1846.
VOUCHER: R.L. Dressler 5928 (MO).

Fernandezia hispidula (Rchb.f.) M.W.Chase, Phytotaxa 20: 30. 2011.

Aeranthes hispidula Rchb.f., Linnaea 41: 31. 1876.

Campylocentrum hispidulum (Rchb.f.) Rolfe, Orchid Rev. 11: 246. 1903.

Pachyphyllum hispidulum (Rchb.f.) Garay \& Dunst., Venez. Orchids Ill. 3: 236. 1965.

Orchidotypus hispidulus (Rchb.f.) Senghas in F.R.R.Schlechter, Orchideen Beschreib. Kult. Zücht., ed. 3, I/B(31): 1923 (1995).

Orchidotypus muscoides Kraenzl., Bot. Jahrb. Syst. 37: 383. 1906.

Pachyphyllum muscoides (Kraenzl.) Schltr., Repert. Spec. Nov. Regni Veg. 15: 216. 1918.

Dichaea microscopica Kraenzl. in H.G.A.Engler (ed.), Pflanzenr., IV, 50(83): 36. 1923.

Pachyphyllum schultesii L.O.Williams, Caldasia 1(3): 15. 1941.

Orchidotypus vareschii Foldats, Acta Biol. Venez. 2: 28.1957.

Orchidotypus schultesii (L.O.Williams) Senghas in F.R.R.Schlechter, Orchideen Beschreib. Kult. Zücht., ed. 3, I/B(31): 1923. 1995.

Voucher: D. Cáceres et al. 531 (UCH).

Fernandezia tica Mora-Ret. \& García Castro, Brenesia 39-40: 164. 1993.

VOUCHER: R.L. Dressler \& J.T. Atwood 6267 (PMA).

Galeandra Lindl.

Galeandra arundinis Garay \& G.A.Romero, Vanishing Beauty, Native Costa Rican Orchids 1: 326. 2005.

VOucher: H. von Wedel 1770 (MO).

Galeandra beyrichii Rchb.f., Linnaea 22: 854. 1850.

Galeandra viridis Barb.Rodr., Gen. Spec. Orchid. 2: 176.1882.

Galeandra coxipoensis Hoehne, Relat. Commiss. Linhas Telegr. Estratég. Matto Grosso Amazonas 4: 15. 1912.

Galeandra fiebrigii Schltr., Repert. Spec. Nov. Regni Veg. Beih. 10: 47. 1922.

VOUCHER: P.H. Allen 1268 (MO).

GaLeottia A.Rich.

Galeottia grandiflora A.Rich., Ann. Sci. Nat., Bot., III, 3: 25.1845. 
Batemannia grandiflora (A.Rich.) Rchb.f., Bonplandia (Hannover) 4: 323. 1856.

Zygopetalum grandiflorum (A.Rich.) Benth. \&

Hook.f. ex Hemsl., Biol. Cent.-Amer., Bot. 3: 251. 1884.

Mendoncella grandiflora (A.Rich.) A.D.Hawkes, Orquídea (Rio de Janeiro) 25: 7. 1963.

Voucher: J. Polanco 3005 (PMA).

\section{GoMPHICHIs Lindl.}

Gomphichis adnata (Ridl.) Schltr., Repert. Spec. Nov. Regni Veg. Beih. 6: 61. 1919.

Stenoptera adnata Ridl., Trans. Linn. Soc. London,

Bot. 2: 284. 1887.

Stenoptera costaricensis Schltr., Beih. Bot. Centralbl. 36(2): 375. 1918.

Gomphichis costaricensis (Schltr.) Ames, F.T.Hubb. \& C.Schweinf., Bot. Mus. Leafl. 3: 37. 1934

Gomphichis merzii Senghas, Orchidee (Hamburg),

Suppl. 2: 26. 1994.

VOUCHER: D. Cáceres et al. 1275 (UCH).

\section{Gongora Ruiz \& Pav.}

Gongora armeniaca (Lind1.) Rchb.f., Xenia Orchid. 1: 52. 1854

Acropera armeniaca Lindl., Paxton's Fl. Gard. 1: 94. 1850.

VOUCHER: G. Gerlach - 04/2485 (M).

Gongora atropurpurea Hook., Exot. F1. 3: t. 178. 1825.

Gongora maculata var. atropurpurea (Hook.) Teusch., Amer. Orchid Soc. Bull. 35: 734. 1966.

Gongora quinquenervis var. atropurpurea (Hook.)

H.G.Jones, Bradea 1: 264. 1972.

Gongora heisteri Rchb.f., Xenia Orchid. 1: 54. 1854.

Voucher: J.H. Kirkbride, Jr. \& N. Bristan 1458 (MO).

Gongora dressleri Jenny, Monogr. Gongora: 90. 1993.

VOuCHer: R.L. Dressler s.n. (K).

Gongora fulva Lindl., Edwards's Bot. Reg. 25: t. 51. 1839.

Gongora maculata var. tricolor Lindl., Edwards's Bot. Reg. 30(Misc.): 26. 1844.

Gongora tricolor (Lindl.) Rchb.f., Bonplandia (Hannover) 2: 93. 1854.
Voucher: C.W. Powell 32 (MO).

Gongora gibba Dressler, Orquideología 7: 72. 1972.

Voucher: R.L. Dressler s.n. (US).

Gongora horichiana Fowlie, Orchid Digest 29: 247. 1965.

Gongora armeniaca var. bicornuta C.Schweinf. \& P.H.Allen, Bot. Mus. Leafl. 13: 139. 1948.

VOUCHER: P.H. Allen 4648 (MO).

Gongora latibasis (C.Schweinf. \& P.H.Allen) Jenny, Monogr. Gongora: 54. 1993.

Gongora maculata var. latibasis C.Schweinf. \& P.H.Allen, Bot. Mus. Leafl. 13: 144. 1948.

Gongora fulva var. latibasis (C.Schweinf. \& P.H.Allen) Whitten \& Jenny, Orchidee (Hamburg) 43: 272. 1992.

VOUCHER: H. Butcher s.n. (AMES).

Gongora leucochila Lem., Fl. Serres Jard. Eur. 1: 207. 1845.

Gongora quinquenervis var. leucochila (Lem.)

C.Schweinf., Fieldiana, Bot. 33: 53. 1970.

Voucher: G. Gerlach 04/2483 (M).

Gongora powellii Schltr., Repert. Spec. Nov. Regni Veg. Beih. 17: 62. 1922.

Voucher: C.W. Powell 76 (AMES).

Gongora unicolor Schltr., Repert. Spec. Nov. Regni Veg. Beih. 19: 299. 1923.

VOUCHER: G. Gerlach 04/2488 (M).

\section{GOODYERA R.Br.}

Goodyera erosa (Ames \& C.Schweinf.) Ames, F.T.Hubb. \& C.Schweinf., Bot. Mus. Leafl. 3: 37. 1934.

Epipactis erosa Ames \& C.Schweinf., Schedul. Orchid. 10: 9. 1930.

VOUCHER: not seen, cited by Dressler (2003c).

Goodyera ovatilabia Schltr., Repert. Spec. Nov. Regni Veg. Beih. 19: 274. 1923.

Voucher: not seen, cited by Dressler (2003c).

Goodyera is a poorly understood genus in Panama.

\section{GovenIA Lindl.}

Govenia ciliilabia Ames \& C.Schweinf., Schedul. Orchid. 10: 80. 1930.

VOUCHER: G. McPherson 9263 (MO).

Govenia powellii Schltr., Repert. Spec. Nov. Regni

Veg. Beih. 17: 51. 1922.

Voucher: C.W. Powell 205 (AMES). 
Govenia quadriplicata Rchb.f., Beitr. Orchid.-K. C. Amer.: 75. 1866.

VOUCHER: B.E. Hammel 4323 (MO).

Govenia viaria Dressler, Lankesteriana 3: 26. 2002.

VOUCHER: R. Villarreal et al. 375 (UCH).

\section{Guarianthe Dressler \& W.E.Higgins}

Guarianthe patinii (Cogn.) Dressler \& W.E.Higgins, Lankesteriana 7: 38. 2003.

Cattleya patinii Cogn. in C.A.Cogniaux \& A.P.G.Goossens, Dict. Icon. Orchid., Cattleya: t. 25.1900.

Cattleya skinneri var. patinii (Cogn.) Schltr., Orchideen Beschreib. Kult. Zücht.: 227. 1914.

Cattleya skinneri var. parviflora Lindl., Bot. Mag. 82: t. 4916. 1856.

Epidendrum huegelianum var. parviflorum (Lind1.) Rchb.f., Xenia Orchid. 2: 29. 1862.

Cattleya patinii var. alba Cogn. in C.A.Cogniaux \& A.P.G.Goossens, Dict. Icon. Orchid., Cattleya: t. 25A. 1900.

Cattleya skinneri var. autumnalis P.H.Allen ex L.O.Williams, Ann. Missouri Bot. Gard. 29: 345. 1942.

VOUCHER: P. H. Allen 2668 (AMES).

\section{Habenaria Willd.}

Habenaria alata Hook., Exot. Fl. 3: t. 169. 1825.

Platantheroides alata (Hook.) Szlach., Richardiana 4: 104. 2004.

Habenella alata (Hook.) Szlach. \& Kras-Lap., Richardiana 6: 34. 2006.

Habenaria laxiflora Poepp. \& Endl., Nov. Gen. Sp. P1. 1: 46.1836.

Habenaria bidentata Poepp. ex Steud., Nomencl. Bot., ed. 2, 1: 716. 1840., nom. inval.

Habenaria stricta A.Rich. \& Galeotti, Ann. Sci. Nat., Bot., sér. 3, 3: 29. 1845.

Habenaria triptera Rchb.f., Linnaea 22: 814. 1850.

Habenaria triptera var. heteroglossa Rchb.f., Linnaea 22: 814. 1850.

VOUCHER: C.A. Luer 1167 (MO).

Habenaria avicula Schltr., Repert. Spec. Nov. Regni Veg. 17: 138. 1921.

VOuCHER: W. Joseph s.n. (B, destroyed); P.H. Allen 2251 (MO).
Habenaria aviculoides Ames \& C.Schweinf., Schedul. Orchid. 10: 1. 1930.

VOUCHER: not seen, cited by Dressler (2003c).

Habenaria bicornis Lindl., Gen. Sp. Orchid. P1.: 309. 1835. Habenaria tricuspis A.Rich. in R.de la Sagra, Hist.

Fis. Cuba, Bot. 11: 249. 1850.

Habenaria goyazensis Cogn. in C.F.P.von Martius $\&$ auct. suc. (eds.), Fl. Bras. 3(4): 77. 1893.

VOUCHER: P.C. Standley 28982 (US).

Habenaria clypeata Lindl., Gen. Sp. Orchid. P1. 311. 1835. VOUCHER: J.P. Folsom 4927 (MO).

Habenaria dentifera C.Schweinf., Bot. Mus. Leafl. 9: 46. 1941.

Platantheroides dentifera (C.Schweinf.) Szlach., Richardiana 4: 106. 2004.

Habenella dentifera (C.Schweinf.) Szlach. \& KrasLap., Richardiana 6: 35. 2006.

VOUCHER: A.H. Gentry 13779 (MO).

Habenaria distans Griseb., Cat. Pl. Cub.: 270. 1866. Habenaria distans var. jamaicensis (Fawc. \& Rendle) Cogn. in I.Urban, Symb. Antill. 6: 300. 1909.

Habenaria jamaicensis Fawc. \& Rendle, J. Bot. 47: 126. 1909.

Habenaria amparoana Schltr., Repert. Spec. Nov. Regni Veg. Beih. 19: 6. 1923.

VOUCHER: H.P. Butcher s.n. (MO).

Habenaria eustachya Rchb.f., Ber. Deutsch. Bot. Ges. 3: 274-275. 1885.

Platantheroides eustachya (Rchb.f.) Szlach., Richardiana 4: 106. 2004.

Habenella eustachya (Rchb.f.) Szlach. \& KrasLap., Richardiana 6: 35. 2006.

Habenaria sanbornii Ames, Proc. Biol. Soc. Wash. 16: 117. 1903.

Habenaria troyana Fawc. \& Rendle, J. Bot. 47: 261. 1909.

Habenaria jimenezii Schltr., Beih. Bot. Centralbl. 36(2): 372. 1918.

VOuCHER: R.L. Dressler 3905 (MO).

Habenaria heptadactyla Rchb.f., Linnaea 22: 812. 1850.

Habenaria leprieurii var. heptadactyla (Rchb.f.) R.E.Schult., Bot. Mus. Leafl. 17: 193. 1956.

Habenaria viridiaurea Lindl. ex Kraenzl., Bot. Jahrb. Syst. 16: 102. 1893.

VOUCHER: R.L. Dressler 2921 (PMA).

Habenaria mediocris Dressler, Bol. Inst. Bot. (Guadalajara) 7: 96. 1999[2000]. 
VOUCHER: R.L. Dressler 5845 (MO).

Habenaria mesodactyla Griseb., F1. Brit. W. I.: 644. 1864.

VOUCHER: R.L. Dressler 5846 (PMA).

Habenaria monorrhiza (Sw.) Rchb.f., Ber. Deutsch. Bot. Ges. 3: 274. 1885.

Orchis monorrhiza Sw., Prodr. Veg. Ind. Occ.: 118. 1788.

Habenaria brachyceratitis Willd., Sp. Pl. 4: 44. 1805., nom. superfl.

Habenaria brachyceras Spreng., Syst. Veg. 3: 692. 1826., nom. superfl.

Platanthera monorrhiza (Sw.) W.J.Schrenk, Orchidee (Hamburg) 28: 69. 1977.

Orchis setacea Jacq., Enum. Syst. P1.: 28. 1760.

Habenaria latifolia Kunth in F.W.H.von Humboldt, A.J.A.Bonpland \& C.S.Kunth, Nov. Gen. Sp. 1: 331. 1816.

Habenaria maculosa Lindl., Gen. Sp. Orchid. Pl.: 309. 1835.

Habenaria speciosa Poepp. \& Endl., Nov. Gen. Sp. P1. 1: 44. 1836.

Habenaria sodiroi Schltr., Repert. Spec. Nov. Regni Veg. 14: 115. 1915.

VOucher: J.A. Duke 6181 (PMA).

Habenaria patentiloba Ames, Proc. Biol. Soc. Wash. 34: 151. 1921.

VOUCHER: P. Killip 3124 (AMES).

Habenaria petalodes Lindl., Gen. Sp. Orchid. Pl.: 316. 1835.

Platantheroides petalodes (Lindl.) Szlach., Richardiana 4: 108. 2004.

Habenella petalodes (Lindl.) Szlach. \& Kras-Lap., Richardiana 6: 38. 2006.

VOUCHER: R.L. Dressler 3905 (PMA).

Habenaria repens Nutt., Gen. N. Amer. Pl. 2: 190. 1818.

Mesicera repens (Nutt.) Raf., Neogenyton: 4. 1825.

Platanthera repens (Nutt.) Alph.Wood, Class-book Bot.: 685. 1861.

Orchis repens (Nutt.) Alph.Wood, Amer. Bot. Fl.: 328. 1870.

Voucher: R.L. Dressler \& J.T. Atwood 6258 (PMA).

Habenaria rodeiensis Barb.Rodr., Gen. Spec. Orchid. 2: 256.1882.

Bertauxia rodeiensis (Barb.Rodr.) Szlach., Richardiana 4: 58. 2004.
Habenaria corcovadensis Kraenzl., Bot. Jahrb. Syst. 16: 120. 1893.

Habenaria carvalhoi Ruschi, Orquid. Nov. Estad. Espirito Santo: 31. 1946.

VOUCHER: R.L. Dressler 3889 (PMA).

Habenaria strictissima Rchb.f., Linnaea 18: 407. 1845.

Platantheroides strictissima (Rchb.f.) Szlach., Richardiana 4: 108. 2004.

Habenella strictissima (Rchb.f.) Szlach. \& KrasLap., Richardiana 6: 39. 2006.

Habenaria pyramidalis Lindl., Ann. Mag. Nat. Hist. 15: 386. 1845.

Habenaria amesiana Schltr., Beih. Bot. Centralbl. 36(2): 371. 1918., nom. illeg.

Habenaria latipetala Schltr., Repert. Spec. Nov. Regni Veg. 15: 194.1918.

Habenaria amblyantha Kraenzl., Vidensk. Meddel. Naturhist. Foren. Kjøbenhavn 71: 179. 1920.

Voucher: J.T. Witherspoon 8795 (PMA).

Habenaria trifida Kunth in F.W.H.von Humboldt, A.J.A.Bonpland \& C.S.Kunth, Nov. Gen. Sp. 1: 330. 1816.

Habenaria angustifolia Kunth in F.W.H.von Humboldt, A.J.A.Bonpland \& C.S.Kunth, Nov. Gen. Sp. 1: 330. 1816.

Bonatea flexuosa Lindl., Gen. Sp. Orchid. P1.: 328. 1835.

Bonatea pauciflora Lindl., Gen. Sp. Orchid. Pl.: 329. 1835.

Orchis longicornu Salzm. ex Lindl., Gen. Sp. Orchid. Pl.: 329. 1835., pro syn.

Habenaria setifera Lindl., Ann. Nat. Hist. 4: 381. 1839.

Habenaria spathacea A.Rich. \& Galeotti, Ann. Sci. Nat., Bot., sér. 3, 3: 29. 1845.

Habenaria pauciflora (Lindl.) Rchb.f., Bonplandia (Hannover) 2: 10. 1854.

Habenaria biflora Barb.Rodr., Gen. Spec. Orchid. 1: 151.1877.

Habenaria allemanii Barb.Rodr., Gen. Spec. Orchid. 2: 254. 1882.

Habenaria flexa Rchb.f. ex Kraenzl., Bot. Jahrb. Syst. 16: 97. 1893.

Habenaria flexa var. rodriguesii Cogn. in C.F.P.von Martius \& auct. suc. (eds.), Fl. Bras. 3(4): 38. 1893. 
Habenaria pauciflora var. pluriflora Cogn., Bull. Soc. Roy. Bot. Belgique 43: 270.1907.

Habenaria caracasana Schltr., Repert. Spec. Nov. Regni Veg. Beih. 6: 26. 1919.

Habenaria caucana Schltr., Repert. Spec. Nov. Regni Veg. Beih. 7: 37. 1920.

Habenaria smithii Schltr., Repert. Spec. Nov. Regni Veg. Beih. 7: 38. 1920.

Habenaria gehrtii Hoehne \& Schltr., Anexos Mem. Inst. Butantan, Secç. Bot. 1(2): 18. 1921.

Habenaria hoehnei Schltr., Anexos Mem. Inst. Butantan, Secç. Bot. 1(2): 17. 1921.

Habenaria endresiana Schltr., Repert. Spec. Nov. Regni Veg. Beih. 19: 272. 1923.

Habenaria duckeana Schltr., Beih. Bot. Centralbl. 42(2): 71.1925.

Habenaria kuhlmannii Schltr., Beih. Bot. Centralbl. 12(2): 72.1925.

Habenaria curti-bradei Hoehne, Bot. Jahrb. Syst. 68: 130. 1937.

Habenaria pickelii Hoehne, Bot. Jahrb. Syst. 68: 129. 1937.

Habenaria dentirostris Pabst, Arq. Bot. Estado São Paulo, n.s., f.m., 3: 118. 1955.

Habenaria flexuosa Rchb.f., Flora 48: 180. 1965., nom. illeg.

VOUCHER: R.J. Seibert 326 (MO).

Habenaria warszewiczii Schltr., Anexos Mem. Inst. Butantan, Secç. Bot. 1(2): 25. 1921.

Habenaria petalodes var. micrantha Rchb.f., Beitr. Orchid.-K. C. Amer.: 5. 1866.

VOUCHER: J. Warscewicz s.n. (W).

Habenaria wercklei Schltr., Repert. Spec. Nov. Regni Veg. Beih. 19: 7. 1923.

Habenaria gymnadenioides Schltr., Repert. Spec.

Nov. Regni Veg. Beih. 19: 271. 1923.

Habenaria verecunda Schltr., Repert. Spec. Nov. Regni Veg. Beih. 19: 6. 1923.

Habenaria hondurensis Ames, Bot. Mus. Leafl. 3: 26. 1934

VOUCHER: J.T. Witherspoon 8456 (MO).

\section{Helleriella A.D.Hawkes}

Helleriella nicaraguensis A.D.Hawkes, Phytologia 14: 4. 1966.

VOUCHER: R.L. Dressler 3868 (FLAS).

\section{Heterotaxis Lindl.}

Heterotaxis discolor (Lodd. ex Lindl.) Ojeda \& Carnevali, Novon 15: 580. 2005.

Dicrypta discolor Lodd. ex Lindl., Edwards's Bot. Reg. 25(Misc.): 91. 1839.

Maxillaria discolor (Lodd. ex Lindl.) Rchb. in W.G.Walpers, Ann. Bot. Syst. 6: 529. 1863.

VOUCHER: R.L. Dressler 3667 (PMA).

Heterotaxis maleolens (Schltr.) Ojeda \& Carnevali, Novon 15: 580. 2005.

Maxillaria maleolens Schltr., Repert. Spec. Nov. Regni Veg. Beih. 19: 233. 1923.

VOuCHER: H. Pittier 5321 (US).

Heterotaxis sessilis (Sw.) F.Barros, Hoehnea 29: 112. 2002.

Epidendrum sessile Sw., Prodr. Veg. Ind. Occ.: 122. 1788 .

Maxillaria sessilis (Sw.) Fawc. \& Rendle, Fl. Jamaica 1: 120. 1910., nom. illeg.

Heterotaxis crassifolia Lindl., Bot. Reg. 12: t. 1028. 1826.

Dicrypta baueri Lindl., Gen. Sp. Orchid. P1.: 44. 1830.

Dicrypta crassifolia (Lind1.) Loudon, Suppl. Hort. Brit. 3: 536. 1850.

Maxillaria crassifolia (Lindl.) Rchb.f., Bonplandia (Hannover) 2: 16. 1854.

Maxillaria gatunensis Schltr., Repert. Spec. Nov. Regni Veg. Beih. 17: 68. 1922.

Heterotaxis gatunensis (Schltr.) Szlach. \& Sitko, Biodivers. Res. Conservation 25: 28. 2012.

VOucher: C.W. Powell 207 (AMES).

Heterotaxis valenzuelana (A.Rich.) Ojeda \& Carnevali, Novon 15: 581. 2005.

Pleurothallis valenzuelana A.Rich. in R.de la Sagra, Hist. Fis. Cuba, Bot. 11: 234. 1850.

Maxillaria valenzuelana (A.Rich.) Nash, Bull. Torrey Bot. Club 34: 121. 1907.

Marsupiaria valenzuelana (A.Rich.) Garay, Arch. Jard. Bot. Rio de Janeiro 12: 183. 1953.

Dicrypta iridifolia Bateman ex Rchb.f., Bonplandia (Hannover) 2: 16. 1854.

Maxillaria iridifolia (Bateman ex Rchb.f.) Rchb.f., Bonplandia (Hannover) 2: 16. 1854.

Dicrypta irisphyta Barb.Rodr., Gen. Spec. Orchid. 1: 126.1877. 
Marsupiaria iridifolia (Bateman ex Rchb.f.) Hoehne, Arq. Bot. Estado São Paulo, n.s., f.m., 2: 71.1947.

Maxillaria valenzuelana subsp. angustata J.T.Atwood, Icon. P1. Trop. 14: t. 1371. 1989.

Marsupiaria valenzuelana var. angustata (J.T.Atwood) Senghas in F.R.R.Schlechter, Orchideen Beschreib. Kult. Zücht., ed. 3, I/B(29): 1779. 1994.

Voucher: C.A. Luer \& H. P. Butcher 1168 (SEL).

\section{Homalopetalum Rolfe}

Homalopetalum pumilio (Rchb.f.) Schltr., Arch. Bot. São Paulo 1: 250. 1926.

Brassavola pumilio Rchb.f., Linnaea 18: 402.1845. Bletia pumilio (Rchb.f.) Rchb.f. in W.G.Walpers, Ann. Bot. Syst. 6: 433. 1862.

Pinelia pumilio (Rchb.f.) Schltr., Beih. Bot. Centralbl. 36(2): 478. 1918.

Pinelia lehmanniana Kraenzl., Bot. Jahrb. Syst. 26: 479. 1899.

Restrepia lehmanniana (Kraenzl.) Schltr., Repert. Spec. Nov. Regni Veg. 3: 277. 1907.

Homalopetalum costaricense Schltr., Repert. Spec.

Nov. Regni Veg. Beih. 19: 47. 1923.

Homalopetalum lehmannianum (Kraenzl.) Schltr., Arch. Bot. São Paulo 1: 250. 1926.

Pinelia tuerckheimii Kraenzl., Ann. Naturhist.

Mus. Wien 44: 326. 1930.

VouCHER: D. Bogarín et al. 11010 (UCH).

\section{Horichia Jenny}

Horichia dressleri Jenny, Orchidee (Hamburg) 32: 108. 1981.

VOUCHER: R. Jenny JE-29(G).

\section{Houlletia Brongn.}

Houlletia odoratissima Linden ex Lindl. \& Paxton, Paxton's Fl. Gard. 3: 172. 1853.

Houlletia picta Linden \& Rchb.f., Gartenflora 1855: 2. 1855.

Houlletia odoratissima var. antioquensis André, Ill. Hort. 17: 59, t. 12. 1870.

Houlletia odoratissima var. xanthina Rchb.f., Gard. Chron., n.s., 22: 38. 1884.

Houlletia odoratissima var. macrosepala Regel,
Gartenflora 34: 147. 1885.

Houlletia juruenensis Hoehne, Relat. Commiss. Linhas Telegr. Estratég. Matto Grosso Amazonas 5: 47. 1910.

Houlletia antioquensis (André) Ames \& Nash in L.H.Bailey, Stand. Cycl. Hort. 3: 1608. 1915.

Houlletia buchtienii Kraenzl., Repert. Spec. Nov. Regni Veg. 25: 28. 1928.

Houlletia boliviana Schltr., Repert. Spec. Nov. Regni Veg. 27: 65. 1929.

Houlletia odoratissima f. antioquiensis (André) O.Gruss \& M.Wolff, Orchid. Atlas: 170. 2007.

Houlletia odoratissima f. xanthina (Rchb.f.) O.Gruss \& M.Wolff, Orchid. Atlas: 170. 2007.

Voucher: R. L. Dressler 3020 (PMA).

Houlletia tigrina Linden ex Lindl., Paxton's F1. Gard. 3: 171.1853.

Voucher: T. M. Antonio 3965 (MO).

\section{Huntleya Bateman ex Lindl.}

Huntleya burtii (Endrés \& Rchb.f.) Pfitzer in H.G.A.Engler \& K.A.E.Prantl, Nat. Pflanzenfam. 2(6): 205. 1889.

Batemannia burtii Endrés \& Rchb.f., Gard. Chron. 1872: 1099. 1872.

Zygopetalum burtii (Endrés \& Rchb.f.) Benth. \& Hook.f. ex Hemsl., Biol. Cent.-Amer., Bot. 3: 251. 1884 .

Huntleya burtii var. wallisii Rolfe, Orchid Rev. 8: 272. 1900 .

Voucher: R. L. Dressler 4638 (PMA).

Huntleya fasciata Fowlie, Orchid Digest 30: 281. 1966.

VOUCHER: E. Jackson J64 P23 (UCLA).

\section{INTı M.A.Blanco}

Inti bicallosa (Rchb.f.) M.A.Blanco, Lankesteriana 7: 524. 2007.

Kefersteinia bicallosa (Rchb.f.) Rchb.f., Otia Bot. Hamburg.: 31. 1878.

Zygopetalum bicallosum Rchb.f., Otia Bot. Hamburg.: 9. 1878.

Maxillaria bicallosa (Rchb.f.) Garay, Caldasia 8: 527. 1962.

Ornithidium dolichophyllum Schltr., Repert. Spec. 
Nov. Regni Veg. Beih. 9: 106. 1921.

Ornithidium cyperifolium Schltr., Repert. Spec.

Nov. Regni Veg. Beih. 27: 100. 1924.

Maxillaria caespitosa C.Schweinf., Bot. Mus.

Leafl. 11: 264. 1945.

Maxillaria cyperifolia (Schltr.) P.Ortiz,

Orquideología 18: 100. 1991.

Voucher: M.A. Blanco \& D. Penneys 3003 (PMA).

Inti chartacifolia (Ames \& C.Schweinf.) M.A.Blanco, Lankesteriana 7: 524. 2007.

Maxillaria chartacifolia Ames \& C.Schweinf., Schedul. Orchid. 10: 92. 1930.

Trigonidium equitans Garay, Svensk Bot. Tidskr.

47: 228. 1953.

Voucher: S. Laube 391 (PMA).

\section{IonOPSIs Kunth}

Ionopsis satyrioides (Sw.) Rchb.f. in W.G.Walpers, Ann. Bot. Syst. 6: 683. 1863.

Epidendrum satyrioides Sw., Prodr. Veg. Ind. Occ.: 123. 1788.

Dendrobium testiculatum Sw., Nova Acta Regiae Soc. Sci. Upsal. 6: 83. 1799.

Cybelion testiculatum (Sw.) Spreng., Syst. Veg. 3: 721.1826.

Ionopsis testiculata (Sw.) Lindl., Gen. Sp. Orchid. P1.: 193. 1833.

Ionopsis teres Lindl., Edwards's Bot. Reg. 24(Misc.): 95. 1838.

Cybelion teres (Lindl.) Steud., Nomencl. Bot., ed. 2, 1: 458. 1840.

Epidendrum acinacifolium Sessé \& Moc., Fl. Mexic., ed. 2: 201. 1894.

Ionopsis pusilla Barb.Rodr., Contr. Jard. Bot. Rio de Janeiro 4: 102. 1907.

Ionopsis costaricensis Schltr., Repert. Spec. Nov. Regni Veg. Beih. 19: 62. 1923.

Voucher: S. Laube et al. 57 (PMA).

Ionopsis utricularioides (Sw.) Lindl., Coll. Bot.: t. 39A. 1826.

Epidendrum utricularioides Sw., Prodr. Veg. Ind. Occ.: 122. 1788.

Dendrobium utricularioides (Sw.) Sw., Nova Acta Regiae Soc. Sci. Upsal. 6: 83. 1799.

Cybelion utriculariae Spreng., Syst. Veg. 3: 721. 1826., nom. superfl.
Ionopsis pulchella Kunth in F.W.H.von Humboldt, A.J.A.Bonpland \& C.S.Kunth, Nov. Gen. Sp. 1: 348. 1816.

Iantha pallidiflora Hook., Exot. Fl. 2: t. 113. 1824.

Cybelion pallidiflorum (Hook.) Spreng., Syst. Veg. 3: 721.1826.

Cybelion pulchellum (Kunth) Spreng., Syst. Veg. 3: 721.1826.

Epidendrum crenatum Vell., Fl. Flumin. 9: t. 6. 1831.

Ionopsis pallidiflora (Hook.) Lindl., Edwards's Bot. Reg. 22: t. 1904. 1836.

Ionopsis paniculata Lindl., Edwards's Bot. Reg. 22: t. 1904. 1836.

Ionopsis tenera Lindl., Edwards's Bot. Reg. 22: t. 1904. 1836.

Scaphyglottis pallidiflora (Hook.) Lindl. in R.Sweet, Hort. Brit., ed. 3: 646. 1839.

Cybelion tenerum (Lindl.) Steud., Nomencl. Bot., ed. 2, 1: 458. 1840.

Ionopsis gardneri Lindl., Paxton's Fl. Gard. 2: 13. 1851.

Ionopsis zonalis Lindl., Paxton's Fl. Gard. 2: 13. 1851.

Ionopsis tenera var. effusa Lindl., Fol. Orchid. 1: 3. 1852.

Ionopsis tenera var. tomentosa Lindl., Fol. Orchid. 1: 3.1852.

Ionopsis tenera var. violacea Lindl., Fol. Orchid. 1: 3.1852.

Epidendrum paniculatum (Lindl.) Rchb.f. in W.G.Walpers, Ann. Bot. Syst. 6: 1166. 1865., nom. illeg.

Ionopsis paniculata var. maxima L.Linden \& Rodigas, Lindenia 3: 39. 1887.

Epidendrum calcaratum Sessé \& Moc., Fl. Mexic., ed. 2: 201. 1894., nom. illeg.

Ionopsis utricularioides f. latifolia Urb., Symb. Antill. 4: 180. 1903.

Ionopsis utricularioides var. angustifolia Cogn. in C.F.P.von Martius \& auct. suc. (eds.), Fl. Bras. 3(6): 175. 1904.

Ionopsis utricularioides var. parviflora Schltr., Repert. Spec. Nov. Regni Veg. Beih. 17: 74. 1922.

Epidendrum sessei Hoehne, Arq. Bot. Estado São Paulo, n.s., f.m., 2: 143. 1952. 
Ionopsis utricularioides var. virginalis L.C.Menezes, Schlechteriana 4: 51. 1993. Ionopsis utricularioides f. virginalis (L.C.Menezes)

Christenson, Lindleyana 11: 17. 1996.

Voucher: C.W. Powell 181 (AMES).

\section{IsOCHILUS R.Br.}

Isochilus carnosiflorus Lindl., Paxton's Mag. Bot. 11: 213. 1844.

Isochilus linearis var. carnosiflorus (Lindl.)

Correll, Bot. Mus. Leafl. 10: 7. 1941.

Isochilus crassiflorus A.Rich. \& Galeotti, Ann. Sci.

Nat., Bot., sér. 3, 3: 22. 1845.

VOUCHER: H. von Wedel 1584 (MO).

Isochilus chiriquensis Schltr., Repert. Spec. Nov. Regni Veg. Beih. 17: 25. 1922.

Isochilus amparoanus Schltr., Repert. Spec. Nov.

Regni Veg. Beih. 19: 27. 1923.

Isochilus major var. amparoanus (Schltr.) Correll,

Bot. Mus. Leafl. 10: 11. 1941.

Voucher: C.W. Powell 98 (AMES).

Isochilus linearis (Jacq.) R.Br. in W.T.Aiton, Hortus Kew. 5: 209. 1813.

Epidendrum lineare Jacq., Enum. Syst. P1.: 29. 1760. Cymbidium lineare (Jacq.) Sw., Nova Acta Regiae

Soc. Sci. Upsal. 6: 72. 1799.

Leptothrium lineare (Jacq.) Kunth in F.W.H.von

Humboldt, A.J.A.Bonpland \& C.S.Kunth, Nov.

Gen. Sp. 1: 340. 1816. nom. inval.

Isochilus leucanthus Barb.Rodr., Gen. Spec.

Orchid. 1: 47.1877.

Isochilus linearis var. leucanthus (Barb.Rodr.)

Cogn. in C.F.P.von Martius \& auct. suc. (eds.),

F1. Bras. 3(5): 4. 1898.

VOUCHER: not seen, cited by Dressler (2003c).

\section{JACQUiNiELla Schltr.}

Jacquiniella aporophylla (L.O.Williams) Dressler, Taxon 15: 242. 1966.

Epidendrum equitantifolium var. aporophyllum L.O.Williams, Bot. Mus. Leafl. 8: 152. 1940. Briegeria aporophylla (L.O.Williams) Senghas, Orchidee (Hamburg) 31: 30. 1980.

Voucher: R.L. Dressler s.n. (FLAS).

Jacquiniella equitantifolia (Ames) Dressler, Taxon 15: 242.1966.
Epidendrum equitantifolium Ames, Schedul. Orchid. 4: 39. 1923.

Briegeria equitantifolia (Ames) Senghas, Orchidee (Hamburg) 31: 30. 1980.

Epidendrum equitans Lindl., Edwards's Bot. Reg. 24(Misc.): 44. 1838., nom. illeg.

Voucher: E. Rodríguez-Quiel \& Z. Samudio 611 (UCH).

Jacquiniella globosa (Jacq.) Schltr., Repert. Spec. Nov. Regni Veg. Beih. 7: 124. 1920.

Epidendrum globosum Jacq., Enum. Syst. P1.: 29. 1760.

Cymbidium globosum (Jacq.) Sw., Nova Acta Regiae Soc. Sci. Upsal. 6: 72. 1799.

Isochilus globosus (Jacq.) Lindl., Gen. Sp. Orchid. P1.: 112. 1831.

Epidendrum kuhlmannii Schltr., Beih. Bot. Centralbl. 42(2): 79. 1925., nom. illeg.

Epidendrum geraldoi Porto \& Brade, Rodriguésia 1(2): 30. 1935.

Epidendrum rudolfianum Hoehne, Ind. Bibl. Pl. Col. Com. Rondon: 172. 1951., nom. illeg.

Voucher: S. Laube et al. 561 (PMA).

Jacquiniella pedunculata Dressler, Orquideología 5: 24. 1970.

VOUCHER: R.L. Dressler s.n. (US)

Jacquiniella standleyi (Ames) Dressler, Taxon 15: 242. 1966.

Epidendrum standleyi Ames, Schedul. Orchid. 9: 52. 1925.

Briegeria standleyi (Ames) Senghas, Orchidee (Hamburg) 31: 30. 1980.

Voucher: R.L. Dressler 5332 (MO).

Jacquiniella teretifolia (Sw.) Britton \& P.Wilson, Sci. Surv. Porto Rico \& Virgin Islands 6: 340. 1926.

Epidendrum teretifolium Sw., Prodr. Veg. Ind. Occ.: 122. 1788.

Cymbidium teretifolium (Sw.) Sw., Nova Acta

Regiae Soc. Sci. Upsal. 6: 72. 1799.

Isochilus teretifolius (Sw.) Lindl., Gen. Sp. Orchid. P1.: 112. 1831.

Briegeria teretifolia (Sw.) Senghas, Orchidee (Hamburg) 31: 30. 1980.

Epidendrum teres Rchb.f., Bonplandia (Hannover) 3: 220. 1855., nom. illeg.

Epidendrum subuliferum Schltr., Beih. Bot. Centralbl. 36(2): 409. 1918. 
Epidendrum teretifolium var. powellianum Schltr., Repert. Spec. Nov. Regni Veg. Beih. 17: 41 1922.

Jacquiniella teres Hamer \& Garay in F.Hamer,

Orquid. El Salvador 2: 30. 1974.

VOuCHer: C.W. Powell 240 (AMES).

\section{KefERSTEINIA Rchb.f.}

Kefersteinia alata Pupulin, Harvard Pap. Bot. 8: 161. 2004.

VOUCHER: F. Pupulin et al. 3119 (USJ).

Kefersteinia alba Schltr., Repert. Spec. Nov. Regni Veg. Beih. 19: 228. 1923.

Chondrorhyncha alba (Schltr.) L.O.Williams, Ceiba 5: 191. 1956.

VOUCHER: J.D. Ackerman 1200 (SEL).

Kefersteinia angustifolia Pupulin \& Dressler, Harvard Pap. Bot. 8: 164. 2004.

VOUCHER: H. Butcher s.n. (MO).

Kefersteinia auriculata Dressler, Orquideología 16: 49. 1983.

Senghasia auriculata (Dressler) Szlach., J. Orchideenfr. 10: 336. 2003.

VOUCHER: R.L. Dressler 4063 (US).

Kefersteinia costaricensis Schltr., Beih. Bot. Centralbl. 36(2): 413. 1918.

Chondrorhyncha costaricensis (Schltr.) P.H.Allen, Ann. Missouri Bot. Gard. 36: 86. 1949.

Senghasia costaricensis (Schltr.) Szlach., J. Orchideenfr. 10: 336. 2003.

VOUCHER: R.L. Dressler 5045 (MO).

Kefersteinia elegans Garay, Orquideología 4: 80. 1969.

Voucher: A. Maduro \& E. Olmos 156 (MO).

Kefersteinia excentrica Dressler \& Mora-Ret., Orquídea (Mexico City), n.s., 13: 261.1993.

Voucher: R.L. Dressler \& J.T. Atwood 6236 (PMA).

Kefersteinia lactea (Rchb.f.) Schltr., Repert. Spec. Nov. Regni Veg. 36: 613. 1918.

Zygopetalum lacteum Rchb.f., Gard. Chron. 1872: 1290. 1872.

Chondrorhyncha lactea (Rchb.f.) L.O.Williams, Caldasia 1(5): 16. 1942.

Senghasia lactea (Rchb.f.) Szlach. \& Romowicz, Richardiana 6: 182. 2006.

VOUCHER: J. Linden S.n. 4 (W).
Kefersteinia maculosa Dressler, Orquideología 16: 52. 1983.

Senghasia maculosa (Dressler) Szlach., J. Orchideenfr. 10: 336. 2003.

VOUCHeR: R.L. Dressler 6061 (US).

Kefersteinia mystacina Rchb.f., Gard. Chron., n.s., 15: 530. 1881.

Zygopetalum mystacinum Rchb.f., Gard. Chron., n.s., 15: 530. 1881.

Voucher: R.L. Dressler s.n. (MO).

Kefersteinia parvilabris Schltr., Repert. Spec. Nov. Regni Veg. Beih. 19: 52. 1923.

Chondrorhyncha parvilabris (Schltr.) L.O.Williams, Ceiba 5: 195. 1956.

Senghasia parvilabris (Schltr.) Szlach., J. Orchideenfr. 10: 336. 2003.

Kefersteinia deflexipetala Fowlie, Orchid Digest 30: 117. 1966.

VOUCHER: R.L. Dressler 5511 (MO).

\section{Kegeliella Mansf}

Kegeliella atropilosa L.O.Williams \& A.H.Heller, Fieldiana, Bot. 31: 39. 1964.

VOUCHER: P.H. Allen 2759 (AMES).

Kegeliella kupperi Mansf., Repert. Spec. Nov. Regni Veg. 36: 60. 1934.

VOUCHER: H.P. Butcher S.n. (AMES).

\section{KoelLensteinia Rchb.f.}

Koellensteinia lilijae Foldats, Bol. Soc. Venez. Ci. Nat. 22: 263. 1961.

VOUCHER: J.D. Dwyer 1974 (MO).

\section{Kraenzlinella Kuntze}

Kraenzlinella erinacea (Rchb.f.) Solano, Icon. Orchid. 5-6: xi. 2002[2003].

Pleurothallis erinacea Rchb.f., Bonplandia (Hannover) 3: 72. 1855.

Humboldtia erinacea (Rchb.f.) Kuntze, Revis. Gen. Pl. 2: 667. 1891.

Kraenzlinella erinacea (Rchb. f.) Luer, Monogr. Syst. Bot. Missouri Bot. Gard. 95: 258. 2004.

Pleurothallis muricata Schltr., Repert. Spec. Nov. Regni Veg. 10: 293. 1912.

Pleurothallis sororia Schltr., Repert. Spec. Nov. Regni Veg. 10: 294. 1912. 
Kraenzlinella muricata (Schltr.) Rolfe, Orchid Rev. 23: 326. 1915.

Kraenzlinella rufescens Rolfe, Orchid Rev. 23: 326. 1915.

Kraenzlinella sororia (Schltr.) Rolfe, Orchid Rev. 23: 326. 1915.

Pleurothallis rufescens (Rolfe) Schltr., Repert. Spec. Nov. Regni Veg. Beih. 9: 142. 1921.

Pleurothallis diuturna Schltr., Repert. Spec. Nov.

Regni Veg. Beih. 17: 19. 1922.

Pleurothallis muricata T.Hashim., Bull. Natl. Sci.

Mus. Tokyo, B 13: 35. 1987., nom. illeg.

VOucher: C.W. Powell 172 (AMES).

\section{KREODANTHus Garay}

Kreodanthus bugabae Ormerod, Harvard Pap. Bot. 13: 59. 2008.

Voucher: H. van der Werff \& J. Herrera 6441 (MO).

Kreodanthus secundus (Ames) Garay, Bradea 2: 199. 1977.

Physurus secundus Ames, Orchidaceae 2: 260. 1908.

Erythrodes secunda (Ames) Ames, Orchidaceae 5: 28. 1915.

VOUCHER: P.H. Allen 1439 (MO).

Kreodanthus sytsmae Ormerod, Harvard Pap. Bot. 13: 61. 2008.

VOUCHER: K. Sytsma et al. 4945 (MO).

\section{LaCaena Lindl.}

Lacaena spectabilis (Klotzsch) Rchb.f., Bonplandia (Hannover) 2: 92.1854.

Nauenia spectabilis Klotzsch, Allg. Gartenzeitung 21: 193.1853.

Lacaena nicaraguensis L.O.Williams, Fieldiana, Bot. 31: 41. 1964.

VOUCHER: not seen, cited by Dressler (2003c).

According to Dressler (2003c), this species ranges from Mexico to western Panama. We have not seen herbarium Vouchers from Panama.

\section{LAELIa Lindl.}

Laelia lueddemannii (Prill.) L.O.Williams, Ann. Missouri Bot. Gard. 27: 282. 1940.

Schomburgkia lueddemannii Prill., J. Soc. Imp. Centr. Hort. 8: 123. 1862.
Schomburgkia undulata var. lueddemannii (Prill.) H.G.Jones, Orchid Rev. 71: 51. 1963.

Bletia undulata var. costaricana Rchb.f., Xenia Orchid. 2: 49. 1863.

Schomburgkia undulata var. costaricana (Rchb.f.) H.G.Jones, Orchid Rev. 71: 51. 1963.

Schomburgkia lueddemannii var. costaricana (Rchb.f.) H.G.Jones, Feddes Repert. 76: 195. 1967.

Voucher: A. Ibáñez et al. 2558 (PMA).

\section{LALEXIa Luer}

Lalexia quadrifida (Lex.) Luer, Harvard Pap. Bot. 16(2): 358. 2011.

Dendrobium quadrifidum La Llave \& Lex., Nov. Veg. Descr. 2(Orch. Opusc.): 40-41. 1825.

Pleurothallis quadrifida (La Llave \& Lex.) Lindl., Edwards's Bot. Reg. 28: Misc. 70. 1842.

Humboldtia quadrifida (La Llave \& Lex.) Kuntze, Revis. Gen. Pl. 2: 668. 1891.

Stelis quadrifida (La Llave \& Lex.) Solano \& Soto Arenas, Icon. Orchid. 5-6: xi 2002. [2003].

Specklinia quadrifida (La Llave \& Lex.) Luer, Monogr. Syst. Bot. Missouri Bot. Gard. 95: 263. 2004.

Loddigesia quadrifida (La Llave \& Lex.) Luer, Monogr. Syst. Bot. Missouri Bot. Gard. 105: 251. 2006.

Pleurothallis racemiflora Lindl. ex G.Lodd., Bot. Cab. 10: t. 949. 1825., nom. illeg.

Stelis racemiflora Lodd. ex W.H.Baxter in J.C.Loudon, Hort. Brit., Suppl. 3: 643. 1839.

Pleurothallis ghiesbreghtiana A.Rich. \& Galeotti, Ann. Sci. Nat., Bot., sér. 2, 3: 16. 1845.

Physosiphon nicaraguensis Liebm., Index Seminum (C) 1853: 20. 1853.

Pleurothallis incompta Rchb.f., Bonplandia (Hannover) 2: 24. 1854.

Pleurothallis longissima Lindl., Fol. Orchid. 9: 31. 1859.

Pleurothallis nicaraguensis (Liebm.) Rchb.f. in W.G.Walpers, Ann. Bot. Syst. 6: 171. 1861.

Humboldtia incompta (Rchb.f.) Kuntze, Revis. Gen. Pl. 2: 667. 1891.

Humboldtia longissima (Lindl.) Kuntze, Revis. Gen. Pl. 2: 667. 1891. 
Humboldtia nicaraguensis (Rchb.f.) Kuntze, Revis. Gen. P1. 2: 668. 1891.

Humboldtia racemiflora Kuntze, Revis. Gen. P1. 2: 668. 1891.

Pleurothallis lyroglossa Schltr., Repert. Spec. Nov. Regni Veg. 8(191-195): 566. 1910.

Pleurothallis tricostata Cogn. in I.Urban, Symb. Antill. 7: 175. 1912.

Pleurothallis niederleinii Schltr., Beih. Bot. Centralbl. 36(2): 396. 1918.

Pleurothallis ghiesbreghtiana var. cleistogama L.O.Williams, Ceiba 2: 103. 1951.

Anathallis racemiflora Pridgeon \& M.W.Chase, Lindleyana 16: 250. 2001.

Stelis racemiflora (Pridgeon \& M.W.Chase) Pridgeon \& M.W.Chase, Lindleyana 16: 266. 2001.

Stelis nicaraguensis (Liebm.) Solano \& Soto Arenas, Icon. Orchid. 5-6: xi. 2002. [2003].

VOUCHER: C. Galdames et al. 3649 (PMA).

According to Luer (2011) and Karremans et al. (2013) this species is not a member of Stelis s.l., thus it should be treated in genus Lalexia or in a broad concept of Pleurothallis.

\section{LANKESTERIANA Karremans}

Lankesteriana casualis (Ames) Karremans, Lankesteriana 13(3): 327. 2014.

Pleurothallis casualis Ames, Schedul. Orchid. 9: 30-33, f. 5. 1925.

Anathallis casualis (Ames) Pridgeon \& M.W.Chase, Lindleyana 16(4): 248. 2001.

Specklinia casualis (Ames) Luer, Monogr. Syst.

Bot. Missouri Bot. Gard. 95: 259. 2004.

Panmorphia casualis (Ames) Luer, Monogr. Syst.

Bot. Missouri Bot. Gard. 105: 149-150. 2006.

VOucher: C. Luer \& J. Luer 1082 (SEL).

Lankesteriana cuspidata (Luer) Karremans, Lankesteriana 13(3): 327. 2014.

Anathallis cuspidata (Luer) Pridgeon \& M.W.Chase, Lindleyana 16: 248. 2001.

Pleurothallis cuspidata Luer, Selbyana 3(3-4): 282-283, f. 241. 1977.

Specklinia cuspidata (Luer) Luer, Monogr. Syst. Bot. Missouri Bot. Gard. 95: 260. 2004.

Panmorphia cuspidata (Luer) Luer, Monogr. Syst. Bot. Missouri Bot. Gard. 105: 152. 2006.

VOUCHer: C.A. Luer et al. 1335 (SEL).
Lankesteriana fractiflexa (Ames \& C.Schweinf.)

Karremans, Lankesteriana 13(3): 327. 2014.

Anathallis fractiflexa (Ames \& C.Schweinf.) Luer, Monogr. Syst. Bot. Missouri Bot. Gard. 115: 258. 2009.

Pleurothallis fractiflexa Ames \& C.Schweinf., Schedul. Orchid. 10: 26-27. 1930.

Pleurothallis corynophora Luer, Phytologia 55(3): 201. 1984.

Anathallis corynophora (Luer) Pridgeon \& M.W.Chase, Lindleyana 16(4): 248. 2001.

Specklinia fractiflexa (Ames \& C.Schweinf.) Luer, Monogr. Syst. Bot. Missouri Bot. Gard. 95: 260. 2004.

Panmorphia fractiflexa (Ames \& C.Schweinf.) Luer, Monogr. Syst. Bot. Missouri Bot. Gard. 105: 154-155. 2006.

Voucher: C.A. Luer et al. 9299 (SEL).

Lankesteriana muricaudata (Luer) Karremans, Lankesteriana 13(3): 327. 2014.

Pleurothallis muricaudata Luer, Selbyana 7: 119. 1982.

Anathallis muricaudata (Luer) Pridgeon \& M.W.Chase, Lindleyana 16: 249. 2001.

Specklinia muricaudata (Luer) Luer, Monogr. Syst.

Bot. Missouri Bot. Gard. 95: 262. 2004.

Panmorphia muricaudata (Luer) Luer, Monogr. Syst. Bot. Missouri Bot. Gard. 105: 168. 2006. Voucher: C.A. Luer 10577 (MO).

LeOchiLus Knowles \& Westc.

Leochilus inconspicuus (Kraenzl.) M.W.Chase \& N.H.Williams, Lindleyana 21(3): 21. 2008.

Rodriguezia inconspicua Kraenzl., Bull. Herb. Boissier 3: 630. 1895.

Hybochilus inconspicuus (Kraenzl.) Schltr., Repert. Spec. Nov. Regni Veg. 16: 430. 1920.

Leochilus parviflorus Standl. \& L.O.Williams, Ceiba 1: 235. 1951.

VOUCHER: R.L. Dressler \& N.H. Williams 3990 (PMA).

Leochilus labiatus (Sw.) Kuntze, Revis. Gen. P1. 2: 656. 1891.

Epidendrum labiatum Sw., Prodr. Veg. Ind. Occ.: 124. 1788.

Liparis labiata (Sw.) Spreng., Syst. Veg. 3: 741. 1826. 
Oncidium labiatum (Sw.) Rchb.f. in W.G.Walpers, Ann. Bot. Syst. 6: 741. 1863.

Leochilus cochlearis (Lindl.) Lindl., Edwards's Bot. Reg. 28(Misc.): 23. 1842.

Rodriguezia cochlearis Lindl., Edwards's Bot. Reg. 28(Misc.): 22. 1842.

Oncidium salvum Rchb.f., Flora 48: 278. 1865.

Leochilus salvus (Rchb.f.) Griseb., Cat. Pl. Cub.: 267. 1866.

Oncidium lansbergii Rchb.f., Gard. Chron., n.s., 5: 460. 1876.

Oncidium depauperatum F.Lehm. \& Kraenzl., Bot. Jahrb. Syst. 26: 496. 1899.

Cyrtochilum depauperatum (F.Lehm. \& Kraenzl.) Kraenzl., Notizbl. Bot. Gart. Berlin-Dahlem 7: 98. 1917.

Leochilus depauperatus (F.Lehm. \& Kraenzl.) Kraenzl. in H.G.A.Engler (ed.), Pflanzenr., IV, 50(80): 295. 1922.

Leochilus gracilis Schltr., Repert. Spec. Nov. Regni Veg. Beih. 19: 152. 1923.

VOUCHER: S. Laube et al 56 (PMA).

Leochilus leochilinus (Rchb.f.) M.W.Chase \& N.H.Williams, Lindleyana 21(3): 21. 2008.

Rodriguezia leochilina Rchb.f., Gard. Chron. 1871: 970. 1871.

Mesospinidium leochilinum (Rchb.f.) Schltr., Repert. Spec. Nov. Regni Veg. Beih. 19: 253. 1923.

Hybochilus leochilinus (Rchb.f.) Mansf., Repert. Spec. Nov. Regni Veg. 44: 58. 1938.

Goniochilus leochilinus (Rchb.f.) M.W.Chase, Contr. Univ. Michigan Herb. 16: 125. 1987.

VOUCHER: H. Butcher S.n. (PMA).

Leochilus scriptus (Scheidw.) Rchb.f., Xenia Orchid. 1: 15.1854.

Cryptosanus scriptus Scheidw., Allg. Gartenzeitung 11: 101. 1843.

Oncidium scriptum (Scheidw.) Rchb.f. in W.G.Walpers, Ann. Bot. Syst. 6: 772. 1863.

Leochilus herbaceus Lindl., Edwards's Bot. Reg. 30(Misc.): 90. 1844.

Oncidium herbaceum (Lindl.) Rchb.f. in W.G.Walpers, Ann. Bot. Syst. 6: 772. 1863.

Leochilus major Schltr., Repert. Spec. Nov. Regni Veg. 15: 209. 1918.

Leochilus powellii Schltr., Repert. Spec. Nov. Regni Veg. Beih. 17: 81. 1922.
Leochilus retusus Schltr., Repert. Spec. Nov. Regni Veg. Beih. 19: 256. 1923.

Voucher: C.W. Powell 129 (AMES).

Leochilus tricuspidatus (Rchb.f.) Kraenzl. in H.G.A.Engler (ed.), Pflanzenr., IV, 50(80): 297. 1922.

Oncidium tricuspidatum Rchb.f., Beitr. Orchid.-K.

C. Amer.: 72. 1866.

VOUCHER: H.P. Butcher s.n. (SEL).

\section{LEPANTHES Sw.}

\section{Lepanthes sp.}

VOUCher: D. Bogarin 8651 (JBL).

This Voucher correspond to a new species of the $L$. guatemalensis group (Pupulin \& Bogarín, in prep.).

Lepanthes ankistra Luer \& Dressler, Orquideología 16(3): 12.1986.

Voucher: C. Luer et al. 10534 (MO).

Lepanthes antilocapra Luer \& Dressler, Orquideología 16(3): 15. 1986.

Voucher: C. Luer et al. 10544 (MO).

Lepanthes blephariglossa Schltr., Beih. Bot. Centralbl. 36(2): 394. 1918.

Lepanthes collaris Luer, Lindleyana 2(4): 185. 1987.

Voucher: C. Luer et al. 10563 (MO).

Lepanthes brunnescens Luer, Phytologia 55: 179. 1984

Voucher: C. Luer et al. 9299 (SEL).

Lepanthes caloptera Luer, Phytologia 55: 180. 1984.

VOUCHER: C. Luer et al. 9194 (SEL).

Lepanthes candida Endres ex Luer, Lindleyana 10: 138. 1995.

VOUCHER: C. Luer et al. 9276 (SEL).

Lepanthes chiriquensis Schltr., Repert. Spec. Nov. Regni Veg. Beih. 17: 17. 1922.

Voucher: C.H. Powell 254 (AMES).

Lepanthes coeloglossa Luer, Phytologia 55: 180. 1984.

VOUCHER: C. Luer et al. 1357 (SEL).

Lepanthes confusa Ames \& C.Schweinf., Schedul. Orchid. 10: 40. 1930.

VOUCHER: not seen, cited by Luer (2003).

Lepanthes confusoides Luer, Monogr. Syst. Bot. Missouri Bot. Gard. 76: 165. 1999.

VOUCHER: C. Luer 18975 (MO).

Lepanthes deformis Luer \& Hirtz, Orchidee (Hamburg) 38: 38. 1987.

VOuCHER: S. Laube et al. 628 (PMA). 
Lepanthes demissa Luer, Phytologia 55: 182. 1984.

Voucher: C. Luer et al. 9292 (MO).

Lepanthes disticha (A.Rich. \& Galeotti) Garay \& R.E.Schult., Rhodora 61: 6. 1959.

Voucher: C. Luer et al. 9205 (MO).

Lepanthes droseroides Luer, Lindleyana 2: 188. 1987.

VOUCHer: C. Luer 11632 (MO).

Lepanthes eciliata Schltr., Repert. Spec. Nov. Regni

Veg. 12: 203. 1913.

Voucher: H. Pittier 3173 (US).

Lepanthes edentula Luer, Lindleyana 10: 143. 1995.

Voucher: C. Luer et al. 10587 (MO).

Lepanthes elata Rchb.f., Beitr. Orchid.-K. C. Amer.:

90. 1866 .

VOUCHER: R.L. Dressler 5090 (PMA).

Lepanthes elegans Luer, Lindleyana 10: 146. 1995.

Voucher: not seen, cited by Luer (2003).

Lepanthes excedens Ames \& Correll, Bot. Mus. Leafl. 10: 72.1942.

Lepanthes empis Luer., Phytologia 55(3): 183-184. 1984.

VOuCHER: C. Luer et al. 9314 (SEL).

Lepanthes eximia Ames, Schedul. Orchid. 5: 21. 1923.

Voucher: E. Rodríguez-Quiel et al. $322(\mathrm{UCH})$.

Lepanthes exposita Luer, Lindleyana 2(4): 191. 1987.

VOUCHER: C. Luer et al. 11634 (SEL).

Lepanthes fimbriata Ames, Schedul. Orchid. 3: 11. 1923.

Lepanthes setos Luer, Phytologia 55(3): 193-194. 1984.

Voucher: C. Luer et al. 9279 (SEL).

Lepanthes gracillima Endrés ex Luer, Lindleyana 10: 154. 1995.

VOUCHER: D. Bogarín 10993 (UCH).

Lepanthes grandiflora Ames \& C. Schweinf., Schedul. Orch. 10: 44-45. 1930.

Voucher: E. Olmos sub D. Bogarín 2976 (JBL).

Lepanthes helleri A.D.Hawkes, Phytologia 14: 6. 1966.

Lepanthes infundibulum Luer, Phytologia 55(3): 186. 1984.

VOUCHER: C. Luer et al. 1331 (SEL).

Lepanthes horrida Rchb.f., Bonplandia (Hannover) 3: 70. 1855 .

VOUCHER: T. M. Antonio 1643 (MO).

Lepanthes incantata Luer, Lindleyana 2: 194. 1987.

VOUCHER: C. Luer et al. 9195 (SEL).
Lepanthes jimenezii Schltr., Repert. Spec. Nov. Regni Veg. Beih. 19: 281. 1923.

Lepanthes crossota Luer, Lindleyana 2(4): 188. 1987.

Voucher: C. Luer et al. 11630 (MO).

Lepanthes jugum Luer, Lindleyana 2(4): 197. 1987.

VOUCHER: C. Luer et al. 18968 (MO).

Lepanthes laevis Luer, Monogr. Syst. Bot. Missouri Bot. Gard. 76: 168. 1999.

VOUCHER: C. Luer et al. 18823 (MO).

Lepanthes lindleyana Oerst. \& Rchb.f. in H.G.Reichenbach, Xenia Orchid. 1: 149. 1858.

Voucher: C. Luer et al. 9282 (SEL).

Lepanthes luerorum B.T.Larsen, Phytotaxa 175 (5)

279-280. 2014.

VOUCHER: P. Peeters s.n. (LISI).

Lepanthes maduroi Luer, Phytologia 55: 186. 1984.

Voucher: C. Luer et al. 9305 (SEL).

Lepanthes mariposa Luer, Phytologia 55: 187. 1984.

VOUCHER: C. Luer et al. 9278 (SEL).

Lepanthes maxonii Schltr., Repert. Spec. Nov. Regni Veg. 12: 204. 1913.

Voucher: W.R. Maxon 5494 (US).

Lepanthes minutilabia Ames \& C.Schweinf., Schedul. Orchid. 10: 49. 1930.

Voucher: D. Bogarín et al. 10738 (JBL).

Lepanthes minutissima Endres ex Luer, Lindleyana 10: 159. 1995.

Voucher: D. Bogarin et al. 11003 (JBL).

Lepanthes minyglossa Luer, Selbyana 23: 15. 2002.

VOUCHER: C. Luer et al. 10540 (MO).

Lepanthes mollis Luer, Selbyana 23: 15. 2002.

VOUCHer: C. Luer et al. 10532 (MO).

Lepanthes myiophora Luer, Lindleyana 2: 204. 1987.

VOUCHER: C. Luer et al. 12879 (MO).

Lepanthes mystax Luer \& R.Escobar, Phytologia 54: 355. 1983.

Voucher: E. Olmos sub D. Bogarín 5963 (JBL).

Lepanthes odontolabis Luer, Lindleyana 2: 207. 1987.

VOUCHER: C. Luer et al. 11633 (MO).

Lepanthes olmosii Bogarín, Orchid Digest 73: 141. 2009.

VOUCHER: D. Bogarín 3005 (PMA).

Lepanthes otopetala Luer, Lindleyana 6: 76. 1991.

VOuCHER: C. Luer et al. 14741 (MO).

Lepanthes pachyglossa Luer, Phytologia 57: 59. 1985.

VOUCHER: C. Luer et al. 10029 (MO). 
Lepanthes pantomima Luer \& Dressler, Orquideología 16: 3.1986.

Lepanthes arachnion Luer \& Dressler, Orquideología 16(3): 6-9, 20-22. 1986.

Voucher: C. Luer et al. 10614 (MO).

Lepanthes pexa Luer, Lindleyana 2: 181. 1987.

Voucher: C. Luer et al. 10622 (MO).

Lepanthes prora Luer, Phytologia 55: 191. 1984.

VOUCHER: C. Luer et al. 9207 (SEL).

Lepanthes psyche Luer, Phytologia 55: 192. 1984.

VOUCHER: C. Luer et al. 9317 (SEL).

Lepanthes pulcherrima Endrés ex Bogarín \& Pupulin, Harvard Pap. Bot. 15: 118. 2010.

VOUCHER: D. Bogarín 5992 (JBL).

Lepanthes pycnogenia Luer, Lindleyana 7(2): 114, f. 10. 1992.

VOUCHER: C. Luer et al. 13782 (MO).

Lepanthes rauhii Luer, Orchidee (Hamburg) 38: 59. 1987.

VOUCHER: C. Luer et al. 11423 (MO).

Lepanthes regularis Luer, Lindleyana 2: 210. 1987.

VOUCHER: C. Luer et al. 11631 (MO).

Lepanthes ridicula Luer, Monogr. Syst. Bot. Missouri Bot. Gard. 76: 168. 1999.

Voucher: C. Luer et al. 18827 (MO).

Lepanthes rotundifolia L.O.Williams, Ann. Missouri

Bot. Gard. 27: 275. 1940.

VOUCHER: P.H. Allen 1835 (MO).

Lepanthes sanjuanensis Bogarín \& Karremans, Lankesteriana 12(2): 113-114, f. 3, 4C. 2012.

Voucher: Z. Serracin et al. 772 (UCH).

Lepanthes schizocardia Luer, Phytologia 55: 193. 1984.

Voucher: C. Luer et al. 9193 (SEL).

Lepanthes seegeri Luer, Orchidee (Hamburg) 38: 60. 1987.

VOUCHER: C. Luer et al. 11424 (MO).

Lepanthes stenorhyncha Luer, Lindleyana 10: 170. 1995.

Voucher: E. Olmos sub D. Bogarín 5974 (JBL).

Lepanthes tetroptera Luer, Lindleyana 2: 214. 1987.

Voucher: C. Luer et al. 11628 (MO).

Lepanthes tipulifera Rchb.f., Beitr. Orchid.-K. C. Amer.: 91. 1866.

Lepanthes insectiflora C.Schweinf., Bot. Mus. Leafl. 7: 153. 1939.

VOUCHER: M.E. Davidson 185 (F).
Lepanthes trichidion Luer, Phytologia 55: 194. 1984. VOUCHER: C. Luer et al. 9307 (SEL).

Lepanthes truncata Luer \& Dressler, Orquideología 16(3): 9. 1986.

VOUCHER: C. Luer et al. 10618 (MO).

Lepanthes turialvae Rchb.f., Bonplandia (Hannover) 3: 255.1855.

Voucher: M. Blanco et al. 2835 (PMA).

Lepanthes wendlandii Rchb.f., Beitr. Orchid.-K. C. Amer.: 91. 1866.

Voucher: C. Galdames et al. 3432 (PMA).

Lepanthopsis (Cogn.) Ames

Lepanthopsis floripecten (Rchb.f.) Ames, Bot. Mus. Leafl. 1(9): 11. 1933.

Pleurothallis floripecten Rchb.f., Bonplandia (Hannover) 2: 25. 1854.

Humboldtia floripecten (Rchb.f.) Kuntze, Revis. Gen. P1. 2: 667. 1891.

Lepanthes secunda Barb.Rodr., Gen. Spec. Orchid. 2: 70.1881.

Pleurothallis unilateralis Cogn. in C.F.P.von Martius \& auct. suc. (eds.), Fl. Bras. 3(4): 592. 1896.

Lepanthopsis unilateralis (Cogn.) Porto \& Brade, Rodriguésia 1(2): 37. 1935.

Lepanthopsis secunda (Barb.Rodr.) Hoehne, Bol. Mus. Nac. Rio de Janeiro 12(2): 29. 1936.

VOUCHER: not seen, cited by Luer (1991).

Although widely distributed in the Neotropics we were unable to see a Voucher from Panama. This species was cited by Luer (1991) based on Dunsterville \& Garay (1961); however, they did not cite any voucher. It is likely that a population of this species will appear in Panama.

Lepanthopsis rinkei Luer, Monogr. Syst. Bot. Missouri Bot. Gard. 88: 104. 2002.

VOUCHER: C. Luer 20048 (MO).

This species is based on a plant without collecting data (ostensibly from Panama), purchased from Andy's Orchids, who had obtained the plant form Maduro in Panama (Luer 2002b).

\section{LIPARIS Rich.}

Liparis arnoglossophylla (Rchb.f.) Rchb.f. ex Hemsl., Biol. Cent.-Amer., Bot. 3: 212. 1884.

Sturmia arnoglossophylla Rchb.f., Bonplandia (Hannover) 4: 217. 1856. 
Leptorkis arnoglossophylla (Rchb.f.) Kuntze, Revis. Gen. Pl. 2: 671. 1891.

Liparis vexillifera var. arnoglossophylla (Rchb.f.)

R.E.Schult., Bot. Mus. Leafl. 7: 123. 1939.

VOUCHER: M.E. Davidson 998 (F).

Liparis elata Lindl., Bot. Reg. 14: t. 1175. 1828.

Leptorkis elata (Lindl.) Kuntze, Revis. Gen. P1. 2: 671. 1891.

Diteilis elata (Lindl.) M.A.Clem. \& D.L.Jones, Orchadian 15: 40. 2005.

VOUCHER: R.L. Dressler 2864 (US).

Liparis vexillifera (Lex.) Cogn. in C.F.P.von Martius $\&$ auct. suc. (eds.), Fl. Bras. 3(4): 289. 1895.

Cymbidium vexilliferum Lex. in P.de La Llave \& J.M.de Lexarza, Nov. Veg. Descr. 2(Orchid. Opusc.): 11. 1825.

Leptorkis vexillifera (Lex.) Kuntze, Revis. Gen. Pl. 3(2): 300. 1898.

Malaxis galeottiana A.Rich. \& Galeotti, Ann. Sci. Nat., Bot., sér. 3, 3: 18.1845.

Liparis galeottiana (A.Rich. \& Galeotti) Hemsl., Gard. Chron., n.s., 11: 559. 1879.

Liparis vexillifera var. latifolia Cogn. in C.F.P.von Martius (ed.), Fl. Bras. 3(4): 290. 1895.

Liparis vexillifera var. galeottiana (A.Rich. \& Galeotti) Ames \& Correll, Bot. Mus. Leafl. 10: 79. 1942.

VOUCHER: not seen, cited by Dressler (2003c).

\section{LOCKHARTIA Hook.}

Lockhartia acuta (Lindl.) Rchb.f., Bot. Zeitung (Berlin) 10: 767. 1852.

Fernandezia acuta Lindl., Edwards's Bot. Reg. 21: t. 1806.1836.

Lockhartia pallida Rchb.f., Bonplandia (Hannover) 2: 14.1854.

Lockhartia lasseri Schnee, Revista Fac. Agron. (Maracay) 1: 120. 1952.

VOUCHER: T.B. Croat 8056 (MO).

Lockhartia amoena Endrés \& Rchb.f., Gard. Chron. 1872: 666. 1872.

Lockhartia costaricensis Schltr., Repert. Spec.

Nov. Regni Veg. 3: 81. 1906.

Voucher: C.A. Luer et al. 10520 (MO).

Lockhartia chocoensis Kraenzl. in H.G.A.Engler (ed.), Pflanzenr., IV, 50(83): 19. 1923.

VOUCHER: D. Bogarín et al. 10908 (UCH).
Lockhartia grandibractea Kraenzl. in H.G.A.Engler (ed.), Pflanzenr., IV, 50(83): 15. 1923.

Lockhartia triangulabia Ames \& C.Schweinf., Schedul. Orchid. 8: 80. 1925.

Lockhartia amoena var. triangulabia (Ames \& C.Schweinf.) C.Schweinf. \& P.H.Allen, Bot. Mus. Leafl. 13: 150. 1948.

VOUCHER: C.W. Powell $362 a$ (AMES).

Lockhartia hercodonta Rchb.f. ex Kraenzl. in H.G.A.Engler (ed.), Pflanzenr., IV, 50(83): 8. 1923.

Voucher: D. Cáceres et al. 572 (UCH).

Lockhartia micrantha Rchb.f., Bot. Zeitung (Berlin) 10: 768.1852.

Lockhartia chiriquiensis Schltr., Repert. Spec. Nov. Regni Veg. 12: 215. 1913.

Lockhartia lankesteri Ames, Schedul. Orchid. 5: 36. 1923.

Lockhartia pandurata Pupulin, Revista Biol. Trop. 46: 998. 1998.

VOUCHER: H. Cuming s.n. (W).

Lockhartia obtusata L.O.Williams, Amer. Orchid Soc. Bull. 9: 209. 1941.

Voucher: P.H. Allen 2160 (AMES).

Lockhartia oerstedii Rchb.f., Bot. Zeitung (Berlin) 10: 767. 1852.

Oncidium mirabile Rchb.f., Bot. Zeitung (Berlin) 10: 697. 1852., nom. utique rej.

Lockhartia mirabilis (Rchb.f.) Rchb.f., Xenia Orchid. 1: 106. 1855.

Lockhartia verrucosa Rchb.f., Hamburger GartenBlumenzeitung 15: 53. 1859.

Lockhartia lamellosa Rchb.f., Hamburger GartenBlumenzeitung 21: 300. 1865.

Fernandezia robusta Bateman, Bot. Mag. 92: t. 5592. 1866.

Lockhartia robusta (Bateman) Schltr., Orchideen Beschreib. Kult. Zücht.: 532. 1914.

VOUCHER: J. Warscewicz S.n. (W).

Lockhartia parthenoglossa Rchb.f., Hamburger Garten- Blumenzeitung 21:300. 1865.

Lockhartia pittieri Schltr., Repert. Spec. Nov. Regni Veg. 12: 216. 1913.

Lockhartia variabilis Ames \& C.Schweinf., Schedul. Orchid. 8: 81. 1925.

Lockhartia integra Ames \& C.Schweinf., Schedul. Orchid. 10: 108. 1930.

VOUCHER: H. Pittier (B.S.P.C.Z.) 3401 (AMES). 
LyCASTE Lindl.

Lycaste brevispatha (Klotzsch) Lindl. \& Paxton, Paxt. Fl. Gard. 3: 44. 1852.

Maxillaria brevispatha Klotzsch, Allg. Gartenzeitung 19: 217. 1851.

Lycaste brevispatha (Klotzsch) Rchb. f., Ann. Bot. Syst. 6: 604. 1863.

Lycaste candida Lindl. ex Rchb.f., Ann. Bot. Syst. 6: 604. 1863.

Lycaste candida var. alba Oakeley, Lycaste, Ida, Anguloa: 48. 2008.

Lycaste candida var. rubra Oakeley, Lycaste, Ida, Anguloa: 49. 2008.

Lycaste angelae Oakeley, Lycaste, Ida, Anguloa: 27. 2008.

Voucher: C.W. Powell 140 (MO).

Lycaste campbellii C.Schweinf., Sargentia 8: 103. 1949.

VOUCHER: I.M. Johnston 1371 (AMES).

Lycaste dowiana Endrés \& Rchb.f., Gard. Chron., n.s., 2: 194.1874.

Voucher: R.J. Seibert 644 (AMES, MO).

Lycaste leucantha (Klotzsch) Lindl., Paxton's F1. Gard. 2: 37. 1851.

Maxillaria leucantha Klotzsch, Allg. Gartenzeitung 18: 402.1850.

Voucher: H.W. Churchill 5420 (MO).

Lycaste panamanensis (Fowlie) Archila, Revista Guatemal. 5(2): s.p. 2002.

Lycaste macrophylla subsp. panamanensis Fowlie, Lasca Leaves 14: 40. 1964.

Lycaste panamanensis Oakeley, Lycaste, Ida, Anguloa: 139. 2008.

Voucher: J.A. Fowlie F62P8 (LASCA).

Lycaste powellii Schltr., Repert. Spec. Nov. Regni Veg.

Beih. 17: 65. 1922.

Voucher: C.W. Powell 15 (AMES).

Lycaste puntarenasensis (Fowlie) Archila, Revista Guatemal. 5(2): s.p. 2002.

Lycaste macrophylla subsp. puntarenasensis Fowlie, Lasca Leaves 14(1): 45, f.. 1964.

Lycaste puntarenasensis (Fowlie) Oakeley, Orchid

Digest 71(4): 207. 2007.

VOUCHER: H.P. Butcher S.n. (MO).

Lycaste schilleriana Rchb.f., Bonplandia (Hannover) 3: 215.1855.
Lycaste schilleriana var. lehmannii Regel, Gartenflora 39: 233. 1890.

Lycaste hennisiana Kraenzl., Orchis 1: 33. 1906.

Lycaste longisepala C.Schweinf., Bot. Mus. Leafl. 15: 157. 1952.

Lycaste schilleriana var. alba Oakeley, Lycaste, Ida, Anguloa: 151. 2008.

Lycaste schilleriana var. rosea Oakeley, Lycaste, Ida, Anguloa: 153. 2008.

VOUCHER: R.L. Dressler 5390 (PMA).

Lycaste tricolor Rchb.f. in W.G.Walpers, Ann. Bot. Syst. 6: 603. 1863.

Maxillaria tricolor Klotzsch, Allg. Gartenzeitung 20: 186. 1852., nom. illeg.

VOUCHER: not seen, cited by Dressler (2003c).

Macradenia R.Br.

Macradenia brassavolae Rchb.f., Bot. Zeitung (Berlin) 10: 734. 1852.

Serrastylis modesta Rolfe, Bull. Misc. Inform. Kew 1894: 158. 1894.

Macradenia modesta (Rolfe) Rolfe, Orchid Rev. 4: 357. 1896.

Macradenia brassavolae var. albiflora Senghas \& Seeger, Orchidee (Hamburg) 47(4) cppo: 832. 1996. VOUCHER: H. Pittier 4816 (US)

\section{MaCroclinium Barb.Rodr.}

Macroclinium alleniorum Dressler \& Pupulin, Lindleyana 11: 34. 1996.

Voucher: T. González Ruíz s.n. (USJ).

Macroclinium cordesii (L.O.Williams) Dodson, Icon. Pl. Trop. 10: t. 938. 1984.

Notylia cordesii L.O.Williams, Ann. Missouri Bot. Gard. 26: 286. 1939.

Voucher: R.E. Woodson et al. 1932 (MO).

Macroclinium doderoi Mora-Ret. \& Pupulin, Selbyana 18: 7. 1997.

VOUCHER: R.L. Dressler 5386 (MO).

Macroclinium junctum (Dressler) Dodson, Icon. Pl. Trop. 10: t. 938. 1984.

Notylia juncta Dressler, Orquideología 4: 164. 1969.

VOUCHER: R.L. Dressler 3092 (US).

Macroclinium lineare (Ames \& C.Schweinf.) Dodson, Icon. Pl. Trop. 10: t. 938. 1984. 
Notylia linearis Ames \& C.Schweinf., Schedul. Orchid. 8: 72. 1925.

Voucher: D. Bogarin et al. 11129 (UCH).

Macroclinium paniculatum (Ames \& C.Schweinf.) Dodson, Icon. P1. Trop. 10: t. 938. 1984.

Notylia paniculata Ames \& C.Schweinf., Schedul. Orchid. 10: 102. 1930.

Voucher: J.P. Folsom \& G. Alonzo de Monte 5025 (MO).

Macroclinium ramonense (Schltr.) Dodson, Icon. Pl. Trop. 10: t. 939. 1984.

Notylia ramonensis Schltr., Repert. Spec. Nov. Regni Veg. Beih. 19: 250. 1923.

VOUCHER: not seen, cited by Dressler (2003c).

Macroclinium robustum Pupulin \& Mora-Ret., Selbyana 18: 7. 1997.

VOUCHER: R.L. Dressler 3751 (US).

Macroclinium simplex (Dressler) Dodson, Icon. P1. Trop. 10: t. 939. 1984.

Notylia simplex Dressler, Orquideología 4: 166. 1969.

VOUCHER: R.L. Dressler 3460 (US).

Macroclinium wullschlaegelianum (H.Focke)

Dodson, Icon. Pl. Trop. 10: t. 939. 1984.

Notylia wullschlaegeliana H.Focke, Bot. Zeitung (Berlin) 11: 343. 1853.

VOUCHER: R.L. Dressler 3373 (MO).

Malaxis Sol. ex Sw.

Malaxis brachyrrhynchos (Rchb.f.) Ames, Proc. Biol. Soc. Wash. 35: 84. 1922.

Microstylis brachyrrhynchos Rchb.f., Flora 71: 152. 1888.

Voucher: J.P. Folsom 4082 (MO).

Malaxis brevis Dressler, Lankesteriana 4(1): 97. 2004.

Malaxis brevis Dressler, Selbyana 24: 141. 2003. nom. inval.

VOUCHER: G.L. Webster \& G. Brecken 16599 (MO).

Malaxis carnosa Correll, Lloydia 10: 210. 1948.

Stelis carnosa Kunth in F.W.H.von Humboldt, A.J.A.Bonpland \& C.S.Kunth, Nov. Gen. Sp. 1: 361.1816.

Dienia calycina Lindl., Gen. Sp. Orchid. Pl.: 23. 1830.

Microstylis calycina (Lind1.) Ridl., J. Linn. Soc., Bot. 24: 331. 1888.
Microstylis gracilis Ridl., J. Linn. Soc., Bot. 24: 321. 1888.

Serapias parisitica Pavone ex Ridl., J. Linn. Soc., Bot. 24: 331. 1888.

Malaxis calycina (Lindl.) Kuntze, Revis. Gen. Pl. 2: 673. 1891.

Malaxis gracilis (Ridl.) Kuntze, Revis. Gen. Pl. 2: 673. 1891.

Microstylis monticola Schltr., Repert. Spec. Nov. Regni Veg. 3: 17. 1906.

Microstylis microtoides Schltr., Beih. Bot. Centralbl. 36(2): 381. 1918.

Malaxis monticola (Schltr.) Ames, Proc. Biol. Soc. Wash. 35: 84. 1922., nom. illeg.

VOucher: not seen, cited by Dressler (2003c).

Malaxis hastilabia (Rchb.f.) Kuntze, Revis. Gen. Pl. 2: 673. 1891.

Microstylis hastilabia Rchb.f., Beitr. Orchid.-K. C. Amer.: 101. 1866.

Microstylis hastilabia var. major Porsch, Denkschr. Kaiserl. Akad. Wiss., Wien. Math.-Naturwiss. K1. 79: 102. 1908.

Malaxis maguirei C.Schweinf., Bull. Torrey Bot. Club 75: 217. 1948.

Malaxis sertulifera (Barb.Rodr.) Pabst, Orquídea (Rio de Janeiro) 29: 112. 1967.

Malaxis uncinata Ames \& C.Schweinf., Schedul. Orchid. 10: 15. 1930.

Voucher: T.B. Croat \& D.M. Porter 16146 (MO).

Malaxis histionantha (Link) Garay \& Dunst., Venez. Orchids Ill. 6: 226. 1976.

Microstylis histionantha Link in J.H.F.Link, J.F.Klotzsch \& C.F.Otto, Icon. Pl. Rar. 1: 11. 1840.

Microstylis brenesii Schltr., Repert. Spec. Nov. Regni Veg. Beih. 19: 167. 1923.

Voucher: R.E. Woodson 1581 (MO).

Malaxis maianthemifolia Cham. \& Schltdl., Linnaea 6: 59.1831.

Microstylis maianthemifolia (Cham. \& Schltdl.) Rchb.f., Linnaea 22: 834. 1850.

Achroanthes maianthemifolia (Cham. \& Schltdl.) Greene, Pittonia 2: 184. 1891.

Dienia cordata (Rchb.f.) Lindl., Edwards's Bot. Reg. 24(Misc.): 73. 1838.

Malaxis cochleariifolia A.Rich. \& Galeotti, Ann. Sci. Nat., Bot., sér. 3, 3: 18. 1845. 
Malaxis ichthiorhynca A.Rich. \& Galeotti, Ann. Sci. Nat., Bot., sér. 3, 3: 18. 1845.

Microstylis cochleariifolia (A.Rich. \& Galeotti) Rchb.f., Linnaea 22: 834. 1850.

Microstylis cordata (Lindl.) Rchb.f. in W.G.Walpers, Ann. Bot. Syst. 6: 207. 1861.

Microstylis ichthyorhyncha (A.Rich. \& Galeotti)

Rchb.f., Beitr. Orchid.-K. C. Amer.: 99. 1866.

Malaxis cordata (Lindl.) Kuntze, Revis. Gen. Pl.

2: 673. 1891.

Microstylis elegantula Schltr., Repert. Spec. Nov.

Regni Veg. 3: 17. 1906.

VOucher: R.E. Woodson, Jr. \& R.W. Schery 474 (MO).

Malaxis pandurata (Schltr.) Ames, Proc. Biol. Soc. Wash. 35: 84. 1922.

Microstylis pandurata Schltr., Repert. Spec. Nov.

Regni Veg. 3: 77. 1906.

VOUCHER: G. McPherson 7255 (MO).

Malaxis pittieri (Schltr.) Ames, Proc. Biol. Soc. Wash.

35: 84. 1922.

Microstylis pittieri Schltr., Repert. Spec. Nov.

Regni Veg. 12: 203. 1913.

Voucher: H. Pittier 3277 (MO).

Malaxis rostratula Dressler, Lankesteriana 4(1): 97. 2004.

Malaxis rostratula Dressler, Selbyana 24: 142. 2003. nom. inval.

VOUCHER: R.L. Dressler 5102 (MO).

Malaxis simillima (Rchb.f.) Kuntze, Revis. Gen. P1.

2: 673. 1891.

Microstylis simillima Rchb.f., Beitr. Orchid.-K. C.

Amer.: 101. 1866.

VOUCHER: S. Knapp et al. 2137 (MO).

Malaxis soulei L.O.Williams, Ann. Missouri Bot. Gard. 21: 343. 1934.

Microstylis montana Engelm. ex Rothr., Rep. U.S.

Geogr. Surv., Wheeler 6: 264. 1879.

Achroanthes montana (Engelm. ex Rothr.) Greene,

Pittonia 2: 183. 1891.

Malaxis montana (Engelm. ex Rothr.) Kuntze,

Revis. Gen. P1. 2: 673. 1891., nom. illeg.

VOUCHER: R.E. Woodson et al. 804 (MO).

Malaxis steyermarkii Correll, Lloydia 10: 210. 1948.

Microstylis steyermarkii (Correll) Marg., Ann.

Naturhist. Mus. Wien, B 109: 184. 2007[2008].

Voucher: not seen, cited by Dressler (2003c).

Malaxis wendlandii (Rchb.f.) L.O.Williams, Ann.

Missouri Bot. Gard. 33: 136. 1946.
Liparis wendlandii Rchb.f., Beitr. Orchid.-K. C. Amer.: 98. 1866.

Leptorkis wendlandii (Rchb.f.) Kuntze, Revis. Gen. P1. 2: 669. 1891.

Crossoliparis wendlandii (Rchb.f.) Marg., Acta Soc. Bot. Poloniae 78: 299. 2009.

Voucher: R.J. Seibert 216 (MO).

Malaxis wercklei (Schltr.) Ames, Proc. Biol. Soc. Wash. 35: 85. 1922.

Microstylis wercklei Schltr., Beih. Bot. Centralbl. 36(2): 382. 1918.

Microstylis parthoni var. denticulata Rchb.f., Beitr. Orchid.-K. C. Amer.: 100. 1866.

Malaxis parthoni var. denticulata (Rchb.f.) Ames, F.T.Hubb. \& C.Schweinf., Bot. Mus. Leafl. 3: 40. 1934.

Voucher: R.J. Seibert 170 (MO).

Malaxis woodsonii L.O.Williams, Ann. Missouri Bot. Gard. 26: 281. 1939.

VOUCHER: R.E. Woodson et al. 831 (MO).

\section{Mapinguari Carnevali \& R.B.Singer}

Mapinguari longipetiolatus (Ames \& C.Schweinf.) Carnevali \& R.B.Singer, Lankesteriana 7: 525. 2007.

Maxillaria longipetiolata Ames \& C.Schweinf., Schedul. Orchid. 8: 61. 1925.

Voucher: C.W. Powell 307 (AMES).

\section{Masdevallia Ruiz \& Pav.}

Masdevallia andresiana Luer \& Maduro, Monogr. Syst. Bot. Missouri Bot. Gard. 103: 283. 2005.

Alaticaulia andresiana (Luer \& Maduro) Luer, Monogr. Syst. Bot. Missouri Bot. Gard. 112: 118. 2007.

VOUCHER: M. Rao 96 (MO).

Masdevallia attenuata Rchb.f., Gard. Chron. 1871: 834. 1871.

Acinopetala attenuata (Rchb.f.) Luer, Monogr. Syst. Bot. Missouri Bot. Gard. 105: 3. 2006.

Masdevallia scabrilinguis Luer, Phytologia 44: 168. 1979.

Acinopetala scabrilinguis (Luer) Luer, Monogr. Syst. Bot. Missouri Bot. Gard. 105: 3. 2006.

VOUCHER: G. Zahn S.n. (W).

Masdevallia chontalensis Rchb.f., Otia Bot. Hamburg.: 17. 1878. 
Acinopetala chontalensis (Rchb.f.) Luer, Monogr.

Syst. Bot. Missouri Bot. Gard. 105: 3. 2006.

Masdevallia diantha Schltr., Beih. Bot. Centralbl. 36(2): 384. 1918.

Voucher: D. Cáceres et al. 523 (UCH).

Masdevallia collina L.O.Williams, Amer. Orchid Soc. Bull. 11: 93. 1942.

Petalodon collinus (L.O.Williams) Luer, Monogr.

Syst. Bot. Missouri Bot. Gard. 105: 11. 2006.

VOUCHER: P.H. Allen 2158 (AMES).

Masdevallia eburnea Luer \& Maduro, Monogr. Syst. Bot. Missouri Bot. Gard. 91: 409a. 2003.

Reichantha eburnea (Luer \& Maduro) Luer, Monogr. Syst. Bot. Missouri Bot. Gard. 105: 13. 2006.

VOucher: E. Olmos 157 (MO).

Masdevallia flaveola Rchb.f., Gard. Chron., n.s., 21: 638. 1884.

Acinopetala flaveola (Rchb.f.) Luer, Monogr. Syst.

Bot. Missouri Bot. Gard. 105: 3. 2006.

VOUCHER: B.E. Hammel 5757 (MO).

Masdevallia gloriae Luer \& Maduro, Monogr. Syst. Bot. Missouri Bot. Gard. 91: 413a. 2003.

Reichantha gloriae (Luer \& Maduro) Luer, Monogr. Syst. Bot. Missouri Bot. Gard. 105: 13. 2006.

VOUCHER: A. Maduro s.n. (MO).

Masdevallia lata Rchb.f., Gard. Chron. 1877(1): 653. 1877.

Alaticaulia lata (Rchb.f.) Luer, Monogr. Syst. Bot. Missouri Bot. Gard. 105: 5. 2006.

Masdevallia borucana P.H.Allen, Ceiba 3: 147. 1952.

VOUCHER: C.A. Luer et al. 9329 (SEL).

Masdevallia livingstoneana Roezl ex Rchb.f., Gard. Chron., n.s., 2: 322. 1874.

Acinopetala livingstoneana (Roezl ex Rchb.f.) Luer, Monogr. Syst. Bot. Missouri Bot. Gard. 112: 118. 2007.

Scaphosepalum panamense Schltr., Repert. Spec. Nov. Regni Veg. 12: 205. 1913.

Masdevallia panamensis (Schltr.) Ames, Schedul. Orchid. 4: 9. 1923.

VOUCHER: B. Roezl s.n. (W).

Masdevallia maduroi Luer, Monogr. Syst. Bot. Missouri Bot. Gard. 72: 110. 1998.

Alaticaulia maduroi (Luer) Luer, Monogr. Syst.
Bot. Missouri Bot. Gard. 112: 118. 2007.

VOUCHER: C.A. Luer 18932 (MO).

Masdevallia marginella Rchb.f., Gard. Chron., n.s., 20: 38. 1883.

Reichantha marginella (Rchb.f.) Luer, Monogr. Syst. Bot. Missouri Bot. Gard. 105: 13. 2006.

Masdevallia costaricensis Rolfe, Gard. Chron., ser. 3, 8: 183. 1890.

Voucher: D. Bogarín et al. 8686 (JBL).

Masdevallia molossoides Kraenzl., Repert. Spec. Nov. Regni Veg. 17: 416. 1921.

Buccella molossoides (Kraenzl.) Luer, Monogr. Syst. Bot. Missouri Bot. Gard. 105: 7. 2006.

Masdevallia anura Kraenzl., Repert. Spec. Nov. Regni Veg. 17: 433. 1921.

Masdevallia rhopalura Schltr., Repert. Spec. Nov. Regni Veg. Beih. 19: 14. 1923.

VOUCHER: C.A. Luer 5425 (MO).

Masdevallia nidifica Rchb.f., Otia Bot. Hamburg.: 18. 1878.

Voucher: D. Cáceres et al. 548 (UCH).

Masdevallia olmosii Königer \& Sijm, Arcula 12: 303. 2003.

Voucher: W. Königer WK-115 (M).

Masdevallia ostaurina Luer, Monogr. Syst. Bot. Missouri Bot. Gard. 95: 249. 2004.

Reichantha ostaurina (Luer) Luer, Monogr. Syst. Bot. Missouri Bot. Gard. 105: 13. 2006.

VOUCHER: V.N.M. Rao 161 (MO).

Masdevallia picturata Rchb.f., Otia Bot. Hamburg.: 16. 1878.

Fissia picturata (Rchb.f.) Luer, Monogr. Syst. Bot. Missouri Bot. Gard. 105: 9. 2006.

Masdevallia meleagris Rchb.f., Xenia Orchid. 1: 198. 1858., nom. illeg.

Masdevallia picturata var. minor Cogn. in C.F.P.von Martius \& auct. suc. (eds.), Fl. Bras. 3(4): 329. 1896.

Masdevallia ocanensis Kraenzl., Repert. Spec. Nov. Regni Veg. 17: 429. 1921.

Masdevallia cryptocopis Rchb.f. ex Kraenzl., Repert. Spec. Nov. Regni Veg. Beih. 34: 182. 1925.

Rodrigoa cryptocopis (Rchb.f. ex Kraenzl.) Braas, Orchidee (Hamburg) 30: 220. 1979.

Masdevallia picturata subsp. minor (Cogn.) Luer, Monogr. Syst. Bot. Missouri Bot. Gard. 16: 12. 1986. 
Voucher: C.A. Luer et al. 1365 (SEL).

Masdevallia pleurothalloides Luer, Selbyana 3: 218. 1977.

Fissia pleurothalloides (Luer) Luer, Monogr. Syst.

Bot. Missouri Bot. Gard. 105: 9. 2006.

Voucher: R.L. Dressler 3155 (SEL).

Masdevallia rafaeliana Luer, Selbyana 5: 149. 1979.

Spilotantha rafaeliana (Luer) Luer, Monogr. Syst.

Bot. Missouri Bot. Gard. 105: 15. 2006.

Voucher: L.D. Gómez 22225 (MO).

Masdevallia schizopetala Kraenzl., Bull. Misc. Inform. Kew 1925: 101. 1925.

Acinopetala schizopetala (Kraenzl.) Luer, Monogr. Syst. Bot. Missouri Bot. Gard. 105: 3. 2006.

Masdevallia morenoi Luer, Phytologia 39: 213. 1978.

Voucher: C.A. Luer 2927 (SEL).

Masdevallia smallmaniana Luer, Monogr. Syst. Bot. Missouri. Bot. Gard. 87: 505a. 2002.

Voucher: D. Bogarín et al. 10984 (UCH).

Masdevallia striatella Rchb.f., Gard. Chron., n.s., 26: 103. 1886.

Reichantha striatella (Rchb.f.) Luer, Monogr. Syst.

Bot. Missouri Bot. Gard. 105: 13. 2006.

Masdevallia chloracra Rchb.f., Flora 69: 560. 1886.

Masdevallia ecaudata Schltr., Beih. Bot. Centralbl. 36: 384. 1918.

Masdevallia superflua Kraenzl., Repert. Spec. Nov. Regni Veg. 17: 414. 1921.

Masdevallia demissa var. superflua (Kraenzl.)

Kraenzl., Repert. Spec. Nov. Regni Veg. Beih. 34: 80. 1925.

VOUCHER: G. White \& P. White 81 (MO).

Masdevallia thienii Dodson, Selbyana 2: 54. 1977.

Reichantha thienii (Dodson) Luer, Monogr. Syst. Bot. Missouri Bot. Gard. 105: 13. 2006.

Alaticaulia thienii (Dodson) Luer, Monogr. Syst.

Bot. Missouri Bot. Gard. 112: 118. 2007.

VOUCHER: G. McPherson 9615 (MO).

Masdevallia tokachiorum Luer, Lindleyana 6: 90. 1991.

Acinopetala tokachiorum (Luer) Luer, Monogr. Syst. Bot. Missouri Bot. Gard. 105: 3. 2006.

Reichantha tokachiorum (Luer) Luer, Monogr.

Syst. Bot. Missouri Bot. Gard. 112: 121. 2007.

Voucher: C. A. Luer 14943 (MO).
Masdevallia tonduzii Woolward, Bull. Herb. Boissier, II, 6: 82. 1906.

Reichantha tonduzii (Woolward) Luer, Monogr. Syst. Bot. Missouri Bot. Gard. 105: 13. 2006.

Voucher: C. A. Luer 2236 (SEL).

Masdevallia utriculata Luer, Phytologia 44: 169. 1979.

Regalia utriculata (Luer) Luer, Monogr. Syst. Bot. Missouri Bot. Gard. 105: 12. 2006.

VOUCHER: R.L Dressler \& J. Kuhn s.n (SEL).

Masdevallia zahlbruckneri Kraenzl., Repert. Spec.

Nov. Regni Veg. 17: 413. 1921.

Voucher: C. A. Luer 18956 (MO).

Maxillaria Ruiz \& Pav.

Maxillaria acostae Schltr., Repert. Spec. Nov. Regni Veg. Beih. 19: 301. 1923.

Voucher: M.A. Blanco \& D. Penneys 2826 (PMA).

Maxillaria angustisegmenta Ames \& C.Schweinf., Schedul. Orchid. 10: 86. 1930.

Voucher: A. Gentry 3624 (PMA).

Maxillaria angustissima Ames, F.T.Hubb. \&

C.Schweinf., Bot. Mus. Leafl. 3: 41. 1934.

Maxillaria acutifolia Schltr., Repert. Spec. Nov. Regni Veg. Beih. 19: 229. 1923., nom. illeg.

Voucher: R.L. Dressler 2946 (PMA).

Maxillaria arachnitiflora Ames \& C.Schweinf., Schedul. Orchid. 10: 87. 1930.

Voucher: M.A. Blanco \& D. Penneys 2898 (PMA).

Maxillaria brachybulbon Schltr., Repert. Spec. Nov.

Regni Veg. Beih. 19: 55. 1923.

Voucher: S.A. Mori et al. 3928 (MO).

Maxillaria chionantha J.T.Atwood, Selbyana 16: 242. 1995.

Voucher: R.L. Dressler 4839 (SEL).

Maxillaria confusa Ames \& C.Schweinf., Schedul. Orchid. 8: 57. 1925.

Voucher: M.D. Correa et al. 1893 (PMA).

Maxillaria curvicolumna M.A.Blanco \& Neubig, Orchids (West Palm Beach) 76: 451. 2007.

VOUCHER: M. Whitten 2933 (FLAS).

Maxillaria darienensis J.T.Atwood, Selbyana 19: 254. 1998[1999].

Camaridium darienensis (J.T. Atwood) Szlach. \& Sitko, Biodivers. Res. Conservation 25: 25. 2012.

VOUCHER: J.P. Folsom 4412 (MO). 
Maxillaria endresii Rchb.f., Gard. Chron., n.s., 26: 680. 1886.

VOucher: R. Pérez 228 (PMA).

Maxillaria galantha J.T.Atwood \& Carnevali, Lindleyana 9: 227. 1994.

VOUCHer: J.P. Folsom 3608 (MO).

Maxillaria hennisiana Schltr., Orchis 6: 117. 1912. Maxillaria shepheardii Rolfe, Bull. Misc. Inform. Kew 1917: 83. 1917.

VOUCHER: J.P. Folsom 5924 (MO).

Maxillaria longiloba (Ames \& C.Schweinf.)

J.T.Atwood, Icon. Pl. Trop. 16: t. 1562. 1993.

Maxillaria brenesii var. longiloba Ames \& C.Schweinf., Schedul. Orchid. 10: 90. 1930.

VOUCHer: J.P. Folsom 7252 (MO).

Maxillaria lueri Dodson, Icon. Pl. Trop. 1: t. 155. 1980.

Pseudocymbidium lueri (Dodson) Szlach. \& Sitko,

Biodivers. Res. Conservation 25: 34. 2012.

Voucher: K.J. Sytsma et al. 2505 (MO).

Maxillaria pachyacron Schltr., Repert. Spec. Nov. Regni Veg. 9(205-207): 165. 1911.

Voucher: D. Cáceres et al. 575 (UCH).

Maxillaria porrecta Lindl., Edwards's Bot. Reg. 24(Misc.): 192. 1838.

Maxillaria brunnea Linden \& Rchb.f., Bonplandia (Hannover) 2: 281. 1854.

Maxillaria mutabilis Rchb.f., Bonplandia (Hannover) 3: 216. 1858.

Maxillaria brenesii Schltr., Repert. Spec. Nov. Regni Veg. Beih. 19: 56. 1923.

Maxillaria lactea Schltr., Repert. Spec. Nov. Regni Veg. Beih. 19: 233. 1923.

Maxillaria trinitatis Ames, Schedul. Orchid. 2: 34. 1923.

Maxillaria aurantiaca Schltr., Repert. Spec. Nov. Regni Veg. Beih. 27: 87. 1924., nom. illeg.

Maxillaria trinitensis Broadway, Orchid Rev. 34: 202. 1926., orth. var.

Maxillaria calendulina Christenson, Phytotaxa 1: 21. 2009.

VOUCHER: G. McPherson 13545 (MO).

Maxillaria powellii Schltr., Repert. Spec. Nov. Regni Veg. Beih. 17: 70. 1922.

VOucher: C.W. Powell 28 (MO).

Maxillaria ramonensis Schltr., Repert. Spec. Nov. Regni Veg. Beih. 19: 235. 1923.
Maxillaria brevipes Schltr., Repert. Spec. Nov. Regni Veg. Beih. 19: 302. 1923.

Voucher: W.D. Stevens 1841 (MO).

Maxillaria reichenheimiana Endrés \& Rchb.f., Gard. Chron. 1871: 78. 1871.

VOUCHER: H.W. Churchill 5426 (MO).

Maxillaria ringens Rchb.f. in W.G.Walpers, Ann. Bot. Syst. 6: 523. 1863.

Maxillaria yzabalana S.Watson, Proc. Amer. Acad. Arts 12: 286. 1888.

Maxillaria tuerckheimii Schltr., Repert. Spec. Nov. Regni Veg. 10: 295. 1912.

Maxillaria rousseauae Schltr., Beih. Bot. Centralbl. 36(2): 413. 1918.

Maxillaria pubilabia Schltr., Repert. Spec. Nov. Regni Veg. Beih. 17: 71. 1922.

Maxillaria amparoana Schltr., Repert. Spec. Nov. Regni Veg. Beih. 19: 54. 1923.

Maxillaria lactea Schltr., Beih. Bot. Centralbl. 42(2): 131. 1925., nom. illeg.

Maxillaria lactiflora Pabst, Orquídea (Rio de Janeiro) 29: 114. 1967.

VOUCHER: Mrs. Rousseau (MO).

Maxillaria rodrigueziana J.T.Atwood \& Mora-Ret., Icon. Pl. Trop. 14: t. 1361. 1989.

VOUCHER: T. Antonio 2137 (MO).

\section{MAxillariella M.A.Blanco \& Carnevali}

Maxillariella acervata (Rchb.f.) M.A.Blanco \& Carnevali, Lankesteriana 7: 528. 2007.

Maxillaria acervata Rchb.f., Bonplandia (Hannover) 3: 217. 1855.

Maxillaria panamensis Schltr., Repert. Spec. Nov. Regni Veg. Beih. 17: 70. 1922.

Maxillariella panamensis (Schltr.) Szlach. \& Sitko, Biodivers. Res. Conservation 25: 31. 2012.

VOucher: C.W. Powell 124 (AMES).

Maxillariella alba (Hook.) M.A.Blanco \& Carnevali, Lankesteriana 7: 528. 2007.

Dendrobium album Hook., Exot. Fl. 2: t. 142. 1825.

Broughtonia alba (Hook.) Spreng., Syst. Veg. 3: 735. 1826.

Maxillaria alba (Hook.) Lindl., Gen. Sp. Orchid. P1.: 143. 1832.

Camaridium album (Hook.) Hoehne, Arq. Bot. Estado São Paulo, n.s., f.m., 2: 72.1947. 
Maxillaria hedyosma Schltr., Repert. Spec. Nov. Regni Veg. Beih. 8: 169. 1921.

Voucher: K. Systma 1560 (PMA).

Maxillariella caucae (Garay) Bogarín, comb. nov.

Bas.: Maxillaria caucae Garay, Bot. Mus. Leafl. 21: 258. 1967. Maxillaria parvula Schltr., Repert. Spec. Nov. Regni Veg. Beih. 27: 176. 1924., nom. illeg. [non Maxillaria parvula Hook. Exot. Fl. t. 217. 1826].

Voucher: F. C. Lehmann 103 (B, destroyed).

Schlechter (1924) described Maxillaria parvula Schltr. nom. illeg. based on a plant collected by F.C. Lehmann in Montijo Bay. Schlechter (1924) stated that he was not sure if that locality belongs to Colombia or Panama; however, he wrote Cauca in the type locality. The name is predated by Maxillaria parvula Hook., so Garay (1967) proposed a new name, M. caucae (although Cauca is actually part of Colombia, and the type locality stated by F.C. Lehmann today belongs to the province of Veraguas, Panama along the Pacific coast (Fig. 18). Schlechter (1924) compared the species with M. acervata because of the foliate pseudobulbs but distinguished it by the black-brownish flowers. This species should be treated in Maxillariella according to Blanco et al. (2007).

Maxillariella costaricensis (Schltr.) M.A.Blanco \& Carnevali, Lankesteriana 7: 528. 2007.

Maxillaria costaricensis Schltr., Repert. Spec. Nov. Regni Veg. Beih. 10: 232. 1923.

Voucher: M.A. Blanco \& D. Penneys 2900 (PMA).

Maxillariella diuturna (Ames \& C.Schweinf.)

M.A.Blanco \& Carnevali, Lankesteriana 7: 528. 2007.

Maxillaria diuturna Ames \& C.Schweinf., Schedul. Orchid. 8: 58. 1925.

Voucher: C.W. Powell 376 (AMES).

Maxillariella foliosa (Ames \& C.Schweinf.) Bogarín, comb. nov.

Bas.: Maxillaria foliosa Ames \& C.Schweinf., Schedul. Orchid. 8: 60. 1925.

VOUCHER: I. Gordon \& J. Him 491 (PMA).

Maxillariella foliosa has been treated as synonym of $M$. acervata however, it differs mainly by the suberect plants with elongated secondary stems (cespitose with short secondary stems), the obtuse sepals (vs. acute) and the pollinarium shape among other features. The species are found at different elevations, with M. foliosa above $1200 \mathrm{~m}$ and M. acervata is found below $800 \mathrm{~m}$ along the Pacific coast of Costa Rica and Panama (Atwood 2003).

Maxillariella linearifolia (Ames \& C.Schweinf.) M.A.Blanco \& Carnevali, Lankesteriana 7: 529. 2007.
Maxillaria linearifolia Ames \& C.Schweinf., Schedul. Orchid. 10: 95. 1930.

Voucher: T.B. Croat 49987 (MO).

Maxillariella oreocharis (Schltr.) M.A.Blanco \& Carnevali, Lankesteriana 7: 529. 2007.

Maxillaria oreocharis Schltr., Repert. Spec. Nov. Regni Veg. Beih. 17: 69. 1922.

Voucher: C.W. Powell 256 (AMES).

Maxillariella ponerantha (Rchb.f.) M.A.Blanco \& Carnevali, Lankesteriana 7: 529. 2007.

Maxillaria ponerantha Rchb.f., Bonplandia (Hannover) 2: 17. 1854.

VOUCHER: R.L. Liesner 405 (MO).

Maxillariella sanguinea (Rolfe) M.A.Blanco \& Carnevali, Lankesteriana 7: 529. 2007.

Maxillaria sanguinea Rolfe, Bull. Misc. Inform. Kew 1895: 8. 1895.

VOUCHer: J. O'Brien S.n. (K).

Maxillariella variabilis (Bateman ex Lindl.)

M.A.Blanco \& Carnevali, Lankesteriana 7: 530. 2007.

Maxillaria variabilis Bateman ex Lindl., Edwards's Bot. Reg. 23: t. 1986. 1837.

Maxillaria variabilis var. unipunctata Lindl., Edwards's Bot. Reg. 24(Misc.): 23. 1838.

Maxillaria angustifolia Hook., Hooker's Icon. P1. 4: t. 348. 1841.

Maxillaria lyonii Lindl., Edwards's Bot. Reg. 31(Misc.): 17. 1845.

Maxillaria revoluta Klotzsch, Allg. Gartenzeitung 20: 186. 1852.

Maxillaria variabilis subvar. lutea A.H.Kent in H.J.Veitch, Man. Orchid. P1. 9: 162. 1893.

Maxillaria chiriquiensis Schltr., Repert. Spec. Nov.

Regni Veg. Beih. 17: 68. 1922.

Maxillariella chiriquiensis (Schltr.) Szlach. \& Sitko, Biodivers. Res. Conservation 25: 31. 2012.

Maxillariella variabilis var. unipunctata (Lindl.)

Solano, Acta Bot. Mex. 97: 52. 2011.

Voucher: C.W. Powell 125 (MO).

\section{Microchilus C.Pres1.}

Microchilus calophyllus (Rchb.f.) Ormerod, Lindleyana 17: 216. 2002.

Physurus calophyllus Rchb.f., Beitr. Orchid.-K. C. Amer.: 64. 1866.

Erythrodes calophylla (Rchb.f.) Ames, Orchidaceae 7: 68. 1922. 
VOUCHER: C. Galdames et al. 1307 (PMA).

Microchilus epiphyticus (Dressler) Ormerod, Lindleyana 17: 216. 2002.

Erythrodes epiphytica Dressler, Orquídea (Mexico City), n.s., 13: 257. 1993.

VOUCHER: R.L. Dressler 4383 (MO).

Microchilus fuscatus Ormerod, Harvard Pap. Bot. 11(2): 161. 2007.

Voucher: S. Mori \& J. Kallunki 3412 (MO).

Microchilus maasii Ormerod, Harvard Pap. Bot. 11(2): 161. 2007.

VOUCHER: B. E. Hammel 4593 (MO).

Microchilus nigrescens (Schltr.) Ormerod, Lindleyana 17: 218. 2002.

Physurus nigrescens Schltr., Beih. Bot. Centralbl. 36(2): 380. 1918.

Erythrodes nigrescens (Schltr.) Ames, Orchidaceae 7: 74. 1922.

Erythrodes killipii Ames, Proc. Biol. Soc. Wash. 34: 150. 1921.

Microchilus killipii (Ames) Ormerod, Lindleyana 17: 217. 2002.

VOUCHER: E P. Killip 3561 (MO).

Microchilus ovalis Ormerod, Harvard Pap. Bot. 11(2): 164. 2007.

VOUCHER: G. McPherson 15047 (MO).

Microchilus panamanicus Ormerod, Harvard Pap. Bot. 9: 414. 2005.

VOUCHER: P.H. Allen 3768 (AMES).

Microchilus subquadratus Ormerod, Harvard Pap. Bot. 11(2): 172. 2007.

Voucher: A. Gentry et al. 16834 (MO).

Microchilus tessellatus Ormerod, Harvard Pap. Bot. 11(2): 172. 2007.

Voucher: C.W. Hamilton \& G. Davidse 2692 (MO).

Microchilus tridax (Rchb.f.) Ormerod, Lindleyana 17: 223. 2002.

Physurus tridax Rchb.f., Beitr. Orchid.-K. C. Amer.: 64. 1866.

Erythrodes tridax (Rchb.f.) Ames, Orchidaceae 7: 76. 1922.

Physurus purpureus Ames, Orchidaceae 2: 259. 1908.

Erythrodes purpurea (Ames) Ames, Orchidaceae 5: 28.1915.

VOUCHER: G. McPherson 11084 (MO).

Microchilus vesicifer (Rchb.f.) Ormerod, Lindleyana 17: 223. 2002.
Physurus vesicifer Rchb.f., Beitr. Orchid.-K. C. Amer.: 63. 1866.

Erythrodes vesicifera (Rchb.f.) Ames, Orchidaceae 7: 78.1922.

Physurus lehmannii Schltr., Beih. Bot. Centralbl. 36(2): 379. 1918.

Erythrodes lehmannii (Schltr.) Ames, Orchidaceae 7: 71.1922.

VOUCHER: W.G. D'Arcy 13151 (MO).

Microchilus whitefoordiae Ormerod, Harvard Pap. Bot. 11(2): 173. 2007.

Voucher: R.J. Hampshire \& C. Whitefoord 867 (BM).

Miltoniopsis God.-Leb.

Miltoniopsis roezlii (Rchb.f.) God.-Leb., Orchidophile (Argenteuil) 9: 148. 1889.

Odontoglossum roezlii W.Bull, Garden (London 1871-1927) 4: 20. 1873.

Miltonia roezlii (W.Bull) G.Nicholson, Ill. Dict. Gard. 2: 369. 1886.

Odontoglossum roezlii var. alba W.Bull ex W.G.Sm., Fl. Mag. (London), n.s., 1875: t. 164. 1875.

Miltoniopsis santanae Garay \& Dunst., Venez. Orchids Ill. 6: 276. 1976.

Miltoniopsis roezlii var. alba (W.Bull ex W.G.Sm.) Lückel, Orchidee (Hamburg) 46: A119. 1995.

VOUCHER: R.L. Dressler 4134 (MO).

Miltoniopsis warszewiczii (Rchb.f.) Garay \& Dunst., Venez. Orchids Ill. 6: 278. 1976.

Odontoglossum warszewiczii Rchb.f., Bot. Zeitung (Berlin) 10: 692. 1852.

Miltonia endresii G.Nicholson, Ill. Dict. Gard. 2: 368. 1886.

Miltonia superba Schltr., Repert. Spec. Nov. Regni Veg. 3: 249. 1907., nom. illeg.

Voucher: J. Warscewicz s.n. (W).

\section{MONOPHYLLORCHIS Schltr.}

Monophyllorchis microstyloides (Rchb.f.) Garay, Caldasia 8: 517. 1962.

Pogonia microstyloides Rchb.f., Flora 69: 547. 1886.

Monophyllorchis colombiana Schltr., Repert. Spec. Nov. Regni Veg. Beih. 7: 39. 1920.

Monophyllorchis maculata Garay, Fl. Ecuador 9(225: 1): 43. 1978.

VOUCHER: R.L. Dressler 5890 (PMA). 


\section{Mormodes Lindl.}

Mormodes colossus Rchb.f., Bot. Zeitung (Berlin) 10: 636. 1852.

Mormodes citrina B.S.Williams, Orch.-Grow. Man., ed. 2: 120. 1862.

Mormodes wendlandii Rchb.f. in W.G.Walpers,

Ann. Bot. Syst. 6: 581. 1863.

Voucher: not seen, cited by Salazar \& Dressler (2003c).

Mormodes fractiflexa Rchb.f., Gard. Chron. 1872: 141. 1872.

VOUCHER: Z. Serracin 87 (UCH).

Mormodes hookeri Lem., Jard. Fleur. 1(Misc.): 116. 1851.

Mormodes atropurpurea Hook., Bot. Mag. 77: t. 4577. 1851., nom. illeg.

Mormodes barbata Lindl. \& Paxton, Paxton's F1. Gard. 2: 57. 1851.

VOUCHER: J. Warscewicz s.n. (W).

Mormodes lancilabris Pabst, Ann. Missouri Bot. Gard. 62: 510.1975.

VOuCHER: R.L. Dressler s.n. (RB).

Mormodes lobulata Schltr., Repert. Spec. Nov. Regni Veg. 8: 456. 1910.

Voucher: not seen, cited by Salazar \& Dressler (2003c).

Mormodes powellii Schltr., Repert. Spec. Nov. Regni Veg. Beih. 17: 55. 1922.

Voucher: C.W. Powell 59 (MO).

Mormodes skinneri Rchb.f., Gard. Chron. 1869: 50. 1869.

VOucher: S. Laube et al. 616 (PMA).

\section{Mormolyca Fenzl}

Mormolyca culebrica Bogarín \& Pupulin, Orchid Digest 74: 44. 2010.

Maxillaria culebrica (Bogarín \& Pupulin)

Christenson, Richardiana 11: 78. 2011.

VOUCHER: D. Bogarín 2951 (PMA).

Mormolyca dressleriana (Carnevali \& J.T.Atwood)

M.A.Blanco, Lankesteriana 7: 531. 2007.

Maxillaria dressleriana Carnevali \& J.T.Atwood, Lindleyana 11: 29. 1996.

Xanthoxerampellia dressleriana (Carnevali \& J.T. Atwood) Szlach. \& Sitko, Biodivers. Res. Conservation 25: 37. 2012.
VOUCHER: M. Akers JJ (see OIC9304) (SEL).

Mormolyca hedwigiae (Hamer \& Dodson)

M.A.Blanco, Lankesteriana 7: 531. 2007.

Maxillaria hedwigiae Hamer \& Dodson, Icon. Pl. Trop. 8: t. 800. 1982[1983].

Xanthoxerampellia hedwigiae (Hamer \& Dodson) Szlach. \& Śmiszek, Biodivers. Res. Conservation 25: 37. 2012.

VOUCHER: R.L. Dressler 3305 (MO).

Mormolyca moralesii (Carnevali \& J.T.Atwood) M.A.Blanco, Lankesteriana 7: 531. 2007.

Maxillaria moralesii Carnevali \& J.T.Atwood, Lindleyana 11: 31. 1996.

Xanthoxerampellia moralesii (Carnevali \& J.T.Atwood) Szlach. \& Sitko, Biodivers. Res. Conservation 25: 37. 2012.

VOUCHER: T.B. Croat 22196 (MO).

Mormolyca ringens (Lindl.) Gentil, Pl. Cult. Serres Jard. Bot. Brux.: 124. 1907.

Trigonidium ringens Lindl., Edwards's Bot. Reg. 26(Misc.): 57. 1840.

VOUCHER: not seen, cited by Dressler (2003c).

The Voucher R.L. Dressler 6375 cited in TROPICOS database was not located at PMA.

\section{Muscarella Luer}

Muscarella aristata (Hook.) Luer, Monogr. Syst. Bot. Missouri Bot. Gard. 105: 96. 2006.

Pleurothallis aristata Hook., Ann. Nat. Hist. 2: 329. 1839.

Humboldtia aristata (Hook.) Kuntze, Revis. Gen. P1. 2: 667. 1891.

Specklinia aristata (Hook.) Pridgeon \& M.W.Chase, Lindleyana 16: 256. 2001.

Pleurothallis barberiana Rchb.f., Gard. Chron., n.s., 16: 6. 1881.

Pleurothallis urbaniana Rchb.f., Ber. Deutsch. Bot. Ges. 3: 279. 1885.

Humboldtia barberiana (Rchb.f.) Kuntze, Revis. Gen. P1. 2: 667. 1891.

Pleurothallis dichotoma Ames, Schedul. Orchid. 6: 58. 1923., nom. illeg.

Pleurothallis divexa Ames, Schedul. Orchid. 7: 20. 1924.

Voucher: M.E. Davidson 187 (not located, cited by Williams \& Allen 1980).

Distributed in the Antilles, Costa Rica, Ecuador, Peru 
and Bolivia. Luer (2006) did not cite records from Panama. The specimen cited by Williams (1946a) is "unsatisfactory" according to them and the locality is odd for the species. The description fits with a species of Muscarella. It may be another species of the genus but we were unable to check the specimen.

Muscarella marginata (Rich.) Luer, Monogr. Syst. Bot. Missouri Bot. Gard. 105: 111. 2006.

Epidendrum marginatum Rich., Actes Soc. Hist. Nat. Paris 1: 112. 1792.

Pleurothallis marginata (Rich.) Cogn. in C.F.P.von Martius \& auct. suc. (eds.), Fl. Bras. 3(4): 478. 1896., nom. illeg.

Voucher: S. Laube et al. 488 (PMA).

Muscarella quinqueseta (Ames) Luer, Monogr. Syst. Bot. Missouri Bot. Gard. 105: 114. 2006.

Pleurothallis quinqueseta Ames, Schedul. Orchid. 9: 35. 1925.

Specklinia quinqueseta (Ames) Luer, Monogr. Syst. Bot. Missouri Bot. Gard. 95: 263. 2004.

VOUCHER: C.A. Luer 9342 (SEL).

\section{Myoxanthus Poepp. \& Endl.}

Myoxanthus colothrix (Luer) Luer, Selbyana 7: 36. 1982.

Pleurothallis colothrix Luer, Phytologia 49: 202. 1981.

VOUCHER: C.A. Luer \& H.P. Butcher 1259 (SEL).

Myoxanthus hirsuticaulis (Ames \& C.Schweinf.) Luer, Selbyana 7: 43. 1982.

Pleurothallis hirsuticaulis Ames \& C.Schweinf.,

Schedul. Orchid. 10: 29. 1930.

VOUCHER: B.E. Hammel 4682 (PMA).

Myoxanthus octomeriae (Schltr.) Luer, Selbyana 7: 47. 1982.

Pleurothallis octomeriae Schltr., Repert. Spec.

Nov. Regni Veg. Beih. 17: 21. 1922.

Myoxanthus octomerioides var. octomeriae

(Schltr.) O.Gruss \& M.Wolff, Orchid. Atlas: 237. 2007.

VOUCHER: C.W. Powell 252 (MO).

Myoxanthus scandens (Ames) Luer, Selbyana 7: 49. 1982.

Pleurothallis scandens Ames, Schedul. Orchid. 5: 18. 1923.

VOUCHER: C.A. Luer et al. 1018 (MO).

Myoxanthus sotoanus Pupulin, Bogarín \& Mel.
Fernández, Lankesteriana 9: 470. 2010.

Voucher: S. Laube et al. 545 (MO).

Myoxanthus speciosus (Luer) Luer, Selbyana 7: 51. 1982.

Pleurothallis speciosa Luer, Selbyana 3: 392. 1977.

Voucher: C.A. Luer \& H.P. Butcher 1371 (SEL).

Myoxanthus trachychlamys (Schltr.) Luer, Selbyana 7: 51.1982.

Pleurothallis trachychlamys Schltr., Repert. Spec. Nov. Regni Veg. Beih. 17: 23. 1922.

Voucher: C.W. Powell 215 (MO).

Nemaconia Knowles \& Westc.

Nemaconia striata (Lindl.) Van den Berg, Salazar \& Soto Arenas, Neodiversity 2: 8. 2007.

Ponera striata Lindl., Edwards's Bot. Reg. 28(Misc.): 18. 1842.

VOUCHER: not found, cited by Dressler (2003c).

\section{Neomoorea Rolfe}

Neomoorea wallisii (Rchb.f.) Schltr., Orchid Rev. 32: 355. 1924.

Lueddemannia wallisii Rchb.f., Linnaea 41: 109. 1876.

Moorea irrorata Rolfe, Gard. Chron., ser. 3, 8: 7. 1890.

Neomoorea irrorata (Rolfe) Rolfe, Orchid Rev. 12: 30. 1904.

VOUCHER: A.A. Hunter s.n. (MO).

Nidema Britton \& Millsp.

Nidema boothii (Lindl.) Schltr., Repert. Spec. Nov. Regni Veg. Beih. 17: 43. 1922.

Maxillaria boothii Lindl., Edwards's Bot. Reg. 24(Misc.): 52. 1838.

Epidendrum boothii (Lind1.) L.O.Williams, Ann. Missouri Bot. Gard. 26: 282. 1939.

Dinema paleaceum Lindl., Edwards's Bot. Reg. 26(Misc.): 51. 1840.

Epidendrum auritum Lindl., Edwards's Bot. Reg. 29(Misc.): 4. 1843.

Epidendrum lindenianum A.Rich. \& Galeotti, Ann. Sci. Nat., Bot., sér. 3, 3: 20. 1845.

Epidendrum paleaceum (Lindl.) Rchb.f., Beitr. Orchid.-K. C. Amer.: 80. 1866. 
Nidema paleacea (Lindl.) Acuña, Bol. Estaçión Exp. Agron. Santiago de las Vegas 60: 70. 1939. Encyclia paleacea (Lindl.) A.Lemée, Fl. Guyane Franç. 1: 419. 1955., no basionym ref.

VOUCHER: S. Laube et al 382 (PMA).

Nidema ottonis (Rchb.f.) Britton \& Millsp., Bahama Fl.: 94. 1920.

Epidendrum ottonis Rchb.f., Hamburger GartenBlumenzeitung 14: 213. 1858.

Encyclia ottonis (Rchb.f.) Pabst, Orquídea (Rio de Janeiro) 29: 276. 1972.

Nidema boothii var. australis Schltr., Repert. Spec.

Nov. Regni Veg. Beih. 19: 211. 1923.

Nidema boothii var. triandrum Schltr., Repert.

Spec. Nov. Regni Veg. Beih. 19: 211. 1923.

Nidema ottonis var. triandrum (Schltr.) Nir, Orchid. Antill.: 407. 2000.

Voucher: C.W. Powell 131 (AMES).

\section{Nitidobulbon Ojeda}

Nitidobulbon nasutum (Rchb.f.) Ojeda \& Carnevali, Novon 19: 99. 2009.

Maxillaria nasuta Rchb.f., Beitr. Orchid.-K. C. Amer.: 104. 1866.

Maxillaria oxysepala Schltr., Repert. Spec. Nov. Regni Veg. 27: 73. 1929.

Maxillaria brevipedunculata Ames \& C.Schweinf., Schedul. Orchid. 10: 91. 1930.

Voucher: M.A. Blanco \& D. Penneys 2829 (PMA).

\section{Notylia Lindl.}

Notylia albida Klotzsch, Allg. Gartenzeitung 19: 281. 1851.

Notylia panamensis Ames, Orchidaceae 7: 112. 1922.

VOUCHER: R. S. Williams 977 (NY).

Notylia latilabia Ames \& C.Schweinf., Schedul. Orchid. 8: 71. 1925.

VOucher: C.W. Powell 406 (AMES).

Notylia pentachne Rchb.f., Bonplandia (Hannover) 2: 90. 1854.

Notylia gracilispica Schltr., Repert. Spec. Nov. Regni Veg. Beih. 17: 75. 1922.

Voucher: Herrn Keferstein sub Lehmann s.n. (W).

Notylia pittieri Schltr., Beih. Bot. Centralbl. 36(2): 418. 1918.

Voucher: Z. Samudio et al. 249 (UCH).

\section{Octomeria R.Br.}

Octomeria costaricensis Schltr., Repert. Spec. Nov. Regni Veg. Beih. 19: 111.1923.

VOUCHER: R.L. Dressler 3882 (MO).

Octomeria graminifolia (L.) R.Br. in W.T.Aiton, Hortus Kew. 5: 211. 1813.

VOUCHER: G. McPherson 12555 (PMA).

Octomeria valeroi Ames \& C.Schweinf., Schedul. Orchid. 10: 51-52. 1930.

Voucher: B. Hammel 2132 (SEL).

Octomeria scirpoidea (Poepp. \& Endl.) Rchb. f., Bot. Zeitung (Berlin) 10: 856. 1852.

Voucher: S. Mori \& J. Kallunki 2158 (MO).

Octomeria yauaperyensis Barb.Rodr., Vellosia, ed. 2, 1: 120.1891.

Octomeria hondurensis Ames, Bot. Mus. Leafl. 1(4): 1.1933.

Octomeria complanata C.Schweinf., Bot. Mus. Leafl. 10: 195. 1942.

VOUCHER: R.L. Dressler 3747 (PMA).

Oeceoclades Lindl.

Oeceoclades maculata (Lindl.) Lindl., Gen. Sp. Orchid. Pl.: 237. 1833.

Angraecum maculatum Lindl., Coll. Bot.: t. 15. 1821.

Limodorum maculatum (Lindl.) G.Lodd., Bot. Cab. 5: t. 496. 1821.

Aerobion maculatum (Lindl.) Spreng., Syst. Veg. 3: 718. 1826.

Eulophia maculata (Lindl.) Rchb.f. in W.G.Walpers, Ann. Bot. Syst. 6: 647. 1863.

Eulophidium maculatum (Lindl.) Pfitzer, Entwurf. Anordn. Orch.: 87. 1887.

Graphorkis maculata (Lindl.) Kuntze, Revis. Gen. P1. 2: 662.1891.

Angraecum monophyllum A.Rich., Mém. Soc. Hist. Nat. Paris 4: 58. 1828.

Epidendrum connivens Vell., Fl. Flumin. 9: t. 44. 1831.

Geodorum pictum Link in J.H.F.Link, J.F.Klotzsch \& C.F.Otto, Icon. Pl. Rar. 1: 14. 1840., nom. illeg.

Eulophia monophylla (A.Rich.) S.Moore in J.G.Baker, Fl. Mauritius: 360. 1877.

Eulophia ledienii Stein ex N.E.Br., Bull. Misc. 
Inform. Kew 1889: 90. 1889., orth. var.

Eulophia liedenii N.E.Br., Bull. Misc. Inform. Kew 1889: 90. 1889.

Eulophia mackenii Rolfe ex Hemsl., Gard. Chron., ser. 3, 12: 583. 1892.

Eulophidium warneckeanum Kraenzl., Bot. Jahrb. Syst. 33: 70. 1902.

Eulophidium liedenii (N.E.Br.) De Wild., Ann. Mus. Congo Belge, Bot., sér. 5, 1: 115. 1903.

Eulophidium mackenii (Rolfe ex Hemsl.) Schltr., Bot. Jahrb. Syst. 53: 593. 1915.

Eulophidium monophyllum (A.Rich.) Schltr., Bot. Jahrb. Syst. 53: 593. 1915.

Eulophidium nyassanum Schltr., Bot. Jahrb. Syst. 53: 593. 1915.

Eulophidium maculatum var. pterocarpum Hauman, Anales Mus. Nac. Hist. Nat. Buenos Aires 29: 381 (1917.

Oeceoclades paraguayensis Hauman, Anales Mus. Nac. Hist. Nat. Buenos Aires 1917: 29. 1917., nom. nud.

Oeceoclades mackenii (Rolfe ex Hemsl.) Garay \& P.Taylor, Bot. Mus. Leafl. 24: 265. 1976.

Oeceoclades maculata var. pterocarpa (Hauman) Garay \& P.Taylor, Bot. Mus. Leafl. 24: 267. 1976.

Oeceoclades monophylla (A.Rich.) Garay \& P.Taylor, Bot. Mus. Leafl. 24: 267. 1976.

Voucher: M.D. Correa et al. 8133 (UCH).

\section{Oncidium Sw.}

Oncidium abortivoides M.W.Chase \& N.H.Williams, Lindleyana 21(3): 22. 2008.

Sigmatostalix abortiva L.O.Williams, Ann. Missouri Bot. Gard. 27: 284. 1940.

VOUCHER: P.H. Allen 2121 (MO).

Oncidium allenii Dressler, Selbyana 22: 9. 2001.

Voucher: P.H. Allen 2938 (AMES).

Oncidium ansiferum Rchb.f., Bot. Zeitung (Berlin) 10: 696. 1852.

Oncidium tenue Lindl., J. Hort. Soc. London 7: 271. 1852., nom. illeg.

Oncidium tenue var. grandiflorum Lindl., J. Hort. Soc. London 8: 271. 1852.

Oncidium delumbe Lindl., Fol. Orchid. 6: 48. 1855.

Oncidium lankesteri Ames, Schedul. Orchid. 4: 53.
1923.

VOUCHER: J. Warscewicz s.n. (W)

Oncidium anthocrene Rchb.f., Linnaea 41: 102. 1876. Cyrtochilum anthocrene Kraenzl., Notizbl. Bot. Gart. Berlin-Dahlem 7: 95. 1917.

Oncidium powellii Schltr., Repert. Spec. Nov. Regni Veg. Beih.17: 86-87. 1922.

VOUCHER: C.W. Powell 58 (AMES).

Oncidium bracteatum Warsz. \& Rchb.f., Bot. Zeitung (Berlin) 10: 695. 1852.

Oncidium henrici-gustavi Kraenzl. in H.G.A.Engler (ed.), Pflanzenr., IV, 50(80): 242. 1922.

Oncidium paleatum Schltr., Repert. Spec. Nov. Regni Veg. Beih. 19: 260. 1923.

VOUCHeR: J. Warscewicz s.n. (W).

Oncidium brownii (Garay) M.W.Chase \& N.H.Williams, Lindleyana 21(3): 22. 2008.

Sigmatostalix brownii Garay, Caldasia 10: 236. 1968.

VOUCHER: H. Brown s.n. (AMES).

Oncidium bryolophotum Rchb.f., Gard. Chron. 1871: 738. 1871.

Oncidium inops Cogn. \& Rolfe, J. Orchidées 3: 346. 1893.

Oncidium megalous Schltr., Repert. Spec. Nov. Regni Veg. 9: 30. 1910.

Oncidium asparagoides Kraenzl. in H.G.A.Engler (ed.), Pflanzenr., IV, 50(80): 175. 1922.

Oncidium microphyton Kraenzl. in H.G.A.Engler (ed.), Pflanzenr., IV, 50(80): 204. 1922.

VOUCHER: P.H. Allen 1381 (MO)

Oncidium cariniferum (Rchb.f.) Beer, Prakt. Stud. Orchid.: 283. 1854.

Odontoglossum cariniferum Rchb.f., Bot. Zeitung (Berlin) 10: 638. 1852.

Collare-stuartense cariniferum (Rchb.f.) Senghas \& Bockemühl, J. Orchideenfr. 4: 75. 1997.

Miltonioides carinifera (Rchb.f.) Senghas \& Lückel, J. Orchideenfr. 4: 75. 1997.

Odontoglossum hastilabium var. fuscatum Hook., Bot. Mag. 82: t. 4919. 1856.

VOUCHER: H. Pittier 2988 (US).

Oncidium cheirophorum Rchb.f., Bot. Zeitung (Berlin) 10: 695. 1852.

Oncidium dielsianum Kraenzl. in H.G.A.Engler (ed.), Pflanzenr., IV, 50(80): 197. 1922.

Oncidium macrorhynchum Kraenzl. in 
H.G.A.Engler (ed.), Pflanzenr., IV, 50(80): 200. 1922.

Oncidium cheirophorum var. exauriculatum Hamer \& Garay in F.Hamer, Orquid. El Salvador 2: 176. 1974.

Oncidium exauriculatum (Hamer \& Garay) R.Jiménez, Orquídea (Mexico City), n.s., 12: 270. 1992.

VOucher: J. Warscewicz s.n. (W).

Oncidium dichromaticum Rchb.f., Bonplandia (Hannover) 3: 215. 1855.

Oncidium cabagrae Schltr., Repert. Spec. Nov. Regni Veg. 9: 292. 1911.

Oncidium castaneum Rchb.f. ex Kraenzl. in H.G.A.Engler (ed.), Pflanzenr., IV, 50(80): 287. 1922.

Oncidium rechingerianum Kraenzl. in H.G.A.Engler (ed.), Pflanzenr., IV, 50(80): 202. 1922.

Oncidium andreae Königer, Arcula 6: 162. 1996.

VOUCHER: B.E. Hammel 4271 (MO).

Oncidium ensatum Lindl., Edwards's Bot. Reg. 28(Misc.): 17. 1842.

Oncidium cerebriferum Rchb.f., Bot. Zeitung (Berlin) 10: 696. 1852.

Oncidium confusum Rchb.f., Xenia Orchid. 1: 234. 1858.

Cyrtopodium verrucosum Griseb., Cat. Pl. Cub.: 265. 1866.

Oncidium floridanum Ames, Schedul. Orchid. 7: 13. 1924.

Voucher: J. Wascewicz s.n. (W).

Oncidium exalatum Hágsater, Orquídea (Mexico City), n.s., 8: 98. 1981.

Heteranthocidium exalatum (Hágsater) Szlach., Mytnik \& Romowicz, Polish Bot. J. 51: 55. 2006.

Voucher: R.L Dressler \& E.Hágsater 4173 (AMO).

Oncidium fuscatum Rchb.f., W.G.Walpers, Ann. Bot. Syst. 6: 763. 1863.

Miltonia warszewiczii Rchb.f., Xenia Orchid. 1: 132. 1856.

Miltonioides warszewiczii (Rchb.f.) Brieger \& Lückel, Orchidee (Hamburg) 34: 132. 1983.

Chamaeleorchis warszewiczii (Rchb.f.) Senghas \& Lückel in F.R.R.Schlechter, Orchideen Beschreib. Kult. Zücht., ed. 3, I/C(33-36):
2305. 1997.

Odontoglossum weltonii Rchb.f., Gard. Chron. 1870: 417. 1870.

Oncidium weltonii (Rchb.f.) Rollisson, Nursery Cat. (Rollisson) 1875-1876: 37. 1875.

Miltonia warszewiczii var. aetherea Rchb.f., Gard. Chron., n.s., 15: 428. 1881.

Voucher: B. E. Hammel et al. 14823 (MO).

Oncidium guatemalenoides M.W.Chase \& N.H.Williams, Lindleyana 21(3): 24. 2008.

Sigmatostalix guatemalensis Schltr., Repert. Spec. Nov. Regni Veg. 10: 253. 1911.

Sigmatostalix costaricensis Rolfe, Bull. Misc. Inform. Kew 1916: 78. 1916.

VOUCHer: T.B. Croat 26813 (MO).

Oncidium integrilabre (Pupulin) M.W.Chase \& N.H.Williams, Lindleyana 21(3): 25. 2008.

Sigmatostalix integrilabris Pupulin, Harvard Pap. Bot. 8: 45. 2003.

VOUCHER: S. Laube et al. 512 (PMA).

Oncidium isthmi Schltr., Repert. Spec. Nov. Regni Veg. Beih. 17: 84. 1922.

Voucher: H. Pittier 5569 (US).

Oncidium klotzschianum Rchb.f., Bot. Zeitung (Berlin) 10: 695. 1852.

Vitekorchis klotzschiana (Rchb.f.) Romowicz \& Szlach., Polish Bot. J. 51: 46. 2006.

Oncidium obryzatum Rchb.f. \& Warsz., Bonplandia (Hannover) 2: 108. 1854.

Vitekorchis obryzata (Rchb.f. \& Warsz.) Romowicz \& Szlach., Polish Bot. J. 51: 46. 2006.

Oncidium meliosmum Rchb.f., Gard. Chron., n.s., 17: 796. 1882.

Oncidium angustisepalum Kraenzl. in H.G.A.Engler (ed.), Pflanzenr., IV, 50(80): 203. 1922.

Oncidium fulgens Schltr., Repert. Spec. Nov. Regni Veg. Beih. 17: 83. 1922.

Oncidium microcachrys Rchb.f. ex Kraenzl. in H.G.A.Engler (ed.), Pflanzenr., IV, 50(80): 237. 1922.

Oncidium sclerophyllum Kraenzl. in H.G.A.Engler (ed.), Pflanzenr., IV, 50(80): 204. 1922.

Oncidium varians Schltr., Repert. Spec. Nov. Regni Veg. Beih. 19: 151. 1923.

Oncidium graciliforme C.Schweinf., Bot. Mus. Leafl. 5(6): 96-98. 1938. 
VOUCHER: J. Warscewicz s.n. (W).

Oncidium dulcineae (Pupulin \& G.A.Rojas) M.W.Chase \& N.H.Williams, Lindleyana 21(3): 24. 2008.

Sigmatostalix dulcineae Pupulin \& G.Rojas, Orchids (West Palm Beach) 75: 681. 2006.

Voucher: D. Bogarín et al. 10365 (UCH).

Oncidium luteum Rolfe, Bull. Misc. Inform. Kew 1893: 172. 1893.

Oncidium pittieri Schltr., Repert. Spec. Nov. Regni Veg. 9: 31. 1910.

Oncidium cheirophoroides Kraenzl. in

H.G.A.Engler (ed.), Pflanzenr., IV, 50(80): 199. 1922.

VOUCHER: R.L Dressler 4843 (MO).

Oncidium macrobulbon (Kraenzl.) M.W.Chase \& N.H.Williams, Lindleyana 21(3): 25. 2008.

Sigmatostalix macrobulbon Kraenzl. in

H.G.A.Engler (ed.), Pflanzenr., IV, 50(80): 307. 1922.

Sigmatostalix reichenbachiana Kraenzl. in H.G.A.Engler (ed.), Pflanzenr., IV, 50(80): 307. 1922.

Oncidium reichenbachianum (Kraenzl.)

M.W.Chase \& N.H.Williams, Lindleyana 21(3): 26. 2008.

Voucher: S. Knapp 5048 (MO).

Oncidium maduroi Dressler, Orchids 69: 762. 2000.

Voucher: R. L. Dressler 6220 (MO).

Oncidium martinezii Königer, Arcula 15: 369. 2005.

Voucher: W. Königer WK-122 (M).

Oncidium panamense Schltr., Repert. Spec. Nov. Regni Veg. Beih. 17: 85. 1922.

Voucher: C.W. Powell 4 (MO).

Oncidium parviflorum L.O.Williams, Amer. Orchid Soc. Bull. 11: 32. 1942.

VOUCHER: P.H. Allen 2937 (AMES).

Oncidium picturatissimum (Kraenzl.) M.W.Chase \& N.H.Williams, Lindleyana 21(3): 26. 2008.

Sigmatostalix picturatissima Kraenzl. in

H.G.A.Engler (ed.), Pflanzenr., IV, 50(80): 312. 1922.

Sigmatostalix racemifera L.O.Williams, Ann. Missouri Bot. Gard. 27: 285. 1940.

Sigmatostalix occultans Christenson \& M.J.Lee, Orchids (West Palm Beach) 71: 314. 2002.

VOUCHER: P.H. Allen 1232 (MO).
Oncidium polycladium Rchb.f. ex Lindl., Fol. Orchid. 6: 47.1855.

Oncidium guttulatum Rchb.f. ex Lindl., Fol. Orchid. 6: 50. 1855.

Oncidium peliogramma Linden \& Rchb.f., Gard. Chron. 1871: 1451. 1871.

Oncidium costaricense Schltr., Repert. Spec. Nov. Regni Veg. 9: 30. 1910.

Oncidium tonduzii Schltr., Repert. Spec. Nov. Regni Veg. 9: 31. 1910.

Oncidium scabripes Kraenzl. in H.G.A.Engler (ed.), Pflanzenr., IV, 50(80): 277. 1922.

Voucher: J. Warczewicz s.n. (W).

Oncidium punctulatum Dressler, Selbyana 22: 10. 2001.

Voucher: R.L. Dressler \& J.T. Atwood 6274 (MO).

Oncidium schroederianum (O'Brien) Garay \& Stacy, Bradea 1: 408. 1974.

Miltonia schroederiana O’Brien, Gard. Chron., ser. 3, 6: 210. 1889.

Miltonioides schroederiana (O’Brien) Lückel, Orchidee (Hamburg) 37: 55. 1986.

Odontoglossum schroederianum Rchb.f., Gard. Chron., ser. 3, 2: 364. 1887., nom. illeg.

Odontoglossum confusum Garay, Orchidee (Hamburg) 13: 217. 1962.

Miltonioides confusa (Garay) Brieger \& Lückel, Orchidee (Hamburg) 34: 131. 1983.

VOUCHER: R.L. Dressler 5465 (MO).

Oncidium sotoanum R.Jiménez \& Hágsater, Lankesteriana 9: 415. 2010.

VOUCHER: T.S. Cochrane 7793 (MO).

Formerly known as $O$. ornithorhynchum Kunth, this species ranges from Mexico to Nicaragua. The Costa Rican Vouchers are dubious (Dressler 2003c). The Panamanian voucher cited here has been allegedly collected alive by James L. Sawyer in Cerro Punta.

Oncidium stenotis Rchb.f., Linnaea 41: 67. 1876.

Oncidium turialbae Schltr., Repert. Spec. Nov. Regni Veg. 9: 32. 1910.

VOUCHER: R.L. Dressler 5980 (MO).

Oncidium warszewiczii Rchb.f., Bot. Zeitung (Berlin) 10: 693. 1852.

Oncidium bifrons Lindl., Gard. Chron. 1857: 84. 1857.

Voucher: J. Warscewicz s.n. (W).

Oncidium zelenkoanum Dressler \& Pupulin, 
Lankesteriana 8: 37. 2003.

Voucher: A. Maduro \& E. Olmos 195 (MO).

OrNithidium Salisb. ex R.Br.

Ornithidium adendrobium (Rchb.f.) M.A.Blanco \& Ojeda, Lankesteriana 7: 532. 2007.

Ponera adendrobium Rchb.f., Flora 48: 278. 1865. Pleuranthium adendrobium (Rchb.f.) Benth. \& Hook.f. ex B.D.Jacks., Index Kew. 2: 562. 1894.

Neo-urbania adendrobium (Rchb.f.) Fawc. \& Rendle, J. Bot. 47: 125. 1909.

Maxillaria adendrobium (Rchb.f.) Dressler, Taxon 13: 248. 1964.

Camaridium parviflorum Fawc. in I.Urban, Symb. Antill. 1: 472. 1900.

Voucher: G. de Nevers et al. 6137 (PMA).

Ornithidium conduplicatum Ames \& C.Schweinf., Schedul. Orchid. 8: 66. 1925.

Maxillaria conduplicata (Ames \& C.Schweinf.) L.O.Williams, Ann. Missouri Bot. Gard. 29: 348. 1942.

Adamanthus conduplicatus (Ames \& C.Schweinf.)

Szlach. \& Sitko, Biodivers. Res. Conservation 25: 21. 2012.

Voucher: C.W. Powell 341 (AMES).

Ornithidium fulgens Rchb.f., Beitr. Orchid.-K. C. Amer.: 76. 1866.

Maxillaria fulgens (Rchb.f.) L.O.Williams, Ann. Missouri Bot. Gard. 28: 425. 1941.

Laricorchis fulgens (Rchb. f.) Szlach. \& Sitko,

Biodivers. Res. Conservation 25: 29. 2012.

Voucher: T. Antonio 1443 (PMA).

Ornithidium nicaraguense (Hamer \& Garay) M.A.Blanco \& Ojeda, Lankesteriana 7: 533. 2007.

Neo-urbania nicaraguensis Hamer \& Garay, Icon.

Pl. Trop. 13: t. 1238. 1985.

Maxillaria nicaraguensis (Hamer \& Garay) J.T.Atwood, Selbyana 10: 60. 1987[1988].

Adamanthus nicaraguensis (Ames \& C.Schweinf.)

Szlach. \& Sitko, Biodivers. Res. Conservation 25: 21.2012.

VOUCHER: R.L. Dressler 5851 (PMA).

Ornithidium pittieri Ames, Schedul. Orchid. 2: 35 1923.

Maxillaria pittieri (Ames) L.O.Williams, Ann.
Missouri Bot. Gard. 29: 349. 1942.

Adamanthus pittieri (Ames) Szlach., Richardiana 7: 32. 2007[2006].

Laricorchis pittieri (Ames) Szlach. \& Sitko, Biodivers. Res. Conservation 25: 29. 2012.

Voucher: G. McPherson 11994 (MO).

Ornithidium repens (L.O.Williams) M.A.Blanco \& Ojeda, Lankesteriana 7: 533. 2007.

Maxillaria repens L.O.Williams, Amer. Orchid Soc. Bull. 10: 273. 1942.

Laricorchis repens (L.O.Williams) Szlach. \& Sitko, Biodivers. Res. Conservation 25: 29. 2012.

VOUCHER: P.H. Allen 2868 (AMES).

\section{OrNithocephaLus Hook.}

Ornithocephalus aristatus Pupulin \& Dressler, Brittonia 58: 314. 2006.

Voucher: A. Maduro \& E. Olmos 327 (PMA).

Ornithocephalus bicornis Lindl. in G.Bentham, Bot. Voy. Sulphur: 172. 1846.

Ornithocephalus oberonia Rchb.f., Gard. Chron. 1869: 988. 1869.

Ornithocephalus stenoglottis Rchb.f., Flora 69: 551. 1886

Ornithocephalus xiphochilus Schltr., Repert. Spec. Nov. Regni Veg. 3: 251. 1907.

Zygostates costaricensis Nash, Bull. Torrey Bot. Club 34: 122. 1907.

Ornithocephalus lanuginosus Ames, Proc. Biol. Soc. Wash. 34: 152. 1921.

Ornithocephalus diceras Schltr., Repert. Spec. Nov. Regni Veg. Beih. 17: 87. 1922.

VOUCHER: A. Sinclair s.n. (K).

Ornithocephalus cochleariformis C.Schweinf., Bot. Mus. Leafl. 4: 124. 1937.

Voucher: A.A. Hunter \& P.H. Allen 383 (AMES).

Ornithocephalus cryptanthus (C.Schweinf. \& P.H.Allen) Toscano \& Dressler, Lindleyana 15: 255. 2000.

Oakes-Amesia cryptantha C.Schweinf. \& P.H.Allen, Bot. Mus. Leafl. 13: 134. 1948.

Sphyrastylis cryptantha (C.Schweinf. \& P.H.Allen) Garay, Bot. Mus. Leafl. 21: 264. 1967.

Voucher: P.H. Allen \& D.O. Allen 4196 (AMES).

Ornithocephalus dressleri (Toscano) Toscano \& Dressler, Lindleyana 15: 255. 2000. 
Sphyrastylis dressleri Toscano, Lindleyana 14: 24. 1999.

Voucher: J.P. Folsom \& R. Page 5942 (MO).

Ornithocephalus inflexus Lindl., Ann. Nat. Hist. 4: 384. 1840.

Ornithocephalus mexicanus A.Rich. \& Galeotti, Ann. Sci. Nat., Bot., sér. 3, 3: 24. 1845.

Ornithocephalus elephas Rchb.f. in W.G.Walpers, Ann. Bot. Syst. 6: 493. 1863.

Ornithocephalus salvinii Rchb.f. ex Hemsl., Biol. Cent.-Amer., Bot. 3: 291. 1884.

Ornithocephalus pottsiae S.Watson, Proc. Amer. Acad. Arts 22: 478. 1887.

Ornithocephalus tonduzii Schltr., Beih. Bot. Centralbl. 36(2): 420. 1918.

VOUCHER: R.L. Dressler s.n. (MO).

Ornithocephalus powellii Schltr., Repert. Spec. Nov. Regni Veg. Beih. 17: 188. 1922.

Voucher: C.H. Powell 231 (AMES).

ОтоGLossum (Schltr.) Garay \& Dunst.

Otoglossum chiriquense (Rchb.f.) Garay \& Dunst., Venez. Orchids Ill. 6: 41. 1976.

Odontoglossum chiriquense Rchb.f., Bot. Zeitung (Berlin) 10: 692. 1852.

Oncidium chiriquense (Rchb.f.) Beer, Prakt. Stud. Orchid.: 283. 1854.

Odontoglossum coronarium var. chiriquense (Rchb.f.) A.H.Kent in H.J.Veitch, Man. Orchid. Pl. 1: 23. 1887.

VOUCHER: J. Warscewicz s.n. (W).

Otoglossum globuliferum (Kunth) N.H.Williams \& M.W.Chase, Lindleyana 16: 138. 2001.

Oncidium globuliferum Kunth in F.W.H.von Humboldt, A.J.A.Bonpland \& C.S.Kunth, Nov. Gen. Sp. 1: 347. 1816.

Brevilongium globuliferum (Kunth) Christenson, Richardiana 6: 47. 2006.

Oncidium globuliferum var. costaricense Rchb.f., Gard. Chron. 1871: 1678. 1871.

Oncidium wercklei Schltr., Repert. Spec. Nov. Regni Veg. Beih. 19: 68. 1923.

Oncidium luerorum Dodson, Orquideología 20: 91. 1996.

Otoglossum luerorum (Dodson) M.W.Chase \& N.H.Williams, Lindleyana 16: 219. 2001.
Brevilongium luerorum (Dodson) Christenson, Richardiana 6: 48. 2006.

Brevilongium globuliferum var. costaricense (Rchb.f.) Jenny, Orchidee (Hamburg) 58: 697. 2007[2008].

Voucher: D. Cáceres \& R. Rincón 1153 (UCH).

\section{Pabstiella Brieger \& Senghas}

Pabstiella tripterantha (Rchb.f.) F.Barros, Bradea 8: 296. 2002.

Pleurothallis tripterantha Rchb.f., Bonplandia (Hannover) 2: 24. 1854.

Humboldtia tripterantha (Rchb.f.) Kuntze, Revis. Gen. Pl. 2: 668. 1891.

Anthereon tripteranthus (Rchb.f.) Pridgeon \& M.W.Chase, Lindleyana 16: 252. 2001.

Specklinia tripterantha (Rchb.f.) Luer, Monogr. Syst. Bot. Missouri Bot. Gard. 95: 264. 2004.

Pleurothallis tripterygia Rchb.f., Bonplandia (Hannover) 2: 24. 1854.

Pleurothallis procumbens Lindl., Fol. Orchid. 9: 35. 1859.

Lepanthes tricarinata Barb.Rodr., Gen. Spec. Orchid. 2: 43. 1881.

Humboldtia procumbens (Lindl.) Kuntze, Revis. Gen. Pl. 2: 668. 1891.

Humboldtia tripterygia (Rchb.f.) Kuntze, Revis. Gen. Pl. 2: 668. 1891.

Pleurothallis trialata Cogn. in C.F.P.von Martius \& auct. suc. (eds.), Fl. Bras. 3(4): 500. 1896.

Masdevallia tricarinata (Barb.Rodr.) F.Lehm. \& Kraenzl., Bot. Jahrb. Syst. 26: 456. 1899.

Pleurothallis medellinensis Schltr., Repert. Spec. Nov. Regni Veg. Beih. 7: 236. 1920.

Masdevallia aperta Kraenzl., Repert. Spec. Nov. Regni Veg. 17: 430. 1921.

Masdevallia carpophora Kraenzl., Repert. Spec. Nov. Regni Veg. 17: 427. 1921.

Pleurothallis hunteriana Schltr., Repert. Spec. Nov. Regni Veg. Beih. 17: 20. 1922.

Pleurothallis hamata Rolfe ex Ames, Schedul. Orchid. 3: 8. 1923.

Pleurothallis aperta (Kraenzl.) Ames, Schedul. Orchid. 7: 17. 1924.

Scaphosepalum carpophorum (Kraenzl.) Garay, Bot. Mus. Leafl. 21: 251. 1967. 
Voucher: C.W. Powell 277 (AMES).

At least two different species have been treated under $P$. tripterantha in Costa Rica, Panama, Colombia and Ecuador. One is characterized by the coriaceous leaves, the yellow flowers with brown spots and the caudate sepals, thickened at the apex. The other species has thick but subcoriaceous leaves, purple abaxially and the flowers are purplemaculated with acute, tailess sepals. A careful review of the heterotypic synonyms and a more accurate morphological characterization are needed to clarify the taxonomy of this group. In this paper, this species is accepted in a broad sense. The voucher cited here is the type of $P$. hunteriana and it fits well with the specimens having yellow flowers, with brown lines or spots and caudate sepals.

\section{Palmorchis Barb.Rodr.}

Palmorchis nitida Dressler, Orchidee (Hamburg) 34: 29. 1983.

Voucher: R.L. Dressler 6015 (US).

Palmorchis powellii (Ames) C.Schweinf. \& Correll, Bot. Mus. Leafl. 8: 119. 1940.

Rolfea powellii Ames, Schedul. Orchid. 7: 32-34, f. 6.1924.

Voucher: C.W. Powell 325 (AMES).

Palmorchis trilobulata L.O.Williams, Ann. Missouri Bot. Gard. 28: 415. 1941.

Voucher: P.H. Allen 2463 (AMES).

Palmorchis trinotata Dressler, Orquideología 20: 263. 1997.

Voucher: R.L. Dressler 4680 (MO).

\section{Paphinia Lindl.}

Paphinia subclausa Dressler, Novon 7: 121. 1997.

Voucher: R. L. Dressler s.n. (MO).

Paphinia vermiculifera G.Gerlach \& Dressler, Lankesteriana 8: 27. 2003.

VOUCHER: R. L. Dressler 6350 (MO).

\section{Pelexia Poit. ex Rich.}

Pelexia callifera (C.Schweinf.) Garay, Fl. Ecuador 9(225: 1): 247. 1978.

Spiranthes callifera C.Schweinf., Bot. Mus. Leafl. 20: 1. 1962.

VOUCHER: H. Butcher s.n. (FLAS).

Pelexia funckiana (A.Rich. \& Galeotti) Schltr., Repert. Spec. Nov. Regni Veg. 15: 197. 1918.

Spiranthes funckiana A.Rich. \& Galeotti, Ann. Sci.
Nat., Bot., sér. 3, 3: 32. 1845.

Gyrostachys funckiana (A.Rich. \& Galeotti)

Kuntze, Revis. Gen. P1. 2: 664. 1891.

Pelexia pringlei Fernald, Proc. Amer. Acad. Arts 35: 562. 1900.

Pelexia guatemalensis Schltr., Repert. Spec. Nov. Regni Veg. 15: 197. 1918.

Pelexia hondurensis Ames, Schedul. Orchid. 2: 4. 1923.

Pelexia subaequalis Ames, Schedul. Orchid. 2: 5. 1923.

Pelexia callosa Ames, Schedul. Orchid. 7: 15. 1924.

Voucher: R. L. Dressler 5294 (FLAS).

Pelexia laxa (Poepp. \& Endl.) Lindl., Gen. Sp. Orchid. P1.: 482. 1840.

Stenorrhynchos laxum Poepp. \& Endl., Nov. Gen. Sp. P1. 2: 7.1836.

Spiranthes laxa (Poepp. \& Endl.) C.Schweinf., Bot. Mus. Leafl. 10: 29. 1941.

VOuCHER: P. Burns-Balogh s.n. (DLSz-spirit).

Pelexia ochyrae Szlach., Fragm. Florist. Geobot. 41: 858. 1996.

VOUCHER: H. Butcher s.n. (FLAS).

\section{Peristeria Hook.}

Peristeria cerina Lindl., Edwards's Bot. Reg. 23: t. 1953. 1837.

Lycomormium cerinum (Lindl.) Benth., J. Linn. Soc., Bot. 18: 321. 1881.

VOUCHER: R.L. Dressler 6358 (MO).

Peristeria elata Hook., Bot. Mag. 58: t. 3116. 1831.

Voucher: H. Barnard ex R. Harrison s.n. (K).

Peristeria pendula Hook., Bot. Mag. 63: t. 3479. 1836.

Peristeria lentiginosa Lodd. ex W.H.Baxter in J.C.Loudon, Suppl. Hort. Brit.: 604. 1850.

Peristeria maculata Loudon, Encycl. Pl., new ed., Suppl. 2: 1474. 1855.

VOUCHER: T. Neudecker s.n. (PMA).

\section{Pescatoria Rchb.f.}

Pescatoria cerina (Lindl. \& Paxton) Rchb.f., Bot. Zeitung (Berlin) 10: 667. 1852.

Huntleya cerina Lindl. \& Paxton, Paxton's F1. Gard. 3: 62. 1852.

Zygopetalum cerinum (Lindl. \& Paxton) Rchb.f. in 
W.G.Walpers, Ann. Bot. Syst. 6: 651. 1863.

Pescatoria costaricensis Schltr., Repert. Spec.

Nov. Regni Veg. Beih. 19: 139. 1923.

VOUCHER: J. Warscewicz s.n. (K).

Pescatoria dayana Rchb.f., Gard. Chron. 1872: 1618. 1872.

Zygopetalum dayanum (Rchb.f.) Rchb.f., Index Kew. 2: 1255. 1895.

Pescatoria dayana var. candidula Rchb.f., Gard. Chron., n.s., 2: 226. 1874.

Pescatoria dayana var. rhodacra Rchb.f., Gard.

Chron., n.s., 2: 226. 1874.

Zygopetalum dayanum candidulum (Rchb.f.)

G.Nicholson, Ill. Dict. Gard. 4: 245. 1887.

Zygopetalum dayanum rhodacrum (Rchb.f.)

G.Nicholson, Ill. Dict. Gard. 4: 245. 1887.

Zygopetalum dayanum splendens G.Nicholson, Ill.

Dict. Gard. 4: 245. 1887.

VOUCHER: K. P. Reading s.n. (MO).

\section{Phalaenopsis Blume}

Phalaenopsis stuartiana Rchb.f., Gard. Chron., n.s., 15: 748. 1881.

Phalaenopsis schilleriana var. stuartiana (Rchb.f.)

Burb., Garden (London 1871-1927) 22: 119. 1882.

Phalaenopsis stuartiana var. nobilis Rchb.f., Gard.

Chron., n.s., 16: 748. 1881.

Phalaenopsis schilleriana var. vestalis Rchb.f., Gard. Chron., n.s., 17: 330. 1882.

Phalaenopsis stuartiana var. punctatissima Rchb.f., Gard. Chron., n.s., 17: 44. 1882.

Phalaenopsis stuartiana var. punctulata Linden, Lindenia 1: 21. 1885.

Phalaenopsis stuartiana var. bella Rchb.f., Gard. Chron., ser. 3, 3: 200. 1888.

Phalaenopsis schilleriana var. alba Roebelen, Gard. Chron., ser. 3, 7: 459. 1890.

Phalaenopsis schilleriana subvar. vestalis (Rchb.f.) A.H.Kent in H.J.Veitch, Man. Orchid. P1. 7: 37. 1891.

Phalaenopsis stuartiana f. nobilis (Rchb.f.) Christenson, Phalaenopsis: a monograph: 215. 2001.

Phalaenopsis stuartiana f. punctatissima (Rchb.f.)

Christenson, Phalaenopsis: a monograph: 215.
2001.

VOUCHER: M. Whitten 3322 (FLAS).

A species from Mindanao, Phillipines currently naturalized in Bocas del Toro. It is the only exotic epiphytic orchid in Panama.

\section{Phloeophila Hoehne \& Schltr.}

Phloeophila pelecaniceps (Luer) Pridgeon \& M.W.Chase, Lindleyana 16: 254. 2001.

Masdevallia pelecaniceps Luer, Selbyana 3: 22. 1976.

Luerella pelecaniceps (Luer) Braas, Orchidee (Hamburg) 30: 108. 1979.

VOUCHER: R.L. Dressler 3028 (SEL).

Phloeophila pleurothallopsis (Kraenzl.) Pridgeon \& M.W.Chase, Lindleyana 16: 254. 2001.

Cryptophoranthus pleurothallopsis Kraenzl., Bull. Misc. Inform. Kew 1925: 115. 1925.

Ophidion pleurothallopsis (Kraenzl.) Luer, Selbyana 7: 80. 1982.

Cryptophoranthus auriculatus Garay,

Orquideología 5: 16. 1970.

VOUCHER: R.L. Dressler 5656 (MO).

\section{Phragmipedium Rolfe}

Phragmipedium longifolium (Warsz. \& Rchb.f.) Rolfe, Orchid Rev. 4: 332.1896.

Cypripedium longifolium Warsz. \& Rchb.f., Bot. Zeitung (Berlin) 10: 690. 1852.

Selenipedium longifolium (Warsz. \& Rchb.f.) Rchb.f. \& Warsz., Bonplandia (Hannover) 2: 116. 1854.

Paphiopedilum longifolium (Warsz. \& Rchb.f.) Pfitzer, Jahrb. Wiss. Bot. 19: 159. 1888.

Cypripedium reichenbachii W.Bull, Nursery Cat. (William Bull) 48: 4. 1869.

Selenipedium dariense Rchb.f., Nov. Actorum Acad. Caes. Leop.-Carol. German. Nat. Cur. 35: t. 8. 1869.

Selenipedium reichenbachii Endrés ex Rchb.f., Gard. Chron. 1869: 1206. 1869., nom. inval.

Selenipedium roezlii Rchb.f., Gartenflora 1871: 164. 1871.

Cypripedium roezlii Regel, Gartenflora 22: 97. 1873.

Selenipedium longifolium var. coloratum Rchb.f., 
Gard. Chron. 1873: 289. 1873.

Cypripedium dariense Rchb.f., Gard. Chron., n.s., 9: 202.1878.

Cypripedium hincksianum Rchb.f., Gard. Chron., n.s., 9: 202. 1878.

Selenipedium hincksianum Rchb.f., Gard. Chron., n.s., 9: 202. 1878.

Cypripedium reichenbachianum auct., Lindenia 3: 90. 1887.

Selenipedium gracile (A.H.Kent) Desbois, Monogr. Cypriped.: 136. 1888.

Cypripedium gracile A.H.Kent in H.J.Veitch, Man. Orchid. Pl. 4: 65. 1889.

Cypripedium longifolium var. gracile (A.H.Kent) Rolfe, Gard. Chron., ser. 3, 8: 729. 1890.

Selenipedium longifolium var. gracile (A.H.Kent) Pucci, Cypripedium: 140. 1891.

Selenipedium longifolium var. hincksianum (Rchb.f.) Pucci, Cypripedium: 140. 1891.

Selenipedium longifolium var. splendidum Pucci, Cypripedium: 140. 1891.

Paphiopedilum longifolium var. hincksianum (Rchb.f.) Stein, Orchid.-Buch: 475. 1892.

Paphiopedilum hincksianum (Rchb.f.) Pfitzer, Bot. Jahrb. Syst. 19: 41. 1894.

Paphiopedilum roezlii (Rchb.f.) Pfitzer, Bot. Jahrb. Syst. 19: 42. 1894.

Selenipedium longifolium var. roezlii (Rchb.f.) Autran \& T.Durand, Hortus Boiss.: 364. 1896.

Phragmipedium longifolium var. darienense (Rchb.f.) Hallier f., Ann. Jard. Bot. Buitenzorg 14: 45. 1897.

Phragmipedium longifolium var. roezlii (Rchb.f.) Hallier f., Ann. Jard. Bot. Buitenzorg 14: 45. 1897.

Phragmipedium longifolium var. coloratum (Rchb.f.) Pfitzer in H.G.A.Engler (ed.), Pflanzenr., IV, 50(12): 49. 1903.

Phragmipedium longifolium var. gracile (A.H.Kent) Pfitzer in H.G.A.Engler (ed.), Pflanzenr., IV, 50(12): 49. 1903.

Phragmipedium longifolium var. hincksianum (Rchb.f.) Pfitzer in H.G.A.Engler (ed.), Pflanzenr., IV, 50(12): 49. 1903.

Phragmipedium longifolium var. splendidum (Pucci) Pfitzer in H.G.A.Engler (ed.), Pflanzenr., IV, 50(12): 50. 1903.
Phragmipedium dariense (Rchb.f.) Garay, Orchid Digest 43: 141. 1979.

Phragmipedium hincksianum (Rchb.f.) Garay, Orchid Digest 43: 144. 1979.

Phragmipedium roezlii (Rchb.f.) Garay, Orchid Digest 43: 145 (1979).

Phragmipedium longifolium f. gracile (A.H.Kent) O.Gruss, Caesiana 16: 40 (2001).

Phragmipedium longifolium f. minutum O.Gruss, Caesiana 16: 40 (2001).

Phragmipedium longifolium f. album O.Gruss \& Koop., Orchid Digest 72: 133 (2008), contrary Art. 37.4 ICBN (2006).

Voucher: H. Herrera et al. 1019 (PMA).

Phragmipedium humboldtii (Warsz. ex Rchb.f.) J.T.Atwood \& Dressler, Selbyana 19: 246. 1998[1999].

Cypripedium humboldtii Warsz., Bot. Zeitung (Berlin) 10: 691. 1852.

Cypripedium caudatum var. roseum Delchev., Rev. Hort. (Paris) 39: 133. 1867.

Selenipedium warscewiczii Rchb.f., Xenia Orchid. 2: 189.1873.

Cypripedium caudatum var. warscewiczii (Rchb.f.) Kerch., Livre Orchid.: 454. 1894., nom. superfl.

Phragmipedium caudatum var. roseum (Delchev.) Pfitzer in H.G.A.Engler (ed.), Pflanzenr. 12: 52. 1903.

Phragmipedium popowii Braem, Ohlund \& Quéné, Richardiana 4: 185. 2004.

Phragmipedium exstaminodium subsp. warszewiczii Dressler, Orchid Digest 69: 89. 2005.

Phragmipedium warscewiczii (Rchb.f.) Christenson, J. Orchideenfr. 13: 142. 2006.

VOUCHER: J. Warszewicz 41 (W).

Platystele Schltr.

Platystele aurea Garay, Orquideología 8(3): 182. 1973.

Pleurothallis rubella Luer, Selbyana 3: 378. 1977.

Rubellia rubella (Luer) Luer, Monogr. Syst. Bot. Missouri Bot. Gard. 95: 258. 2004.

Voucher: R.L. Dressler s.n. (SEL).

Platystele brenneri Luer, Selbyana 3: 24. 1976.

Voucher: R.L. Dressler 5593 (MO).

Platystele calymma Luer, Selbyana 3: 226. 1977. 
VOucher: R.L. Dressler s.n. (SEL).

Platystele caudatisepala (C.Schweinf.) Garay, Orquideología 9: 119. 1974.

Pleurothallis caudatisepala C.Schweinf., Bot. Mus. Leafl. 4: 113. 1937.

VOuCHER: R.L. Dressler 5800 (MO).

Platystele compacta (Ames) Ames, Proc. Biol. Soc. Wash. 35: 85. 1922.

Stelis compacta Ames, Orchidaceae 3: 76. 1908.

Pleurothallis compacta (Ames) Ames \&

C.Schweinf., Schedul. Orchid. 10: 24. 1930.

Platystele bulbinella Schltr., Repert. Spec. Nov.

Regni Veg. 8: 565. 1910.

VOUCHER: R.L. Dressler 5100 (UCH).

Platystele dressleri Luer, Selbyana 3: 26. 1976.

Voucher: C. Luer et al. 963 (SEL).

Platystele hampshireae Luer, Monogr. Syst. Bot. Missouri Bot. Gard. 65: 113. 1998.

VOUCHER: R.J. Hampshire \& C. Whitefoord 414 (BM).

Platystele jungermannioides (Schltr.) Garay, Orquideología 9: 120. 1974.

Pleurothallis jungermannioides Schltr., Repert. Spec. Nov. Regni Veg. 11: 42. 1912.

VOUCHER: J.P. Folsom 2884 (MO).

Platystele microtatantha (Schltr.) Garay, Bot. Mus. Leafl. 21: 251. 1967.

Pleurothallis microtatantha Schltr., Repert. Spec.

Nov. Regni Veg. 3(44-45): 276. 1907.

VOUCHER: A.P. Karremans et al. 6133 (JBL).

Platystele minimiflora (Schltr.) Garay, Orquideología 9: 120.1974.

Pleurothallis minimiflora Schltr., Repert. Spec.

Nov. Regni Veg. Beih. 19: 288. 1923.

Pleurothallis halbingeriana R.E.Schult., Bot. Mus.

Leafl. 9: 30. 1941.

Platystele halbingeriana (R.E.Schult.) Garay,

Orquideología 9: 119. 1974.

Voucher: F.L. Stevenson 81974-1 (SEL).

Platystele ortiziana Luer \& R.Escobar, Monogr. Syst. Bot. Missouri Bot. Gard. 38: 80. 1990.

Voucher: R.L. Dressler s.n., photo cited by Luer (1990).

Platystele ovalifolia (H.Focke) Garay \& Dunst., Venez. Orchids Ill. 2: 268. 1961.

Stelis ovalifolia H.Focke, Tijdschr. Natuurk. Wetensch. Kunsten 2: 202. 1849.

Pleurothallis ovalifolia (H.Focke) Rchb.f. in
W.G.Walpers, Ann. Bot. Syst. 6: 188. 1861.

Pleurothallis rhomboglossa Rchb.f., Flora 48: 276. 1865.

Pleurothallis vaginulata Griseb., Cat. Pl. Cub.: 259. 1866.

Voucher: B.E. Hammel 7209 (MO).

Platystele ovatilabia (Ames \& C.Schweinf.) Garay, Orquideología 9: 120. 1974.

Pleurothallis ovatilabia Ames \& C.Schweinf., Schedul. Orchid. 10: 33. 1930.

VOUCHER: I. Martínes \& R. Rincón 717 (UCH).

Platystele oxyglossa (Schltr.) Garay, Orquideología 9: 120. 1974.

Pleurothallis oxyglossa Schltr., Repert. Spec. Nov. Regni Veg. 10: 354. 1912.

Pleurothallis lancilabris var. oxyglossa (Schltr.) C.Schweinf., Bot. Mus. Leafl. 6: 200. 1938.

Pleurothallis schulzeana Schltr., Beih. Bot. Centralbl. 36(2): 396. 1918.

Pleurothallis pygmaea Hoehne, Bol. Agric. (São Paulo) 34: 604. 1933[1934].

Platystele brasiliensis Brade, Arch. Jard. Bot. Rio de Janeiro 11: 73. 1951.

Platystele pygmaea (Hoehne) Pabst, Rodriguésia 18-19: 30. 1956

Platystele schulzeana (Schltr.) Garay, Orquideología 9: 120. 1974.

VOUCHER: C.A. Luer et al. 1452 (SEL).

Platystele perpusilla (Rchb.f.) Garay, Orquideología 9: 120. 1974.

Pleurothallis perpusilla Rchb. f., Bot. Voy. Herald 215. 1854.

Humboldtia perpusilla (Rchb. f.) Kuntze, Revis. Gen. Pl. 2: 668. 1891.

VOUCHer: B.C. Seemann 1565 (K).

Platystele resimula Luer \& Hirtz, Monogr. Syst. Bot. Missouri Bot. Gard. 38: 105. 1990.

Voucher: C.A. Luer et al. 10566 (MO).

Platystele speckmaieri Luer \& Sijm, Monogr. Syst. Bot. Missouri Bot. Gard. 120: 146, f. 17. 2010.

VOUCHER: A.P. Sijm 290815 (MO).

Platystele stenostachya (Rchb.f.) Garay, Caldasia 8: 520. 1962.

Pleurothallis stenostachya Rchb.f., Linnaea 18: 399. 1845.

Humboldtia stenostachya (Rchb.f.) Kuntze, Revis. Gen. Pl. 2: 668. 1891. 
Pleurothallis dubia A.Rich. \& Galeotti, Ann. Sci. Nat., Bot., sér. 3, 3: 16. 1845.

Pleurothallis minutiflora S.Watson, Proc. Amer. Acad. Arts 23: 286. 1888.

Humboldtia dubia (A.Rich. \& Galeotti) Kuntze, Revis. Gen. Pl. 2: 667. 1891.

Pleurothallis myriantha F.Lehm. \& Kraenzl., Bot. Jahrb. Syst. 26: 445. 1899.

Pleurothallis lankesteri Rolfe, Bull. Misc. Inform. Kew 1914: 210. 1914.

Pleurothallis dubia var. myriantha (F.Lehm. \& Kraenzl.) Schltr., Repert. Spec. Nov. Regni Veg. Beih. 17: 20. 1922.

Pleurothallis stenostachya var. lankesteri (Rolfe)

Ames, Schedul. Orchid. 7: 31. 1924.

VOUCHER: C.A. Luer et al. 1316 (SEL).

Platystele sylvestrei Karremans \& Bogarín, Lankesteriana 13(3): 268. 2014.

Voucher: D. Bogarin 10744 (JBL).

Platystele taylorii Luer, Selbyana 3: 30. 1976.

Voucher: C. Luer et al. 741 (SEL).

\section{Platythelys Garay}

Platythelys maculata (Hook.) Garay, Bradea 2: 197. 1977.

Physurus maculatus Hook., Bot. Mag. 88: t. 5305. 1862.

Erythrodes maculata (Hook.) Ames, Orchidaceae 7: 72.1922.

Voucher: R.L. Dressler 5664 (PMA).

Platythelys vaginata (Hook.) Garay, Bradea 2: 198. 1977.

Physurus vaginatus Hook., Hooker's Icon. Pl. 5: t. 449. 1842.

Erythrodes vaginata (Hook.) Ames, Orchidaceae 5: 29. 1915.

Voucher: T.M. Antonio 2084 (MO).

Platythelys venustula (Ames) Garay, Bradea 2: 198. 1977.

Physurus venustulus Ames, Orchidaceae 2: 261. 1908.

Erythrodes venustula (Ames) Ames, Orchidaceae 5: 28.1915.

Erythrodes querceticola var. venustula (Ames) Correll, Lloydia 10: 210. 1947.

VOUCHER: R.L. Dressler 5871 (PMA).

\section{Plectrophora H.Focke}

Plectrophora alata (Rolfe) Garay, Bot. Mus. Leafl. 21: 261. 1967.

Trichocentrum alatum Rolfe, Bull. Misc. Inform. Kew 1898: 197. 1898.

VOUCHER: H. Teuscher s.n. (MO).

Pleurothallis R.Br.

Pleurothallis allenii L.O.Williams, Ann. Missouri Bot. Gard. 27: 275. 1940.

Zosterophyllanthos allenii (L.O.Williams) Szlach. \& Marg., Polish Bot. J. 46: 118. 2001.

Acronia allenii (L.O.Williams) Luer, Monogr. Syst. Bot. Missouri Bot. Gard. 103: 57. 2005.

VOUCHER: P.H. Allen 1240 (MO).

Pleurothallis annectens Luer, Selbyana 3: 240. 1977. Acronia annectens (Luer) Luer, Monogr. Syst. Bot. Missouri Bot. Gard. 103: 57. 2005.

Voucher: C. Luer et al. 1583 (SEL).

Pleurothallis archicolonae Luer, Selbyana 3: 52. 1976.

Acronia archicolonae (Luer) Luer, Monogr. Syst. Bot. Missouri Bot. Gard. 103: 57. 2005.

Voucher: C. Luer et al. 964 (MO).

Pleurothallis bivalvis Lindl., Orchid. Linden.: 2. 1846.

Humboldtia bivalvis (Lindl.) Kuntze, Revis. Gen.

P1. 2: 667. 1891.

Acronia bivalvis (Lindl.) Luer, Monogr. Syst. Bot. Missouri Bot. Gard. 103: 93. 2005.

Zosterophyllanthos bivalvis (Lindl.) Szlach., Ill.

Field Guide Orchids Yotoco Forest Reserve: 276. 2011.

Pleurothallis cardium Rchb.f., Bonplandia (Hannover) 2: 26. 1854.

Pleurothallis lansbergiana Regel, Gartenflora 6: t. 193, f. d. 1856.

Pleurothallis lansbergii Regel, Index Seminum (LE) 1856: 17. 1856.

Pleurothallis cardiantha Rchb.f., Linnaea 41: 15. 1876.

Humboldtia cardium (Rchb.f.) Kuntze, Revis. Gen. P1. 2: 667. 1891.

Pleurothallis stenocardium Schltr., Notizbl. Königl. Bot. Gart. Berlin 6: 123. 1914.

Pleurothallis pichinchae Schltr., Repert. Spec. 
Nov. Regni Veg. 14: 132. 1915., nom. illeg.

Pleurothallis chanchamayoensis Schltr., Repert.

Spec. Nov. Regni Veg. Beih. 9: 73. 1921.

Pleurothallis ignivomi Schltr., Repert. Spec. Nov.

Regni Veg. Beih. 8: 129. 1921.

Pleurothallis sigsigensis Schltr., Repert. Spec.

Nov. Regni Veg. Beih. 8: 63. 1921.

Pleurothallis fuscata Braid, Bull. Misc. Inform.

Kew 1924: 200. 1924.

Pleurothallis phaeantha Schltr., Repert. Spec. Nov.

Regni Veg. Beih. 27: 52. 1924.

Pleurothallis angusta Ames \& C.Schweinf.,

Schedul. Orchid. 8: 23. 1925.

Pleurothallis archidiaconi Ames, Schedul. Orchid. 9: 29. 1925.

Pleurothallis antonensis L.O.Williams, Ann. Missouri Bot. Gard. 29: 341. 1942.

Pleurothallis harlingii Garay, Svensk Bot. Tidskr. 47: 208. 1953.

Zosterophyllanthos ignivomi (Schltr.) Szlach. \& Marg., Polish Bot. J. 46: 119. 2001.

Pleurothallis lilianiae Luer, Orquideología 22: 264. 2003.

Zosterophyllanthos lilianiae (Luer) Szlach. \& Kulak, Richardiana 6: 134. 2006.

VOUCHER: P.H. Allen 2701 (AMES).

Pleurothallis cardiochila L.O.Williams, Ann. Missouri Bot. Gard. 29: 343. 1942.

Acronia cardiochila (L.O.Williams) Luer, Monogr. Syst. Bot. Missouri Bot. Gard. 103: 103. 2005.

Zosterophyllanthos cardiochilus (L.O.Williams)

Szlach. \& Kulak, Richardiana 6: 187. 2006.

Voucher: M.E. Davidson 119 (AMES).

Pleurothallis cardiothallis Rchb.f., Bot. Zeitung (Berlin) 15: 158. 1857.

Humboldtia cardiothallis (Rchb.f.) Kuntze, Revis.

Gen. P1. 2: 667. 1891.

Acronia cardiothallis (Rchb.f.) Luer, Monogr. Syst.

Bot. Missouri Bot. Gard. 103: 106. 2005.

Zosterophyllanthos cardiothallis (Rchb.f.) Szlach.

\& Kulak, Richardiana 6: 187. 2006.

Pleurothallis subulata Rolfe, Bull. Misc. Inform.

Kew 1892: 137. 1892.

Pleurothallis acutipetala Schltr., Repert. Spec.

Nov. Regni Veg. 10: 353. 1912.

Pleurothallis costaricensis Schltr., Beih. Bot.

Centralbl. 36(2): 395. 1918., nom. illeg.
Pleurothallis schlechteriana Ames, Schedul. Orchid. 2: 24. 1923.

VOUCHER: R.L. Dressler 5121 (SEL).

Pleurothallis chloroleuca Lindl., Orchid. Linden.: 2. 1846.

Humboldtia chloroleuca (Lind1.) Kuntze, Revis. Gen. P1. 2: 667. 1891.

Pleurothallis ventricosa Lindl., Fol. Orchid. 9: 16. 1859.

Pleurothallis rhomboglossa Rchb.f., Flora 71: 154. 1888., nom. illeg.

Pleurothallis wendlandiana Rchb.f., Flora 71: 153. 1888.

Humboldtia ventricosa (Lindl.) Kuntze, Revis. Gen. P1. 2: 668. 1891.

Pleurothallis reichenbachiana Schltr., Repert. Spec. Nov. Regni Veg. Beih. 8: 131. 1921.

VOUCHER: not seen, cited by Luer (2003).

Pleurothallis cleistogama Luer, Monogr. Syst. Bot. Missouri Bot. Gard. 72: 41. 1998.

VOuCHER: C.A. Luer et al. 10527 (MO).

Pleurothallis cobriformis L.O.Williams, Ann. Missouri Bot. Gard. 27: 277. 1940.

Acronia cobriformis (L.O.Williams) Luer, Monogr. Syst. Bot. Missouri Bot. Gard. 103: 111, f. 48. 2005.

VOUCHER: P.H. Allen 2027 (MO).

Pleurothallis colossus Kraenzl. ex Kerch., Livre Orchid.: 376. 1894.

Pleurothallis praegrandis Ames, Schedul. Orchid. 5: 17.1923.

Pleurothallis saccata Ames, Schedul. Orchid. 4: 25. 1923.

Pleurothallis hirtzii Luer, Phytologia 47: 76. 1980.

VOUCHER: R.S. Williams 973 (AMES).

Pleurothallis cordifolia Rchb.f. \& H.Wagener, Bonplandia (Hannover) 2: 26. 1854.

Humboldtia cordifolia (Rchb.f. \& H.Wagener) Kuntze, Revis. Gen. P1. 2: 667. 1891.

Acronia cordifolia (Rchb.f. \& H.Wagener) Luer, Monogr. Syst. Bot. Missouri Bot. Gard. 103: 117. 2005.

Zosterophyllanthos cordifolius (Rchb.f. \& H.Wagener) Szlach. \& Kulak, Richardiana 6: 188. 2006.

Pleurothallis veraguacensis Luer, Selbyana 3(34): 402-403, f. 301. 1977. 
Voucher: C.A. Luer \& R.L. Dressler 1141 (SEL).

Pleurothallis crescentilabia Ames, Schedul. Orchid. 4: 20.1923.

VOUCHER: C.A. Luer 2280 (MO).

Pleurothallis crocodiliceps Rchb.f., Bonplandia (Hannover) 3: 72. 1855.

Humboldtia crocodiliceps (Rchb.f.) Kuntze, Revis. Gen. P1. 2: 667. 1891.

Ancipitia crocodiliceps (Rchb.f.) Luer, Monogr. Syst. Bot. Missouri Bot. Gard. 95: 254. 2004.

Pleurothallis arietina Ames, Schedul. Orchid. 4: 16. 1923.

Pleurothallis nelsonii Ames, Schedul. Orchid. 4: 22. 1923.

Pleurothallis microchila L.O.Williams, Fieldiana, Bot. 31: 259. 1967.

Voucher: J.P. Folsom 2187 (MO).

Pleurothallis dariensis (Kolan. \& Szlach.) Bogarín, comb. nov.

Bas.: Zosterophyllanthos dariensis Kolan. \& Szlach., Richardiana 14: 106. 2014.

VOUCHER: R.L. Dressler 5589 (FLAS).

Pleurothallis dentipetala Rolfe ex Ames, Schedul. Orchid. 3: 7. 1923.

Acronia dentipetala (Rolfe ex Ames) Luer, Monogr. Syst. Bot. Missouri Bot. Gard. 103: 57. 2005.

Pleurothallis cooperi Schltr., Repert. Spec. Nov. Regni Veg. Beih. 19: 286. 1923.

Pleurothallis stelidiformis Schltr., Repert. Spec.

Nov. Regni Veg. Beih. 19: 195. 1923.

Pleurothallis stevensii Luer, Selbyana 5: 181. 1979.

VOUCHER: G. McPherson 15954 (MO).

Pleurothallis discoidea Lindl., Edwards's Bot. Reg.

21: t. 1797. 1835.

Voucher: T.B. Croat 49217 (MO).

Pleurothallis divaricans Schltr., Repert. Spec. Nov. Regni Veg. 10: 387. 1912.

VOUCHER: C.A. Luer 1388 (MO).

Pleurothallis dressleriana Bogarín, nom. nov.

Bas.: Ancipitia dressleri Kolan. \& Szlach., Richardiana 14: 104, f. 1A-F. 2014.

Non Pleurothallis dressleri Luer, Selbyana 3: 98. 1976.

VOUCHER: R.L. Dressler 5591 (FLAS).

Pleurothallis dukei Luer, Lindleyana 12: 45. 1997.

Acronia dukei (Luer) Luer, Monogr. Syst. Bot. Missouri Bot. Gard. 103: 57. 2005.
Voucher: J.A. Duke \& T.S. Elias 13845 (MO).

Pleurothallis eumecocaulon Schltr., Repert. Spec. Nov. Regni Veg. Beih. 19: 187. 1923.

Ancipitia eumecocaulon (Schltr.) Luer, Monogr. Syst. Bot. Missouri Bot. Gard. 95: 254. 2004.

Voucher: B.E. Hammel 786 (MO).

Pleurothallis excavata Schltr., Repert. Spec. Nov. Regni Veg. Beih. 19: 287. 1923.

Acronia excavata (Schltr.) Luer, Monogr. Syst.

Bot. Missouri Bot. Gard. 103: 133. 2005.

Zosterophyllanthos excavatus (Schltr.) Szlach. \&

Kulak, Richardiana 6: 189. 2006.

Pleurothallis concaviflora C.Schweinf., Bot. Mus. Leafl. 4: 114. 1937.

Pleurothallis imago Luer, Selbyana 3: 318. 1977.

Voucher: C.A. Luer 732 (SEL).

Pleurothallis fantastica Ames, Bot. Mus. Leafl. 4: 41. 1936.

Acronia fantastica (Ames) Luer, Monogr. Syst.

Bot. Missouri Bot. Gard. 103: 134. 2005.

Pleurothallis horichii Luer, Orchidee (Hamburg) 36: 23. 1985.

Zosterophyllanthos horichii (Luer) Szlach. \& Marg., Polish Bot. J. 46: 119. 2001.

Voucher: D. Bogarín et al. 10960 (UCH).

Pleurothallis folsomii (Luer \& Endara) Bogarín, comb. nov.

Bas.: Acronia folsomii Luer \& Endara, Monogr. Syst. Bot. Missouri Bot. Gard. 103: 134. 2005.

Voucher: J.A. Folsom 6096 (MO).

Pleurothallis hammelii Luer, Lindleyana 12: 48. 1997. Acronia hammelii (Luer) Luer, Monogr. Syst. Bot. Missouri Bot. Gard. 103: 58. 2005.

VOUCHER: B. Hammel 868 (SEL).

Pleurothallis harpago Luer, Selbyana 5: 167. 1979. Ancipitia harpago (Luer) Luer, Monogr. Syst. Bot. Missouri Bot. Gard. 95: 254. 2004.

VOUCHER: C.A. Luer 2798 (SEL).

Pleurothallis hemileuca Luer, Selbyana 7: 118. 1982. Acronia hemileuca (Luer) Luer, Monogr. Syst. Bot. Missouri Bot. Gard. 103: 142. 2005.

Zosterophyllanthos hemileucus (Luer) Szlach. \& Kulak, Richardiana 14: 108. 2014.

VOUCHER: C.A. Luer 1274 (SEL).

Pleurothallis homalantha Schltr., Repert. Spec. Nov. Regni Veg. Beih. 19: 24. 1923.

Zosterophyllanthos homalanthus (Schltr.) Szlach. 
\& Marg., Polish Bot. J. 46: 119. 2001.

Acronia homalantha (Schltr.) Luer, Monogr. Syst.

Bot. Missouri Bot. Gard. 103: 143. 2005.

Pleurothallis homalanthoides Schltr., Repert. Spec.

Nov. Regni Veg. Beih. 19: 190. 1923.

Pleurothallis nemorum Schltr., Repert. Spec. Nov.

Regni Veg. Beih. 19: 191. 1923.

Pleurothallis nervosa Braid, Bull. Misc. Inform.

Kew 1924: 201. 1924.

Voucher: T.B. Croat 76497 (MO).

Pleurothallis instar Luer, Selbyana 3: 320. 1977.

VOuCHER: C. Luer et al. 1389 (SEL).

Pleurothallis isthmica Luer, Selbyana 3: 322. 1977.

Acronia isthmica (Luer) Luer, Monogr. Syst. Bot.

Missouri Bot. Gard. 103: 146. 2005.

Zosterophyllanthos isthmicus (Luer) Szlach. \&

Kulak, Richardiana 6: 189. 2006.

VOuCHer: C. Luer \& R.L. Dressler 1140 (SEL).

Pleurothallis knappiae Luer, Monogr. Syst. Bot. Missouri Bot. Gard. 79: 128. 2000.

Acronia knappiae (Luer) Luer, Monogr. Syst. Bot.

Missouri Bot. Gard. 103: 58. 2005.

VOUCHER: S. Knapp \& K. Sytsma 2653 (MO).

Pleurothallis leucantha Schltr., Repert. Spec. Nov. Regni Veg. 10: 353. 1912.

Zosterophyllanthos leucanthus (Schltr.) Szlach. \&

Marg., Polish Bot. J. 46: 119. 2001.

Acronia leucantha (Schltr.) Luer, Monogr. Syst.

Bot. Missouri Bot. Gard. 103: 149. 2005.

Voucher: C.A. Luer et al. 10636 (MO).

Pleurothallis longipedicellata Ames \& C.Schweinf., Schedul. Orchid. 8: 27. 1925.

Acronia longipedicellata (Ames \& C.Schweinf.)

Luer, Monogr. Syst. Bot. Missouri Bot. Gard. 103: 58. 2005.

Voucher: C.A. Luer \& H. Butcher 1358 (SEL).

Pleurothallis loranthophylla Rchb.f., Bot. Zeitung (Berlin) 10: 674. 1852.

Humboldtia loranthophylla (Rchb.f.) Kuntze,

Revis. Gen. P1. 2: 667. 1891.

Rhynchopera loranthophylla (Rchb.f.) Szlach. \&

Marg., Polish Bot. J. 46: 118. 2001.

Rhynchopera punctata H.Karst., Auswahl Gew.

Venez. 2: 21. 1849.

Pleurothallis subpellucida Klotzsch, Index

Seminum (B) 1852(App.): 1. 1852.

Pleurothallis loranthophylla var. pellucida Regel,
Ann. Sci. Nat., Bot., sér. 4, 6: 373. 1856.

Pleurothallis navicularis Lindl., Fol. Orchid. 8: 6. 1859.

Humboldtia navicularis (Lindl.) Kuntze, Revis. Gen. P1. 2: 668. 1891.

Humboldtia subpellucida (Klotzsch) Kuntze, Revis. Gen. P1. 2: 668. 1891.

Pleurothallis intermedia Schltr., Repert. Spec. Nov. Regni Veg. Beih. 6: 85. 1919.

Pleurothallis punctata (H.Karst.) Schltr., Repert. Spec. Nov. Regni Veg. Beih. 6: 63. 1919., nom. illeg.

Pleurothallis violaceorosea Schltr., Repert. Spec. Nov. Regni Veg. Beih. 19: 198. 1923.

Pleurothallis spathata Schltr., Repert. Spec. Nov. Regni Veg. 27: 54. 1929.

Voucher: C.A. Luer \& R.L. Dressler 1256 (SEL).

Pleurothallis maduroi Luer, Lindleyana 12: 51. 1997. Acronia maduroi (Luer) Luer Monogr. Syst. Bot. Missouri Bot. Gard. 103: 156, f. 123. 2005.

Zosterophyllanthos maduroi (Luer) Szlach. \& Kulak, Richardiana 6: 190. 2006.

VOUCHER: A. Maduro 18 (MO).

Pleurothallis mammillata Luer, Selbyana 3: 138. 1976.

Voucher: C.A. Luer et al. 951 (SEL).

Pleurothallis nitida Luer, Selbyana 3(3-4): 352. 1977.

Acronia nitida (Luer) Luer, Monographs Monogr. Syst. Bot. Missouri Bot. Gard. 103: 164. 2005.

VOUCHer: C. Luer et al. 1337 (SEL).

Pleurothallis orygmoglossa (Luer \& Dressler) Bogarín, comb. nov.

Bas.: Acronia orygmoglossa Luer \& Dressler, Monogr. Syst. Bot. Missouri Bot. Gard. 103: 167. 2005.

VOUCHER: R.L. Dressler 5351 (SEL).

Pleurothallis pallida Luer, Selbyana 3: 358. 1977.

Acronia pallida (Luer) Luer, Monogr. Syst. Bot. Missouri Bot. Gard. 103: 58. 2005.

VOUCHER: C.A. Luer et al. 954 (SEL).

Pleurothallis palliolata Ames, Proc. Biol. Soc. Wash. 35: 86. 1922.

Acronia palliolata (Ames) Luer, Monogr. Syst. Bot. Missouri Bot. Gard. 103: 168. 2005.

Zosterophyllanthos palliolatus (Ames) Szlach. \& Kulak, Richardiana 6: 191. 2006.

VOucher: H. van der Werff \& C. van Hardeveld 6619 
(MO).

Pleurothallis peculiaris Luer, Selbyana 3: 158. 1976. Acronia peculiaris (Luer) Luer, Monogr. Syst. Bot. Missouri Bot. Gard. 103: 170, f. 150. 2005.

Zosterophyllanthos peculiaris (Luer) Szlach. \& Marg., Polish Bot. J. 46(2): 120. 2001.

Voucher: C.A. Luer \& R.L. Dressler 1142 (SEL).

Pleurothallis phyllocardia Rchb.f., Beitr. Orchid.-K. C. Amer.: 97. 1866.

Humboldtia phyllocardia (Rchb.f.) Kuntze, Revis. Gen. P1. 2: 668. 1891.

Zosterophyllanthos phyllocardius (Rchb.f.) Szlach. \& Marg., Polish Bot. J. 46: 120. 2001.

Acronia phyllocardia (Rchb.f.) Luer, Monogr. Syst.

Bot. Missouri Bot. Gard. 103: 173. 2005.

Pleurothallis triangulabia C.Schweinf., Ann. Missouri Bot. Gard. 24: 183. 1937.

VOUCHER: R.J. Seibert 269 (MO).

Pleurothallis phyllocardioides Schltr., Repert. Spec. Nov. Regni Veg. Beih. 19: 193. 1923.

Zosterophyllanthos phyllocardioides (Schltr.)

Szlach. \& Marg., Polish Bot. J. 46: 120. 2001. Acronia phyllocardioides (Schltr.) Luer, Monogr.

Syst. Bot. Missouri Bot. Gard. 103: 174. 2005. Pleurothallis acostaei Schltr., Repert. Spec. Nov.

Regni Veg. Beih. 19: 285. 1923.

Pleurothallis rhaphidopus Schltr., Repert. Spec.

Nov. Regni Veg. Beih. 27: 54. 1924.

Pleurothallis graciliscapa C.Schweinf., Bot. Mus. Leafl. 15: 93. 1951.

Voucher: C.A. Luer et al. 10608 (MO).

Pleurothallis polysticta Luer, Selbyana 5: 176. 1979. Acronia polysticta (Luer) Luer Monogr. Syst. Bot.

Missouri Bot. Gard. 103: 58. 2005.

VOUCHER: C.A. Luer 2799 (SEL).

Pleurothallis pruinosa Lindl., Edwards's Bot. Reg. 28(Misc.): 75. 1842.

Humboldtia pruinosa (Lindl.) Kuntze, Revis. Gen. P1. 2: 667. 1891.

Stelis flavida H.Focke, Tijdschr. Natuurk. Wetensch. Kunsten 2: 202. 1849.

Pleurothallis flavida (H.Focke) Lindl., Fol. Orchid. 9: 44.1859.

Pleurothallis pauciflora Schltr., Repert. Spec. Nov.

Regni Veg. Beih. 19: 192. 1923.

VOUCHER: T.M. Antonio 2628 (MO).

Pleurothallis rectipetala Ames \& C.Schweinf.,
Schedul. Orchid. 8: 32. 1925.

Acronia rectipetala (Ames \& C.Schweinf.) Luer, Monogr. Syst. Bot. Missouri Bot. Gard. 103: 180. 2005.

Zosterophyllanthos rectipetalus (Ames \& C.Schweinf.) Szlach. \& Kulak, Richardiana 6: 192. 2006.

Pleurothallis scitula Luer, Selbyana 3(3-4): 384, f. 292. 1977.

VOUCHER: C.A. Luer 1361 (SEL).

Pleurothallis rhodoglossa Schltr., Repert. Spec. Nov. Regni Veg. Beih. 17: 22.1922.

Zosterophyllanthos rhodoglossus (Schltr.) Szlach. \& Marg., Polish Bot. J. 46: 120. 2001.

Acronia rhodoglossa (Schltr.) Luer, Monogr. Syst.

Bot. Missouri Bot. Gard. 103: 182. 2005.

Voucher: C.W. Powell 182 (AMES).

Pleurothallis rowleei Ames, Schedul. Orchid. 1: 11. 1922.

Acronia rowleei (Ames) Luer, Monogr. Syst. Bot. Missouri Bot. Gard. 103: 58. 2005.

Pleurothallis cucullata Ames, Schedul. Orchid. 3: 5. 1923.

Pleurothallis rhodocardia Garay, Svensk Bot. Tidskr. 47: 204. 1953.

VOUCHER: T.B. Croat 48773 (MO).

Pleurothallis ruscifolia (Jacq.) R.Br. in W.T.Aiton, Hortus Kew. 5: 211. 1813.

Epidendrum ruscifolium Jacq., Enum. Syst. Pl.: 29. 1760.

Dendrobium ruscifolium (Jacq.) Sw., Nova Acta Regiae Soc. Sci. Upsal. 6: 84. 1799.

Humboldtia ruscifolia (Jacq.) Kuntze, Revis. Gen. P1. 2: 668. 1891.

Pleurothallis laurifolia Kunth in F.W.H.von Humboldt, A.J.A.Bonpland \& C.S.Kunth, Nov. Gen. Sp. 1: 364. 1816.

Humboldtia succosa Pav. ex Lindl., Gen. Sp. Orchid. P1.: 5. 1830.

Pleurothallis succosa Lindl., Gen. Sp. Orchid. Pl.: 5. 1830.

Pleurothallis multicaulis Poepp. \& Endl., Nov. Gen. Sp. Pl. 1: 47. 1836.

Humboldtia laurifolia (Kunth) Kuntze, Revis. Gen. P1. 2: 667. 1891.

Pleurothallis glomerata Ames, Schedul. Orchid. 4: 21. 1923. 
Pleurothallis ruscifolia var. caquetana Schltr., Repert. Spec. Nov. Regni Veg. Beih. 27: 55. 1924.

VOUCHER: P.H. Allen 3814 (MO).

Pleurothallis sanchoi Ames, Schedul. Orchid. 4: 26. 1923.

Acronia sanchoi (Ames) Luer, Monogr. Syst. Bot. Missouri Bot. Gard. 103: 186. 2005.

Zosterophyllanthos sanchoi (Ames) Szlach. \& Marg., Polish Bot. J. 46(2): 120. 2001.

Voucher: C.A. Luer 10636 (SEL).

Pleurothallis simulans L.O.Williams, Ann. Missouri Bot. Gard. 27: 281. 1940.

Zosterophyllanthos simulans (L.O.Williams) Szlach. \& Marg., Polish Bot. J. 46: 121. 2001. Acronia simulans (L.O.Williams) Luer, Monogr. Syst. Bot. Missouri Bot. Gard. 103: 58. 2005.

VOUCHER: P.H. Allen 1912 (MO).

Pleurothallis telamon Luer, Selbyana 5: 185. 1979. Acronia telamon (Luer) Luer, Monogr. Syst. Bot. Missouri Bot. Gard. 103: 198. 2005.

Zosterophyllanthos telamon (Luer) Szlach. \& Kulak, Richardiana 6: 193. 2006.

VOUCHER: C.A. Luer 2112 (SEL).

Pleurothallis titan Luer, Selbyana 3: 400. 1977. Acronia titan (Luer) Luer, Monogr. Syst. Bot. Missouri Bot. Gard. 103: 198, f. 199. 2005.

Zosterophyllanthos titan (Luer) Szlach. \& Kulak, Richardiana 6: 135. 2006.

Voucher: R.L. Dressler s.n. (SEL).

Pleurothallis tonduzii Schltr., Beih. Bot. Centralbl. 36(2): 397. 1918.

Acronia tonduzii (Schltr.) Luer, Monogr. Syst. Bot.

Missouri Bot. Gard. 103: 199, f. 200. 2005.

Zosterophyllanthos tonduzii (Schltr.) Szlach. \&

Kulak, Richardiana 6(4): 193. 2006.

Voucher: C.A. Luer \& H. Butcher 1229 (SEL).

Pleurothallis volcanica Luer, Selbyana 3: 406. 1977. Acronia volcanica (Luer) Luer, Monogr. Syst. Bot.

Missouri Bot. Gard. 103: 58. 2005.

VOucher: C.A. Luer \& H. Butcher 1260 (SEL).

Pleurothallopsis Porto \& Brade

Pleurothallopsis tubulosa (Lindl.) Pridgeon \&

M.W.Chase, Lindleyana 16: 255. 2001.

Pleurothallis tubulosa Lindl., Fol. Orchid. 9: 19.
1859.

Humboldtia tubulosa (Lindl.) Kuntze, Revis. Gen. P1. 2: 668. 1891.

Restrepiella tubulosa (Lindl.) Garay \& Dunst., Venez. Orchids Ill. 4: 266. 1966.

Restrepiopsis tubulosa (Lind1.) Luer, Selbyana 2: 200. 1978.

Pleurothallis viridula Lindl., Fol. Orchid. 9: 19. 1859.

Humboldtia viridula (Lindl.) Kuntze, Revis. Gen. P1. 2: 668. 1891.

Pleurothallis caliensis Schltr., Repert. Spec. Nov. Regni Veg. Beih. 7: 102. 1920.

Pleurothallis flavescens Schltr., Repert. Spec. Nov. Regni Veg. Beih. 19: 188. 1923.

Restrepiella viridula (Lindl.) Garay \& Dunst., Venez. Orchids Ill. 4: 268. 1966.

Restrepiopsis viridula (Lindl.) Luer, Selbyana 2: 200. 1978.

VOUCHER: G. McPherson 8993 (MO).

Pleurothallopsis ujarensis (Rchb.f.) Pridgeon \& M.W.Chase, Lindleyana 16: 255. 2001.

Restrepia ujarensis Rchb.f., Bonplandia (Hannover) 3: 225. 1855.

Pleurothallis ujarensis (Rchb.f.) Lindl., Fol. Orchid. 9: 19. 1859.

Humboldtia ujarensis (Rchb.f.) Kuntze, Revis. Gen. P1. 2: 668. 1891.

Restrepiella ujarensis (Rchb.f.) Garay \& Dunst., Venez. Orchids Ill. 4: 266. 1966.

Restrepiopsis ujarensis (Rchb.f.) Luer, Selbyana 2: 200. 1978.

Restrepiella lenkenhoffii Braas \& H.Mohr, Orchidee (Hamburg) 33: 154. 1982.

Voucher: M.A. Blanco \& D. Penneys 2942 (PMA).

PolyCyCNIS Rchb.f.

Polycycnis barbata (Lindl.) Rchb.f., Bonplandia (Hannover) 3: 218. 1855.

Cycnoches barbatum Lindl., J. Hort. Soc. London 4: 268.1849.

Voucher: J. S. Miller \& L. Miller 970 (MO).

Polycycnis blancoi G.Gerlach, Lankesteriana 4: 67. 2004.

VOUCHER: L. Martínez et al. 406 (PMA).

Polycycnis gratiosa Endrés \& Rchb.f., Gard. Chron. 
1871: 1451. 1871.

Polycycnis lepida var. gratiosa (Endrés \& Rchb.f.)

Stein, Orchid.-Buch: 527. 1892.

Voucher: S.A. Mori \& J.A. Kallunki 7039 (MO).

Polycycnis ornata Garay, Canad. J. Bot. 34: 256. 1956.

Polycycnopsis ornata (Garay) Szlach., Polish Bot. J. 51: 34. 2006.

VOUCHER: J.A. Duke 13796 (MO).

Polycycnis tortuosa Dressler, Orquideología 12: 120. 1977.

VOUCHER: R.L. Dressler 5731 (US).

\section{Polystachya Hook.}

Polystachya foliosa (Hook.) Rchb.f. in W.G.Walpers, Ann. Bot. Syst. 6: 640. 1863.

Stelis foliosa Hook., Ann. Nat. Hist. 2: 330. 1839.

Dendrorkis foliosa (Hook.) Kuntze, Revis. Gen. Pl. 2: 658. 1891.

Encyclia polystachya Poepp. \& Endl., Nov. Gen. Sp. Pl. 2: 10. 1836.

Polystachya estrellensis Rchb.f., Linnaea 25: 231. 1852.

Polystachya weigeltii Rchb.f., Linnaea 25: 230. 1852.

Polystachya nana Klotzsch, Index Seminum (B) 1853: 12. 1853.

Polystachya caracasana Rchb.f., Bonplandia (Hannover) 2: 15. 1854.

Polystachya abbreviata Rchb.f., Gard. Chron., n.s., 2: 291. 1874.

Dendrorkis caracasana (Rchb.f.) Kuntze, Revis. Gen. Pl. 2: 658. 1891.

Dendrorkis estrellensis (Rchb.f.) Kuntze, Revis. Gen. Pl. 2: 658. 1891.

Polystachya minor Fawc. \& Rendle, J. Bot. 48: 106. 1910.

Polystachya altilamellata Schltr., Repert. Spec. Nov. Regni Veg. 10: 385. 1912.

Polystachya ecuadorensis Schltr., Repert. Spec. Nov. Regni Veg. Beih. 8: 90. 1921.

Polystachya guatemalensis Schltr., Repert. Spec. Nov. Regni Veg. 16: 141. 1921.

Polystachya poeppigii Schltr., Repert. Spec. Nov. Regni Veg. Beih. 9: 155. 1921.

Polystachya panamensis Schltr., Repert. Spec. Nov. Regni Veg. Beih. 17: 49. 1922.
Polystachya costaricensis Schltr., Repert. Spec. Nov. Regni Veg. Beih. 19: 223. 1923.

Polystachya powellii Ames, Schedul. Orchid. 7: 31. 1924.

Polystachya amazonica Schltr., Beih. Bot. Centralbl. 42(2): 77. 1925.

Polystachya huebneri Schltr., Beih. Bot. Centralbl. 42(2): 11a. 1925.

Polystachya micrantha Schltr., Repert. Spec. Nov. Regni Veg. Beih. 35: 81. 1925.

Polystachya edwallii Hoehne \& Schltr., Arch. Bot. São Paulo 1: 256. 1926.

Polystachya bradei Schltr. ex Mansf., Repert. Spec. Nov. Regni Veg. 24: 245. 1928.

Polystachya rupicola Brade, Arch. Jard. Bot. Rio de Janeiro 6: 76. 1951.

Polystachya foliosa var. triandra Sauleda \& R.M.Adams, Brittonia 31: 294. 1979.

Polystachya fariae Campacci, Colet. Orquídeas Brasil. 8: 294. 2010.

VOucher: C.W. Powell 122 (AMES).

Polystachya masayensis Rchb.f., Bonplandia (Hannover) 3: 217. 1855.

Dendrorkis masasayensis (Rchb.f.) Kuntze, Revis. Gen. Pl. 2: 658. 1891.

VOUCHER: M.H. Nee 10733 (MO).

\section{Ponthieva R.Br}

Ponthieva brenesii Schltr., Repert. Spec. Nov. Regni Veg. Beih. 19: 165. 1923.

VOUCHER: J.P. Folsom et al. 5492 (MO).

Ponthieva formosa Schltr., Repert. Spec. Nov. Regni Veg. Beih. 19: 12. 1923.

VOUCHER: J.P. Folsom et al. 5769 (MO).

Ponthieva racemosa (Walter) C.Mohr, Contr. U. S. Natl. Herb. 6: 460. 1901.

Arethusa racemosa Walter, Fl. Carol.: 222. 1788.

Neottia pubera Steud., Nomencl. Bot., ed. 2, 2: 189. 1841., nom. superfl.

Ophrys pubera Michx., Fl. Bor.-Amer. 2: 158. 1803.

Neottia glandulosa Sims, Bot. Mag. 22: t. 842. 1805.

Epipactis pubescens Pursh, Fl. Amer. Sept. 2: 591. 1813.

Ponthieva glandulosa (Sims) R.Br. in W.T.Aiton, 
Hortus Kew. 5: 200. 1813.

Cranichis multiflora Elliott ex Nutt., Gen. N. Amer. P1. 2: 191. 1818.

Serapias pubescens (Pursh) Steud., Nomencl. Bot.: 767. 1821.

Listera pubescens (Pursh) Elliott, Sketch Bot. S. Carolina 2: 494. 1823.

Nerissa glandulosa (Sims) Raf., Fl. Tellur. 2: 89. 1837.

Ponthieva oblongifolia A.Rich. \& Galeotti, Ann. Sci. Nat., Bot., sér. 3, 3: 30. 1845.

Ponthieva lancifolia A.Rich. in R.de la Sagra, Hist. Fis. Cuba, Bot. 11: 251. 1850.

Ponthieva glandulosa var. macra Rchb.f., Linnaea 26: 142. 1854.

Ponthieva guatemalensis Rchb.f., Beitr. Orchid.-K. C. Amer.: 63. 1866.

Ponthieva orchioides Schltr., Repert. Spec. Nov. Regni Veg. 15: 50. 1917.

Ponthieva costaricensis Schltr., Repert. Spec. Nov. Regni Veg. Beih. 19: 84. 1923.

Voucher: Z. Serracin et al. 175 (UCH).

Ponthieva tuerckheimii Schltr., Repert. Spec. Nov. Regni Veg. 3: 47. 1906.

Ponthieva graciliscapa Schltr., Repert. Spec. Nov.

Regni Veg. Beih. 19: 166. 1923.

Voucher: B.E. Hammel 7525 (MO).

\section{Prescottia Lindl.}

Prescottia oligantha (Sw.) Lindl., Gen. Sp. Orchid. P1.: 454. 1840.

Cranichis oligantha Sw., Prodr. Veg. Ind. Occ.: 120. 1788.

Prescottia panamensis Schltr., Repert. Spec. Nov. Regni Veg. 16: 357. 1920.

VOUCHER: C. Galdames et al. 7305 (PMA).

Prescottia stachyodes (Sw.) Lindl., Edwards's Bot. Reg. 22: t. 1915. 1836.

Cranichis stachyodes Sw., Prodr. Veg. Ind. Occ.: 120. 1788.

Prescottia colorans Lindl., Edwards's Bot. Reg. 22: t. 1915. 1836.

Prescottia galeottii Rchb.f., Linnaea 19: 377. 1846.

Prescottia gigantea Lodd. ex W.H.Baxter in J.C.Loudon, Suppl. Hort. Brit.: 618. 1850., nom. nud.
Prescottia longipetiolata Barb.Rodr., Gen. Spec. Orchid. 1: 177. 1877.

Prescottia paulensis Cogn. in C.F.P.von Martius \& auct. suc. (eds.), Fl. Bras. 3(6): 548. 1906.

Prescottia longifolia Schltr., Repert. Spec. Nov. Regni Veg. Beih. 7: 51. 1920.

Prescottia smithii Schltr., Repert. Spec. Nov. Regni Veg. Beih. 7: 52. 1920.

Prescottia colorans var. macrophylla Hoehne, Revista Soc. Brasil. Agron. 8(2): 222. 1945.

Voucher: R. Méndez 148 (PMA).

Prosthechea Knowles \& Westc.

Prosthechea abbreviata (Schltr.) W.E.Higgins, Phytologia 82: 376.1997 [1998].

Epidendrum abbreviatum Schltr., Repert. Spec. Nov. Regni Veg. 3: 107. 1906.

Encyclia abbreviata (Schltr.) Dressler, Brittonia 13: 264.1961

Anacheilium abbreviatum (Schltr.) Withner \& P.A.Harding, Cattleyas \& Relatives: Debatable Epidendrums: 40. 2004.

Epidendrum prorepens Ames, Schedul. Orchid. 2: 33. 1923.

Voucher: R.L. Dressler \& N.H. Williams 3998 (PMA). Prosthechea aemula (Lindl.) W.E.Higgins, Phytologia 82: 376.1997 [1998].

Epidendrum aemulum Lindl., Edwards's Bot. Reg. 22: t. 1898.1836.

Epidendrum fragrans var. aemulum (Lindl.) Barb. Rodr., Gen. Spec. Orchid. 2: 136. 1881.

Encyclia fragrans subsp. aemula (Lindl.) Dressler, Phytologia 21: 440. 1971.

Encyclia aemula (Lindl.) Carnevali \& I.Ramírez, Monogr. Syst. Bot. Missouri Bot. Gard. 45: 1257. 1993.

Anacheilium aemulum (Lindl.) Withner \& P.A.Harding, Cattleyas \& Relatives: Debatable Epidendrums: 42. 2004.

Epidendrum cordatum Vell., Fl. Flumin. 9: t. 38. 1831., nom. illeg.

Epidendrum cordifolium Steud., Nomencl. Bot., ed. 2, 1: 557. 1840.

Epidendrum aemulum var. brevistriatum Rchb.f., Linnaea 41: 37. 1876.

Epidendrum fragrans var. alticallum Barb.Rodr., 
Gen. Spec. Orchid. 2: 138. 1882.

Epidendrum fragrans var. janeirense Barb.Rodr.,

Gen. Spec. Orchid. 2: 137. 1882.

Epidendrum fragrans var. micranthum Barb.Rodr.,

Gen. Spec. Orchid. 2: 138. 1882.

Epidendrum fragrans var. rivularium Barb.Rodr.,

Gen. Spec. Orchid. 2: 137. 1882.

Epidendrum fragrans var. brevistriatum (Rchb.f.)

Cogn. in C.F.P.von Martius \& auct. suc. (eds.),

Fl. Bras. 3(5): 85. 1898.

Voucher: H. Pittier 3496 (US).

Prosthechea baculus (Rchb.f.) W.E.Higgins, Phytologia 82: 376. 1997 [1998].

Epidendrum baculus Rchb.f., Bonplandia (Hannover) 4: 214. 1856.

Encyclia baculus (Rchb.f.) Dressler \& G.E.Pollard, Phytologia 21: 436. 1971.

Anacheilium baculus (Rchb.f.) Withner \& P.A.Harding, Cattleyas \& Relatives: Debatable Epidendrums: 49. 2004.

Epidendrum fragrans var. megalanthum Lindl., J.

Hort. Soc. London 4: 223. 1849.

Epidendrum pentotis Rchb.f., Linnaea 41: 81. 1876.

Epidendrum acuminatum Sessé \& Moc., Fl. Mexic., ed. 2: 202. 1894., nom. illeg.

Epidendrum confusum Rolfe, Orchid Rev. 7: 197. 1899.

Epidendrum beyrodtianum Schltr., Orchis 9: 49. 1915.

Encyclia pentotis (Rchb.f.) Dressler, Brittonia 13: 265. 1961.

Anacheilium confusum (Rolfe) Withner \& P.A.Harding, Cattleyas \& Relatives: Debatable Epidendrums: 72. 2004.

Prosthechea confusa (Rolfe) W.E.Higgins, Selbyana 29: 214. 2009.

VOUCHER: not seen, cited by Dressler (2003c).

Prosthechea brassavolae (Rchb.f.) W.E.Higgins, Phytologia 82: 376. 1997 [1998].

Epidendrum brassavolae Rchb.f., Bot. Zeitung (Berlin) 10: 729. 1852.

Encyclia brassavolae (Rchb.f.) Dressler, Brittonia 13: 264. 1961.

Pseudencyclia brassavolae (Rchb.f.) V.P.Castro \& Chiron, Richardiana 4: 32. 2003.

Panarica brassavolae (Rchb.f.) Withner \&
P.A.Harding, Cattleyas \& Relatives: Debatable Epidendrums: 208. 2004.

Voucher: J. Warszewicz s.n. (W).

Prosthechea campylostalix (Rchb.f.) W.E.Higgins, Phytologia 82: 377.1997 [1998].

Epidendrum campylostalix Rchb.f., Bot. Zeitung (Berlin) 10: 730. 1852.

Encyclia campylostalix (Rchb.f.) Schltr., Repert. Spec. Nov. Regni Veg. Beih. 17: 45. 1922.

Pseudencyclia campylostalix (Rchb.f.) V.P.Castro \& Chiron, Richardiana 4: 32. 2003.

Pollardia campylostalix (Rchb.f.) Withner \& P.A.Harding, Cattleyas \& Relatives: Debatable Epidendrums: 220. 2004.

Voucher: J. Warszewicz s.n. (W).

Prosthechea chacaoensis (Rchb.f.) W.E.Higgins, Phytologia 82: 377. 1997 [1998].

Epidendrum chacaoense Rchb.f., Bonplandia (Hannover) 2: 20. 1854.

Encyclia chacaoensis (Rchb.f.) Dressler \& G.E.Pollard, Phytologia 21: 436. 1971.

Anacheilium chacaoense (Rchb.f.) Withner \& P.A.Harding, Cattleyas \& Relatives: Debatable Epidendrums: 63. 2004.

Epidendrum pachycarpum Schltr., Repert. Spec. Nov. Regni Veg. 3: 109. 1906.

VOUCHer: C.W. Powell 187 (MO).

Prosthechea chimborazoensis (Schltr.) W.E.Higgins, Phytologia 82: 377.1997 [1998].

Epidendrum chimborazoense Schltr., Repert. Spec.

Nov. Regni Veg. 14: 389. 1916.

Encyclia chimborazoensis (Schltr.) Dressler, Phytologia 21: 440. 1971.

Anacheilium chimborazoense (Schltr.) Withner \&

P.A.Harding, Cattleyas \& Relatives: Debatable Epidendrums: 65. 2004.

Voucher: G. McPherson 7965 (MO).

Prosthechea cochleata (L.) W.E.Higgins, Phytologia 82: 377.1997 [1998].

Epidendrum cochleatum L., Sp. Pl. ed. 2: 1351. 1763. Anacheilium cochleatum (L.) Hoffmanns., Linnaea 16(Litt.): 229. 1842.

Encyclia cochleata (L.) Dressler, Brittonia 13: 264. 1961.

Epidendrum cochleatum var. grandiflorum Mutel, Mém. Soc. Roy. Centr. Agric. Dépt. N. 1838: 86. 1838. 
Prosthechea cochleata var. grandiflora (Mutel)

Christenson, Richardiana 8: 30. 2008.

Voucher: S. Laube 184 (PMA).

Prosthechea crassilabia (Poepp. \& Endl.) Carnevali \& I.Ramírez in J.A.Steyermark \& al. (eds.), Fl. Venezuelan Guayana 7: 538. 2003.

Epidendrum crassilabium Poepp. \& Endl., Nov. Gen. Sp. P1. 2: 1. 1836.

Epidendrum variegatum var. crassilabium (Poepp.

\& Endl.) Lindl., Fol. Orchid. 3: 38. 1853.

Encyclia crassilabia (Poepp. \& Endl.) Dressler,

Brittonia 13: 264. 1961.

Anacheilium crassilabium (Poepp. \& Endl.)

Withner, P.A.Harding \& Campacci, Cattleyas

\& Relatives: Debatable Epidendrums: 74. 2004.

Epidendrum variegatum Hook., Bot. Mag. 59: t.

3151. 1832., nom. illeg.

Epidendrum coriaceum C.Parker ex Hook., Bot.

Mag. 64: t. 3595. 1837., nom. illeg.

Epidendrum coriaceum Focke, Bot. Zeitung

(Berlin) 11: 228. 1853., nom. illeg.

Epidendrum variegatum var. coriaceum Lindl.,

Fol. Orchid. 3: 38. 1853.

Epidendrum variegatum var. leopardinum Lindl.,

Fol. Orchid. 3: 38. 1853.

Epidendrum variegatum var. virens Lindl., Fol.

Orchid. 3: 38. 1853.

Epidendrum pachysepalum Klotzsch, Allg. Gartenzeitung 23: 274. 1855.

Epidendrum variegatum var. lineatum Rchb.f., Bonplandia (Hannover) 4: 326. 1856.

Epidendrum christii Rchb.f., Linnaea 41: 112. 1876.

Epidendrum leopardinum Rchb.f., Linnaea 41: 112. 1876.

Epidendrum longipes Rchb.f., Otia Bot. Hamburg.: 10. 1881.

Epidendrum feddeanum Kraenzl., Repert. Spec.

Nov. Regni Veg. 1: 188. 1905.

Epidendrum saccharatum Kraenzl., Orchis 18: 113. 1908.

Epidendrum rhabdobulbon Schltr., Repert. Spec.

Nov. Regni Veg. Beih. 7: 146. 1920.

Epidendrum baculibulbum Schltr., Repert. Spec.

Nov. Regni Veg. Beih. 19: 116. 1923.

Epidendrum rhopalobulbon Schltr., Repert. Spec.

Nov. Regni Veg. Beih. 27: 72. 1924.
Auliza wilsonii Acuña, Bol. Estaçión Exp. Agron. Santiago de las Vegas 60: 85. 1939.

Epidendrum variegatum var. angustipetalum Hoehne, Arq. Bot. Estado São Paulo, n.s., 2: 82. 1947.

Hormidium baculibulbum (Schltr.) Brieger, Proc. World Orchid Conf. 3: 315. 1960.

Hormidium coriaceum (Lindl.) Brieger, Publ. Ci. Inst. Genét. Esc. Super. Agric. Luis de Queiroz 1: 20.1960.

Hormidium variegatum Brieger, Publ. Ci. Inst. Genét. Esc. Super. Agric. Luis de Queiroz 1: 20. 1960.

Hormidium virens (Lindl.) Brieger, Proc. World Orchid Conf. 3: 315. 1960.

Encyclia vespa subsp. triandra Dod, Orquídea (Mexico City), n.s., 13: 215. 1993.

Encyclia christii (Rchb.f.) Dodson, Orquideología 19: 149. 1994.

Encyclia leopardina (Rchb.f.) Dodson \& Hágsater, Orquideología 19: 149. 1994.

Prosthechea christii (Rchb.f.) Dodson \& Hágsater, Monogr. Syst. Bot. Missouri Bot. Gard. 75: 956. 1999.

Prosthechea leopardina (Rchb.f.) Dodson \& Hágsater, Monogr. Syst. Bot. Missouri Bot. Gard. 75: 956. 1999.

Prosthechea vespa subsp. triandra (Dod) Nir, Orchid. Antill.: 407. 2000.

Prosthechea pachysepala (Klotzsch) Chiron \& V.P.Castro, Richardiana 3: 174. 2003.

Prosthechea longipes (Rchb.f.) Chiron, Richardiana 5: 148. 2005.

Prosthechea vespa subsp. duartiana Chiron \& V.P.Castro, Richardiana 5: 91. 2005.

VOUCHER: R. Blas et al. 660 (UCH).

Prosthechea fortunae (Dressler) W.E.Higgins, Phytologia 82: 377.1997 [1998].

Encyclia fortunae Dressler, Orquídea (Mexico City), n.s., 7: 355. 1980.

Voucher: R.L. Dressler 5520 (US).

Prosthechea fragrans (Sw.) W.E.Higgins, Phytologia 82: 377.1997 [1998].

Epidendrum fragrans Sw., Prodr. Veg. Ind. Occ.: 123. 1788.

Anacheilium fragrans (Sw.) Acuña, Bol. Estaçión Exp. Agron. Santiago de las Vegas 60: 86. 1939. 
Encyclia fragrans (Sw.) Dressler, Brittonia 13: 264. 1961.

Epidendrum cochleatum Curtis, Bot. Mag. 5: t. 152. 1791., sensu auct.

Epidendrum lineatum Salisb., Prodr. Stirp. Chap. Allerton: 10. 1796.

Epidendrum ionoleucum Hoffmanns. ex Rchb.f., Linnaea 24: 244. 1851.

Epidendrum fragrans var. ionoleucum (Hoffmanns. ex Rchb.f.) Barb.Rodr., Gen. Spec. Orchid. 2: 136. 1881.

Epidendrum fragrans var. magnum Stein, Orchid.Buch: 230. 1891.

Epidendrum vaginatum Sessé \& Moc., Fl. Mexic., ed. 2: 201. 1894.

Epidendrum fragrans var. pachypus Schltr., Repert. Spec. Nov. Regni Veg. Beih. 17: 32. 1922.

VOUCHER: C.W. Powell 208 (MO).

Prosthechea ionocentra (Rchb.f.) W.E.Higgins, Phytologia 82: 378. 1997 [1998].

Epidendrum ionocentrum Rchb.f., Gard. Chron., n.s., 20: 8. 1883.

Epidendrum prismatocarpum var. ionocentrum (Rchb.f.) Teusch., Amer. Orchid Soc. Bull. 38: 398. 1969.

Encyclia ionocentra (Rchb.f.) Mora-Ret. \& García Castro, Brenesia 33: 124. 1990.

Pseudencyclia ionocentra (Rchb.f.) V.P.Castro \& Chiron, Richardiana 4: 32. 2003.

Panarica ionocentra (Rchb.f.) Withner \& P.A.Harding, Cattleyas \& Relatives: Debatable Epidendrums: 211. 2004.

Encyclia prismatocarpa var. ionocentra (Rchb.f.) M.Wolff \& O.Gruss, Orchid. Atlas: 146. 2007.

Epidendrum auriculigerum Rchb.f., Gard. Chron., ser. 3, 4: 34. 1888.

VOucher: T.B. Croat 26804 (MO).

Prosthechea ionophlebia (Rchb.f.) W.E.Higgins, Phytologia 82: 378. 1997 [1998].

Epidendrum ionophlebium Rchb.f., Beitr. Orchid.-K. C. Amer.: 103. 1866.

Encyclia ionophlebia (Rchb.f.) Dressler, Brittonia 13: 265.1961.

Anacheilium ionophlebium (Rchb.f.) Withner \& P.A.Harding, Cattleyas \& Relatives: Debatable Epidendrums: 93. 2004.

Epidendrum hoffmannii Schltr., Repert. Spec. Nov.
Regni Veg. 16: 444. 1920.

Voucher: I.O. Gordon 439 (MO).

Prosthechea livida (Lindl.) W.E.Higgins, Phytologia 82: 379. 1997[1998].

Epidendrum lividum Lindl., Edwards's Bot. Reg. 24(Misc.): 51. 1838.

Encyclia livida (Lind1.) Dressler, Brittonia 13: 265. 1961.

Anacheilium lividum (Lindl.) Pabst, Moutinho \& A.V.Pinto, Bradea 3: 184. 1981.

Pseudencyclia livida (Lindl.) V.P.Castro \& Chiron, Richardiana 4: 33. 2003.

Pollardia livida (Lindl.) Withner \& P.A.Harding, Cattleyas \& Relatives: Debatable Epidendrums: 231. 2004.

Epidendrum articulatum Klotzsch, Allg. Gartenzeitung 6: 297. 1838., nom. illeg.

Epidendrum tessellatum Bateman ex Lindl., Edwards's Bot. Reg. 24(Misc.): 7. 1838., nom. illeg.

Epidendrum chondrochilum F.Lehm. \& Kraenzl., Bot. Jahrb. Syst. 26: 467. 1899.

Epidendrum condylochilum F.Lehm. \& Kraenzl., Bot. Jahrb. Syst. 26: 459. 1899.

Epidendrum henrici Schltr., Repert. Spec. Nov. Regni Veg. 3: 108. 1906.

Encyclia tessellata Schltr., Beih. Bot. Centralbl. 36(2): 474. 1918.

Epidendrum deamii Schltr., Beih. Bot. Centralbl. 36(2): 402.1918.

Epidendrum dasytainia Schltr., Repert. Spec. Nov. Regni Veg. Beih. 8: 71. 1921.

Encyclia deamii (Schltr.) Hoehne, Arq. Bot. Estado São Paulo, n.s., f.m., 2: 151. 1952.

VOUCHER: D. Cáceres et al. 642 (UCH).

Prosthechea prismatocarpa (Rchb.f.) W.E.Higgins, Phytologia 82: 380.1997 [1998].

Epidendrum prismatocarpum Rchb.f., Bot. Zeitung (Berlin) 10: 729. 1852.

Encyclia prismatocarpa (Rchb.f.) Dressler, Brittonia 13: 265. 1961.

Pseudencyclia prismatocarpa (Rchb.f.) V.P.Castro \& Chiron, Richardiana 4: 33. 2003.

Panarica prismatocarpa (Rchb.f.) Withner \& P.A.Harding, Cattleyas \& Relatives: Debatable Epidendrums: 213. 2004.

VOUCHER: J. Warszewicz s.n. (W). 
Prosthechea pseudopygmaea (Finet) W.E.Higgins, Phytologia 82: 380. 1997 [1998].

Hormidium pseudopygmaeum Finet, Bull. Herb. Boissier 7: 121. 1899.

Encyclia pseudopygmaea (Finet) Dressler \& G.E.Pollard, Orquídea (Mexico City), n.s., 3: 313. 1974.

Epidendrum pseudopygmaeum (Finet) Hamer \& Garay in F.Hamer, Orquid. El Salvador 1: 242. 1974.

VOUCHER: R.L. Dressler 5405 (PMA).

Prosthechea pygmaea (Hook.) W.E.Higgins, Phytologia 82: 380. 1997 [1998].

Epidendrum pygmaeum Hook., J. Bot. (Hooker) 1: 49. 1834.

Hormidium pygmaeum (Hook.) Benth. \& Hook.f. ex Hemsl., Biol. Cent.-Amer., Bot. 3: 218. 1884.

Encyclia pygmaea (Hook.) Dressler, Brittonia 13: 265. 1961.

Anacheilium pygmaeum (Hook.) Baptista, Bol. CAOB 58: 49. 2005.

Coelogyne triptera Brongn. in L.I.Duperrey, Voy. Monde: 201. 1834.

Epidendrum caespitosum Poepp. \& Endl., Nov. Gen. Sp. P1. 2: 1. 1836., nom. illeg.

Epidendrum uniflorum Lindl., Edwards's Bot. Reg. 25(Misc.): 15. 1839., nom. illeg.

Epidendrum monanthum Steud., Nomencl. Bot., ed. 2, 1: 558. 1840.

Hormidium uniflorum Heynh., Nom. Bot. Hort.: 880. 1841., nom. superfl.

Hormidium tripterum (Brongn.) Cogn. in C.F.P.von Martius \& auct. suc. (eds.), Fl. Bras. 3(5): 29. 1898.

Microstylis humilis Cogn. in C.F.P.von Martius \& auct. suc. (eds.), Fl. Bras. 3(6): 550. 1906.

Hormidium humile (Cogn.) Schltr., Repert. Spec. Nov. Regni Veg. 16: 331. 1920.

Hormidium hioramii Acuña \& Roíg, Mem. Soc. Cub. Hist. Nat. ''Felipe Poey" 10: 51. 1936.

Epidendrum hioramii (Acuña \& Ró́g) Acuña \& Alain, Mem. Soc. Cub. Hist. Nat. 'Felipe Poey" 24: 110. 1960.

Encyclia triptera (Brongn.) Dressler \& G.E.Pollard, Phytologia 21: 438. 1971.

Lanium hioramii (Acuña \& Roíg) H.Dietr., Wiss.
Z. Friedrich-Schiller-Univ. Jena, Math.Naturwiss. Reihe 29: 524 (1980).

VOUCHER: B.E. Hammel 6949 (MO).

Prosthechea racemifera (Dressler) W.E.Higgins, Phytologia 82: 380. 1997 [1998].

Encyclia racemifera Dressler, Lindleyana 11: 37. 1996.

Hormidium racemiferum (Dressler) Withner \& P.A.Harding, Cattleyas \& Relatives: Debatable Epidendrums: 157. 2004.

VOucher: Carl A. Luer \& Henry P. Butcher 1171 (SEL).

Prosthechea sima (Dressler) W.E.Higgins, Phytologia 82: 381.1997 [1998].

Encyclia sima Dressler, Orquideología 4: 91.1969.

Epidendrum simum (Dressler) P.Taylor, Bot. Mag. 182: 39. 1978.

Anacheilium simum (Dressler) Withner \& P.A.Harding, Cattleyas \& Relatives: Debatable Epidendrums: 116. 2004.

Voucher: R.L. Dressler 3160 (US).

Prosthechea spondiada (Rchb.f.) W.E.Higgins, Phytologia 82: 381.1997 [1998].

Epidendrum spondiadum Rchb.f., Bot. Zeitung (Berlin) 10: 731. 1852.

Encyclia spondiada (Rchb.f.) Dressler, Phytologia 21: 441. 1971.

Anacheilium spondiadum (Rchb.f.) Withner \& P.A.Harding, Cattleyas \& Relatives: Debatable Epidendrums: 118. 2004.

Epidendrum platycardium Schltr., Repert. Spec.

Nov. Regni Veg. Beih. 17: 36. 1922.

Voucher: C.W. Powell 131 (B, destroyed; AMES).

Prosthechea varicosa (Bateman ex Lindl.) W.E.Higgins, Phytologia 82: 381.1997 [1998].

Prosthechea varicosa (Bateman ex Lindl.) W.E.Higgins, Phytologia 82: 381. 1997.

Epidendrum varicosum Bateman ex Lindl., Edwards's Bot. Reg. 24(Misc.): 30. 1838.

Encyclia varicosa (Bateman ex Lindl.) Schltr., Beih. Bot. Centralbl. 36(2): 474. 1918.

Pseudencyclia varicosa (Bateman ex Lindl.) V.P.Castro \& Chiron, Richardiana 4: 33. 2003.

Pollardia varicosa (Bateman ex Lindl.) Withner \& P.A.Harding, Cattleyas \& Relatives: Debatable Epidendrums: 245. 2004.

Epidendrum leiobolbon Hook., J. Bot. (Hooker) 3: 308. 1841. 
Epidendrum quadratum Klotzsch, Allg. Gartenzeitung 18: 402. 1850.

Epidendrum chiriquense Rchb.f., Bot. Zeitung (Berlin) 10: 730. 1852.

Epidendrum phymatoglossum Rchb.f., Bot. Zeitung (Berlin) 10: 731. 1852.

Epidendrum lunaeanum A.Rich. ex Lindl., Fol. Orchid. 3: 23. 1853.

Encyclia chiriquensis (Rchb.f.) Schltr., Beih. Bot. Centralbl. 36(2): 472. 1918.

Encyclia phymatoglossa (Rchb.f.) Schltr., Beih. Bot. Centralbl. 36(2): 473. 1918.

Epidendrum ramirezii Gojon Sánchez, Mejores Orquideas Mexico: 46. 1930.

Encyclia varicosa subsp. leiobolbon (Hook.) Dressler \& G.E.Pollard, Phytologia 21: 438. 1971.

Encyclia varicosa var. leiobolbon (Hook.) M.Wolff

\& O.Gruss, Orchid. Atlas: 148. 2007.

VOUCHER: J. Warscewicz s.n. (W).

\section{Pseudocentrum Lindl.}

Pseudocentrum hoffmannii (Rchb.f.) Rchb.f., Linnaea 41: 53. 1876.

Pelexia hoffmannii Rchb.f., Beitr. Orchid.-K. C. Amer.: 102. 1866.

Voucher: B.E. Hammel 5777 (MO).

Psilochilus Barb.Rodr.

Psilochilus dressleri Kolan., Phytotaxa 175 (1): 5557. 2014.

VOUCHER: R.L. Dressler 5663 (FLAS).

Psilochilus macrophyllus (Lindl.) Ames, Orchidaceae 7: 45.1922.

Pogonia macrophylla Lindl., Ann. Mag. Nat. Hist., ser. 3, 1: 335. 1858.

VOUCHER: R.L. Dressler 2959 (PMA).

Psilochilus physurifolius (Rchb.f.) Løjtnant, Bot. Not. 130: 168. 1977.

Pogonia physurifolia Rchb.f., Ned. Kruidk. Arch. 4: 324. 1859.

VOUCHER: J.P. Folsom et al. 5409 (MO).

\section{Psychopsis Raf.}

Psychopsis krameriana (Rchb.f.) H.G.Jones, Novosti Sist. Vyssh. Rast. 12: 141. 1975.
Oncidium kramerianum Rchb.f., Allg. Gartenzeitung 23: 9. 1855.

Oncidium krameri J.Muir, Garden (London 18711927) 6: 384. 1874., orth. var.

Oncidium nodosum E.Morren, Ann. Hort. Belge Étrangère 24: 258. 1874.

Papiliopsis nodosus E.Morren ex Cogn. \& Marchal, P1. Feuill. Ornem. 2: t. 55. 1874.

Oncidium papilioniforme Regel, Trudy Imp. S.Peterburgsk. Bot. Sada 6: 292. 1879.

Oncidium kramerianum var. resplendens Rchb.f., Gard. Chron., ser. 3, 3: 360. 1888.

Voucher: not seen, cited by Williams \& Allen (1980) and Dressler (2003c).

Williams \& Allen (1980) cited this species from cultivated plants likely collected in the area of Laguna de Chiriquí; however, we have not yet found a herbarium oucher from Panama. Recent collections in the GandocaManzanillo area in Costa Rica, near the border with Panama, suggest the presence of this species at least in the vicinity of Almirante and Bocas del Toro. It is frequently found in cultivation in Panama.

\section{Pterichis Lindl.}

Pterichis habenarioides (F.Lehm. \& Kraenzl.) Schltr., Repert. Spec. Nov. Regni Veg. Beih. 7: 214. 1920.

Goodyera habenarioides F.Lehm. \& Kraenzl., Bot. Jahrb. Syst. 26: 499. 1899.

Pterichis costaricensis Ames \& C.Schweinf., Schedul. Orchid. 10: 10. 1930.

Voucher: G. Davidse et al. 23852 (MO).

\section{Restrepia Kunth.}

Restrepia aberrans Luer, Orquideología 20: 117. 1996. VOUCHer: C.A. Luer 10612 (MO).

Restrepia muscifera (Lindl.) Rchb.f. ex Lindl., Fol. Orchid. 8: 7. 1859.

Pleurothallis muscifera Lindl., Edwards's Bot. Reg. 28(Misc.): 79. 1842.

Restrepia lansbergii Hook., Bot. Mag. 87: t. 5257.

1861., nom. illeg.

Restrepia xanthophthalma Rchb.f., Hamburger Garten- Blumenzeitung 21: 300. 1865.

Restrepia dayana Rchb.f., Gard. Chron., n.s., 4: 257. 1875.

Restrepia shuttleworthii Rolfe, Bull. Misc. Inform. Kew 1892: 138. 1892. 
Restrepia powellii Schltr., Repert. Spec. Nov. Regni Veg. Beih. 17: 25. 1922.

Restrepia tonduzii Schltr., Repert. Spec. Nov. Regni Veg. Beih. 19: 291. 1923.

Pleurothallis dayana (Rchb.f.) L.O.Williams, Bot. Mus. Leafl. 8: 144. 1940.

Pleurothallis xanthophthalma (Rchb.f.) L.O.Williams, Bot. Mus. Leafl. 8: 144. 1940.

Restrepia muscifera subsp. shuttleworthii (Rolfe) H.Mohr, Leafl. Schlechter Inst. 2: 8. 1996.

Restrepia muscifera f. dayana (Rchb.f.) O.Gruss \& M.Wolff, Orchid. Atlas: 387. 2007.

VOUCHER: C.W. Powell 123 (MO).

Restrepia trichoglossa F.Lehm. ex Sander, Sander's Orch. Guide: 215. 1901.

Restrepia leontoglossa Schltr., Repert. Spec. Nov. Regni Veg. Beih. 7: 120. 1920.

Restrepia serrilabia Schltr., Repert. Spec. Nov. Regni Veg. Beih. 7: 121. 1920.

Restrepia angustilabia Schltr., Repert. Spec. Nov. Regni Veg. Beih. 19: 290. 1923.

Restrepia subserrata Schltr., Repert. Spec. Nov.

Regni Veg. Beih. 19: 291. 1923.

Restrepia filamentosa Ames \& C.Schweinf., Schedul. Orchid. 8: 19. 1925.

Restrepia lankesteri Ames \& C.Schweinf., Schedul. Orchid. 10: 20. 1930.

Pleurothallis amesiana L.O.Williams, Bot. Mus.

Leafl. 8: 143. 1940.

Pleurothallis filamentosa (Ames \& C.Schweinf.)

L.O.Williams, Bot. Mus. Leafl. 8: 144. 1940.

Pleurothallis subserrata (Schltr.) L.O.Williams,

Bot. Mus. Leafl. 8: 144. 1940.

Restrepia angustilabia subsp. subserrata (Schltr.)

H.Mohr, Leafl. Schlechter Inst. 2: 14. 1996.

Restrepia antennifera subsp. leontoglossa (Schltr.)

H.Mohr, Leafl. Schlechter Inst. 2: 13. 1996.

Restrepia brachypus subsp. serrilabia (Schltr.)

H.Mohr, Leafl. Schlechter Inst. 2: 14. 1996.

VOUCHER: C.W. Powell 344 (AMES).

\section{RheTinantha M.A.Blanco}

Rhetinantha aciantha (Rchb.f.) M.A.Blanco, Lankesteriana 7: 534. 2007.

Maxillaria aciantha Rchb.f., Bot. Zeitung (Berlin) 10: 858. 1852.
Lycaste aciantha Rchb.f., Bonplandia (Hannover) 3: 216.1855.

Voucher: T.B. Croat \& D.M. Porter 15651 (MO).

Rhetinantha friedrichsthalii (Rchb.f.) M.A.Blanco, Lankesteriana 7: 534. 2007.

Maxillaria friedrichsthalii Rchb.f., Bot. Zeitung (Berlin) 10: 858. 1852.

Maxillaria turialbae Schltr., Beih. Bot. Centralbl. 36(2): 414. 1918.

Voucher: C.W. Powell 136 (MO).

Rhetinantha scorpioidea (Kraenzl.) M.A.Blanco, Lankesteriana 7: 535. 2007.

Maxillaria scorpioidea Kraenzl., Kongl. Svenska Vetensk. Acad. Handl., n.s., 46(10): 71. 1911.

Camaridium scorpioideum (Kraenzl.) Hoehne, Arq. Bot. Estado São Paulo, n.s., f.m., 2: 72. 1947.

Maxillaria rhodosticta Kraenzl., Repert. Spec. Nov. Regni Veg. 24: 223. 1928.

VOUCHER: R.L. Dressler 4874 (PMA).

\section{RHYNChOSTELE Rchb.f.}

Rhynchostele bictoniensis (Bateman) Soto Arenas \& Salazar, Orquídea (Mexico City), n.s., 13: 147. 1993.

Cyrtochilum bictoniense Bateman, Orchid. Mexico Guatemala: t. 6. 1838 .

Odontoglossum bictoniense (Bateman) Lindl., Edwards's Bot. Reg. 26: t. 66. 1840.

Lemboglossum bictoniense (Bateman) Halb. ex Soto Arenas, Orquídea (Mexico City), n.s., 11: 271. 1988.

Amparoa bictoniensis (Bateman) Archila, Revista Guatemal. 12(2): 12. 2009.

Zygopetalum africanum Hook., Bot. Mag. 67: t. 3812. 1840.

Odontoglossum bictoniense var. splendens Lem., Ill. Hort. 12: t. 449. 1865.

VOUCHER: D. Bogarín et al. 10363 (UCH).

RoDriguezia Ruiz \& Pav.

Rodriguezia compacta Schltr., Repert. Spec. Nov. Regni Veg. Beih. 19: 144. 1923.

VOUCHER: S. Laube et al. 136 (PMA).

Rodriguezia lanceolata Ruiz \& Pav., Syst. Veg. Fl. Peruv. Chil.: 219. 1798.

Rodriguezia secunda Kunth in F.W.H.von 
Humboldt, A.J.A.Bonpland \& C.S.Kunth, Nov. Gen. Sp. 1: 367. 1816.

Pleurothallis coccinea Hook., Exot. Fl. 2: t. 129. 1824.

Burlingtonia rosea Rand, J. Orchidées 5: 14. 1894.

Rodriguezia secunda var. panamensis Schltr.,

Repert. Spec. Nov. Regni Veg. Beih. 17: 75. 1922.

VOUCHER: C.W. Powell 79 (MO).

Rossioglossum (Schltr.) Garay \& G.C.Kenn.

Rossioglossum ampliatum (Lind1.) M.W.Chase \& N.H.Williams, Lindleyana 21(3): 31. 2008.

Oncidium ampliatum Lindl., Gen. Sp. Orchid. Pl.: 202. 1833.

Chelyorchis ampliata (Lindl.) Dressler \& N.H.Williams in G.A.Romero \& G.Carnevali, Orchids Venezuela, ed. 2: 1130. 2000.

Oncidium gyrobulbon Rchb.f., Gard. Chron. 1869: 838. 1869.

Oncidium ampliatum var. majus Van Houtte, Fl. Serres 20: 147. 1874.

Oncidium bernoullianum Kraenzl. in H.G.A.Engler (ed.), Pflanzenr., IV, 50(80): 231. 1922.

Voucher: H. Cuming 1312 (K).

Rossioglossum krameri (Rchb.f.) M.W.Chase \& N.H.Williams, Lindleyana 21(3): 31. 2008.

Odontoglossum krameri Rchb.f., Gard. Chron. 1868: 98. 1868.

Ticoglossum krameri (Rchb.f.) Halb., Orquídea (Mexico City), n.s., 9: 5. 1983.

Odontoglossum krameri var. smithianum Rchb.f., Gard. Chron., n.s., 19: 242. 1883.

Odontoglossum krameri var. album Rolfe, Orchid Rev. 1: 200. 1893.

Odontoglossum krameri var. album Lucas Rodr. ex Halb., Orquídea (Mexico City), n.s., 8: 186. 1982, nom. illeg.

Ticoglossum krameri var. album (Lucas Rodr. ex Halb.) Halb., Orquídea (Mexico City), n.s., 9: 5. 1983.

Ticoglossum krameri var. smithianum (Rchb.f.) Christenson, Lindleyana 6: 47. 1991.

Ticoglossum krameri f. album (Lucas Rodr. ex Halb.) M.Wolff \& O.Gruss, Orchid. Atlas: 419. 2007.

Voucher: R.L. Dressler s.n. (MO).
Rossioglossum oerstedii (Rchb.f.) M.W.Chase \& N.H.Williams, Lindleyana 21(3): 31. 2008.

Odontoglossum oerstedii Rchb.f., Bonplandia (Hannover) 3: 214. 1855.

Ticoglossum oerstedii (Rchb.f.) Halb., Orquídea (Mexico City), n.s., 9: 5. 1983.

Odontoglossum oerstedii var. majus B.S.Williams, Orchid Album 8: t. 376. 1889.

Voucher: W. D'Arcy et al. 13221 (PMA).

Rossioglossum schlieperianum (Rchb.f.) Garay \& G.C.Kenn., Orchid Digest 40: 143. 1976.

Odontoglossum schlieperianum Rchb.f., Gard. Chron. 1865: 1082. 1865.

Odontoglossum grande-pallidum Klotzsch ex Rchb.f., Gard. Chron. 1865: 1082. 1865.

Odontoglossum schlieperianum var. flavidum

Rchb.f., Gard. Chron., n.s., 20: 135. 1883.

Odontoglossum powellii Schltr., Repert. Spec. Nov. Regni Veg. Beih. 17: 78. 1922.

Rossioglossum powellii (Schltr.) Garay \& G.C.Kenn., Orchid Digest 40: 142. 1976.

Rossioglossum schlieperianum var. flavidum (Rchb.f.) Garay \& G.C.Kenn., Orchid Digest 40: 143.1976.

Rossioglossum schlieperianum f. flavidum (Rchb.f.) Pupulin, Lankesteriana 4: 66. 2001.

Voucher: M.E. Davidson 797 (F).

\section{Rudolfiella Hoehne}

Rudolfiella picta (Schltr.) Hoehne, Fl. Brasilica 12(7): 51. 1953.

Lindleyella picta Schltr., Repert. Spec. Nov. Regni Veg. Beih. 27: 173. 1924.

Bifrenaria picta (Schltr.) C.Schweinf., Bot. Mus. Leafl. 11(8): 246. 1944.

Schlechterella picta (Schltr.) Hoehne, Arq. Bot. Estado São Paulo 2: 14. 1944.

Rudolfiella picta (Schltr.) L.O.Williams, Ceiba 5(3): 188. 1956., nom. illeg. hom.

VOUCHER: C.W. Powell 75 (K).

\section{SACOILA Raf.}

Sacoila lanceolata (Aubl.) Garay, Bot. Mus. Leafl. 28: 352. 1980[1982].

Limodorum lanceolatum Aubl., Hist. P1. Guiane 2: 821.1775. 
Neottia lanceolata (Aubl.) Willd., Sp. Pl. 4: 73. 1805.

Stenorrhynchos lanceolatum (Aubl.) Rich., De Orchid. Eur.: 37. 1817.

Gyrostachys lanceolata (Aubl.) Kuntze, Revis. Gen. P1. 2: 664. 1891.

Spiranthes lanceolata (Aubl.) León, Contr. Ocas. Mus. Hist. Nat. Colegio 'De Le Salle” 8: 358. 1946.

Satyrium orchioides Sw., Prodr. Veg. Ind. Occ.: 118. 1788.

Neottia orchioides (Sw.) Sw., Fl. Ind. Occid. 3: 1411. 1806.

Stenorrhynchos orchioides (Sw.) Rich., De Orchid. Eur.: 37. 1817.

Spiranthes orchioides (Sw.) A.Rich. in R.de la Sagra, Hist. Fis. Cuba, Bot. 11: 252. 1850.

Gyrostachys orchioides (Sw.) Kuntze, Revis. Gen.

P1. 2: 664.1891.

VOUCHER: P.H. Allen 3513 (MO).

\section{SARCoglottis C.Presl.}

Sarcoglottis hunteriana Schltr., Repert. Spec. Nov. Regni Veg. Beih. 17: 13. 1922.

Voucher: C.W. Powell 147 (AMES).

Sarcoglottis neglecta Christenson, Lindleyana 6: 133. 1991.

Voucher: C.W. Powell 388 (AMES).

Sarcoglottis powellii Schltr., Repert. Spec. Nov. Regni Veg. Beih. 17: 14. 1922.

VOUCHER: C.W. Powell 179 (AMES).

Sarcoglottis woodsonii (L.O.Williams) Garay, Bot. Mus. Leafl. 28: 355. 1980[1982].

Spiranthes woodsonii L.O.Williams, Ann. Missouri

Bot. Gard. 29: 337. 1942.

VOUCHER: R.E. Woodson et al. 1148 (MO).

\section{Scaphosepalum Pfitzer}

Scaphosepalum anchoriferum (Rchb.f.) Rolfe, J. Bot. 28: 136. 1890.

Masdevallia anchorifera Rchb.f., Gard. Chron., n.s., 21: 577. 1884.

Masdevallia punctata Rolfe, Gard. Chron., ser. 3, 4: 323.1888.

Scaphosepalum punctatum (Rolfe) Rolfe, J. Bot. 28: 137. 1890.
Scaphosepalum endresianum Kraenzl., Repert. Spec. Nov. Regni Veg. 17: 435. 1921.

Scaphosepalum naviculare Kraenzl., Repert. Spec. Nov. Regni Veg. 17: 435. 1921.

Voucher: C.A. Luer 2660 (SEL).

Scaphosepalum clavellatum Luer, Selbyana 3: 32. 1976.

VOUCHER: C.A. Luer et al. 748 (SEL).

Scaphosepalum microdactylum Rolfe, Bull. Misc. Inform. Kew 1893: 335. 1893.

Scaphosepalum pittieri Schltr., Repert. Spec. Nov. Regni Veg. 3: 78. 1906.

Scaphosepalum elasmatopus Schltr., Repert. Spec. Nov. Regni Veg. 12: 204. 1913.

Scaphosepalum longirepens Ames, Proc. Biol. Soc. Wash. 34: 153. 1921.

Scaphosepalum standleyi Ames, Schedul. Orchid. 9: 24. 1925

VOUCHer: H. Pittier \& W.R. Maxon 3229 (US).

Scaphosepalum viviparum Luer, Selbyana 2: 390. 1978.

VouCHER: C. Luer 1820 (SEL).

Scaphyglottis Poepp. \& Endl.

Scaphyglottis acostae (Schltr.) C.Schweinf., Bot. Mus. Leafl. 10: 27. 1941.

Hexadesmia acostae Schltr., Repert. Spec. Nov. Regni Veg. Beih. 19: 293. 1923.

Hexadesmia powellii Schltr., Repert. Spec. Nov. Regni Veg. Beih. 17: 27.1922.

Voucher: C.W. Powell 225 (MO).

Scaphyglottis amparoana (Schltr.) Dressler, Taxon 13: 246. 1964.

Costaricaea amparoana Schltr., Repert. Spec. Nov. Regni Veg. Beih. 19: 31. 1923.

Hexisea amparoana (Schltr.) Ames, F.T.Hubb. \& C.Schweinf., Bot. Mus. Leafl. 3: 40. 1934.

VOUCHER: R.L. Dressler 5577 (PMA).

Scaphyglottis arctata (Dressler) B.R.Adams, Phytologia 64: 257. 1988.

Hexisea arctata Dressler, Orquídea (Mexico City), n.s., 7: 223. 1979.

VOUCHER: R.L. Dressler 5185 (US).

Scaphyglottis behrii (Rchb.f.) Benth. \& Hook.f. ex Hemsl., Biol. Cent.-Amer., Bot. 3: 219. 1884.

Ponera behrii Rchb.f., Bonplandia (Hannover) 3: 220. 1855. 
Hexadesmia stenopetala Rchb.f., Bonplandia (Hannover) 3: 221. 1855.

Ponera albida Rchb.f., Beitr. Orchid.-K. C. Amer.: 103. 1866.

Scaphyglottis guatemalensis Schltr., Repert. Spec. Nov. Regni Veg. 2: 133. 1906.

Scaphyglottis pauciflora Schltr., Repert. Spec. Nov. Regni Veg. 3: 47. 1906.

Scaphyglottis alba Rolfe, Bull. Misc. Inform. Kew 1908: 415. 1908.

Scaphyglottis albida (Rchb.f.) Schltr., Beih. Bot. Centralbl. 36(2): 456. 1918.

Scaphyglottis sanctae-martae Schltr., Repert. Spec. Nov. Regni Veg. Beih. 7: 122. 1920.

Scaphyglottis bradeorum Schltr., Repert. Spec. Nov. Regni Veg. Beih. 19: 113. 1923.

Scaphyglottis stenotepala (Rchb.f.) C.Schweinf., Bot. Mus. Leafl. 10: 28. 1941.

VOUCHER: H.A. Behr s.n. (W).

Scaphyglottis bidentata (Lindl.) Dressler, Lankesteriana 3: 28. 2002.

Hexisea bidentata Lindl., J. Bot. (Hooker) 1: 8. 1834.

VOUCHER: H. Cuming s.n. (K).

Scaphyglottis bilineata (Rchb.f.) Schltr., Beih. Bot. Centralbl. 36(2): 456. 1918.

Ponera bilineata Rchb.f., Beitr. Orchid.-K. C. Amer.: 88. 1866.

Scaphyglottis lueckelii I.Bock, Orchidee (Hamburg) 35: 178. 1984.

Voucher: G. Davidse \& C.W. Hamilton 23577 (PMA).

Scaphyglottis boliviensis (Rolfe) B.R.Adams, Phytologia 64: 257. 1988.

Hexadesmia boliviensis Rolfe, Mem. Torrey Bot. Club 6: 122. 1896.

Scaphyglottis stricta Schltr., Repert. Spec. Nov. Regni Veg. Beih. 7: 123. 1920.

Scaphyglottis exilis Schltr., Repert. Spec. Nov. Regni Veg. Beih. 27: 60. 1924.

Scaphyglottis amazonica Schltr., Beih. Bot. Centralbl. 42(2): 94. 1925.

Scaphyglottis huebneri Schltr., Beih. Bot. Centralbl. 42(2): 95.1925.

Ornithidium flaccidum Kraenzl., Repert. Spec. Nov. Regni Veg. 25: 31. 1928.

Scaphyglottis matogrossensis Brade, Arq. Serv. Florest. 1(1): 44. 1939.
Scaphyglottis decipiens C.Schweinf., Bot. Mus. Leafl. 17: 43. 1955.

Scaphyglottis flaccida (Kraenzl.) Garay, Canad. J. Bot. 34: 255. 1956.

Scaphyglottis suarezii Dodson, Icon. Pl. Trop., ser. 2, 6: t. 579. 1989.

VOucher: G. de Nevers \& C. Todzia 3528 (PMA).

Scaphyglottis cernua Dressler, J. Orchideenfr. 11: 310. 2004.

Voucher: R.L. Dressler 6373 (MO).

Scaphyglottis chlorantha B.R.Adams, Phytologia 64: 249. 1988.

Voucher: K.J. Sytsma 2003 (MO).

Scaphyglottis clavata Dressler, J. Orchideenfr. 11: 311. 2004.

VOUCHER: R.L. Dressler 6287 (MO).

Scaphyglottis corallorrhiza (Ames) Ames, F.T.Hubb. \& C.Schweinf., Bot. Mus. Leafl. 3: 40. 1934.

Pachystele corallorrhiza Ames, Schedul. Orchid. 9: 45. 1925.

Pachystelis corallorrhiza (Ames) Rauschert, Feddes Repert. 94: 456. 1983.

VOUCHER: R.L. Dressler 5934 (MO).

Scaphyglottis coriacea (L.O.Williams) Dressler, Brittonia 56: 64. 2004.

Platyglottis coriacea L.O.Williams, Ann. Missouri Bot. Gard. 29: 347. 1942.

VOUCHER: P.H. Allen 2936 (AMES).

Scaphyglottis crurigera (Bateman ex Lindl.) Ames \& Correll, Bot. Mus. Leafl. 10: 85. 1942.

Hexadesmia crurigera (Bateman ex Lindl.) Lindl., Edwards's Bot. Reg. 30(Misc.): 2. 1844.

Hexopia crurigera Bateman ex Lindl., Edwards's Bot. Reg. 30(Misc.): 2. 1844.

VOUCHER: T.B. Croat 33055 (MO).

Scaphyglottis cuniculata (Schltr.) Dressler, Lankesteriana 3: 28. 2002.

Fractiunguis cuniculatus Schltr., Repert. Spec. Nov. Regni Veg. Beih. 19: 31. 1923.

Hexisea cuniculata (Schltr.) Ames, Schedul. Orchid. 9: 48. 1925.

Reichenbachanthus cuniculatus (Schltr.) Pabst, Rodriguésia 16-17: 138. 1955.

VOUCHER: R.L. Dressler s.n. (MO).

Scaphyglottis densa (Schltr.) B.R.Adams, Phytologia 64: 258. 1988.

Pachystele densa Schltr., Repert. Spec. Nov. Regni 
Veg. Beih. 19: 29. 1923.

Pachystelis densa (Schltr.) Rauschert, Feddes Repert. 94: 456. 1983.

VOUCHER: J.P. Folsom et al. 5585 (MO).

Scaphyglottis fusiformis (Griseb.) R.E.Schult., Bot. Mus. Leafl. 17: 205. 1957.

Hexadesmia fusiformis Griseb., Fl. Brit. W. I.: 623. 1864.

Hexadesmia brachyphylla Rchb.f., Beitr. Orchid.-K. C. Amer.: 89. 1866.

Hexadesmia cearensis Schltr., Notizbl. Königl. Bot. Gart. Berlin 6: 124. 1914.

Hexadesmia brachyphylla var. longior Schltr., Repert. Spec. Nov. Regni Veg. Beih. 19: 205. 1923.

Scaphyglottis bilobulata Schltr., Repert. Spec. Nov. Regni Veg. Beih. 19: 113. 1923.

Scaphyglottis brachyphylla (Rchb.f.) C.Schweinf., Bot. Mus. Leafl. 10: 28. 1941.

Voucher: G. McPherson \& J.E. Aranda B. 10162 (MO).

Scaphyglottis gigantea Dressler, Orquídea (Mexico City), n.s., 7: 234. 1979.

Voucher: R.L. Dressler 5533 (US).

\section{Scaphyglottis imbricata (Lindl.) Dressler,}

Lankesteriana 3: 28. 2002.

Diothonea imbricata Lindl., Sert. Orchid.: 40. 1841.

Euothonaea imbricata (Lindl.) Rchb.f., Bot. Zeitung (Berlin) 10: 772. 1852.

Hexisea imbricata (Lindl.) Rchb.f. in W.G.Walpers, Ann. Bot. Syst. 6: 470. 1862.

Hexisea bidentata var. imbricata (Lindl.) C.Schweinf., Bot. Mus. Leafl. 15: 106. 1951.

Epidendrum oppositifolium A.Rich. \& Galeotti, Ann. Sci. Nat., Bot., sér. 3, 3: 21. 1845.

Diothonea oppositifolia (A.Rich. \& Galeotti) Rchb.f., Linnaea 22: 842. 1850.

Euothonaea oppositifolia (A.Rich. \& Galeotti) Rchb.f., Bot. Zeitung (Berlin) 10: 772. 1852.

Hexisea oppositifolia (A.Rich. \& Galeotti) Rchb.f. in W.G.Walpers, Ann. Bot. Syst. 6: 470. 1862.

VOuCHER: M.D. Correa \& R.L. Dressler 1058 (PMA).

Scaphyglottis jimenezii Schltr., Beih. Bot. Centralbl. 36(2): 399. 1918.

Pachystele jimenezii (Schltr.) Schltr., Repert. Spec.

Nov. Regni Veg. Beih. 19: 30. 1923.
Pachystelis jimenezii (Schltr.) Rauschert, Feddes Repert. 94: 456. 1983.

VOucher: not seen, cited by Dressler (2003c).

Scaphyglottis laevilabium Ames, Proc. Biol. Soc. Wash. 34: 154. 1921.

Scaphyglottis dolichophylla Schltr., Repert. Spec. Nov. Regni Veg. Beih. 17: 28. 1922.

VOUCHER: E.P. Killip 3113 (AMES).

Scaphyglottis lindeniana (A.Rich. \& Galeotti) L.O.Williams, Ann. Missouri Bot. Gard. 28: 423. 1941.

Hexadesmia lindeniana A.Rich. \& Galeotti, Ann. Sci. Nat., Bot., sér. 3, 3: 23. 1845.

Hexadesmia fasciculata Brongn., Ann. Sci. Nat., Bot., sér. 2, 17: 45. 1842.

Hexadesmia rhodoglossa Rchb.f., Bonplandia (Hannover) 4: 328. 1856.

Hexadesmia rigidipes Schltr., Repert. Spec. Nov. Regni Veg. Beih. 19: 206. 1923.

VOUCHER: P.H. Allen 3291 (AMES).

Scaphyglottis longicaulis S.Watson, Proc. Amer. Acad. Arts 23: 286. 1888.

Scaphyglottis unguiculata Schltr., Repert. Spec. Nov. Regni Veg. 12: 206. 1913.

Scaphyglottis chocoana I.Bock, Orchidee (Hamburg) 35: 176. 1984.

VOUCHER: H. Pittier 4090 (B, destroyed).

Scaphyglottis mesocopis (Endrés \& Rchb.f.) Benth. \& Hook.f. ex Hemsl., Biol. Cent.-Amer., Bot. 3: 220. 1884.

Ponera mesocopis Endrés \& Rchb.f., Xenia Orchid. 2: 222.1874.

Scaphyglottis powellii Schltr., Repert. Spec. Nov. Regni Veg. Beih. 17: 28. 1922.

Voucher: C.W. Powell 134 (AMES).

Scaphyglottis micrantha (Lindl.) Ames \& Correll, Bot. Mus. Leafl. 10: 85. 1942.

Hexadesmia micrantha Lindl., Edwards's Bot. Reg. 30(Misc.): 2. 1844.

Pseudohexadesmia micrantha (Lindl.) Brieger in F.R.R.Schlechter, Orchideen Beschreib. Kult. Zücht., ed. 3, 8(29-32): 489. 1976, nom. inval.

Epidendrum micranthum Lindl., J. Bot. (Hooker) 3: 88. 1841., nom. illeg.

VOUCHER: not seen, cited by Dressler (2003c).

Scaphyglottis minutiflora Ames \& Correll, Bot. Mus. Leafl. 10: 83. 1942.

VOUCHER: P.H. Allen 2258 (AMES). 
Scaphyglottis modesta (Rchb.f.) Schltr., Repert. Spec. Nov. Regni Veg. 23: 46. 1926.

Tetragamestus modestus Rchb.f., Bonplandia (Hannover) 2: 21. 1854.

Ponera modesta (Rchb.f.) Rchb.f., Linnaea 41: 85. 1876.

Ponera striolata Rchb.f., Linnaea 41: 39. 1876.

Tetragamestus antillanus Schltr., Anexos Mem.

Inst. Butantan, Secç. Bot. 1(4): 57. 1922.

Scaphyglottis striolata (Rchb.f.) Correll, Bot. Mus.

Leafl. 9: 148. 1941.

VOUCHER: T.B. Croat 27758 (MO).

Scaphyglottis monspirrae Dressler, Novon 10: 199. 2000.

VOUCHER: R.L. Dressler 5668 (MO).

Scaphyglottis pachybulbon (Schltr.) Dressler, Novon 10: 199. 2000.

Hexadesmia pachybulbon Schltr., Repert. Spec.

Nov. Regni Veg. Beih. 17: 26. 1922.

VOUCHER: C.W. Powell 229 (AMES).

Scaphyglottis panamensis B.R.Adams, Phytologia 64: 251. 1988.

VOUCHER: H.W. Churchill 3942 (MO).

Scaphyglottis prolifera (R.Br.) Cogn. in C.F.P.von Martius \& auct. suc. (eds.), Fl. Bras. 3(5): 15. 1898.

Isochilus proliferus R.Br. in W.T.Aiton, Hortus Kew. 5: 209. 1813.

Scaphyglottis cuneata Schltr., Beih. Bot. Centralbl. 36(2): 398. 1918.

Tetragamestus gracilis Schltr., Beih. Bot. Centralbl. 36(2): 400. 1918.

Scaphyglottis gracilis (Schltr.) Schltr., Repert. Spec. Nov. Regni Veg. Beih. 19: 28. 1923.

Scaphyglottis wercklei Schltr., Repert. Spec. Nov. Regni Veg. Beih. 19: 28. 1923.

Ponera mapiriensis Kraenzl., Repert. Spec. Nov. Regni Veg. 25: 22. 1928.

Voucher: S. Laube et al. 496 (PMA).

Scaphyglottis pulchella (Schltr.) L.O.Williams, Ann. Missouri Bot. Gard. 28: 424. 1941.

Ramonia pulchella Schltr., Repert. Spec. Nov. Regni Veg. Beih. 19: 295. 1923.

Voucher: T. Antonio 2623 (PMA).

Scaphyglottis punctulata (Rchb.f.) C.Schweinf., Bot. Mus. Leafl. 17: 47. 1955.

Ponera punctulata Rchb.f., Bonplandia (Hannover) 3: 220. 1855.
Helleriella punctulata (Rchb.f.) Garay \& H.R.Sweet in R.A.Howard, Fl. Lesser Antilles, Orchid.: 167. 1974.

Pseudoponera punctulata (Rchb.f.) Brieger in F.R.R.Schlechter, Orchideen Beschreib. Kult. Zücht., ed. 3, 8(29-32): 475. 1976., nom. inval. Scaphyglottis arundinacea Regel, Ann. Sci. Nat., Bot., sér. 4, 6: 376. 1856.

Tetragamestus isochiloides Regel, Index Seminum (LE) 1856: 20. 1856.

Epidendrum dussii Cogn. in I.Urban, Symb. Antill. 6: 482.1910.

Scaphyglottis isochiloides (Regel) Foldats, Acta Biol. Venez. 2: 381. 1959.

Pseudoponera dussii (Cogn.) Brieger in F.R.R.Schlechter, Orchideen Beschreib. Kult. Zücht., ed. 3, 8(29-32): 475 (1976), nom. inval. Voucher: W.D. Stevens 18382 (MO).

Scaphyglottis reflexa Lindl., Edwards's Bot. Reg. 25(Misc.): 20. 1839.

Hexisea reflexa (Lindl.) Rchb.f. ex Griseb., Fl. Brit. W. I.: 623. 1864.

Reichenbachanthus reflexus (Lindl.) Porto \& Brade, Rodriguésia 1(2): 55. 1935.

VOUCHER: R.L. Dressler 4527 (MO).

Scaphyglottis robusta B.R.Adams, Phytologia 64: 253. 1988.

Voucher: J.L. Luteyn \& R.L. Wilbur 4679 (DUKE).

Scaphyglottis sessiliflora B.R.Adams, Phytologia 64: 254. 1988.

Voucher: H. van der Werff \& C. van Hardeveld 6227 (MO).

Scaphyglottis sigmoidea (Ames \& C.Schweinf.) B.R.Adams, Phytologia 64: 257. 1988.

Hexisea sigmoidea Ames \& C.Schweinf., Schedul. Orchid. 8: 39. 1925.

VOUCHER: G. McPherson 13576 (PMA).

Scaphyglottis spathulata C.Schweinf., Bot. Mus. Leafl. 10: 28. 1941.

Hexadesmia jimenezii Schltr., Repert. Spec. Nov. Regni Veg. Beih. 19: 293. 1923.

Voucher: S. Knapp \& R.L. Dressler 3443 (PMA).

Scaphyglottis stellata Lodd. ex Lindl., Edwards's Bot. Reg. 25(Misc.): 44. 1839.

Ponera stellata (Lodd. ex Lindl.) Rchb.f. in W.G.Walpers, Ann. Bot. Syst. 6: 454. 1862.

Ponera amethystina Rchb.f. in W.W.Saunders, 
Refug. Bot. 2(1): 93. 1872.

Scaphyglottis brachiata Schltr., Repert. Spec. Nov.

Regni Veg. 9: 432. 1911.

Scaphyglottis ochroleuca Schltr., Notizbl. Königl.

Bot. Gart. Berlin 6: 123. 1914.

Scaphyglottis amethystina (Rchb.f.) Schltr., Beih.

Bot. Centralbl. 36(2): 456. 1918.

Scaphyglottis floribunda Mansf., Notizbl. Bot.

Gart. Berlin-Dahlem 10: 378. 1928.

VOUCHER: G. Skinner s.n. (W).

Scaphyglottis tenella L.O.Williams, Ann. Missouri Bot. Gard. 28: 423. 1941.

Voucher: P.H. Allen 2276 (AMES).

\section{SELENiPEdium Rchb.f.}

Selenipedium chica Rchb.f., Xenia Orchid. 1: 3. 1854. Voucher: E.P. Duchassaing de Fontbressin s.n. (W).

\section{Sievekingia Rchb.f.}

Sievekingia butcheri Dressler, Orquideología 13: 221. 1979.

VOUCHER: H. Butcher s.n. (US).

Sievekingia fimbriata Rchb.f., Flora 69: 449. 1886.

Voucher: N.H. Williams 78-480 (SEL).

Sievekingia suavis Rchb.f., Beitr. Syst. Pflanzenk.: 3. 1871.

VOUCHER: S. Laube et al. 311 (PMA).

\section{Sobralia Ruiz \& Pav.}

Sobralia allenii L.O.Williams, Ann. Missouri Bot. Gard. 29: 336. 1942.

VOUCHER: P.H. Allen 2686 (AMES).

Sobralia amabilis (Rchb.f.) L.O.Williams, Ann. Missouri Bot. Gard. 33: 30. 1946.

Fregea amabilis Rchb.f., Bot. Zeitung (Berlin) 10: 712. 1852.

Sobralia lepida Rchb.f., Beitr. Orchid.-K. C. Amer:: 68. 1866.

Fregea wercklei Schltr., Repert. Spec. Nov. Regni Veg. Beih. 19: 9. 1923.

Sobralia wercklei (Schltr.) L.O.Williams, Ceiba 5: 25. 1956.

VOucher: J. Warscewicz s.n. (W).

Sobralia atropubescens Ames \& C.Schweinf., Schedul. Orchid. 10: 2. 1930.
Sobralia decora var. aerata C.K.Allen \& L.O.Williams, Ann. Missouri Bot. Gard. 29: 337. 1942.

Sobralia melanothrix Løjtnant, Bot. Not. 130: 170. 1977.

Sobralia atropubescens f. aurea Christenson, Bol. CAOB 66/67: 57. 2007.

Sobralia aerata (C.K.Allen \& L.O.Williams) Garay, Bol. CAOB 66-67: 56. 2007.

VOucher: P.H. Allen 2755 (MO).

Sobralia bletiae Rchb.f., Bot. Zeitung (Berlin) 10: 713. 1852.

Sobralia suaveolens Rchb.f., Gard. Chron., n.s., 9: 622.1878.

Sobralia epiphytica Schltr., Repert. Spec. Nov. Regni Veg. 12: 213. 1913.

VOUCHER: J. Warscewicz s.n. (W).

Sobralia callosa L.O.Williams, Ann. Missouri Bot. Gard. 33: 31. 1946.

Lindsayella amabilis Ames \& C.Schweinf., Bot. Mus. Leafl. 5: 34. 1937.

Voucher: W.R. Lindsay \& G.H. Bevins s.n. (AMES).

Sobralia carazoi C.H.Lank. \& Ames, Schedul. Orchid. 7: 34.1924.

Voucher: C.A. Luer \& H.P. Butcher 1165 (SEL).

Sobralia chrysostoma Dressler, Orchids 70: 750. 2001. VOUCHER: G. McPherson 12477 (PMA).

Sobralia citrea Dressler, Orchids (West Palm Beach) 74: 937.2005.

Sobralia mireyae Dressler, Orchids (West Palm Beach) 76: 700. 2007.

VOUCHER: R.L. Dressler 6338 (MO).

Sobralia crispissima Dressler, Lankesteriana 5: 10. 2002.

Voucher: A. Maduro \& E. Olmos 223 (MO).

Sobralia dissimilis Dressler, Novon 5: 142. 1995.

Voucher: A. Maduro \& E. Olmos 145 (PMA).

Sobralia doremiliae Dressler, Novon 5: 142. 1995.

Voucher: M.A. Blanco et al 2973 (PMA).

Sobralia exigua Dressler, Orchids (West Palm Beach) 74: 938. 2005.

VOUCHER: R.L. Dressler 6365B (MO).

Sobralia fenzliana Rchb.f., Bot. Zeitung (Berlin) 10 : 714. 1852.

Sobralia panamensis Schltr., Repert. Spec. Nov. Regni Veg. Beih. 17: 11. 1922.

Sobralia panamensis var. albiflos Schltr., Repert. 
Spec. Nov. Regni Veg. Beih. 17: 11. 1922.

Sobralia neglecta Schltr., Repert. Spec. Nov. Regni

Veg. Beih. 19: 161. 1923.

VOUCHER: Z. Serracín 101 (UCH).

Sobralia fragrans Lindl., Gard. Chron. 1853: 598. 1853.

VOUCHER: R.L. Dressler 3688 (PMA).

Sobralia gloriana Dressler, Lankesteriana 5: 11. 2002.

Voucher: A. Maduro \& E. Olmos 212 (MO).

Sobralia helleri A.D.Hawkes, Phytologia 14: 16. 1966.

Voucher: M.A. Blanco \& D. Penneys 2840 (PMA).

Sobralia $\times$ intermedia P.H.Allen, Amer. Orchid Soc. Bull. 27: 534. 1958.

Voucher: P.H. Allen 2670 (not stated).

Sobralia kerryae Dressler, Orchid Digest 62: 90. 1998.

Voucher: M.A. Blanco \& D. Penneys 2943 (PMA).

Sobralia kruskayae Dressler, Orchids (West Palm Beach) 74: 939. 2005.

VOUCHER: R.L. Dressler 6383 (MO).

Sobralia labiata Warsz. \& Rchb.f., Bot. Zeitung (Berlin) 10: 714. 1852.

VOUCHER: J. Warscewicz s.n. (W).

Sobralia leucoxantha Rchb.f., Beitr. Orchid.-K. C. Amer:: 68. 1866.

Voucher: M.A. Blanco \& D. Penneys 2914 (PMA).

Sobralia lindleyana Rchb.f., Bot. Zeitung (Berlin) 10: 713. 1852.

VOUCHER: J. Warscewicz s.n. (W).

Sobralia macrophylla Rchb.f., Bot. Zeitung (Berlin) 10: 713.1852.

Sobralia chlorantha Hook., Bot. Mag. 78: t. 4682. 1852.

Cattleya chlorantha (Hook.) Beer, Prakt. Stud. Orchid.: 209. 1854.

Cyathoglottis macrantha Lem., Jard. Fleur. 4(1): 855. 1854.

Sobralia rolfeana Schltr., Repert. Spec. Nov. Regni Veg. Beih. 17: 12. 1922.

VOUCHER: J. Warscewicz s.n. (W).

Sobralia maduroi Dressler, Orchids (West Palm Beach) 73: 776. 2004.

Voucher: A. Maduro \& E. Olmos 206 (MO).

Sobralia marianneae Dressler, Lankesteriana 5: 13. 2002.

VOUCHER: R.L. Dressler 6352 (MO).

Sobralia mucronata Ames \& C.Schweinf., Schedul.
Orchid. 8: 54. 1925.

Voucher: R.L. Dressler 4753 (PMA).

Sobralia nutans Dressler, Lankesteriana 5: 13. 2002. Voucher: A. Maduro \& E. Olmos 236 (MO).

Sobralia powellii Schltr., Repert. Spec. Nov. Regni Veg. Beih. 17: 11. 1922.

Voucher: C.W. Powell 2 (MO).

Sobralia quinata Dressler, Lankesteriana 6: 27. 2003. Sobralia fuzukiae Dressler \& Bogarín, Orchids (West Palm Beach) 76: 696. 2007.

VOUCHER: R.L. Dressler 6810 (PMA).

Sobralia recta Dressler, Orchids (West Palm Beach) 74: 939. 2005.

Voucher: A. Maduro \& E. Olmos 295 (MO).

Sobralia sanfelicis Dressler, Orchids (West Palm Beach) 73: 777. 2004.

Voucher: A. Maduro \& E. Olmos 269 (MO).

Sobralia sororcula Dressler, Orquideología 25: 39. 2007.

VOUCHER: R.L. Dressler 6415 (MO).

Sobralia sotoana Dressler \& Bogarín, Lankesteriana 9: 482. 2010.

VOUCHER: R.L. Dressler 7168 (CR).

Sobralia theobromina Dressler, Orchids (West Palm Beach) 74: 940. 2005.

Voucher: A. Maduro \& E. Olmos 293 (MO).

Sobralia tricolor Dressler, Orchids (West Palm Beach) 74: 941. 2005.

Voucher: A. Maduro \& E. Olmos 297 (MO).

Sobralia undatocarinata C.Schweinf., Bot. Mus. Leafl. 6: 197. 1938.

Voucher: R.L. Dressler \& J.T. Atwood 6261 (PMA).

Sobralia valida Rolfe, Bull. Misc. Inform. Kew 1909: 65. 1909.

Voucher: Sander \& Sons s.n. (K).

Sobralia warszewiczii Rchb.f., Bot. Zeitung (Berlin) 10: 714.1852.

VOUCHER: J. Warscewicz s.n. (W).

Sobralia wilsoniana Rolfe, Gard. Chron., III, 8: 378. 1890.

Sobralia sanderae Rolfe, Gard. Chron., ser. 3, 8: 494. 1890.

Sobralia bouchei Ames \& C.Schweinf., Schedul. Orchid. 10: 4. 1930.

Sobralia triandra A.H.Heller \& A.D.Hawkes, Phytologia 14: 17. 1966.

Voucher: G. de Nevers \& H. Herrera 6955 (PMA). 


\section{Solenocentrum Schltr.}

Solenocentrum costaricense Schltr., Repert. Spec. Nov. Regni Veg. 9: 163. 1911.

Voucher: S.A. Mori \& A. Bolten 7277 (MO).

\section{Spathoglottis Blume}

Spathoglottis plicata Blume, Bijdr. Fl. Ned. Ind.: 401. 1825.

Bletia angustifolia Gaudich., Voy. Uranie: 421. 1829.

Paxtonia rosea Lindl., Edwards's Bot. Reg. 24(Misc.): 61. 1838.

Spathoglottis lilacina Griff., Not. P1. Asiat. 3: 323. 1851.

Phaius rumphii Blume, Mus. Bot. 2: 179. 1856.

Spathoglottis spicata Lindl., J. Proc. Linn. Soc., Bot. 3: 9. 1858.

Spathoglottis deplanchei Rchb.f., Linnaea 41: 86. 1876.

Spathoglottis vieillardii Rchb.f., Linnaea 41: 85. 1876.

Spathoglottis angustifolia (Gaudich.) Benth. \& Hook.f., Gen. Pl. 3: 512. 1883.

Spathoglottis augustorum Rchb.f., Gard. Chron., n.s., 25: 9. 1886.

Spathoglottis rosea (Lindl.) G.Nicholson, Ill. Dict. Gard. 3: 467. 1886.

Spathoglottis plicata var. minahassae Schltr. Repert. Spec. Nov. Regni Veg. 21: 180. 1925.

Spathoglottis daenikeri Kraenzl., Vierteljahrsschr. Naturf. Ges. Zürich 74: 80. 1929.

Calanthe poilanei Gagnep., Bull. Mus. Natl. Hist. Nat., sér. 2, 22: 625. 1951.

Voucher: T.B. Croat \& G. Zhu 76939 (PMA).

A species from Asia is currently naturalized in the wild in Panama.

\section{SPECKLINIA Lindl.}

Specklinia absurda Bogarín, Karremans \& R.Rincón, Phytotaxa 115 (2): 31-41. 2013.

Voucher: D. Bogarín et al. 8711 (CR).

Specklinia acrisepala (Ames \& C.Schweinf.) Pridgeon \& M.W.Chase, Lindleyana 16: 256. 2001.

Pleurothallis acrisepala Ames \& C.Schweinf., Schedul. Orchid. 8: 22. 1925.
Sarcinula acrisepala (Ames \& C.Schweinf.) Luer, Monogr. Syst. Bot. Missouri Bot. Gard. 105: 203. 2006.

VOUCHER: C.W. Powell 420 (AMES).

Specklinia areldii (Luer) Pridgeon \& M.W.Chase, Lindleyana 16: 256. 2001.

Pleurothallis areldii Luer, Selbyana 2: 383. 1978.

Sarcinula areldii (Luer) Luer, Monogr. Syst. Bot. Missouri Bot. Gard. 105: 204. 2006.

Voucher: C. Luer 1653 (SEL).

Specklinia barbae (Schltr.) Luer, Monogr. Syst. Bot. Missouri Bot. Gard. 95: 259. 2004.

Pleurothallis barbae Schltr., Repert. Spec. Nov. Regni Veg. Beih. 19: 104. 1923.

Sarcinula barbae (Schltr.) Luer, Monogr. Syst. Bot. Missouri Bot. Gard. 105: 205. 2006.

VOUCHER: C. Luer 18780 (MO).

Specklinia bicornis (Luer) Pridgeon \& M.W.Chase, Lindleyana 16(4): 256. 2001.

Acostaea bicornis Luer, Phytologia 54(5): 379. 1983.

Acostaea costaricensis subsp. bicornis (Luer) Luer, Monogr. Syst. Bot. Missouri Bot. Gard. 24: 8. 1987.

VOUCHER: C. Luer et al. 744 (SEL).

Specklinia brighamella (Luer) Pridgeon \& M.W.Chase, Lindleyana 16: 256. 2001.

Pleurothallis brighamella Luer, Monogr. Syst. Bot. Missouri Bot. Gard. 76: 171. 1999.

Sarcinula brighamella (Luer) Luer, Monogr. Syst. Bot. Missouri Bot. Gard. 105: 206. 2006.

Pleurothallis brighamella Luer, Orquideología 21: 323 (2000), nom. illeg.

VOUCHer: C.A. Luer 19005 (MO).

Specklinia brighamii (S.Watson) Pridgeon \& M.W.Chase, Lindleyana 16: 256. 2001.

Pleurothallis brighamii S.Watson, Proc. Amer. Acad. Arts 23: 285. 1888.

Sarcinula brighamii (S.Watson) Luer, Monogr. Syst. Bot. Missouri Bot. Gard. 105: 206. 2006. Voucher: C.W. Powell 274 (AMES).

Specklinia cactantha (Luer) Pridgeon \& M.W.Chase, Lindleyana 16: 257. 2001.

Pleurothallis cactantha Luer, Selbyana 3: 72. 1976. Sylphia cactantha (Luer) Luer, Monogr. Syst. Bot. Missouri Bot. Gard. 105: 228. 2006.

VOUCHER: C.A. Luer 743 (SEL). 
Specklinia calyptrostele (Schltr.) Pridgeon \& M.W.Chase, Lindleyana 16: 257. 2001.

Pleurothallis calyptrostele Schltr., Repert. Spec. Nov. Regni Veg. Beih. 19: 23. 1923.

Pleurothallis biflora Schltr., Repert. Spec. Nov.

Regni Veg. Beih. 19: 181. 1923., nom. illeg.

Pleurothallis geminiflora Ames, F.T.Hubb. \&

C.Schweinf., Bot. Mus. Leafl. 3: 39. 1934.

Specklinia geminiflora (Ames, F.T.Hubb. \&

C.Schweinf.) Pridgeon \& M.W.Chase,

Lindleyana 16: 257. 2001.

VOUCHER: R.L. Dressler 2927 (SEL).

Specklinia condylata (Luer) Pridgeon \& M.W.Chase, Lindleyana 16: 257. 2001.

Pleurothallis condylata Luer, Selbyana 3: 80. 1976. Sarcinula condylata (Luer) Luer, Monogr. Syst.

Bot. Missouri Bot. Gard. 105: 208. 2006.

VOuCHER: C.A. Luer \& H. Butcher 1198 (SEL).

Specklinia corniculata (Sw.) Steud., Nomencl. Bot., ed. 2, 2: 489. 1841.

Epidendrum corniculatum Sw., Prodr. Veg. Ind. Occ.: 123. 1788.

Dendrobium corniculatum (Sw.) Sw., Nova Acta

Regiae Soc. Sci. Upsal. 6: 83. 1799.

Cymbidium corniculatum (Sw.) Spreng., Syst. Veg. 3: 722.1826.

Pleurothallis corniculata (Sw.) Lindl., Edwards's

Bot. Reg. 28(Misc.): 83. 1842.

Humboldtia corniculata (Sw.) Kuntze, Revis. Gen.

P1. 2: 667. 1891.

Sarcinula corniculata (Sw.) Luer, Monogr. Syst.

Bot. Missouri Bot. Gard. 105: 209. 2006.

Epidendrum alpestre Sw., Prodr. Veg. Ind. Occ.: 125. 1788.

Dendrobium alpestre (Sw.) Sw., Nova Acta Regiae Soc. Sci. Upsal. 6: 84. 1799.

Epidendrum monophyllum Hook., Exot. Fl. 2: t. 109. 1824.

Liparis monophylla (Hook.) Spreng., Syst. Veg. 3: 741. 1826).

Pleurothallis alpestris (Sw.) Lindl., Gen. Sp.

Orchid. P1.: 7. 1830.

Specklinia emarginata Lindl., Gen. Sp. Orchid. Pl.: 8. 1830.

Physosiphon emarginatus (Lindl.) Lindl.,

Edwards's Bot. Reg. 24: t. 1797. 1838.

Pleurothallis vilipensa Rchb.f., Hamburger Garten-
Blumenzeitung 13: 3. 1857.

Pleurothallis nubigena Lindl., Ann. Mag. Nat. Hist., ser. 3, 1: 326. 1858.

Pleurothallis emarginata (Lindl.) Lindl., Fol. Orchid. 9: 25. 1859), nom. illeg.

Pleurothallis pyrsodes Rchb.f., Gard. Chron., n.s., 6: 386.1876.

Humboldtia alpestris (Sw.) Kuntze, Revis. Gen. Pl. 2: 667. 1891.

Humboldtia monophylla (Hook.) Kuntze, Revis. Gen. Pl. 2: 668. 1891.

Humboldtia pyrsodes (Rchb.f.) Kuntze, Revis. Gen. P1. 2: 668. 1891.

Humboldtia vilipensa (Rchb.f.) Kuntze, Revis. Gen. Pl. 2: 668. 1891.

Pleurothallis monophylla (Hook.) Fawc. \& Rendle, Fl. Jamaica 1: 60. 1910.

Pleurothallis barboselloides Schltr., Repert. Spec. Nov. Regni Veg. Beih. 17: 18. 1922.

Pleurothallis jocolensis Ames, Schedul. Orchid. 2: 19. 1923.

Specklinia barboselloides (Schltr.) Pridgeon \& M.W.Chase, Lindleyana 16: 256. 2001.

Specklinia pyrsodes (Rchb.f.) Pridgeon \& M.W.Chase, Lindleyana 16: 259. 2001.

Antilla alpestris (Sw.) Luer, Monogr. Syst. Bot. Missouri Bot. Gard. 95: 255. 2004.

VOUCHer: C.A. Luer \& H. Butcher 1244 (SEL).

Specklinia costaricensis (Rolfe) Pridgeon \& M.W.Chase, Lindleyana 16: 257. 2001.

Pleurothallis costaricensis Rolfe, Bull. Misc. Inform. Kew 1917: 80. 1917.

Pleurothallis ehrhartiiflora Schltr., Repert. Spec. Nov. Regni Veg. Beih. 19: 187. 1923.

VOUCHER: R.L. Dressler 5367 (UCH).

Specklinia cucumeris (Luer) Bogarín \& Karremans, comb. nov.

Bas.: Pleurothallis cucumeris Luer, Selbyana 5: 162. 1979.

Cucumeria cucumeris (Luer) Luer, Monogr. Syst. Bot. Missouri Bot. Gard. 95: 257. 2004.

VOuCHer: C. Luer et al. 2258 (SEL).

This species should be treated in Specklinia. The phylogenetic relationships of Specklinia and closely related genera will be treated in a further paper (Karremans et al. in prep).

Specklinia displosa (Luer) Pridgeon \& M.W.Chase, Lindleyana 16: 257. 2001. 
Pleurothallis displosa Luer, Monogr. Syst. Bot. Missouri Bot. Gard. 76: 172. 1999.

Sarcinula displosa (Luer) Luer, Monogr. Syst. Bot. Missouri Bot. Gard. 105: 212. 2006.

Voucher: C. Luer et al. 6533 (MO).

Specklinia dressleri (Luer) Bogarín \& Karremans, comb. nov.

Bas.: Pleurothallis dressleri Luer, Selbyana 3: 98. 1976.

Areldia dressleri (Luer) Luer, Monogr. Syst. Bot. Missouri Bot. Gard. 95: 255. 2004.

VOUCHER: R.L. Dressler 3033 (SEL).

Plants available for study (Fig. 26J) show characters resembling species related to Acostaea (=Specklinia) such as the elongated lip callus made up by papillose glands, the winged column and the naked pollinia. The phylogenetic relationships of Specklinia and closely related genera will be treated in a further paper (Karremans et al. in prep).

Specklinia fuegi (Rchb.f.) Solano \& Soto Arenas, Icon. Orchid. 5-6: x. 2002[2003].

Pleurothallis fuegi Rchb.f., Beitr. Orchid.-K. C. Amer.: 97. 1866.

Humboldtia fuegi (Rchb.f.) Kuntze, Revis. Gen. Pl. 2: 667. 1891.

Anathallis fuegi (Rchb.f.) Pridgeon \& M.W.Chase, Lindleyana 16: 248. 2001.

Sylphia fuegi (Rchb.f.) Luer, Monogr. Syst. Bot. Missouri Bot. Gard. 105: 228. 2006.

Pleurothallis fuegi var. echinata L.O.Williams, Ann. Missouri Bot. Gard. 33: 120. 1946.

Pleurothallis hagsateri Luer, Orquídea (Mexico City), n.s., 6: 168. 1977.

Specklinia echinata (L.O.Williams) Soto Arenas \& Solano, Icon. Orchid. 5-6: t. 670. 2002 [2003].

Voucher: M.E. Davidson 981 (AMES).

Specklinia fulgens (Rchb.f.) Pridgeon \& M.W.Chase, Lindleyana 16: 257. 2001.

Pleurothallis fulgens Rchb.f., Gard. Chron., n.s., 4: 516. 1875.

Humboldtia fulgens (Rchb.f.) Kuntze, Revis. Gen. P1. 2: 667. 1891.

Sarcinula fulgens (Rchb.f.) Luer, Monogr. Syst. Bot. Missouri Bot. Gard. 105: 213. 2006.

Pleurothallis brenesii Schltr., Repert. Spec. Nov. Regni Veg. Beih. 19: 182. 1923.

VOUCHER: C.W. Powell 335 (MO).

Specklinia glandulosa (Ames) Pridgeon \& M.W.Chase, Lindleyana 16: 257. 2001.
Pleurothallis glandulosa Ames, Schedul. Orchid. 6: 60. 1923.

Sarcinula glandulosa (Ames) Luer, Monogr. Syst. Bot. Missouri Bot. Gard. 105: 214. 2006.

Pleurothallis vittariifolia Schltr., Repert. Spec. Nov. Regni Veg. Beih. 19: 26. 1923.

Pleurothallis pertenuis C.Schweinf., Bot. Mus. Leafl. 3: 83. 1935).

Specklinia vittariifolia (Schltr.) Pridgeon \& M.W.Chase, Lindleyana 16: 259. 2001

VOUCHER: C.W. Powell 306 (AMES)

Specklinia grobyi (Bateman ex Lindl.) F.Barros, Hoehnea 10: 110. 1983[1984].

Pleurothallis grobyi Bateman ex Lindl., Edwards's Bot. Reg. 21: t. 1797. 1835.

Humboldtia grobyi (Bateman ex Lindl.) Kuntze, Revis. Gen. P1. 2: 667. 1891.

Pleurothallis marginalis Rchb.f., Bonplandia (Hannover) 3: 224. 1855.

Pleurothallis perplexa Rchb.f., Hamburger GartenBlumenzeitung 16: 15. 1860.

Pleurothallis trilineata Barb.Rodr., Gen. Spec. Orchid. 1: 6. 1877.

Pleurothallis crepidophylla Rchb.f., Otia Bot. Hamburg.: 18. 1878.

Lepanthes marmorata Barb.Rodr., Gen. Spec. Orchid. 2: 42. 1881.

Lepanthes trilineata (Barb.Rodr.) Barb.Rodr., Gen. Spec. Orchid. 2: 42.1881.

Pleurothallis choconiana S.Watson, Proc. Amer. Acad. Arts 23: 285. 1888.

Humboldtia crepidophylla (Rchb.f.) Kuntze, Revis. Gen. Pl. 2: 667. 1891.

Humboldtia marginalis (Rchb.f.) Kuntze, Revis. Gen. Pl. 2: 668. 1891.

Humboldtia trilineata (Barb.Rodr.) Kuntze, Revis. Gen. P1. 2: 668. 1891.

Pleurothallis pergracilis Rolfe, Bull. Misc. Inform. Kew 1893: 334. 1893.

Pleurothallis grobyi var. trilineata (Barb.Rodr.) Cogn. in C.F.P.von Martius \& auct. suc. (eds.), Fl. Bras. 3(4): 496. 1896.

Pleurothallis marmorata (Barb.Rodr.) Cogn. in C.F.P.von Martius \& auct. suc. (eds.), Fl. Bras. 3(4): 490. 1896.

Pleurothallis marmorata var. concolor Cogn. in C.F.P.von Martius \& auct. suc. (eds.), Fl. Bras. 
3(6): 559. 1906.

Pleurothallis barbosae Schltr., Repert. Spec. Nov. Regni Veg. Beih. 9: 143. 1921.

Pleurothallis biglandulosa Schltr., Notizbl. Bot. Gart. Berlin-Dahlem 8: 119. 1922.

Pleurothallis ezechiasi Hoehne, Arq. Bot. Estado São Paulo, n.s., f.m., 2: 21. 1946.

Pleurothallis grobyi var. marmorata (Barb.Rodr.) Garay, Arch. Jard. Bot. Rio de Janeiro 11: 54. 1951.

Specklinia biglandulosa (Schltr.) Pridgeon \& M.W.Chase, Lindleyana 16: 256. 2001.

Specklinia marginalis (Rchb.f.) F.Barros, Hoehnea 10: 110. 1983[1984].

Specklinia ezechiasi (Hoehne) Luer, Monogr. Syst. Bot. Missouri Bot. Gard. 95: 260. 2004.

Pabstiella ezechiasi (Hoehne) Luer, Monogr. Syst. Bot. Missouri Bot. Gard. 112: 119. 2007.

Voucher: C.A. Luer et al. 10600 (MO).

Specklinia microphylla (A.Rich. \& Galeotti) Pridgeon \& M.W.Chase, Lindleyana 16: 258. 2001.

Pleurothallis microphylla A.Rich. \& Galeotti, Ann. Sci. Nat., Bot., sér. 3, 3: 17. 1845.

Humboldtia microphylla (A.Rich. \& Galeotti) Kuntze, Revis. Gen. P1. 2: 668. 1891.

Pleurothallis rotundifolia Rolfe, Bull. Misc. Inform. Kew 1895: 191. 1895.

Pleurothallis panamensis Schltr., Repert. Spec. Nov. Regni Veg. 17: 140. 1921.

Voucher: M. Wagner 24 (AMES).

At least two species have been treated under $S$. microphylla in Costa Rica and Panama (Luer 2003b). One species is restricted to the seasonally dry Pacific forest of Costa Rica and Panama. It is characterized by the creeping, repent plants with prostrate, superposed, rounded, granuloseverrucose leaves with lax inflorescences. The flowers are yellow with red stripes on the dorsal sepal and petals and the lip is stained with red purple (Bogarín \& Pupulin in prep.). It has not been possible to locate the holotype of the Mexican P. microphylla to confirm the identity of the populations from southern Central America. An illustration of type at AMES differs in flower details with the specimens studied from Costa Rica and Panama.

Specklinia cf. microphylla (A.Rich. \& Galeotti) Pridgeon \& M.W.Chase, Lindleyana 16: 258. 2001.

VOUCHER: S. Laube et al. 532 (PMA).

This is the other species treated under S. microphylla. It is restricted to the Caribbean lowlands of Costa Rica and
Panama (and possibly Nicaragua) (Luer 2003b). Plants differ in the erect or suberect leaves (vs. prostrate, creeping) and the subpandurate lip (vs. oblong), among other floral details. A careful taxonomic study is needed to clarify the status of these taxa from southern Central America.

Specklinia mirifica Pridgeon \& W.M. Chase, Lindleyana 16: 258. 2001.

Acostaea costaricensis Schltr., Repert. Sp. Nov. Regni Veg. Beih. 19: 284. 1923.

Acostaea pleurothalloides Schltr., Repert. Sp. Nov. Regni Veg. Beih. 19: 285. 1923.

Voucher: C.A. Luer et al. 1444 (SEL).

Specklinia pfavii (Rchb.f.) Pupulin \& Karremans, Phytotaxa 63: 8. 2012.

Pleurothallis pfavii Rchb.f., Flora 69 (n. s. 44): 555. 1886.

Masdevallia platyrachis Rolfe, Gard. Chron. 4(86): 178-179. 1888.

Pleurothallis platyrachis (Rolfe) Rolfe, J. Bot. 28: 136. 1890, comb. inval.

Pleurothallis platyrachis (Rolfe) Rolfe ex Hooker f., Curtis' Bot. Mag. 116: sub pl. 7129. 1890.

Kraenzlinella platyrachis (Rolfe) Rolfe, Orchid Rev. 23: 326. 1915.

Voucher: R. Pfau. s.n. (W).

Specklinia picta (Lindl.) Pridgeon \& M.W.Chase, Lindleyana 16: 259. 2001.

Pleurothallis picta Lindl., Edwards's Bot. Reg. 21: t. 1797.1835.

Humboldtia picta (Lindl.) Kuntze, Revis. Gen. Pl. 2: 668. 1891.

Pleurothallis marginata Lindl., Edwards's Bot. Reg. 24(Misc.): 42. 1838.

Pleurothallis surinamensis H.Focke, Tijdschr. Natuurk. Wetensch. Kunsten 2: 194. 1849.

Pleurothallis florulenta Linden \& Rchb.f., Bonplandia (Hannover) 3: 223. 1855.

Humboldtia florulenta (Linden \& Rchb.f.) Kuntze, Revis. Gen. P1. 2: 667. 1891.

Humboldtia marginata (Lind1.) Kuntze, Revis. Gen. Pl. 2: 668. 1891.

Lepanthes plurifolia Barb.Rodr., Vellosia, ed. 2, 1: 118. 1891.

Pleurothallis densifolia Rolfe, Mem. Torrey Bot. Club 4: 260. 1895.

Pleurothallis lindleyana Cogn. in C.F.P.von Martius \& auct. suc. (eds.), Fl. Bras. 3(4): 
489.1896., nom. superfl.

Pleurothallis pluriflora Cogn. in C.F.P.von Martius \& auct. suc. (eds.), Fl. Bras. 3(4): 492.1896., nom. illeg.

Pleurothallis dryadum Schltr., Repert. Spec. Nov. Regni Veg. Beih. 19: 186. 1923., nom. illeg.

Pleurothallis arevaloi Schltr., Repert. Spec. Nov. Regni Veg. Beih. 27: 43. 1924.

Pleurothallis integrilabia Ames, F.T.Hubb. \& C.Schweinf., Bot. Mus. Leafl. 3: 39. 1934.

Specklinia florulenta (Linden \& Rchb.f.) Pridgeon \& M.W.Chase, Lindleyana 16: 257. 2001.

Specklinia marginata (Lindl.) Pridgeon \&

M.W.Chase, Lindleyana 16: 258. 2001.

VOUCHER: H. Pittier 4787 (AMES).

Specklinia recula (Luer) Luer, Monogr. Syst. Bot. Missouri Bot. Gard. 95: 263. 2004.

Pleurothallis recula Luer, Lindleyana 11: 92. 1996. VOUCHER: C. A. Luer at al. 742 (SEL).

Specklinia remotiflora Pupulin \& Karremans, Phytotaxa 63: 11. 2012.

VOUCHER: D. Bogarin 8656 (JBL).

Specklinia segregatifolia (Ames \& C.Schweinf.) Solano \& Soto Arenas, Icon. Orchid. 5-6: xi. 2002 [2003].

Pleurothallis segregatifolia Ames \& C.Schweinf., Schedul. Orchid. 8: 33. 1925.

Pleurothallis calyptrosepala L.O.Williams, Amer. Orchid Soc. Bull. 11: 65. 1942.

VOUCHER: C. A. Luer at al. 10576 (SEL).

Specklinia cf. simmleriana (Rendle) Luer, Monogr. Syst. Bot. Missouri Bot. Gard. 95: 263. 2004.

Pleurothallis simmleriana Rendle, J. Bot. 38: 274. 1900.

Sarcinula simmleriana (Rendle) Luer, Monogr. Syst. Bot. Missouri Bot. Gard. 105: 218. 2006.

Pleurothallis periodica Ames, Schedul. Orchid. 7: 21. 1924.

Specklinia periodica (Ames) Pridgeon \& M.W.Chase, Lindleyana 16: 258. 2001.

Voucher: S. Laube et al. 211 (PMA).

Specklinia simmleriana (Rendle) Luer, Monogr. Syst.

Bot. Missouri Bot. Gard. 95: 263. 2004.

Pleurothallis simmleriana Rendle, J. Bot. 38: 274. 1900.

Sarcinula simmleriana (Rendle) Luer, Monogr. Syst. Bot. Missouri Bot. Gard. 105: 218. 2006.
Pleurothallis periodica Ames, Schedul. Orchid. 7: 21. 1924.

Specklinia periodica (Ames) Pridgeon \& M.W.Chase, Lindleyana 16: 258. 2001.

VOUCHER: H. Wedel 185 (AMES).

Specklinia spectabilis (Ames \& C.Schweinf.) Pupulin \& Karremans, Phytotaxa 63: 15. 2012.

Pleurothallis spectabilis Ames \& C.Schweinf., Schedul. Orchid. 8: 34. 1925.

Voucher: C.W. Powell 382 (AMES).

Specklinia striata (H.Focke) Luer, Monogr. Syst. Bot. Missouri Bot. Gard. 95: 264. 2004.

Pleurothallis striata H.Focke, Tijdschr. Natuurk. Wetensch. Kunsten 4: 63. 1851.

Humboldtia striata (H.Focke) Kuntze, Revis. Gen. P1. 2: 668. 1891.

Sarcinula striata (H.Focke) Luer, Monogr. Syst. Bot. Missouri Bot. Gard. 105: 219. 2006.

VOUCHER: C.A. Luer 1389 (AMES).

Specklinia tribuloides (Sw.) Pridgeon \& M.W.Chase, Lindleyana 16: 259. 2001.

Epidendrum tribuloides Sw., Prodr. Veg. Ind. Occ.: 123. 1788.

Dendrobium tribuloides (Sw.) Sw., Nova Acta Regiae Soc. Sci. Upsal. 6: 83. 1799.

Cymbidium tribuloides (Sw.) Spreng., Syst. Veg. 3: 721.1826.

Pleurothallis tribuloides (Sw.) Lindl., Gen. Sp. Orchid. P1.: 6. 1830.

Humboldtia tribuloides (Sw.) Kuntze, Revis. Gen. P1. 2: 668. 1891.

Cryptophoranthus tribuloides (Sw.) H.Dietr., Revista Jard. Bot. Nac. Univ. Habana 5: 48. 1984.

Tribulago tribuloides (Sw.) Luer, Monogr. Syst. Bot. Missouri Bot. Gard. 95: 265. 2004.

Pleurothallis spathulata A.Rich. \& Galeotti, Ann. Sci. Nat., Bot., sér. 3, 3: 17. 1845.

Pleurothallis fallax Rchb.f., Bonplandia (Hannover) 3: 224. 1855.

Cryptophoranthus acaulis Kraenzl., Repert. Spec. Nov. Regni Veg. Beih. 34: 232. 1925.

VOUCHER: C. Galdames et al. 4562 (PMA).

Specklinia turrialbae (Luer) Luer, Monogr. Syst. Bot. Missouri Bot. Gard. 95: 264. 2004.

Pleurothallis turrialbae Luer, Lindleyana 6: 105. 1991. 
Sylphia turrialbae (Luer) Luer, Monogr. Syst. Bot. Missouri Bot. Gard. 105: 229. 2006.

VOUCHER: R.L. Dressler 5099 (PMA).

Specklinia unicornis (Luer) Pridgeon \& M.W.Chase, Lindleyana 16(4): 259. 2001.

Acostaea costaricensis subsp. unicornis (Luer)

Luer, Monogr. Syst. Bot. Missouri Bot. Gard. 24: 14. 1987.

Acostaea unicornis Luer, Phytologia 54(5): 379380. 1983.

Voucher: C. Luer et al. 760 (SEL).

\section{Stanhopea J.Frost ex Hook.}

Stanhopea avicula Dressler, Amer. Orchid Soc. Bull. 58: 885. 1989.

VOUCHER: K.R. Reading \& R.R. Tasker s.n. (MO).

Stanhopea cirrhata Lindl., J. Hort. Soc. London 5: 37. 1850.

VOUCHER: Z. Serracín 107 (UCH).

Stanhopea confusa G.Gerlach \& Beeche, Lankesteriana 4: 217. 2004.

VOUCHER: M. Whitten 94082 (FLAS).

Stanhopea costaricensis Rchb.f., Hamburger GartenBlumenzeitung 16: 424. 1860.

VOUCHER: C.W. Powell 295 (MO).

Stanhopea ecornuta Lem., Fl. Serres Jard. Eur. 2: t. 181. 1846.

VOUCHER: S. Laube et al. 183 (PMA).

Stanhopea maduroi Dodson \& Dressler, Orquideología

21: 62.1998.

VOUCHER: C.H. Dodson 16259 (MO).

Stanhopea panamensis N.H.Williams \& W.M.Whitten, Lindleyana 3: 9. 1988.

VOUCHER: M. Whitten 909 (FLAS).

Stanhopea pulla Rchb.f., Gard. Chron. 1877(1): 850. 1877.

Voucher: S. Laube et al. 586 (PMA).

Stanhopea wardii Lodd. ex Lindl., Sert. Orchid.: t. 20. 1838.

VOUCHER: C.W. Powell 103 (MO).

Stanhopea warszewicziana Klotzsch, Allg. Gartenzeitung 20: 274. 1852.

VOUCHER: J. Warszewicz s.n. (B).

\section{Stelis Sw.}

Stelis adriananijhuisae Bogarín \& Serracín, nom. nov.
Bas.: Salpistele adrianae Luer \& Sijm, Selbyana 30: 18. 2009.

VOUCHER: A.P. Sijm 220411 (MO).

This combination is needed in order to treat this species in Stelis s.l. (Pridgeon et al. 2001).

Stelis aemula Schltr., Beih. Bot. Centralbl. 36(2): 385. 1918.

VOUCHer: P. H. Allen 2154 (AMES).

Stelis allenii L.O.Williams, Ann. Missouri Bot. Gard. 29: 338. 1942.

VOUCHER: P. H. Allen 2952 (AMES).

Stelis argentata Lindl., Edwards's Bot. Reg. 28(Misc.): 64. 1842.

Stelis endresii Rchb.f., Gard. Chron. 1870: 1373. 1870.

Stelis parvibracteata Ames, Orchidaceae 7: 131. 1922.

Stelis glandulosa Ames, Schedul. Orchid. 3: 3. 1923.

Stelis praesecta Schltr., Repert. Spec. Nov. Regni Veg. Beih. 19: 175. 1923.

Stelis violascens Schltr., Repert. Spec. Nov. Regni Veg. Beih. 19: 176. 1923.

Stelis huebneri Schltr., Beih. Bot. Centralbl. 42(2): 88. 1925.

VOUCHER: R.S. Williams 972 (AMES).

Stelis atrorubens L.O.Williams, Ann. Missouri Bot. Gard. 29: 339. 1942.

VOUCHER: P. H. Allen 2876 (AMES).

Stelis biserrula Lindl., Fol. Orchid. 8: 16. 1859.

VOUCHER: R.L. Dressler 5075 (PMA).

Stelis bracteata Schltr., Repert. Spec. Nov. Regni Veg. Beih. 19: 171. 1923.

VOUCHER: S. Knapp 5026 (MO).

Stelis brunnea (Dressler) Pridgeon \& M.W.Chase, Lindleyana 16: 261. 2001.

Salpistele brunnea Dressler, Orquideología 14: 6. 1979.

Voucher: R.L. Dressler 5124 (US).

Stelis butcheri Luer, Phytologia 49: 224. 1981.

VOuCHER: C.A. Luer 1101 (SEL).

Stelis canae (Ames) Pridgeon \& M.W.Chase, Lindleyana 16: 261. 2001.

Pleurothallis canae Ames, Schedul. Orchid. 2: 18. 1923.

Unciferia canae (Ames) Luer, Monogr. Syst. Bot. Missouri Bot. Gard. 95: 265. 2004. 
VOUCHER: R.S. Williams 971 (AMES).

Stelis carnosiflora Ames \& C.Schweinf., Schedul. Orchid. 8: 15. 1925.

Voucher: not seen, cited by Luer (2003).

Stelis carnosilabia (A.H.Heller \& A.D.Hawkes) Pridgeon \& M.W.Chase, Lindleyana 16: 261. 2001.

Pleurothallis carnosilabia A.H.Heller \& A.D.Hawkes, Phytologia 14: 9. 1966.

Dracontia carnosilabia (A.H.Heller \& A.D.Hawkes) Luer, Monogr. Syst. Bot. Missouri Bot. Gard. 95: 257. 2004.

Pleurothallis caligularis Luer, Selbyana 3: 74. 1976.

VOUCHER: R.L. Dressler 4808 (SEL).

Stelis carpinterae (Schltr.) Pridgeon \& M.W.Chase, Lindleyana 16: 261. 2001.

Pleurothallis carpinterae Schltr., Repert. Spec.

Nov. Regni Veg. Beih. 19: 105. 1923.

Elongatia carpinterae (Schltr.) Luer, Monogr. Syst.

Bot. Missouri Bot. Gard. 95: 257. 2004.

VOUCHER: R.L. Dressler 1418 (SEL).

Stelis ciliaris Lindl., Companion Bot. Mag. 2: 353. 1837.

Apatostelis ciliaris (Lindl.) Garay, Bot. Mus. Leafl. 27: 188. 1979.

Stelis atropurpurea Hook., Bot. Mag. 69: t. 3975. 1842.

Stelis micrantha var. atropurpurea (Hook.) Josst,

Beschr. Cult. Orchid.: 38. 1851.

Stelis confusa Schltr., Beih. Bot. Centralbl. 36(2): 386. 1918.

Stelis jimenezii Schltr., Beih. Bot. Centralbl. 36(2): 389. 1918.

Stelis pendulispica Ames, Bot. Mus. Leafl. 2: 85. 1934.

Stelis fimbriata R.K.Baker, Ann. Missouri Bot. Gard. 55: 68. 1968.

Apatostelis jimenezii (Schltr.) Garay, Bot. Mus. Leafl. 27: 189. 1979.

Apatostelis pendulispica (Ames) Garay, Bot. Mus. Leafl. 27: 190. 1979.

Stelis gratiosa Luer, Selbyana 5: 192. 1979.

Apatostelis garayi Dunst., Amer. Orchid Soc. Bull. 50: 1075. 1981

Stelis garayi (Dunst.) Carnevali \& I.Ramírez, Ann.

Missouri Bot. Gard. 77: 556. 1990.

VOUCHER: R.K. Baker 249 (MO).
Stelis collina Schltr., Repert. Spec. Nov. Regni Veg. Beih. 17: 15. 1922.

Voucher: C.W. Powell 180 (MO).

Stelis convallaria (Schltr.) Pridgeon \& M.W.Chase, Lindleyana 16: 262. 2001.

Pleurothallis convallaria Schltr., Repert. Spec. Nov. Regni Veg. Beih. 19: 185. 1923

Specklinia convallaria (Schltr.) Luer, Monogr. Syst. Bot. Missouri Bot. Gard. 95: 259. 2004.

Effusiella convallaria (Schltr.) Luer, Monogr. Syst. Bot. Missouri Bot. Gard. 112: 106. 2007.

Voucher: C.A. Luer \& R.L. Dressler 1133 (SEL).

Stelis costaricensis Rchb.f., Bonplandia (Hannover) 3: 225. 1855.

Apatostelis costaricensis (Rchb.f.) Garay, Bot. Mus. Leafl. 27: 188. 1979.

Stelis minutiflora Ames \& C.Schweinf., Schedul. Orchid. 8: 18-19. 1925. nom. illeg. hom.

VOUCHER: D. Bogarín et al. 11170 (UCH).

Stelis crescentiicola Schltr., Repert. Spec. Nov. Regni Veg. 16: 442. 1920.

VOUCHER: F.C. Lehmann 4540 (AMES).

Stelis cucullata Ames, Schedul. Orchid. 6: 52. 1923.

Voucher: C.A. Luer \& H.P. Butcher 1334 (SEL).

Stelis cylindrata Pridgeon \& M.W.Chase, Lindleyana 17: 98. 2002.

Pleurothallis macrantha L.O.Williams, Ann. Missouri Bot. Gard. 28: 417. 1941.

Stelis macrantha (L.O.Williams) Pridgeon \& M.W.Chase, Lindleyana 16: 264. 2001, nom. illeg.

Dracontia macrantha (L.O.Williams) Luer, Monogr. Syst. Bot. Missouri Bot. Gard. 95: 257. 2004

VOucher: R.E. Woodson \& R.W. Schery 666 (MO).

Stelis cymbisepala Pridgeon \& M.W.Chase, Lindleyana 17: 98. 2002.

Salpistele dressleri Luer, Monogr. Syst. Bot. Missouri Bot. Gard. 39: 128. 1991.

Stelis dressleri (Luer) Pridgeon \& M.W.Chase, Lindleyana 16: 262. 2001., nom. illeg.

Voucher: C.simulansA. Luer 12820 (MO).

Stelis decurrens Pridgeon \& M.W.Chase, Lindleyana 17: 99. 2002.

Pleurothallis croatii Luer, Monogr. Syst. Bot. Missouri Bot. Gard. 65: 18. 1998.

Stelis croatii (Luer) Pridgeon \& M.W.Chase, 
Lindleyana 16: 262. 2001., nom. illeg.

Crocodeilanthe croatii (Luer) Luer, Monogr. Syst.

Bot. Missouri Bot. Gard. 95: 256. 2004.

VOuCher: T.B. Croat 48506 (MO).

Stelis deregularis Barb.Rodr., Gen. Spec. Orchid. 2: 94. 1881.

Physosiphon deregularis (Barb.Rodr.) Cogn. in C.F.P.von Martius \& auct. suc. (eds.), Fl. Bras. 3(4): 341. 1896.

Pseudostelis deregularis (Barb.Rodr.) Schltr., Anexos Mem. Inst. Butantan, Secç. Bot. 1(4): 38. 1922.

Pleurothallis deregularis (Barb.Rodr.) Luer, Selbyana 2: 385. 1978.

Physosiphon spiralis Lindl., Edwards's Bot. Reg. 21: t. 1797. 1835.

Pleurothallis clausa De Puydt, Orchidées 32-33: f. 44-53. 1880.

Pseudostelis bradei Schltr., Anexos Mem. Inst. Butantan, Secç. Bot. 1(4): 38. 1922.

Pseudostelis spiralis (Lindl.) Schltr., Anexos Mem. Inst. Butantan, Secç. Bot. 1(4): 38. 1922.

Physosiphon minutiflorus Ames \& C.Schweinf., Schedul. Orchid. 8: 11. 1925.

Pleurothallis schweinfurthiana L.O.Williams, Ceiba 5: 92. 1956.

Voucher: C.W. Powell 345 (AMES).

Stelis despectans Schltr., Repert. Spec. Nov. Regni Veg. 8: 453. 1910.

Stelis chiriquensis Schltr., Beih. Bot. Centralbl. 36(2): 386. 1918.

Stelis nutantiflora Schltr., Beih. Bot. Centralbl. 36(2): 390. 1918.

Voucher: C.A. Luer 10518A (MO).

Stelis dressleri Luer, Phytologia 49: 227. 1981.

Voucher: C.A. Luer \& R.L. Dressler 1146 (SEL).

This species has been treated as synonym of Stelis morganii Dodson \& Garay (Luer 2003c). However, plants of $S$. morganii from Ecuador differ from $S$. dressleri in floral details, so we prefer to treat $S$. dressleri as a separate species. No vouchers that match the concept of $S$. morganii have been seen from Panama.

Stelis falcatiloba (Ames) Bogarín \& Serracín, comb. nov.

Bas.: Pleurothallis falcatiloba Ames, Proc. Biol. Soc. Wash. 34: 152. 1921.

VOUCHER: P. Killip 3540 (AMES).
This species should be treated in Stelis s.l. instead of Pleurothallis (Pridgeon et al. 2001).

Stelis fortunae (Luer \& Dressler) Pridgeon \& M.W.Chase, Lindleyana 16: 263. 2001.

Pleurothallis fortunae Luer \& Dressler, Lindleyana 6: 97.1991.

Dracontia fortunae (Luer \& Dressler) Luer, Monogr. Syst. Bot. Missouri Bot. Gard. 95: 257. 2004.

Voucher: R.L. Dressler s.n. (SEL).

Stelis furculifera (Dressler \& Bogarín) Bogarín, comb. nov.

Bas.: Condylago furculifera Dressler \& Bogarín, Harvard Pap. Bot. 12: 2. 2007.

Voucher: R.L. Dressler 6835 (PMA).

This combination is needed in order to threat this species in Stelis s.l. (Pridgeon et al. 2001).

Stelis gelida (Lindl.) Pridgeon \& M.W.Chase, Lindleyana 16: 263. 2001.

Pleurothallis gelida Lindl., Edwards's Bot. Reg. 27(Misc.): 91. 1841.

Humboldtia gelida (Lind1.) Kuntze, Revis. Gen. P1. 2: 667. 1891.

Specklinia gelida (Lindl.) Luer, Monogr. Syst. Bot. Missouri Bot. Gard. 95: 260. 2004.

Niphantha gelida (Lindl.) Luer, Monogr. Syst. Bot. Missouri Bot. Gard. 120: 154. 2010.

Pleurothallis univaginata Lindl., Ann. Mag. Nat. Hist., ser. 3, 1: 326. 1858.

Pleurothallis polyliria Endrés \& Rchb.f., Gard. Chron. 1871: 1483. 1871.

Anathallis hebesepala Barb.Rodr., Gen. Spec. Orchid. 2: 75.1881.

Humboldtia polyliria (Endrés \& Rchb.f.) Kuntze, Revis. Gen. P1. 2: 668. 1891.

Humboldtia univaginata (Lindl.) Kuntze, Revis. Gen. Pl. 2: 668. 1891.

Pleurothallis puberula Rolfe, Bull. Misc. Inform. Kew 1893: 169. 1893.

Pleurothallis hebesepala (Barb.Rodr.) Cogn. in C.F.P.von Martius \& auct. suc. (eds.), Fl. Bras. 3(4): 568. 1896.

Pleurothallis chiquindensis F.Lehm. \& Kraenzl., Bot. Jahrb. Syst. 26: 438. 1899.

Pleurothallis tenuispica Schltr., Repert. Spec. Nov. Regni Veg. 14: 133. 1915.

Pleurothallis chiriquensis Schltr., Repert. Spec. 
Nov. Regni Veg. Beih. 17: 19. 1922.

Pleurothallis pidax Luer, Selbyana 5: 174. 1979. Anathallis pidax (Luer) Pridgeon \& M.W.Chase, Lindleyana 16: 250. 2001.

Specklinia pidax (Luer) Luer, Monogr. Syst. Bot.

Missouri Bot. Gard. 95: 263. 2004.

Niphantha pidax (Luer) Luer, Monogr. Syst. Bot.

Missouri Bot. Gard. 120: 154. 2010.

Voucher: C.W. Powell 227 (AMES).

Stelis gigantea Pridgeon \& M.W.Chase, Lindleyana 17: 99. 2002.

Pleurothallis powellii Schltr., Repert. Spec. Nov. Regni Veg. Beih. 17: 22. 1922.

Stelis powellii (Schltr.) Pridgeon \& M.W.Chase, Lindleyana 16: 265. 2001., nom. illeg.

Dracontia powellii (Schltr.) Luer, Monogr. Syst.

Bot. Missouri Bot. Gard. 95: 257. 2004.

VOuCHER: C.W. Powell 146 (MO).

Stelis glossula Rchb.f., Gard. Chron. 1870: 1373. 1870.

Stelis inaequalis Ames, Schedul. Orchid. 4: 12. 1923.

Apatostelis inaequalis (Ames) Garay, Bot. Mus.

Leaff. 27: 189. 1979.

VOUCHER: C.A. Luer 9505 (MO).

Stelis gnoma Pridgeon \& M.W.Chase, Lindleyana 17: 99. 2002.

Salpistele parvula Luer \& Dressler, Monogr. Syst.

Bot. Missouri Bot. Gard. 39: 132. 1991.

Stelis parvula (Luer \& Dressler) Pridgeon \&

M.W.Chase, Lindleyana 16: 265. 2001., nom. illeg.

Voucher: R.L. Dressler 5832 (MO).

Stelis gracilifolia C.Schweinf., Bot. Mus. Leafl. 17: 38. 1955.

Apatostelis gracilifolia (C.Schweinf.) Garay, Bot.

Mus. Leafl. 27: 188. 1979.

VOUCHER: not seen, cited by Luer (2003).

Stelis gracilis Ames, Orchidaceae 2: 266. 1908.

Stelis panamensis Schltr., Beih. Bot. Centralbl. 36(2): 391. 1918.

VOUCHER: H. Pittier 4490 (AMES).

Stelis guttata (Luer) Pridgeon \& M.W.Chase, Lindleyana 16: 263. 2001.

Pleurothallis guttata Luer, Selbyana 3: 116. 1976.

Elongatia guttata (Luer) Luer, Monogr. Syst. Bot.

Missouri Bot. Gard. 95: 257. 2004.

VOUCHER: R.L. Dressler 3086 (SEL).
Stelis hymenantha Schltr., Repert. Spec. Nov. Regni Veg. 10: 291. 1912.

Stelis cuspidilabia Schltr., Repert. Spec. Nov. Regni Veg. Beih. 19: 17. 1923.

Stelis seleniglossa Schltr., Repert. Spec. Nov. Regni Veg. Beih. 19: 97. 1923.

Voucher: C.A. Luer \& H.P. Butcher 1240 (SEL).

Stelis immersa (Linden \& Rchb.f.) Pridgeon \& M.W.Chase, Lindleyana 16: 263. 2001.

Pleurothallis immersa Linden \& Rchb.f., Bonplandia (Hannover) 3: 224. 1855.

Humboldtia immersa (Linden \& Rchb.f.) Kuntze, Revis. Gen. P1. 2: 667. 1891.

Specklinia immersa (Linden \& Rchb.f.) Luer, Monogr. Syst. Bot. Missouri Bot. Gard. 95: 261. 2004.

Effusiella immersa (Linden \& Rchb.f.) Luer, Monogr. Syst. Bot. Missouri Bot. Gard. 112: 106. 2007.

Pleurothallis krameriana Rchb.f., Hamburger Garten- Blumenzeitung 21: 294. 1865.

Humboldtia krameriana (Rchb.f.) Kuntze, Revis. Gen. P1. 2: 667. 1891.

Pleurothallis lasiosepala Schltr., Repert. Spec. Nov. Regni Veg. 11: 43. 1912.

Pleurothallis calerae Schltr., Repert. Spec. Nov. Regni Veg. Beih. 19: 184.1923.

Voucher: C.A. Luer \& H. Butcher 1231 (SEL).

Stelis imraei (Lindl.) Pridgeon \& M.W.Chase, Lindleyana 16: 263. 2001.

Pleurothallis imraei Lindl., Fol. Orchid. 9: 9. 1859.

Humboldtia imraei (Lindl.) Kuntze, Revis. Gen. Pl. 2: 667. 1891.

Specklinia imraei (Lindl.) Luer, Monogr. Syst. Bot. Missouri Bot. Gard. 95: 261. 2004.

Effusiella imraei (Lindl.) Luer, Monogr. Syst. Bot. Missouri Bot. Gard. 112: 106. 2007.

Pleurothallis vaginata Schltr., Repert. Spec. Nov. Regni Veg. Beih. 19: 197. 1923.

Pleurothallis umbraticola Schltr., Repert. Spec. Nov. Regni Veg. 27: 56. 1929.

Stelis vaginata (Schltr.) Pridgeon \& M.W.Chase, Lindleyana 16: 267. 2001.

Specklinia vaginata (Schltr.) Luer, Monogr. Syst. Bot. Missouri Bot. Gard. 95: 264. 2004.

Effusiella vaginata (Schltr.) Luer, Monogr. Syst. Bot. Missouri Bot. Gard. 112: 107. 2007. 
Pleurothallis imraei var. vaginata (Schltr.) O.Gruss \& M.Wolff, Orchid. Atlas: 359. 2007.

VOUCHER: P.H. Allen 3808 (K).

Stelis isthmi Schltr., Repert. Spec. Nov. Regni Veg. Beih. 17: 16. 1922.

Voucher: C.W. Powell 232 (MO).

Stelis lamprophylla (Schltr.) Karremans, Lankesteriana 13(3): 329. 2014.

Pleurothallis lamprophylla Schltr., Repert. Spec.

Nov. Regni Veg. 15: 205. 1918.

Pleurothallis dolichopus Schltr., Repert. Spec.

Nov. Regni Veg. 10(257-259): 394. 1912.

Anathallis dolichopus (Schltr.) Pridgeon \&

M.W.Chase, Lindleyana 16: 248. 2001.

Specklinia dolichopus (Schltr.) Luer, Monogr. Syst.

Bot. Missouri Bot. Gard. 95: 260. 2004.

Pleurothallis dolichopus var. bradeorum Schltr.,

Repert. Spec. Nov. Regni Veg. Beih. 19: 106. 1923.

Voucher: R.E. Woodson, Jr. \& R.W. Schery 392 (MO).

Stelis lankesteri Ames, Schedul. Orchid. 3: 4. 1923.

VOUCHER: H. van der Werff 22211 (MO).

Stelis leucopogon Rchb. f., Beitr. Orchid.-K. C. Amer. 95-96, t. 9, f. I, 1-4. 1866.

VOUCHER: R.L. Dressler 5358 (PMA).

Stelis listerophora (Schltr.) Pridgeon \& M.W.Chase, Lindleyana 16: 264. 2001.

Pleurothallis listerophora Schltr., Repert. Spec.

Nov. Regni Veg. 3: 107. 1906.

Specklinia listerophora (Schltr.) Luer, Monogr.

Syst. Bot. Missouri Bot. Gard. 95: 261. 2004.

Effusiella listerophora (Schltr.) Luer, Monogr.

Syst. Bot. Missouri Bot. Gard. 112: 106. 2007.

Pleurothallis campicola Luer, Selbyana 5: 161. 1979.

Voucher: C. Luer et al. 1830 (SEL).

Stelis loculifera Luer, Phytologia 49: 229. 1981.

Voucher: C. Luer et al. 1614 (SEL).

Stelis longipetiolata Ames, Schedul. Orchid. 1: 6. 1922.

Voucher: W.R. Maxon 5460 (US).

Stelis maculata Pridgeon \& M.W.Chase, Lindleyana 17: 99. 2002.

Salpistele lutea Dressler, Orquideología 14: 8. 1979.

Stelis lutea (Dressler) Pridgeon \& M.W.Chase, Lindleyana 16: 264. 2001., nom. illeg.
VOUCHER: R.L. Dressler 5125 (US).

Stelis maduroi Luer \& Sijm, Monogr. Syst. Bot. Missouri Bot. Gard. 112: 111. 2007.

VOUCHER: T. Sijm 220728 (MO).

Stelis maxonii Schltr., Beih. Bot. Centralbl. 36(2): 389. 1918.

VOUCHER: W.R. Maxon 5697 (AMES).

Stelis megachlamys (Schltr.) Pupulin, Lankesteriana 4: 74. 2002.

Pleurothallis megachlamys Schltr., Repert. Spec.

Nov. Regni Veg. Beih. 19: 108. 1923.

Stelis megachlamys (Schltr.) Pridgeon \& M.W.Chase, Lindleyana 17(2): 99-100. 2002.

Pleurothallis tuerckheimii Schltr., Repert. Spec.

Nov. Regni Veg. 10: 292. 1912.

Stelis tuerckheimii (Schltr.) Pridgeon \& M.W.Chase,

Lindleyana 16: 267. 2001., nom. illeg.

Stelis megachlamys subsp. teotepecensis Soto

Arenas, Icon. Orchid. 5-6: t. 685. 2002 [2003].

Dracontia tuerckheimii (Schltr.) Luer, Monogr.

Syst. Bot. Missouri Bot. Gard. 95: 257. 2004.

Dracontia tuerckheimii f. viridiflavens Roeth \&

Baumbach, Orchidee (Hamburg) 58: 98. 2007.

Voucher: T.B. Croat 26347 (SEL).

Stelis meganthera Luer, Selbyana 22: 122. 2001.

VOUCHER: C.A. Luer et al. 1453 (SEL).

Stelis megapetala Luer, Selbyana 22: 124. 2001.

VOUCHER: C.A. Luer \& H. Butcher 1264 (SEL).

Stelis micragrostis Schltr., Repert. Spec. Nov. Regni

Veg. Beih. 19: 95. 1923.

Voucher: C.A. Luer et al. 10567 (SEL).

Stelis microchila Schltr., Repert. Spec. Nov. Regni Veg. 9: 289. 1911.

Stelis barbata Rolfe, Bull. Misc. Inform. Kew 1913: 141. 1913.

Stelis cinerea Schltr., Beih. Bot. Centralbl. 36(2): 444. 1918.

Stelis costaricensis Schltr., Beih. Bot. Centralbl. 36(2): 388. 1918., nom. illeg.

Stelis bryophila Schltr., Repert. Spec. Nov. Regni

Veg. Beih. 19: 16. 1923.

Voucher: C.A. Luer 1020 (SEL).

Stelis montana L.O.Williams, Ann. Missouri Bot. Gard. 27: 272. 1940.

Apatostelis montana (L.O.Williams) Garay, Bot.

Mus. Leafl. 27: 190. 1979.

VOUCHER: P.H. Allen 1463 (MO). 
Stelis montis-mortense (Karremans \& Bogarín) Bogarín \& Karremans, comb. nov.

Bas.: Dracontia montis-mortense Karremans \& Bogarín, Syst. Bot. 38(2) 307-309. 2013.

Voucher: R.L. Dressler 5089 (PMA).

This combination is needed in order to threat this species in Stelis s.l. (Pridgeon et al. 2001).

Stelis mystax (Luer) Pridgeon \& M.W.Chase, Lindleyana 16: 264. 2001.

Pleurothallis mystax Luer, Selbyana 3(1-2): 146, f. 176. 1976.

Mystacorchis mystax (Luer) Szlach. \& Marg., Polish Bot. J. 46(2): 117. 2001.

VOUCHER: C.A. Luer \& R.L. Dressler 1261 (SEL).

Stelis oscitans Luer, Lindleyana 11: 105. 1996.

Voucher: C.A. Luer et al. 10575 (MO).

Stelis pardipes Rchb.f., Beitr. Orchid.-K. C. Amer.: 96. 1866.

Stelis triseta var. pardipes (Rchb.f.) C.Schweinf., Bot. Mus. Leafl. 15: 24. 1951.

Stelis longicuspis Schltr., Repert. Spec. Nov. Regni Veg. 9: 28. 1910.

Stelis albertii Schltr., Repert. Spec. Nov. Regni Veg. Beih. 19: 170. 1923.

Stelis vagans Schltr., Repert. Spec. Nov. Regni Veg. 27: 48. 1929., nom. illeg.

VOUCHER: C.A. Luer 1039 (MO).

Stelis parvula Lindl., Fol. Orchid. 8: 7. 1859.

Stelis bidentata Schltr., Repert. Spec. Nov. Regni Veg. 10: 358. 1912.

Voucher: C.A. Luer et al. 10570 (MO).

Stelis pilosa Pridgeon \& M.W.Chase, Lindleyana 17: 100. 2002.

Pleurothallis amparoana Schltr., Repert. Spec. Nov. Regni Veg. Beih. 19: 23. 1923.

Stelis amparoana (Schltr.) Pridgeon \& M.W.Chase,

Lindleyana 16: 261. 2001., nom. illeg.

Specklinia amparoana (Schltr.) Luer, Monogr.

Syst. Bot. Missouri Bot. Gard. 95: 258. 2004.

Effusiella amparoana (Schltr.) Luer, Monogr. Syst.

Bot. Missouri Bot. Gard. 112: 106. 2007.

Voucher: S. Knapp \& M.R. Vodicka 5504 (MO).

Stelis piperina Lindl., Fol. Orchid. 8: 17. 1859.

VOUCHER: C.A. Luer 14828 (MO).

Stelis pompalis (Ames) Pridgeon \& M.W.Chase, Lindleyana 16: 265. 2001.

Pleurothallis pompalis Ames, Schedul. Orchid. 7:
23. 1924.

Unciferia pompalis (Ames) Luer, Monogr. Syst. Bot. Missouri Bot. Gard. 95: 265. 2004.

Voucher: C.A. Luer et al. 1387 (SEL).

Stelis powellii Schltr., Repert. Spec. Nov. Regni Veg. Beih. 17: 16. 1922.

VOucher: C.W. Powell 234 (AMES).

Stelis praemorsa Schltr., Repert. Spec. Nov. Regni Veg. Beih. 17: 17. 1922.

Voucher: C.W. Powell 247 (AMES).

Stelis pulchella Kunth in F.W.H.von Humboldt, A.J.A.Bonpland \& C.S.Kunth, Nov. Gen. Sp. 1: 364. 1816.

Pleurothallis pulchella (Kunth) Lindl., Gen. Sp. Orchid. P1.: 7. 1830.

Humboldtia pulchella (Kunth) Kuntze, Revis. Gen. P1. 2: 668. 1891.

Crocodeilanthe pulchella (Kunth) Luer, Monogr. Syst. Bot. Missouri Bot. Gard. 95: 256. 2004.

Pleurothallis sphenochila Lindl., Fol. Orchid. 9: 22. 1859.

Pleurothallis naraniensis Rchb.f., Beitr. Orchid.-K. C. Amer.: 96. 1866.

Humboldtia naraniensis (Rchb.f.) Kuntze, Revis. Gen. Pl. 2: 668. 1891.

Humboldtia sphenochila (Lindl.) Kuntze, Revis. Gen. Pl. 2: 668. 1891.

Pleurothallis ecuadorensis Schltr., Repert. Spec. Nov. Regni Veg. 14: 129. 1915.

Pleurothallis pteroglossa Schltr., Repert. Spec. Nov. Regni Veg. Beih. 7: 112. 1920.

Pleurothallis lloensis Schltr., Repert. Spec. Nov. Regni Veg. Beih. 8: 59. 1921.

Pleurothallis bogotensis Schltr., Repert. Spec. Nov. Regni Veg. Beih. 27: 162. 1924., nom. illeg.

Pleurothallis tepuiensis Carnevali \& I.Ramírez, Novon 3: 121. 1993.

Crocodeilanthe tepuiensis (Carnevali \& I.Ramírez) Carnevali \& I.Ramírez in O.Hokche, P.E.Berry \& O.Huber (eds.), Nuevo Cat. Fl. Vasc. Venezuela: 758. 2008.

VOUCHER: P.H. Allen 2245 (AMES).

Stelis purpurascens A.Rich. \& Galeotti, Ann. Sci. Nat., Bot., III, 3: 18. 1845.

Stelis bourgeaui Schltr., Repert. Spec. Nov. Regni Veg. 9: 284. 1911.

Stelis curvata Schltr., Repert. Spec. Nov. Regni 
Veg. 10: 358. 1912.

Stelis carioi Schltr., Repert. Spec. Nov. Regni Veg. 15: 202. 1918.

Stelis fulva Schltr., Beih. Bot. Centralbl. 37(2): 388. 1918.

Stelis purpusii Schltr., Beih. Bot. Centralbl. 36(2): 391. 1918.

Stelis alfaroi Ames \& C.Schweinf., Schedul. Orchid. 8: 14. 1925.

Stelis chiapensis Solano, Orquídea (Mexico City), n.s., 13: 23. 1993.

Stelis thermatica Luer \& Dodson, Monogr. Syst.

Bot. Missouri Bot. Gard. 112: 53. 2007.

VOUCHER: H. Pittier 3242 (AMES).

Stelis purpurea (Ruiz \& Pav.) Willd., Sp. Pl. 4: 140. 1805.

Humboldtia purpurea Ruiz \& Pav., Syst. Veg. Fl. Peruv. Chil.: 235. 1798.

Pleurothallis purpurea (Ruiz \& Pav.) Schltr., Repert. Spec. Nov. Regni Veg. Beih. 9: 142. 1921., nom. illeg.

VOUCHER: R.L. Dressler 4376 (PMA).

Stelis sanchoi Ames, Schedul. Orchid. 4: 13. 1923.

Voucher: C.A. Luer 985 (SEL).

Stelis sarcophylla Luer, Phytologia 49: 238. 1981.

Voucher: C.A. Luer \& H. Butcher 1245 (SEL).

Stelis segoviensis (Rchb.f.) Pridgeon \& M.W.Chase, Lindleyana 16: 266. 2001.

Pleurothallis segoviensis Rchb.f., Bonplandia (Hannover) 3: 223. 1855.

Humboldtia segoviensis (Rchb.f.) Kuntze, Revis. Gen. P1. 2: 668. 1891.

Unciferia segoviensis (Rchb.f.) Luer, Monogr. Syst. Bot. Missouri Bot. Gard. 95: 265. 2004.

Pleurothallis wercklei Schltr., Repert. Spec. Nov. Regni Veg. 9: 213. 1911.

Pleurothallis johannis Schltr., Repert. Spec. Nov. Regni Veg. 17: 140. 1921.

Pleurothallis amethystina Ames, Schedul. Orchid. 4: 14. 1923.

Pleurothallis melicoides Schltr., Repert. Spec. Nov. Regni Veg. Beih. 19: 24. 1923.

Pleurothallis vinacea Ames, Schedul. Orchid. 6: 69. 1923.

VOUCHER: R.L. Dressler 5507 (PMA).

Stelis semperflorens Luer, Phytologia 49: 238. 1981.

Voucher: C.A. Luer et al. 966 (SEL).
Stelis skutchii Ames, Bot. Mus. Leafl. 6: 17. 1938.

Apatostelis skutchii (Ames) Garay, Bot. Mus. Leafl. 27: 190. 1979.

VOUCHER: M.E. Davidson 270 (MO).

Stelis spathulata Luer, Phytologia 49: 238. 1981.

Stelis tricuspis Schltr., Repert. Spec. Nov. Regni

Veg. 3: 276. 1907.

VOUCHER: M.A. Blanco et al. 2833 (FLAS).

Stelis standleyi Ames, Schedul. Orchid. 9: 21. 1925.

Apatostelis standleyi (Ames) Garay, Bot. Mus. Leafl. 27: 191. 1979.

Stelis persimilis Ames, Bot. Mus. Leafl. 2: 14. 1934.

Apatostelis persimilis (Ames) Garay, Bot. Mus. Leafl. 27: 190. 1979.

Voucher: C.A. Luer et al. 1276 (SEL).

Stelis storkii Ames, Bot. Mus. Leafl. 3: 54. 1935.

VOUCHER: P.H. Allen 1828 (MO).

Stelis superbiens Lindl., Fol. Orchid. 8: 8. 1859.

Stelis cascajalensis Ames, Schedul. Orchid. 4: 11. 1923.

Stelis eximia Ames, Schedul. Orchid. 6: 54. 1923.

Stelis propinqua Ames, Schedul. Orchid. 6: 55. 1923.

Stelis convallarioides Garay, Canad. J. Bot. 34: 351. 1956.

VOUCHer: C.W. Powell 312 (AMES).

Stelis tenuissima Schltr., Repert. Spec. Nov. Regni Veg. 15: 204. 1918.

Voucher: T.B. Croat 37139 (MO).

Stelis thecoglossa Rchb.f., Beitr. Orchid.-K. C. Amer.: 93. 1866.

Voucher: C.A. Luer \& H.P. Butcher 1238 (SEL).

Stelis thymochila (Luer) Pridgeon \& M.W.Chase, Lindleyana 16: 267. 2001.

Pleurothallis thymochila Luer, Selbyana 3: 398. 1977.

Dracontia thymochila (Luer) Luer, Monogr. Syst. Bot. Missouri Bot. Gard. 95: 257. 2004.

VOUCHER: C.A. Luer 992 (SEL).

Stelis transversalis Ames, Bot. Mus. Leafl. 2: 91. 1934.

Voucher: C.A. Luer \& H.P. Butcher 1384 (SEL).

Stelis triaristata Luer, Selbyana 5: 193. 1979.

Voucher: C.A. Luer et al. 2109 (SEL).

Stelis umbelliformis Hespenh. \& Dressler, Orquideología 6: 21. 1971. 
Voucher: H.A. Hespenheide \& R.L. Dressler 2266 (US).

Stelis cf. uniflora Luer \& Hirtz, Monogr. Syst. Bot. Missouri Bot. Gard. 112: 57. 2007.

Voucher: S. Laube et al. 531 (PMA).

This species is allied to $S$. uniflora from Ecuador. In Costa Rica there are at least more than one species related to $S$. uniflora. The group is currently in study (Bogarín et al. in prep.). This is the first record of this group in Panama.

Stelis veraguasensis Luer, Selbyana 22: 126. 2001.

Voucher: C.A. Luer \& R. Dressler 1235 (SEL).

Stelis vestita Ames, Schedul. Orchid. 6: 56. 1923.

Voucher: S.A. Mori \& J.A. Kallunki 5988 (MO).

Stelis vulgaris Pridgeon \& M.W.Chase, Lindleyana 17: 100. 2002.

Pleurothallis floribunda Poepp. \& Endl., Nov. Gen. Sp. P1. 1: 48. 1836.

Humboldtia floribunda (Poepp. \& Endl.) Kuntze, Revis. Gen. P1. 2: 667. 1891.

Stelis floribunda (Poepp. \& Endl.) Pridgeon \& M.W.Chase, Lindleyana 16: 263. 2001., nom. illeg.

Crocodeilanthe floribunda (Poepp. \& Endl.) Luer, Monogr. Syst. Bot. Missouri Bot. Gard. 95: 256. 2004.

Pleurothallis pittieri Schltr., Repert. Spec. Nov. Regni Veg. 3: 247. 1907.

Voucher: S.Knapp \& R.L. Dressler 5411 (MO).

Stelis wagneri (Schltr.) Pridgeon \& M.W.Chase, Lindleyana 16: 267. 2001.

Pleurothallis wagneri Schltr., Repert. Spec. Nov. Regni Veg. 17: 141. 1921.

Unciferia wagneri (Schltr.) Luer, Monogr. Syst. Bot. Missouri Bot. Gard. 95: 265. 2004.

Pleurothallis aguilarii Ames, Schedul. Orchid. 9: 28. 1925.

Voucher: M. Wagner 25 (AMES).

Stelis williamsii Ames, Orchidaceae 7: 133. 1922.

VOUCHER: R.S. Williams 970 (AMES).

\section{STENORRHYnChos Rich. ex Spreng.}

Stenorrhynchos speciosum (Jacq.) Rich. ex Spreng., Syst. Veg. 3: 709. 1826.

Serapias speciosa (Jacq.) J.F.Gmel., Syst. Nat. ed. 13[bis]: 59. 1791.

Neottia speciosa Jacq., Icon. Pl. Rar. 3: t. 600. 1794.
Ibidium speciosum (Jacq.) Salisb., Trans. Hort. Soc. London 1: 291. 1812.

Stenorrhynchos speciosum (Jacq.) Rich., De Orchid. Eur.: 37. 1817.

Spiranthes speciosa (Jacq.) Lilja, Handb. Odl. Vext. Fl. Kult. 1: 113. 1842, nom. illeg.

Gyrostachys speciosa (Jacq.) Kuntze, Revis. Gen. P1. 2: 664. 1891.

Stenorrhynchos nutans Kunth \& C.D.Bouché, Index Seminum (B) 1848: 12. 1848.

Spiranthes colorata N.E.Br., Gard. Chron., n.s., 19: 210. 1883., nom. illeg.

Spiranthes colorata var. maculata N.E.Br., Gard. Chron., n.s., 19: 210. 1883.

Spiranthes colorans Hemsl., Biol. Cent.-Amer., Bot. 3: 300. 1884.

Ibidium coloratum House in L.H.Bailey, Stand. Cycl. Hort. 3: 1636. 1915.

Stenorrhynchos millei Schltr., Repert. Spec. Nov. Regni Veg. 15: 51.1917.

Spiranthes nutans (Kunth \& C.D.Bouché) Garay \& Dunst., Venez. Orchids Ill. 6: 39. 1976.

Stenorrhynchos speciosum var. nutans (Kunth \&

C.D.Bouché) Szlach., Fragm. Florist. Geobot. 39: 419. 1994.

Voucher: T.B. Croat 48846 (MO).

Stenotyla Dressler

Stenotyla lankesteriana (Pupulin) Dressler, Lankesteriana 5: 96. 2005.

Chondrorhyncha lankesteriana Pupulin, Lindleyana 15: 21. 2000.

Voucher: not seen, cited by Dressler (2003c).

Stenotyla panamensis Pupulin, Orchid Rev. 117: 162. 2009.

VOUCHER: F. Pupulin 5867 (PMA).

Stenotyla picta (Rchb.f.) Dressler, Lankesteriana 5: 96. 2005.

Warczewiczella picta Rchb.f., Gard. Chron., n.s., 20: 8. 1883 .

Zygopetalum pictum (Rchb. f.) Rchb.f., Gard. Chron., n.s., 20: 8. 1883.

Cochleanthes picta (Rchb. f.) Garay, Bot. Mus. Leafl. 21(9): 256. 1967.

Chondrorhyncha picta (Rchb. f.) Senghas, Orchidee (Hamburg) 41(3): 94. 1990.

Warczewiczella caloglossa Schltr., Repert. Spec. 
Nov. Regni Veg. 12: 216. 1913.

Chondrorhyncha caloglossa (Schltr.) P.H. Allen,

Ann. Missouri Bot. Gard. 36(1): 85. 1949.

Chondrorhyncha estrellensis Ames, Schedul.

Orchid. 4: 54. 1923.

VOUCHER: P.H. Allen 1462 (MO).

\section{Systeloglossum Schltr.}

Systeloglossum costaricense Schltr., Repert. Spec. Nov. Regni Veg. Beih. 19: 252. 1923.

Voucher: A. Maduro \& E. Olmos 320 (MO).

Systeloglossum panamense Dressler \& N.H.Williams, Amer. Orchid Soc. Bull. 39: 326. 1970.

Voucher: R.L. Dressler \& N.H. Williams 3642 (US).

\section{Telipogon Kunth}

\section{Telipogon sp.}

VOuCHER: S. Laube et al. 493 (PMA).

This Voucher belongs to the group of Telipogon formerly treated under Stellilabium. It seems different from the other species of the genus recorded in Panama (Williams et al. 2005). A comparison with the Costa Rican species of Telipogon is needed to confirm its identity.

Telipogon acicularis (Dressler) N.H.Williams \& Dressler, Lankesteriana 5: 168. 2005.

Stellilabium aciculare Dressler, Harvard Pap. Bot. 4: 471.1999.

Voucher: R.L. Dressler 3617 (AMES).

Telipogon biolleyi Schltr., Repert. Spec. Nov. Regni Veg. 9: 293. 1911.

VOUCHER: H.P. Butcher s.n (MO).

Telipogon bombiformis Dressler, Orchids 72: 114 2003.

Voucher: A. Maduro \& E. Olmos s.n. (MO).

Telipogon butcheri Dodson \& R.Escobar, Orquideología 18: 278. 1993.

VOUCHER: H.P. Butcher s.n. (MO).

Telipogon butchii N.H.Williams \& Dressler, Lankesteriana 5: 169. 2005.

Stellilabium butcheri Dressler, Harvard Pap. Bot. 4: 472.1999.

VOUCHER: H.P. Butcher 654 (SEL).

Telipogon caulescens Dressler, Orchids 72: 114. 2003.

Voucher: A. Maduro \& E. Olmos 168 (MO).

Telipogon chiriquiensis Dodson \& R.Escobar, Orquideología 18: 281. 1993.

Voucher: C.A. Luer \& H. Butcher 1208 (RPSC).
Telipogon fortunae (Dressler) N.H.Williams \& Dressler, Lankesteriana 5: 169. 2005.

Stellilabium fortunae Dressler, Harvard Pap. Bot. 4: 473. 1999.

VOUCHER: R.L. Dressler 5464 (MO).

Telipogon fractus Dressler, Orchideen J. 14: 12. 2007. VOuCHER: A. Maduro \& E. Olmos 243 (MO).

Telipogon griesbeckii Dressler, Orchids 72: 115. 2003. Voucher: A. Maduro \& E. Olmos 169 (MO).

Telipogon maduroi Dressler, Orchids 72: 116. 2003.

VOucher: A. Maduro \& E. Olmos 190 (MO).

Telipogon medusae Dressler, J. Orchideenfr. 13: 211. 2006.

Voucher: A. Maduro \& E. Olmos 209 (MO).

Telipogon monticola L.O.Williams, Fieldiana, Bot. 32: 202. 1970.

Voucher: A. Maduro \& E. Olmos 241 (MO).

Telipogon morii (Dressler) N.H.Williams \& Dressler, Lankesteriana 5: 170. 2005.

Stellilabium morii Dressler, Harvard Pap. Bot. 4: 473. 1999.

Voucher: S. Mori \& J. Kallunki 5813 (MO).

Telipogon nobilis Dressler, Orchideen J. 14: 12. 2007. Voucher: A. Maduro \& E. Olmos 305 (PMA).

Telipogon nunezii Dressler, Orchideen J. 14: 14. 2007. Voucher: A. Maduro \& J. Nuñez 249 (PMA).

Telipogon olmosii Dressler, J. Orchideenfr. 13: 212. 2006.

Voucher: A. Maduro \& E. Olmos 213 (MO).

Telipogon panamensis Dodson \& R.Escobar, Orquideología 18: 284. 1993.

Voucher: R.E. Woodson \& R.W. Schery 964 (MO).

Telipogon parvulus C.Schweinf., Bot. Mus. Leafl. 4: 123. 1937.

Voucher: A. Maduro \& E. Olmos 193 (PMA).

Telipogon personatus Dressler, J. Orchideenfr. 13: 214. 2006.

Voucher: A. Maduro \& E. Olmos 214 (MO).

Telipogon reticulatus Dressler, Orchideen J. 14: 14. 2007.

VOUCHER: A. Maduro \& E. Olmos 310 (MO).

Telipogon seibertii Dodson \& R.Escobar, Orquideología 18: 288. 1993.

Voucher: R.E. Woodson et al. 961 (MO).

TeusCheria Garay

Teuscheria horichiana Jenny \& Braem, Orchid Digest 51: 187.1987. 
Voucher: R.L. Dressler \& J.T. Atwood 6245 (PMA).

Teuscheria pickiana (Schltr.) Garay, Rhodora 61: 41. 1959.

VOUCHER: H.P. Butcher s.n. (MO).

Trichocentrum Poepp. \& Endl.

Trichocentrum ascendens (Lindl.) M.W.Chase \& N.H.Williams, Lindleyana 16: 137. 2001.

Oncidium ascendens Lindl., Edwards's Bot. Reg. 28: t. 4. 1842.

Stilifolium ascendens (Lindl.) Königer \& D.Pongratz, Arcula 7: 186. 1997.

Cohniella ascendens (Lind1.) Christenson, Lindleyana 14(4): 177. 1999.

Voucher: Z. Serracin 83 (UCH).

Trichocentrum capistratum Linden \& Rchb.f., Gard. Chron. 1871: 1257. 1871.

Trichocentrum panamense Rolfe, Bull. Misc. Inform. Kew 1913: 341. 1913.

VOUCHER: L.J. Lipscomb s.n. (K).

Trichocentrum crispiflorum (Schltr.) Bogarín, stat. nov.

Bas.: Oncidium oerstedii var. crispiflorum Schltr., Repert. Spec. Nov. Regni Veg. Beih. 17: 85. 1922.

Lophiaris crispiflora (Schltr.) Balam \& Cetzal, J.

Torrey Bot. Soc. 139: 14. 2012.

Voucher: C.W. Powell 1 (AMES).

This name is needed in order to treat this species in Trichocentrum s.l. (Williams et al. 2001).

Trichocentrum helicanthum (Kraenzl.) J.M.H.Shaw, Orchid Rev. 120(1297, Suppl.): 16. 2012.

Oncidium helicanthum Kraenzl. in H.G.A.Engler (ed.), Pflanzenr., IV, 50(80): 95. 1922.

Cohniella helicantha (Kraenzl.) Cetzal \& Carnevali, J. Torrey Bot. Soc. 137: 210. 2010.

Oncidium teres Ames \& C.Schweinf., Schedul. Orchid. 8: 78. 1925.

Stilifolium teres (Ames \& C.Schweinf.) Königer \& D.Pongratz, Arcula 7: 190. 1997.

Cohniella teres (Ames \& C.Schweinf.) Christenson, Lindleyana 14: 177. 1999.

Trichocentrum teres (Ames \& C.Schweinf.) M.W.Chase \& N.H.Williams, Lindleyana 16: 138. 2001.

Voucher: C.W. Powell 383 (AMES).
Trichocentrum lacerum (Lindl.) J.M.H.Shaw, Orchid Rev. 120(1297, Suppl.): 16. 2012.

Oncidium lacerum Lindl., Bot. Reg. 30. Misc. 38. 1844.

Oncidium stipitatum Lindl. in G.Bentham, Bot. Voy. Sulphur: 172. 1846.

Oncidium stipitatum Lindl. var. platyonyx Rchb. f., Gard. Chron. N.S. 9: 788. 1878.

Stilifolium stipitatum (Lindl.) Königer \& D.Poncischweinfgratz, Arcula 7: 189. 1997.

Cohniella stipitata (Lindl.) Christenson, Lindleyana 14: 177. 1999.

Trichocentrum stipitatum (Lindl.) M.W.Chase \& N.H.Williams, Lindleyana 16: 138. 2001.

Trichocentrum nudum subsp. stipitatum (Lindl.) Dressler \& N.H.Williams, Selbyana 24: 45. 2003.

Voucher: ex Hort. Loddigges (K).

Trichocentrum nudum (Bateman ex Lindl.) M.W.Chase \& N.H.Williams, Lindleyana 16: 138. 2001 .

Oncidium nudum Bateman ex Lindl., Edwards's Bot. Reg. 23: t. 1994. 1837.

Stilifolium nudum (Bateman ex Lindl.) Königer \& D.Pongratz, Arcula 7: 189. 1997.

Cohniella nuda (Bateman ex Lindl.) Christenson, Lindleyana 14: 177. 1999.

Oncidium ebrachiatum Ames \& C.Schweinf., Schedul. Orchid. 2: 75. 1923.

Voucher: R. S. Williams 975 (AMES).

Trichocentrum pfavii Rchb.f., Gard. Chron., n.s., 16: 70. 1881 .

Voucher: R. Pfau 80 (W).

Trichocentrum pupulinianum Bogarín \& Karremans, Lindleyana in Orchids (West Palm Beach) 82(2): 106108. 2013.

Voucher: C.A. Luer et al. 9280 (SEL).

Trichocentrum silverarum (Carnevali \& Cetzal) J.M.H.Shaw, Orchid Rev. 122(1306): 37. 2014.

Lophiaris silverarum Carnevali \& Cetzal, Phytotaxa 162(3): 166. 2014.

Voucher: G. Silvera \& B. Rodríguez s.n. (PMA).

Trichopilia Lindl.

Trichopilia amabilis Dressler, Orquideología 22: 245. 2003.

VOUCHER: R.L. Dressler 6346 (MO). 
Trichopilia eneidae Dressler, Orquideología 22: 246. 2003.

VOUCHER: R.L. Dressler 6348 (MO).

Trichopilia galeottiana A.Rich., Ann. Sci. Nat., Bot., sér. 3, 3: 26. 1845.

Voucher: A. Maduro \& E. Olmos 226 (MO).

Trichopilia leucoxantha L.O.Williams, Amer. Orchid Soc. Bull. 10: 137. 1941.

VOUCHER: P.H. Allen 2401 (AMES).

Trichopilia maculata Rchb.f., Bonplandia (Hannover) 3: 215.1855.

Trichopilia powellii Schltr., Repert. Spec. Nov.

Regni Veg. Beih. 17: 77. 1922.

VOUCHER: H.A. Behr s.n. (W).

Trichopilia marginata Henfr., Gard. Mag. Bot. 3: 185. 1851.

Trichopilia marginata var. lepida (W.G.Sm.) A.H.Kent in H.J.Veitch, Man. Orchid. P1. 9: 183. 1893.

Trichopilia marginata var. olivacea Rchb.f., Xenia Orchid. 2: 102. 1865.

Voucher: J. Warscewicz s.n. (W).

Trichopilia olmosii Dressler, Selbyana 22: 11. 2001.

VOUCHER: R.L. Dressler 6288 (MO).

Trichopilia similis Dressler, Selbyana 22: 12. 2001.

VOuCHer: R.L. Dressler 3907 (MO).

Trichopilia suavis Lindl. \& Paxton, Paxton's Fl. Gard. 1: 44.1850.

Trichopilia kienastiana Rchb.f., Gard. Chron., n.s., 20: 166. 1883.

Trichopilia suavis var. alba L.Linden \& Rodigas, Lindenia 1: 9. 1885.

Voucher: P.H. Allen 912 (AMES).

Trichopilia subulata (Sw.) Rchb.f., Flora 48: 278. 1865.

Epidendrum subulatum Sw., Prodr. Veg. Ind. Occ.: 123. 1788.

Cymbidium subulatum (Sw.) Sw., Nova Acta

Regiae Soc. Sci. Upsal. 6: 73. 1799.

Leucohyle subulata (Sw.) Schltr., (Sw.) Schltr., Orchideen Beschreib. Kult. Zücht.: 469. 1914.

Aerides subulata (Sw.) Schltr., Repert. Spec. Nov.

Regni Veg. 19: 382. 1924., nom. illeg.

Voucher: M.D. Correa \& E. Montenegro 9598 (PMA).

Trichopilia tortilis Lindl., Edwards's Bot. Reg. 22: t. 1863. 1836.

VOUCHER: W.R. Maxon 5028 (US).
Trichopilia turialbae Rchb.f., Hamburger GartenBlumenzeitung 19: 11. 1863.

VOUCHER: M. Whitten 93176 (FLAS).

\section{TrichosalPinx Luer}

Trichosalpinx arbuscula (Lind1.) Luer, Phytologia 54: 394. 1983.

Pleurothallis arbuscula Lindl., Edwards's Bot. Reg. 28(Misc.): 72. 1842.

Humboldtia arbuscula (Lindl.) Kuntze, Revis. Gen. Pl. 2: 667. 1891.

Tubella arbuscula (Lindl.) Archila, Revista Guatemal. 3(1): 49. 2000.

Pleurothallis dinotherii Rchb.f. \& Warsz., Bonplandia (Hannover) 2: 114. 1854.

Pleurothallis diptera Lindl., Fol. Orchid. 9: 44. 1859.

Pleurothallis moschata Rchb.f., Xenia Orchid. 3: 42. 1881.

Humboldtia dinotherii (Rchb.f. \& Warsz.) Kuntze, Revis. Gen. P1. 2: 667. 1891.

Humboldtia diptera (Lindl.) Kuntze, Revis. Gen. P1. 2: 667. 1891.

Humboldtia moschata (Rchb.f.) Kuntze, Revis. Gen. P1. 2: 668. 1891.

Pleurothallis lepanthoides Schltr., Repert. Spec. Nov. Regni Veg. Beih. 7: 106. 1920.

Pleurothallis tricaudata Schltr., Repert. Spec. Nov. Regni Veg. Beih. 9: 77. 1921.

Pleurothallis arbusculoides T.Hashim., Bull. Natl. Sci. Mus. Tokyo, B 4: 9. 1978.

Trichosalpinx arbusculoides (T.Hashim.) Luer, Phytologia 54: 394. 1983.

Trichosalpinx dinotherii (Rchb.f. \& Warsz.) Luer, Phytologia 54: 395. 1983.

Trichosalpinx moschata (Rchb.f.) Luer, Phytologia 54: 396. 1983.

Tubella moschata (Rchb.f.) Archila, Revista Guatemal. 3(1): 60. 2000.

Specklinia lepanthoides (Schltr.) Luer, Monogr. Syst. Bot. Missouri Bot. Gard. 95: 261. 2004.

VOUCHER: R.L. Dressler 6046 (MO).

Trichosalpinx blaisdellii (S. Watson) Luer, Phytologia 54: 394. 1983.

Pleurothallis blaisdellii S.Watson, Proc. Amer. Acad. Arts 23: 284. 1888.

Pleurothallis peraltensis Ames, Schedul. Orchid. 
6: 65.1923.

Pleurothallis standleyi Ames, Schedul. Orchid. 9: 37. 1925.

Trichosalpinx tamayoana Soto Arenas, Orquídea (Mexico City), n.s., 10: 264. 1987.

Trichosalpinx lankesteriana Luer, Lindleyana 11: 108. 1996.

VOuCHER: M.E. Davidson 169 (MO).

Trichosalpinx carinilabia (Luer) Luer, Phytologia 54: 394. 1983.

Pleurothallis carinilabia Luer, Selbyana 3: 256. 1977.

Tubella carinilabia (Luer) Archila, Revista Guatemal. 3(1): 51. 2000.

Pleurothallis broadwayi var. tricarinata C.Schweinf., Bot. Mus. Leafl. 8(2): 42. 1940.

VOUCHER: G. McPherson 8959 (MO).

Trichosalpinx caudata Luer \& R.Escobar, Monogr. Syst. Bot. Missouri Bot. Gard. 64: 20. 1997.

Voucher: C.A. Luer \& H.P. Butcher 1246 (MO).

Trichosalpinx cedralensis (Ames) Luer, Phytologia 54: 394. 1983.

Pleurothallis cedralensis Ames, Schedul. Orchid. 4: 18.1923.

Tubella cedralensis (Ames) Archila, Revista Guatemal. 3(1): 52. 2000.

Pleurothallis myrtillus Schltr., Repert. Spec. Nov. Regni Veg. Beih. 19: 25. 1923.

Voucher: T.B. Croat \& D. Porter 15685 (MO).

Trichosalpinx dressleri Luer, Monogr. Syst. Bot. Missouri Bot. Gard. 64: 54. 1997.

Tubella dressleri (Luer) Archila, Revista Guatemal. 3(1): 54. 2000.

VOUCher: R. L. Dressler 5855 (MO).

Trichosalpinx dura (Lindl.) Luer, Phytologia 54: 395. 1983.

Pleurothallis dura Lindl., Fol. Orchid. 9: 32. 1859.

Humboldtia dura (Lindl.) Kuntze, Revis. Gen. Pl. 2: 667. 1891.

Pleurothallis foliata Griseb., Fl. Brit. W. I.: 610. 1864.

Humboldtia foliata (Griseb.) Kuntze, Revis. Gen. P1. 2: 667. 1891.

Pleurothallis corazonica F.Lehm. \& Kraenzl., Bot. Jahrb. Syst. 26: 443. 1899.

Pleurothallis broadwayi Ames, Orchidaceae 2: 267. 1908.
Pleurothallis guadalupensis Cogn. in I.Urban, Symb. Antill. 6: 432. 1909.

Pleurothallis lepanthopsis Schltr., Repert. Spec. Nov. Regni Veg. 14: 386. 1916.

Pleurothallis williamsii Ames, Orchidaceae 7: 120. 1922.

Pleurothallis anomala Hoehne, Arch. Inst. Biol. Defesa Agric. 2: 43. 1929.

Pleurothallis broadwayi subsp. anomala (Hoehne) Garay, Arch. Jard. Bot. Rio de Janeiro 11: 53. 1951.

Trichosalpinx foliata (Griseb.) Luer, Phytologia 54: 395. 1983.

Tubella broadwayi (Ames) Archila, Revista Guatemal. 3(1): 51. 2000.

Tubella foliata (Griseb.) Archila, Revista Guatemal. 3(1): 56. 2000.

VOUCHER: R.S. Williams 976 (AMES).

Trichosalpinx macphersonii Luer, Monogr. Syst. Bot. Missouri Bot. Gard. 64: 66. 1997.

Tubella macphersonii (Luer) Archila, Revista Guatemal. 3(1): 59. 2000.

VOUCHER: G. McPherson 12777 (MO)

Trichosalpinx membraniflora (C.Schweinf.) Luer, Phytologia 54: 396. 1983.

Pleurothallis membraniflora C.Schweinf., Bot. Mus. Leafl. 5: 91. 1938.

Tubella membraniflora (C.Schweinf.) Archila, Revista Guatemal. 3(1): 59. 2000.

VOUCHER: C.A. Luer et al. 11393 (MO).

Trichosalpinx memor (Rchb.f.) Luer, Phytologia 54: 396. 1983.

Pleurothallis memor Rchb.f., Bonplandia (Hannover) 4: 330. 1856

Humboldtia memor (Rchb.f.) Kuntze, Revis. Gen. P1. 2: 668. 1891.

Pleurothallis brevis Schltr., Repert. Spec. Nov. Regni Veg. Beih. 19: 183. 1923.

Pleurothallis gnomonifera Ames, Schedul. Orchid. 6: 61. 1923

Trichosalpinx greenwoodiana Soto Arenas, Orquídea (Mexico City), n.s., 10: 257. 1987.

Trichosalpinx nageliana Soto Arenas, Orquídea (Mexico City), n.s., 10: 259. 1987.

VOUCHER: C.A. Luer et al. 1362 (SEL).

Trichosalpinx minutipetala (Ames \& C.Schweinf.) Luer, Phytologia 54(5): 396. 1983. 
Pleurothallis minutipetala Ames \& C.Schweinf., Schedul. Orchid. 10 (32-33). 1930.

Voucher: D. Bogarín et al. 8703 (JBL).

Trichosalpinx orbicularis (Lindl.) Luer, Phytologia 54: 396. 1983.

Specklinia orbicularis Lindl., Edwards's Bot. Reg. 25(Misc.): 31. 1839.

Pleurothallis orbicularis (Lindl.) Lindl., Edwards's Bot. Reg. 28(Misc.): 79. 1842.

Humboldtia orbicularis (Lindl.) Kuntze, Revis. Gen. Pl. 2: 668. 1891.

Pleurothallis biflora H.Focke, Tijdschr. Natuurk. Wetensch. Kunsten 2: 197. 1849.

Lepanthes orbiculata Lindl. ex Rchb.f., Xenia Orchid. 1: 152. 1856.

Humboldtia biflora (H.Focke) Kuntze, Revis. Gen. P1. 2: 667. 1891.

Pleurothallis trachytheca F.Lehm. \& Kraenzl., Bot. Jahrb. Syst. 26: 444. 1899.

Pleurothallis lancifera Schltr., Repert. Spec. Nov. Regni Veg. Beih. 27: 48. 1924.

Trichosalpinx lancifera (Schltr.) Luer, Phytologia 54: 396. 1983.

Trichosalpinx oxychilos Carnevali \& G.A.Romero, Novon 3: 21. 1993.

Voucher: C.A. Luer et al. 11394 (MO).

Trichosalpinx pergrata (Ames) Luer, Phytologia 54: 396. 1983.

Pleurothallis pergrata Ames, Schedul. Orchid. 4: 24. 1923.

Tubella pergrata (Ames) Archila, Revista Guatemal. 3(1): 62. 2000.

Pleurothallis falcipetala Schltr., Repert. Spec. Nov. Regni Veg. Beih. 27: 163. 1924.

Trichosalpinx falcipetala (Schltr.) Luer, Phytologia 54: 395. 1983.

VOUCHer: C.A. Luer 192 (SEL).

Trichosalpinx ringens Luer, Lindleyana 11: 108. 1996. Voucher: C.A. Luer et al. 10539 (MO).

Trichosalpinx rotundata (C.Schweinf.) Dressler, Novon 7: 124. 1997.

Pleurothallis rotundata C.Schweinf., Bot. Mus. Leafl. 4: 115. 1937.

Pleurothallis operculata Luer, Phytologia 49: 213. 1981. Trichosalpinx operculata (Luer) Luer, Phytologia 54: 396. 1983.

Voucher: A.A. Hunter \& P.H. Allen 561 (AMES).
Trichosalpinx tantilla (Luer) Luer, Monogr. Syst. Bot. Missouri Bot. Gard. 64: 81. 1997.

Pleurothallis tantilla Luer, Selbyana 5: 183. 1979.

Tubella tantilla (Luer) Archila, Revista Guatemal. 3(1): 65. 2000.

VOUCHER: C.A. Luer et al. 1078 (SEL).

Trichosalpinx todziae Luer, Lindleyana 11: 111. 1996. Tubella todziae (Luer) Archila, Revista Guatemal. 3(1): 66. 2000.

VOUCHER: F.L. Stevenson 53177-1 (SEL).

Trichosalpinx tropida (Luer) Luer, Phytologia 54: 398/ 1983.

Pleurothallis tropida Luer, Phytologia 49: 219. 1981.

Tubella tropida (Luer) Archila, Revista Guatemal. 3(1): 67. 2000.

VOUCHER: C. Luer \& H. Butcher 1120 (SEL).

Trigonidium Lindl.

Trigonidium egertonianum Bateman ex Lindl., Edwards's Bot. Reg. 24(Misc.): 73. 1838.

Maxillaria brachyglossa A.Rich. \& Galeotti, Ann. Sci. Nat., Bot., sér. 3, 3: 24. 1845.

Trigonidium seemannii Rchb.f. in B.Seemann, Bot. Voy. Herald: 214. 1854.

Trigonidium spatulatum Linden \& Rchb.f., Bonplandia (Hannover) 2: 280. 1854.

Trigonidium callistele Rchb.f., Hamburger GartenBlumenzeitung 16: 178. 1860.

Trigonidium brachyglossum (A.Rich. \& Galeotti) Schltr., Beih. Bot. Centralbl. 36(2): 499. 1918.

VOUCHER: Walter H. Lewis 687 (MO).

Trigonidium lankesteri Ames, Schedul. Orchid. 5: 32. 1923.

Trigonidium amparoanum Schltr., Repert. Spec. Nov. Regni Veg. Beih. 19: 61. 1923.

Voucher: G. McPherson 19522 (MO).

Trigonidium riopalenquense Dodson, Selbyana 2: 56. 1977.

VOUCHER: S. Laube et al. 382 (PMA).

\section{Triphora Nutt.}

Triphora debilis (Schltr.) Schltr., Repert. Spec. Nov. Regni Veg. 17: 139. 1921.

Pogonia debilis Schltr., Repert. Spec. Nov. Regni Veg. 15: 196. 1918.

Voucher: T.B. Croat 15567 (MO). 
Triphora gentianoides (Sw.) Nutt. ex Ames \& Schltr. in O.Ames, Orchidaceae 7: 5. 1922.

Limodorum gentianoides Sw., Prodr. Veg. Ind. Occ.: 119. 1788.

Arethusa gentianoides (Sw.) Sw., Fl. Ind. Occid. 3: 1436. 1806.

Pogonia gentianoides (Sw.) Spreng., Syst. Veg. 3: 706. 1826.

Pogonia cubensis Rchb.f., Ned. Kruidk. Arch. 4: 322. 1859.

Pogonia lutea Schltr., Repert. Spec. Nov. Regni Veg. 9: 24. 1910.

Triphora cubensis (Rchb.f.) Ames, Schedul. Orchid. 7: 35. 1924.

Triphora lutea (Schltr.) Schltr. ex Mansf., Repert. Spec. Nov. Regni Veg. Beih. 58: 7. 1930.

Triphora gentianoides f. albidaviridis P.M.Br. \& Steph.Jones, N. Amer. Native Orchid J. 15: 45. 2009.

VOUCHER: L.A. Kenoyer 250 (US)

Triphora ravenii (L.O.Williams) Garay, Bot. Mus. Leafl. 26: 3. 1978.

Pogonia ravenii L.O.Williams, Fieldiana, Bot. 32: 200. 1970.

VOUCHER: H.P. Butcher 662 (F).

Triphora wagneri Schltr., Repert. Spec. Nov. Regni Veg. 17: 139. 1921.

Pogonia wagneri (Schltr.) L.O.Williams, Ann.

Missouri Bot. Gard. 33: 19. 1946.

Voucher: M. Wagner 1778 (B, destroyed).

\section{Trisetella Luer}

Trisetella dressleri (Luer) Luer, Phytologia 47: 57. 1980.

Masdevallia dressleri Luer, Selbyana 3: 20. 1976.

Triaristella dressleri (Luer) Luer, Selbyana 2: 205. 1978.

Triaristellina dressleri (Luer) Rauschert, Feddes Repert. 94: 469. 1983.

VOUCHER: C. Luer 730 (SEL).

Trisetella tenuissima (C.Schweinf.) Luer, Phytologia 47: 58.1980.

Masdevallia tenuissima C.Schweinf., Bot. Mus. Leafl. 4: 111. 1937.

Triaristella tenuissima (C.Schweinf.) Luer, Selbyana 2: 206. 1978.
Triaristellina tenuissima (C.Schweinf.) Rauschert, Feddes Repert. 94: 469. 1983.

Masdevallia butcheri Luer, Selbyana 3: 16. 1976.

Voucher: A.A. Hunter \& P.H. Allen 587 (AMES).

Trisetella triaristella (Rchb.f.) Luer, Phytologia 47: 58. 1980.

Masdevallia triaristella Rchb.f., Gard. Chron., n.s., 6: 226.1876.

Triaristella reichenbachii Brieger ex Luer, Selbyana 2: 206. 1978.

Triaristellina triaristella (Rchb.f.) Rauschert, Feddes Repert. 94: 469. 1983.

Voucher: C.A. Luer \& R.L. Dressler 1149 (SEL).

Trisetella triglochin (Rchb.f.) Luer, Phytologia 47: 58. 1980.

Masdevallia triglochin Rchb.f., Gard. Chron., n.s., 8: 648. 1877.

Triaristella triglochin (Rchb.f.) Luer, Selbyana 2: 206. 1978.

Triaristellina triglochin (Rchb.f.) Rauschert, Feddes Repert. 94: 469. 1983.

Masdevallia trichaete Rchb.f., Gard. Chron., n.s., 20: 360. 1883.

Masdevallia tridactylites Rchb.f., Gard. Chron., n.s., 19: 784. 1883.

Masdevallia huebneri Schltr., Beih. Bot. Centralbl. 42(2): 88. 1925.

Masdevallia triseta Rchb.f. ex Kraenzl., Repert. Spec. Nov. Regni Veg. Beih. 34: 201. 1925.

Masdevallia allenii L.O.Williams, Ann. Missouri Bot. Gard. 27: 273. 1940.

Triaristella huebneri (Schltr.) Luer, Selbyana 2: 205. 1978.

Triaristella trichaete (Rchb.f.) Luer, Selbyana 2: 206. 1978.

Triaristella tridactylites (Rchb.f.) Luer, Selbyana 2: 206. 1978.

Trisetella huebneri (Schltr.) Luer, Phytologia 47: 57. 1980.

Trisetella trichaete (Rchb.f.) Luer, Phytologia 47: 58. 1980.

Trisetella tridactylites (Rchb.f.) Luer, Phytologia 47: 58. 1980.

Triaristellina huebneri (Schltr.) Rauschert, Feddes Repert. 94: 469. 1983.

Triaristellina trichaete (Rchb.f.) Rauschert, Feddes Repert. 94: 469. 1983. 
Triaristellina tridactylites (Rchb.f.) Rauschert, Feddes Repert. 94: 469. 1983.

VOUCHER: P.H. Allen 1230 (MO).

\section{TrIzeuXIs Lindl}

Trizeuxis falcata Lindl., Coll. Bot.: t. 2. 1821.

VOUCHER: H. Einzmann 177 (PMA).

\section{UlEIORCHIS Hoehne}

Uleiorchis ulei (Cogn.) Handro, Arq. Bot. Estado São Paulo, n.s., f.m., 3: 175. 1958.

Wullschlaegelia ulei Cogn. in C.F.P.von Martius \& auct. suc. (eds.), Fl. Bras. 3(4): 244. 1895.

Uleiorchis cogniauxiana Hoehne, Arq. Bot. Estado

São Paulo, n.s., f.m., 1: 129. 1944.

VOUCHER: R. L. Liesner 1367 (MO).

\section{VAnilla Plum. ex Mill.}

Vanilla dressleri Soto Arenas, Lankesteriana 9: 303. 2010. Voucher: S. Knapp \& J. Mallet 4621 (MO).

Vanilla hartii Rolfe, Bull. Misc. Inform. Kew 1899: 133. 1899.

Vanilla leprieurii Portères, Bull. Soc. Bot. France 98: 94.1951.

Voucher: P.C. Standley 29353 (AMES).

Vanilla inodora Schiede, Linnaea 4: 574. 1829.

Notylia inodora (Schiede) Conz., Fl. Taxon. Mex. 3: 151.1947.

Vanilla pfaviana Rchb.f., Gard. Chron., n.s., 20: 230. 1883.

Vanilla preussii Kraenzl., Notizbl. Bot. Gart. Berlin-Dahlem 7: 320. 1919.

VOUCHER: H. von Wedel 2880 (AMES).

Vanilla odorata C.Presl, Reliq. Haenk. 1: 101. 1826.

Vanilla ensifolia Rolfe, Bull. Misc. Inform. Kew 1892: 141. 1892.

Epidendrum vermifugum Sessé \& Moc., Fl. Mexic., ed. 2: 201.1894.

Vanilla uncinata Huber ex Hoehne, Arch. Inst. Biol. (São Paulo) 8: 269. 1937.

Vanilla denticulata Pabst, Anais Soc. Bot. Brasil 23: 109. 1972[1973].

VOUCHER: F.W. Hunnewell 16433 (AMES).

Vanilla phaeantha Rchb.f., Flora 48: 274. 1865.

Vanilla planifolia var. macrantha Griseb., Cat. P1. Cub.: 267. 1866.
Voucher: C.W. Powell 412 (AMES).

Vanilla planifolia Jacks. ex Andrews, Bot. Repos. 8: t. 538. 1808.

Notylia planifolia (Jacks. ex Andrews) Conz., Fl. Taxon. Mex. 3: 151. 1947.

Epidendrum rubrum Lam., Encycl. 1: 178. 1783.

Vanilla aromatica Willd., Sp. Pl. 4: 121. 1805., nom. illeg.

Myrobroma fragrans Salisb., Parad. Lond.: t. 82. 1807, nom. illeg.

Vanilla viridiflora Blume, Bijdr. Fl. Ned. Ind.: 422. 1825.

Vanilla sativa Schiede, Linnaea 4: 573. 1829.

Vanilla sylvestris Schiede, Linnaea 4: 573. 1829.

Vanilla duckei Huber, Bol. Mus. Goeldi Hist. Nat. Ethnogr. 5: 327. 1909.

Vanilla rubra (Lam.) Urb., Repert. Spec. Nov. Regni Veg. Beih. 5: 157. 1920.

Vanilla fragrans Ames, Schedul. Orchid. 7: 36. 1924., nom. illeg.

Vanilla planifolia var. angusta Costantin \& Poiss. ex C.Henry, Agric. Colon. 83: 135. 1924.

Notylia sativa (Schiede) Conz., Fl. Taxon. Mex. 3: 151. 1947.

Notylia sylvestris (Schiede) Conz., Fl. Taxon. Mex. 3: 151. 1947, nom. illeg.

Vanilla bampsiana Geerinck, Bull. Jard. Bot. Natl. Belg. 52: 345. 1982.

Voucher: P.C. Standley 40897 (US).

Vanilla pompona subsp. pittieri (Schltr.) Dressler, Lankesteriana 9: 341. 2010.

Vanilla pittieri Schltr., Repert. Spec. Nov. Regni Veg. 3: 106. 1906.

VOUCHER: H. Pittier 6600 (AMES).

Soto Arenas \& Dressler (2010) treated V. pompona Schiede as a variable, widely distributed species. They recognized three subspecies: V. pompona subsp. pompona restricted to Mexico; V. pompona subsp. grandiflora (Lindl.) Soto Arenas for populations restricted to the Amazon basin, Guyanas, Venezuela and Brazil; and V. pompona subsp. pittieri for populations ranging from Honduras to the Pacific coast of Panama.

Vanilla trigonocarpa Hoehne, Arq. Bot. Estado São Paulo, n.s., f.m., 1: 126. 1944.

Vanilla pauciflora Dressler, Orquideología 13: 229. 1979.

VOUCHER: R.L. Dressler 4571 (PMA). 


\section{WARCZEWICZELLA Rchb.f.}

Warczewiczella discolor (Lindl.) Rchb.f., Bot. Zeitung (Berlin) 10: 636. 1852.

Warrea discolor Lindl., J. Hort. Soc. London 4: 265. 1849.

Zygopetalum discolor (Lindl.) Rchb.f. in W.G.Walpers, Ann. Bot. Syst. 6: 655. 1863.

Chondrorhyncha discolor (Lindl.) P.H.Allen, Ann. Missouri Bot. Gard. 36: 87. 1949.

Cochleanthes discolor (Lindl.) R.E.Schult. \& Garay, Bot. Mus. Leafl. 18: 324. 1959.

VOUCHER: R. González \& Z. Martínez 48 (UCH).

Warczewiczella lipscombiae (Rolfe) Fowlie, Orchid Digest 33: 229. 1969.

Chondrorhyncha lipscombiae Rolfe, Bull. Misc. Inform. Kew 1912: 133. 1912.

Cochleanthes lipscombiae (Rolfe) Garay, Orquideología 4: 152. 1969.

VOUCHER: Mrs. Lipscomb (K).

\section{WARREA Lindl.}

Warrea costaricensis Schltr., Repert. Spec. Nov. Regni Veg. 16: 446. 1920.

VOUCHER: R.L. Dressler 3250 (MO).

\section{WARREOPSIS Garay}

Warreopsis parviflora (L.O.Williams) Garay, Orquideología 8: 51. 1973.

Zygopetalum parviflorum L.O.Williams, Ann.

Missouri Bot. Gard. 28(4): 424, pl. 25. 1941.

VOUCHER: R.E. Woodson \& R.W. Schery 605 (AMES).

\section{WulLSCHLAEgelia Rchb.f.}

Wullschlaegelia aphylla (Sw.) Rchb.f., Bot. Zeitung (Berlin) 21: 131. 1863.

Cranichis aphylla Sw., Prodr. Veg. Ind. Occ.: 120. 1788.

Voucher: Thomas F. Daniel et al. 5547 (MO).

Wullschlaegelia calcarata Benth., J. Linn. Soc., Bot. 18: 342. 1881.

VOUCHER: Barry E. Hammel 2729 (MO).

\section{Xуцовium Lindl.}

Xylobium colleyi (Bateman ex Lindl.) Rolfe, Gard. Chron. 7: 288. 1890.
Maxillaria colleyi Bateman ex Lindl., Edwards's Bot. Reg. 24(Misc.): 87. 1838.

Maxillaria brachypus Rchb.f., Bot. Zeitung (Berlin) 10: 734. 1852.

Maxillaria rebellis Rchb.f., Fl. Serres Jard. Eur. 9: 102. 1854.

Xylobium brachypus (Rchb.f.) Hemsl., Biol. Cent.Amer., Bot. 3: 252. 1884.

Xylobium brachystachyum Kraenzl., Gard. Chron., ser. 3, 40: 302. 1906.

Xylobium rebellis (Rchb.f.) Schltr., Orchis 7: 23. 1913.

VOUCHER: R.L. Dressler 3748 (MO)

Xylobium elongatum (Lindl. \& Paxton) Hemsl., Biol. Cent.-Amer., Bot. 3: 252. 1884.

Maxillaria elongata Lindl. \& Paxton, Paxton's Fl. Gard. 3: 69. 1852.

Voucher: M.A. Blanco \& D. Penneys 2937 (PMA).

Xylobium foveatum (Lindl.) G.Nicholson, Ill. Dict. Gard. 4: 225. 1887.

Maxillaria foveata Lindl., Edwards's Bot. Reg. 25(Misc.): 2. 1839.

Maxillaria concava Lindl., Edwards's Bot. Reg. 30(Misc.): 4. 1844.

Maxillaria stachyobiorum Rchb.f., Bot. Zeitung (Berlin) 10: 735. 1852.

Xylobium concavum (Lindl.) Hemsl., Biol. Cent.Amer., Bot. 3: 252. 1884.

Xylobium stachyobiorum (Rchb.f.) Hemsl., Biol.

Cent.-Amer., Bot. 3: 252. 1884.

Xylobium ecuadorense Rolfe, Bull. Misc. Inform.

Kew 1913: 341. 1913.

Xylobium filomenoi Schltr., Repert. Spec. Nov.

Regni Veg. Beih. 9: 100. 1921.

VOUCHER: J. Warscewicz s.n. (W).

Xylobium squalens (Lindl.) Lindl., Bot. Reg. 11: t. 897. 1825.

Dendrobium squalens Lindl., Bot. Reg. 9: t. 732. 1823.

Maxillaria squalens (Lindl.) Hook., Bot. Mag. 56: t. 2955.1829.

Cyrtopera scabrilinguis Lindl., Gen. Sp. Orchid. P1.: 189. 1833.

Maxillaria squalens var. taffinii Mutel, Mém. Orchid. 1: 16. 1842.

Maxillaria scabrilinguis (Lindl.) Lindl., Edwards's Bot. Reg. 30(Misc.): 71. 1844. 
Maxillaria squalens var. obscura Regel, Index Seminum (LE) 1856: 20. 1856.

Maxillaria squalens var. stenopetala Regel, Index Seminum (LE) 1856: 20. 1856.

Xylobium squalens var. obscura (Regel) Cogn. in C.F.P.von Martius \& auct. suc. (eds.), Fl. Bras. 3(5): 469. 1902.

Xylobium squalens var. stenopetala (Regel) Cogn. in C.F.P.von Martius \& auct. suc. (eds.), Fl. Bras. 3(5): 469. 1902.

Xylobium squalens var. taffinii (Mutel) Cogn. in C.F.P.von Martius \& auct. suc. (eds.), Fl. Bras. 3(5): 469. 1902.

Xylobium scabrilingue (Lindl.) Schltr., Orchis 7: 23. 1913.

Xylobium squalens f. major Hoehne, Relat.

Commiss. Linhas Telegr. Estratég. Matto Grosso Amazonas 5(9): 38. 1919.

Voucher: not seen, cited by Dressler (2003c).

Xylobium sulfurinum (Lem.) Schltr., Beih. Bot. Centralbl. 36(2): 493. 1918.

Maxillaria sulfurina Lem., Fl. Serres Jard. Eur. 4: t. 330.1848.

Xylobium powellii Schltr., Repert. Spec. Nov. Regni Veg. Beih. 17: 66. 1922.
Xylobium sublobatum Schltr., Repert. Spec. Nov. Regni Veg. Beih. 19: 51. 1923.

Xylobium tuerckheimii Kraenzl., Ann. Naturhist. Mus. Wien 44: 325. 1930.

Voucher: C.W. Powell 117 (AMES).

\section{ZoOTROPHION Luer}

Zootrophion endresianum (Kraenzl.) Luer, Selbyana 7: 82. 1982.

Cryptophoranthus endresianus Kraenzl., Repert. Spec. Nov. Regni Veg. 17: 437. 1921.

VOUCHER: R.L. Dressler 6072 (MO).

Zootrophion gracilentum (Rchb.f.) Luer, Selbyana 7: 82. 1982.

Masdevallia gracilenta Rchb.f., Gard. Chron., n.s., 4: 98.1875.

Cryptophoranthus gracilentus (Rchb.f.) Rolfe, Gard. Chron., ser. 3, 2: 693. 1887.

Cryptophoranthus lehmannii Rolfe, Orchid Rev. 11: 303. 1903.

Cryptophoranthus lepidotus L.O.Williams, Ann. Missouri Bot. Gard. 29: 340.1942.

VOUCHER: P.H.Allen 2718 (AMES).

Zootrophion williamsii Luer, Selbyana 23: 41. 2002.

Voucher: C. Luer 0676 (SEL). 

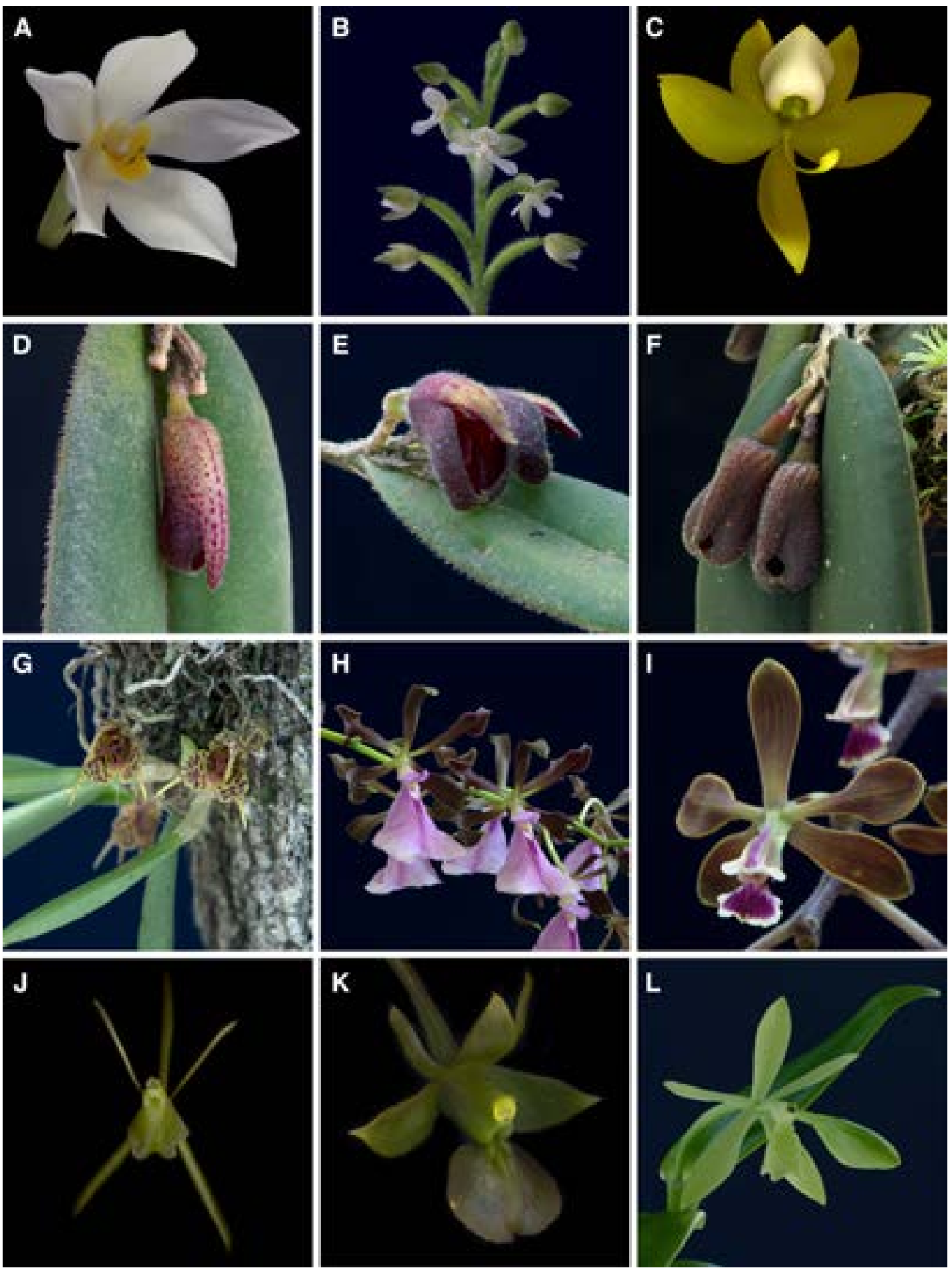

FIgURE 21. A - Camaridium ochroleucum. B - Cranichis diphylla. C - Cycnoches warscewiczii . D - Dresslerella elvallensis. E - D. hispida. F - D. pertusa. G - Dryadella butcheri. H - Encyclia cordigera f. rosea. I - E. elegantula. J - Epidendrum atwoodii. K - E. bisulcatum. L- E. candelabrum. Photos by D. Bogarín and Z. Serracín. 

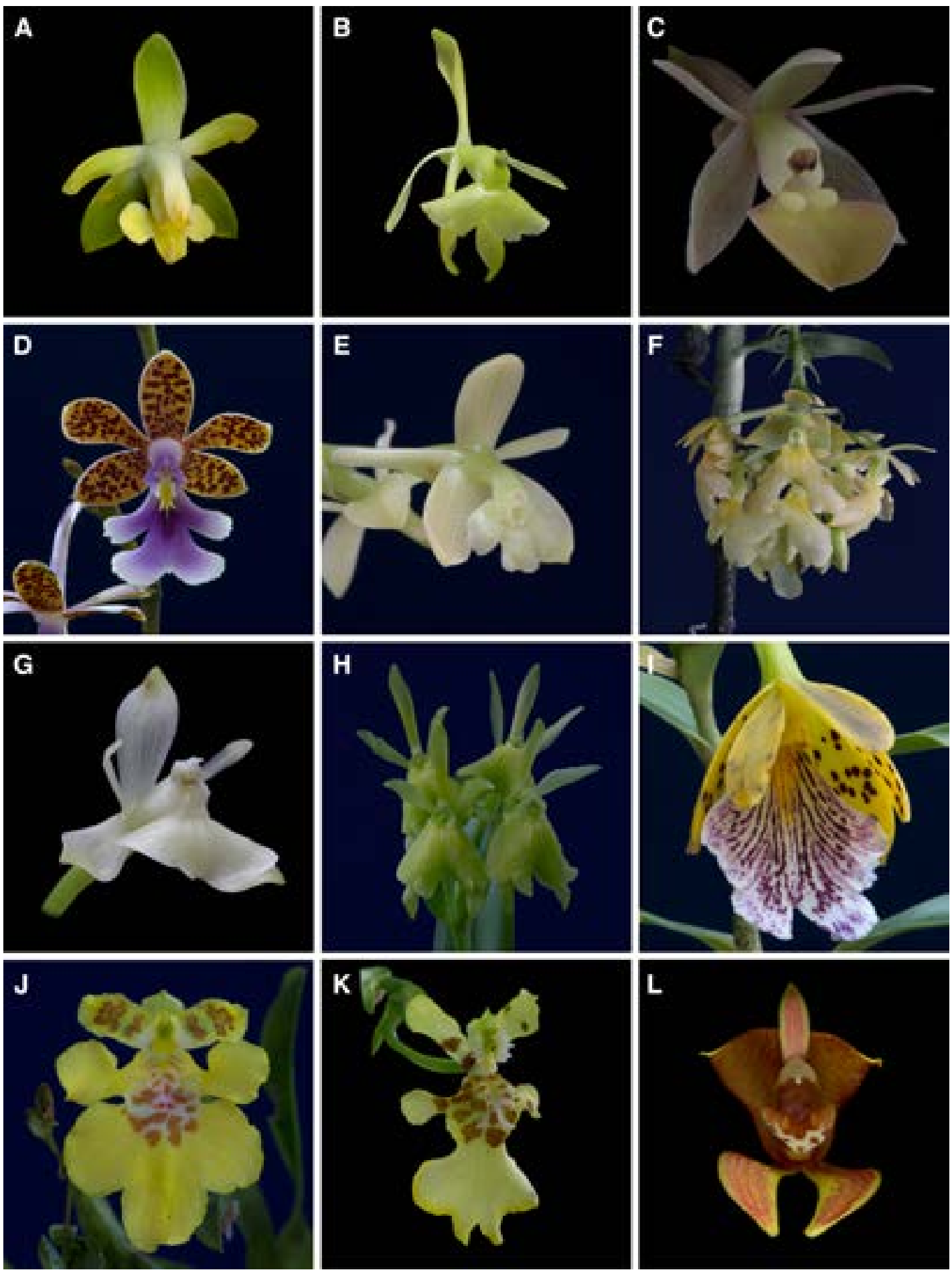

Figure 22. A - Epidendrum intermixtum. B - E. montis-narae. C - E. physodes. D - E. pseudoshumannianum. E - Epidendrum rousseauae. $\mathrm{F}-$ E. sotoanum. $\mathrm{G}-$ E. trialatum. $\mathrm{H}-$ E. vulgoamparoanum. I - E. wallisii. J Erycina crista-galli. K - E. pusilla. L- Govenia viaria. Photos by D. Bogarín and Z. Serracín. 

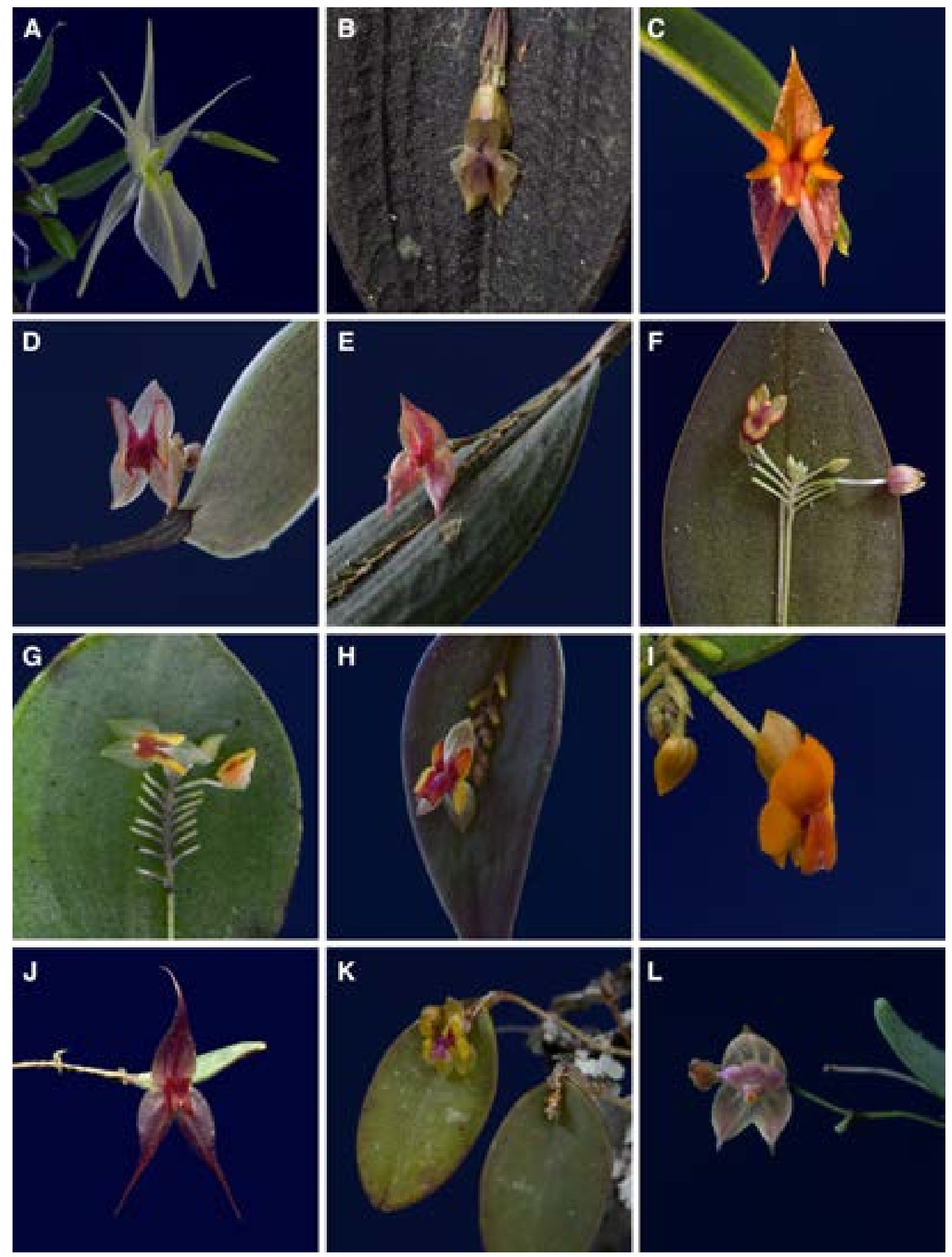

L

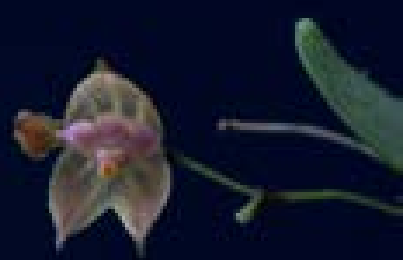

FiguRE 23. A - Homalopetalum pumilio. B - Lepanthes ankistra. C - L. antilocapra. D - L. candida. E - L. demissa. $\mathrm{F}$ - L. disticha. $\mathrm{G}$ - L. droseroides. $\mathrm{H}-$ L. exasperata. $\mathrm{I}$ - L. fimbriata. J - L. grandiflora. $\mathrm{K}-$ L. mariposa. L- L. minutilabia. Photos by D. Bogarín. 

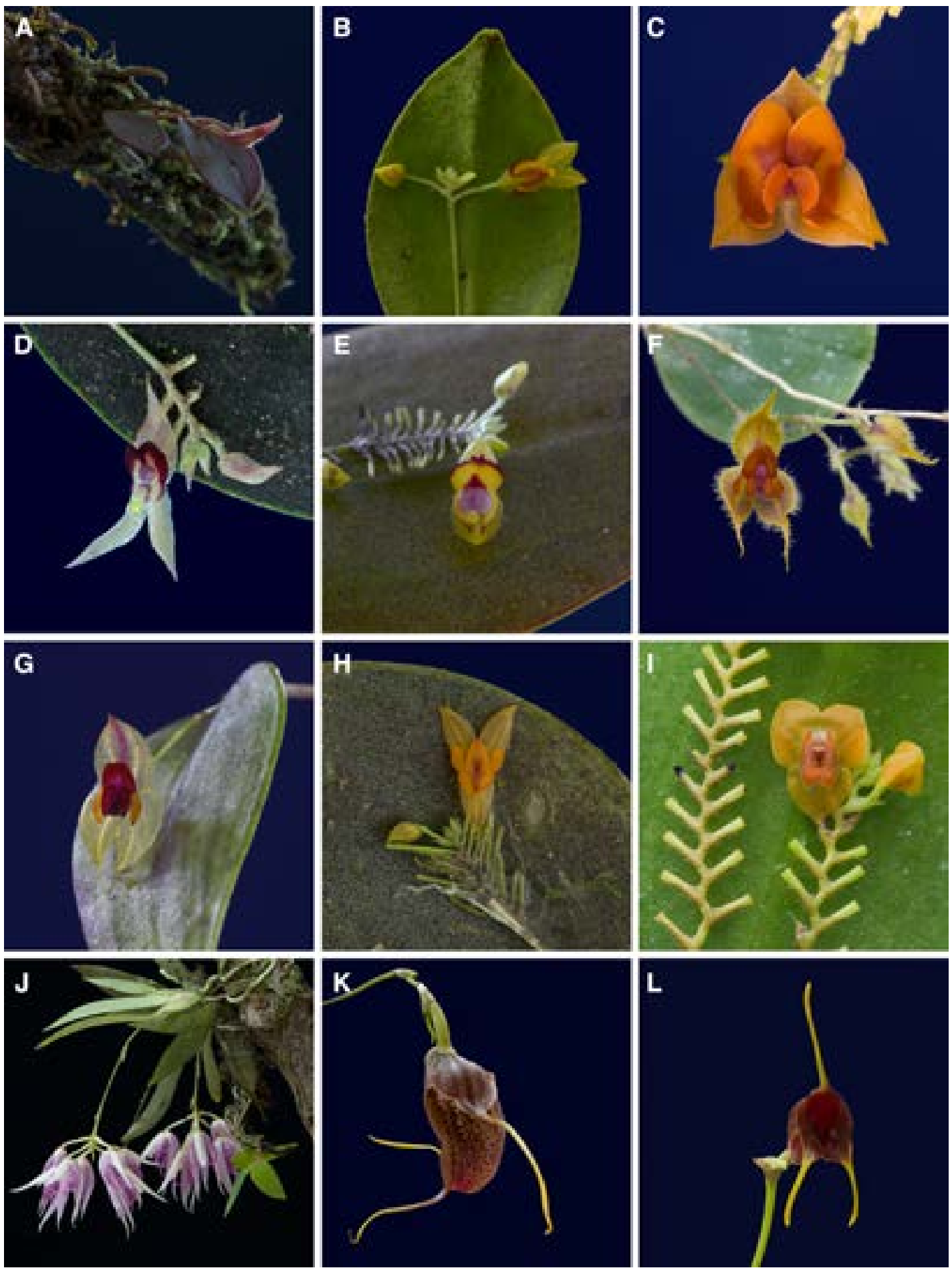

Figure 24. A - Lepanthes minutissima. B - L. minyglossa. C - L. olmosii. D - L. otopetala. E - L. pexa. F - L. L. pulcherrima. $\mathrm{G}$ - L. regularis. $\mathrm{H}$ - L. schizocardia. $\mathrm{I}$ - L. seegeri. J - Macroclinium lineare. $\mathrm{K}-$ Masdevallia andresiana. L- M. collina. Photos by D. Bogarín. 

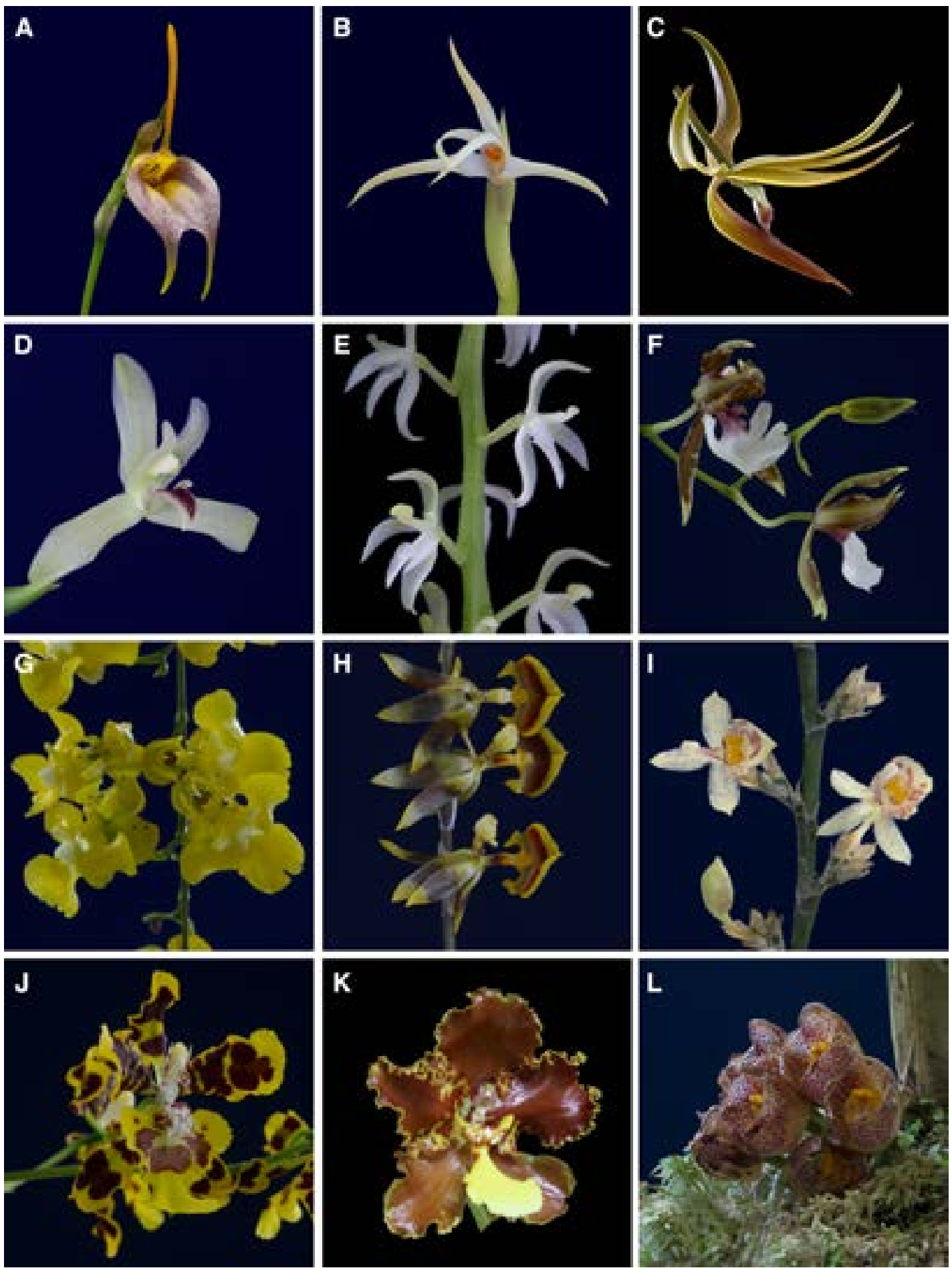

FiguRE 25. Masdevallia ostaurina. B - Maxillaria galantha. C - Mormodes powellii. D - Mormolyca culebrica. E Notylia pittieri. F - Oncidium cariniferum. G - Oncidium cheirophorum. $\mathrm{H}$ - O. dulcineae. I - O. picturatissimum. J - O. zelenkoanum. K - Otoglossum chiriquense. L- Peristeria cerina. Photos by D. Bogarín and Z. Serracín. 

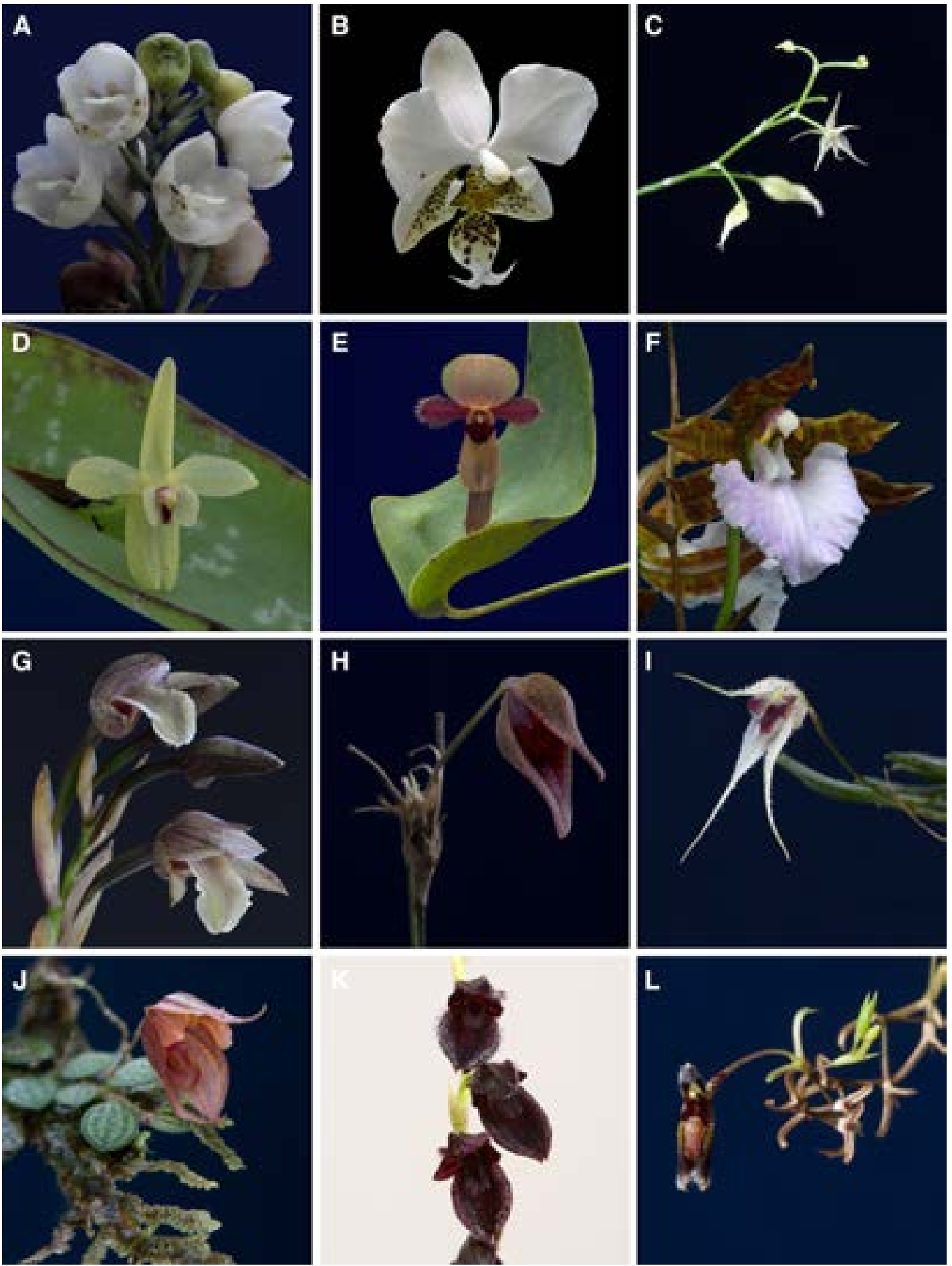

Figure 26. A - Peristeria elata. B - Phalaenopsis stuartiana. C - Platystele sylvestrei. D - Pleurothallis fantastica. E - P. peculiaris. F - Rhynchostele bictoniense. G - Scaphyglottis coriacea. H - Specklinia areldii. I - S. cactantha. J - Specklinia dressleri. K - Stelis falcatiloba. L-S. furculifera. Photos by D. Bogarín and Z. Serracín. 


\section{APPENDIX 1}

\section{List of basionyms of the orchid flora of Panama}

Acineta mireyae G.Gerlach \& M.H.Weber, J. Orchideenfr. 10(3): 230. 2003.

TyPe: Panama. Coclé: El Valle de Antón, Jun 2000, A. Maduro 330 (holotype, PMA; isotype, M).

Acineta sella-turcica Rchb.f., Bot. Zeit. (Berlin) 10: 705. 1852.

TyPe: Panama. Chiriquí: Cordillereuketten. Juli. 6 -8000', J. Warszewicz s.n. (holotype, W; drawings of type, $\mathrm{K}, \mathrm{AMES})$.

Acostaea bicornis Luer, Phytologia 54: 379. 1983. = Specklinia bicornis (Luer) Pridgeon \& M.W.Chase

Type: Panama. Panama: Epiphytic in cloud forest, Cerro Jefe, alt. 1000 m, 2 Mar 1976, C. Luer, J. Luer, P. Taylor \& R. Dressler 744 (holotype, SEL).

Acostaea unicornis Luer, Phytologia 54: 379. 1983. = Specklinia unicornis (Luer) Pridgeon \& M.W.Chase

TyPE: Panama. Coclé: Epiphytic in cloud forest above El Valle, alt. 1000 m, 6 Mar 1976, C. Luer, J. Luer, R.L.

Dressler \& P. Taylor 760 (holotype, SEL).

Acronia folsomii Luer \& Endara, Monogr. Syst. Bot. Missouri Bot. Gard. 103: 134. 2005. = Pleurothallis folsomii (Luer \& Endara) Bogarín

TYPE: Panama. Chiriquí: Cerro Colorado, along ridge road near border with Bocas del Toro, 1400-1700 m, 24

Oct 1977, J.A. Folsom 6096 (holotype, MO).

Acronia orygmoglossa Luer \& Dressler, Monogr. Syst. Bot. Missouri Bot. Gard. 103: 167. 2005. Pleurothallis orymoglossa (Luer \& Dressler) Bogarín

Type: Panama. Chiriquí: Camp Hornito. Fortuna dam site, 1000-1200 m, 13 Aug 1976, R.L. Dressler 5351 (holotype, SEL).

Acrorchis roseola Dressler, Orquídea (Mexico City) 12: 14. 1990.

TyPe: Panama. Chiriquí: Cerro Colorado, approx. 1650 m, 15 Feb 1985, R.L. Dressler 6103 (holotype, MO; isotypes, MO, AMO, F, FLAS, PMA, US).

Ancipitia dressleri Kolan. \& Szlach., Richardiana 14: 104. 2014. = Pleurothallis dressleriana Bogarín

TyPe: Panama. San Blas/Darién: mountains between Tubualá (San Blas) and Masargandi (Darién), 400-500 m, 2 Feb 1997, R. L. Dressler 5591 (holotype, FLAS).

Aspasia epidendroides Lindl., J. Bot. (Hooker) 1: 6. 1834.

TyPE: Habitat in Panama et Columbia occidentali, Cuming 1297 (holotype, K).

Aspasia principissa Rchb.f., Bot. Zeitung (Berlin) 10: 637. 1852.

TYPE: Panama. Veraguas, J. Warscewicz s.n. (holotype, W; drawing of type, AMES).

Aspasia pusilla C.Schweinf., Bot. Mus. Leafl. 10: 21. 1941. = Cischweinfia pusilla (C.Schweinf.) Dressler \& N.H.Williams

Type: Panama. Darien Province: Chepigana District, Cana Cuasi Trail (Camp 2), epiphyte "4 to 8 inches high “, at 2000 feet altitude, March 11, 1940, M.E. \& R.A. Terry 1502 (holotype, AMES; isotypes, AMES, F, MO).

Aspasia rousseauae Schltr., Gartenflora 71: 76. 1922. = Aspasia principissa Rchb.f.

TYPe: Panama. Mrs. Rousseau s.n. (holotype, B, destroyed; lectotype cited by Mora de Retana (1993), illustration in Gartenflora 72: t. 10 (1922)). 
Barbosella circinata Luer, Selbyana 3(3/4): 204. 1977.

TYPE: Panama. Chiriquí: epiphytic in the cloud forest along the continental divide, above Camp Escopeta, Cerro Colorado, alt. 1700 m, 16 Feb 1977, C. Luer, J. Luer \& R.L. Dressler 1575 (holotype, SEL).

Barbosella orbicularis Luer, Selbyana 3(1/2): 10. 1976.

TYPe: Panama. Panama: epiphytic, La Eneida, region of Cerro Jefe, alt. 1000 m, 25 Dec 1967, R. L. Dressler 3285 (holotype, SEL).

Baskervilla stenopetala Dressler, Bol. Inst. Bot. Univ. Guadalajara 5: 70. 1998.

TyPe: Panama. Darién: Cerro Pirre, 1050-1400 m, 10 Nov 1977, flowers very pale green, J. Folsom s.n. (holotype, MO).

Bletia purpurea var. pittieri Schltr., Repert. Spec. Nov. Regni Veg. Beih. 17: 50. 1922.= Bletia purpurea (Lam.) A.DC.

TyPe: Panama. Auf Hügeln bei Panama-City, C.W. Powell 128 (holotype, B, lectotype designated by Christenson (1991b), K; isolectotype, AMES).

Brachionidium calypso Luer, Monogr. Syst. Bot. Missouri Bot. Gard. 57: 24. 1995.

TYPE: Panama. Chiriquí: La Chumblada above Guadalupe, 15 July 1979, R.L. Dressler 5838 (holotype, MO).

Brachionidium dentatum Luer \& Dressler, Monogr. Syst. Bot. Missouri Bot. Gard. 57: 34. 1995.

TyPE: Panama. Chiriquí: above Cerro Punta, alt. ca. $2800 \mathrm{~m}$, collected and cultivated by A. Maduro, flowered in cultivation at Cerro Punta, July 1979, R.L. Dressler s.n. (holotype, MO).

Brachionidium dressleri Luer, Monogr. Syst. Bot. Missouri Bot. Gard. 57: 40. 1995.

TyPE: Panama. Chiriquí: La Chumbada, above Guadalupe, 15 July 1979, R. L. Dressler 5837 (holotype, MO).

Brachionidium folsomii Dressler, Orquideologia 15: 154. 1982.

TyPe: Panama. Coclé: vertiente norte del Alto del Calvario, al norte del Aserradero El Copé, aprox. $9 \mathrm{~km} \mathrm{al}$ norte de El Copé, elev. 800-850 m, 23 Feb 1982, R.L. Dressler 6032 (holotype, US; isotype, PMA).

Brachionidium lucanoideum Luer, Monogr. Syst. Bot. Missouri Bot. Gard. 57: 78. 1995.

TYPE: Panama. Chiriquí: elfin forest along the ridge of the continental divide northeast of Cerro Pate Macho above Palo Alto, alt. 2200 m, 24 Apr 1982, S. Knapp \& R. Schmalzel 4829 (holotype, MO; isotype, AMES, K, US).

Brachionidium peltarion Luer, Monogr. Syst. Bot. Missouri Bot. Gard. 57: 92-93. 1995.

TYPE: Panama. Coclé: Cerro Gaital, mesa north of El Valle de Antón, near western peak, alt. ca. 900 m, 14 Mar 1981, R.L. Dressler \& E. Hagsater 6005 (holotype, MO).

Brachionidium polypodium Luer, Monogr. Syst. Bot. Missouri Bot. Gard. 57: 98. 1995.

Type: Panama. Chiriquí: Cerro Colorado, alt. 1400 m, growing in dense moss on side of tree, 25 Jul 1979, T. Antonio 1413 (holotype, MO).

Brachionidium satyreum Luer, Monogr. Syst. Bot. Missouri Bot. Gard. 57: 112. 1995.

TYPE: Panama. Bocas del Toro: Trocha 3 de noviembre, near Paso de la Zorra over the continental divide, alt. 1500 m, 5 April 1977, R.L. Dressler, K. Dressler \& N. H. Williams 5809 (holotype, MO).

Brassia allenii L.O.Williams ex C.Schweinf., Bot. Mus. Leafl. 13: 145. 1948.

TYPe: Panama. Panama: summit of Cerro Campana near Campana, ca. $1000 \mathrm{~m}$ alt., in cloud forest, 2 Oct 1947, P.H. Allen 5150 (holotype, AMES).

Brassia longissima (Rchb.f.) Nash var. minor Schltr., Repert. Spec. Nov. Regni Veg. Beih. 17: 80. 1922. = Brassia caudata (L.) Lindl.

TYPE: Panama. auf Hügeln bei Panama City, C.W. Powell 72 (holotype, B, destroyed; lectotype designated by 
Christenson (1991b), AMES-23903; isolectotypes, K, MO-955925).

Bulbophyllum wagneri Schltr., Repert. Spec. Nov. Regni Veg. 17: 142. 1921. = Bulbophyllum pachyrachis (A.Rich.) Griseb.

Syntypes: Panama. Muame and Gorgone, in Januar 1858, M. Wagner s.n. (B, destroyed). Panama. vicinity of San Felix, eastern Chiriquí, 0-120 m, Dec 1911, H. Pittier 5285 (B, destroyed), lectotype designated by Hamer \& Garay (1995) [1997].

Camaridium affine Schltr., Repert. Spec. Nov. Regni Veg. Beih. 17: 72. 1922.= Camaridium ochroleucum Lindl. Type: Panama. Auf Hügeln bei Panama City, C.W. Powell 7 (holotype, B, destroyed; lectotype designated by Christenson (1991b), AMES-23884; isolectotypes, AMES-43843, MO-955879; drawing of type, AMES26830; drawing published in Mansfeld [1931: t. 66, nr. 264].).

Camaridium arachnites Schltr., Repert. Spec. Nov. Regni Veg. Beih. 17: 73. 1922. = Camaridium ctenostachys (Rchb.f.) Schltr.

Type: Panama. Chiriquí, c. 4000 Fuss ü. M., C.W. Powell 210 (holotype, B, destroyed, lectotype designated by Christenson (1991b), AMES-23886; isolectotypes, AMES-23885, AMES-30308, AMES-30309, AMES30310, MO-956027; drawing of type, AMES-26893; drawing published in Mansfeld [1931: t. 67, nr. 265].).

Camaridium grandiflorum Ames, Proc. Biol. Soc. Washington 34: 149. 1921. nom. illeg. hom. $=$ Camaridium ampliflorum (C.Schweinf.) M.A.Blanco

Type: Panama. Chirirquí: in humid forest of the Cordillera, east of the Río Caldera, 17-19 Feb 1918, $2000 \mathrm{~m}$ alt., P. Killip 3565 (holotype, AMES; isotype, AMES, US; photo of isotype ex US, AMES).

Camaridium latifolium Schltr., Repert. Spec. Nov. Regni Veg. Beih. 17: 74. 1922.

Type: Panama. Auf Hügeln bei Panama City, C.W. Powell 8 (holotype, B, destroyed; lectotype designated by Christenson (1991b), AMES; isolectotypes, AMES, K-K000079347, K-K000079346, MO, S-0707871, S-0707870; drawing published in Mansfeld [1931: t. 68, nr. 269].).

Campylocentrum dressleri H.Dietr. \& M.A.Díaz, Orchidee (Hamburg) 35(1): 28-30, f. 1984. = Campylocentrum tyrridion Garay \& Dunst.

TYPE: Panama. Darién: margins of the Tschkonake river, $5 \mathrm{~km} \mathrm{~W}$ of Yavisa, tropical rain forest, April 1980, flowering in cultivation in Jardín Botánico Nacional de Cuba, May 1983, J. Bisse, A. Álvarez \& A. Díaz s.n. sub H. Dietrich s.n. (holotype, HAJB).

Campylocentrum panamense Ames, Orchidaceae 7: 88. 1922.

TYPE: [Panama]. In woods near Gatún, on trees, Jan 10, 1860, Sutton Hayes 958 (holotype, NY; illustration of type, AMES).

Campylocentrum peniculus Schltr., Repert. Spec. Nov. Regni Veg. Beih. 17: 91. 1922. = Campylocentrum micranthum (Lindl.) Maury

TYPe: Panama. auf Hügeln bei Panama City, C.W. Powell 184 (holotype,B, destroyed; lectotype designated by Christenson (1991b), AMES-23936; detail of type, AMES; drawing of type, AMES-26798, isolectotype, MO-956017; drawing published in Mansfeld [1931: t. 80, nr. 318].).

Campylocentrum tenellum Todzia, Ann. Missouri Bot. Gard. 72: 877. 1985.

TyPe: Panama. Panama. La Eneida, region of Cerro Jefe, 26 Oct 1969, R.L Dressler 3758 (holotype, CR; isotype, CR).

Catasetum warczewitzii Lindl. \& Paxton, Paxton's Fl. Gard. 1: 45. 1850. Clowesia warczewitzii (Lindl. \& Paxton) Dodson

TYPE: A most fragrant terrestrial orchid from Panama. Introduced by Mr. Skinner. Flowers pale green.

Discovered by Warczewitz; blossomed at Penllergaré in April with J. D. Llewelyn, Esq. (holotype, K). 
Cattleya skinneri var. autumnalis P.H.Allen ex L.O.Williams, Ann. Missouri Bot. Gard. 29: 345. 1942.= Guarianthe patinii (Cogn.) Dressler \& W.E. Higgins

TYPE: Panama. vicinity of Bejuca, alt. ca. 30 m, 15 Aug 1941, P. H. Allen 2668 (holotype, AMES; isotypes, AMES-217940, AMES-217941, AMES-217942).

Chondrorhyncha crassa Dressler, Orchidee (Hamb.) 34: 222. 1983. = Daiotyla crassa (Dressler) Dressler

TYPE: Panama. Chiriquí: Fortuna Valley, north side of river near La Sierpe, 11 May 1982, flowered in cult. 20 May through July, R.L. Dressler 6055 (holotype, US; isotype, PMA).

Chondrorhyncha eburnea Dressler, Orchidee (Hamburg) 34: 224. 1983. = Chondroscaphe eburnea (Dressler) Dressler

TYPe: Panama. Coclé: Cerro Caracoral, hills north of El Valle de Antón, ca. 850 m, 9 July 1982, R.L. Dressler 6070 (holotype, US; isoytpe, PMA).

Chondrorhyncha lipscombiae Rolfe, Bull. Misc. Inform. Kew 1912: 133. = Warczewiczella lipscombiae (Rolfe) Fowlie

TYPE: Panama. "It was found by ... Mr. Lancelot Lipscomb, when helping to clear some bush for rubber and cocoa planting, and was sent home with a good many other in 1910", flowered in the collection of Mrs. Lipscomb, Milton Grove, Wimbledon, Dec. 1911, Lipscomb s.n. (holotype, K).

Chondroscaphe atrilinguis Dressler, Orquideología 22(1): 16. 2001.

TYPE: Panama. Bocas del Toro: floreció en cultivo, 8 Sept. 2000, R.L. Dressler 6289 (holotype, MO).

Chysis pluricostata Dressler, Orquideología 24(2): 142. 2006.

TyPe: Panama. Darién: cerca de Metetí, de Porfirio Martínez, prensado de cultivo, 8 Jun 2005, M. Whitten 2968 (holotype, PMA).

Chysis violacea Dressler, Orquideología 22(3): 237. 2003.

TYPE: Panama, prov. Panama, Dist. de Chimán, Río Tuti, Cerro Chucantí, creciendo en risco, colectado por Neal G. Smith; preparado de cultivo Sept. 2001, R. L. Dressler 6340 (holotype, MO; isotype, PMA; clonotype, SEL; photo of type).

Cischweinfia nana Dressler, Selbyana 22(1): 9. 2001.

TyPE: Panama. Chiriquí: Camp Hornito, Fortuna dam site, $82^{\circ} 10^{\prime} \mathrm{W}, 8^{\circ} 44^{\prime} \mathrm{N}$, elev. 1000-1200 m, 17 September 1977, R.L. Dressler 5700 (holotype, MO; isotype, PMA).

Cischweinfia pusilla (C.Schweinf.) Dressler \& N.H.Williams subsp. furcata Dressler \& Dalström, Selbyana 25(1): 5. 2004.

Type: Panama. Veraguas: EI Pantano, N.E. of Santa Fe; flowered in cult. 16 Oct. 2002, R.L. Dressler $6396 A$ (holotype, MO).

Cischweinfia sheehaniae Christenson, Orchids (West Palm Beach) 72: 126. 2003. = Cischweinfia pusilla (C.Schweinf.) Dressler \& N.H.Williams

TYPE: Hort. Andy's Orchids (2553): Panama and Colombia, but may also occur in Costa Rica, Christenson 2057 (holotype, NY).

Coccineorchis cristata Szlach., Rutk. \& Mytnik, Ann. Bot. Fenn. 41(6): 482. 2004

TYPe: Panama. Panama: Cerro Jefe, ca. 800 m, 16 Dec 1972, R.L. Dressler 4233 (holotype, FLAS).

Coccineorchis dressleri Szlach., Rutk. \& Mytnik, Ann. Bot. Fenn. 41(6): 481. 2004

TyPe: Panama. Chiriquí: Cerro Hornito, 1700 m, 14 Dec 1976, C.A. Luer, J. Luer, R.L. Dressler \& N.H. Williams 1322 (holotype, SEL).

Cochleanthes anatona Dressler, Orchidee (Hamburg) 34: 160. 1983. = Euryblema anatonum (Dressler) Dressler TYPE: Panama. Coclé, near Aserradero El Copé; ca. 8 km. NO of El Copé, 750-850 m, very wet cloud forest, 
1 September 1977, epiphyte, R.L. Dressler 5690 (holotype, US; isotype, PMA).

Cocleorchis sarcoglottidis Szlach., Fragm. Florist. Geobot. 39: 558-560. 1994. = Cyclopogon dressleri Szlach.

TyPE: Panama. Cocle: El Cope, Atlantic slope E of sawmill, high ridge E of sawmill, 26 Feb 1979, B.E. Hammel 6275 (holotype, MO; isotype, MO).

Coeliopsis hyacinthosma Rchb. f., Gard. Chron. 9. 1872.

TYPE: Panama. Flowered in the garden of W. Wilson Saunders, who got it from Panama, W. Wilson Saunders s.n. (holotype, W; illustration of type, AMES).

Condylago furculifera Dressler \& Bogarín, Harvard Pap. Bot. 12(1): 2. 2007. = Stelis furculifera (Dressler \& Bogarín) Bogarín

Type: Panama. Veraguas: Chilagro, near Cerro Arizona, west of Santa Fé, 1000-1200 m, flowered in cultivation at Finca Dracula, Cerro Punta, Chiriquí, 14 December 2006, R. L. Dressler 6835 (holotype, PMA; isotype, JBL-spirit).

Coryanthes hunteriana Schltr., Repert. Spec. Nov. Regni Veg. Beih. 17: 63. 1922.

TyPe: Panama. An Wasserläufen zwischen Hügeln bei Panama City, C.W. Powell 19 (holotype, B, destroyed, lectotype designated by Christenson (1991b), AMES-26894; isolectotypes, AMES-25007, AMES-25008, AMES-31853[?], AMES-s.n. [?], K, MO-955890; illustration of type, AMES).

Coryanthes maduroana G.Gerlach, Lankesteriana 4(1): 70. 2004.

TYPe: Panama. Coclé: El Valle de Antón, floreció en cultivo en Panama, 4 de deciembre 2002, A. Maduro \& E. Olmos 512 (holotype, PMA).

Coryanthes panamensis G.Gerlach, Trop. Subtrop. Pflanzenwelt 83: 141. 1993.

TyPe: Panama. Kanal Zone, Rio Piedras, kultiviert in Botanischen Garten Heidelberg unter der Nr. O-18594, H.G. Seeger O-18594 (holotype, HEID).

Coryanthes powellii Schltr., Repert. Spec. Nov. Regni Veg. Beih. 17: 64. 1922.= Coryanthes hunteriana Schltr. TyPe: Panama. Auf Hügeln bei Panama-City, C.W. Powell 156 (holotype, B, destroyed; lectotype designated by Christenson (1991b), K; isolectotype, AMES-26893).

Crossoglossa bifida Dressler, Orquideología 20: 255. 1997.

Type: Panama. Panama: Cerro Jefe, 22 Sept. 1970; on mossy trunk in cloud forest; sepals and petals pale green, lip orange-tan; R.L. Dressler 3890 (holotype, MO; isotype, PMA).

Crossoglossa elliptica Dressler, Orquideología 20: 256, fig. 1997.

TYPE: Panama. Coclé: near Aserradero El Copé, ca. 8 km N of El Copé, Jan 1978, J.P. Folsom s.n. (holotype, $\mathrm{MO}$; isotype, FLAS).

Cryptocentrum caespitosum Carnevali, Harvard Pap. Bot. 5(2): 472. 2001.

TYPE: Panama. Coclé: North rim of El Valle, 9 July 1939, P.H. Allen 1911 (holotype, US; isotypes, AMES, F, MO, NY, SEL, VEN).

Cryptophoranthus lepidotus L.O.Williams, Ann. Missouri Bot. Gard. 29: 340. 1942.=Zootrophion gracilentum (Rchb.f.) Luer

TyPe: Panama. Coclé: trail to Las Minas, hills north of El Valle de Antón, alt. ca. 1000 m, 1 Sep 1941, epiphytic, lower sides of many leaves deep purple, flowers white with very heavy purple stripes, $P$. H. Allen 2718 (holotype, AMES; isotype, MO).

Cryptophoranthus powellii Ames, Schedul. Orchid. 4: 8. 1925. = Dresslerella powellii (Ames) Luer

TYPE: Panama. Foothills east of Panama. Grows in shady places. Leaves dark velvety green, lying flat on the limbs of trees. Flowers in February. Sea level. C.W. Powell 278 (holotype, AMES [not located]; isotype, $\mathrm{MO})$. 
Cyclopogon dressleri Szlach., Novon 4: 172-173. 1994.

TYPe: Panama. Cocle: upper Rio Blanco, NW of El Cope (Caribbean slope), 600-800 m, R.L. Dressler 5986 (holotype, FLAS).

Cycnoches dianae Rchb.f., Bot. Zeitung (Berlin) 10: 636. 1852.

TYPE: Mittelamerika. J. Warszewicz s.n. (holotype, W).

Cycnoches guttulatum Schltr., Repert. Spec. Nov. Regni Veg. Beih. 17: 56. 1922.

TYPE: Panama. Auf Hügeln bei Panama-City, C.W. Powell 14 (holotype, B, destroyed; lectotype designated by Christenson (1991b), AMES-23843; isolectotypes, K, MO-955885; drawing published in Mansfeld [1931: t. 61, nr. 242].).

Cycnoches pachydactylon Schltr., Repert. Spec. Nov. Regni Veg. Beih. 17: 57. 1922.

Type: Panama. Chiriquí, c. 5000 fuss u.m., am Vulkan, C.W. Powell 159 (holotype, B, destroyed; lectotype designated by Christenson (1991b), K; drawing of type, AMES-26807; drawing published in Mansfeld [1931: t. 61, nr. 243].).

Cycnoches powellii Schltr., Repert. Spec. Nov. Regni Veg. Beih. 17: 58. 1922.

TYPE: Panama. Ohne nähere Herkunftsangabe, C.W. Powell 111 (holotype, B, destroyed; lectotype designated by Christenson (1991b), drawing of type, AMES-26761).

Cycnoches stenodactylon Schltr., Repert. Spec. Nov. Regni Veg. Beih. 17: 59. 1922.

TYPE: Panama. Chiriquí, 3000-3500 fuss um.m., C.W. Powell 173 (holotype, B, destroyed; lectotype designated by Christenson (1991b), K; drawing of type, AMES-26823; drawing published in Mansfeld [1931: t. 61, nr. 244].).

Cycnoches warszewiczii Rchb.f., Bot. Zeitung (Berlin) 10: 734. 1852.

TYPE: [Panama]: Chiriquí, J. Warszewicz s.n. (holotype, W).

Cypripedium dariense Rchb.f., Gard. Chron., n.s., 9: 202. 1878. = Phragmipedium longifolium (Warsz. \& Rchb.f.) Rolfe

TYPE: [Panama]. (holotype, W).

Cypripedium humboldtii Warsz., Bot. Zeitung (Berlin) 10: 691. 1852. = Phragmipedium humboldtii (Warsz. ex Rchb.f.) J.T.Atwood \& Dressler

TYPE: Panama. In Quercubus montium Chiriquí; lectotype designated by Atwood \& Dressler (1998), W: "Mai Juni Juli [1848-1851]/Cordill. Chiriquí”, J. Warszewicz 41, W).

Diacrium bilamellatum var. reichenbachiana Schltr., Repert. Spec. Nov. Regni Veg. Beih. 17:47. 1922. = Caularthron bilamellatum (Rchb.f.) R.E.Schult.

TyPe: Panama. von Panama langs der pazifischen Küste ziemlich haufig, C.W. Powell 67 (holotype, B, destroyed; lectotype designated by Christenson (1991b), AMES-23966; isolectotype, AMES-23965).

Dichaea dressleri Folsom, Novon 16(3): 336. 2006.

TYPE: Panama. Chiriquí: forest near Fortuna Lake, just beyond bridge, 1100 m, 9 Apr. 1995, J.P. Folsom11278A (holotype, PMA; isotypes, HNT, MO).

Dichaea eligulata Folsom, Orchid Digest 58(4): 185-186. 1994.

TYPE: Costa Rica[or adjacent Panama]. locality of original collection unknown--growing on trees at Las Cruces Botanical Garden, probably collected in Costa Rica or adjacent Panama, 8 Jul 1981, J.R. Folsom 10261 (holotype, CR; isotype, TEX).

Dichaea elliptica Dressler \& Folsom, Lankesteriana 3: 25. 2002.

TYPe: Panama. Coclé, Aserradero El Copé, ca. 8 km N of El Copé, 800-900 m, 9-10 April 1977, R.L. Dressler 5641 (holotype, MO; isotype, PMA). 
Dichaea obovatipetala Folsom, Orchid Digest 58: 186. 1994.

TyPe: Panama. Bocas del Toro: $15 \mathrm{~km} \mathrm{~N}$ of border with Chiriquí, road from Fortuna to Chiriquí Grande, 100 m, 10 Apr 1985, J.P. Folsom 11302M (holotype, PMA; isotypes, HNT, TEX).

Dichaea panamensis Lindl., Gen. Sp. Orchid. Pl. 209. 1833.

TyPe: Hab. in Panama, et Columbia occidentali, H. Cuming 1292 (holotype, K).

Dichaea powellii Schltr., Repert. Spec. Nov. Regni Veg. Beih. 17: 90. 1922. = Dichaea trulla Rchb.f.

TyPe: Panama, auf Hügeln bei Alt. Bohio, unweit Panama City, in einem Tal., C. W. Powell 23 (holotype, B, destroyed, lectotype designated by Christenson (1991b), AMES; drawing of type, AMES-26859; drawing published in Mansfeld [1931: t. 79, nr. 316].).

Dichaea retroflexiligula Folsom, Orchid Digest 58: 187. 1994.

TYPe: Panama. Bocas del Toro: $12 \mathrm{~km} \mathrm{~N}$ of Chiriquí border, en route to Chiriquí Grande, 340 m, 10 Apr 1985, J.P. Folsom $11301 K$ (holotype, PMA; isotypes, HNT, PMA, TEX).

Dichaea violacea Folsom, Orchid Digest 58: 190. 1994.

TYPE: Panama. Cocle: at continental divide, 7 km N of El Cope, 850-950 m, 6 Apr 1985, J.P. Folsom 11283MM (holotype, PMA; isotypes, HNT, TEX).

Dimerandra isthmi Schltr., Repert. Spec. Nov. Regni Veg. Beih. 17: 44. 1922. = Dimerandra emarginata (G.Mey.) Hoehne

TYPE: Panama. Auf Hügeln bei Panama City, C.W. Powell 17 (holotype, B, destroyed; lectotype designated by Christenson (1991b), AMES-23816; isolectotypes, K, MO, S; drawing of type, AMES-26749; drawing published in Mansfeld [1931: t. 59, nr. 235].).

Dracula agnosia A.Doucette, Phytotaxa 56: 23. 2012.

TyPe: Panama. Chiriquí: Guadalupe, gathered from material cultivated at Finca Dracula, 1 Nov 2011, $A$. Doucette 4011 (holotype, BH; isotypes, PMA, WIS).

Dracula immunda A.Doucette, Phytotaxa 16: 38. 2011.

TYPE: Panama. Veraguas: gathered from material cultivated at Finca Dracula, reported from the Province of Veraguas from cloud forest above Santa Fé, A. Doucette 3389 (holotype, BH; isotype, PMA; photo of type).

Dracula maduroi Luer, Monogr. Syst. Bot. Missouri Bot. Gard. 95: 234. 2004.

TYPE: Panama. Bocas del Toro: Velorio area, alt. 1100-1500 m, 10 August 2003, A. Maduro \& E. Olmos 410 (holotype, MO).

Dracula olmosii Luer \& Maduro, Monogr. Syst. Bot. Missouri Bot. Gard. 76: 136. 1999.

TyPE: Panama. Bocas del Toro: alt. 2000 m, collected by E. Olmos, 1994, cultivated at Finca Dracula, Cerro Punta, C. Luer 18990 (holotype, MO).

Dresslerella elvallensis Luer, Selbyana 3(1/2): 2. 1976.

TYPE: Panama. Coclé: epiphytic in the cloud forest in the hills above El Valle, ca. 1000 m, 6 Mar. 1976, C. Luer, J. Luer, P. Taylor \& R.L. Dressler 1079 (holotype, SEL, not located; clonotype, K).

Dressleria allenii H.G.Hills, Lindleyana 15(3): 171. 2000.

TyPE: Panama. Without locality, N.H. Williams field collection, ex hort. Selby Gardens living accession 7656-19, 18 Sep 1978, J. Ackerman 1336 (holotype, SEL).

Dressleria kerryae H.G.Hills, Lindleyana 15(3): 175. 2000.

Type: Panama. Prov. Panama: Cerro Jefe, 1968, R.L. Dressler 3515 (holotype, FLAS).

Dressleria severiniana H.G.Hills, Amer. Orchid Soc. Bull. 62: 616, figs. 1993.

Type: Panama. Prov. Coclé: El Valle de Antón, leg. R.L. Dressler, 12 May 1990, hort. FLAS \#F1757, H.G. Hills 90015 (holotype, AMES). 
Dryadella butcheri Luer, Monogr. Syst. Bot. Missouri Bot. Gard. 76: 160. 1999.

TYPE: Panama. Chiriquí: without locality, collected by H. Butcher, flowered in cultivation by P. \& A. Jesup in Bristol, CT., 10 December 1988, C. Luer 13804A (holotype, MO).

Dryadella dressleri Luer, Monogr. Syst. Bot. Missouri Bot. Gard. 76: 162. 1999.

TYPE: Panama. Canal Zone: epiphytic in forest along Río Piedras near Colón, alt. ca. 100 m, collected by R.L. Dressler, cultivated in Balboa, 18 Feb. 1985, C. Luer 10627 (holotype, MO).

Dryadella odontostele Luer, Lindleyana 11: 54, fig. 1996.

TyPe: Panama. Panama: Cerro Jefe, epiphytic, alt. ca. 1000 m, 22 Sep 1972, A. Gentry 6178 (holotype, MO).

Dryadella sororcula Luer, Lindleyana 11: 56, fig. 1996.

TYPE: Panama. Bocas del Toro: epiphytic in tall, wet forest between Fortuna and Chiriquí Grande, alt. $350 \mathrm{~m}$, 17 Feb 1985, C. Luer, J. Luer, R. Dressler \& K. Dressler 10606 (holotype, MO).

Elleanthus capitatellus Dressler, J. Orchideenfr. 11(2): 143. 2004.

TyPe: Panama. Coclé: Area of El Valle de Antón, flowered in cult. 3 July 2003, R.L. Dressler 6387 (holotype, MO; isotype, PMA).

Elleanthus decipiens Dressler, J. Orchideenfr. 11(2): 146. 2004.

TYPE: Panama. Chiriquí, along trail to summit ridge of Cerro Pate Macho, ca. 6-8 km NE of Boquete, cloud forest and wind-swept ridge, corolla cream, pollinarium purple, infrequent at summit, elev. 1750-2100 m, 13 Mar 1988, T.F. Daniel, F. Almeda \& G. McPherson 5574 (holotype, CAS).

Elleanthus laxus Schltr., Repert. Spec. Nov. Regni Veg. 12: 213. 1913. = Elleanthus lancifolius C.Presl.

TYPE: Panama. Cerro de Garagara, Samba basin (Süddarien) zwischen 500 und 974 m, blühend im Feb 1911, H. Pittier 5640 (holotype, B, destroyed; drawing of type, AMES).

Elleanthus stolonifer Barringer, Brittonia 37: 287. 1985.

TyPE: Panama. Prov. Chiriquí: La Fortuna Hydroelectric project, in cloud forest on ridge behind camp, 13001400 m, 23 Mar 1978, B. Hammel 2280 (holotype, MO).

Elleanthus trilobatus Ames \& C.Schweinf., Schedul. Orchid. 8: 53. 1925. = Elleanthus longibracteatus (Lindl. ex Griseb.) Fawc.

TYPE: Panama. San Juan. Epiphyte at sea level. April 1923, C.W. Powell 3267 (Hort. Powell 97) (holotype, AMES).

Encyclia atropurpurea var. leucantha Schltr., Repert. Spec. Nov. Regni Veg. Beih. 17: 45. 1922. = Encyclia cordigera (Kunth) Dressler

Type: Panama. sehr selten auf Hügeln bei Panama City, C.W. Powell 149 (holotype, B, destroyed; lectotype designated by Christenson (1991b), AMES-23942, isolectotypes, K, MO-955992).

Encyclia atropurpurea Schltr. var. rhodoglossa Schltr., Repert. Spec. Nov. Regni Veg. Beih. 17: 45. 1922. = Encyclia cordigera

TYPE: Panama. sehr häufig auf Hügeln bei Panama City, C.W. Powell 80 (holotype, B, destroyed; lectotype designated by Christenson (1991b), AMES-23940; isolectotypes, AMES-23941, K).

Encyclia elegantula Dressler, Orchid Digest 68(4): 244. 2004.

TYPe: Panama. Panama, Altos de Pacora, 930 m, cloud forest, 18 Sept 2002, A. Maduro \& E. Olmos 303 (holotype, MO).

Encyclia fortunae Dressler, Orquídea (Mexico City) 7:359. 1980.=Prosthechea fortunae (Dressler) W.E.Higgins TyPE: Panama. Chiriquí: near Camp Hornito, Fortuna dam site, $82^{\circ} 13$ ' W, $8^{\circ} 44$ ' N, 1000-1400 m, 27 Sept. 1976, R.L. Dressler 5520 (holotype, US; isotype, PMA).

Encyclia hunteriana Schltr., Repert. Spec. Nov. Regni Veg. Beih. 17: 46. 1922. = Encyclia stellata (Lind1.) Schltr. TyPE: Panama. Bei David, im Chiriquí-Distrikt, etwa in Meereshöhe, C.W. Powell 84 (holotype, B, destroyed; 
lectotype designated by Christenson (1991b), AMES-23937; drawing, AMES; isolectotypes, AMES-955939, $\mathrm{K}, \mathrm{MO})$.

Encyclia powellii Schltr., Repert. Spec. Nov. Regni Veg. Beih. 17: 46. 1922. = Encyclia ceratistes (Lindl.) Schltr. TYPE: Panama. Chiriquí-Bezirk; 4000-5000 fuss, C.W. Powell 83 (holotype, B, destroyed, lectotype designated by Christenson (1987), AMES-23812; isolectotypes, AMES-26874, AMES-27875, K, MO-955937, MO-955938).

Encyclia sima Dressler, Orquideología 4: 91, fig. 1969. = Prosthechea sima (Dressler) W.E.Higgins

Type: Panama. Panama, Cerro Jefe, 12 Nov 1967. Selva nublada a aprox. 950 m, R. L. Dressler 3160 (holotype, US; isotypes, MO, PMA).

Encyclia silverarum Leopardi \& Carnevali, Phytotaxa 183 (3): 161. 2014.

TYPE: Panama. Panamá: Capira, Bajo Bonito, 13 April 2012, G. \& K. Silvera, s.n. (holotype, PMA).

Epidanthus crassus Dressler, Orquídea (Mexico City) 9: 15. 1983. = Epidendrum insolatum Barringer

TYPE: Panama. Coclé: al norte del Asserradero El Copé, 9-10 km al norte de El Copé, vertiente del Atlático, elev. 800-850m, selva de neblina muy húmeda, 1 Sep 1977, epífita, R.L. Dressler 5686 (holotype, US; isotypes, $\mathrm{F}, \mathrm{MO}$, PMA).

Epidendrum acrostigma Hágsater \& García-Cruz, Icon. Orchid. 3(2): t. 301. 1999. = Epidendrum gibbosum L.O.Williams

TyPe: Panama. Chiriquí: W end of high N of Barú summit, ca. 3000 m, col. 8 Apr. 1979, B. Hammel 6910, W. D'Arcy, E. C. Hills, S. Schwartz \& O. \&. H. Wolcott (holotype, AMO; isotype, MO).

Epidendrum alanjense Ames, Schedul. Orchid. 1: 13. 1922. = Encyclia stellata (Lind1.) Schltr.

TyPe: Panama. Chiriquí: Alanje, H. Pittier s.n. (holotype, US).

Epidendrum allenii L.O.Williams in Woodson \& Schery, Ann. Missouri Bot. Gard. 28: 418. 1941.

TYPE: Panama. Coclé: epiphytic, leaves and stem red, sepals and petals pale pink, labellum white, hills north of El Valle de Antón, ca. 1000 m, 14 Jul 1940, P. Allen 2203 (holotype, AMES).

Epidendrum amandum Ames, Schedul. Orchid. 4: 36. 1923. = Encyclia chloroleuca (Hook.) Neumann

Type: Panama. foothills east of Panama. Flowers in January and February, sea level, C.W. Powell 271 (holotype, AMES; isotype, MO).

Epidendrum antonense Hágsater, Icon. Orchid. 2: 108 fig. 1993.

TyPe: Panama. Colón: near Río Guanche, 10 Aug 1971, R.L. Dressler 4071 (holotype, AMO).

Epidendrum atropurpureum var. laciniatum Ames, F.T.Hubb. \& C.Schweinf., Bot. Mus. Leafl. 3: 63. 1935. = Encyclia cordigera

TYPE: Panama, foothills east of the city. "Sepals and petals are the same as Epid. atropurpureum, lip is a pink with dentate or notehed margin, the lateral lobes are very small and do not meet over the column. color rose pink.” At sea-level. C. W. Powell 287 (holotype, AMES).

Epidendrum bilamellatum Rchb.f. in W.G.Walpers, Ann. Bot. Syst. 6: 345. 1862.=Caularthron bilamellatum (Rchb.f.) R.E.Schult.

Syntypes: [Venezuela]. Wagener s.n. (syntype, W; fragment of syntype, AMES), [Panama] P. Chagres, Behr s.n. (syntype, W).

Epidendrum bisulcatum Ames, Schedul. Orchid. 5: 24.1923.

Type: Panama. Chiriquí: Palo Alto Hill, 6000 feet altitude, February to March 1923, C.W. Powell 280 (holotype, AMES; isotype, AMES).

Epidendrum brachybotrys Ackerman \& Montalvo, Selbyana 9: 129. 1986.

TYPE: Panama. Coclé: N of El Copé, near sawmill, edge of continental divide, elev. 1,200-1,400 m, windward slope, 22 Jan 1980, A. Montalvo 383 (holotype, SEL). 
Epidendrum brassavolae Rchb.f., Bot. Zeitung (Berlin) 10: 729. 1852. = Prosthechea brassavolae (Rchb.f.) W.E.Higgins

TYPe: Panama. Chiriquí Vulcan. 8000', J. Warszewicz s.n. (holotype, W).

Epidendrum brevicaule Schltr., Repert. Spec. Nov. Regni Veg. Beih. 17: 30. 1922.=Epidendrum schlechterianum Ames

TYPE: Panama. hills east of the [PanamaJ City, at sea level, C. W. Powell 18 (holotype, B, destroyed, lectotype designated by Christenson (1991b), AMES-23944; isolectotypes, K, MO-955889, S; illustration of type, AMES; drawing published in Mansfeld [1931: t. 47, nr. 187].). Superfluously designated by Santiago \& Hágsater (2007), MO-955889.

Epidendrum bugabense Hágsater, Icon. Orchid. 2: 115. 1993.

TyPe: Panama. Chiriquí: Distrito Bugaba: Cerro Punta, $8^{\circ} 52^{\prime} \mathrm{N}, 82^{\circ} 33^{\prime} \mathrm{W}, 2200 \mathrm{~m}$, along ridge to watershed to Bocas del Toro, 26 January 1985, H. van der Werff \& J. Herrera 6427 (holotype, AMO; isotypes, MO, PMA).

Epidendrum caligarium Rchb.f., Gard. Chron. 1869: 1110. 1869.

TYPE: New Granada. Wentworth W. Buller s.n. (holotype, W; isotypes, K, W; drawing of type, AMES).

Epidendrum caluerorum Hágsater, Icon. Orchid. 2: 116. 1993.

TYPE: Panama. Coclé: north of El Copé, $700 \mathrm{~m}$, vertiente atlántica, preparado de material cultivado 23 junio 1986, R.L Dressler sub E. Hágsater 6299 (holotype, AMO).

Epidendrum campylostalix Rchb.f., Bot. Zeitung (Berlin) 10: 730. 1852. = Prosthechea campylostalix (Rchb.f.) W.E.Higgins

SyntyPes: "Costa Rica und Chiriquí”; Costa Rica, Warszewicz s.n. (W; isosyntype, K); Panama. Chiriquí, J. Warszewicz s.n. (W).

Epidendrum chiriquense Rchb.f., Bot. Zeitung (Berlin) 10: 730. 1852. = Prosthechea varicosa (Bateman ex Lindl.) W.E.Higgins

TYPE: Costa Rica un [Panama] Chiriquí, J. Warszewicz s.n. (holotype, W).

Epidendrum chlorocorymbos Schltr., Repert. Spec. Nov. Regni Veg. Beih. 17: 30.1922.

TYPe: Panama. Am Gatun-See, unweit Panama-City, C.W. Powell 82 (holotype, B, destroyed, lectotype designated by Christenson (1991b), AMES-23950, illustration of type, AMES-26780; isolectotypes BM, K, MO-955935; drawing published in Mansfeld [1931: t. 48, nr. 190].).

Epidendrum centropetalum Rchb.f., Bot. Zeitung (Berlin) 10: 732.1852.

Type: Panama. Chiriquí: Vulkan, 4000', J. Warszewicz s.n. (holotype, W).

Epidendrum cirrhochiloides Hágsater \& E.Santiago, Icon. Orchid. 11: t. 1112. 2008.

Type: Panama. Coclé: Continental Divide above El Copé, 650-750 m, 27 Nov 1985, G. de Nevers, A. Henderson, H. Herrera, G. McPherson \& L. Brako 6396 (holotype, MO; isotype, AMO).

Epidendrum cocleense Ames, F.T.Hubb. \& C.Schweinf., Bot. Mus. Leafl. 4: 6. 1936.= Epidendrum guanacastense Ames \& C.Schweinf.

TYPE: Panama. Coclé: lower portion of valley and marshes along R. Antón, alt. $500 \mathrm{~m}$, Feb 1935, A.A. Hunter \& P.H. Allen 389 (holotype, AMES; isotypes, BM, BR, CAS, F, G, K, MICH, MO, NY, P, S, SEL, U, UC, US).

Epidendrum colonense Ames, Schedul. Orchid. 1: 14. 1922. = Epidendrum sculptum Rchb.f.

Type: Panama. Colón: Río Indio de Fató, near sea level, 24 Aug 1911, H. Pittier 4265 (holotype, US; illustration of type, AMES).

Epidendrum coriifolium var. purpurascens Schltr., Repert. Spec. Nov. Regni Veg. 17: 31. $19222490=$ Epidendrum allochronum Hágsater 
TYPE: Panama. Chiriquí, 3800-4500', C.W. Powell 119 (holotype, B, destroyed, lectotype designated by Christenson (1991b), AMES-23949; isolectotype, K; photo and drawing, AMES). Superfluously designated by Hágsater (1993).

Epidendrum croatii Hágsater, Icon. Orchid. 3(2): t. 328. 1999.

TYPE: Panama. Coclé: north slope and summit od Cerro Pilón, 900-1173 m, 16 Mar 1973, T.B. Croat 23240 (holotype, MO).

Epidendrum cryptanthum L.O.Williams, Amer. Orchid Soc. Bull. 11: [249], tab. 7. 1942.

TYPE: Panama. Coclé: hills north of El Valle de Antón, epiphytic, alt. 800-1000 m., 21 Nov 1942, P.H. Allen 2262 (holotype, AMES).

Epidendrum curvicolumna Ames, F.T.Hubb. \& C.Schweinf., Bot. Mus. Leafl. 3: 67. 1935. = Epidendrum simulacrum Ames

Type: Panama. Chiriquí: "Lino Hill," at 4000-4500 ft. alt. Grows in exposed places in considerable sun. Flowers green. Flowers May-July, C.W. Powell 296 (holotype, AMES; isotype, MO).

Epidendrum curvisepalum Hágsater \& Dressler, Icon. Orchid. 2: 125 fig. 1993.

TyPE: Panama. Chiriquí: Cerro Colorado, about 50 km north of San Félix, ca. 1400 m, 17 Aug 1975, R.L. Dressler 5087 (holotype, AMO).

Epidendrum cycnostalix Rchb.f., Bot. Zeitung (Berlin) 10: 731. 1852. = Epidendrum stamfordianum Bateman TyPe: Pamama. Chiriquí, J. Warscewicz s.n. (holotype, W).

Epidendrum cymbiglossum Hágsater, Icon. Orchid. 2: 126 fig. 1993.

TYPE: Panama. Chiriquí: Distr. Bugaba: Santa Clara, $8^{\circ} 50^{\prime} \mathrm{N}, 82^{\circ} 44^{\prime} \mathrm{W}$, to Cerro Pando, $H$. van der Werff \& $J$. Herrera 7186 (holotype, SEL).

Epidendrum dariense Hágsater, Kolan. \& L.Sánchez, Icon. Orchid. 14(10): t. 1421. 2013.

TYPE: Panama. Prov. Darién: About 10 km from Pavarandó, about 150-200 m, 29 March 2012, M. Kolanowska 410, (holotype, AMO; isotype, UGDA, flower in spirit).

Epidendrum dentilobum Ames, F.T.Hubb. \& C.Schweinf., Bot. Mus. Leafl. 3: 69. 1935.

TYPE: Panama. range of hills back of San Juan, upper reaches of the Chagres River. About sea-level. Late September, C.W. Powell 342 (holotype, AMES).

Epidendrum dosbocasense Hágsater, Orquídea (Mexico City) 12: 282. 1992.

TYPe: Panama. Veraguas: Guabal, Río Dos Bocas, about 16 km NW of Santa Fé, ca. 500 m., 15-16 Nov 1974, R.L. Dressler 4814 (holotype, AMO; isotypes, MO, PMA).

Epidendrum dunnii A.D.Hawkes, Orchid J. 1: 39. 1952. = Epidendrum pansamalae Schltr.

TYPE: Panama. Coclé, rain forest on side of Cerro Grande at headwaters of Río Ceri, $2000 \mathrm{ft}$. alt., 15 Oct 1950, H.A. Dunn s.n. (Alex D. Hawkes 2392) (holotype, UC).

Epidendrum dwyeri Hágsater, Icon. Orchid. 2: 130 fig. 1993.

TyPE: Panama. Chiriquí: Boquete, Cerro Horqueta, 5000-6000 ft, 8 Aug 1967, J.D. Dwyer \& S.M.V. Hayden 7733 (holotype, MO).

Epidendrum dwyerioides Hágsater \& E.Santiago, Icon. Orchid. 9: t. 929. 2007.

TyPE: Panama. Bocas del Toro: Changuinola, Sierra Madre, 2200 m, 5 Mar 2000, Jardín Finca Drácula 483 y 476, E. Olmos \& J. Nuñez 202 (holotype, AMO).

Epidendrum elephantinum Hágsater, Icon. Orchid. 7: t. 734. 2005.

TyPE: Panama. Darién: Parque Nacional del Darién W slope of Cerro Mali, on ridge between N \& S branches of Río Púcuro; ca. 18 km E of Púcuro, 84’N 77¹6’W, 1000-1200 m., 22 Oct 1987, B. Hammel, G. de Nevers, H. Cuadros \& H. Herrera 16421 (holotype, MO). 
Epidendrum ellipsophyllum L.O.Williams in Woodson \& Schery, Ann. Missouri Bot. Gard. 28: 419, tab. 22, fig. 1, 2. 1941.

TYPE: Panama. Coclé: epiphytic, flowers green, hills north of El Valle de Antón, 1000 m alt., 23 Jun 1940, $P$. Allen 2178 (holotype, AMES).

Epidendrum erythrostigma Hágsater, Icon. Orchid. 3(2): t. 331. 1999.

TYPE: Panama. Chiriquí, northwest of Cerro Punta, at INRENARE station in Parque Amistad, $8^{\circ} 54^{\prime} \mathrm{N}$, 82³5'W, 2300 m, 17 Oct 1992, M. Richardson sub G. McPherson 15905 (holotype, AMO; isotypes, MO, PMA).

Epidendrum flexuosissimum C.Schweinf., Bot. Mus. Leafl. 14: 55, tab. 14. 1949.

Type: Panama. Coclé, Cerro Pajita, hills north of El Valle, at 1100 m. alt., 27 Oct 1946, Paul H. Allen 3780 (holotype, AMES; isotypes, BM, G, MO).

Epidendrum folsomii Hágsater \& E.Santiago, Icon. Orchid. 8: 832. 2006.

TYPE: Panama. Coclé: sawmill 7 km NE of El Copé, 1800 ft., 22 Jun 1979, T. Antonio 1123 (holotype, AMO).

Epidendrum fortunae Hágsater \& Dressler, Icon. Orchid. 8: 833. 2006.

TYPE: Panama. Chiriquí: Boquete, Fortuna dam site, epiphytic, in cloud forest, 10 Feb 1985, H. van der Werff \& Cor van Hardeveld 6855 (holotype, AMO; isotype, MO).

Epidendrum fragrans var. pachypus Schltr., Repert. Spec. Nov. Regni Veg. Beih. 17: 32. 1922. = Prosthechea fragrans (Sw.) W.E. Higgins

TyPE: Panama. Chiriquí: ca. 4000 ft, C.W. Powell 208 (holotype, B, destroyed; lectotype designated by Christenson (1991b), AMES-23961; isolectotype, MO-956025).

Epidendrum fuscopurpureum Schltr., Repert. Spec. Nov. Regni Veg. Beih. 17: 32. 1922. = Epidendrum coriifolium Lindl.

TyPE: Panama. Chiriquí, c. 4500', blühend im Oktober, C.W. Powell 257 (holotype, B, destroyed; lectotype designated by Christenson (1991b), photograph at AMES-39206).

Epidendrum galeochilum Hágsater \& Dressler, Icon. Orchid. 7: t. 738. 2005.

TYPe: Panama. Coclé: El Copé, April 1978, R.L. Dressler s.n. (holotype, AMO (color illustration prepared from slides; photo, AMES, COL, CR, JAUM, K, MO, PMA, SEL).

Epidendrum gibbosum L.O.Williams in Woodson \& Schery, Ann. Missouri Bot. Gard. 28: 420. 1941.

TYPE: Panama. Chiriquí: terrestrial, flowers maroon, Casita Alta to Cerro Copete, alt. 2300-3300 m, 10 Jul 1940, Woodson \& Schery 367 (holotype, MO).

Epidendrum glandulosum Ames, Schedul. Orchid. 7: 5, pl. 20. 1924. = Epidendrum stangeanum Rchb.f.

TYPE: Panama. on trail from Colon, 1923. Sepals and petals olive green, labellum white. Flowering in September. Sea level, C.W. Powell 338 (holotype, AMES).

Epidendrum gregorii Hágsater, Icon. Orchid. 2: 138 fig. 1993.

TYPE: Panama. Valle de Antón, comprada a Gregorio [Ruiz] en la Mesa, prensada 30 agosto 1981, E. Hágsater 6410 (holotype, AMO; isotype, MEXU).

Epidendrum gymnochlamys Hágsater \& E.Santiago, Icon. Orchid. 8: 839. 2006.

TYPE: Panama. Chiriquí: aboye Boquete, on trail to Pate Macho on continental divide, 1850-2100 m, 13 March 1988, G. McPherson 12323 (holotype, MO).

Epidendrum gymnopodum Hágsater, Icon. Orchid. 7: t. 741. 2005. = Epidendrum cancanae (P.Ortiz) Hágsater

TYPE: Panama. Darién: Parque Nacional del Darién Ridge between N \& S branches of Río Púcuro, across river from old Kuna village of Tacarcuna; ca. 18 km E of Púcuro, 804' N 7r16’ W, 600-1000 m, 21 October 1987, B. Hammel, G. de Nevers, H. Cuadros \& H. Herrera 16342 (holotype, MO). 
Epidendrum hammelii Hágsater \& L.Sánchez, Icon. Orchid. 2: 143 fig. 1993.

TYPE: Panama. Coclé: near sawmill $16.7 \mathrm{~km}$ north of turnoff to Coclesito from Llano Grande, alt. $700 \mathrm{ft}, 6$ March 1978, B. Hammel 1809 (holotype, MO).

Epidendrum hornitense Hágsater \& L.Sánchez, Icon. Orchid. 8: 841. 2006.

TYPe: Panama. Chiriquí: Camp Hornito, Fortuna Dam site, 844’'N 82²13’W, 1000-1200 m, 12 Aug 1976, R.L. Dressler 5326 (holotype, AMO; isotype, PMA).

Epidendrum hunterianum Schltr., Repert. Spec. Nov. Regni Veg. Beih. 17: 33. 1922.

TyPE: Panama. Im Überschwemmungsgebiete des Gatun-Sees, C.W. Powell 29 (holotype, B, destroyed, lectotype designated by Christenson (1991b), K; drawing of type, AMES-26778; drawing published in Mansfeld [1931 : t. 50, nr. 198]).

Epidendrum incomptum Rchb.f., Bot. Zeitung (Berlin) 10: 733. 1852.

TYPE: [Panama]. Chiriquí: J. Warszewicz s.n. (holotype, W; illustration of type, AMES).

Epidendrum infundibulum Hágsater \& E.Santiago, Icon. Orchid. 9: t. 946. 2007.

TYPE: Panama. Chiriquí: Vicinity of Fortuna Dam along trail below highway and across the valley of the Hornito, $8^{\circ} 45^{\prime} \mathrm{N} 82^{\circ} 15^{\prime} \mathrm{W}, 1100-1350$ m, 24 Jan 1988, G. McPherson 11986 (holotype, MO).

Epidendrum isthmi Schltr., Repert. Spec. Nov. Regni Veg. Beih. 17: 34. 1922.

TYPE: Panama. Auf Hügelm bei Panama-City, C.W. Powell 104 (holotype, B, destroyed; lectotype designated by Christenson (1991b), AMES-23956; isolectotypes, AMES-23957, K, MO; drawing of type, AMES-26750; drawing published in Mansfeld [1931: t. 51, nr. 201].).

Epidendrum jefestigma Hágsater \& García-Cruz, Icon. Orchid. 3(2): t. 340. 1999.

TYPe: Panama. Panama: Cerro Jefe, ca. 800 m, col. 11 Apr. 1977, W. G. D’Arcy 11379 (holotype, MO).

Epidendrum jefeallenii Hágsater \& García-Cruz, Icon. Orchid. 3(2): t. 339. 1999.

TyPe: Panama. Provincia Panama: Cerro Jefe, alt. 1000 m, 13 Feb. 1977, C. Luer \& J. Luer 1400 (holotype, SEL; isotype, F).

Epidendrum kerichilum Hágsater, Orquídea (Mexico City), n.s., 11: 26. 1988.

TyPE: Panama. Chiriquí: Cerro Horqueta, al E de Volcán Barú, 1700-2300 m, selva alta. Colectada March 1981. Preparado de material cultivado, 4 June 1981, E. Hágsater \& R.L. Dressler 6519 (holotype, AMO; isotypes, $\mathrm{K}, \mathrm{MO})$.

Epidendrum lacteum Dressler, Orquideología 11(2): 108-109, photo. 1976.

TyPE: Panama. Veraguas, cerca de la divisa continental, aprox. 12-15 km al noroeste de Santa Fé; alt. aprox. 700 m.; selva muy húmeda, 17 May 1975, R.L. Dressler 5030 (holotype, US).

Epidendrum leucocardium Schltr., Repert. Spec. Nov. Regni Veg. 12: 206. 1913. = Epidendrum eburneum Rchb.f.

TyPe: Panama. Colón: Río Fato, etwa bei Meereshïhe, blühend im Aug 1911, H. Pittier 4266 (holotype, B, destroyed; isotype, US).

Epidendrum longibracteatum Hágsater, Icon. Orchid. 3(2): t. 347. 1999.

TYPE: Panama. Chiriquí: Cerro Horqueta, detrás de Boquete, al E del Volcán Barú, ca. 2000 m, col 15 Mar 1981, pressed 7 June 1981, E. Hágsater, R.L. Dressler \& C. Castro 6526 (holotype, AMO; isotypes, PMA, SEL, AMES, COL, CR, ENCB, F, G, INB, K, MEXU, MO, NY, P, QCNE, S, SCZ, US, USM, WIS).

Epidendrum lorifolium Schltr., Repert. Spec. Nov. Regni Veg. Beih. 17: 35. 1922. = Epidendrum flexuosum G.Mey

TYPE: Panama. Auf Hügeln bei Panama City, C.W. Powell 138 (holotype, B, destroyed; lectotype designated by Christenson (1991b), AMES-23962; isolectotypes, AMES-27945, K, MO-955980; drawing of type, AMES-26766; drawing published in Mansfeld [1931: t. 52, nr. 205].). 
Epidendrum lutheri Hágsater, Icon. Orchid. 2: 155 fig. 1993.

Type: Panama. Chiriquí-Bocas del Toro: Cerro Colorado, above the Copper Mine, ca. $1600 \mathrm{~m}$, June 1986 [as 1989], H. Luther, Besse, Halton \& Kress 1059 (holotype, SEL; isotypes, AMES, AMO, AMO, US).

Epidendrum maduroi Hágsater \& García-Cruz, Icon. Orchid. 3(2): t. 352. 1999.

TYPe: Panama. Chiriquí: Norte de Guadalupe, Cerro Punta, Volcán Barú, 2000-3000 M, collected 15 Mar 1981, pressed 22 Feb 1983, E. Hágsater \& R.L. Dressler 6468 (holotype, AMO; isotype, MO).

Epidendrum mantis-religiosae Hágsater, Orquídea (Mexico City), n.s., 11: 27. 1988.

Type: Panama. Panama: El Llano-Cartí. Colectado 18 Mar 1981. Preparado de material cultivado. 25 Sep 1982, R.L Dressler sub Hágsater 6304 (holotype, AMO; isotype, MO).

Epidendrum microanoglossum Hágsater \& Karremans, Icon. Orchid. 14(10): t. 1457. 2013.

TYPE: Costa Rica-Panama. Puntarenas-Chiriquí: Coto Brus-Renacimiento, línea fronteriza entre Cerro Quijada del Diablo y Cerro Pando, entre mojones N.336-338, 854’51.9” N 8243’59.13” W, 2205 m, bosque muy húmedo premontano, epífitas en bosque primario, "in itinere per limitem Costa Rica et Panama inter montis Quijada del Diablo et montis Pando”, 19 Abril 2011, A. P. Karremans, D. Bogarín \& D. Jiménez 4010 (holotype, CR; isotype JBL-Spirit).

Epidendrum microrigidiflorum Hágsater, Icon. Orchid. 8: 853. 2006.

TYPE: Panama. Chiriquí: Valle de Fortuna, 1100-1200 m, 27 Oct. 1999, R.L. Dressler 6259 (holotype, AMO).

Epidendrum mora-retanae Hágsater, Icon. Orchid. 2: 161 fig. 1993.

TYPE: Panama. Chiriquí: Cuesta de Piedra-Finca El Milagro, 800-1000 m, prepared from cultivated material, 27 February 1984, E. Hágsater \& R.L. Dressler 6499 (holotype, AMO).

Epidendrum niveocaligarium Hágsater, Icon. Orchid. 8: 860. 2006.

TYPE: Panama. Darién: 10 km NE of Jaqué, ridge between Río Tabuelita and Río Pavarandó, 1400-1600 ft., 1 Feb 1981, W.G. D'Arcy \& K. Sytsma 14547 (holotype, MO).

Epidendrum $\times$ nocteburneum Hágsater \& L.Sánchez, Icon. Orchid. 11: t. 1148. 2008.

TYPe: Panama. Comarca de San Blas, Nusagandi, El Llano Cartí, carretera, pressed 5 Mar 1981, R.L. Dressler sub E. Hágsater 6285 (holotype, AMO).

Epidendrum nocturnum Jacq. var. panamense Schltr., Repert. Spec. Nov. Regni Veg. Beih. 17: 36. 1922.= Epidendrum buenaventurae F.Lehm. \& Kraenzl.

TYPE: Panama. Gatun-See, ungefähr in Seehöhe, C.W. Powell 35 (holotype, B, destroyed; lectotype designated by Christenson (1991b), AMES-23803; isolectotypes, K, MO).

Epidendrum orthodontum Hágsater \& L.Sánchez, Icon. Orchid. 3(2): t. 361. 1999.

TYPe: Panama. Chrirquí: Cuesta de Las Palmas, southern slopes of Cerro de La Horqueta, humid forest, 17002100 m, 17 Mar 1911, H. Pittier 3220 (holotype, US; isotype, AMES).

Epidendrum pajitense C.Schweinf., Bot. Mus. Leafl. 14: 56, t. 1949.

TYPE: Panama. Coclé: Cerro Pajita, hills north of El Valle, at 1100 m. alt.,27 Oct 1946, Paul H. Allen 3784 (holotype, AMES).

Epidendrum panamense Schltr., Repert. Spec. Nov. Regni Veg. 12: 212. 1913.

TYPE: Panama. Cerro de Garagara, Sambu-basin (Süd-Darién), 500-974 m, blühend in Feb 1902, H. Pittier 5635 (holotype, B, destroyed; isotypes, US, AMES).

Epidendrum pendens L.O.Williams, Ann. Missouri Bot. Gard. 28: 421, tab. 23. 1941.

TYPE: Panama. Coclé: epiphytic, pendant plants, foliage gray, flower terminal, green, La Mesa, El Valle de Antón, alt. ca. 1000 m, 22 Jun 1941, Allen 2570 (holotype, AMES). 
Epidendrum philowercklei Hágsater \& E.Santiago, Icon. Orchid. 8: 870. 2006.

TyPE: Panama. Bocas del Toro: Changuinola, Sierra Madre, 2200 m, 5 Feb. 2000, Jardín Finca Drácula 482, A. Maduro \& E. Olmos 200 (holotype, AMO).

Epidendrum piliferum Rchb.f., Linnaea 41: 83. 1876.

TyPe: Costa Rica. [Panama]. Chiriquí v. J. Warscewicz s.n. Cultum miserunt dom Linden a Wallisio, dom. Veitch a Zahnio lectum, lectotype designated by Santiago \& Hágsater (2008), W-49570; isolectotype, W-49582).

Epidendrum pirrense Hágsater, Icon. Orchid. 4(3): t. 471. 2001.

TyPE: Panama. Darién: Top of ridge leading to Cerro Pirre. Area near Rancho Plástico, 1200-1400 m, 13 Nov 1977, J.P. Folsom, José Contrerra \& Brijilio (of Piji Vasal) 6276 (holotype, MO).

Epidendrum plagiophyllum Hágsater, Icon. Orchid. 3(2): t. 370. 1999.

TyPE: Panama. Chiriquí: Volcán Barú, Cerro Punta, al N de Guadalupe, 2000-2300 m, collected 15 Mar 1981, pressed 19 Dec 1981, E. Hágsater, R.L. Dressler \& C. Castro 6481 (holotype, AMO; isotype, STRI).

Epidendrum platycardium Schltr., Repert. Spec. Nov. Regni Veg. Beih. 17: 36. 1922. = Prosthechea spondiada (Rchb.f.) W.E.Higgins

TyPE: Panama. Chiriquí: ca. 4000 ft Panama, C.W. Powell 141 (holotype, B, destroyed; lectotype designated by Christenson (1991b), AMES; isolectotypes, AMES, K; drawing of type, AMES-26748; drawing published in Mansfeld [1931: t. 54, nr. 216].).

Epidendrum platyphyllostigma Hágsater \& García-Cruz, Icon. Orchid. 3(2): t. 372. 1999.

TyPE: Panama. Chiriquí: Cerro Colorado, ca. 50 km NO of San Félix, 1400-1450 m, 20 Sep. 1977, R. L. Dressler 5713 (holotype, AMO).

Epidendrum platystomum Hágsater \& L.Sánchez, Icon. Orchid. 8: 873. 2006.

TyPE: Panama. Chiriquí: along Boquete Trail, Cerro Respingo, East of town of Cerro Punta, ca. 2000-2500 m., 11 Sept 1972, A. Gentry 5948 (holotype, MO; isotype, MO).

Epidendrum pleurothalloides Hágsater, Icon. Orchid. 2: t. 174. 1993.

TyPE: Panama. Chiriquí: Cerro Horqueta, detrás de Boquete, 1700-2300 m, prensado en cultivo 25 septiembre 1983, E. Hágsater \& R.L. Dressler 6524 (holotype, AMO; isotypes, F, K, MO, US).

Epidendrum porphyrophyllum Schltr., Repert. Spec. Nov. Regni Veg. Beih. 17: 37. 1922. = Epidendrum porpax Rchb.f.

TyPE: Panama. Auf Hügeln bei Panama City, C.W. Powell 183 (holotype, B, destroyed; lectotype designated by Christenson (1991b), AMES-23806; isolectotype, MO-956016; drawing published in Mansfeld [1931: t. 55, nr. 217].).

Epidendrum powellii Schltr., Repert. Spec. Nov. Regni Veg. Beih. 17: 38. 1922.

TyPE: Panama. Chiriquí, c. 4000', C.W. Powell 228 (holotype, B, destroyed; neotype designated by Santiago \& Hágsater (2008), lectotype designated by Christenson (1991b), AMES-23808; illustration of type, AMES26768; drawing published in Mansfeld [1931: t. 55, nr. 218].).

Epidendrum prismatocarpum Rchb.f., Bot. Zeitung (Berlin) 10: 729. 1852. = Prosthechea prismatocarpa (Rchb.f.) W.E.Higgins

TYPE: [Panama]. Chiriquí, J. Warszewicz s.n. (holotype, W).

Epidendrum probiflorum Schltr., Repert. Spec. Nov. Regni Veg. Beih. 17: 39. 1922.

TYPE: Panama. Chiriquí gegen 4500', C.W. Powell 250 (holotype, B, destroyed; lectotype designated by Christenson (1991b), AMES-27066; isolectotype, MO-956056).

Epidendrum pseudoschumannianum Fowlie, Orchid Digest 33: 255. 1969. 
TYPE: Panama. Coclé: Beyond la Loma del Tigre, District of El Valle de Anton, on very tall trees of a warm, humid jungle, 800-1000 m, C.K. Horich s.n. (holotype, UCLA).

Epidendrum pudicum Ames, Schedul. Orchid. 6: 71, fig. 10. 1923.

Type: Panama. Chiriquí: Palo Alto, flowered under cultivation at Balboa in Aug 1923, C.W. Powell 319 (holotype, AMES).

Epidendrum radicans var. chiriquense Schltr., Repert. Spec. Nov. Regni Veg. Beih. 17: 39. 1922.=Epidendrum radicans Pav. ex Lindl.

TYPE: Panama. Chiriquí, terrestrisch an Wegrändern zwischen Kraut und Gebüsch, c. $4000 \mathrm{ft}$. und darunter, C.W. Powell 61 (holotype, B, destroyed; lectotype designated by Hágsater \& Salazar (1990), MO-955940; isolectotypes, AMES-23811, K).

Epidendrum radlkoferianum Schltr., Repert. Spec. Nov. Regni Veg. 17: 142. 1921. = Epidendrum volutum Lindl. \& Paxton

TyPE: Panama. Provinz Chiriquí, April 1858, Dr. Moritz Wagner s.n. (holotype, B, destroyed; photo of type, AMES; isotype, M).

Epidendrum rafael-lucasii Hágsater, Icon. Orchid. 2: 179. 1993.

TYPE: Panama. Sin localidad, preparado de material cultivado 15 abril 1983, R.L. Dressler sub E. Hágsater 6293 (holotype, AMO).

Epidendrum resectum Rchb.f., Linnaea 41: 83. 1876.

TyPE: Ex America centrali, vix dubie ex [Panama] Chiriquí vivum misit b. Zahn ad hortulanos Veitch, Londinenses, qui bis specimina mecum communicavere viva. Zahn ex Veitch s.n. (holotype, W; illustration of type, AMES).

Epidendrum rigidum Jacq. var. angustisegmentum L.O.Williams, Ann. Missouri Bot. Gard. 33: 371. 1946. = Epidendrum angustisegmentum (L.O. Williams) Hágsater

TYPE: Panama. Bocas del Toro: Water Valley, vicinity of Chiriquí Lagoon, von Wedel 1708 (holotype, AMES).

Epidendrum rousseauae Schltr., Beih. Bot. Centralbl. 36, Ab. 2: 407. 1918.

TYPE: Panama. Am Isthmus. Mrs. Rousseau s.n. (holotype, B, destroyed).

Epidendrum schistostemum Hágsater, Laube \& L.Sánchez, Icon. Orchid. 11: t. 1177. 2008.

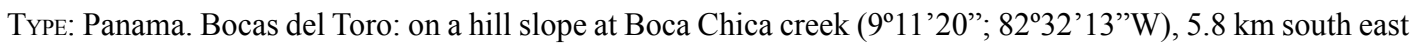
of the community Charco La Pava at the Río Changuinola, 550 m., Oct 2007, S. Laube 563 (holotype, PMA).

Epidendrum sculptum Rchb.f., Bonplandia (Hannover) 2: 89. 1854.

TYPE: Panama. von Chagres von Herrn Keferstein eingeführt von Herrn Lehmann cultivirt, Lehmann s.n. (holotype, W; illustration of type, AMES).

Epidendrum simulacrum Ames, Schedul. Orchid. 6: 75. 1923.

TyPE: Panama. Chiriquí: Lino Hill, alt 4000-4500 ft, growing in exposed place, flowering in May and June, C.W. Powell 298 (holotype, AMES).

Epidendrum spathulipetalum Hágsater \& Dressler, Icon. Orchid. 4(3): t. 486. 2001.

TYPE: Panama. Chiriquí: de arriba de Boquete, cultivada en Finca Dracula, Cerro Punta, 20 Aug 1998, Robert L. Dressler 6230 (holotype, AMO; isotype, PMA).

Epidendrum sphenostele Hágsater \& E.Santiago, Icon. Orchid. 9: t. 989. 2007.

TYPE: Panama. Chiriquí: Boquete, Valle Fortuna; received flowers 1 Oct 1987, cultivated by Andres Maduro, sent to Robert L. Dressler, who in turn sent it to E. Hágsater, E. Hágsater 9361 (holotype, AMO).

Epidendrum stangeanum Rchb.f., Gard. Chron., n.s., 15: 462. 1881.

TYPE: Panama. F.F. Stange s.n. (holotype, W; drawing of type, AMES). 
Epidendrum subpatens Schltr., Repert. Spec. Nov. Regni Veg. Beih. 17: 40. 1922. = Epidendrum coronatum Ruiz \& Pav.

TYPe: Panama. Im Gebiete des Gatun-Sees und auch in Veraguas bei Santiago, C.W. Powell 86 (holotype, B, destroyed lectotype designated by Christenson (1991b), AMES-23943; isolectotypes, MO-955940, illustration of type, AMES-26812; drawing published in Mansfeld [1931: t. 56, nr. 223].).

Epidendrum suturatum Hágsater \& Dressler, Icon. Orchid. 2: 190 fig. 1993.

TYPE: Panama. Veraguas: ridge east of Cerro Tute, northwest of Santa Fé, 800-1000 m elev., 12 October 1975, R.L. Dressler 5187 (holotype, AMO; isotype, AMES).

Epidendrum tacarcunense Hágsater, Icon. Orchid. 3(2): t. 384. 1999.

TYPE: Panama. Darién: vicinity of Cerro Tacarcuna summit camp, 1500-1750 m, 31 Jan 1975, A. Gentry \& S. Mori 14043 (holotype, MO).

Epidendrum teretifolium Sw. var. powellianum Schltr., Repert. Spec. Nov. Regni Veg. Beih. 17: 41. 1922. = Jacquiniella teretifolia (Sw.) Britton \& P. Wilson

TYPE: Panama. Chiriquí, 4000-4500 ft., C.W. Powell 240 (holotype, B, destroyed; lectotype designated by Christenson (1991b), AMES-27067; isolectotype, MO-956048).

Epidendrum tetraceros Rchb.f., Bot. Zeitung (Berlin) 10: 733. 1852.

TYPE: [Panama]. Chiriquí, J. Warscewicz 2721 (holotype, W; illustration of type, AMES; photo of type, AMO; isotype, $\mathrm{K}$; photo of isotype, AMES).

Epidendrum transversellipticum Hágsater, Icon. Orchid. 4(3): t. 492. 2001.

TYPE: Panama. Chiriquí/Bocas del Toro: Cerro Colorado, along intersection of Bocas Rd. with main ridge rd., $15.4 \mathrm{~km}$ form Chami, 1400-1700 m, 24 Oct 1977, J. P. Folsom 6101 (holotype, $\mathrm{MO})$.

Epidendrum trialatum Hágsater, Orquídea (Mexico City), n.s., 9: 356. 1984.

TYPe: Panama. Coclé: Valle de Antón, Nov 1981, R.L. Dressler sub Hágsater 4674 (holotype, AMO; isotypes, AMES, CR, K, PMA, US; illustration of type; photo of type).

Epidendrum unicallosum Hágsater \& E.Santiago, Icon. Orchid. 8: 895. 2006.

TYPE: Panama. Chiriquí: Guadalupe, 20 Feb 2001, A. Maduro \& E. Olmos 179 (holotype, AMO).

Epidendrum veraguasense Hágsater, Orquídea (Mexico City) 12: 286. 1992.

TYPE: Panama. Veraguas: Along steep trail to summit of Cerro Tute, ca. $3 \mathrm{~km}$ above Escuela Agricultura Alto Piedra near Santa Fé, 3000-3100 ft., 4 Jan 1981, K. Sytsma \& T. Antonio 3046 (holotype, AMO; isotype, MO).

Epidendrum verecundum Schltr., Repert. Spec. Nov. Regni Veg. Beih. 17: 42. 1922. = Epidendrum strobiliferum Rchb.f.

TYPE: Panama. Auf Hügeln bei Panama City, C.W. Powell 209 (holotype, B, destroyed; lectotype designated by Christenson (1991b), AMES-23817; isolectotype, MO-956026; drawing of type, AMES-26767; drawing published in Mansfeld [1931: t. 58, nr. 232].).

Epidendrum warszewiczii Rchb.f., Bot. Zeitung (Berlin) 10: 742. 1852.

TYPE: Costa Rica. [Panama] Veraguas, J. Warszewicz s.n. (holotype, W-50158 (drawing, AMES)).

Epidendrum witherspooniorum Hágsater \& Dressler, Icon. Orchid. 7: t. 798. 2005.

Type: Panama. Panama, Cerro Jefe, trail leading W from summit, 24 Sept 1975, J.T. \& F. Witherspoon 8489 (holotype, MO).

Erythrodes epiphytica Dressler, Orquídea (Mexico City) 13: 257. 1993. = Microchilus epiphyticus (Dressler) Ormerod

TyPE: Panama. Coclé: Hills N of El Valle de Antón, 12 May 1973, R.L. Dressler 4383 (holotype, MO; isotype, PMA). 
Erythrodes killipii Ames, Proc. Biol. Soc. Washington 34: 150. 1921. = Microchilus nigrescens (Schltr.) Ormerod TYPe: Panama. Chiriquí: valley of the Río Caldera, from El Boquete to the Cordillera, 1 Feb 1918, Killip 3561 (holotype, AMES; isotypes, AMES, US, Rochester Academy of Science; illustration and photo of type).

Erythrodes roseoalba Dressler, Orquídea (Mexico City) 13: 257. 1993. = Aspidogyne roseoalba (Dressler) Ormerod

TYPE: Panama. Veraguas: Ridge east of Cerro Tute [actually C. Arizona], NW of Santa Fé, elev. 1000-1200 m; 30 Oct 1977, R.L. Dressler 5736 (holotype, MO).

Eurystyles cornu-bovis Szlach., Fragm. Florist. Geobot. 37: 16. 1992.

TYPE: Panama. Veraguas: between second and third branches of Río Santa María, 10-12 km NW of Santa Fe, 4 Aug 1975, R.L. Dressler 5077 (holotype, FLAS).

Evelyna hymenophora Rchb.f., Bot. Zeitung (Berlin) 10: 710. 1852. = Elleanthus hymenophorus (Rchb.f.) Rchb.f.

TYPE: [Panama]. “Chiriquí Cordilleren. 6-7000'. Decbr.”, J. Warszewicz s.n. (holotype, W).

Fregea amabilis Rchb.f., Bot. Zeitung (Berlin) 10: 712. 1852. = Sobralia amabilis (Rchb.f.) L.O.Williams

TYPe: [Panama]: Cordilleren von Chiriquí, Herrn Dr. Klotzsch s.n. (holotype, W).

Gongora armeniaca var. bicornuta C.Schweinf. \& P.H.Allen, Bot. Mus. Leafl. 13: 139. 1948. = Gongora horichiana Fowlie

TYPE: Panama. Veraguas: region west of Santa Fé, Cerro Tute, at 1000 m alt., 24 Jun 1947, P.H. Allen 4648 (holotype, AMES; isotype, MO).

Gongora dressleri Jenny, Monogr. Gen. Gongora 90. 1993.

TyPE: Panama. Tacaruma [Tacarcuna?]: 1500 m, R.L. Dressler s.n., 1980, ex cult. R. Jenny, Bern (holotype, $\mathrm{G}$; isotype, K).

Gongora gibba Dressler, Orquideología 7: 72. 1972.

TYPE: Panama. Colón camino madero de Santa Rita, aprox. 15 km al este de Colón; floreció en cultivo 16 Apr 1968, R.L. Dressler s.n. (holotype, US; isotype, PMA).

Gongora maculata var. latibasis C.Schweinf. \& P.H.Allen, Bot. Mus. Leafl. 13: 144. 1948. = Gongora latibasis (C.Schweinf. \& P.H.Allen) Jenny

TYPe: Panama. Canal Zone: ca. 2 mi W of Gatún Dam, at ca. 184 m alt., 1 Apr 1939, blooming time, $H$. Butcher s.n. (holotype, AMES).

Gongora powellii Schltr., Repert. Spec. Nov. Regni Veg. Beih. 17: 62. 1922.

TYPe: Panama. Auf Hügeln bei Panama-City, C.W. Powell 76 (holotype, B, destroyed; lectotype designated by Christenson (1991b), AMES-23856; isolectotypes, AMES-23855, MO; drawing of type, AMES-26848; drawing published in Mansfeld [1931: t. 62, nr. 247].).

Govenia powellii Schltr., Repert. Spec. Nov. Regni Veg. Beih. 17: 51. 1922.

TyPE: Panama. Chiriquí, in Schluchten, c. 4000 fuss, C.W. Powell 205 (holotype, B, destroyed, lectotype designated by, Christenson (1991b), AMES-23837; isolectotype, MO-956022; drawing of type, AMES26851; drawing published in Mansfeld [1931: t. 60, nr. 240].).

Habenaria avicula Schltr., Repert. Spec. Nov. Regni Veg. 17: 138. 1921.

TYPE: Panama. Ohne nähere Standortsangabe, Dec 1900, W. Joseph s.n. (holotype, B, destroyed).

Habenaria mediocris Dressler, Bol. Inst. Bot. Univ. Guadalajara 7(1-3): 96. 1999.

TYPE: Panama. Chiriquí: Llanos del Volcán, in rocky grassland, flowers pale green, lip yellowish, 16 Jul 1979, R.L. Dressler 5845 (holotype, MO). 
Habenaria patentiloba Ames, Proc. Biol. Soc. Washington 34: 151. 1921.

TYPE: Panama. Panama. Orange River Valley, 7 Oct 1917, 60 m alt., dense forest, Killip 3124 (holotype, AMES; illustration of type, AMES; isotype, Rochester Academy of Science, US).

Habenaria petalodes var. micrantha Rchb.f., Beitr. Orchid.-K. C. Amer. 5. 1866. = Habenaria warszewiczii Schltr. TyPE: Lectam in Panama. J. Warscewicz s.n. (holotype, W).

Hexadesmia pachybulbon Schltr., Repert. Spec. Nov. Regni Veg. Beih. 17: 26. 1922. = Scaphyglottis pachybulbon (Schltr.) Dressler

TyPe: Panama. Chiriquí, 4500 ft., C.W. Powell 229 (holotype, B, destroyed; lectotype designated by Christenson (1991b), AMES-23833; drawing of type, AMES-26779; isolectotype, MO-956040; drawing published in Mansfeld [1931: t. 45, nr. 180].).

Hexadesmia powellii Schltr., Repert. Spec. Nov. Regni Veg. Beih. 17: 27. 1922. = Scaphyglottis acostae (Schltr.) C.Schweinf.

TyPe: Panama. Chiriquí, c. 5000 Fuss ü. M., C.W. Powell 225 (holotype, B, destroyed; lectotype designated by Christenson (1991b), AMES-23822; isolectotypes, AMES, MO-956038; drawing of type, AMES-26769; drawing published in Mansfeld [1931: t. 46, nr. 181].).

Hexisea arctata Dressler, Orquídea (Mexico City) 7: 233. 1979. = Scaphyglottis arctata (Dressler) B.R.Adams TyPE: Panama. Veraguas: ridge of Cerro Tute, about $1000 \mathrm{~m}$, about $7 \mathrm{~km} \mathrm{NW}$ of Santa Fe, 12 Oct 1975, R.L. Dressler 5185 (holotype, US; isotypes, AMO, PMA, SEL, MO).

Hexisea bidentata Lindl., J. Bot. (Hooker) 1: 8. 1834. = Scaphyglottis bidentata (Lindl.) Dressler TYPE: Panama et Columbia occidentali, H. Cuming s.n. (holotype, K).

Horichia dressleri Jenny, Orchidee (Hamburg) 32: 108. 1981.

TYPE: Panama. Aus Kultur (JE-29, Cons. Jard. Bot. Chambesy, Genf). Pflanze aus Panama, Provinz Bocas, 8 km nördlich von Almirante, nahe der Strasse von Almirante nach Changuinola, $200 \mathrm{~m}$ höhe, 10 Dec 1979, $R$. Jenny JE-29 (holotype, G).

Huntleya cerina Lindl. \& Paxton, Paxton's Fl. Gard. 3: 62. 1852. = Pescatoria cerina (Lindl. \& Paxton) Rchb.f. TyPE: [Panama]. It was found in Veragua, by Mr. Warczewicz, on the Chiriquí Volcano, at 8000 feet above the level of the sea, and was sold by auction by Mr. Stevens sometime in 1851. Mr. Rucker has been the first to flower it, J. Warscewicz s.n. (holotype, K).

Huntleya fasciata Fowlie, Orchid Digest 30: 281. 1966.

TYPe: Panama. Cocle. El Valle de Anton, 3000 ft., Sept 1964, Edna Jackson J64 P23 (holotype, UCLA).

Ionopsis utricularioides var. parviflora Schltr., Repert. Spec. Nov. Regni Veg. Beih. 17: 74. 1922. = Ionopsis utricularioides (Sw.) Lindl.

TyPE: Panama. auf Hügeln bei Panama City, C.W. Powell 181 (holotype, B, destroyed; lectotype designated by Christenson (1991b), AMES-23889; isolectotypes, MO-956014, MO-961312).

Isochilus chiriquensis Schltr., Repert. Spec. Nov. Regni Veg. Beih. 17: 25. 1922.

TyPE: Panama. Chiriquí-Distrikt, c. 4000', C.W. Powell 98 (holotype, B, destroyed, lectotype designated by Christenson (1991b), AMES-23831; isolectotypes, K, MO-955949; drawing of type, AMES-26817; drawing published in Mansfeld [1931: t. 45, nr. 178].).

Jacquiniella pedunculata Dressler, Orquideología 5: 24. 1970.

TyPE: Panama. Colón: Camino maderero de Santa Rita; preparada de cultivo 28 Jan 1966, R.L. Dressler 2986 (holotype, US; topotype, K).

Kefersteinia alata Pupulin, Harvard Pap. Bot. 8(2):161. 2004.

TyPE: Panama. Bocas del Toro: road from David to Chiriquí Grande, around km. 74, ca. 450 m, epiphytic in 
premontane moist forest, 10 April 2001, flowered in cultivation at Gaia Botanical Garden, 18 August 2001, $F$. Pupulin, D. Castelfranco \& E. Salas 3119 (holotype, USJ; isotype, USJ-Spirit).

Kefersteinia angustifolia Pupulin \& Dressler, Harvard Pap. Bot. 8(2):164. 2004.

TyPE: Panama. Chiriquí: Laguna, near El Hato del Volcán, 21 Oct. 1967, H. Butcher s.n. (holotype, MO).

Kefersteinia auriculata Dressler, Orquideología 16: 49. 1983.

TYPE: Panama. Coclé: cerros al N de El Valle de Antón, preparada de cultivo, junio, julio, 1971, R.L. Dressler 4063 (holotype, US: isotype, PMA).

Kefersteinia maculosa Dressler, Orquideología 16: 52. 1983.

TYPe: Panama. Veraguas: filo de Cerro Arizona, arriba de Escuela Alto de Piedra, al Oeste de Santa Fe, 850$950 \mathrm{~m}$; flores de cultivo, junio y Julio, R.L. Dressler 6061 (holotype, US; isotype, PMA).

Kreodanthus bugabae Ormerod, Harvard Pap. Bot. 13(1): 59. 2008.

TYPE: Panama. Chiriquí: Distrito Bugaba, Cerro Punta, along ridge to watershed to Bocas del Toro Province, 2200 m, 26 Jan 1985, H. van der Werff \& J. Herrera 6441 (holotype, MO).

Kreodanthus sytsmae Ormerod, Harvard Pap. Bot. 13(1): 61. 2008.

TYPE: Panama. Bocas del Toro: NW ridge of Cerro Pate Macho, from summit to Finca Serrano, 1200-2100 m, 27 May 1981, K. Sytsma, S. Knapp \& L. Andersson 4945 (holotype, $\mathrm{MO})$.

Leochilus powellii Schltr., Repert. Spec. Nov. Regni Veg. Beih. 17: 81. 1922.= Leochilus scriptus (Scheidw.) Rchb.f. TYPE: Panama. Veragua-Provinz und auf Calabash-Bäumen auf der Westseite des Kanals C.W. Powell 129 (holotype, B, destroyed; lectotype designated by Chase (1986), AMES-23926; isolectotypes, K, MO-955972; drawing of type, AMES-26796; drawing published in Mansfeld [1931: t. 81, nr. 324].).

Lepanthes ankistra Luer \& Dressler, Orquideología 16(3): 12. 1986.

TYPE: Panama. Prov. of Chiriquí: epiphytic in cloud forest on Cerro Colorado, alt. 1500 m, 15 February 1985, C. Luer, J. Luer, R.L. Dressler \& K. Dressler 10534 (holotype, MO).

Lepanthes antilocapra Luer \& Dressler, Orquideología 16(3): 15. 1986.

TYPE: Panama. Prov. of Chiriquí: epiphytic in cloud forest on Cerro Colorado, alt. 1750 m, 15 February 1985,

C. Luer, J. Luer, R.L. Dressler \& K. Dressler 10544 (holotype, MO).

Lepanthes arachnion Luer \& Dressler, Orquideología 16(3): 6-9, 20-22. 1986. = Lepanthes pantomima Luer \& Dressler

TYPE: Panama. Prov. of Bocas del Toro: epiphytic in wet forest between Fortuna and Chiriquí Grande, alt. 350 m, 17 February 1985, C. Luer, J. Luer, R.L. Dressler \& K. Dressler 10615 (holotype, MO).

Lepanthes brunnescens Luer, Phytologia 55: 179. 1984.

TYPE: Panama. Prov. of Panama: epiphytic in cloud forest, Cerro Jefe, alt. 900 m, 1 Dec 1983, C. Luer, J. Luer, A. Maduro \& H. Butcher 9200 (holotype, SEL).

Lepanthes caloptera Luer, Phytologia 55: 180. 1984.

TYPE: Panama. Prov. of Panama: epiphytic in cloud forest, Cerro Jefe, alt. 900 m, 1 Dec. 1983, C. Luer, J. Luer, A. Maduro \& H. Butcher 9194 (holotype, SEL).

Lepanthes chiriquensis Schltr., Repert. Spec. Nov. Regni Veg. Beih. 17: 17. 1922.

TYPE: Panama. Chiriquí: c. $5000 \mathrm{ft}$ in feuchten Schluchten, C.W. Powell 254 (holotype, B; lectotype designated by Christenson (1991b), AMES-23781; drawing published in Mansfeld [1931: t. 39, nr. 154].).

Lepanthes coeloglossa Luer, Phytologia 55: 180. 1984.

TyPE: Panama. Prov. of Chiriquí: epiphytic in elfin forest on Cerro Hornito, alt. 1700 m, 15 Dec. 1976, C. Luer, A. Luer, R.L. Dressler \& N.H. Williams 1357 (holotype, SEL). 
Lepanthes collaris Luer, Lindleyana 2(4): 185. 1987. = Lepanthes blephariglossa Schltr.

TYPe: Panama. Prov. of Chiriquí: epiphytic in forest on Cerro Colorado, alt. 1500 m, 15 February 1986, $C$. Luer, J. Luer, R.L. Dressler \& K. Dressler 10563 (holotype, MO).

Lepanthes confusoides Luer, Monogr. Syst. Bot. Missouri Bot. Gard. 76: 165. 1999.

TYPE: Panama. Bocas del Toro, forest at alt. 900 m, collected by E. Olmos, cultivated at finca Dracula, Cerro Punta, 15 November 1998., C. Luer 18975 (holotype, MO).

Lepanthes crossota Luer, Lindleyana 2(4): 188. 1987. = Lepanthes jimenezii Schltr.

TyPE: Panama. Prov. of Chiriquí: Cerro Punta, collected and cultivated by A. Maduro, 1986, C. Luer 11630 (holotype, MO).

Lepanthes demissa Luer, Phytologia 55: 182. 1984.

TyPE: Panama. Chiriquí: epiphytic in cloud forest above Guadalupe, 2300 m, 8 Dec. 1983, C. Luer, J. Luer \& A. Maduro 9292 (holotype, SEL).

Lepanthes droseroides Luer, Lindleyana 2: 188. 1987.

TyPE: Panama. Prov. of Chiriquí: Cerro Punta, collected by A. Maduro 19-M., C. Luer 11632 (holotype, MO).

Lepanthes eciliata Schltr., Repert. Spec. Nov. Regni Veg. 12: 203. 1913.

TyPE: Panama. Chiriquí: Epiphyte im feuchten Walde bei Los Signas Camp, am Südabhang des Cerro de la Horqueta, 1700 m, bkühend im Mar 1911, H. Pittier 3173 (holotype, B, destroyed; drawing of type, AMES; isotype, US).

Lepanthes edentula Luer, Lindleyana 10: 143, fig. 1995.

TyPE: Panama. Chiriquí: epiphytic near the Fortuna sldam site, alt. 1100 m, 16 Feb 1985, C. Luer, R. Dressler \& K. Dressler 10587 (holotype, MO).

Lepanthes empis Luer, Phytologia 55: 183. 1984. = Lepanthes excedens Ames \& Correll

TYPE: Panama. Prov. of Chiriquí: epiphytic in small trees near Volcan, "La Cordillera," alt. 1300 m, 9 Dec. 1983, C. Luer, J. Luer, A. Maduro \& H. Butcher 9314 (holotype, SEL).

Lepanthes exposita Luer, Lindleyana 2(4): 191. 1987.

Type: Panama. Prov. of Chiriquí: Cerro Punta, collected by A. Maduro 23-M., C. Luer 11634 (holotype, MO).

Lepanthes incantata Luer, Lindleyana 2: 194. 1987.

TYPE: Panama. Prov. of Panama: epiphytic in cloud forest on Cerro Jefe, alt. 900 m, 1 Dec. 1983., C. Luer, J. Luer \& A. Maduro 9195 (holotype, SEL).

Lepanthes infundibulum Luer, Phytologia 55: 186. 1984. = Lepanthes helleri A.D.Hawkes

TyPe: Panama. Prov. oc Chiriquí: epiphytic in elfin forest, Cerro Hornito, alt. 1700 m, 15 Dec 1976, C. Luer, A. Luer, R.L. Dressler \& N.H. Williams 1331 (holotype, SEL).

Lepanthes insectiflora C.Schweinf., Bot. Mus. Leafl. 7: 152. $1939=$ Lepanthes tipulifera Rchb.f.

TYPE: Panama. Chiriquí: Boquete: Bajo Chorro, at $6000 \mathrm{ft}$ alt., epiphyte in rain forest, 2-4 inches high, flowers burnt orange (?), 22 Jan 1938, M.E. Davidson 185 (holotype, F; isotype, AMES).

Lepanthes laevis Luer, Monogr. Syst. Bot. Missouri Bot. Gard. 76: 168. 1999.

TYPE: Panama. Chiriquí, obtained from A. Maduro, cultivated at J \& L Orchids, Easton, CT, 19 June 1998., C. Luer 18823 (holotype, MO).

Lepanthes luerorum B.T.Larsen, Phytotaxa 175(5) 279-280. 2014.

TYPE: Panama. Prov. of Chiriquí: epiphytic in forest above Boquete, 1,300-1,400 m, 6 May 2013, collected in the wild by Peter H. Peeters \& André De Jonghe 023, flowered in cultivation in Bertem, Belgium, 9 July 2013, P. Peeters s.n. (holotype, LISI).

Lepanthes maduroi Luer, Phytologia 55: 186. 1984. 
TYPE: Panama. Prov. of Chiriquí: epiphytic in cloud forest above Guadalupe, alt. 2300 m, 8 Dec. 1983, $C$. Luer, J. Luer, A. Maduro 9305 (holotype, SEL).

Lepanthes mariposa Luer, Phytologia 55: 187. 1984.

TyPE: Panama. Prov. of Chiriquí: epiphytic in scrubby trees near Volcan, alt. 1350 m, 7 Dec 1983, C. Luer, J. Luer \& H. Butcher 9278 (holotype, SEL).

Lepanthes maxonii Schltr., Repert. Spec. Nov. Regni Veg. 12: 204.1913.

TYPE: Panama. An Gaumstämmen in feuchten Wäldern zwischen Alto de Las Palmas und dem Gipfel des Cerro de Horqueta (Chiriquí), 2100-2268 m, blühend im Mar 1911, W.R. Maxon 5494 (holotype, B; destroyed, illustration of type, AMES, isotypes, NY, US).

Lepanthes minyglossa Luer, Selbyana 23: 15. 2002.

Type: Panama. Chiriquí: epiphytic in cloud forest, Cerro Colorado, alt. 1650 m, 15 February 1985, C. Luer et al. 10540 (holotype, MO).

Lepanthes mollis Luer, Selbyana 23: 15. 2002.

TYPe: Panama. Chiriquí: epiphytic in forest, Cerro Colorado, alt. 1500 m, 15 February 1985, C. Luer et al. 10532 (holotype, MO; isotype, MO).

Lepanthes myiophora Luer, Lindleyana 2: 204. 1987.

TYPE: Panama. Prov. of Chiriquí: near the Fortuna dam site, alt. ca. 1000 m, collected Dec. 1976 by C. Luer et al., flowered in cultivation by P. \& A. Jesup in Bristol, CT., 26 April 1987., C. Luer 12879 (holotype, MO).

Lepanthes odontolabis Luer, Lindleyana 2: 207. 1987.

TYPe: Panama. Prov. of Chiriquí: Cerro Punta, collected by A. Maduro 20-M., C. Luer 11633 (holotype, MO).

Lepanthes olmosii Bogarín, Orchid Digest 73(3): 141. 2009.

TYPE: Panama. Chiriquí: Boquete, Bajo Chorro, Alto Chiquero, collected by Erick Olmos, without further locality data, flowered in cultivation at Finca Dracula, Cerro Punta, Guadalupe, 12 December 2006, D. Bogarín 3005 (holotype, PMA; isotypes, CR, JBL).

Lepanthes otopetala Luer, Lindleyana 6: 76. 1991.

TYPE: Panama. Chiriquí, collected by A. Maduro, without locality, flowered in cultivation by J \& L Orchids, Easton, CT, May 1990, C. Luer 14741 (holotype, MO).

Lepanthes pachyglossa Luer, Phytologia 57: 59. 1985.

TYPE: Panama. Prov. of Panama: epiphytic in cloud forest on Cerro Jefe, alt. 880 m, 1 Dec. 1983, flowered in cultivation in Sarasota, FL. 27 April 1984., C. Luer, J. Luer, H. Butcher \& A. Maduro 10029 (holotype, MO).

Lepanthes pexa Luer, Lindleyana 2: 181. 1987.

TYPE: Panama. Prov. of Bocas del Toro: epiphytic in cloud forest between Fortuna and Chiriquí Grande, alt. 1000 m. 17 February 1985, C. Luer, J. Luer, R. Dressler \& K. Dressler 10622 (holotype, MO).

Lepanthes prora Luer, Phytologia 55: 191. 1984.

TYPE: Panama. Prov. of Panama: epiphytic in cloud forest, Cerro Jefe, alt. 900 m, 1 Dec. 1983, C. Luer, J. Luer, A. Maduro \& H. Butcher 9207 (holotype, SEL).

Lepanthes psyche Luer, Phytologia 55: 192. 1984.

TYPE: Panama. Prov. of Chiriquí, epiphytic in small trees near Volcan, "La Cordillera," alt. 1300 m, 9 Dec. 1983, C. Luer, J. Luer, A. Maduro \& H. Butcher 9317 (holotype, SEL).

Lepanthes pycnogenia Luer, Lindleyana 7: 114. 1992.

TYPe: Panama. Prov. of Chiriquí collected by R. \& K. Dressler \& C. \& J. Luer in Feb. 1985, flowered in cultivation by P. \& A. Jesup in Bristol, CT, 10 Dec. 1988., C. Luer 13782 (holotype, MO). 
Lepanthes rauhii Luer, Orchidee (Hamburg) 38: 59. 1987.

TYPE: Panama. Prov. of Panama: epiphytic in forest above Chepo, La Margarita, south of the Cordillera San Blas, alt. ca. 800 m, May 1980, collected by W. Rauh 65025, flowered in cultivation at Heidelberg University 16 Oct. 1985, C. Luer 11423 (holotype, MO).

Lepanthes regularis Luer, Lindleyana 2: 210. 1987.

TyPe: Panama. Prov. of Chiriquí: Cerro Punta, Las Nubes, collected by A. Maduro 6-B., C. Luer 11631 (holotype, MO).

Lepanthes ridicula Luer, Monogr. Syst. Bot. Missouri Bot. Gard. 76: 168. 1999.

TYPE: Panama. Chiriquí, obtained from A. Maduro, cultivated at J \& L Orchids, Easton, CT, 19 June 1998., C. Luer 18827 (holotype, MO).

Lepanthes rotundifolia L.O.Williams in Woodson \& Schery, Ann. Missouri Bot. Gard. 27: 275. 1940.

TyPE: Panama. Coclé: epiphytic, wet north rim, vicinity of el Valle, 800-1000 m alt., 21 May 1939, P.H. Allen 1835 (holotype, MO).

Lepanthes schizocardia Luer, Phytologia 55: 193. 1984.

TYPE: Panama. Panama: epiphytic in cloud forest, Cerro Jefe, 900 m, 1 Dec. 1983, C. Luer, J. Luer, H. Butcher \& A. Maduro 9193 (holotype, SEL).

Lepanthes seegeri Luer, Orchidee (Hamburg) 38: 60, fig. 1987.

TYPE: Panama. Prov. of Chiriquí: epiphytic in forest above Boquete, alt. 1600-1700 m, April 1985, collected by H. Seeger 678A, flowered in cultivation at Heidelberg University 16 Oct. 1985., C. Luer 11424 (holotype, $\mathrm{MO})$.

Lepanthes setos Luer, Phytologia 55: 193. 1984. = Lepanthes fimbriata Ames

TYPE: Panama. Prov. of Chiriquí: epiphytic in scrubby trees near Volcan, alt. 1350 m, 7 Dec. 1983, C. Luer, J. Luer \& H. Butcher 9279 (holotype, SEL).

Lepanthes tetroptera Luer, Lindleyana 2: 214. 1987.

TYPe: Panama. Prov. of Chiriquí: Cerro Punta, collected by A. Maduro 2-B, C. Luer 11628, (holotype, MO).

Lepanthes trichidion Luer, Phytologia 55: 194. 1984.

TyPE: Panama. Prov. of Chiriquí: epiphytic in cloud forest above Guadalupe, alt. 2300 m, 8 Dec. 1983, C. Luer, J. Luer \& A. Maduro 9307 (holotype, SEL).

Lepanthes truncata Luer \& Dressler, Orquideología 16(3): 9. 1986.

TYPE: Panama. Prov. of Bocas del Toro: epiphytic in wet forest between Fortuna and Chiriquí Grande, alt. 350 m, 17 February 1985., C. Luer, J. Luer, R.L. Dressler \& K. Dressler 10618 (holotype, MO).

Lepanthopsis rinkei Luer, Monogr. Syst. Bot. Missouri Bot. Gard. 88: 104. 2002.

TYPE: Panama?: without collection data, purchased from Andy's Orchids, who had obtained the plant form Maduro in Panama, cultivated in Wichita, Kansas by Bryon Rinke, C. Luer 20048 (holotype, MO).

Ligeophila grandis Ormerod, Harvard Pap. Bot. 9(2): 395. 2005. = Aspidogyne grandis (Ormerod) Ormerod TyPE: Panama. Bajo Mono to Roblado trail, 2135 m, 24 Jul 1947, P.H. Allen 4857 (holotype, AMES; isotype, US).

Lindsayella amabilis Ames \& C.Schweinf., Bot. Mus. Leafl. 5: 34. 1937. = Sobralia callosa L.O.Williams

TYPE: Panama. Chiriquí: El Valle, alt. $2000 \mathrm{ft}$, in crevices of rocks or more rarely on a very large dead tree. 22 Jul 1935, W.R. Lindsay \& G.H. Bevins s.n. (holotype, AMES).

Lockhartia chiriquiensis Schltr., Repert. Spec. Nov. Regni Veg. 12: 215. 1913. = Lockhartia micrantha Rchb.f. TYPE: Panama. in der Nähe von San Félix, am östlichen Chiriquí, bis 120 m, blühend im Dec 1911, H. Pittier 5286 (holotype, B, destroyed; illustration of type, AMES; isotype, NY). 
Lockhartia micrantha Rchb.f., Bot. Zeitung (Berlin) 10: 768. 1852.

TYPE: Panama. Veragua Hinds. Cuming (holotype, W).

Lockhartia obtusata L.O.Williams, Amer. Orchid Soc. Bull. 9: [209], tab. 8. 1941.

TYPE: Panama. Coclé: hills north of El Valle de Antón, at $1000 \mathrm{~m}$. alt., flowers bright yellow, the lip with raised orange ridges at the base, 23 Jun 1940, P.H. Allen 2160 (holotype, AMES

Lockhartia pittieri Schltr., Repert. Spec. Nov. Regni Veg. 12: 216. 1913. = Lockhartia parthenoglossa Rchb.f.

TYPE: Panama. kultiviert in Culebra von Mrs. Rousseau, ursprünglich von Bohis stammend, blühend im Apr 1911, H. Pittier (B.S.P.C.Z.) 3401 (holotype, B, destroyed; illustration of type, AMES).

Lockhartia triangulabia Ames \& C.Schweinf., Schedul. Orchid. 8: 80. 1925. = Lockhartia grandibractea Kraenzl.

TYPE: Panama. Chiriquí: alt. 4000-5000 ft, C.W. Powell $362 a$ (holotype, AMES).

Lockhartia variabilis Ames \& C.Schweinf., Schedul. Orchid. 8: 81. 1925. = Lockhartia parthenoglossa

TYPE: Panama. wooded hills near Frijoles, Jan-Feb 1924, sea level, C.W. Powell 355 (holotype, AMES).

Lophiaris silverarum Carnevali \& Cetzal, Phytotaxa 162(3): 166. 2014. = Trichocentrum silverarum (Carnevali \& Cetzal) J.M.H.Shaw

TyPe: Panama. Veraguas: Distrito de La Mesa, Palo Verde, Río Subí, Costa Pacífica, $8^{\circ} 13^{\prime} \mathrm{N}, 81^{\circ} 10^{`} \mathrm{~W}$, approx. 180 m, December 2005, vegetación primaria a orillas del río, Silvera \& Rodríguez s.n. (holotype, PMA; isotypes, AMES, CICY).

Lycaste campbellii C.Schweinf. in I.M.Johnst., Sargentia 8: 103. 1949.

TYPE: Panama. San Jose Island, epiphyte from streamside forest in Area 11B, I.M. Johnston 1371 (holotype, AMES; paratype, AMES).

Lycaste macrophylla Lindl. subsp. panamanensis Fowlie, Lasca Leaves 14: 40. 1964. = Lycaste panamanensis (Fowlie) Archila

TYPE: Panama. Coclé: El Valle de Antón, 3,000ft., "F62P8” (holotype, LASCA).

Lycaste powellii Schltr., Repert. Spec. Nov. Regni Veg. Beih. 17: 65. 1922.

TYPE: Panama. Auf Hügeln bei Panama-City, C. Powell 15 (holotype, B, destroyed, lectotype designated by Christenson (1991b), AMES-23869; isolectotypes, K, MO-955886).

Malaxis brevis Dressler, Selbyana 24(2): 141.2003.

TYPE: Panama. Chiriquí: W slopes of Cerro Respingo, NE of Cerro Punta Village, approx. $82^{\circ} 34^{\prime} \mathrm{W}, 08^{\circ} 52^{\prime} \mathrm{N}$, 8000-8500 ft, flowers yellowish green, 15 June 1971, G.L. Webster \& G. Brecken 16599 (holotype, MO).

Malaxis rostratula Dressler, Selbyana 24(2): 142. 2003

TYPE: Panama. San Félix: Cerro Colorado, about $50 \mathrm{~km} \mathrm{~N}$ of San Félix, ca. 1400, 17 August 1975, cloud forest, flowers pale green, R.L. Dressler 5102 (holotype, MO).

Malaxis woodsonii L.O.Williams in Woodson \& Seibert, Ann. Missouri Bot. Gard. 26: 281. 1939.

TyPE: Panama. Chiriquí: terrestrial, vic. of Casita Alta, Volcán de Chiriquí, alt. about 1500-2000 m, June 28July 2, 1938, R.E. Woodson, P.H. Allen \& R.J. Seibert 831 and 832 (holotype, AMES; isotype, MO).

Masdevallia allenii L.O.Williams in Woodson \& Schery, Ann. Missouri Bot. Gard. 27: 273. 1940. = Trisetella triglochin (Rchb.f.) Luer

TYPE: Panama. Coclé: vicinity of El Valle, 600-1000 m alt., 8 Dec 1938, P.H. Allen 1230 (holotype, MO).

Masdevallia andresiana Luer \& Maduro, Monogr. Syst. Bot. Missouri Bot. Gard. 103: 283. 2005.

TYPE: Panama. Bocas del Toro: alt. $1200 \mathrm{~m}$, collected and Andrew Maduro and son, cultivated by A. Maduro at Finca Dracula, Cerro Punta, Chiriquí, Panama, flowered in cultivation in Wilmington, DE, 16 Oct 1998, M. Rao 96 (holotype, MO). 
Masdevallia attenuata Rchb.f., Gard. Chron. 1871: 834. 1871.

SynTYPES: Costa Rica [?]. “Z[ahn?] 178”, cultivated by Veitch 192; Panama [?], Zahn s.n. lectotype designated by Smith \& Pupulin (2012), W: Panama [?]. Without collecting data, drawing of a syntype, based on Zahn s.n. (W 28837, Rchb-Orch 50775, upper part of the drawing).

Masdevallia butcheri Luer, Selbyana 3(1/2): 16. 1976. = Trisetella tenuissima (C.Schweinf.) Luer TYPE: Panama. Coclé: epiphytic in old orange trees above El Valle, alt. ca. 1000 m, 2 Sept 1976, C. Luer \& H. Butcher 1119 (holotype, SEL).

Masdevallia collina L.O.Williams, Amer. Orchid Soc. Bull. 11: [93], tab. 3. 1942.

TYPE: Panama. Coclé: epiphytic, hills north of El Valle de Antón, alt. ca. $1000 \mathrm{~m}$, sepals and petals maroonpurple, sepaline tails yellow, 23 Jun 1940, P.H. Allen 2158 (holotype, AMES; isotype, MO).

Masdevallia dressleri Luer, Selbyana 3: 20. 1976. = Trisetella dressleri (Luer) Luer

Type: Panama. Panama: epiphytic along the Altos de Pacora road, alt. 650 m, 4 Mar 1976, C. Luer, J. Luer, P. Taylor \& R.L. Dressler 730 (holotype, SEL).

Masdevallia eburnea Luer \& Maduro, Monogr. Syst. Bot. Missouri Bot. Gard. 91: 409a. 2003.

TYPE: Panama. Chiriquí: alt. 1900-2000 m, collected by E. Olmos, spring 2000, cultivated at Finca Dracula, Cerro Punta, spring 2001, E. Olmos 157 (holotype, MO).

Masdevallia gloriae Luer \& Maduro, Monogr. Syst. Bot. Missouri Bot. Gard. 91: 413a. 2003.

TyPE: Panama. Chiriquí: Cerro Punta, Guadalupe, alt. $2000 \mathrm{~m}$, collected by A. Maduro and E. Olmos, cultivated at Finca Dracula at Cerro Punta, A. Maduro s.n. (holotype, MO).

Masdevallia livingstoneana Rchb.f. \& Roezl, Gard. Chron. n.s., 2: 322. 1874.

TyPE: Panama. It was discovered in Panama by M. Roezl, and was sold lately at a sale in Mr. Stevens' rooms. B. Roezl s.n. (holotype, W).

Masdevallia maduroi Luer, Monogr. Syst. Bot. Missouri Bot. Gard. 72: 110. 1998.

TYPE: Panama. Chiriquí: "Fortuna," alt. 1200 m, collected \& cultivated by Maduro's Tropical Flowers, flowered in cultivation at J \& L Orchids, Easton, CT, 16 October 1998, C. Luer 18932 (holotype, MO).

Masdevallia olmosii Königer \& Sijm, Arcula 12: 303. 2003.

TYPE: Panama. Bocas del Toro: Culebra, 1000 m, gesammelt von Sr. Erick Olmos, in Kultur bei Sr. Andrés Maduro auf der Finca Dracula in Cerro Punta, Panama, in Kultur in Blüte bei Herrn Ton Sijm in Hem, Niederlande, W. Königer WK-115 (holotype, M; isotype, PMA).

Masdevallia ostaurina Luer \& V.N.M.Rao, Monogr. Syst. Bot. Missouri Bot. Gard. 95: 249. 2004.

TYPE: Panama. Chiriquí: without more exact locality, alt. 1900-2000 m, collected by A. Maduro and E. Olmos, cultivated at Finca Dracula since 2000, flowered in cultivation in Wilmington, DE, 6 December 2003, V.N.M. Rao 161 (holotype, MO).

Masdevallia pelecaniceps Luer, Selbyana 3: 22. 1976. = Phloeophila pelecaniceps (Luer) Pridgeon

TYPE: Panama. Panama: epiphytic in the cloud forest of Cerro Jefe, alt. 1000 m, 19 Aug 1967, R.L. Dressler 3028 (holotype, SEL).

Masdevallia pleurothalloides Luer, Selbyana 3 (3/4): 218. 1977.

TYPE: Panama. epiphytic in cloud forest on Cerro Jefe, alt. 1000 m, 12 Nov 1967, R.L. Dressler 3155 (holotype, SEL).

Masdevallia scabrilinguis Luer, Phytologia 44: 168. 1979. = Masdevallia attenuata Rchb.f.

TYPE: Panama. Chiriquí: Cerro Gordo, collected by R.L. Dressler s.n., 1977, cultivated at SEL-77-1707, flowered in cult. 6 Mar. 1978, C. Luer 2788 (holotype, SEL).

Masdevallia tenuissima C.Schweinf., Bot. Mus. Leafl. 4: 11. 1937. = Trisetella tenuissima (C.Schweinf.) Luer 
TYPE: Panama. Coclé: mountains beyond La Pintada, alt 400-600 m, Feb 1935, A.A. Hunter \& P.H. Allen 587 (holotype, AMES; isotype, MO).

Masdevallia tokachiorum Luer, Lindleyana 6: 90. 1991.

TYPE: Panama. Chiriquí: epiphytic in forest near the Fortuna dam site, alt. ca. 1000 m, collected March 1985 by R. Tokach, flowered in cultivation by K. Tokach in Tacoma, Washington, Sept. 1990, C. Luer 14943 (holotype, MO).

Masdevallia utriculata Luer, Phytologia 44: 169. 1979.

TyPE: Panama. Chiriquí: epiphytic in cloud forest on Cerro Pate Macho, alt. ca. 2200 m, 27 Feb 1979, R.L. Dressler \& J. Kuhn s.n. (holotype, SEL).

Maxillaria allenii L.O.Williams in Woodson \& Schery, Ann. Missouri Bot. Gard. 27: 282, tab. 35. 1940. = Camaridium allenii (L.O. Williams) M.A. Blanco

TYPE: Panama. Coclé: epiphytic, N rim of El Valle de Antón, 600-1000 m alt., flowers yellow, 12 Feb 1939, P.H. Allen 1650 (holotype, MO).

Maxillaria chionantha J.T.Atwood, Selbyana 16: 242, fig. 1995.

TyPE: Panama. Prov. Veraguas: Vicinity of Continental Divide, third branch of Río Santa María to drop-off to lowlands, 12-15 km NW of Santa Fe, 650-750 m, 16-17 Nov 1974, R.L. Dressler 4839 (holotype, SEL).

Maxillaria chiriquiensis Schltr., Repert. Spec. Nov. Regni Veg. Beih. 17: 68. 1922. = Maxillariella variabilis (Bateman ex Lindl.) M.A.Blanco \& Carnevali

Type: Panama. Chiriquí, c. 4000 ft., C.W. Powell 125 (holotype, B, destroyed; lectotype designated by Christenson (1991b), AMES-23878; isolectotypes, K, MO-955970, US; drawing of type, AMES-26773; drawing published in Mansfeld [1931: t. 64, nr. 254].).

Maxillaria curvicolumna M.A.Blanco \& Neubig, Orchids (West Palm Beach) 76(6): 451. 2007.

TYPE: Panama. Bocas del Toro. Fortuna area? (cultivated in roadside garden north of Fortuna Dam site), $M$. Whitten 2933 (holotype, FLAS).

Maxillaria darienensis J.T.Atwood, Selbyana 19: 254. 1998[1999].

TyPE: Panama. Prov. Darién: Cerro Pirre, valley between Pirre and next most southerly peak, 10-20 Jul 1977, J. Folsom 4412 (holotype, MO; isotype, SEL).

Maxillaria diuturna Ames \& C.Schweinf., Schedul. Orchid. 8: 58. 1925. = Maxillariella diuturna (Ames \& C.Schweinf.) M.A.Blanco \& Carnevali

TyPE: Panama. Cativa-Porto Bello Trail, Feb 1924, sea level, C.W. Powell 376 (holotype, AMES).

Maxillaria dressleriana Carnevali \& J.T.Atwood, Lindleyana 11:29. 1996. = Mormolyca dressleriana (Carnevali \& J.T.Atwood) M.A.Blanco

TYPE: Panama. Chiriquí: Fortuna Dam, near Rambola. Flowering in cultivation at the Marie Selby Botanical Gardens on January 3, 1995, Akers JJ (See OIC9304) Prepared by Atwood 95-1; s.d.; Cultivated at the Marie Selby Botanical Garden from material collected in Panama. Prov. Chiriquí, Fortuna (holotype, SEL; isotypes, AMES, INB, MO).

Maxillaria fragrans J.T.Atwood, Selbyana 22(2): 131. 2001. = Camaridium fragrans (J.T.Atwood) M.A.Blanco TYPE: Panama. Prov. Panama: Cerro Jefe, cloud forest dominated by Clusia spp. and Colpothrinax cookii, premontane rain forest along trail on ridge running NE from summit, elev. ca. 1000 m, 18 Dec 1974, Mori \& Kallunki 3770 (holotype, SEL; isotypes, MO, WIS).

Maxillaria galantha J.T.Atwood \& Carnevali, Lindleyana 9: 227. 1994.

TYPE: Panama. Top of Cerro Jefe, epiphytic herb, 9 June 1977, J.P. Folsom 3608 (holotype, MO; isotype, SEL). 
Maxillaria gatunensis Schltr., Repert. Spec. Nov. Regni Veg. Beih. 17: 68. 1922. = Heterotaxis sessilis (Sw.) F.Barros

TYPe: Panama. Gatun-See-Gebiet bei Panama, C. Powell 207 (holotype, B, destroyed, lectotype designated by Christenson (1991b), AMES-26946; isolectotype, MO-956024; drawing published in Mansfeld [1931: t. 64, nr. 255].).

Maxillaria insolita Dressler, Orquideología 14: 204. 1981. = Camaridium insolitum (Dressler) M.A.Blanco TyPe: Panama. Panama: La Eneida, Altos de Pácora, Jul 1971, flores producidas en cultivo de junio a septiembre, R.L. Dressler 4064 (holotype, US; photo of the flower from the plant that served as the holotype).

Maxillaria longicolumna J.T.Atwood, Selbyana 22(2): 132. 2001. = Camaridium longicolumna (J.T.Atwood) M.A.Blanco

TYPE: Panama. Prov. Verguas: road from Santa Fe $1.5 \mathrm{~km}$ past Ag School to base of Cerro Tute, ca. 1000-1200 m elev., 4 Feb 1977, J.P. Folsom 1574 (holotype, MO; isotype, MO).

Maxillaria longipetiolata Ames \& C.Schweinf., Schedul. Orchid. 8: 61. 1925. = Mapinguari longipetiolatus (Ames \& C.Schweinf.) Carnevali \& R.B.Singer

TYPE: Panama. Hill east of Corozal, sea level, C.W. Powell 307 (holotype, AMES).

Maxillaria lutheri J.T.Atwood, Selbyana 19: 257. 1998[1999]. = Camaridium lutheri (J.T.Atwood) M.A.Blanco TYPE: Panama. Prov. Bocas del Toro: Cerro Colorado, above the Copper Mine, elev. ca. 1600 m, Jun 1986, H. Luther et al. 1068 (holotype, SEL).

Maxillaria obscura Linden \& Rchb.f., Beitr. Orchid.-K. C. Amer.: 31. 1866. = Camaridium obscurum (Linden \& Rchb.f.) M.A.Blanco

TyPE: Floes obtinui ex horto Lindeniano Bruxellensi. Planta ex Columbia introducta fertur, J. Linden s.n. (holotype, W).

Maxillaria oreocharis Schltr., Repert. Spec. Nov. Regni Veg. Beih. 17: 69. 1922. = Maxillariella oreocharis (Schltr.) M.A.Blanco \& Carnevali

TyPE: Panama. Chiriquí, 4000-5000 ft, C.W. Powell 256 (holotype, B, destroyed, lectotype designated by Christenson (1991b), AMES-23877; isolectotype, MO-956060).

Maxillaria panamensis Schltr., Repert. Spec. Nov. Regni Veg. Beih. 17: 70. 1922.= Maxillariella acervata (Rchb.f.) M.A.Blanco \& Carnevali

TyPE: Panama. Auf Hügeln bei Panama City, C.W. Powell 124 (holotype, B; lectotype designated by Christenson (1991b), K; isolectotype, K; drawing of type, AMES-26813; drawing published in Mansfeld [1931: t. 65, nr. 257].).

Maxillaria parvula Schltr., Repert. Spec. Nov. Regni Veg. Beih. 27: 176. 1924., nom. illeg. = Maxillariella caucae (Garay) Bogarín

TYPE: Colombia. Cauca: Pflanze gleich kletternden Rasen an den Baumstämmen nahe dem Boden, in den feuchtwarmen Ebenen nahe [Panama]. Bahia de Montijo (Veragua); häufig, F. C. Lehmann no. 103, blühend im Mai 1879 (B, destroyed).

Maxillaria powellii Schltr., Repert. Spec. Nov. Regni Veg. Beih. 17: 70. 1922.

TYPE: Panama. Auf Hügeln bei Panama City, C.W Powell 28 (holotype, B, destroyed; lectotype designated by Christenson (1991b), AMES-23880; isolectotypes, K, MO-955897; drawing of type, AMES-26794; drawing published in Mansfeld [1931: t. 65, nr. 258].).

Maxillaria pubilabia Schltr., Repert. Spec. Nov. Regni Veg. Beih. 17: 71. 1922. = Maxillaria ringens Rchb.f.

TYPE: Panama. Auf Hügeln bei Panama City, C.W. Powell 214 (holotype, B, destroyed; lectotype designated by Christenson (1991b), AMES-23881; isolectotype, MO-956030; drawing of type, AMES-26751; drawing published in Mansfeld [1931: t. 65, nr. 259].). 
Maxillaria repens L.O.Williams, Amer. Orchid Soc. Bull. 10: [273], tab. 9. 1942. = Ornithidium repens (L.O.Williams) M.A.Blanco \& Ojeda

TYPE: Panama. Coclé: Epiphytic, trail to Las Minas, north of El Valle de Antón, alt. 1000 m, 2 Dec 1941, $P$. Allen 2868 (holotype, AMES; isotype, MO).

Maxillaria rodrigueziana J.T.Atwood \& Mora-Ret., Icon. P1. Trop. 14(1): t. 1361. 1989.

TYPE: Panama. Panama: El Cope, T. Antonio 2137 (holotype, MO; isotype, SEL; detail of isotype, SEL).

Maxillaria rousseauae Schltr., Beih. Bot. Centralbl. 36, Ab. 2: 413. 1918. = Maxillaria ringens Rchb.f.

TyPE: Panama. Im Gebiete des Panama-Kanals, 1914, Rousseau s.n. (holotype, B, destroyed, neotype designated by Atwood (1999), C.W. Powell 115, MO).

Maxillaria sanguinea Rolfe, Bull. Misc. Inform. Kew 1895: 8. 1895. = Maxillariella sanguinea (Rolfe)

M.A.Blanco \& Carnevali

TYPE: Panama. Chiriquí: Chiriquí Lagoon, James O’Brien s.n. (holotype, K).

Maxillaria scalariformis J.T.Atwood, Selbyana 19: 257. 1998[1999]. = Camaridium scalariforme (J.T.Atwood) M.A.Blanco

TYPE: Panama. Prov. Chiriquí: near "Vivero,” 2-3 km S. of Fortuna dam site, Valle de Hornito, 10 May 1982, R.L. Dressler 6050 (holotype, SEL).

Maxillaria stachyobiorum Rchb.f., Bot. Zeitung (Berlin) 10: 735. 1852. = Xylobium foveatum (Lindl.) G.Nicholson

TYPE: Panama. Chiriquí, J. Warscewicz s.n. (holotype, W).

Maxillaria synsepala J.T.Atwood, Selbyana 19: 260. 1998[1999]. = Camaridium synsepalum (J.T.Atwood) M.A.Blanco

TyPE: Panama. Prov. Chiriquí: Distrito Bugaba: Cerro Punta, 2200 m, 26 Jan 1985, van der Werff \& Herrera 6435 (holotype, SEL; isotype, MO).

Maxillaria tricarinata J.T.Atwood, Selbyana 19: 260. 1998[1999]. = Camaridium tricarinatum (J.T.Atwood)

M.A.Blanco

TYPe: Panama. Prov. Bocas del Toro: Trocha 3 de noviembre, near pass over continental divide (Paso de la Zorra), elev. ca. 1500 m, 5 Apr 1978, R.L. Dressler 5810 (holotype, SEL).

Maxillaria tubercularis J.T.Atwood, Lindleyana 9(4): 229. 1994. Camaridium tuberculare (J.T.Atwood) M.A.Blanco

TyPE: Panama. Chiriquí: Fortuna Dam region, along trail to Cerro Hornito (Pate de Macho) on southern ridge of watershed, short dense forest, 1800-1950 m, G. McPherson 13567 (holotype, MO).

Maxillaria tutae J.T.Atwood, Selbyana 19: 262. 1998[1999]. = Camaridium tutae (J.T.Atwood) M.A.Blanco

TYPE: Panama. Prov. Veraguas: trail on ridge to summit of Cerro Tute [possibly Cerro Arizona Cordillera de Tute, $1 \mathrm{~km}$ past Escuela la Agrícola Alto de Piedras, W of Santa Fe, upper montane and elfin forest, 12501410 m, S. Knapp \& K. Sytsma 2641 (holotype, SEL; isotype, MO).

Maxillaria vagans Ames \& C.Schweinf., Schedul. Orchid. 8: 65. 1925. = Camaridium bracteatum (Schltr.) Schltr. TYPe: Panama. Chiriquí, Palo Alto, alt 4500-5000 ft, Nov 1923, C.W. Powell 348 (holotype, AMES).

Mesospinidium panamense Garay, Orchidee (Hamburg) 24: 185. 1973. = Brassia panamensis (Garay) M.W.Chase TYPE: Panama. prov. Coclé, im Norden von El valle de Antón, in der Nähe von La Mesa, 1000 m, P.H. Allen 2785 (holotype, AMES).

Microchilus fuscatus Ormerod, Harvard Pap. Bot. 11(2): 161. 2007.

TYPE: Panama. Panama: Gorgas Memorial Labs, Yellow Fever research camp, "Campamento Quatro", 5-10 km NE of Altos de Pacora, 600 m, 21-24 Nov 1974, S. Mori \& J. Kallunki 3412 (holotype, MO). 
Microchilus ovalis Ormerod, Harvard Pap. Bot. 11(2): 164. 2007.

TYPE: Panama. Darien: Cana region, on trail above Cana, leading to Pirre Massif, 800-900 m, 5 May 1990, G. McPherson 15047 (holotype, MO).

Microchilus panamanicus Ormerod, Harvard Pap. Bot. 9(2): 414. 2005.

TyPE: Panama. Panama: Cerro Trinidad, 800-1000 m, 20 Oct 1946, P.H. Allen 3768 (holotype, AMES; isotype, $\mathrm{MO})$.

Microchilus subquadratus Ormerod, Harvard Pap. Bot. 11(2): 172. 2007.

TyPe: Panama. Darien: Serrania del Darien, track from Cerro Mali to Río Pucuro base camp, 20 Jul 1976, A. Gentry, H. Leon \& L. Forero 16834 (holotype, MO).

Microchilus whitefoordiae Ormerod, Harvard Pap. Bot. 11(2): 173. 2007.

TYPE: Panama. Chiriquí: Fortune Lake to Chiriquí Grande road, trail W of continental divide, 1200 m, 22 Mar 1985, R.J. Hampshire \& C. Whitefoord 867 (holotype, BM).

Microstylis blephariglottis Schltr., Repert. Spec. Nov. Regni Veg. 12(317-321): 202. 1913. = Crossoglossa blephariglottis (Schltr.) Dressler ex Dodson

Syntypes: Costa Rica. Brade s.n. (B, destroyed). Panama. Chiriquí: an Baumstämmen in feuchten Wäldern der oberen Caldera Wasserschiede, oberhalb El Boquete, 1650-1925, Mar 1911, W.R. Maxon 5667 (B, destroyed, US; illustration of type, AMES-21081; AMES-24380; photo of type, AMES).

Microstylis pittieri Schltr., Repert. Spec. Nov. Regni Veg. 12: 203. 1913. = Malaxis pittieri (Schltr.) Ames

TyPE: Panama. Chiriquí: feuchte Wälder zwischen Alto de las Palmas und dem Gipfel des Cerro de Horqueta (Chiriquí), 2100-2268 m, blühend Mar 1911, H. Pittier 3277 (holotype, US; illustration of type, AMES101737, illustration of type, AMES-24153).

Mormodes hookeri Lem., Jard. Fleur 1: 116. 1851.

TYPE: Panama. (holotype, K).

Mormodes lancilabris Pabst, Ann. Missouri Bot. Gard. 62: 510, f. 1. 1975.

TyPe: Panama. Coclé: Atlantic side of El Valle de Antón, floruit in cultura, 22 Nov 1965, R. Dressler s.n. (holotype, RB).

Mormodes powellii Schltr., Repert. Spec. Nov. Regni Veg. Beih. 17: 55. 1922.

TYPE: Panama. an den Hinterwässern des Gatun-Sees, C.W. Powell 59 (holotype, B, destroyed; lectotype designated by Christenson (1991b), MO-955917; isolectotype, AMES-23840, K; drawing of type, AMES-26826).

Mormolyca culebrica Bogarín \& Pupulin, Orchid Digest 74(1): 44. 2010.

TYPE: Panama. Bocas del Toro: Culebra, between rivers Estrella and Changuinola, $1200 \mathrm{~m}$, a plant collected by Erick Olmos, without further locality data, in cultivation at Finca Dracula, Cerro Punta, Chiriquí, Panama, 12 Dec. 2006, D. Bogarín 2951 (holotype, PMA; isotypes, JBL-spirit).

Neowilliamsia cuneata Dressler, Orquídea (Mexico City) 8: 24. 1983. = Epidendrum cuneatoides Dodson ex Hágsater

TyPe: Panama. Veraguas: Pico de Cerro Arizona, arriba de la Escuela Alto de Piedra, oeste de Santa Fé, alt. 1300 m, 5 Jun 1982, R.L. Dressler 6066 (holotype, US; isotypes, AMO, F, MO, PMA, SEL).

Neowilliamsia tenuisulcata Dressler, Orquídea (Mexico City) 8: 29. 1981. = Epidendrum tenuisulcatum (Dressler) Hágsater

TyPE: Panama. Coclé: cerca del Aserradero El Copé, a unos 8 km al norte de El Copé; 12 Aug 1977, R.L. Dressler 5673 (holotype, US).

Nidema boothii Schltr. var. triandrum Schltr., Repert. Spec. Nov. Regni Veg. Beih. 17: 43. 1922. = Nidema ottonis (Rchb.f.) Britton \& Millsp 
TYPE: Panama. auf Hügeln óstlich Panama City, etwa in Meereshöhe, C.W. Powell 131. (holotype, B, destroyed; lectotype designated by Christenson (1991b), AMES-23804; isolectotypes, K, MO-955974).

Notylia cordesii L.O.Williams in Woodson \& Seibert, Ann. Missouri Bot. Gard. 26: 286. 1939. = Macroclinium cordesii (L.O.Williams) Dodson

TyPE: Panama. Bocas Del Toro: epiphytic, Mosquito Hill, 12-16 Aug 1938, Woodson, Allen \& Seibert 1932 (holotype, MO; illustration of type, AMES).

Notylia gracilispica Schltr., Repert. Spec. Nov. Regni Veg. Beih. 17: 75. 1922. = Notylia pentachne Rchb.f.

TYPE: Panama. auf Hügeln bei Panama City, C.W. Powell 185 (holotype,B, destroyed; lectotype designated by Christenson (1991b), MO-956018; drawing of type, AMES-26829; drawing published in Mansfeld [1931: t. 81, nr. 322].).

Notylia juncta Dressler, Orquideología 4: 164. 1969. = Macroclinium junctum (Dressler) Dodson

TyPE: Panama. Panama: La Eneida, región de Cerro Jefe, 15 Oct .1967, R.L. Dressler 3092 (holotype, US).

Notylia latilabia Ames \& C.Schweinf., Schedul. Orchid. 8: 71. 1925.

TYPE: Panama. Frijoles, Apr 1924, sea level. Grows in full sunlight, usually on Calabash trees. Petals white without the orange dot on them characteristic of N. pentachne Reichb. f., C.W. Powell 406 (holotype, AMES).

Notylia panamensis Ames, Orchidaceae 7: 112. 1922. = Notylia albida Klotzsch

TYPE: Panama. Marraganti and vicinity, 10-200 feet altitude, April 3-9, 1908, R. S. Williams 977 (holotype, NY; photo of type, AMES).

Notylia pentachne Rchb.f., Bonplandia (Hannover) 2: 90. 1854.

TYPE: [Panama]. Von Chagres von Herrn Keferstein bezogen, von Herrn Lehmann cultivirt (holotype, W).

Notylia simplex Dressler, Orquideología 4: 166. 1969. = Macroclinium simplex (Dressler) Dodson

TYPe: Panama. Panama: La Eneida, región de Cerro Jefe; 25 Mar. 1968, R.L. Dressler 3460 (holotype, US).

Oakes-amesia cryptantha C.Schweinf. \& P.H.Allen, Bot. Mus. Leafl. 13(6): 134. 1948. = Ornithocephalus cryptanthus (C.Schweinf. \& P.H.Allen) Toscano \& Dressler

TYPE: Panama. Coclé: summit of Cerro Pajita, hills north of El Valle de Antón, 1000-1200 m alt., epiphyte on small trees in dwarf cloud forest, 7 Jan 1947, P.H. Allen \& D.O. Allen 4196 (holotype, AMES).

Odontoglossum chiriquense Rchb.f., Bot. Zeitung (Berlin) 10: 692. 1852. = Otoglossum chiriquense (Rchb.f.) Garay \& Dunst.

TYPE: [Panama]. Cordilleren von Chiriquí. 9000’. Auf verfaulten Baumstämmen. October (holotype, W).

Odontoglossum powellii Schltr., Repert. Spec. Nov. Regni Veg. Beih. 17: 129. 1922. Rossioglossum schlieperianum (Rchb.f.) Garay \& G.C.Kenn

TyPE: Panama. Chiriquí Distrikt: etwa 3800 ft, C.W. Powell 178 (holotype, B, destroyed; lectotype designated by Christenson (1991b), AMES-25014, drawing of type; isolectotypes, MO-956010, MO-956011; drawing of type, AMES-26840; drawing published in Mansfeld [1931: t. 72, nr. 285].).

Odontoglossum warszewiczii Rchb.f., Bot. Zeitung (Berlin) 10: 692. 1852. = Miltoniopsis warscewiczii (Rchb.

f.) Garay \& Dunst.

TYPE: [Panama]. Cordillera de Chiriquí. 8000' auf Leguminosen. October-Januar, J. Warscewicz s.n. (holotype, $\mathrm{W})$.

Oerstedella ornata Dressler, Orquídea (Mexico City), n.s., 8: 345. 1982. = Epidendrum stolidium Hágsater

TyPE: Panama. Chiriquí: arriba de Guadalupe, alt. 2200-2300 m, Oct 1980, R.L. Dressler 5930 (holotype, US; isotypes, $\mathrm{AMO}, \mathrm{MO}, \mathrm{MO}, \mathrm{PMA}, \mathrm{U})$.

Oerstedella fuscina Dressler, Orquídea (Mexico City), n.s., 8: 347. 1982. = Epidendrum fuscinum (Dressler) Hágsater 
TYPE: Panama. Coclé: comprado en el mercado, El Valle de Antón, se dice que proviene de las montañas al norte de El Valle, 22, 29 Mar 1981, R.L. Dressler 6010 (holotype, US; isotypes, AMO, MO, PMA).

Oncidium allenii Dressler, Selbyana 22(1): 9. 2001.

TYPE: Panama. Coclé: region north of El Valle de Antón. elev. ca. 1000 m. 20 February 1942, P.H. Allen s.n. (holotype, AMES; isotypes, SEL, US).

Oncidium ampliatum Lindl., Gen. Sp. Orchid. Pl. 202. 1833. = Rossioglossum ampliatum (Lind1.) M.W.Chase \& N.H.Williams

TyPE: Hab. in Panama, et Columbia occidentali, H. Cuming 1312 (holotype, K).

Oncidium ansiferum Rchb.f., Bot. Zeitung (Berlin) 10: 696. 1852.

TYPE: [Panama]. Chiriquí, J. Warscewicz s.n. (holotype, W).

Oncidium bracteatum Warsz. \& Rchb.f., Bot. Zeitung (Berlin) 10: 695. 1852.

TyPE: [Panama]. Chiriquí Cordilleren 6-9000' auf Bäumen, J. Warscewiczii s.n (holotype, W).

Oncidium castaneum Rchb.f. ex Kraenzl. in H.G.A.Engler (ed.), Pflanzenr., IV, 50(80): 287. 1922. = Oncidium dichromaticum Rchb.f.

Syntypes: Subäquatoriale andine Provinz. Costa Rica, A.R. Endres s.n. [Panama]. Subäquatoriale andine Provinz. Chiriquí, R. Pfau s.n. (lectotype designated by Pupulin (2011), W, Costa Rica. Without specific locality, 1867, A.R. Endrés s.n. «N.o 108 Oncd.m» [W 0019929 / Rchb. Orch. 18381]; isolectotypes, W-0019925, W-0019930).

Oncidium cerebriferum Rchb.f., Bot. Zeitung (Berlin) 10: 696. 1852. = Oncidium ensatum Lindl. TYPE: [Panama]. Chiriquí, J. Warscewicz s.n. (holotype, W).

Oncidium cheirophorum Rchb.f., Bot. Zeitung (Berlin) 10: 695. 1852.

TYPE: [Panama]. Chiriquí Vulkan, J. Warscewicz s.n. (holotype, W).

Oncidium confusum Rchb.f., Xenia Orchid. 1: 234. 1858. = Oncidium ensatum

TYPE: Panama. Chiriquí, October. 3-6000', J. Warscewicz s.n. (holotype, W).

Oncidium ebrachiatum Ames \& C.Schweinf., Schedul. Orchid. 8: 75. 1925. = Trichocentrum nudum (Bateman ex Lindl.) M.W.Chase \& N.H.Williams

TYPE: Panama. Cana and vicinity, 4 Apr 1908, alt 2000-6500 ft., R.S. Williams 975 (holotype, AMES).

Oncidium exalatum Hágsater, Orquídea (Mexico City) 8: 98.1981.

TYPE: Panama. Veraguas: Cerro Tute, Dec 1975. Preparado de material cultivado, 1 Oct 1980, R.L Dressler \& Hágsater 4173 (holotype, AMO; isotype, MO).

Oncidium fulgens Schltr., Repert. Spec. Nov. Regni Veg. Beih. 17: 83. 1922.= Oncidium klotzschianum Rchb.f. TYPE: Panama. Chiriquí, 4000-5000 fuss, blühend im Februar-März, C.W. Powell 157 (holotype, B, destroyed; lectotype designated by Christenson (1991b), AMES-23918; isolectotypes, K, MO-955998, MO-955999; drawing of type, AMES-26799; drawing published in Mansfeld [1931: t. 73, nr. 290].).

Oncidium graciliforme C.Schweinf., Bot. Mus. Leafl. 5: 96. 1938. = Oncidium klotzschianum

TyPe: Panama. Chiriquí: alt. 4000-5000 ft, Mar 1923, C.W. Powell 3227 (referred to Powell 157) (holotype, AMES).

Oncidium isthmi Schltr., Repert. Spec. Nov. Regni Veg. Beih. 17: 84. 1922.

TyPe: Panama. Sambo River, Süd-Darien, 200 m., blühend im Feb 1912, H. Pittier 5569 (holotype, B, destroyed).

Oncidium klotzschianum Rchb.f., Bot. Zeitung (Berlin) 10: 695. 1852.

TYPE: Panama. Chiriquí Cordilleren 4-5000', J. Warscewicz s.n. (holotype, W). 
Oncidium lacerum Lindl., Bot. Reg. 30. Misc. 38. 1844. = Trichocentrum lacerum (Lindl.) J.M.H.Shaw TYPE: [Panama. Without precise locality] ex Hort. Loddigges (holotype, K).

Oncidium maduroi Dressler, Orchids (West Palm Beach) 69(8): 762. 2000.

TYPE: Panama. Bocas del Toro, elev. 1200-1400 m, cultivated in Finca Dracula, Cerro Punta, 17 Aug 1998, $R$. L. Dressler 6220 (holotype, MO; isotype, PMA).

Oncidium martinezii Königer, Arcula 15: 369. 2005.

TYPE: Panama. Darién: Chepigana: Cerro Pechito Berroa, 600-800 m, var einigen Jahren schon entdeckt von Sr. Porfirio Martínez aus Concepción, Panama, in Kultur bei Sr. Andrés Maduro, Panama, in Kultur in Blüte bei $W$. Königer $W K-122$ (holotype, M; isotypes, PMA, Herb. H. Königer).

Oncidium mirabile Rchb.f., Bot. Zeitung (Berlin) 10: 697. 1852., nom. utique rej. = Lockhartia oerstedii Rchb.f. TYPE: Panama. [Without locality] J. Warszewicz s.n. (holotype, W).

Oncidium ochmatochilum Rchb.f., Bot. Zeitung (Berlin) 10: 698. 1852.= Cyrtochiloides ochmatochila (Rchb.f.) N.H.Williams \& M.W.Chase

TYPE: Panama. [Without locality] J. Warszewicz s.n. (holotype, W).

Oncidium oerstedii Rchb.f. var. crispiflorum Schltr., Repert. Spec. Nov. Regni Veg. Beih. 17: 85. 1922. = Trichocentrum crispiflorum (Schltr.) Bogarín

TyPe: Panama. An der Küste des Pacifischen Ozeans bei Panama-City, C.W. Powell 1 (holotype, B, destroyed; lectotype designated by Christenson (1991b), AMES-23920; isolectotypes, K, MO-955973).

Oncidium panamense Schltr., Repert. Spec. Nov. Regni Veg. Beih. 17: 85. 1922.

TYPE: Panama. Auf Hügeln bei Panama City, C.W. Powell 4 (holotype, B, destroyed; lectotype designated by Christenson (1991b), AMES-23921; isolectotypes, AMES-23922, MO-955877, MO-955878; drawing of type, AMES-26800; drawing published in Mansfeld [1931: t. 74, nr. 294].).

Oncidium parviflorum L.O.Williams, Amer. Orchid Soc. Bull. 11: [32], tab. 1. 1942.

TYPE: Panama. Coclé: region north of El Valle de Antón, alt. about 1000 m, epiphyte, 20 Feb 1942, P.H.Allen 2937 (holotype, AMES).

Oncidium peliogramma Linden \& Rchb.f., Gard. Chron. 1871: 1451. 1871. = Oncidium polycladium Rchb.f. ex Lindl.

TYPE: [Panama]. It flowered with Director Linden, who obtained it from Chiriquí through Mr. Wallis s.n. (holotype, W).

Oncidium polycladium Rchb.f. ex Lindl., Fol. Orchid. 6: 47. 1855.

TYPE: [Panama]. Wild in Costa Rica, Veragua, Chiriquí, Warczewicz s.n. (holotype, K; isotype, W; illustration of type, K).

Oncidium powellii Schltr., Repert. Spec. Nov. Regni Veg. Beih. 17: 86. 1922. = Oncidium anthocrene Rchb.f. TYPE: Panama. an den Hinterwässern des Gatun-Sees unweit Panama-City, C.W. Powell 58 (holotype, B, destroyed; lectotype designated by Christenson (1991b), AMES-23923; isolectotypes K, MO-955916; drawing of type, AMES-26814; drawing published in Mansfeld [1931: t. 74, nr. 296].).

Oncidium punctulatum Dressler, Selbyana 22(1): 10. 2001.

TyPe: Panama. Chiriquí: Cerro Colorado, arriba de Chami, elev. 1300-1700 m. bosque nuboso, 1 November 1999, R.L. Dressler \& J.T. Atwood 6274 (holotype, MO; isotype, PMA).

Oncidium stipitatum Lindl. in Benth., Bot. Voy. Sulphur [Bentham] 172. 1846. = Trichocentrum lacerum (Lindl.) J.M.H.Shaw

TYPE: [Panama. Island of Taboga, 30 Jan.-11 Feb. 1837], G.W. Barclay 958 (holotype, BM).

Oncidium stipitatum var. platyonyx Rchb.f., Gard. Chron. (n. s.) 9: 788. 1878. = Trichocentrum lacerum 
TYPE: [Panama. Without precise locality] ex Hort. W. Bull. (holotype,W).

Oncidium teres Ames \& C.Schweinf., Schedul. Orchid. 8: 78. 1925. = Trichocentrum helicanthum (Kraenzl.) J.M.H.Shaw

TyPe: Panama. Veraguas: San Francisco, Feb 1924, alt. 1000 ft., C.W. Powell 383 (holotype, AMES).

Oncidium warszewiczii Rchb.f., Bot. Zeitung (Berlin) 10: 693. 1852.

Syntypes: [Panama]: Veragua, Chiriquí Vulkan, J. Warscewicz s.n., lectotype designated by Mora de Retana (1993), W; Costa Rica: [Cartago]. Carthago Vulkan. An Eichen. 8-10,000', J. Warscewicz s.n. (W).

Oncidium zelenkoanum Dressler \& Pupulin, Lankesteriana 8: 37. 2003.

TYPE: Panama. Bocas del Toro: Culebra (Velorio), 1000 m, Nov. 2000, epífita en árboles muy altos, floreció en cultivo julio 2001, A. Maduro \& E. Olmos 195 (holotype, MO; isotype, PMA).

Ornithidium conduplicatum Ames \& C.Schweinf., Schedul. Orchid. 8: 66. 1925.

TyPe: Panama. Chiriquí: Palo Alto Hill, Sept-Oct 1923, alt. 4000-5000 ft, C.W. Powell 341 (holotype, AMES).

Ornithocephalus aristatus Pupulin \& Dressler, Brittonia 58(4): 314. 2006.

TyPE: Panama. Bocas del Toro: Changuinola, Valle Risco, $800 \mathrm{~m}$, primary forest; at $3 \mathrm{~m}$ from the ground epiphytic on shrub, flowered in cult. Finca Drácula, 26 Sep. 2003, A. Maduro \& E. Olmos 327 (Holotype, PMA; isotype, CR).

Ornithocephalus cochleariformis C.Schweinf., Bot. Mus. Leafl. 4: 124. 1937.

TYPE: Panama. Coclé: lower portion of valley and marshes along Río Antón, El Valle de Antón, alt. 500 m, Feb 1935, A.A. Hunter \& P.H. Allen 383 (holotype, AMES).

Ornithocephalus diceras Schltr., Repert. Spec. Nov. Regni Veg. Beih. 17: 87. 1922. = Ornithocephalus bicornis Lindl.

TyPe: Panama. Rep. of Panama, hills east of the city, C.W. Powell 174 (holotype, B; destroyed, lectotype designated by Christenson (1991b), AMES-23935, isolectotype, MO-956007; drawing of type, AMES26821; photo of the isolectotype ex MO, K; drawing published in Mansfeld [1931: t. 76, nr. 304].).

Ornithocephalus lanuginosus Ames, Proc. Biol. Soc. Washington 34: 152. 1921. = Ornithocephalus bicornis TYPE: Panama. Panama: edge of forest along Panama-Pecora Road, near Tocumen River, 30 Dec 1917, 75 m alt., on trees, E. Killip 3314 (holotype, AMES; isotype, ROCH).

Ornithocephalus powellii Schltr., Repert. Spec. Nov. Regni Veg. Beih. 17: 88. 1922.

TYPE: Panama. Auf Hügeln östlich von Panama-City. 100-300 Fuss, blühend Februar bis März, C.W. Powell 231, lectotype designated by Christenson (1991b), AMES-23934; isolectotype, MO-956042; photo of isolectotype ex MO, K; drawing published in Mansfeld [1931: t. 77, nr. 305].).

Osmoglossum acuminatum Schltr., Repert. Spec. Nov. Regni Veg. Beih. 17: 79. 1922. Cuitlauzina egertonii (Lindl.) Dressler \& N.H.Williams

TyPE: Panama. Chiriquí: $5000 \mathrm{ft}$, C.W. Powell 255 (holotype, B destroyed; lectotype designated by Christenson (1991b), AMES-23899; isolectotype, MO-956059; drawing of type, K). Superfluously designated by Yañez \& Soto (2003).

Palmorchis trilobulata L.O.Williams in Woodson \& Schery, Ann. Missouri Bot. Gard. 28: 415. 1941.

TYPE: Panama. Coclé: terrestrial, flowers pure white, region north of El Valle de Antón, trail to Las Minas, alt. ca. 1000 m, 10 May 1941, P. Allen 2463 (holotype, AMES).

Palmorchis trinotata Dressler, Orquideología 20: 263. 1997.

TYPE: Panama. Coclé: terrestrial, flowers pure white, region north of El Valle de Antón, trail to Las Minas, alt. ca. 1000 m, 10 May 1941, P. Allen 2463 (holotype, AMES).

Paphinia vermiculifera G.Gerlach \& Dressler, Lankesteriana 8: 27. 2003. 
TYPe: Panama. Coclé: El Valle de Antón, 800-1000 m, floreció en cultivo el 6 de julio 2002, R. L. Dressler 6350 (holotype, MO).

Pelexia ochyrae Szlach., Fragm. Florist. Geobot. 41: 858. 1996.

TyPe: Panama. Chiriquí: Buenavista, 30 Mar 1974, H. Butcher s.n. (holotype, FLAS).

Peristeria elata Hook., Bot. Mag. 58: t. 3116. 1831.

TYPE: Panama. In the year 1826, Henry Barnard, Esq. of Truxillo in Peru, communicated to Richard Harrison, Esq. of Liverpool, a bulb of a remarkable, parasitical, orchideous plant, which he had found in the neighbourhood of Panama (holotype, K).

Phragmipedium exstaminodium Castaño, Hágsater \& E.Aguirre subsp. warszewiczii Dressler, Orchid Digest 69(2): 89. 2005. = Phragmipedium humboldtii (Warsz. ex Rchb.f.) J.T.Atwood \& Dressler

TYPe: Panama. Bocas del Toro: Cerro Colorado, along road between Río San Felix and mining exploration camp, $7 \mathrm{ml} \mathrm{W}$ of Chame, along trail through Guaymi village, $8^{\circ} 35^{\prime} \mathrm{N} 81^{\circ} 50 \mathrm{~W}, 1500 \mathrm{~m}, 8$ July 1988 , T. Croat 69170 (holotype, MO).

Phragmipedium popowii Braem, Ohlund \& Quéné, Richardiana 4(4): 185. 2004. nom. illeg. = Phragmipedium humboldtii

TYPE: Panama. Chiriquí, Warszewicz 41 (holotype, W).

Phymatidium panamense Dressler, Orquideología 6: 142. 1971. = Eloyella panamensis (Dressler) Dodson

Type: Panama. prov. Panama, La Eneida, región de Cerro Jefe; 6 Aug 1968; R.L. Dressler 3570 (holotype, US).

Physosiphon minutiflorus Ames \& C.Schweinf., Schedul. Orchid. 8: 11. 1925. = Stelis deregularis Barb.Rodr

Type: Panama. Chiriquí: Jaramillo, Oct 1923, alt 5000 ft, C.W. Powell 345 (holotype, AMES).

Platyglottis coriacea L.O.Williams, Ann. Missouri Bot. Gard. 29: 347. 1942. = Scaphyglottis coriacea (L.O.Williams) Dressler

TYPE: Panama. Coclé: region north of El Valle de Antón, alt. c. 1000 m, 20 Feb 1942, P. H. Allen 2936 (holotype, AMES).

Platystele calymma Luer, Selbyana 3(3/4): 226. 1977.

TYPE: Panama. Panama: epiphytic in the cloud forest along the El Llano-Carti road, alt. ca. 650 m, Feb. 1977, R.L. Dressler s.n. (holotype, SEL; isotype, MO).

Platystele dressleri Luer, Selbyana 3(1/2): 26, fig. 116. 1976.

TYPE: Panama. Panama: epiphytic along the Altos de Pacora road, alt. ca. 650 m., collected 4 Mar 1976, $C$. Luer, J. Luer, P. Taylor \& R.L. Dressler 963 (holotype, SEL).

Platystele hampshireae Luer, Monogr. Syst. Bot. Missouri Bot. Gard. 65: 113, fig. 1998.

TYPE: Panama. Chiriquí: wet forest between Fortuna Lake and Chiriquí Grande, $1 \mathrm{~km}$ south of the continental divide, alt. 1200 m, 10 Mar 1985, R.J. Hampshire \& C. Whitefoord 414 (holotype, BM).

Platystele speckmaieri Luer \& Sijm, Monogr. Syst. Bot. Missouri Bot. Gard. 120: 146. 2010.

Type: Panama. Panama: EI Valle de Anton, flowered in cultivation at Wien, Sept. 2009, A.P Sijm 290815 (holotype, MO).

Platystele taylorii Luer, Selbyana 3(1/2): 30. 1976.

TyPe: Panama. Panama: Epiphytic along the Altos de Pacora road, alt. 650 m., 4 Mar 1976, C. Luer, J. Luer, P. Taylor \& R.L. Dressler 741 (holotype, SEL).

Pleurothallis aberrans Luer, Selbyana 2: 382. 1978. = Acianthera aberrans (Luer) Pupulin \& Bogarín

TYPE: Panama. Veraguas: epiphytic in tree north of the continental divide, alt. ca. 700 m., above Santa Fé, 6 Sept 1976, C. Luer \& R.L. Dressler 1628 (holotype, SEL). 
Pleurothallis acrisepala Ames \& C.Schweinf., Schedul. Orchid. 8: 22. 1925. = Specklinia acrisepala (Ames \& C.Schweinf.) Pridgeon \& M.W.Chase

TYPE: Panama. without exact locality, C.W. Powell 420 (holotype, AMES; isotype, AMES).

Pleurothallis allenii L.O.Williams in Woodson \& Schery, Ann. Missouri Bot. Gard. 27: 275. 1940.

TyPe: Panama. Coclé: vicinity of El Valle, alt. 600-1000 m, 8 Dec. 1938, P.H. Allen 1240 (holotype, MO; isotype, AMES; drawing of type, AMES).

Pleurothallis alpina Ames, Schedul. Orchid. 5: 14. 1923. = Acianthera sicaria (Lind1.) Pridgeon \& M.W.Chase TyPe: Panama. Chiriquí: "Caramillia” [Jaramillo?], alt 4500 ft, 1923, C.W. Powell 282 (holotype, AMES; isotype, MO).

Pleurothallis annectens Luer, Selbyana 3 (3/4): 240. 1977.

TYPE: Panama. Chiriquí: epiphytic in cloud forest along the continental divide above Camp Escopeta, Cerro Colorado, alt. 1700 m, 16 Feb 1977, C. Luer, J. Luer \& R.L. Dressler 1583 (holotype, SEL).

Pleurothallis antonensis L.O.Williams, Ann. Missouri Bot. Gard. 29: 341. 1942. = Pleurothallis bivalvis Lindl. TYPE: Panama. Coclé: hills north of El Valle de Antón, alt. ca. 1000 m, 1 Sep 1941, P.H. Allen 2701 (holotype, AMES).

Pleurothallis archicolonae Luer, Selbyana 3(1/2): 52. 129. 1976.

TyPe: Panama. Panama: epiphytic on Cerro Jefe, alt. 1000 m, 2 Mar 1976, C. Luer, J. Luer, P. Taylor \& H. Butcher 964 (holotype, SEL).

Pleurothallis areldii Luer, Selbyana 2: 383. 1978. = Specklinia areldii (Luer) Pridgeon \& M.W.Chase

TYPE: Panama. Chiriquí: epiphytic in cloud forest at the Fortuna dam site, alt. ca. 750 m, Feb 1977, R.L. Dressler s.n., greenhouse acc. no. 77-1787, flowered in cult. 9 Aug 1977, C. Luer 1653 (holotype, SEL).

Pleurothallis balaeniceps Luer \& Dressler, Orquideología 16: 47. 1986. = Echinosepala balaeniceps (Luer \& Dressler) Pridgeon \& M.W.Chase

TYPE: Panama. Veraguas: epiphytic in cloud forest on Cerro Arizona above Escuela Alto de Piedra, west of Santa Fe, alt. 850-950 m, June 5, 1982, flowered in cultivation 23 May 1984, R.L. Dressler 6063 (holotype, $\mathrm{MO}$; isotype, MO).

Pleurothallis barboselloides Schltr., Repert. Spec. Nov. Regni Veg. Beih. 17: 18. 1922. = Specklinia corniculata (Sw.) Steud

TYPE: Panama. Auf Hügeln bei Panama City, C.W. Powell 98 (holotype, B, destroyed; lectotype designated by Christenson (1991b), AMES-23792; isolectotypes, K, MO-955946; drawing of type, AMES-26760; drawing published in Mansfeld [1931: t. 29, nr. 113].).

Pleurothallis brighamella Luer, Monogr. Syst. Bot. Missouri Bot. Gard. 76: 171. 1999.= Specklinia brighamella (Luer) Pridgeon \& M.W.Chase

TYPE: Panama. without collection data, cultivated by Maduro's Tropical Flowers at Cerro Punta, 18 Nov 1998, C. Luer 19005 (holotype, MO).

Pleurothallis butcheri L.O.Williams, Fieldiana, Bot. 29(6): 346. 1961. = Acianthera butcheri (L.O.Williams) Pridgeon \& M.W.Chase

TYPE: Panama. Chiriquí: in mossy cloud forest along branch Río Machomonte, Cordillera, vicinity Volcán, slopes of Chiriquí volcano, alt.4000 m, 19 Jan 1961, H.P. Butcher 651 (holotype, F).

Pleurothallis cactantha Luer, Selbyana 3(1/2): 72. 1976. = Specklinia cactantha (Luer) Pridgeon \& M.W.Chase TYPE: Panama. Panama: epiphytic along the Altos de Pacora road, alt. 650 m., 4 Mar 1976, C. Luer, J. Luer, P. Taylor \& R.L. Dressler 743 (holotype, SEL).

Pleurothallis caligularis Luer, Selbyana 3(1/2): 74. 1976. = Stelis carnosilabia (A.H.Heller \& A.D.Hawkes) 
Pridgeon \& M.W.Chase

TyPe: Panama. Veraguas: Guabal, Río Dos Bocas, 16 km NW of Santa Fé, alt. 500 m, 15 Nov 1974, R.L. Dressler 4808 (holotype, SEL).

Pleurothallis campicola Luer, Selbyana 5: 161. 1979. = Stelis listerophora (Schltr.) Pridgeon \& M.W.Chase

TYPE: Panama. Chiriquí: epiphytic in scrubby trees on the llanos del volcán, a few $\mathrm{km} \mathrm{W}$ of El Hato del Volcán, alt. 1500 m, 20 Feb 1977, greenhouse acc. no. 77-1754, flowered in cult. 15 Sept 1977, C. Luer, J. Luer \& H. Butcher 1830 (holotype, SEL).

Pleurothallis canae Ames, Schedul. Orchid. 2: 18. 1923. = Stelis canae (Ames) Pridgeon \& M.W.Chase

TYPE: Panama. Cana and vicinity, April 27, 1908. On trees. Flowers purplish. 6000 feet altitude, R. S. Williams 971 (holotype, AMES).

Pleurothallis cardiochila L.O.Williams, Ann. Missouri Bot. Gard. 29: 343. 1942.

TYPe: Panama. Chiriquí: rain forest, Bajo Chorro, Boquete District, alt. ca. 1800 m, 13 Jan 1938, M.E. Davidson 119 (holotype, AMES; isotype, MO).

Pleurothallis chiriquensis Schltr., Repert. Spec. Nov. Regni Veg. Beih. 17: 19. 1922. = Stelis gelida (Lindl.) Pridgeon \& M.W.Chase

TyPE: Panama. Chiriquí, 4000-5000 Fuss.ü M., blühend im Feb, C.W. Powell 227 (holotype, B, destroyed lectotype designated by Christenson (1991b), AMES-26783).

Pleurothallis citrophila Luer, Selbyana 3 (3/4): 266. 1977. = Acianthera lojae (Schltr.) Luer

TYPE: Panama. Coclé: epiphytic in old orange grove near the mesa above El Valle, alt. ca. $1000 \mathrm{~m}, 2$ Sept 1976, C. Luer \& H. Butcher 1375 (holotype, SEL).

Pleurothallis cleistogama Luer, Monogr. Syst. Bot. Missouri Bot. Gard. 72: 41. 1998.

Type: Panama. Chiriquí: Bajo Grande, above Cerro Punta, alt. 2400 m, 13 Feb 1985, C. Luer, J. Luer, R. Dressler \& K. Dressler 10527 (holotype, MO).

Pleurothallis cobriformis L.O.Williams in Woodson \& Schery, Ann. Missouri Bot. Gard. 27: 277. 1940.

TYPE: Panama. Coclé: epiphytic, vicinity of El Valle de Antón, ca. $600 \mathrm{~m}$ alt, flower tan spotted with maroon, 19 Dec 1939, P.H. Allen 2057 (holotype, AMES).

Pleurothallis condylata Luer, Selbyana 3(1/2): 80. 1976. = Specklinia condylata (Luer) Pridgeon \& M.W.Chase TYPE: Panama. Chiriquí: epiphytic in trees along the stream near San Vicente, alt. 500 m., between Concepción and Volcán, 11 Sept 1976, C. Luer \& Butcher 1198 (holotype, SEL).

Pleurothallis congruens Luer, Selbyana 2: 391. 1978. = Acianthera cogniauxiana (Schltr.) Pridgeon \& M.W.Chase

TYPE: Panama. Panama: epiphytic in cloud forest along the El Llano-Carti road, alt. 300 m, 3 Mar 1976, $C$. Luer, J. Luer, P. Taylor \& R.L. Dressler 987 (holotype, SEL).

Pleurothallis corynophora Luer, Phytologia 55: 201. 1984. = Lankesteriana fractiflexa (Ames \& C.Schweinf.) Karremans

TyPE: Panama. Prov. of Chiriquí: epiphytic in cloud forest above Guadalupe, alt. 2300 m, 8 Dec. 1983, $C$. Luer, J. Luer \& A. Maduro 9299 (holotype, SEL).

Pleurothallis croatii Luer, Monogr. Syst. Bot. Missouri Bot. Gard. 65: 18. 1998. = Stelis decurrens Pridgeon \& M.W.Chase

TYPE: Panama. Chiriquí: Cerro Pate Macho, along the continental divide, northeast of Boquete, alt. ca. 2200 m, 23 Nov 1979, T.B. Croat 48506 (holotype, MO).

Pleurothallis cucumeris Luer, Selbyana 5: 162. 1979. = Specklinia cucumeris (Luer) Bogarín \& Karremans TYPE: Panama. Chiriquí: epiphytic in cloud forest, Cerro Colorado, alt. ca. 1700 m, Feb 1977, greenhouse acc. 
no. 77-1825, flowered in cult. 10 Dec 1977, C. Luer, J. Luer \& R.L. Dressler 2258 (holotype, SEL).

Pleurothallis cuspidata Luer, Selbyana 3(3/4): 282. 1977. = Lankesteriana cuspidata (Luer) Karremans

TyPE: Panama. Chiriquí: epiphytic in elfin cloud forest on Cerro Hornito, alt. ca. 1700 m, 15 Dec 1976, C.

Luer, R.L. Dressler, N.H. Williams \& F.L. Stevenson 1335 (holotype, SEL).

Pleurothallis displosa Luer, Monogr. Syst. Bot. Missouri Bot. Gard. 76: 172. 1999. = Specklinia displosa (Luer) Pridgeon \& M.W.Chase

TYPE: Panama. Coclé: El Valle del Antón, alt. ca. 1000 m, collected by George Kennedy, flowered in cultivation,

20 Oct 1981, C. Luer 6533 (holotype, SEL).

Pleurothallis diuturna Schltr., Repert. Spec. Nov. Regni Veg. Beih. 17: 19. 1922. = Kraenzlinella erinacea (Rchb.f.) Solano

TyPE: Panama. Veragua-Distrikt, etwa in Meereshöhe, C.W. Powell 172 (holotype, B, destroyed; lectotype designated by Christenson (1991b), AMES-23787; isolectotypes, K, MO-956006; drwing of type, AMES26864; drawing published in Mansfeld [1931: t. 30, nr. 119].).

Pleurothallis dressleri Luer, Selbyana 3(1/2): 98. 1976. = Specklinia dressleri (Luer) Bogarín \& Karremans

TyPE: Panama. Panama: epiphytic on mossy limbs, Cerro Jefe, alt. 1000 m., 20 Aug 1967, R.L. Dressler 3033 (holotype, SEL).

Pleurothallis dukei Luer, Lindleyana 12: 45. 1997.

TyPe: Panama. Darién: cloud forest, Cerro Pirre, alt. 2500-4500 ft, 9-10 Aug 1967, J.A. Duke \& T.S. Elias 13845 (holotype, MO; isotypes, GH, SEL, US).

Pleurothallis ellipsophylla L.O.Williams, Ann. Missouri Bot. Gard. 29: 344. 1942. = Acianthera ellipsophylla (L.O.Williams) Pridgeon \& M.W.Chase

TyPE: Panama. Bocas del Toro: epiphyte, SW of Bocas at Maccaw Hill, Isla Colón, alt. 0-125 m, 25 Aug 1940, flowers greenish-brown, H. von Wedel 560 (holotype, AMES).

Pleurothallis falcatiloba Ames, Proc. Biol. Soc. Washington 34: 152. 1921. = Stelis falcatiloba (Ames) Bogarín \& Serracín

TyPE: Panama. Chiriquí: valley of the Río Quebrada, 8 Feb 1918, 1700 m alt., on trees in deep woods, Killip 3540 (holotype, AMES; isotype, ROCH).

Pleurothallis fortunae Luer \& Dressler, Lindleyana 6: 97. 1991. = Stelis fortunae (Luer \& Dressler) Pridgeon \& M.W.Chase

TyPE: Panama. Prov. of Chiriquí: epiphytic in cloud forest around the Fortuna dam site, alt. ca. $1000 \mathrm{~m}$, collected Aug. 1976, flowered in cultivation 13 Nov. 1976, R.L. Dressler s.n. (holotype, SEL).

Pleurothallis fuegi Rchb.f. var. echinata L.O.Williams, Ann. Missouri Bot. Gard. 33: 120. 1946. = Specklinia fuegi (Rchb.f.) Solano \& Soto Arenas

TYPE: Panama. Chiriquí: Volcán de Chiriquí, alt. ca. 2720 m, M.E. Davidson 981 (holotype, AMES; isotype, AMES).

Pleurothallis glandulosa Ames, Schedul. Orchid. 6: 60. 1923. = Specklinia glandulosa (Ames) Pridgeon \& M.W.Chase

TyPe: Panama. Juna Grande range, sea level, C.W. Powell 306 (holotype, AMES; isotypes, AMES, MO).

Pleurothallis gnomonifera Ames, Schedul. Orchid. 6: 61. 1923. = Trichosalpinx memor (Rchb.f.) Luer

TyPe: Panama. Chiriquí, Cerro Norquita [Horqueta], alt 5000-6000 ft, C.W. Powell 311 (holotype, AMES).

Pleurothallis guttata Luer, Selbyana 3(1/2): 116. 1976. = Stelis guttata (Luer) Pridgeon \& M.W.Chase

TYPE: Panama. Panama: epiphytic in the region of Cerro Jefe, alt. 1000 m, 15 Oct 1967, R.L. Dressler 3086 (holotype, SEL). 
Pleurothallis hammelii Luer, Lindleyana 12: 48. 1997.

TYPE: Panama. Panama: epiphytic in forest along the Llano-Cartí road, alt. 1200 m, 13 Jan 1978, B. Hammel 868 (holotype, SEL; isotype, MO).

Pleurothallis harpago Luer, Selbyana 5: 167. 1979.

TYPe: Panama. Darien: Cerro Pirre, July 1977, R.L. Dressler s.n., cultivated at SEL, greenhouse acc. no. 772600A, flowered in cult. 20 Mar 1978, C. Luer 2798 (holotype, SEL).

Pleurothallis hemileuca Luer, Selbyana 7: 118. 1982.

TYPE: Panama. Chiriquí: epiphytic in cloud forest at the Fortuna dam site, alt. ca. 1000 m, Aug 1976, R.L. Dressler s.n., cultivated at SEL, 114-76-11, flowered in cult. 23 Dec 1976, C. Luer 1274 (holotype, SEL).

Pleurothallis hispida L.O.Williams, Ann. Missouri Bot. Gard. 27: 277. 1940. = Dresslerella hispida (L.O.Williams) Luer

TYPe: Panama. Coclé: vicinity of El Valle, 800-1000 m, 5 Sept. 1939, P.H. Allen 782 (holotype, AMES).

Pleurothallis hunteriana Schltr., Repert. Spec. Nov. Regni Veg. Beih. 17: 20. 1922. = Pabstiella tripterantha (Rchb.f.) F.Barros

TyPe: Panama. Chiriquí, 4000 ft., C.W. Powell 245 (holotype, B, destroyed, lectotype designated by Christenson (1991b), AMES-27296; isolectotype, K). Superfluously designated by Luer (2000).

Pleurothallis imago Luer, Selbyana 3 (3/4): 318. 1977. = Pleurothallis excavata Schltr.

TyPE: Panama. Panama: epiphytic along the Altos de Pacora road, 650 m, 4 Mar. 1976, C.A. Luer 732, J. Luer, R.L. Dressler \& P. Taylor (holotype, SEL).

Pleurothallis instar Luer, Selbyana 3 (3/4): 320. 1977.

TYPE: Panama. Chiriquí: epiphytic in cloud forest on Cerro Hornito, alt. ca. 1700 m, 15 Dec 1976, C. Luer, A. Luer, R.L. Dressler, N.H. Williams \& F.L. Stevenson 1389 (holotype, SEL).

Pleurothallis isthmica Luer, Selbyana 3(3/4): 322. 1977.

TYPE: Panama. Veraguas: near continental divide above Santa Fe, 750 m, 4 Sept. 1976, C.A. Luer 1140 \& R.L Dressler (holotype, SEL).

Pleurothallis juxtaposita Luer, Lindleyana 6: 103-105. 1991. = Acianthera juxtaposita (Luer) Luer

TYPE: Panama. Chiriquí: epiphytic in could forest near the Fortuna dam site, alt. 1100 m, 16 Feb 1985, C. Luer, J. Luer, R.L. Dressler \& K. Dressler 10585 (holotype, SEL; isotype MO).

Pleurothallis knappiae Luer, Monogr. Syst. Bot. Missouri Bot. Gard. 79: 128. 2000.

TYPE: Panama. Veraguas: Cerro Tute, Altos de Piedras, west of Santa Fe, alt. 1250-1410 m, 15 Dec 1981, S. Knapp \& K. Sytsma 2653 (holotype, MO; isotype, PMA).

Pleurothallis lepidota L.O.Williams in Woodson \& Schery, Ann. Missouri Bot. Gard. 27: 279. 1940. = Acianthera lepidota (L.O.Williams) Pridgeon \& M.W.Chase

TYPE: Panama. Chiriquí: epiphytic, Llanos del Volcán, ca. $1300 \mathrm{~m}$ alt., flowers green striped maroon, 23 Jan 1939, P.H. Allen 1552 (holotype, AMES).

Pleurothallis macrantha L.O.Williams in Woodson \& Schery, Ann. Missouri Bot. Gard. 28: 417. 1941. = Stelis cylindrata Pridgeon \& M.W.Chase

TYPE: Panama. Chiriquí: terrestrial, flowers pale greenish-yellow, spotted chocolate, vicinity of Bajo Chorro, alt. 1900 m, 20-22 jul 1940, R. E. Woodson, Jr. \& R. W. Schery 666 (holotype, AMES; isotype, MO).

Pleurothallis maduroi Luer, Lindleyana 12: 51.1997.

TYPE: Panama. Chiriquí: Guadalupe, May 1992, A. Maduro 18 (holotype, MO).

Pleurothallis mammillata Luer, Selbyana 3(1/2): 138. 1976.

TyPe: Panama. Panama: epiphytic on Cerro Jefe, alt. 1000 m, 2 Mar 1976, C. Luer, H. Butcher, J. Luer \& P. 
Taylor 951 (holotype, SEL).

Pleurothallis mystax Luer, Selbyana 3(1/2): 146. 1976. = Stelis mystax (Luer) Pridgeon \& M.W.Chase

TYPE: Panama. Veraguas: epiphytic in cloud forest near the continental divide, above Santa Fé, alt. 650-750 m, 5 Sept 1976, C. Luer \& R.L. Dressler 1261 (holotype, SEL).

Pleurothallis nitida Luer, Selbyana 3 (3/4): 352. 1977.

TYPE: Panama. Chiriquí: epiphytic in elfin cloud forest on Cerro Hornito, ca. 1700 m, 15 Dec. 1976, C. Luer, A. Luer, R.L. Dressler, N.H. Williams \& F.L. Stevenson 1337 (holotype, SEL).

Pleurothallis octomeriae Schltr., Repert. Spec. Nov. Regni Veg. Beih. 17: 21. 1922.=Myoxanthus octomeriae (Schltr.) Luer

Type: Panama. An Bäumen in Schluchten des Chiriquí, c. 5000 ft., C.W. Powell 252 (holotype, B, destroyed; lectotype designated by Christenson (1991b), AMES-23789; isolectotypes, K, MO-956057).

Pleurothallis operculata Luer, Phytologia 49: 213. 1981. = Trichosalpinx rotundata (C.Schweinf.) Dressler TYPE: Panama. Coclé: epiphytic in cloud forest, El Copé, R.L. Dressler s.n., cultivated at SEL-78-480, flowered 18 Jul 1979, C. Luer 4075 (holotype, SEL).

Pleurothallis pallida Luer, Selbyana 3(3/4): 358. 1977.

TYPE: Panama. Coclé: epiphytic in the hills north of El Valle, alt. 1000 m, 6 Mar 1976, C. Luer, R. Dressler, J. Luer \& P. Taylor 954 (holotype, SEL).

Pleurothallis pan Luer, Selbyana 3(3/4): 360. 1977. = Echinosepala pan (Luer) Pridgeon \& M.W.Chase TYPE: Panama. Veraguas: epiphytic in cloud forest NW of Santa Fé, alt. ca. $750 \mathrm{~m}$, R.L. Dressler s.n. (holotype, SEL).

Pleurothallis panamensis Schltr., Repert. Spec. Nov. Regni Veg. 17: 140. 1921. = Specklinia microphylla (A.Rich. \& Galeotti) Pridgeon \& M.W.Chase

TyPE: Panama. Auf Pampasbäumen in der Provinz Chiriquí und Veragua, blühend im Apr 1858, M. Wagner 24 (holotype, B, destroyed; illustration of type, AMES).

Pleurothallis peculiaris Luer, Selbyana 3(1/2): 158. 1976.

TYPE: Panama. Veraguas: epiphytic in the cloud forest near the continental divide above Santa Fé, alt. ca. 700 m, Sept 1976, C. Luer \& R.L. Dressler 1142 (holotype, SEL).

Pleurothallis perpusilla Rchb.f. in Seem., Bot. Voy. Herald [Seemann] 6: 215. 1854. = Platystele perpusilla (Rchb.f.) Garay

TYPE: Panama. Near Panama, on trees, Feb. 1848, B. Seemann 1565 (holotype, W; isotypes, BR, K).

Pleurothallis pertusa Dressler, Orquideología 5: 76. 1970. = Dresslerella pertusa (Dressler) Luer

TyPE: Panama. Panama: Cerro Jefe, 12 Nov 1967, epífita péndula en selva nebulosa, R.L. Dressler 3152 (holotype, US; isotypes, MO, PMA).

Pleurothallis pfavii Rchb.f., Flora 69 (n. s. 44): 555. 1886. = Specklinia pfavii (Rchb.f.) Pupulin \& Karremans Type: [Costa Rica or Panama]. Chiriquí, R. Pfau. s.n. (holotype, W).

Pleurothallis polysticta Luer, Selbyana 5: 176. 1979.

TYPe: Panama. Darien: Cerro Pirre, Jul 1977, R.L. Dressler s.n., cult. at SEL, C. Luer 2799 (holotype, SEL).

Pleurothallis powellii Schltr., Repert. Spec. Nov. Regni Veg. Beih. 17: 22. 1922. = Stelis gigantea Pridgeon \& M.W.Chase

TYPE: Panama. Chiriquí, $4000 \mathrm{ft}$, C.W. Powell 146 (holotype, B, destroyed; lectotype designated by Christenson (1991b), AMES-23785; isolectotypes K, MO-955986, MO-961317; drawing of type, AMES-26775; drawing published in Mansfeld [1931: t. 35, nr. 138].). 
Pleurothallis praegrandis Ames, Schedul. Orchid. 5: 17. 1923.= Pleurothallis colossus Kraenzl. ex Kerch TYPE: Panama. Cana and vicinity, 17 Apr-8 Jun 1908, on trees, $6000 \mathrm{ft}$ alt., R.S. Williams 973 (holotype, NY; illustration of type, AMES).

Pleurothallis pterocaulis L.O.Williams in Woodson \& Schery, Ann. Missouri Bot. Gard. 27: 280. 1940. = Acianthera decipiens (Ames \& C.Schweinf.) Pridgeon \& M.W.Chase

TYPE: Panama. Coclé: vicinity of El Valle, 600-1000 m alt., flowers dull red, 8 Dec 1938, P.H. Allen 1239 (holotype, AMES; isotype, MO).

Pleurothallis rhodoglossa Schltr., Repert. Spec. Nov. Regni Veg. Beih. 17:22. 1922.

TYPE: Panama, auf Hügeln bei Panama City, C.W. Powell 182 (holotype, B, destroyed; lectotype designated by Christenson (1991b), MO-956015; isolectotypes AMES-23793?; drawing of type, AMES-26845).

Pleurothallis rotundata C.Schweinf., Bot. Mus. Leafl. 4: 115. 1937. = Trichosalpinx rotundata (C.Schweinf.) Dressler

TYPE: Panama. Coclé: mountains beyond La Pintada, alt. 400-600 m, Feb 1935, A.A. Hunter \& P.H. Allen 561 $D$ (holotype, AMES; isotype, MO).

Pleurothallis rubella Luer, Selbyana 3 (3/4): 378. 1977. = Platystele aurea Garay

TYPE: Panama. Chiriquí: epiphytic, Fortuna dam site, alt. ca. 1000 m, Oct 1976, R.L. Dressler s.n. (holotype, SEL).

Pleurothallis scitula Luer, Selbyana 3 (3/4): 384. 1977. = Pleurothallis rectipetala Ames \& C.Schweinf.

Type: Panama. Chiriquí: epiphytic in cloud forest on Cerro Hornito, alt. ca. 1700 m, 15 Dec 1976, C. Luer, A. Luer, R.L. Dressler, N.H. Williams \& F.L. Stevenson 1361 (holotype, SEL).

Pleurothallis sempergemmata Luer, Selbyana 3: 386. 1977. = Echinosepala sempergemmata (Luer) Pridgeon \& M.W.Chase

TyPE: Panama. Chiriquí: epiphytic in valley of cloud forest above Guadalupe, alt. ca. 2200 m, 17 Dec 1976, C. Luer, A. Luer, R.L. Dressler, N.H. Williams \& F.L. Stevenson 1360 (holotype, SEL).

Pleurothallis simulans L.O.Williams in Woodson \& Schery, Ann. Missouri Bot. Gard. 27: 281. 1940.

TYPE: Panama. Coclé: epiphyte, north rim of El Valle, flowers maroon, 9 Jul 1939, Allen 1912 (holotype, MO).

Pleurothallis speciosa Luer, Selbyana 3: 392. 1977. = Myoxanthus speciosus (Luer) Luer

TYPE: Panama. Chiriquí: epiphytic in cloud forest above Guadalupe, alt. ca. 2000 m, 13 Sep 1976, C. Luer \& H. Butcher 1371 (holotype, SEL).

Pleurothallis spectabilis Ames \& C.Schweinf., Schedul. Orchid. 8: 34. 1925. = Specklinia spectabilis (Ames \& C.Schweinf.) Pupulin \& Karremans

Type: Panama. Veraguas: Santa Fé, Feb. 1924, 1500 ft, C.W. Powell 382 (holotype, AMES; isotype, MO).

Pleurothallis tantilla Luer, Selbyana 5: 183. 1979. = Trichosalpinx tantilla (Luer) Luer

Type: Panama. Coclé: epiphytic in cloud forest above El Valle, alt. 1000 m, 6 Mar 1976, C. Luer, J. Luer, P. Taylor \& R.L. Dressler 1078 (holotype, SEL).

Pleurothallis telamon Luer, Selbyana 5: 185. 1979.

TYPE: Panama. Coclé: epiphytic in cloud forest near El Copé, May 1977, R.L. Dressler s.n., cultivated at SEL, C. Luer 2112 (holotype, SEL).

Pleurothallis thymochila Luer, Selbyana 3(3/4): 398. 1977. = Stelis thymochila (Luer) Pridgeon \& M.W.Chase

TyPE: Panama. Panama: epiphytic along the El Llano-Carti road, alt. ca. 350 m, 3 Mar 1976, C. Luer, J. Luer, P. Taylor \& R.L. Dressler 992 (holotype, SEL; detail of type, SEL).

Pleurothallis titan Luer, Selbyana 3 (3/4): 400. 1977. 
TYPE: Panama. Chiriquí: epiphytic in cloud forest above the Fortuna dam camp site, alt. 1000 m, Aug 1976, cult. at SEL, greenhouse acc. no. 123-76-20, R.L. Dressler s.n. (holotype, SEL).

Pleurothallis trachychlamys Schltr., Repert. Spec. Nov. Regni Veg. Beih. 17: 23. 1922. = Myoxanthus trachychlamys (Schltr.) Luer

TYPE: Panama. Panama: "auf Hügeln bei Panama-City", C.W. Powell 215 (holotype, B, destroyed), lectotype designated by Christenson (1991b), AMES; isolectotypes, AMES, MO). Superfluously designated by Luer (1992).

Pleurothallis tropida Luer, Phytologia 49: 219. 1981. = Trichosalpinx tropida (Luer) Luer

TYPE: Panama. Coclé: epiphytic in an old orange grove in the hills above El Valle, alt. 1000 m, 2 Sept 1976, C. Luer \& H. Butcher 1120 (holotype, SEL).

Pleurothallis veraguacensis Luer, Selbyana 3(3/4): 402. 1977. = Pleurothallis cordifolia Rchb.f. \& H.Wagener

TyPE: Panama. Veraguas: epiphytic in the cloud forest near the continental divide above Santa Fé, alt. ca. 700 m, 5 Sept 1976, C. Luer \& R.L. Dressler 1141 (holotype, SEL).

Pleurothallis verecunda Schltr., Repert. Spec. Nov. Regni Veg. Beih. 17: 24. 1922. = Acianthera verecunda (Schltr.) Pridgeon \& M.W.Chase

TyPE: Panama. Auf Hügeln bei Panama City, C.W. Powell 91 (holotype, B, destroyed, lectotype designated by Christenson (1991b), AMES-23795; isolectotype, MO-955945; drawing of type, AMES-26852; drawing published in Mansfeld [1931: t. 37, nr. 147].).

Pleurothallis volcanica Luer, Selbyana 3(3/4): 406. 1977.

TYPE: Panama. Chiriquí: epiphytic in cloud forest near Río Chiriquí west of Volcán, alt. 1500 m, 12 Sept 1976,

C. Luer \& H. Butcher 1260 (holotype, SEL).

Pleurothallis wagneri Schltr., Repert. Spec. Nov. Regni Veg. 17: 141. 1921. = Stelis wagneri (Schltr.) Pridgeon \& M.W.Chase

TyPE: Panama. Chiriquí, Apr 1858, M. Wagner 25 (holotype, B, destroyed; lectotype designated by Luer (1998), AMES).

Pleurothallis williamsii Ames, Orchidaceae 7: 120. 1922. = Trichosalpinx dura (Lindl.) Luer

TYPE: Panama. Cana and vicinity, 17 Apr-8 Jun 1908, 2000-6500 ft alt., R.S. Williams 976 (holotype, AMES; isotype, NY).

Polycycnis tortuosa Dressler, Orquideología 12: 120. 1977.

TyPe: Panama. Coclé, cerca del Aserradero El Copé, aprox. $9 \mathrm{~km}$ al norte de El Copé; 2 Oct 1977, R.L. Dressler 5731 (holotype, US).

Polystachya panamensis Schltr., Repert. Spec. Nov. Regni Veg. Beih. 17: 49. 1922. = Polystachya foliosa (Hook.) Rchb.f.

TYPE: Panama. auf Hügeln bei Panama, C.W. Powell 122 (holotype, B, destroyed; lectotype designated by Christenson (1991b), AMES-23834; isolectotype, MO-955968).

Polystachya powellii Ames, Schedul. Orchid. 7: 31. 1924. = Polystachya foliosa

TyPe: Panama. Chiriquí, west side of Caldera River, in gulch opposite Lino, alt 4000 ft, C.W. Powell 343 (holotype, AMES).

Ponera amethystina Rchb.f. in W.W.Saunders, Refug. Bot. 2(1): 93. 1872. = Scaphyglottis stellata Lodd. ex Lindl.

TYPE: [Panama]. Sent from Santa Fé de Veraguas, by the late Mr. Skinner, to W. Wilson Saunders, G.U. Skinner s.n. (holotype, W).

Ponera behrii Rchb.f., Bonplandia 3: 220. 1855. = Scaphyglottis behrii (Rchb.f.) Benth. \& Hook.f. ex Hemsl. 
TYPE: [Panama]. Chagres: Dr. Med. Behr. Eingeführt durch Hrn. Keferstein, cultivirt von Hrn. Lehmann (holotype, W; illustration of type, AMES).

Prescottia panamensis Schltr., Repert. Spec. Nov. Regni Veg. 16: 357. 1920. = Prescottia oligantha (Sw.) Lindl. Type: Panama. In savannas, Cerro Vaca, Chiriquí, 900-1100 m, Dez. 1911, H. Pittier 5358 (holotype, B, destroyed; illustration of type, AMES).

Pseudepidendrum spectabile Rchb.f., Bot. Zeitung (Berlin) 10: 733. 1852. = Epidendrum pseudepidendrum Rchb.f.

TYPE: Panama. J. Warscewicz s.n. (holotype, W).

Psilochilus dressleri Kolan., Phytotaxa 175 (1): 55-57. 2014.

TYPE: Panama. Darién: Ridge north of Cerro Pirre, 1050-1200 m, 12 July 1977. R.L. Dressler 5663 (holotype, FLAS).

Restrepia aberrans Luer, Orquideología 20: 117. 1996.

TYPE: Panama. Bocas del Toro: epiphytic in forest above Chiriquí Grande, alt. 350 m, 17 Feb 1985, collected by C Luer, J. Luer, R. Dressler \& K. Dressler, flowered in cultivation by A. \& P. Jesup in Bristol, CT., 26 Apr 1987, C. Luer 10612 (holotype, MO).

Restrepia filamentosa Ames \& C.Schweinf., Schedul. Orchid. 8: 19, fig. 3. 1925. = Restrepia trichoglossa F.Lehm. ex Sander

TYPe: Panama. Chiriquí: Cerro Horquita, Oct 1923, C.W. Powell 344 (holotype, AMES).

Restrepia powellii Schltr., Repert. Spec. Nov. Regni Veg. Beih. 17: 25. 1922. = Restrepia muscifera (Lindl.) Rchb.f. ex Lindl.

TYPE: Panama. Chiriquí auf umgefallenen Bäumen, 4000 ft., C.W. Powell 123 (holotype, B, destroyed, lectotype designated by Christenson (1991b), AMES-26759; isolectotypes, AMES, MO).

Rodriguezia secunda var. panamensis Schltr., Repert. Spec. Nov. Regni Veg. Beih. 17: 75. 1922.= Rodriguezia lanceolata Ruiz \& Pav.

TYPE: Panama. auf Hügeln bei Panama City, C.W. Powell 79 (holotype, B, destroyed; lectotype designated by Christenson (1991b), AMES-28148; isolectotypes, AMES, K, MO-955932).

Rolfea powellii Ames, Schedul. Orchid. 7: 32, fig. 6. 1924. = Palmorchis powellii (Ames) C.Schweinf. \& Correll TyPE: Panama. Juan Diaz: near Panama City, found in damp places in dense shade. Aug-Sept 1923, C.W. Powell 325 (holotype, AMES; isotype, AMES).

Salpistele adrianae Luer \& Sijm, Selbyana 30(1): 18. 2009. = Stelis adriananijhuisae Bogarín \& Serracín TYPE: Panama. Chiriquí: near San Felix, alt. 1700 m., collected by T. \& A. Sijm, 16 Oct 2001, flowered in cult. in Vanhuizen, the Netherlands, Oct 2008, A.P. Sijm 220411 (holotype, MO).

Salpistele brunnea Dressler, Orquideología 14: 6. 1979. = Stelis brunnea (Dressler) Pridgeon \& M.W.Chase TyPE: Panama. Chiriquí: Cerro Colorado, aprox. $50 \mathrm{~km}$ al norte de San Félix, aprox. $1300 \mathrm{~m}$ alt.; 19 Aug 1975, R.L. Dressler 5124 (holotype, US; isotype, PMA).

Salpistele dressleri Luer, Monogr. Syst. Bot. Missouri Bot. Gard. 39: 128. 1991. = Stelis cymbisepala Pridgeon \& M.W.Chase

TYPE: Panama. Chiriquí: Fortuna, collected by R.L. Dressler, sent to J\&L Orchids, Easton, CT, ca. 1979, flowered in cult. 25 Apr 1987, C. Luer 12820 (holotype, MO).

Salpistele lutea Dressler, Orquideología 14: 8. 1979. = Stelis maculata Pridgeon \& M.W.Chase

TyPE: Panama. Chiriquí: Cerro Colorado, aprox. 50 km al norte de San Félix, alt. 1300 m, 19 Aug 1975, R.L.

Dressler 5125 (holotype, US).

Salpistele parvula Luer \& Dressler, Monogr. Syst. Bot. Missouri Bot. Gard. 39: 132. 1991. = Stelis gnoma 
Pridgeon \& M.W.Chase

TYPE: Panama. Prov. of Coclé: hills north of El Valle de Antón, alt. ca. 900 m, 3 Dec 1978, R.L. Dressler 5832 (holotype, MO; isotype, FLAS).

Sarcoglottis hunteriana Schltr., Repert. Spec. Nov. Regni Veg. Beih. 17: 13. 1922.

TyPE: Panama. In Tälern zwischen Hügeln bei Panama City, C.W. Powell 147 (holotype, B, destroyed, lectotype designated by Christenson (1991a), AMES-23776; isolectotypes, K, MO-955987; drawing of type, AMES-26776).

Sarcoglottis neglecta Christenson, Lindleyana 6: 133. 1991.

TYPE: Panama. San Juan, upper reaches of the Chagres river, sea level, flowers green, Feb 1924, C.W. Powell 388 (holotype, AMES; isotypes, AMES, MO).

Sarcoglottis powellii Schltr., Repert. Spec. Nov. Regni Veg. Beih. 17: 14. 1922.

TYPE: Panama. Auf Hügeln bei Panama City, C.W. Powell 179 (holotype, B, destroyed, lectotype designated by Christenson (1991a), AMES-25015; isolectotype, MO-956012; drawing published in Mansfeld [1931: t. 11, nr. 41].).

Scaphosepalum clavellatum Luer, Selbyana 3(1/2): 32. 1976.

TyPe: Panama. Panama: epiphytic along the Altos de Pacora road, alt. 650 m, 4 Mar 1976, C. Luer, J. Luer, P. Taylor \& R.L. Dressler 748 (holotype, SEL).

Scaphosepalum elasmatopus Schltr., Repert. Spec. Nov. Regni Veg. 12: 204. 1913. = Scaphosepalum microdactylum Rolfe

SynTYPes: Panama. Chiriquí: epiphytic in damp forest between Alto de las Palmas and Cerro de Horqueta, alt. 2100-2300 m, Mar 1911, H. Pittier \& W.R. Maxon 3229 , H. Pittier \& W.R. Maxon 5730, H. Pittier \& W.R. Maxon 5484 (B, destroyed; lectotype designated by Luer (1988), drawing of type, AMES; isolectotype, US).

Scaphosepalum longirepens Ames, Proc. Biol. Soc. Washington 34: 153. 1921. = Scaphosepalum microdactylum Rolfe

TYPE: Panama. Chiriquí: humid forest of the Cordillera, east of the Río Caldera, 17-19 Feb 1918, 2000 m alt., Killip 3567 (holotype, AMES; isotype, Rochester Academy of Science, US).

Scaphosepalum panamense Schltr., Repert. Spec. Nov. Regni Veg. 12: 205. 1913. = Masdevallia livingstoneana Roezl ex Rchb.f.

TyPE: Panama. im Wäldern am Río Indio de Gatún, ungefähr bei Meereshïhe, blühend im Feb 1911, H. Pittier 2803 (holotype, B, destroyed; drawing of type, AMES).

Scaphosepalum viviparum Luer, Selbyana 2: 390. 1978.

TYPE: Panama. Veraguas: epiphytic in cloud forest on Cerro Tute, alt. 900 m, Jan 1977, R.L. Dressler s.n., cultivated at SEL-77-3, flowered in cult. 21 Aug 1977, C. Luer 1820 (holotype, SEL).

Scaphyglottis chlorantha B.R.Adams, Phytologia 64: 249. 1988.

TyPe: Panama. Panama: Cerro Jefe, 29 Oct 1980, K. Sytsma 2003 (holotype, MO).

Scaphyglottis clavata Dressler, J. Orchideenfr. 11(4): 311. 2004.

Type: Panama. Chiriquí: San Félix, arriba de San Félix, flowered in cult. April 2000, R.L. Dressler 6287 (holotype, MO; isotype, PMA).

Scaphyglottis dolichophylla Schltr., Repert. Spec. Nov. Regni Veg. Beih. 17: 28. 1922. = Scaphyglottis laevilabium Ames

TYPe: Panama. auf Hügeln östlich von Panama City, C.W. Powell 126 (holotype, B, destroyed, lectotype designated by Christenson (1991b), K; drawing of type, AMES-26781; drawing published in Mansfeld [1931: t. 43 , nr. 171].). 
Scaphyglottis gigantea Dressler, Orquídea (Mexico City) 7: 234. 1979.

TYPE: Panama. Chiriquí, Cerro Hornito, NNE of Gualaca, about 1700 m., semi-cloud forest, 15 Dec 1976, R.L. Dressler 5533 (holotype, US; isotypes, PMA, SEL).

Scaphyglottis laevilabium Ames, Proc. Biol. Soc. Washington 34: 154. 1921.

TYPE: Panama. on branch of tree in dense forest, 2 miles east of Juan Diaz, 60 m, 2 October 1917, Ellsworth P. Killip 3113 (holotype, AMES; isotype, AMES, Rochester Academy of Science, US).

Scaphyglottis monspirrae Dressler, Novon 10(3): 199. 2000.

TYPE: Panama. Darién: Cerro Pirre, elev. 1200-1400 m, 15-16 July 1977, R.L. Dressler 5668 (holotype, MO).

Scaphyglottis panamensis B.R.Adams, Phytologia 64: 251. 1988.

TyPE: Panama. Panama: summit of Cerro Jefe near Radio Towers, 30 Nov 1983, H.W. Churchill 3942 (holotype, MO),

Scaphyglottis powellii Schltr., Repert. Spec. Nov. Regni Veg. Beih. 17: 28. 1922. = Scaphyglottis mesocopis (Endrés \& Rchb.f.) Benth. \& Hook.f. ex Hemsl.

TYPe: Panama. Chiriquí, 3000-4000 ft. C.W. Powell 134 (holotype, B, destroyed; lectotype designated by Christenson (1991b), AMES; isotypes, AMES, MO-955976, K; drawing published in Mansfeld [1931: t. 44, nr. 173].).

Scaphyglottis robusta B.R.Adams, Phytologia 64: 253. 1988.

TYPE: Panama. Panama: El Llano-Carti-Tupile road, 10-12 km N of Inter-Am. Highway, 9 Jan 1975, J.L. Luteyn \& R.L. Wilbur 4679 (holotype, DUKE).

Scaphyglottis tenella L.O.Williams in Woodson \& Schery, Ann. Missouri Bot. Gard. 28: 423, tab. 24. 1941.

TYPE: Panama. Coclé: epiphytic, hills north of El Valle de Antón, alt. 800-1000 m, 21 Nov 1940, P.H. Allen 2276 (holotype, AMES; photo of type, K).

Scaphyglottis unguiculata Schltr., Repert. Spec. Nov. Regni Veg. 12: 206. 1913. = Scaphyglottis longicaulis S.Watson

TyPE: Panama. Colón: Loma de la Gloria, bei Fato, in Wäldern, 10-104 m, blühend im Jul-Aug 1911, H. Pittier 4090 (holotype, B, destroyed; isotype, US).

Selenipedium chica Rchb.f., Xenia Orchid. 1: 3. 1854.

TYPE: Panama. Vanilla en árbol des Panaméniens. E.P. Duchassaing s.n. misit 1851 (holotype, W; isotype, P; illustration of type Xenia Orch. 1: 3, t. 2. 1854; GOET).

Sievekingia butcheri Dressler, Orquideología 13: 221. 1979.

TYPe: Panama. Coclé, La Mesa, al norte de El Valle de Antón, 6 nov 1976, H. Butcher s.n. (holotype, US).

Sigmatostalix abortiva L.O.Williams in Woodson \& Schery, Ann. Missouri Bot. Gard. 27: 284. 1940. = Oncidium abortivoides M.W.Chase \& N.H.Williams

TYPE: Panama. Canal Zone: Quebrada López, $30 \mathrm{~m}$ alt., sepals and petals yellow, lip white, 11 Feb 1940, P.H. Allen 2121 (holotype, MO).

Sigmatostalix brownii Garay, Caldasia 10: 236. 1968. = Oncidium brownii (Garay) M.W.Chase \& N.H.Williams TYPE: Panama. without proper locality. Cultivated by Mr. Henry Brown, Miami, H. Brown s.n. (holotype, AMES).

Sigmatostalix racemifera L.O.Williams in Woodson \& Schery, Ann. Missouri Bot. Gard. 27: 285 (t. 36). 1940. = Oncidium picturatissimum (Kraenzl.) M.W.Chase \& N.H.Williams

TYPE: Panama. Coclé: vicinity of El Valle, 600-100 m alt., flowers yellow, 8 Dec 1938, P.H. Allen 1232 (holotype, MO).

Sobralia allenii L.O.Williams, Ann. Missouri Bot. Gard. 29: 336. 1942. 
TYPE: Panama. Coclé: epiphytic, trail to La Mesa, hills north of El Valle de Antón, alt. ca. 1000 m, 31 Aug 1941, sepals and petals white, labellum pale yellow, P.H. Allen 2686 (holotype, AMES).

Sobralia bletiae Rchb.f., Bot. Zeitung (Berlin) 10: 713. 1852.

TYPE: "Chiriquí” Panama, Warszewicz s.n. (holotype, W; illustration of type, AMES).

Sobralia bouchei Ames \& C.Schweinf., Schedul. Orchid. 10: 4. 1930. = Sobralia wilsoniana Rolfe

TyPE: Panama. March-April 1930, alt 2200 ft, terrestrial and epiphytic, A.M. Bouché s.n. (holotype, AMES).

Sobralia citrea Dressler, Orchids (West Palm Beach) 74(12): 937. 2005.

Type: Panama. Coclé: El Valle de Antón, 800-1000 m, flowered in cult. Aug 2001, R.L. Dressler 6338 (holotype, MO; isotypes, FLAS, PMA, SEL).

Sobralia crispissima Dressler, Lankesteriana 5: 10. 2002.

TYPe: Panama. Chiriquí: al oeste de Volcán, carretera a Río Sereno, alt. 1200 m, 25 August 2001, A. Maduro \& E. Olmos 223 (holotype, MO; isotypes, PMA, FLAS).

Sobralia decora var. aerata P.H.Allen \& L.O.Williams, Ann. Missouri Bot. Gard. 29: 337, tab. 31. 1942. = Sobralia atropubescens Ames \& C.Schweinf.

TYPE: Panama. Coclé: bad lands south of El Valle de Antón, alt. ca. 500 m, 18 Sep 1941, flowered in collection of A. M. Bouché, Pedro Miguel, Canal Zone, P. H. Allen 2755 (holotype, AMES).

Sobralia epiphytica Schltr., Repert. Spec. Nov. Regni Veg. 12: 213. 1913. = Sobralia bletiae Rchb.f.

Syntypes: Panama. in der Umgebung von Culebra, Kanalzone, 50-150 m, H. Pittier 4780 (B, destroyed, US, W). Panama. an Baumstämmen in den Wäldern am Río Indio de Gatún, Kanalzone, R. Maxon 4829 (B, destroyed, US, W).

Sobralia exigua Dressler, Orchids (West Palm Beach) 74(12): 938. 2005.

TYPE: Panama. Chiriquí: Cordillera, epiphytic, 800-1100 m, flowered in cult. 12 Jul 2003, R.L. Dressler 6365B (holotype, MO).

Sobralia fenzliana Rchb.f., Bot. Zeitung (Berlin) 10: 714. 1852.

TYPE: Panama. "Chiriquí Cordilleren 1-2000”, J. Warszewicz s.n. (holotype, W; illustration of type, AMES).

Sobralia fuzukiae Dressler \& Bogarín, Orchids (West Palm Beach) 76(9): 696. 2007. = Sobralia quinata Dressler TYPE: Panama. Bocas del Toro: Culebra, pressed from cult. 9 Jan 2007, R. L. Dressler 6810 (holotype, PMA; isotype, JBL-spirit).

Sobralia gloriana Dressler, Lankesteriana 5: 11. 2002.

TyPE: Panama. Coclé: Area de El Valle de Antón, Alt. 800-1000 m, 23 julio 2001, A. Maduro \& E. Olmos 212 (holotype, MO; isotype, PMA).

Sobralia $\times$ intermedia P.H.Allen, Amer. Orchid Soc. Bull. 27: 534. 1958.

Type: Panama. Cabeceras del Río Mata Ahogado, near El Valle de Antón, Rep. de Panama, 1200 ft. P.H. Allen 2670 (holotype, not stated).

Sobralia kruskayae Dressler, Orchids (West Palm Beach) 74(12): 939. 2005.

TYPE: Panama. Coclé: El Valle de Antón, flowered in cultivation Jul-Aug 2003, R.L. Dressler 6383 (holotype, MO; isotype, PMA).

Sobralia labiata Warsz. \& Rchb.f., Bot. Zeitung (Berlin) 10: 714. 1852.

TYPE: Panama. "Schorche Berg in Chiriquí, J. Warszewicz s.n. (holotype, W; illustration of type, AMES).

Sobralia lindleyana Rchb.f., Bot. Zeitung (Berlin) 10: 713. 1852.

TYPE: [Panama]. "Chiriquí”, J. Warszewicz s.n. (holotype, W).

Sobralia macrophylla Rchb.f., Bot. Zeitung (Berlin) 10: 713. 1852.

TyPE: [Panama]. Chiriquí, J. Warszewicz s.n. (holotype, W). 
Sobralia maduroi Dressler, Orchids (West Palm Beach) 73(10): 776. 2004.

TYPE: Panama. Chiriquí, region of Boquete, 1800-2200 m, 19 Jul 2001, A. Maduro \& E. Olmos 206 (holotype, MO; isotype, PMA).

Sobralia mariannae Dressler, Lankesteriana 5: 13. 2002.

TYPE: Panama. Panama: Cerro Jefe, flowered in cultivation 24 May 2002, epiphyte, flower white, callus yellow, brown at base, fragrant, R.L. Dressler 6352 (holotype, MO).

Sobralia mireyae Dressler, Orchids (West Palm Beach) 76: 700-701. 2007. = Sobralia citrea Dressler

TyPe: Panama. Coclé: La Pintada; flowered in cult. 30 May 2006, R. L. Dressler 6707 (holotype, PMA; isotype, JBL-spirit).

Sobralia nutans Dressler, Lankesteriana 5: 13. 2002.

TyPe: Panama. Bocas del Toro: Km. 63, al norte de Fortuna, alt. 1050 m, 9 sep. 2001, A. Maduro \& E. Olmos 236 (holotype, MO; isotypes, PMA, SEL).

Sobralia panamensis Schltr., Repert. Spec. Nov. Regni Veg. Beih. 17: 11. 1922. = Sobralia fenzliana Rchb.f.

TYPE: Panama. Bei Panama-City, in Tälern; auch längs der Hinterwässer des Gatun-Sees, ziemlich häufig, C.W. Powell 21 (holotype, B, destroyed; lectotype designated by Christenson (1991b), AMES-23770; isolectotypes, AMES-23769, K-K000463288, K000463289, MO-955891, MO-961330).

Sobralia panamensis Schltr. var. albiflos Schltr., Repert. Spec. Nov. Regni Veg. Beih. 17: 11. 1922. = Sobralia fenzliana

TYPE: Panama. Auf Hügeln bei Panama-City, Powell 31 (holotype, B, destroyed; lectotype designated by Christenson (1991b), AMES-23711; isolectotype, K).

Sobralia powellii Schltr., Repert. Spec. Nov. Regni Veg. Beih. 17: 11. 1922.

TyPE: Panama. An den Hinterwässern des Gatun-Sees, selten C.W. Powell 2. (holotype, B, destroyed; lectotype designated by Christenson (1991b), AMES-23774, isolectotypes, AMES-23773, MO-955874, MO-955875).

Sobralia recta Dressler, Orchids (West Palm Beach) 74(12): 939. 2005.

TYPE: Panama. Bocas del Toro, 800 m., floreció en cultivo 12 Sept 2002, A. Maduro \& E. Olmos 295 (holotype, MO; isotype, PMA).

Sobralia rolfeana Schltr., Repert. Spec. Nov. Regni Veg. Beih. 17: 12. 1922. = Sobralia macrophylla Rchb.f. Type: Panama. Am Gatun-See, C.W. Powell 3 (holotype, B, lectotype designated by Christenson (1991b), AMES-23768; isolectotype, MO-955876).

Sobralia sanfelicis Dressler, Orchids (West Palm Beach) 73(10): 777. 2004.

TYPE: Panama. Chiriquí: Cerro Colorado, Dist. San Félix, cult. Finca Drácula, flowered in cultivation 20 Aug 2002, A. Maduro \& E. Olmos 269 (holotype,MO; isotype, PMA).

Sobralia sororcula Dressler, Orquideología 25(1): 39. 2007.

TyPE: Panama. Veraguas: Los González, Noreste de Santa Fé, probablemente de 800-1000 m, floreció en cult. Aug 2004, R.L. Dressler 6415 (holotype, PMA; illustration of type; photo of type).

Sobralia sotoana Dressler \& Bogarín, Lankesteriana 9(3): 482. 2010.

TYPE: Panama. Veraguas, collected near road north of Santa Fé de Veraguas, flowered in culture in Jardín Botánico Lankester, pressed from cultivation, 8 August 2009, R.L. Dressler 7168 (holotype, CR; isotype, JBL-spirit).

Sobralia suaveolens Rchb.f., Gard. Chron., n.s., 9: 622. 1878. = Sobralia bletiae

TYPE: [Panama]. It was introduced from Colon (Aspinwell) in Central America by Mr. Stanger (once the Orchid grower of late Consul Schiller, now professor of a good nursery, and a member of the directorial comittee of our Gartenbau Gesellschaft), who presented it to the Botanical Garden, where it flowered in March under M. Donat's care (holotype, W). 
Sobralia theobromina Dressler, Orchids (West Palm Beach) 74(12): 940. 2005.

TyPE: Panama. Coclé: El Valle de Antón, 800-1000 m, cult. en Finca Drácula, floreció 12 Sept 2002 "la chocolatosa", A. Maduro \& E. Olmos 293 (holotype, MO; isotype, PMA).

Sobralia tricolor Dressler, Orchids (West Palm Beach) 74(12): 941. 2005.

TyPE: Panama. Coclé: El Valle de Antón, 800-1000 m, floreció en Finca Drácula, 12 Sept 2002, Maduro \& Olmos 297 (holotype, MO; isotype, PMA).

Sobralia valida Rolfe, Bull. Misc. Inform. Kew 1909: 65.

TYPE: Panama. near the Darien Gold Mines. Received from Messrs. Sander \& Sons and flowered at Kew in June 1907. It was obtained by Dr. Hodgkinson, The Grange, Wilmslow, Sander \& Sons s.n (holotype, K).

Sobralia warszewiczii Rchb.f., Bot. Zeitung (Berlin) 10: 714. 1852.

TyPE: Panama. "Chiriquí 6000”", J. Warscewicz s.n. (holotype, W).

Specklinia absurda Bogarín, Karremans \& R.Rincón, Phytotaxa 115 (2): 31-41. 2013.

TYPE: Costa Rica-Panama. Puntarenas-Chiriquí: Coto Brus-Renacimiento, línea fronteriza hacia el Cerro Pando, después del mojón N.338, 855'11.22” N 8243’18.18” W, 2446 m, bosque muy húmedo montano bajo, epífita en bosque primario, "in sylvis virginis versus montium Pando in itinere ad summum Costa Rica austro-orientalis in finibus utrimque Costa Rica et Panama”, 19 April 2011, D. Bogarín 8711, D. Jiménez \& A. Karremans (holotype, CR; isotypes, JBL, PMA, UCH, USJ).

Sphyrastylis dressleri Toscano, Lindleyana 14: 24. 1999. = Ornithocephalus dressleri (Toscano) Toscano \& Dressler

TYPE: Panama. Province of Panama: top of Cerro Jefe near antenna, elev. 1000 m, 17 Oct 1977, J.P. Folsom \& R. Page 5942 (holotype, MO).

Spiranthes subpandurata Ames \& C.Schweinf., Schedul. Orchid. 8: 4. 1925. = Beloglottis subpandurata (Ames \& C.Schweinf.) Garay

TyPE: Panama. Upper Chagres River Range, Jan-Feb 1924, C.W. Powell 377 (holotype, AMES).

Spiranthes woodsonii L.O.Williams, Ann. Missouri Bot. Gard. 29: 337. 1942. = Sarcoglottis woodsonii (L.O.Williams) Garay

TYPE: Panama. Chiriquí: vicinity of Boquete, alt. 1200-1500 m, 24-26 Jul 1940, flowers pale green, R.E. Woodson, Jr. \& R.W. Schery 753 (holotype, AMES).

Stanhopea avicula Dressler, Amer. Orchid Soc. Bull. 58: 885. 1989.

TYPE: Panama. Darién: Cerro Tacarcuna, elev. about 1500 m.; Feb 1980; flowered in cult. 13 Feb 1982, K.R. Reading \& R.R. Tasker s.n. (holotype, MO; isotype, FLAS; photo of the plant that served as the holotype).

Stanhopea maduroi Dodson \& Dressler, Orquideología 21: 62. 1998.

TYPE: Panama. collected by Andrés Maduro at Valle de Antón, ca. 1000 m, 28 Nov 1985, C.H. Dodson 16259 (holotype, RPSC).

Stanhopea panamensis N.H.Williams \& Whitten, Lindleyana 3(1): 9. 1988.

TYPE: Panama. Panama: epiphytic in cloud forest on Cerro Campana approx. $50 \mathrm{~km} \mathrm{SW}$ of Panama City, elev. ca. 900 m, Flowered in cultivation at University of Florida, accession number UF-80, M.W. Whitten 909 (holotype, FLAS; isotype, SEL).

Stanhopea warszewicziana Klotzsch, Allg. Gartenzeitung 20: 274. 1852.

TYPE: [Panama]. Der Herr Kunzt und handelsgärtner Mathieu, in dessen Orchideenhause diese Pflanze ende Juli in voller Blüthe steht, erhielt sie von dem Herrn von Warszewicz, dessen Namen sie sührt, aus dem Chiriquí Gebirge in Central America, Warszewicz s.n. (holotype, B).

Steliopsis anneliesae Brieger, Die Orchideen 8(1A): 458. 1976. nom. nud. 
TYPE: Panama. 1972, Anon s.n. (holotype, not stated).

Stelis allenii L.O.Williams, Ann. Missouri Bot. Gard. 29: 338. 1942.

TYPE: Panama. Coclé: hills north of El Valle de Antón, alt. 800 m, 10 Apr 1942, flowers nearly black, P. H. Allen 2952 (holotype, AMES; isotype, AMES).

Stelis atrorubens L.O.Williams, Ann. Missouri Bot. Gard. 29: 239. 1942.

TYPE: Panama. Coclé: hills north of El Valle de Antón, trail to LAs Minas, 2 Dec 1941, flowers maroon, P. H. Allen 2876 (holotype, AMES; isotype, MO).

Stelis butcheri Luer, Phytologia 49: 224. 1981.

TYPE: Panama. Chiriquí: epiphytic in cloud forest above Guadalupe, alt. ca. 2000 m, 1 Sept 1976, H. Butcher s.n. sub C. Luer 1101 (holotype, SEL).

Stelis chiriquensis Schltr., Beih. Bot. Centralbl. 36, Ab. 2: 386. 1918. = Stelis despectans Schltr.

Type: Panama. Chiriquí: Humid forest between Alto de las Palmas and top of Cerro de la Horqueta, 21002268 m, Mar 1911, H. Pittier 3228 (holotype, B, destroyed; isotype, AMES; drawing and photo of type, AMES).

Stelis collina Schltr., Repert. Spec. Nov. Regni Veg. Beih. 17: 15. 1922.

TYPE: Panama. Auf Hügeln bei Panama City, C.W. Powell 180 (holotype, B, destroyed; lectotype designated by Christenson (1991b), AMES-23780, AMES; isolecotype, MO-956013; drawing published in Mansfeld [1931: t. 21, nr. 83].).

Stelis crescentiicola Schltr., Repert. Spec. Nov. Regni Veg. 16: 442. 1920.

TyPE: Panama. An Bäumen, besonders Crescentia, am Río Chagres, April, F.C. Lehmann 4540 (holotype, B, destroyed, illustration of type, AMES).

Stelis dressleri Luer, Phytologia 49: 227. 1981.

TYPE: Panama. Veraguas: epiphytic in cloud forest above Santa Fé, alt. ca. 700 m, 5 Sept 1976, C. Luer \& R.L. Dressler 1146 (holotype, SEL; detail of holotype, SEL).

Stelis eximia Ames, Schedul. Orchid. 6: 54. 1923. = Stelis superbiens Lindl.

TYPE: Panama. Chiriquí, Caramilla [Jaramillo?], C.W. Powell 312 (holotype, AMES).

Stelis fimbriata R.K.Baker, Ann. Missouri Bot. Gard. 55: 68, fig. 1. 1968. = Stelis ciliaris Lindl.

TYPE: Panama. Cerro Jefe, in mossy forest near summit, alt. 2900 ft, 11 Feb 1967, R.K. Baker 249 (holotype, $\mathrm{MO})$.

Stelis gratiosa Luer, Selbyana 5: 192. 1979. = Stelis ciliaris

TYPE: Panama. Panama: epiphytic in cloud forest on Cerro Jefe, alt.1000 m, 2 March 1976, cult. at SEL, greenhouse acc. no. 43-76-155, flowered in cult. 8 May 1977, C. Luer. J. Luer \& P. Taylor 1619 (holotype, SEL).

Stelis inaequalis Ames, Schedul. Orchid. 4: 12. 1923. = Stelis glossula Rchb.f.

TYPE: Panama. Near San Juan in the foothills of the upper reaches of the Chagres River, sea level, C.W. Powell 272 (holotype, AMES).

Stelis isthmi Schltr., Repert. Spec. Nov. Regni Veg. Beih. 17: 16. 1922.

TYPE: Panama. Bei Arrajan, westlich des Kanals, ungefähr auf Seehöhe, C.W. Powell 232 (holotype, B, destroyed; lectotype designated by Christenson (1991b), AMES-23777; isolectotype, MO-956043).

Stelis loculifera Luer, Phytologia 49: 229. 1981.

TYPE: Panama. Chiriquí: epiphytic in cloud forest on Cerro Colorado above Camp Escopeta, alt. 1700 m, Feb 1977, cult. at SEL-77-1901, flowered in cult. 4 May 1977, C. Luer, J. Luer \& R.L. Dressler 1614 (holotype, SEL; detail of the holotype, SEL). 
Stelis longipetiolata Ames, Schedul. Orchid. 1: 6. 1922.

TYPE: Panama. Chiriquí, humid forest between Alto de las Palmas and top of Cerro de la Horqueta, alt 21002268 m, 18 Mar 1911, W.R. Maxon 5460 (holotype, US).

Stelis maduroi Luer \& Sijm, Monogr. Syst. Bot. Missouri Bot. Gard. 112: 111. 2007.

TYPE: Panama. Bocas del Toro, obtained from Finca Dracula 7 Oct.2001, cultivated in the Netherlands, Oct 2006, T. Sijm 220728 (holotype, MO).

Stelis maxonii Schltr., Beih. Bot. Centralbl. 36, Ab. 2: 389. 1918.

TYPE: Panama. Humid forest along 'the upper Caldera-River, Holcombs trail, above El Boquete, Chiriquí, 1450-1650 m, March 1911, William R. Maxon 5697 (holotype, B, destroyed; drawing and photo of type, AMES).

Stelis meganthera Luer, Selbyana 22: 122. 2001.

TYPe: Panama. Chiriquí: Cerro Colorado, alt. 650-700 m, 16 Feb 1977, C. Luer, J. Luer \& R. \& K. Dressler 1453 (holotype, SEL).

Stelis megapetala Luer, Selbyana 22(2): 124. 2001.

Type: Panama. Coclé: El Valle, alt. ca. 1000 m, Sept 1976, C. Luer \& H. Butcher 1264 (holotype, SEL).

Stelis montana L.O.Williams, Ann. Missouri Bot. Gard. 27: 272. 1940.

TYPE: Panama. Chiriquí: trail from Cerro Punta to headwaters of Río Caldera, 2250-2500 m, 4 Jan 1939, P.H. Allen 1463 (holotype, MO).

Stelis oscitans Luer, Lindleyana 11: 105. 1996.

TYPE: Panama. Chiriquí: forest near the Fortuna Dam, alt. 1100 m, 16 Feb 1985, C. Luer, J. Luer, R. Dressler \& K. Dressler 10575 (holotype, MO).

Stelis panamensis Schltr., Beih. Bot. Centralbl. 36, Ab. 2: 391. 1918. = Stelis gracilis Ames

TYPE: Panama. Forests of the upper Mamoni-River, 150-400 m, Oct 1911, H. Pittier 4490 (holotype, B, destroyed; isotype, AMES).

Stelis parvibracteata Ames, Orchidaceae 7: 131. 1922. = Stelis argentata Lindl.

TYPE: Panama. Cana and vicinity, 27 Apr 1908. On trees, flowers purplish, 6000 ft. alt., R.S. Williams 972 (holotype, AMES).

Stelis powellii Schltr., Repert. Spec. Nov. Regni Veg. Beih. 17:16. 1922. = Stelis gigantea Pridgeon \& M.W.Chase TyPe: Panama, Chiriquí, c. 4000 Fuss ü. M., C. W. Powell 247 (holotype, B, destroyed; lectotype designated by Christenson (1991b), AMES-23778; isolectotype, MO-956053).

Stelis praemorsa Schltr., Repert. Spec. Nov. Regni Veg. Beih. 17: 17. 1922.

TyPe: Panama. Auf Hügeln, östlich von Panama City, C.W. Powell 234 (holotype, B, destroyed, lectotype designated by Christenson (1991b), AMES-23799; drawing of type, AMES-26805).

Stelis sarcophylla Luer, Phytologia 49: 238. 1981.

TYPE: Panama. Chiriquí: epiphytic in scrubby trees on the Llanos del Volcán, alt. 1500 m, 10 Sept 1976, cult. at SEL, flowered in cult. 15 Oct 1976, C. Luer \& H. Butcher 1245 (holotype, SEL; detail of holotype, SEL).

Stelis semperflorens Luer, Phytologia 49: 238. 1981.

TYPe: Panama. Panama: epiphytic in cloud forest on Cerro Jefe, alt. 1000 m, 2 Mar 1976, C. Luer, J. Luer, P. Taylor \& R.L. Dressler 966 (holotype, SEL; detail of the holotype).

Stelis triaristata Luer, Selbyana 5: 193. 1979.

TYPE: Panama. Panama: epiphytic in cloud forest along the Altos de Pacora road, alt. ca. 350 m, March 1976,

C. Luer, J. Luer, R.L. Dressler \& P. Taylor 2109 (holotype, SEL; detail of the holotype, SEL). 
Stelis umbelliformis Hespenh. \& Dressler, Orquideología 6: 21. 1971.

TYPe: Panama. Colón: camino madero de Santa Rita, aprox. 20 kms al este de Colón; 15 Oct 1969, con baño morado, H.A. Hespenheide \& R.L. Dressler 2266 (holotype, US; isotypes, K, MO, PH).

Stelis veraguasensis Luer, Selbyana 22(2): 126. 2001.

TYPe: Panama. Veraguas: alt. ca. 500 m, 5 Sept 1976, C. Luer \& R. Dressler 1235 (holotype, SEL).

Stelis williamsii Ames, Orchidaceae 7: 133. 1922.

TYPE: Panama. Cana and vicinity, 27 Apr 1908. On trees, flowers purplish, 2000-6500 ft. alt., R.S. Williams 970 (holotype, AMES).

Stellilabium aciculare Dressler, Harvard Pap. Bot. 4(2): 471. 1999. = Telipogon acicularis (Dressler) N.H.Williams \& Dressler

TYPE: Panama. Panama: Cerro Jefe (lower cafetal), 12 Mar 1969, on smaller branches of tree near stream, inflorescence to $15 \mathrm{~cm}$, unbranched, flowers wine-red, R.L. Dressler 3617 (holotype, AMES; isotypes, MO, PMA).

Stellilabium butcheri Dressler, Harvard Pap. Bot. 4(2): 472. 1999. = Telipogon butchii N.H.Williams \& Dressler TYPE: Panama. Chiriquí: epiphytic on orange tree about 1/2 mi SW of highway near Cuesta Piedra, 9 Apr 1961, flowers dull purple, H.P. Butcher 654 (holotype, SEL).

Stellilabium fortunae Dressler, Harvard Pap. Bot. 4(2): 473. 1999. = Telipogon fortunae (Dressler) N.H.Williams \& Dressler

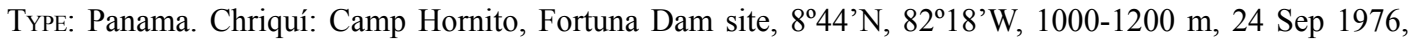
epiphytic, flowers wine-red, R.L. Dressler 5464 (holotype, MO).

Stellilabium morii Dressler, Harvard Pap. Bot. 4(2): 473. 1999. = Telipogon morii (Dressler) N.H.Williams \& Dressler

TYPE: Panama. Chiriquí: N of San Félix at Chiriquí-Bocas del Toro border on Cerro Colorado copper mine road along continental divide, lower montane rain forest (cloud forest, trees to $5 \mathrm{~m}$ tall, $5000-5500 \mathrm{ft}$ alt., 3 May 1977., epiphyte, leaves absent, flowers purple, nonresupinate, S. Mori \& J. Kallunki 5813 (holotype, MO).

Stenotyla panamensis Pupulin, Orchid Rev. 117(1287): 162. 2009.

TYPE: Panama. Without exact provenance, cultivated in san Vito de Java, Costa Rica, by W. Chacón at Happy Garden greenhouses, fowered on October 27, 2005, F. Pupulin 5867 (holotype, PMA; isotype, JBL).

Systeloglossum panamense Dressler \& N.H.Williams, Amer. Orchid Soc. Bull. 39: 326. 1970.

TyPE: Panama. Panama, La Eneida, region of Cerro Jefe; pressed 25 June 1969; epiphyte in tall forest; flowers bronzy green, R.L. Dressler \& N.H. Williams 3642 (holotype, US).

Telipogon bombiformis Dressler, Orchids 72: 114. 2003.

TyPe: Panama. Chiriquí: Cerro Punta, Bajo Grande, 2300 m, 10 Dic 2001, A. Maduro \& E. Olmos s.n. (holotype, MO).

Telipogon butcheri Dodson \& R.Escobar, Orquideología 18: 278. 1993.

TYPE: Panama. Chiriquí: Cordillera, unas 4 millas al este del Volcán, ca. 1500 m, 2 Oct 1960, H.P. Butcher s.n. (holotype, RPSC; isotype, AMES).

Telipogon caulescens Dressler, Orchids 72: 114. 2003.

TyPE: Panama. Chiriquí: Boquete, por la frontera con Bocas del Toro, 2000 m, 30 Jun 2000, A. Maduro y E. Olmos 168 (holotype, MO; isotype, PMA).

Telipogon chiriquiensis Dodson \& R.Escobar, Orquideología 18: 281. 1993.

TyPE: Panama. Chiriquí: Epífita, pocos km arriba de Guadalupe, 2500 m, 13 sept. 1976, C.A. Luer \& H. Butcher 1208 (holotype, RPSC). 
Telipogon fractus Dressler, Orchideen J. 14(1): 12. 2007.

TYPE: Panama. Bocas del Toro: oberhalb Guadalupe (Chiriquí), 2000-2300 m, 10 Sept 2001, A. Maduro \& E. Olmos 243 (holotype, MO; isotype, PMA).

Telipogon griesbeckii Dressler, Orchids 72: 115. 2003.

TyPe: Panama. Chiriquí: Cerro Punta, Entre Ríos, 2100-2200 m, Aug. 1993, A. Maduro \& E. Olmos 169 (holotype, MO; isotype, PMA).

Telipogon maduroi Dressler, Orchids 72: 116. 2003.

TyPe: Panama. Chiriquí: Cerro Punta, Altos de Respingo, 2000-2500 m, 6 Jul 2001, A. Maduro y E. Olmos 190 (holotype, MO; isotype, PMA).

Telipogon medusae Dressler, J. Orchideenfr. 13(3): 211. 2006.

TyPE: Panama. Chiriquí: Cerro Punta, arriba de Guadalupe, 2000-2200 m, 19 Jul. 2001, A. Maduro \& E. Olmos 209 (holotype, MO; isotypes, PMA, FLAS, SEL).

Telipogon nobilis Dressler, Orchideen J. 14(1): 12. 2007.

TyPe: Panama. Chiriquí: Cerro Punta, Las Nubes, Parque Internacional La Amistad, 3 Apr 2003, A. Maduro \& E. Olmos 305 (holotype, PMA).

Telipogon nunezii Dressler, Orchideen J. 14(1): 14. 2007.

TYPe: Panama. Chiriquí: San Félix, Cerro Colorado, prensado de cultivo, 11 Sept 2001, A. Maduro \& J. Nunez 249 (holotype, PMA).

Telipogon olmosii Dressler, J. Orchideenfr. 13(3): 212. 2006.

TyPE: Panama. Bocas del Toro: Sierra Madre, 1500-1750 m, 23 Jul. 2001, A. Maduro \& E. Olmos 213 (holotype, MO; isotype, PMA).

Telipogon panamensis Dodson \& R.Escobar, Orquideología 18: 284. 1993.

TyPE: Panama. Chiriquí: Bajo Choro, 1900 m, 20 Jul 1940, R.E. Woodson, Jr. \& R.W. Schery 694 (holotype, $\mathrm{MO})$.

Telipogon personatus Dressler, J. Orchideenfr. 13(3): 214. 2006.

TyPE: Panama. Chiriquí: Cerro Punta, arriba de Guadalupe, 2100-2200 m, 23 Jul. 2001, A. Maduro \& E. Olmos 214 (holotype, MO; isotype, PMA).

Telipogon reticulatus Dressler, Orchideen J. 14(1): 14. 2007.

TyPE: Panama. Bocas del Toro: above Guadalupe (Chiriquí), 2000-2300 m., Sept 2002, A. Maduro \& E. Olmos 310 (holotype, MO; isotype, PMA).

Telipogon seibertii Dodson \& R.Escobar, Orquideología 18: 288. 1993.

TyPE: Panama. Chiriquí: Casita Alta, Volcán de Chiriquí, 1500-2000 m, 28 Jun-21 Jul 1938, R.E. Woodson, P. Allen \& R.J. Seibert 961 (holotype, MO).

Trichocentrum panamense Rolfe, Bull. Misc. Inform. Kew 1913: 341. = Trichocentrum capistratum Linden \& Rchb.f.

TYPE: Panama. on bush-covered hills east of the Panama Canal, 255 m, L.J. Lipscomb s.n. (holotype, K).

Trichocentrum pfavii Rchb.f., Gard. Chron., n.s., 16: 70. 1881.

Type: Panama. Chiriquí, without specific locality, 19 Nov. 1980, R. Pfau 80 (holotype, W).

Trichopilia amabilis Dressler, Orquideología 22(3): 245. 2003.

TyPe: Panama. prov. Panama: Dist. de Chimán, Río Tuti, Cerro Chucantí, colectado por N.G. Smith, floreció en cultivo 5 marzo 2000, R.L. Dressler 6346 (holotype, MO).

Trichopilia eneidae Dressler, Orquideología 22(3): 246. 2003. 
TYPE: Panama. Bocas del Toro: Culebra, colectada por Eneida Hurtado; floreció en cult. 28 marzo 2002, R.L. Dressler 6348 (holotype, MO).

Trichopilia leucoxantha L.O.Williams, Amer. Orchid Soc. Bull. 10: 137. 1941.

TYPE: Panama. Coclé, hills south of El Valle de Antón, about $700 \mathrm{~m}$. alt., flowers pure white with yellow in throat, 15 Aug 1940, P.H. Allen 2401 (holotype, AMES).

Trichopilia maculata Rchb. f., Bonplandia 3(15-16): 215. 1855.

TYPE: [Panama] Port Chagres, gesammelt von Herrn Dr. Behr. Blühte in Herrn Keferstein's Sammlung zu Kröllwitz unter Hrn. Lehmann's Cultur, H.A. Behr s.n. (holotype, W).

Trichopilia marginata var. olivacea Rchb.f., Xenia Orchid. 2: 102. 1865. = Trichopilia marginata Henfr.

TYPE: [Panama]. Vulcano de Chiriquí auf Quercus und Cupania glabra, Januar, b. ebenda; Bilde von $J$. Warscewicz (holotype, W).

Trichopilia olmosii Dressler, Selbyana 22(1): 11. 2001.

TYPE: Panama. Bocas del Toro: region of Culebra, 1000-1200 m, collected by Erick Olmos; flowered in cultivation 4 August 2000, R.L. Dressler 6288 (holotype, MO).

Trichopilia powellii Schltr., Repert. Spec. Nov. Regni Veg. Beih. 17: 77. 1922. = Trichopilia maculata Rchb.f.

TYPE: Panama. gewönlich auf Mango-Bäumen, in der Umgebung von Panama City, C.W. Powell 9 (holotype, B, destroyed; lectotype designated by Christenson (1991b), AMES-23895; isolectotype K, MO-955882; drawing of type, AMES-26818; drawing published in Mansfeld [1931: t. 71, nr. 283].).

Trichopilia similis Dressler, Selbyana 22(1): 12. 2001.

TYPe: Panama. Panama: Cerro Jefe, 800-900 m, 3 December 1970, R.L. Dressler 3907 (holotype, MO).

Trichosalpinx dressleri Luer, Monogr. Syst. Bot. Missouri Bot. Gard. 64: 54. 1997.

TYPe: Panama. Prov. of Panama: epiphytic in forest of Cerro Jefe, alt. ca. 1000 m, 2 Dec. 1979, R. L. Dressler 5855 (holotype, MO).

Trichosalpinx macphersonii Luer, Monogr. Syst. Bot. Missouri Bot. Gard. 64: 66. 1997.

TYPE: Panama. Panama: vicinity of Cerro Jefe, alt. 700-800 m, 23 Jul 1988, G. McPherson 12777 (holotype, MO).

Trichosalpinx ringens Luer, Lindleyana 11: 108. 1996.

TyPE: Panama. Chiriquí: epiphytic in cloud forest, Cerro Colorado, alt. 1670 m, 15 Feb 1985, C. Luer, J. Luer, R. Dressler \& K. Dressler 10539 (holotype, MO).

Trigonidium seemannii Rchb.f., Bot. Voy. Herald [Seemann] 6: 214. 1854. = Trigonidium egertonianum Bateman ex Lindl.

TYPE: Panama. Panama: rara videtur, B. Seemann 539 (holotype, BM).

Triphora wagneri Schltr., Repert. Spec. Nov. Regni Veg. 17: 139. 1921.

TYPe: Panama. Provinz Chiriquí und Veragua. Blühend im April 1858, Moritz Wagner 1778 (holotype, B, destroyed; illustration of type, AMES).

Vanilla dressleri Soto Arenas, Lankesteriana 9(3): 303. 2010.

TyPe: Panama. Colón: end of Pipeline road on Río Agua Salud, 9-10 mi N of Gamboa. Tropical wet forest. Elev. 20-50 m. Vine. Flowers greenish-yellow, lower lip white. Column yellow. Sweet smelling, 15 April 1982, S. Knapp \& J. Mallet 4621 (holotype, MO).

Vanilla pauciflora Dressler, Orquideología 13: 229. 1979. = Vanilla trigonocarpa Hoehne

TYPE: Panama. Panama: carretera El Llano-Cartí, 10-15 km al N de El Llano; 3 Mar 1976, R.L. Dressler 5290 (holotype, US). 
Warczewiczella caloglossa Schltr., Repert. Spec. Nov. Regni Veg. Beih. 12: 216. 1913. = Stenotyla picta (Rchb.f.) Dressler

Syntypes: Panama. Chiriquí: feuchte Wälder von Cuesta de las Palmas an der Südseite von Cerro de la Horqueta, 1700-2100 m, blühend im Marz 1911, H. Pittier 3214 (B, destroyed; drawings of type, AMES); blühend im Marz 1911, W.R. Maxon 5510 (B, destroyed).

Xylobium powellii Schltr., Repert. Spec. Nov. Regni Veg. Beih. 17: 66. 1922. = Xylobium sulfurinum (Lem.) Schltr.

Type: Panama. Chiriquí, c. 4000', C.W. Powell 117 (holotype, B, destroyed, lectotype designated by Christenson (1991b), AMES-23866; isolectotypes, K, MO-955964; drawing of type, AMES-26865; drawing published in Mansfeld [1931: t. 63, nr. 649].).

Zootrophion williamsii Luer, Selbyana 23: 41. 2002.

TYPE: Panama. Cerro Campana, collected by N.H. Williams, 20 March 1976, flowered in cultivation 30 May 1976, C. Luer 0676 (holotype, SEL).

Zosterophyllanthos dariensis Kolan. \& Szlach., Richardiana 14: 106-108. 2014. = Pleurothallis dariensis (Kolan. \& Szlach.) Bogarín

TyPE: Panama. San Blas/Darién: mountains between Tubualá (San Blas) and Masargandi (Darién), 300-500 m, 2 Feb 1997, R. L. Dressler 5589 (holotype, FLAS).

Zygopetalum lacteum Rchb.f., Gard. Chron. 2: 1290. 1872. = Kefersteinia lactea (Rchb.f.) Schltr.

Syntypes: Costa Rica [?]. [Panama]. Chiriquí [Panama, Wallis s.n., not located]. Later, it was collected by M. Endrés [Costa Rica, A. R. Endrés 334, W 0019731 / Rchb. Orch. 49620]. It flow-ered some years ago with Director Linden [Panama or Costa Rica, J. Linden s.n., W 0019731 / Rchb. Orch. 49620], and is now once more in flower with Messrs. Veitch [without locality, cult. Veitch s.n., W 0019731 / Rchb. Orch. 49620]". Lectotype designated by Pupulin (2001), W, Panama or Costa Rica. Chiriquí, cultivated by Linden s.n. [W 0019731 / Rchb. Orch. 49620].

Zygopetalum parviflorum L.O.Williams, Ann. Missouri Bot. Gard. 28(4): 424, pl. 25. 1941. = Warreopsis parviflora (L.O.Williams) Garay

TYPE: Panama. Chiriquí: terrestrial, flowers purple, lip violet, column white, vicinity of Bajo Chorro, alt. 1900 m, 20-22 Jul 1940, R R.E. Woodson, Jr. \& R.W. Schery 605 (holotype, AMES; isotypes, MO, US). 


\section{APPENDIX 2}

\section{List of orchid species endemic to Panama}

Acianthera juxtaposita (Luer) Luer, Monogr. Syst. Bot. Missouri Bot. Gard. 95: 253. 2004.

Acineta mireyae G.Gerlach \& M.H.Weber, J. Orchideenfr. 10: 230. 2003.

Aspidogyne grandis (Ormerod) Ormerod, Harvard Pap. Bot. 11(2): 147. 2007.

Barbosella circinata Luer, Selbyana 3: 204. 1977.

Baskervilla stenopetala Dressler, Bol. Inst. Bot. (Guadalajara) 5: 70. 1998.

Brachionidium calypso Luer, Monogr. Syst. Bot. Missouri Bot. Gard. 57: 24. 1995.

Brachionidium dentatum Luer \& Dressler, Monogr. Syst. Bot. Missouri Bot. Gard. 57: 34. 1995.

Brachionidium lucanoideum Luer, Monogr. Syst. Bot. Missouri Bot. Gard. 57: 78. 1995.

Brachionidium peltarion Luer, Monogr. Syst. Bot. Missouri Bot. Gard. 57: 92. 1995.

Brachionidium satyreum Luer, Monogr. Syst. Bot. Missouri Bot. Gard. 57: 112. 1995.

Brassia panamensis (Garay) M.W.Chase, Phytotaxa 20: 28. 2011.

Camaridium allenii (L.O.Williams) M.A.Blanco, Lankesteriana 7: 519. 2007.

Camaridium fragrans (J.T.Atwood) M.A.Blanco, Lankesteriana 7: 520. 2007.

Camaridium longicolumna (J.T.Atwood) M.A.Blanco, Lankesteriana 7: 520. 2007.

Camaridium lutheri (J.T.Atwood) M.A.Blanco, Lankesteriana 7: 5202007.

Camaridium scalariforme (J.T.Atwood) M.A.Blanco, Lankesteriana 7: 521. 2007.

Camaridium synsepalum (J.T.Atwood) M.A.Blanco, Lankesteriana 7: 521. 2007.

Camaridium tricarinatum (J.T.Atwood) M.A.Blanco, Lankesteriana 7: 522. 2007.

Camaridium tutae (J.T.Atwood) M.A.Blanco, Lankesteriana 7: 522. 2007.

Chondroscaphe eburnea (Dressler) Dressler, Orquideología 22: 22. 2001.

Chysis pluricostata Dressler, Orquideología 24(2): 142. 2006.

Chysis violacea Dressler, Orquideología 22: 237. 2003.

Cischweinfia nana Dressler, Selbyana 22: 9. 2001.

Cischweinfia pusilla subsp. furcata Dressler \& Dalström, Selbyana 25(1): 5, 9, f. 12. 2004.

Coryanthes maduroana G.Gerlach, Lankesteriana 4: 70. 2004.

Coryanthes panamensis G.Gerlach, Trop. Subtrop. Pflanzenwelt 83: 141. 1993.

Crossoglossa bifida Dressler, Orquideología 20: 255. 1997.

Crossoglossa elliptica Dressler, Orquideología 20: 256. 1997.

Cryptocentrum caespitosum Carnevali, Harvard Pap. Bot. 5: 472. 2001.

Cyclopogon dressleri Szlach., Novon 4: 172. 1994.

Cycnoches dianae Rchb.f., Bot. Zeitung (Berlin) 10: 636. 1852.

Cycnoches powellii Schltr., Repert. Spec. Nov. Regni Veg. Beih. 17: 58. 1922.

Cycnoches stenodactylon Schltr., Repert. Spec. Nov. Regni Veg. Beih. 17: 59. 1922.

Dichaea dressleri Folsom, Novon 16: 336. 2006.

Dichaea retroflexiligula Folsom, Orchid Digest 58: 187. 1994.

Dichaea violacea Folsom, Orchid Digest 58: 190. 1994.

Dracula agnosia A.Doucette, Phytotaxa 56: 23. 2012.

Dracula immunda A.Doucette, Phytotaxa 16: 38. 2011.

Dracula olmosii Luer \& Maduro, Monogr. Syst. Bot. Missouri Bot. Gard. 76: 136. 1999.

Dresslerella elvallensis Luer, Selbyana 3: 2. 1976.

Dresslerella pertusa (Dressler) Luer, Selbyana 3: 6. 1976.

Dresslerella powellii (Ames) Luer, Selbyana 3: 8. 1976. 
Dressleria allenii H.G.Hills, Lindleyana 15: 171. 2000.

Dressleria kerryae H.G.Hills, Lindleyana 15: 173. 2000.

Dressleria severiniana H.G.Hills, Amer. Orchid Soc. Bull. 62: 616. 1993.

Dryadella butcheri Luer, Monogr. Syst. Bot. Missouri Bot. Gard. 76: 160. 1999.

Dryadella dressleri Luer, Monogr. Syst. Bot. Missouri Bot. Gard. 76: 162. 1999.

Echinosepala balaeniceps (Luer \& Dressler) Pridgeon \& M.W.Chase, Lindleyana 17: 101. 2002.

Elleanthus capitatellus Dressler, J. Orchideenfr. 11: 143. 2004.

Elleanthus decipiens Dressler, J. Orchideenfr. 11: 146. 2004.

Encyclia silverarum Leopardi \& Carnevali, Phytotaxa 183 (3): 161. 2014

Epidendrum antonense Hágsater, Icon. Orchid. 2: t. 108. 1993.

Epidendrum brachybotrys Ackerman \& Montalvo, Selbyana 9: 129. 1986.

Epidendrum caligarium Rchb.f., Gard. Chron. 1869: 1110. 1869.

Epidendrum caluerorum Hágsater, Icon. Orchid. 2: t. 116. 1993.

Epidendrum cirrhochiloides Hágsater \& E.Santiago, Icon. Orchid. 11: t. 1112. 2008.

Epidendrum croatii Hágsater, Icon. Orchid. 3: t. 328. 1999.

Epidendrum cuneatoides Dodson ex Hágsater, Orquídea (Mexico City), n.s., 13: 217. 1993.

Epidendrum curvisepalum Hágsater \& Dressler, Icon. Orchid. 2: t. 125. 1993.

Epidendrum cymbiglossum Hágsater, Icon. Orchid. 2: t. 126. 1993.

Epidendrum dariense Hágsater, Kolan. \& L.Sánchez, Icon. Orchid. 14(10): t. 1421. 2013.

Epidendrum dosbocasense Hágsater, Orquídea (Mexico City), n.s., 12: 282. 1992.

Epidendrum dwyeri Hágsater, Icon. Orchid. 2: t. 130. 1993.

Epidendrum dwyerioides Hágsater \& E.Santiago, Icon. Orchid. 9: t. 929. 2007.

Epidendrum elephantinum Hágsater, Icon. Orchid. 7: t. 734. 2004.

Epidendrum folsomii Hágsater \& E.Santiago, Icon. Orchid. 8: 832. 2006.

Epidendrum fortunae Hágsater \& Dressler, Icon. Orchid. 8: 833. 2006.

Epidendrum fuscinum (Dressler) Hágsater, Lankesteriana 5: 73. 2005.

Epidendrum galeochilum Hágsater \& Dressler, Icon. Orchid. 7: t. 738. 2004.

Epidendrum gregorii Hágsater, Icon. Orchid. 2: t. 138. 1993.

Epidendrum gymnochlamys Hágsater \& E.Santiago, Icon. Orchid. 8: 839. 2006.

Epidendrum hornitense Hágsater \& L.Sánchez, Icon. Orchid. 8: 841. 2006.

Epidendrum infundibulum Hágsater \& E.Santiago, Icon. Orchid. 9: t. 946. 2007.

Epidendrum insolatum Barringer, Brittonia 43: 245. 1991[1992].

Epidendrum jefeallenii Hágsater \& García-Cruz, Icon. Orchid. 3: t. 339. 1999.

Epidendrum jefestigma Hágsater \& García-Cruz, Icon. Orchid. 3: t. 340. 1999.

Epidendrum kerichilum Hágsater, Orquídea (Mexico City), n.s., 11: 26. 1988.

Epidendrum lacteum Dressler, Orquideología 11: 108. 1978.

Epidendrum longibracteatum Hágsater, Icon. Orchid. 3: t. 347. 1999.

Epidendrum lutheri Hágsater, Icon. Orchid. 2: t. 154. 1993.

Epidendrum mantis-religiosae Hágsater, Orquídea (Mexico City), n.s., 11: 27. 1988.

Epidendrum niveocaligarium Hágsater, Icon. Orchid. 8: 860. 2006.

Epidendrum pajitense C.Schweinf., Bot. Mus. Leafl. 14: 56. 1949.

Epidendrum panamense Schltr., Repert. Spec. Nov. Regni Veg. 12: 212. 1913.

Epidendrum pirrense Hágsater, Icon. Orchid. 4: t. 471. 2001.

Epidendrum plagiophyllum Hágsater, Icon. Orchid. 3: t. 370. 1999.

Epidendrum platyphyllostigma Hágsater \& García-Cruz, Icon. Orchid. 3: t. 372.1999.

Epidendrum platystomum Hágsater \& L.Sánchez, Icon. Orchid. 8: 873. 2006.

Epidendrum pleurothalloides Hágsater, Icon. Orchid. 2: t. 174. 1993. 
Epidendrum probiflorum Schltr., Repert. Spec. Nov. Regni Veg. Beih. 17: 39. 1922.

Epidendrum pseudoschumannianum Fowlie, Orchid Digest 33: 255. 1969.

Epidendrum pudicum Ames, Schedul. Orchid. 6: 71, fig. 10. 1923.

Epidendrum schistostemum Hágsater, Laube \& L.Sánchez, Icon. Orchid. 11: t. 1177. 2008.

Epidendrum simulacrum Ames, Schedul. Orchid. 6: 75. 1923.

Epidendrum spathulipetalum Hágsater \& Dressler, Icon. Orchid. 4: t. 486. 2001.

Epidendrum sphenostele Hágsater \& E.Santiago, Icon. Orchid. 9: t. 989. 2007.

Epidendrum suturatum Hágsater \& Dressler, Icon. Orchid. 2: t. 190. 1993.

Epidendrum tacarcunense Hágsater, Icon. Orchid. 3: t. 384. 1999.

Epidendrum tenuisulcatum (Dressler) Hágsater, Orquídea (Mexico City), n.s., 13: 217. 1993.

Epidendrum transversellipticum Hágsater, Icon. Orchid. 4: t. 492. 2001.

Epidendrum tritropianthum Hágsater \& E.Santiago, Icon. Orchid. 9: t. 998. 2007.

Epidendrum veraguasense Hágsater, Orquídea (Mexico City), n.s., 12: 286. 1992.

Epidendrum warszewiczii Rchb.f., Bot. Zeitung (Berlin) 10: 742. 1852.

Epidendrum witherspooniorum Hágsater \& Dressler, Icon. Orchid. 7: t. 798. 2004.

Euryblema anatonum (Dressler) Dressler, Lankesteriana 5: 94. 2005.

Gongora powellii Schltr., Repert. Spec. Nov. Regni Veg. Beih. 17: 62. 1922.

Govenia powellii Schltr., Repert. Spec. Nov. Regni Veg. Beih. 17: 51. 1922.

Habenaria avicula Schltr., Repert. Spec. Nov. Regni Veg. 17: 138. 1921.

Habenaria mediocris Dressler, Bol. Inst. Bot. (Guadalajara) 7: 96.1999 [2000].

Habenaria patentiloba Ames, Proc. Biol. Soc. Washington 34: 151. 1921.

Habenaria warszewiczii Schltr., Anexos Mem. Inst. Butantan, Secç. Bot. 1(2): 25.1921.

Jacquiniella pedunculata Dressler, Orquideología 5: 24. 1970.

Kefersteinia alata Pupulin, Harvard Pap. Bot. 8: 161. 2004.

Kefersteinia angustifolia Pupulin \& Dressler, Harvard Pap. Bot. 8: 164. 2004.

Kefersteinia auriculata Dressler, Orquideología 16: 49. 1983.

Kefersteinia maculosa Dressler, Orquideología 16: 52. 1983.

Kreodanthus bugabae Ormerod, Harvard Pap. Bot. 13(1): 59. 2008.

Kreodanthus sytsmae Ormerod, Harvard Pap. Bot. 13(1): 61. 2008.

Lepanthes brunnescens Luer, Phytologia 55: 179. 1984.

Lepanthes caloptera Luer, Phytologia 55: 180. 1984.

Lepanthes confusoides Luer, Monogr. Syst. Bot. Missouri Bot. Gard. 76: 165. 1999.

Lepanthes eciliata Schltr., Repert. Spec. Nov. Regni Veg. 12: 203. 1913.

Lepanthes exposita Luer, Lindleyana 2(4): 191. 1987.

Lepanthes incantata Luer, Lindleyana 2: 194. 1987.

Lepanthes laevis Luer, Monogr. Syst. Bot. Missouri Bot. Gard. 76: 168. 1999.

Lepanthes luerorum B.T.Larsen, Phytotaxa 175 (5) 279-280. 2014.

Lepanthes maduroi Luer, Phytologia 55: 186. 1984.

Lepanthes minyglossa Luer, Selbyana 23: 15. 2002.

Lepanthes mollis Luer, Selbyana 23: 15. 2002.

Lepanthes olmosii Bogarín, Orchid Digest 73(3): 141. 2009.

Lepanthes pachyglossa Luer, Phytologia 57: 59. 1985.

Lepanthes prora Luer, Phytologia 55: 191. 1984.

Lepanthes pycnogenia Luer, Lindleyana 7(2): 114, f. 10. 1992.

Lepanthes rauhii Luer, Orchidee (Hamburg) 38: 59. 1987.

Lepanthes ridicula Luer, Monogr. Syst. Bot. Missouri Bot. Gard. 76: 168. 1999.

Lepanthes schizocardia Luer, Phytologia 55: 193. 1984. 
Lepanthes seegeri Luer, Orchidee (Hamburg) 38: 60, fig. 1987.

Lepanthes tetroptera Luer, Lindleyana 2: 214. 1987.

Lepanthes trichidion Luer, Phytologia 55: 194. 1984.

Lepanthopsis rinkei Luer, Monogr. Syst. Bot. Missouri Bot. Gard. 88: 104. 2002.

Lycaste panamanensis (Fowlie) Archila, Revista Guatemal. 5(2): s.p. 2002.

Macroclinium junctum (Dressler) Dodson, Icon. Pl. Trop. 10: t. 938. 1984.

Macroclinium simplex (Dressler) Dodson, Icon. Pl. Trop. 10: t. 939. 1984.

Malaxis brevis Dressler, Selbyana 24(2): 141 .2003.

Malaxis rostratula Dressler, Lankesteriana 4(1): 97. 2004.

Masdevallia andresiana Luer \& Maduro, Monogr. Syst. Bot. Missouri Bot. Gard. 103: 283. 2005.

Masdevallia collina L.O.Williams, Amer. Orchid Soc. Bull. 11: 93.1942.

Masdevallia eburnea Luer \& Maduro, Monogr. Syst. Bot. Missouri Bot. Gard. 91: 409a. 2003.

Masdevallia gloriae Luer \& Maduro, Monogr. Syst. Bot. Missouri Bot. Gard. 91: 413a. 2003.

Masdevallia maduroi Luer, Monogr. Syst. Bot. Missouri Bot. Gard. 72: 110. 1998.

Masdevallia olmosii Königer \& Sijm, Arcula 12: 303. 2003.

Masdevallia ostaurina Luer, Monogr. Syst. Bot. Missouri Bot. Gard. 95: 249. 2004.

Masdevallia pleurothalloides Luer, Selbyana 3: 218. 1977.

Masdevallia tokachiorum Luer, Lindleyana 6: 90. 1991.

Maxillaria curvicolumna M.A.Blanco \& Neubig, Orchids (West Palm Beach) 76(6): 451. 2007.

Maxillaria darienensis J.T.Atwood, Selbyana 19: 254. 1998 [1999].

Maxillaria galantha J.T.Atwood \& Carnevali, Lindleyana 9: 227. 1994.

Microchilus fuscatus Ormerod, Harvard Pap. Bot. 11(2): 161. 2007.

Microchilus maasii Ormerod, Harvard Pap. Bot. 11(2): 161. 2007.

Microchilus ovalis Ormerod, Harvard Pap. Bot. 11(2): 164. 2007.

Microchilus panamanicus Ormerod, Harvard Pap. Bot. 9: 414. 2005.

Microchilus subquadratus Ormerod, Harvard Pap. Bot. 11(2): 172. 2007.

Microchilus whitefoordiae Ormerod, Harvard Pap. Bot. 11(2): 173. 2007.

Mormodes lancilabris Pabst, Ann. Missouri Bot. Gard. 62: 510.1975.

Mormodes powellii Schltr., Repert. Spec. Nov. Regni Veg. Beih. 17: 55. 1922.

Mormolyca culebrica Bogarín \& Pupulin, Orchid Digest 74(1): 44. 2010.

Notylia latilabia Ames \& C.Schweinf., Schedul. Orchid. 8: 71. 1925.

Oncidium abortivoides M.W.Chase \& N.H.Williams, Lindleyana 21(3): 22. 2008.

Oncidium allenii Dressler, Selbyana 22: 9. 2001.

Oncidium exalatum Hágsater, Orquídea (Mexico City), n.s., 8: 98. 1981.

Oncidium martinezii Königer, Arcula 15: 369. 2005.

Oncidium punctulatum Dressler, Selbyana 22: 10. 2001.

Oncidium zelenkoanum Dressler \& Pupulin, Lankesteriana 8: 37. 2003.

Ornithidium repens (L.O.Williams) M.A.Blanco \& Ojeda, Lankesteriana 7: 533.2007.

Ornithocephalus aristatus Pupulin \& Dressler, Brittonia 58: 314. 2006.

Ornithocephalus cryptanthus (C.Schweinf. \& P.H.Allen) Toscano \& Dressler, Lindleyana 15: 255. 2000.

Ornithocephalus dressleri (Toscano) Toscano \& Dressler, Lindleyana 15: 255. 2000.

Paphinia vermiculifera G.Gerlach \& Dressler, Lankesteriana 8: 27. 2003.

Pelexia ochyrae Szlach., Fragm. Florist. Geobot. 41: 858. 1996.

Phloeophila pelecaniceps (Luer) Pridgeon \& M.W.Chase, Lindleyana 16: 254. 2001.

Platystele calymma Luer, Selbyana 3: 226. 1977.

Platystele dressleri Luer, Selbyana 3: 26. 1976.

Platystele hampshireae Luer, Monogr. Syst. Bot. Missouri Bot. Gard. 65: 113. 1998. 
Pleurothallis allenii L.O.Williams, Ann. Missouri Bot. Gard. 27: 275. 1940.

Pleurothallis annectens Luer, Selbyana 3: 240. 1977.

Pleurothallis archicolonae Luer, Selbyana 3: 52. 1976.

Pleurothallis cardiochila L.O.Williams, Ann. Missouri Bot. Gard. 29: 343. 1942.

Pleurothallis cleistogama Luer, Monogr. Syst. Bot. Missouri Bot. Gard. 72: 41. 1998.

Pleurothallis cobriformis L.O.Williams, Ann. Missouri Bot. Gard. 27: 277. 1940.

Pleurothallis dariensis (Kolan. \& Szlach.) Bogarín, Lankesteriana 14 (3): 241. 2014.

Pleurothallis dressleriana Bogarín, Lankesteriana 14 (3): 241. 2014.

Pleurothallis dukei Luer, Lindleyana 12: 45. 1997.

Pleurothallis folsomii (Luer \& Endara) Bogarín, Lankesteriana 14 (3): 241. 2014.

Pleurothallis hammelii Luer, Lindleyana 12: 48. 1997.

Pleurothallis harpago Luer, Selbyana 5: 167. 1979.

Pleurothallis hemileuca Luer, Selbyana 7: 118. 1982.

Pleurothallis knappiae Luer, Monogr. Syst. Bot. Missouri Bot. Gard. 79: 128. 2000.

Pleurothallis maduroi Luer, Lindleyana 12: 51. 1997.

Pleurothallis mammillata Luer, Selbyana 3: 138. 1976.

Pleurothallis nitida Luer, Selbyana 3(3-4): 352. 1977.

Pleurothallis orygmoglossa (Luer \& Dressler) Bogarín, Lankesteriana 14 (3): 242. 2014.

Pleurothallis pallida Luer, Selbyana 3: 358. 1977.

Pleurothallis polysticta Luer, Selbyana 5: 176. 1979.

Pleurothallis simulans L.O.Williams, Ann. Missouri Bot. Gard. 27: 281. 1940.

Pleurothallis telamon Luer, Selbyana 5: 185. 1979.

Pleurothallis titan Luer, Selbyana 3: 400. 1977.

Polycycnis tortuosa Dressler, Orquideología 12: 120. 1977.

Prosthechea fortunae (Dressler) W.E.Higgins, Phytologia 82: 377.1997 [1998].

Prosthechea sima (Dressler) W.E.Higgins, Phytologia 82: 381. 1997 [1998].

Psilochilus dressleri Kolan., Phytotaxa 175 (1): 55-57. 2014.

Sarcoglottis woodsonii (L.O.Williams) Garay, Bot. Mus. Leafl. 28: 355.1980 [1982].

Scaphosepalum viviparum Luer, Selbyana 2: 390. 1978.

Scaphyglottis arctata (Dressler) B.R.Adams, Phytologia 64: 257. 1988.

Scaphyglottis clavata Dressler, J. Orchideenfr. 11: 311. 2004.

Scaphyglottis chlorantha B.R.Adams, Phytologia 64: 249. 1988.

Scaphyglottis monspirrae Dressler, Novon 10: 199. 2000.

Scaphyglottis panamensis B.R.Adams, Phytologia 64: 251. 1988.

Sievekingia butcheri Dressler, Orquideología 13: 221. 1979.

Sobralia callosa L.O.Williams, Ann. Missouri Bot. Gard. 33: 31. 1946.

Sobralia citrea Dressler, Orchids (West Palm Beach) 74: 937. 2005.

Sobralia exigua Dressler, Orchids (West Palm Beach) 74: 938. 2005.

Sobralia gloriana Dressler, Lankesteriana 5: 11. 2002.

Sobralia kruskayae Dressler, Orchids (West Palm Beach) 74: 939. 2005.

Sobralia maduroi Dressler, Orchids (West Palm Beach) 73: 776. 2004.

Sobralia marianneae Dressler, Lankesteriana 5: 13. 2002.

Sobralia nutans Dressler, Lankesteriana 5: 13. 2002.

Sobralia powellii Schltr., Repert. Spec. Nov. Regni Veg. Beih. 17: 11. 1922.

Sobralia recta Dressler, Orchids (West Palm Beach) 74: 939. 2005.

Sobralia sanfelicis Dressler, Orchids (West Palm Beach) 73: 777. 2004.

Sobralia sororcula Dressler, Orquideología 25: 39. 2007. 
Sobralia sotoana Dressler \& Bogarín, Lankesteriana 9(3): 482. 2010.

Sobralia theobromina Dressler, Orchids (West Palm Beach) 74: 940. 2005.

Sobralia tricolor Dressler, Orchids (West Palm Beach) 74: 941. 2005.

Sobralia $\times$ intermedia P.H.Allen, Amer. Orchid Soc. Bull. 27: 534. 1958.

Specklinia bicornis (Luer) Pridgeon \& M.W.Chase, Lindleyana 16(4): 256. 2001.

Specklinia brighamella (Luer) Pridgeon \& M.W.Chase, Lindleyana 16: 256. 2001.

Specklinia cactantha (Luer) Pridgeon \& M.W.Chase, Lindleyana 16: 257. 2001.

Specklinia cucumeris (Luer) Bogarín \& Karremans, Lankesteriana 14 (3): 261. 2014.

Specklinia displosa (Luer) Pridgeon \& M.W.Chase, Lindleyana 16: 257. 2001.

Specklinia dressleri (Luer) Bogarín \& Karremans, Lankesteriana 14 (3): 262. 2014.

Specklinia unicornis (Luer) Pridgeon \& M.W.Chase, Lindleyana 16(4): 259. 2001.

Stanhopea maduroi Dodson \& Dressler, Orquideología 21: 62. 1998.

Stanhopea panamensis N.H.Williams \& W.M.Whitten, Lindleyana 3: 9. 1988.

Stelis adriananijhuisae Bogarín \& Serracín, Lankesteriana 14 (3): 265. 2014.

Stelis atrorubens L.O.Williams, Ann. Missouri Bot. Gard. 29: 339. 1942.

Stelis collina Schltr., Repert. Spec. Nov. Regni Veg. Beih. 17: 15. 1922.

Stelis cymbisepala Pridgeon \& M.W.Chase, Lindleyana 17: 98. 2002.

Stelis decurrens Pridgeon \& M.W.Chase, Lindleyana 17: 99. 2002.

Stelis falcatiloba (Ames) Bogarín \& Serracín, Lankesteriana 14 (3): 262. 2014.

Stelis fortunae (Luer \& Dressler) Pridgeon \& M.W.Chase, Lindleyana 16: 263. 2001.

Stelis furculifera (Dressler \& Bogarín) Bogarín, Lankesteriana 14 (3): 267. 2014.

Stelis gnoma Pridgeon \& M.W.Chase, Lindleyana 17: 99. 2002.

Stelis guttata (Luer) Pridgeon \& M.W.Chase, Lindleyana 16: 263. 2001.

Stelis isthmi Schltr., Repert. Spec. Nov. Regni Veg. Beih. 17: 16. 1922.

Stelis loculifera Luer, Phytologia 49: 229. 1981.

Stelis longipetiolata Ames, Schedul. Orchid. 1: 6. 1922.

Stelis maculata Pridgeon \& M.W.Chase, Lindleyana 17: 99. 2002.

Stelis maduroi Luer \& Sijm, Monogr. Syst. Bot. Missouri Bot. Gard. 112: 111. 2007.

Stelis meganthera Luer, Selbyana 22: 122. 2001.

Stelis megapetala Luer, Selbyana 22: 124. 2001.

Stelis montana L.O.Williams, Ann. Missouri Bot. Gard. 27: 272. 1940.

Stelis mystax (Luer) Pridgeon \& M.W.Chase, Lindleyana 16: 264. 2001.

Stelis semperflorens Luer, Phytologia 49: 238. 1981.

Stelis thymochila (Luer) Pridgeon \& M.W.Chase, Lindleyana 16: 267. 2001.

Stelis triaristata Luer, Selbyana 5: 193. 1979.

Stelis williamsii Ames, Orchidaceae 7: 133. 1922.

Stenotyla panamensis Pupulin, Orchid Rev. 117(1287): 162. 2009.

Systeloglossum panamense Dressler \& N.H.Williams, Amer. Orchid Soc. Bull. 39: 326. 1970.

Telipogon bombiformis Dressler, Orchids 72: 114. 2003.

Telipogon butcheri Dodson \& R.Escobar, Orquideología 18: 278. 1993.

Telipogon butchii N.H.Williams \& Dressler, Lankesteriana 5: 169. 2005.

Telipogon caulescens Dressler, Orchids 72: 114. 2003.

Telipogon chiriquiensis Dodson \& R.Escobar, Orquideología 18: 281. 1993.

Telipogon fractus Dressler, Orchideen J. 14(1): 12. 2007.

Telipogon griesbeckii Dressler, Orchids 72: 115. 2003.

Telipogon maduroi Dressler, Orchids 72: 116. 2003.

Telipogon medusae Dressler, J. Orchideenfr. 13(3): 211. 2006. 
Telipogon morii (Dressler) N.H.Williams \& Dressler, Lankesteriana 5: 170. 2005.

Telipogon nobilis Dressler, Orchideen J. 14(1): 12. 2007.

Telipogon nunezii Dressler, Orchideen J. 14(1): 14. 2007.

Telipogon olmosii Dressler, J. Orchideenfr. 13(3): 212. 2006.

Telipogon panamensis Dodson \& R.Escobar, Orquideología 18: 284. 1993.

Telipogon personatus Dressler, J. Orchideenfr. 13(3): 214. 2006.

Telipogon reticulatus Dressler, Orchideen J. 14(1): 14. 2007.

Telipogon seibertii Dodson \& R.Escobar, Orquideología 18: 288. 1993.

Trichocentrum silverarum (Carnevali \& Cetzal) J.M.H.Shaw, Orchid Rev. 122(1306): 37. 2014.

Trichopilia amabilis Dressler, Orquideología 22: 245. 2003.

Trichopilia eneidae Dressler, Orquideología 22(3): 246. 2003.

Trichopilia leucoxantha L.O.Williams, Amer. Orchid Soc. Bull. 10: 137. 1941.

Trichopilia olmosii Dressler, Selbyana 22: 11. 2001.

Trichopilia similis Dressler, Selbyana 22: 12. 2001.

Trichosalpinx dressleri Luer, Monogr. Syst. Bot. Missouri Bot. Gard. 64: 54. 1997.

Trichosalpinx macphersonii Luer, Monogr. Syst. Bot. Missouri Bot. Gard. 64: 66. 1997.

Trichosalpinx tantilla (Luer) Luer, Monogr. Syst. Bot. Missouri Bot. Gard. 64: 81. 1997.

Trichosalpinx tropida (Luer) Luer, Phytologia 54: 398/ 1983.

Trisetella dressleri (Luer) Luer, Phytologia 47: 57. 1980. 


\section{APPENDIX 3}

\section{Excluded taxa}

Acineta superba (Kunth) Rchb.f. in W.G.Walpers, Ann. Bot. Syst. 6: 609. 1863.

This is a species from South America not yet recorded in Panama.

Acianthera lanceana (Lodd. ex Lind1.) Pridgeon \& M.W.Chase, Lindleyana 16: 244. 2001.

A species widely distributed in Central and South America. Although expected in Panama, no vouchers have been found or cited for the country (Luer 2004).

Anathallis polygonoides (Griseb.) Pridgeon \& M.W.Chase, Lindleyana 16: 250. 2001.

This species is distributed in Trinidad \& Tobago, Venezuela, Suriname, French Guiana, Ecuador and Peru. No records have been found from Panama (Luer 2006). It can be confused with $A$. sertularioides.

Bletia reflexa Lindl., Edwards's Bot. Reg. 21: t. 1760. 1835.

We have not seen specimens recorded in Panama.

Brassia lanceana Lindl., Edwards's Bot. Reg. 21: t. 1754. 1835.

This is a species from South America not yet recorded in Panama.

Brassia longissima (Rchb.f.) Nash, L.H.Bailey, Stand. Cycl. Hort. 1: 541. 1914.

Specimens collected in Panama correspond to $B$. arcuigera Rchb.f.

Campylocentrum schneeanum Foldats, Bol. Soc. Venez. Ci. Nat. 22: 274. 1961.

We have not seen specimens recorded in Panama. Specimens under this name might correspond to $C$. tenellum Todzia (Correa et al. 2004).

Chysis aurea Lindl., Edwards's Bot. Reg. 23: t. 1937. 1837.

This is a species from South America not yet recorded in Panama.

Coryanthes maculata Hook., Bot. Mag. 58: t. 3102. 1831.

This is a species from South America not yet recorded in Panama.

Coryanthes picturata Rchb.f., Bot. Zeitung (Berlin) 22: 332.1864.
This name was applied to Panama and Costa Rica populations now known as C. kaiseriana (Gerlach \& Dressler 2003)

Coryanthes speciosa Hook., Bot. Mag. 58: t. 3102. 1831.

This is a species from South America not yet recorded in Panama.

Cryptocentrum gracillimum Ames \& C. Schweinf., Schedul, Orch. 8: 68. 1925.

Specimens under this name correspond to Cryptocentrum caespitosum Carnevali.

Cryptocentrum inaequisepalum C.Schweinf., Bot. Mus. Leafl. 12: 186. 1946.

This is a South American species not recorded in Panama (Carnevali 2001).

Cryptocentrum peruvianum subsp. minus (Schltr.) Carnevali, Harvard Pap. Bot. 5: 482. 2001.

This is a subspecies from Colombia not yet recorded in Panama (Carnevali 2001).

Cyclopogon millei (Schltr.) Schltr., Beih. Bot. Centralbl. 37(2): 391. 1920.

This is a South American species not recorded in Panama.

Cyclopogon olivaceous (Rolfe) Schltr., Beih. Bot. Centralbl. 37(2): 392. 1920.

This species is restricted to Ecuador and Peru. According to Szlachetko et al. (2005) this name has been applied to Mesoamerican populations; however, they do not correspond to the type and protologue of $C$. olivaceous.

Cycnoches ventricosum Bateman, Orchid. Mexico Guatemala: t. 5. 1838.

This is a species from South America not yet recorded in Panama.

Cyrtopodium punctatum (L.) Lindl., Gen. Sp. Orchid. P1.: 188. 1833.

According to Romero-González \& CarnevaliFernández (1999) the correct name for the Panamanian populations is Cyrtopodium macrobulbon.

Dichaea brachypoda Rchb.f., Beitr. Orchid.-K. C. Amer.: 78. 1866. 
This species is a synonym of Dichaea panamensis Lindl.. Other specimens in herbaria correspond to $D$. elliptica Dressler \& Folsom (Correa et al. 2004).

Dichaea graminoides (Sw.) Lindl., Gen. Sp. Orchid. P1.: 209. 1833.

We have not seen specimens in herbaria from Panama.

Dichaea tenuifolia Schltr., Repert. Spec. Nov. Regni Veg. Beih. 7: 203. 1920.

This is a poorly known species distributed from Colombia to Ecuador (Pupulin 2005a, 2007). Costa Rican populations of Dichaea with undulate, thintextured leaves and smooth ovary were recognized as distinct from South American material and, thus, described as D. viridula (Pupulin 2005a). We have not yet seen specimens of this group from Panama.

Dressleria dilecta (Rchb.f.) Dodson, Selbyana 1: 132. 1975.

According to Hills (2012), this species is endemic to Costa Rica.

Dressleria eburnea (Rolfe) Dodson, Selbyana 1: 132. 1975. = Dressleria suavis (Ames \& C.Schweinf.) Dodson

According to Hills (2012), this species ranges from Nicaragua to Costa Rica.

Dressleria helleri Dodson, Selbyana 1: 132. 1975.

This species is native to Nicaragua and Costa Rica. It has not yet been recorded in Panama.

Dryadella linearifolia (Ames) Luer, Selbyana 2(2-3): 208. 1978.

A species from Guatemala not yet recorded in Panama.

Dryadella pusiola (Rchb. f.) Luer, Selbyana 2(2/3): 209. 1978.

A species from South America not yet recorded in Panama.

Dryadella simula (Rchb.f.) Luer, Selbyana 2: 209. 1978.

This species is distributed from Colombia to Peru. It has not yet been reported from Panama.

Elleanthus capitatus (Poepp. \& Endl.) Rchb.f., in W.G.Walpers, Ann. Bot. Syst. 6: 475. 1862.

This is a species from the Antilles. The name has been applied to specimens of E. cynarocephalus (Rchb.f.) Rchb.f. in Panama.

Elleanthus linifolius C.Pres1, Reliq. Haenk. 1: 97. 1827.

The name was applied to E. graminifolius (Barb.Rodr.) Løjtnant.
Elleanthus robustus (Rchb.f.) Rchb.f., in W.G.Walpers, Ann. Bot. Syst. 6: 474. 1862.

A species from South America not yet recorded in Panama.

Encyclia gravida (Lindl.) Schltr., Beih. Bot. Centralbl. 36(2): 472.1918.

This name must be applied with some prudence because the paucity specimens examined in Costa Rica and in literature (Pupulin \& Bogarín 2011, 2012) seem to correspond to self-pollinating forms of different taxa. This phenomenon is common within the species complex close to E. ceratistes, E. chloroleuca and other "greenish" Encyclias. Thus, E. gravida might not be considered strictly as a distinct species. This name has been erroniously applied to some fruiting specimens of E. stellata (Correa et al. 2004).

Epidendrum bangii Rolfe, Bull. New York Bot. Gard. 4: 451.1907.

According to Hágsater (2013) this species is found in the Andean region of Colombia and Ecuador.

Epidendrum blepharistes Barker ex Lindl., Edwards's Bot. Reg. 30(Misc.): 20. 1844.

Although this species most likely occur in Panama, no voucher exists from Panama (Hágsater 2008).

Epidendrum carpophorum Barb.Rodr., Gen. Spec. Orchid. 2: 148. 1882.

According to Hágsater (2010) this species is restricted to Venezuela, Guyanas and Brazil. It has been confused with Epidendrum angustilobum, which is found in Costa Rica and Panama and in the Pacific coast of Colombia and Venezuela.

Epidendrum concavilabium C.Schweinf., Bot. Mus. Leafl. 4: 118. 1937.

This species has been traditionally treated as synonym of E. circinatum (Hágsater et al. 2003). According to Karremans et al. (2012), both species are distinct, with E. circinatum restricted to the middle elevations areas of Valle Central in Costa Rica, while E. circinatum is distributed along the Caribbean lowlands of Nicaragua, Costa Rica and Panama. The Panamanian voucher studied corresponds to E. circinatum (S. Laube et al. 590, PMA).

Epidendrum congestum Rolfe, Bull. Misc. Inform. Kew 1913: 29. 1913.

According to Hágsater (2007) this species is restricted to Costa Rica.

Epidendrum crescentilobum Ames, Schedul. Orchid. 4: 38.1923. 
This species is endemic to Costa Rica (Hágsater \& Sánchez-Saldaña 2006).

Epidendrum difforme Jacq., Enum. Syst. P1.: 29. 1760.

This is a species from the Antilles. It is not recorded in Panama.

Epidendrum ibaguense Kunth in F.W.H.von Humboldt, A.J.A.Bonpland \& C.S.Kunth, Nov. Gen. Sp. 1: 352. 1816.

A species from South America not yet recorded in Panama.

Epidendrum microphyllum Lindl., J. Bot. (Hooker) 3:

85. 1841.

This species is restricted to South America.

Epidendrum obliquifolium Ames, F.T.Hubb. \& C.Schweinf., Bot. Mus. Leafl. 3: 73. 1935.

According to Hágsater (1999), the voucher Dressler 5535 (PMA) determined as E. obliquifolium is E. plagiophyllum. Thus, this species is at the present only found in Costa Rica.

Epidendrum paniculatum Ruiz \& Pav., Syst. Veg. F1. Peruv. Chil.: 243. 1798.

A species from South America. It has not yet been recorded in Panama.

Epidendrum peperomia Rchb.f., Bonplandia (Hannover) 2: 20. 1854.

This species is only found in Colombia and Venezuela. The name has been used in Panama for specimens that correspond to E. porpax.

Epidendrum polyanthum Lindl., Gen. Sp. Orchid. P1.: 106. 1831.

According to Hágsater (2008), this species ranges from Mexico to Honduras.

Epidendrum ramonianum Schltr., Repert. Spec. Nov. Regni Veg. Beih. 19: 217. 1923.

According to Hágsater (2003) this species is endemic to Costa Rica.

Epidendrum repens Cogn., Repert. Spec. Nov. Regni Veg. 7: 122. 1909.

Although widely distributed in the Neotropics, Hágsater \& Sánchez-Saldaña (2006) did not record this species in Panama.

Epidendrum schumannianum Schltr., Repert. Spec. Nov. Regni Veg. 9: 215. 1911.

An endemic species to Costa Rica. It has not yet been recorded in Panama. Its sister species is Epidendrum pseudoschumannianum, an endemic to Panama.

Epidendrum smaragdinum Lindl., Edwards's Bot. Reg. 24(Misc.): 32. 1838.
This is a South American species not found in Panama (Hágsater \& Sánchez-Saldaña 2006).

Erycina glossomystax (Rchb.f.) N.H.Williams \& M.W.Chase, Lindleyana 16: 136. 2001.

Widely distributed in South America; however, we have not seen vouchers from Panama.

Galeandra batemannii Rolfe, Gard. Chron., ser. 3, 12: 431. 1892.

This is a species from Mexico. It has not yet been recorded in Panama.

Galeandra bauerii Lindl., in F.A.Bauer, Ill. Orch. P1.: t. 8.1830 .

This is a species from Guyana. It has not yet been recorded in Panama.

Galeandra styllomisantha (Vell.) Hoehne, Arq. Bot. Estado São Paulo, n.s., f.m., 2: 146. 1952.

This is a South American species. The Panamanian specimen corresponds to $G$. arundinis (Garay \& G.A.Romero 2005).

Gomphichis hetaerioides Schltr., Repert. Spec. Nov. Regni Veg. Beih. 7: 54. 1920.

This species ranges from Colombia to Ecuador. The correct name for the populations from western Panama is G. adnata (Dressler 2003c).

Gongora charontis Rchb.f., Linnaea 41: 110. 1876.

This species is from Colombia. We have not seen vouchers of this species based on Panamanian material.

Gongora claviodora Dressler, Orquideologia 7: 75. 1972.

According to Dressler (2003c), this species ranges from Nicaragua to Costa Rica. No vouchers of this species have been seen from Panama.

Gongora maculata Lindl., Edwards's Bot. Reg. 19: t. 1616. 1833.

A species from South America. It has not yet been recorded in Panama.

Gongora quinquenervis Ruiz \& Pav., Syst. Veg. Fl. Peruv. Chil.: 227. 1798.

A species from South America. It has not yet been recorded in Panama.

Goodyera bradeorum Schltr., Repert. Spec. Nov. Regni Veg. Beih. 19: 88. 1923.

The type material of $G$. bradeorum is lost, and the application of this concept is still uncertain (Dressler 2003c).

Goodyera striata Rchb.f., Linnaea 18: 409. 1845.

This species is recorded from Mexico to Nicaragua 
(Govaerts 2003).

Govenia liliacea (Lex.) Lindl., Edwards's Bot. Reg. 21: t. 1795.1835.

A species from northern Mesoamerica. It has not yet been recorded in Panama.

Guarianthe deckeri (Klotzsch) Roeth, Orchidee (Hamburg) 57: 612. 2006.

Specimens collected in Panama correspond to Guarianthe patinii (Cogn.) Dressler \& W.E.Higgins.

Habenaria bractescens Lindl., Gen. Sp. Orchid. P1.: 308. 1835.

This species has not yet been recorded in Panama.

Habenaria entomantha (Lex.) Lindl., Gen. Sp. Orchid. P1.: 311. 1835.

This species has not yet been recorded in Panama.

Habenaria floribunda Lindl., Gen. Sp. Orchid. P1.: 316. 1835.

This species has not yet been recorded in Panama.

Habenaria quinqueseta (Michx.) Eaton, Man. Bot., ed. 5: 235.1829.

This species has not yet been recorded in Panama.

Huntleya meleagris Lindl., Edwards's Bot. Reg. 23: t. 1991.1837.

The specimen cited in Flora of Panama (Williams \& Allen 1980) corresponds to H. burtii (Endrés \& Rchb.f.) Pfitzer (Correa et al. 2004).

Isochilus major Cham. \& Schltdl., Linnaea 6: 60. 1831.

This species was described from Mexico. We were unable to locate or identify Panamanian specimens of this species.

Kefersteinia microcharis Schltr., Repert. Spec. Nov. Regni Veg. Beih. 19: 300. 1923.

According to Pupulin (2001) this species is endemic to Costa Rica.

Kegeliella houtteana (Rchb.f.) L.O.Williams, Ann. Missouri Bot. Gard. 29: 347. 1942.

This name has been erroneously applied to specimens of $K$. atropilosa L.O. Williams \& A.H. Heller.

Laelia rubescens Lindl., Edwards's Bot. Reg. 26(Misc.): 20. 1840.

The voucher quoted by Williams \& Allen (1980) comes from a cultivated plant from C.W. Powell apparently collected in the Chiriquí province, near the border with Costa Rica close to Golfo Dulce. However, this area is outside of the natural distribution of the species which ranges from Mexico to the Central Pacific of
Costa Rica. In Costa Rica, we have not seen specimens along the southern Pacific coast (from Carara to Burica) towards Panama. No collections appeared in Panama other than the voucher cited.

Laelia undulata (Lindl.) L.O. Williams, Darwiniana 5: 76.1941.

This species is known from South America (Dressler 2003). We have not been able to see specimens from Panama.

Lankesteriana barbulata (Lind1.) Karremans, Lankesteriana 13(3): 326. 2014.

Although widely distributed through Central and South America, we have not seen vouchers from Panama. Luer (2006) did not cite any collection for the country. It is expected to be found in Panama.

Lepanthes pristidis Rchb.f., Linnaea 22: 820. 1850.

This species was described from Mexico. Its is considered a synonym of L. disticha (Luer 2003a).

Liparis nervosa (Thunb.) Lindl., Gen. Sp. Orchid. P1.: 26. 1830 .

Specimens from Costa Rica and Panama (Dressler 1993) correspond to L. elata (Dressler 2003c).

Lycaste xytriophora Linden \& Rchb.f. in W.W.Saunders, Refug. Bot. 2: t. 131. 1872.

Although it has been recorded from Costa Rica and Panama, this species is restricted to South America (Dressler 2003c). No vouchers of this species have been seen from Panama.

Malaxis excavata (Lindl.) Kuntze, Revis. Gen. P1. 2: 673. 1891.

This species was described from Mexico. In Costa Rica and Panama the name was applied to populations of M. hastilabia, which is considered a distinct species (Dressler 2003c).

Malaxis fastigiata (Rchb.f.) Kuntze, Revis. Gen. Pl. 2: 673.1891.

This name was misapplied to specimens of $M$. brachyrrhynchos (Dressler 2003c).

Malaxis parthoni C.Morren, Bull. Acad. Roy. Sci. Bruxelles 5: 485. 1839.

This species is from Brazil. The name has been erroneously applied to specimens of $M$. histionantha (Link) Garay \& Dunst.

Maxillariella caespitifica (Rchb.f.) M.A.Blanco \& Carnevali, Lankesteriana 7: 528. 2007.

This species ranges from Nicaragua to Costa Rica. It has not yet been recorded in Panama (Atwood 2003). 
Maxillaria luteoalba Lindl., Orchid. Linden.: 20. 1846.

A species from South America. It has not yet been recorded in Panama.

Maxillaria meridensis Lindl., Orchid. Linden.: 19. 1846. This species known from Costa Rica, Venezuela, Colombia, Ecuador and Bolivia has not yet been recorded in Panama.

Maxillaria speciosa Rchb.f., Gard. Chron., n.s., 6: 197. 1876.

This species is known from Colombia and Ecuador.

Maxillaria splendens Poepp. \& Endl., Nov. Gen. Sp. P1. 1: 38. 1836.

This species is known from Colombia and Venezuela to Peru and Brazil.

Mormodes atropurpurea Lindl., Intr. Nat. Syst. Bot., ed. 2: 446. 1836.

A species from South America and has not yet been recorded in Panama. The name has been erroneously applied to specimens of M. lobulata Schltr.

Mormodes cartonii Hook., Bot. Mag. 72: t. 4214. 1846.

This is a species from Sierra Nevada de Santa Marta Colombia. No vouchers have been seen from Panama.

Mormodes ignea Lindl. \& Paxton, Paxton's Fl. Gard. 3: 97.1852.

A species from South America. It has not yet been recorded in Panama. The name has been erroneously applied to specimens of M. fractiflexa Rchb.f.

Mormodes punctata Rolfe, Gard. Chron., ser. 3, 10: 696. 1891.

This name was published based on a plant of unknown origin. We have not seen the type specimen at $\mathrm{K}$. The application of this name is obscure and its presence in Panama is uncertain.

Mormolyca rufescens (Lindl.) M.A.Blanco, Lankesteriana 7: 531. 2007.

A species from South America. It has not yet been recorded in Panama.

Muscarella zephyrina (Rchb.f.) Luer, Monogr. Syst. Bot. Missouri Bot. Gard. 105: 124. 2006.

This species is widely distributed in South America but it has not yet been recorded in Panama (Luer 2006).

Myoxanthus exasperatus (Lindl.) Luer, Selbyana 7: 36. 1982.

This species is only known from South America (Luer 1997a).
Notylia barkeri Lindl., Edwards's Bot. Reg. 24(Misc.): 90. 1838

This species ranges from Mexico to Honduras. No specimens were found in herbaria from Panama.

Notylia lankesteri Ames, Schedul. Orchid. 5: 34. 1923. This is an endemic species to Costa Rica. It has not yet been recorded in Panama.

Oncidium abortivum Rchb.f., Linnaea 22: 847. 1850. This species is restricted to South America (Govaerts 2003).

Oncidium baueri Lindl., Gen. Sp. Orchid. P1.: 200. 1833.

No vouchers of this species has been seen from Panama. The species is restricted to South America.

Oncidium heteranthum Poepp. \& Endl., Nov. Gen. Sp. Pl. 1: 34. 1836.

This species is restricted to South America. The name was misapplied to specimens of $O$. bryolophotum (Dressler 2003c).

Oncidium hymenanthum (Schltr.) M.W.Chase \& N.H.Williams, Lindleyana 21(3): 25. 2008.

This is an enigmatic endemic species from Costa Rica. The specimens that we studied from Panama fit better with the concept of Oncidium integrilabre $=$ Sigmatostalix integrilabris proposed by Pupulin (2003).

Oncidium ornithorhynchum Kunth in F.W.H.von Humboldt, A.J.A.Bonpland \& C.S.Kunth, Nov. Gen. Sp. 1: 345. 1816.

According to Jiménez \& Hágsater (2010), this species is from South America and the name was misapplied to the populations of Mexico and Central America. The correct name for this species in Panama is $O$. sotoanum.

Oncidium planilabre Lindl., J. Hort. Soc. London 6: 59. 1851

This species is restricted to South America (Govaerts 2003).

Oncidium stenoglossum (Schltr.) Dressler \& N.H.Williams, Orquídea (Mexico City), n.s., 4: 339. 1975.

This species ranges from Mexico to Costa Rica (Dressler 2003c). It is likely found in western Panama but no herbarium vouchers have been seen.

Ornithidium aureum Poepp. \& Endl., Nov. Gen. Sp. Pl. 1: 57. 1836.

This species is restricted to South America (Govaerts 2003). 
Ornithocephalus gladiatus Hook., Exot. Fl. 2: t. 127. 1824.

This species is species from Trinidad and South America. The name has been applied to several specimens along the Neotropics. We have not seen Panamanian material regarding this species.

Pabstiella uniflora (Lindl.) Luer, Monogr. Syst. Bot. Missouri Bot. Gard. 112: 121. 2007.

This species is restricted to South America and it is not recorded in Panama (Luer 1998b).

Palmorchis sylvicola L.O.Williams, Fieldiana, Bot. 32: 199. 1970.

Specimens treated under this name correspond to $P$. trinotata Dressler.

Paphinia clausula Dressler, Taxon 15: 242. 1966.

The specimens recorded in Panama under this name correspond to $P$. subclausa Dressler.

\section{Phloeophila peperomioides (Ames) Garay,} Orquideologia 9: 118. 1974.

This species has been recorded only from Belize, Honduras and Costa Rica (Luer 2006). It has not been yet recorded from Panama. A voucher is cited in TROPICOS database (J.P. Folsom 1496A, MO); however, we were not able to study it.

Phragmipedium caudatum (Lindl.) Rolfe, Orchid Rev. 4: 332. 1896.

This is a South American species. The name has been misapplied to specimens of Phragmipedium humboldtii (Warsz.) J.T.Atwood \& Dressler (Atwood \& Dressler 1998).

Platythelys querceticola (Lind1.) Garay, Bradea 2: 197. 1977.

Although widely distributed in the Neotropics, Ormerod (2013) did not cite any specimen from Panama. We were unable to locate Panamanian specimens in the field or in herbaria.

Platystele lancilabris (Rchb.f.) Schltr., Repert. Spec. Nov. Regni Veg. Beih. 19: 102. 1923.

This species is endemic to Costa Rica (Luer 1990).

Pleurothallis crenata Lindl., Gard. Chron. 1846: 207. 1846.

This is a species from Mexico. This species was cited erroneously in Flora of Panama (Williams \& Allen 1908, Correa et al. 2004).

Pleurothallis luctuosa Rchb.f., Linnaea 41: 48. 1876.

Though no herbarium vouchers have been seen for Panama, it is expected to be found in the country (Luer 2003b, Pupulin et al. 2010a).

Pleurothallis monocardia Rchb.f., Bonplandia (Hannover) 3: 72. 1855.

This species ranges from Colombia to Ecuador. It is not present in Panama (Govaerts 2003).

Pleurothallis platysepala Schltr., Repert. Spec. Nov. Regni Veg. Beih. 7: 111. 1920.

This species is found in Colombia and it has not yet been recorded in Panama (Govaerts 2003).

Polycycnis lehmanni Rolfe, Bull. Misc. Inform. Kew 365. 1894.

This species is known from Colombia. It has not been recorded in Panama (Gerlach 2004).

Polycycnis muscifera (Lindl. \& Paxton) Rchb. f., Bonplandia 3(15-16): 218. 1855.

This species is known from Venezuela, Colombia and Bolivia. It has not been recorded in Panama (Gerlach 2004).

Polystachya cerea Lindl., Edwards's Bot. Reg. 26(Misc.): 86. 1840.

This species has not yet been recorded in Panama.

Ponthieva ephippium Rchb.f., Linnaea 28: 382. 1857. This species was described from Mexico and it is also present in Guatemala and Honduras (Govaerts 2003). Specimens from Panama may correspond to $P$. racemosa.

Ponthieva maculata Lindl., Ann. Mag. Nat. Hist. 15: 385.1845.

According to Dressler (2003c), this name based on Colombian material has been wrongly applied to Central American populations of $P$. brenesii Schltr. and P. formosa Schltr.

Prescottia cordifolia Rchb.f., Bonplandia (Hannover) 3: 66.1855.

The taxonomy of this genus in Panama is still unclear. This species is recorded from Colombia to Ecuador. We were unable to identify specimens in herbaria corresponding to this concept.

Prosthechea vespa (Vell.) W.E.Higgins, Phytologia 82: 381.[1998].

The species complex related to $P$. vespa is still in need of a taxonomic revision. We have observed several specimens of this complex in the field with highly morphological variation in Panama. Some authors suggest the name $P$. crassilabia for the Central American species (Carnevali \& Ramírez-Morillo 2003).

Rhynchostele stellata (Lindl.) Soto Arenas \& Salazar, Orquídea (Mexico City), n.s., 13: 151. 1993. 
A species from Mexico, Guatemala, Honduras and El Salvador. A record from Costa Rica is dubius (Hágsater $\&$ Soto-Arenas 2003). No herbarium records from Panama were found for this species.

Sarcoglottis acaulis (Sm.) Schltr., Repert. Spec. Nov. Regni Veg. Beih. 6: 53. 1919.

In Costa Rica and Panama, some specimens have been treated erroneously as Sarcoglottis acaulis (J. E. Sm.) Schltr., a species from South America no longer considered to occur in Mesoamerica (Christenson 1991c).

Scaphyglottis graminifolia (Ruiz \& Pav.) Poepp. \& Endl., Nov. Gen. Sp. P1. 1: 59. 1836.

A species from South America. It has not yet been recorded in Panama.

Sigmatostalix picta Rchb.f. in W.G.Walpers, Ann. Bot. Syst. 5: 859. 1864.

A species from South America. It has not yet been recorded in Panama.

Sobralia candida (Poepp. \& Endl.) Rchb.f., Fl. Serres Jard. Eur. 8: 247. 1853.

A species from Venezuela, Colombia, Ecuador and Peru not yet found in Panama (Govaerts 2003).

Sobralia decora Bateman, Orchid. Mexico Guatemala: t. 26.1841 .

According to Dressler (2012), S. decora is a species restricted to southern Mexico, Belize, Guatemala and northern Honduras, whereas the material identified as S. decora in Nicaragua, Costa Rica and Panama better correspond to S. fenzliana.

Specklinia alexii (A.H.Heller) Pridgeon \& M.W.Chase, Lindleyana 16: 256. 2001.

This species is only known from Nicaragua (Luer 2006).

Specklinia endotrachys (Rchb.f.) Pridgeon \& M.W.Chase, Lindleyana 16: 257. 2001.

The S. endotrachys complex was revised by Pupulin et al. (2013). Specklinia endotrachys is an endemic species to Costa Rica. Although $S$. endotrachys is not recorded in Panama, three species previously treated under that name are recognized: S. pfavii, S. remotiflora and $S$. spectabilis.

Specklinia lanceola (Sw.) Lindl., Gen. Sp. Orchid. P1.: 8. 1830.

This species has been recorded from Mexico, Jamaica and Costa Rica (Luer 2006). No vouchers from Panama have been found.
Stanhopea graveolens Lindl., Edwards's Bot. Reg. 26(Misc.): 59. 1840.

This is a species distributed from Mexico to Honduras (Govaerts 2003).

Stanhopea oculata (Lodd.) Lindl., Gen. Sp. Orchid. Pl.: 158. 1832.

This species ranges from Mexico to northern Nicaragua (Hágsater \& Soto-Arenas 2003). We have not seen specimens in herbaria of $S$. oculata. It was cited by Williams \& Whitten (1988) in the key to the species of Stanhopea from Panama; however, no voucher was cited.

Stelis aprica Lindl., Companion Bot. Mag. 2: 353. 1837.

This is a South American species not found in Panama (Govaerts 2003).

Stelis alajuelensis Pridgeon \& M.W. Chase, Lindleyana 17(2): 98. 2002.

According to Karremans (2012), several speciemens in herbaria have been erroneously identified as $S$. alajuelensis $(=D$. ramonensis), an endemic species to Costa Rica. At the moment, no specimens referable to this concept have been found in Panama.

Stelis cooperi Schltr., Repert. Spec. Nov. Regni Veg. 3: 276.1907.

This species is endemic to Costa Rica (Luer 2003c).

Stelis janetiae (Luer) Pridgeon \& M.W.Chase, Lindleyana 16: 264. 2001.

This species is endemic to Costa Rica. It is likely found in Panama as the type specimen was collected in the southern region of Costa Rica near the border with Panama. It is related to the Panamanian endemic $S$. guttata.

Stelis maxima Lindl., Ann. Mag. Nat. Hist. 15: 106. 1845.

A South American species not recorded in Panama (Govaerts 2003).

Stelis rodrigoi (Luer) Pridgeon \& M.W.Chase, Lindleyana 16: 266. 2001.

Specimens from Panama correspond to S. furculifera (Dressler \& Bogarín 2007a). No vouchers of $S$. rodrigoi have been seen from Panama.

Stelis sclerophylla (Lind1.) Karremans, Lankesteriana 13(3): 330. 2014.

According to Luer (2003c) this name was applied erroniously in Costa Rica and Panama for specimens that correspond to P. dolichopus=Stelis lamprophylla. 
Stelis velaticaulis (Rchb.f.) Pridgeon \& M.W.Chase, Lindleyana 16: 267. 2001.

This is a South American species not yet recorded in Panama (Luer 1998a).

Telipogon dendriticus Rchb.f., Otia Bot. Hamburg.: 6. 1878.

A species from South America. It has not yet been recorded in Panama.

Telipogon minutiflorus Kraenzl., Ann. Naturhist. Mus. Wien 33: 14. 1920.

This species is endemic to Costa Rica (Dressler 2003c).

Telipogon radiatus Rchb.f., Linnaea 41: 70. 1876.

A species from South America. It has not yet been recorded in Panama.

Trichocentrum carthagenense (Jacq.) M.W.Chase \& N.H.Williams, Lindleyana 16: 137. 2001.

This is a species from the Pacific coast of Colombia and Venezuela. Panamanian specimens correspond to Trichocentrum crispiflorum (Schltr.) Bogarín.

Trichosalpinx ciliaris (Lindl.) Luer, Phytologia 54: 395. 1983.

This species ranges from Mexico to Costa Rica. It has not been recorded in Panama (Luer 1997a).

Trichosalpinx intricata (Lindl.) Luer, Phytologia 54: 396. 1983.

This species is restricted to South America. Luer (1997a) did not record specimens from Panama.

Triphora trianthophora subsp. mexicana (S.Watson) Medley, Selbyana 12: 102. 1991.

The genus Triphora is poorly known in Panama. This species has been recorded in the country (Correa et al. 2004) as T. mexicana; however, we were unable to study specimens in herbaria that fit with this concept. Goaverts (2003) accepted this species as T. triantophora subsp. mexicana. Dressler (2003c) treated the Costa Rican vouchers of $T$. triantophora subsp. mexicana
(Pupulin 2002) as T. debilis.

Trevoria glumacea Garay, Orquideologia 5: 8. 1970. García-Castro et al. (1993) and Correa et al. (2004) recorded this species from Panama; however, no vouchers were cited. We have not found specimens in herbaria. Dressler (2003c) cited T. glumacea only from Nicaragua to Costa Rica. Although the genus is likely to be found in Panama it is excluded in absence of herbarium vouchers.

Trevoria zahlbruckneriana (Schltr.) Garay, Orquideología 5: 8. 1970.

Dressler (2003c) cited T. zahlbruckneriana only from Nicaragua to Costa Rica. See the above discussion.

Vanilla insignis Ames, Bot. Mus. Leafl. 2: 101. 1934. This species ranges from Mexico, Belize, Guatemala and Honduras. It was recorded from Panama (Dressler 2003c, Correa et al. 2004); however, the report may correspond to $V$. dressleri (Soto-Arenas \& Dressler 2010).

Warczewiczella marginata Rchb.f., Bot. Zeitung (Berlin) 10: 636. 1852.

Warczewiczella marginata is a species found in Colombia and Venezuela. It has not yet recorded in Panama. Specimens of W. lipscombiae from Panama were erroniously treated under W. marginata (D'Arcy 1987, Correa et al. 2004).

Zootrophion atropurpureum (Lind1.) Luer, Selbyana 7: 80. 1982.

According to (Luer 2004), this species is recorded from Jamaica, Cuba, Haití, Dominican Republic and Brazil. It has not been recorded in Central America and Panama.

Zootrophion hypodiscus (Rchb.f.) Luer, Selbyana 7: 84. 1982.

This species is known from Colombia and Ecuador. It has not been recorded in Panama (Luer 2004). 


\section{Literature Cited}

Ackerman, J.D. 2007. Invasive orchids: weeds we hate to love?. Lankesteriana 7: 19-21.

Ackerman, J.D. 2014. Rapid transformation of orchid floras. Lankesteriana 13(3): 157-164. doi: http://dx.doi.

org/10.15517/lank.v13i3.14349

Allen, P.H. 1949a. Orchidaceae. Pp. 1-132 in: R.E. Jr. Woodson \& R.W. Schery (eds.), Flora of Panama. Part III, Fascicle

4. Ann. Missouri Bot. Gard. 36. doi: http://dx.doi.org/10.2307/2394411

Allen, P.H. 1949b. Orchidaceae. Pp. 133-245 in: R.E. Jr. Woodson \& R.W. Schery (eds.), Flora of Panama. Part III, Fascicle

5. Ann. Missouri Bot. Gard. 36. doi: http://dx.doi.org/10.2307/2394574

Allen, P.H. 1953. The Orchids of Panama, annotated checklist of genera and species. Part 7. Orchid Journ. 2: 319.

Ames, O. \& C. Schweinfurth. 1925. New or noteworthy species of orchids from the American Tropics. Schedul. Orch. 8: 41.

Ames, O. \& C. Schweinfurth. 1930. New or noteworthy orchids. Schedul. Orch. 10: 1-112.

Ames, O. \& C. Schweinfurth. 1937. A new genus of the Sobraliae. Bot. Mus. Leaf. 5(2): 33-35.

Ames, O. 1921. Additions to the orchid flora of Panama. Proc. Biol. Soc. Wash. 34: 149-154.

Ames, O. 1922a. New or noteworthy Orchids from different parts of the world. Orchidaceae (Ames) 7: 88.

Ames, O. 1922b. Orchidaceae quaedam Americanae. Schedul. Orch. 1: 1-24.

Ames, O. 1928. Orchidaceae. In: P.C. Standley (ed.), Flora of the Panama canal zone. Contributions from the United States

National Herbarium. Vol. 27. Doi 10.1126/science.67.1738.423

Ames, O. 1944. Destruction of the Schlechter Herbarium by bombing. Amer. Orchid Soc. Bull. 13: 105-106.

ANAM, 2000. Mapa de vegetación de Panamá - escala 1:500,000. ANAM/Corredor Biológico Mesoamericano del Atlántico Panameño.

ANAM, 2010. Atlas ambiental de la República de Panamá. Editora Novo Art, S.A. Primera edición. Panamá.

Archila, F. 2000. Estudio taxonómico - morfológico y delimitación de tres géneros de la subtribu Pleurothallidinae (Orchidaceae). Revista Guatemal. 3(1): 33-88.

Archila, F. 2010. Selbyana Archila un nuevo género en la Lycastinae Schltr. Revista Guatemal. 13(1): s.n.

Atwood, J.T. 2003. Maxillaria. Pp. 291-332 in: B. E. Hammel, M. H. Grayum, C. Herrera \& N. Zamora (eds.), Manual de plantas de Costa Rica - Volumen III, monocotiledóneas (Orchidaceae-Zingiberaceae). Monogr. Syst. Bot. Missouri Bot. Gard. 93.

Atwood, J.T. \& R.L. Dressler. 1998. Clarifications and new combinations in the Phragmipedium caudatum complex from Central America. Selbyana (2): 245-248

Beurling, P.J. 1800-1866. Primitiae florae portobellensis, sive, Enumeratio plantarum vascularium quas juxta oppidum Portobello in Isthmo Panamensi Americae Centralis, mense Aprili anno 1826, legit doct. Joh. Eman. Billberg / Stockholm. doi: http://dx.doi.org/10.5962/bhl.title. 9043

Blanco, M.A. \& G. Barboza. 2005. Pseudocopulatory pollination in Lepanthes (Orchidaceae: Pleurothallidinae) by fungus gnats. Annals of Botany 95: 763-772. doi: 10.1093/aob/mci090

Blanco, M.A., G. Carnevali Fernández-Concha, W.M. Whitten, R.B. Singer, S. Koehler, N.H. Williams, I. Ojeda, K.M. Neubig \& L. Endara. 2007. Generic realignments in Maxillariinae (Orchidaceae). Lankesteriana 7: 515-537. doi: http:// dx.doi.org/10.15517/lank.vi.7935

Bogarín, D., A.P. Karremans \& F. Pupulin. 2008. New records and species of Orchidaceae from Costa Rica. Lankesteriana 8(2): 53-74. doi: http://dx.doi.org/10.15517/lank.v0i0.7927

Bogarín, D., A.P. Karremans, R. Rincón \& B. Gravendeel. 2013a. A new Specklinia (Orchidaceae: Pleurothallidinae) from Costa Rica and Panama. Phytotaxa 115(2): 31-41. doi: http://dx.doi.org/10.11646/phytotaxa.115.2.1

Bogarín, D., F. Pupulin, C. Arrocha \& J. Warner. 2013b. Orchids without borders: studying the hotspot of Costa Rica and Panama. Lankesteriana 13(1-2): 13-26. doi: http://dx.doi.org/10.15517/lank.v0i0.11529

Bogarín, D., Z. Serracín \& Z. Zamudio. 2014. Illustrations and studies in Neotropical Orchidaceae. The Specklinia condylata group (Orchidaceae) in Costa Rica and Panama. Lankesteriana 13(3): 185-206. ttp://dx.doi.org/10.15517/ lank.v13i3.14352

Brundu, G. \& I. Camarda. 2013. The Flora of Chad: a checklist and brief analysis. PhytoKeys, 23: 1-18. doi: 10.3897/ phytokeys.23.4752.

Burger, W.C. 1980. Why are there so many kinds of flowering plants in Costa Rica? Brenesia 17: 371-388.

Cameron, K.M. 2001. An expanded phylogenetic analysis of Orchidaceae using three plastid genes: rbcL, atpB, and psbA. 
Am. J. Bot. 88: Supplement, [abstract 2].

Cameron, K.M., M.W. Chase, W.M. Whitten, P.J. Kores, D.C. Jarrell, V.A. Albert, T. Yukawa, H.G. Hills \& D.H. Goldman. 1999. A phylogenetic analysis of the Orchidaceae: evidence from rbcL nucleotide sequences. Am. J. Bot. 86: 208-224.

Carnevali Fernández-Concha, G. 2001. A synoptical view of the classification of Cryptocentrum (Orchidaceae), new taxa, and a key to the genus. Harvard Pap. Bot. 5(2): 467-486.

Carnevali Fernández-Concha, G., R. Duno de Stefano, G. A. Romero-González, R. Balam, W. Cetzal Ix, J. L. Tapia-Muñoz \& I. M. Ramírez. 2009. A reappraisal of the turtle-orchids, genus Chelyorchis (Oncidiinae: Orchidaceae): molecular, phylogenetic, and morphometric approaches. J. Torrey Bot. Soc. 136: 164-185.

Carnevali Fernández-Concha, G. \& I.M. Ramírez-Morillo. 2003. Prosthechea crassilabia. P. 7 in: P. Berry, B. Holst \& K. Yatskievych (eds.), Flora of the Venezuelan Guayana Missouri Botanical Garden Press, St. Louis, Missouri, USA.

Carnevali, G., P. Ortiz V., \& L. Peraza-Flores. 2012. The "lady in white", a showy new species of Cryptocentrum (Orchidaceae, Maxillariinae) from Colombia. Phytotaxa 56: $1-8$.

Chase, M. W. 1986. A monograph of Leochilus (Orchidaceae). ASPT, Ann Arbor, Michigan.

Chase, M.W. \& J.D. Palmer. 1989. Chloroplast DNA systematics of lilioid of the lilioid monocots: feasibility, resources, and an example from the Orchidaceae. Am. J. Bot. 76: 1720-1730. doi: 10.2307/2444471

Chase, M.W. \& J.D. Palmer. 1992. Floral morphology and chromosome number in subtribe Oncidiinae (Orchidaceae): evolutionary insights from a phylogenetic analysis of chlomplast DNA restriction site variation. Pp. 324-339 in: S. Soltis, D. E. Soltis, and J. J. Doyle (eds.), Molecular systematics of plants. New York, NY: Chapman and Hall.

Chase, M.W., J.V. Freudenstein, K.M. Cameron \& R.L. Barrett. 2003. DNA data and Orchidaceae systematics: a new phylogenetic classification. Pp. 69-89 in: K. W. Dixon, S. P. Kell, R. L. Barrett, \& P. J. Cribb (eds.), Orchid conservation. Kota Kinabalu: Natural History Publications.

Chase, M.W., N.H. Williams, K.M. Neubig \& W.M. Whitten. 2008. Taxonomic transfers in Oncidiinae to accord with Genera Orchidacearum, Volume 5. Lindleyana 21(3) in Orchids 77: 20-31.

Chase, M.W. \& W.M. Whitten. 2011. Further taxonomic transfers in Oncidiinae (Orchidaceae). Phytotaxa 20: 26-32.

Chiron, G.R. \& V.P. Castro Neto. 2003. Révision du genre
Prosthechea Knowles \& Wescott [sic] et nouveau genre dans la tribu Laeliinae (Orchidaceae). Richardiana 4: 9-35.

Christenson, E.A. 1991a. Mesoamerican orchid studies I: Orchids of Panama. Lindleyana. 6(1): 42-48.

Christenson, E.A. 1991b. Mesoamerican orchid studies II: Powell's Panamanian Orchidaceae. Lindleyana 6(3): 124-132.

Christenson, E.A. 1991c. Mesoamerican orchid studies III: A new Sarcoglottis. Lindleyana 6(3): 133.

Christenson, E.A. 2006. Brevilongium, un nouveau genre néotropical (Orchidaceae: Oncidiinae). Richardiana 6: 45-49.

Coates, A.G. \& J.A. Obando 1996. The geologic evolution of the Central American isthmus. Pp. 21-56 in: J.B.C. Jackson, A.F. Budd \& A.G. Coates (eds.), Evolution and environments in tropical America. Chicago, IL: Chicago University Press.

Coates, A.G., J.B.C. Jackson, L.S. Collins, T.M. Cronin, H.J. Dowsett, L.M. Bybell, P. Jung \& J.A. Obando. 1992. Closure of the Isthmus of Panama: The nearshore marine record of Costa Rica and western Panama. Geol. Soc. Am. Bull. 104: 814-828.

Coates, A.G., L.S. Collins, M.P. Aubry \& W.A. Berggren. 2004. The geology of the Darien, Panama, and the late Miocene-Pliocene collision of the Panama arc with northwestern South America. Geol. Soc. Am. Bull. 116: 1327-1344.

Cohen, I.M. \& J.D. Ackerman. 2009. Oeceoclades maculata, an alien tropical orchid in a Caribbean rainforest. Ann. Bot. 104: 557-563. doi: 10.1093/aob/mcn191

Correa, M.A., C. Galdames \& M.S. De Stapf. 2004. Catálogo de las plantas vasculares de Panamá. 1. ANAM. STRI y UP. Editorial Novoart. Panamá. Pp. 599.

Croat, T. 1978. The flora of Barro Colorado Island. Stanford University, Stanford, CA.

D'Arcy, W.G. 1987. Flora of Panama. Checklist and Index. Part 1: The introduction and checklist. Monogr. Syst. Bot. Missouri Bot. Gard. 17: v-xxx, 1-328.

Dodson, C.H. 2003. Why are there so many orchid species? Lankesteriana 7: 99-103.

Dodson, C.H. \& R. Escobar. 1993. El género Teliopogon en Panama. Orquideología 18(3): 273-290.

Dressler, R.L. 1993. Field guide to the Orchids of Costa Rica and Panama. Comstock Publishing Associates, Ithaca, New York.

Dressler, R.L. 1997. Novedades en orquídeas Mesoamericanas - I / Mesoamerican orchid novelties I. Orquideología 20(3): 253-265

Dressler, R.L. 2000. Oncidium maduroi: an attractive new species from Panama. Orchids, Mag. Amer. Orchid Soc. 69(8): 761-763.

Dressler, R.L. 2002. The major sections or groups within 
Sobralia, with four new species from Panama and Costa Rica, S. crispissima, S. gloriana, S. mariannae and $S$. nutans. Lankesteriana 5: 9-15.

Dressler, R.L. 2003a. Dos especies Panameñas nuevas y llamtivas de Trichopilia / Two attractive new species of Trichopilia from Panama. Orquideología 22(3): 245249 / 250-253.

Dressler, R.L. 2003b. Mesoamerican orchid novelties 4, Malaxis. Selbyana 24(2): 141-143.

Dressler, R.L. 2003c. Orchidaceae. Pp. 1-595 in: B. E. Hammel, M. H. Grayum, C. Herrera \& N. Zamora (eds.). Manual de plantas de Costa Rica - Volumen III, monocotiledóneas (Orchidaceae-Zingiberaceae). Monogr. Syst. Bot. Missouri Bot. Gard. 93.

Dressler, R.L. 2003d. Chysis violacea, una especie nueva y llamativa de Panamá oriental, con el pronóstico de otra aún no descrita / Chysis violacea, an attractive new species from eastern Panama, with the prediction of another to come. Orquideología 22(3): 237-240 / 241-243

Dressler, R.L. 2003e. The genus Telipogon. Delightful Andean dwarfs with new species from Panama. Orchids, Mag. Amer. Orchid Soc. 72(2): 112-117.

Dressler, R.L. 2004a. Der Scaphyglottis pulchella Komplex/ The Scaphyglottis pulchella complex. J. Orchideenfr. 11: 305-315.

Dressler, R.L. 2004b. Encyclia elegantula: an attractive and unexpected new species from Panama. Orchid Digest 68(4): 243-244.

Dressler, R.L. 2004c. Una clave preliminar para las Trichopilia centroamericanas/A preliminary key to the species of Trichopilia in Mesoamerica. Orquideología 23: $35-48$.

Dressler, R.L. 2004d. Two new species of Elleanthus from Panama. J. Orchideenfr. 11: 142-148.

Dressler, R.L. 2005a. La Chinela, alive and well in western Panama: notes on the Panamanian long-tailed Phragmipedium. Orchid Digest 69 (2): 86-91.

Dressler, R.L. 2005b. Six more new species of Sobralia from Panama. Orchids 74(12): 937-941.

Dressler, R.L. 2006a. New species of Telipogon in western Panama II. J. Orchideenfr. 13(3): 209-217.

Dressler, R.L. 2006b. Chysis pluricostata Dressler, una especie nueva y distintiva de Panamá. Orquideología 24: 141-151.

Dressler, R.L. 2006c. New species of Telipogon from western Panama II. J. Orchideenfr. 3(3): 209-217.

Dressler, R.L. 2007. New species of Telipogon from western Panama III. Orchideen J. 14(1): 12-16.

Dressler, R.L. 2012. Sobralia decora. The species and its cousins in Mexico and Central America. Orchids (West Palm Beach) 81(5): 308-310.

Dressler, R.L. \& D. Bogarín. 2007a. A new and bizarre species in the genus Condylago (Orchidaceae: Pleurothallidinae) from Panama. Harvard. Pap. Bot. (12)1: 1-5. doi: http://dx.doi.org/10.3100/10434534(2007)12[1:ANABSI]2.0.CO;2

Dressler, R.L. \& D. Bogarín. 2007b. Two attractive new species of Sobralia from Panama. Orchids 76 (9): 696-701.

Dressler, R.L. \& D. Bogarín. 2010. Some new Sobraliae from Costa Rica and Panama. Lankesteriana 9(3): 475-485. doi: http://dx.doi.org/10.15517/lank. v0i0. 12109

Dressler, R.L. \& F. Pupulin. 2003. Oncidium zelenkoanum (Orchidaceae), an unusual new species from Panama. Lankesteriana 8: 37-39.

Dressler, R.L. \& N.H. Williams. 2000. Chelyorchis, a new genus. P. 1130 in: G. A. Romero-Gonzalez \& G. Carnevali Fernández-Concha. Orchids of Venezuela: An illustrated field guide. Second edition. Armitano Editores. Caracas, Venezuela.

Dressler, R.L. \& W.E. Higgins. 2003. Guarianthe, a generic name for the "Cattleya" skinneri complex. Lankesteriana 3(2): 37-38.

Dudek, M. \& D.L. Szlachetko. 2010. New combinations in subtribe Elleanthinae (Orchidaceae, Epidendroideae). Richardiana 11: 1-12.

Dunsterville, G.C.K. \& L.A. Garay. 1961. Venezuelan orchids illustrated. Vol 2. André Deutsch Limited. Londres. 360 pp.

Fernández, M., D. Bogarín, A.P. Karremans \& D. Jiménez. 2014. New species and records of Orchidaceae from Costa Rica III. Lankesteriana 13(3): 259-282. doi: http://dx.doi.org/10.15517/lank.v13i3.14363

Funk, V., T. Hollowell, P.E. Berry, C. Kelloff \& S.N. Alexander. 2007. Checklist of the plants of the Guiana Shield (Venezuela: Amazonas, Bolivar, Delta Amacuro; Guyana, Surinam, French Guiana). Contr. U.S. Natl. Herb. 55: 1-584.

Garay, L.A. 1967. Studies in American orchids, 6. Bot. Mus. Leafl. 21: 249-264.

Garay, L.A. \& G. Romero-González. 2005. Galeandra. Pp. 324-327 in: F. Pupulin. 2005 (ed.), Vanishing beauty: native Costa Rican orchids. Vol. 1. AciantheraKegeliella. Editorial Universidad de Costa Rica, San José, Costa Rica. Pp. 421.

García-Castro, J.B., D.E. Mora-Retana \& M.E.R. Rossi. 1993. Lista de orquídeas comunes a Costa Rica y Panama. Brenesia 39-40: 93-107.

Gerlach, G. 2004. Stanhopeinae mesoamericanae II (Orchidaceae). Dos especies nuevas: Polycycnis blancoi y Coryanthes maduroana. Lankesteriana 4: 67-73.

Gerlach, G. \& R.L. Dressler. 2003. Stanhopeinae Mesoamericanae I. Lankesteriana 8: 23-30.

Govaerts, R. 2003. World Checklist of Monocotyledons 
Database in ACCESS: 1-71827. The Board of Trustees of the Royal Botanic Gardens, Kew.

Guo, Y.-Y., Y.-B. Luo, Z.-J. Liu \& X.-Q. Wang. 2012. Evolution and biogeography of the slipper orchids: Eocene vicariance of the conduplicate genera in the Old and New World tropics. PLoS ONE 7: e38788. doi:10.1371/journal.pone.0038788

Gustafsson, A.L., C.F. Verola \& A. Antonelli. 2010. Reassessing the temporal evolution of orchids with new fossils and a Bayesian relaxed clock, with implications for the diversification of the rare South American genus Hoffmannseggella (Orchidaceae:Epidendroideae). BMC Evol. Biol. 10: 177. doi10.1186/1471-2148-10177

Hágsater, E. 1993. Epidendrum allochronum Hágsater P1. 104 in: E. Hágsater \& G. Salazar (eds.), The genus Epidendrum. Part 1. A century of new species in Epidendrum. Icon. Orchid. (Mexico). 2: pl. 101-200.

Hágsater, E. 1999. The genus Epidendrum. Part 2. "A second century of new species in Epidendrum". Icon. Orchid. (Mexico) 3: pl. 301-400.

Hágsater, E. 2001. The genus Epidendrum. Part 3. A third century of new species in Epidendrum. Icon. Orchid. (Mexico). 4: pl. 401-500.

Hágsater, E. 2003. Epidendrum. Pp. 101-169 in: B. E. Hammel, M. H. Grayum, C. Herrera \& N. Zamora (eds.). Manual de plantas de Costa Rica - Volumen III, monocotiledóneas (Orchidaceae-Zingiberaceae). Monogr. Syst. Bot. Missouri Bot. Gard. 93.

Hágsater, E. 2010. The genus Epidendrum. Part 9. "Species new \& old in Epidendrum". Icon. Orchid. (Mexico) 13: i-xxii, pl. 1301-1400.

Hágsater, E. 2013. The genus Epidendrum. Part 10. "Species new \& old in Epidendrum". Icon. Orchid. (Mexico) 14: i-iii, pl. 1401-1500.

Hágsater, E. \& G.A. Salazar. 1990. Epidendrum radicans Pavón ex Lindl. P1. 40 in: E. Hágsater \& G.A. Salazar. Orchids of Mexico. Part 1. Icon. Orchid. (Mexico). Herbario AMO, México, D.F.

Hágsater, E \& G.A. Salazar. 1993. The genus Epidendrum. Part 1. A century of new species in Epidendrum. Icon. Orchid. (Mexico). 2: pl. 101-200.

Hágsater, E. \& L. Sánchez-Saldaña (eds.). 2004. The genus Epidendrum. Part 4. "A fourth century of new species in Epidendrum". Icon. Orchid. (Mexico) 7: pl. 701-800.

Hágsater, E. \& L. Sánchez-Saldaña (eds.). 2006. The genus Epidendrum. Part 5. Species new \& old in Epidendrum. Icon. Orchid. (Mexico) 8: i-xi, pl. 801-900.

Hágsater, E. \& L. Sánchez-Saldaña (eds.). 2007. The genus Epidendrum. Part 6. Species new \& old in Epidendrum. Icon. Orchid. (Mexico) 9: i-xiii, pl. 901-1000.

Hágsater, E. \& L. Sánchez-Saldaña (eds.). 2008. The genus Epidendrum. Part 7. Species new \& old in Epidendrum.
Icon. Orchid. (Mexico) 11: i-xxvii, pl. 1101-1200.

Hágsater, E. \& L. Sánchez-Saldaña (eds.). 2009. The genus Epidendrum. Part 8. Species new \& old in Epidendrum. Icon. Orchid. 12: pl. 1201-1300.

Hágsater, E. \& M. Soto (eds.). 2003. Orchids of Mexico. Parts $2 \&$ 3. Icon. Orchid. 5-6: pls. 501-700.

Hágsater, E \& M.A. Soto Arenas. 2005. Transfers to Epidendrum L. from Oerstedella Rchb. f. Lankesteriana 5(1): 73-75.

Hamer, F. \& L.A. Garay. 1995 [1997]. Bulbophyllum section Bulbophyllaria in the Neotropics B o 1 . Inform. Inst. Bot. Univ. Guadalajara 3(1-3): 5-26, figs. $1-25$.

Hemsley, W.B. 1884. Orchidaceae. Pp. 197-308 in: F. D. Godman \& O. Salvin (eds.), Biologia Centrali Americana. 56 (Bot. 3). London, R. H. Porter.

Hills, H.G. 2012. Taxonomic revision of Dressleria (Orchidaceae, Catasetinae). Phytoneuron 2012-48: $1-28$.

Hinds, R.B. 1844. The botany of the voyage of H.M.S. Sulphur under the command of Captain Sir Edward Belcher; R. N., C. B., F. R., G. S., etc. during the years 1836-1842. Smith, Elder \& Co., London. http://dx.doi. org/10.5962/bhl.title.908

IGTG, 2007. Atlas nacional de la República de Panamá. 4th ed. Panama : Instituto Geografico Nacional "Tommy Guardia", Panama.

IPNI, 2014. The international plant names index. Available from: http:// http://www.ipni.org (August 2014).

Karremans, A.P. 2012. Illustrations and Studies in Neotropical Orchidaceae. 3. On the identity of Dracontia pachyglossa and D. ramonensis; with a new species, D. lueriana (Pleurothallidinae). Ann. Naturhist. Mus. Wien, B. 113: 119-132.

Karremans, A.P. 2014. Lankesteriana, a new genus in the Pleurothallidinae (Orchidaceae). Lankesteriana 13(3): 319-332. doi: http://dx.doi.org/10.15517/lank. v13i3. 14368

Karremans, A.P., F.T. Bakker, F. Pupulin, R. Solano-Gómez, \& M.J.M. Smulders. 2013. Phylogenetics of Stelis and closely related genera (Orchidaceae: Pleurothallidinae). Plant Syst. Evol. 29(1): 69-86. doi: 10.1007/s00606012-0712-7

Karremans, A.P., D. Bogarín, M. Fernández, C.M. Smith \& M.A. Blanco. 2012. New species and records of Orchidaceae from Costa Rica. II. Lankesteriana 12(1): 19-51.

Kirby, S.H. 2007. Geological processes and orchid biogeography with applications to southeast Central America. Lankesteriana 7: 53-55.

Kirby, S.H. 2011. Active mountain building and the distribution of "core" Maxillariinae species in tropical Mexico and Central America. Lankesteriana 11: 275- 
291.

Kohlmann, B., J. Wilkinson, O. García Valverde \& K. Lulla. 2002. Costa Rica desde el espacio. Costa Rica from the Space. Editorial Heliconia, Fundacion Neotropica, San José, Costa Rica.

Leigh, E.G., A. O'Dea \& G.J. Vermeij. 2014. Historical biogeography of the Isthmus of Panama. Biol Rev Camb Philos Soc. 89(1): 148-72. doi: 10.1111/brv.12048.

Lindley, J. 1830-1840. The genera and species of orchidaceous plants. Ridgways, London, pp. 1-553. http://dx.doi.org/10.5962/bhl.title.499

Luer, C.A. 1986. Icones Pleurothallidinarum III. Systematics of the Pleurothallidinae (Orchidaceae). Monogr. Syst. Bot. Missouri Bot. Gard. 20: 1-109.

Luer, C.A. 1987. New Lepanthes species from Costa Rica and Panama. Lindleyana 2: 185-217.

Luer, C.A. 1990. Icones Pleurothallidinarum VII. Systematics of Platystele (Orchidaceae). Monogr. Syst. Bot. Missouri Bot. Gard. 38: 1-184.

Luer, C.A. 1991. Icones Pleurothallidinarum VIII. Systematics of Lepanthopsis, Octomeria subgenus Pleurothallopsis, Restrepiella, Restrepiopsis, Salpistele, and Teagueia. Addenda to Platystele, Porroglossum, and Scaphosepalum. Monogr. Syst. Bot. Missouri Bot. Gard. 39: 1-161.

Luer, C. A. 1992. Icones Pleurothallidinarum IX. Systematics of Myoxanthus. Addenda to Platystele, Pleurothallis subgenus Scopula, and Scaphosepalum (Orchidaceae). Monogr. Syst. Bot. Missouri Bot. Gard. 44: $1-128$.

Luer, C.A. 1996. Icones Pleurothallidinarum XIV: The genus Lepanthes, subgenus Lepanthes in Ecuador. Monogr Syst Bot Missouri Bot Gard 61: 1-255.

Luer, C.A. 1997a. Icones Pleurothallidinarum XV. Systematics of Trichosalpinx. Addenda to Dracula, Masdevallia, Myoxanthus, and Scaphosepalum. Corrigenda to Lepanthes of Ecuador (Orchidaceae). Monogr. Syst. Bot. Missouri Bot. Gard. 64: 1-136.

Luer, C.A. 1997b. New species of Lepanthes and Pleurothallis (Orchidaceae) from Guatemala, Panama, Peru, Suriname, and Venezuela. Lindleyana 12(1): 34-55.

Luer, C.A. 1998a. Icones Pleurothallidinarum XVI. Systematics of Pleurothallis subgenera Crocodeilanthe, Rhynchopera, Talpinaria. Addenda to Lepanthes of Ecuador, Masdevallia, Platystele, Pleurothallis, Restrepia, and Scaphosepalum (Orchidaceae). Monogr. Syst. Bot. Missouri Bot. Gard. 65: 1-122.

Luer, C.A. 1998b. Icones Pleurothallidinarum XVII. Systematics of subgen. Pleurothallis sect. Abortivae, sect. Truncatae, sect. Pleurothallis, subsect. Acroniae, subsect. Pleurothallis, subgen. Dracontia, subgen. Unciferia. Addenda to Dracula, Lepanthes,
Masdevallia, Porroglossum and Scaphosepalum (Orchidaceae). Monogr. Syst. Bot. Missouri Bot. Gard. 72: $1-121$.

Luer, C.A. 1999. lcones Pleurothallidinarum XVIII. Systematics of Pleurothallis Subgen. Pleurothallis Sect. Pleurothallis Subsect. Antenniferae, Subsect. Longiracemosae, Subsect. Macrophyllae-Racemosae, Subsect. Perplexae, Subgen. Pseudostelis, Subgen. Acuminatia. Mongr. Syst. Bot. Missouri Bot. Gard. 76: $1-182$.

Luer, C. A. 2000. Icones Pleurothallidinarum XX. Systematics of Jostia, Andinia, Barbosella, Barbrodria, Pleurothallis subgen. Antilla, subgen. Effusa, subgen. Restrepioidia. Addenda to Lepanthes, Masdevallia, Platystele, Pleurothallis, Restrepiopsis, Scaphosepalum and Teagueia. Monogr. Syst. Bot. Missouri Bot. Gard. 79: $1-140$.

Luer, C.A. 2001a. Icones Pleurothallidinarum XXI. Systematics of Masdevallia part three. Monogr. Syst. Bot. Missouri Bot. Gard. 86: 519-780.

Luer, C.A. 2001b. Miscellaneous new species in the Pleurothallidinae (Orchidaceae). Selbyana 22(2): 103-127.

Luer, C.A. 2002a. A systematic method of classification of the Pleurothallidinae versus a strictly phylogenetic method. Selbyana 23(1): 57-110.

Luer, C. A. 2002b. Icones Pleurothallidinarum XXIV. A First Century of New Species of Stelis of Ecuador, Part One. Addenda to Barbosella, Dracula, Dresslerella, Lepanthopsis, Platystele, Pleurothallis, Restrepia, Scaphosepalum, Teagueia, and Trichosalpinx. Monogr. Syst. Bot. Missouri Bot. Gard. 88: 1-122.

Luer, C.A. 2003a. Lepanthes. Pp. 216-255 in: B. E. Hammel, M. H. Grayum, C. Herrera \& N. Zamora (eds.), Manual de plantas de Costa Rica - Volumen III, monocotiledóneas (Orchidaceae-Zingiberaceae). Monogr. Syst. Bot. Missouri Bot. Gard. 93.

Luer, C.A. 2003b. Pleurothallis. Pp. 386-452 in: B. E. Hammel, M. H. Grayum, C. Herrera \& N. Zamora (eds.), Manual de plantas de Costa Rica - Volumen III, monocotiledóneas (Orchidaceae-Zingiberaceae). Monogr. Syst. Bot. Missouri Bot. Gard. 93.

Luer, C.A. 2003c. Stelis. Pp. 521-545 in: B. E. Hammel, M. H. Grayum, C. Herrera \& N. Zamora (eds.), Manual de plantas de Costa Rica - Volumen III, monocotiledóneas (Orchidaceae-Zingiberaceae). Monogr. Syst. Bot. Missouri Bot. Gard. 93.

Luer, C.A. 2004. Icones Pleurothallidinarum XXVI: Pleurothallis subgenus Acianthera and three allied subgenera; A Second Century of New Species of Stelis of Ecuador; Epibator, Ophidion, Zootrophion. Monogr. Syst. Bot. Missouri Bot. Gard. 95: 1-265.

Luer, C.A. 2005. Icones Pleurothallidinarum XXVII. 
Dryadella and Acronia sect. MacrophyllaceaeFasciculatae. Monogr. Syst. Bot. Missouri Bot. Gard. 103: 1-311.

Luer, C.A. 2006. Icones Pleurothallidinarum XXVIII. Reconsideration of Masdevallia, and the Systematics of Specklinia and vegetatively similar genera (Orchidaceae). Monogr. Syst. Bot. Missouri Bot. Gard. 105: 21-244.

Luer, C.A. 2007. Icones Pleurothallidinarum XXIX. A Third Century of Stelis of Ecuador, Systematics of Apoda-Prorepentia, Systematics of Miscellaneous Small Genera, Addenda New Genera, Species and Combinations (Orchidaceae). Monogr. Syst. Bot. Missouri Bot. Gard 112: 1-130.

Luer, C.A. 2009. Icones Pleurothallidinarum XXX. Lepanthes of Jamaica and Systematics of Stelis, Stelis of Ecuador, part four and addenda: systematic of Masdevallia, new species of Lepanthes from Ecuador, and miscellaneous new combinations. Mongr. Syst. Bot. Missouri Bot. Gard. 115.

Luer, C.A. 2010. Icones Pleurothallidinarum XXXI: Lepanthes of Bolivia; Systematics of Octomeria Species North and West of Brazil; Addenda and Corrigenda. Monogr. Syst. Bot. Missouri Bot. Gard. Botany 120.

Luer, C.A. 2011. Miscellaneous new species in the Pleurothallidinae (Orchidaceae) excluding species from Brazil. Harvard Pap. Bot 16(2): 311-360. doi: http:// dx.doi.org/10.3100/0.25.016.0206

Mansfeld, R. 1931. Blutenanalysen neuer Orchideen von R. Schlechter. Repert. Spec. Nov. Regni Veg. Beih. 59: tt. $1-81$.

Meneguzzo, T.E.C. 2012. Mudanças nomenclaturais em Goodyerinae do Novo Mundo (Orchidaceae). Orquidário 26, 3: 86-91.

Misas, G. 2005. Orquídeas de la Serranía del Baudó, Chocó, Colombia. Corporación Capitalina de Orquideología. Colombia. Pp.1-755.

Myers, N., R.A. Mittermeier, C.G. Mittermeier, G.A. da Fonseca \& J. Kent. 2000. Biodiversity hotspots for conservation priorities. Nature 403, 853-858. doi:10.1038/35002501

Ojeda, I., G. Carnevali Fernández-Concha \& G. A. RomeroGonzález. 2005. New species and combinations in Heterotaxis Lindley (Orchidaceae: Maxillariinae). Novon 15: 572-582.

Ojeda, I., G. Carnevali Fernández-Concha \& G. A. Romero-González. 2009. Nitidobulbon, a new genus of Maxillariinae (Orchidaceae). Novon 19: 96-101. doi: http://dx.doi.org/10.3417/2007039

Ormerod, P. 2002. Taxonomic changes in Goodyerinae (Orchidaceae: Orchidoideae). Lindleyana 17: 189238.

Ormerod, P. 2004. Notulae Goodyerinae. Oasis (Dora
Creek) Suppl. 3: 3-19.

Ormerod, P. 2005. Studies of neotropical Goodyerinae (Orchidaceae). Harvard Pap. Bot. 9(2): 391-423.

Ormerod, P. 2007. Studies of Neotropical Goodyerinae (Orchidaceae) 2. Harvard Pap. Bot. 11(2): 145-177.

Ormerod, P. 2008. Studies of Neotropical Goodyerinae (Orchidaceae) 3. Harvard Pap. Bot. 13(1): 55-87.

Ormerod, P. 2009. Studies of Neotropical Goodyerinae (Orchidaceae) 4. Harvard Pap. Bot. 14(2): 111-128. http://dx.doi.org/10.3100/025.014.0203

Ormerod, P. 2013. Studies of Neotropical Goodyerinae (Orchidaceae) 5. Harvard Pap. Bot. 18(1): 51-60. http:// dx.doi.org/10.3100/025.018.0108

Ossenbach, C. 2009. Orchids and orchidology in Central America: 500 years of history. Lankesteriana 9(12): 1-268. doi: http://dx.doi.org/10.15517/lank. v0i0.12019

Ossenbach, C., Dressler, R.L., F. Pupulin. 2007. Orchids of the American isthmus: checklist and conservation status. Ed. Orquideario 25 de mayo, San José, Costa Rica.

Pridgeon, A.M. \& M.W. Chase. 2001. A phylogenetic reclassification of Pleurothallidinae (Orchidaceae). Lindleyana 16(4): 235-271.

Pridgeon, A. M. \& M. W. Chase. 2002. Nomenclatural notes on Pleurothallidinae (Orchidaceae). Lindleyana 17: 98-101.

Pridgeon, A.M., P.J. Cribb, M.W. Chase \& F.N. Rasmussen. 1999. Genera Orchidacearum. Volume 1. General introduction, Apostasioideae, Cypripedioideae. Oxford: Oxford University Press.

Pridgeon, A.M., P.J. Cribb, M.W. Chase \& F.N. Rasmussen. 2001b. Genera Orchidacearum. Volume 2. Orchidoideae (Part 1). Oxford: Oxford University Press.

Pridgeon, A.M., P.J. Cribb, M.W. Chase \& F.N. Rasmussen. 2003. Genera Orchidacearum. Volume 3. Orchidoideae (Part 2), Vanilloideae. Oxford: Oxford University Press.

Pridgeon, A.M., P.J. Cribb, M.W. Chase \& F.N. Rasmussen. 2005a. Genera Orchidacearum. Volume 4. Epidendroideae (Part 1). Oxford: Oxford University Press.

Pridgeon, A.M., P.J. Cribb, M.W. Chase \& F.N. Rasmussen. 2005b. Genera Orchidacearum. Volume 5. Epidendroideae (Part 2). Oxford: Oxford University Press.

Pridgeon, A.M., P.J. Cribb, M.W. Chase \& F.N. Rasmussen. 2005c. Genera Orchidacearum. Volume 6. Epidendroideae (Part 3). Oxford: Oxford University Press.

Pridgeon, A.M., Solano-Gomez, R. \& Chase, M.W. 2001a. Phylogenetic relationships in Pleurothallidinae (Orchidaceae): combined evidence from nuclear and plastid DNA sequences. Am. J. Bot. 88: 2286-2308. 
http://dx.doi.org/10.2307/3558390

Pupulin, F. 2002. Catálogo revisado y anotado de las Orchidaceae de Costa Rica. Lankesteriana 4: 1-88.

Pupulin, F. 2003. A second look at the genus Sigmatostalix (Orchidaceae: Oncidiinae) in Costa Rica. Harvard Pap. Bot. 8(1): 35-59.

Pupulin, F. 2005a. Dichaea viridula (Orchidaceae: Zygopetalinae), a new species in the $D$. tenuifolia complex. Harvard Pap. Bot. 10(1): 83-87. doi: http://dx.doi.org/10.3100/10434534(2005)10[83:DVOZAN]2.0.CO;2

Pupulin, F. 2005b. Vanishing beauty: native Costa Rican orchids. Vol. 1. Acianthera-Kegeliella. Editorial Universidad de Costa Rica, San José, Costa Rica. Pp.421.

Pupulin, F. 2007. Contributions toward a reassessment of Costa Rican Zygopetalinae (Orchidaceae). A systematic revision of Dichaea in Costa Rica. Harvard Pap. Bot. 12(1): 15-154.

Pupulin, F. \& D. Bogarín. 2011. Of greenish Encyclia: natural variation, taxonomy, cleistogamy, and a comment on DNA barcoding. in: A. M. Pridgeon \& H. G. Navarrete Zambrano (eds.), Proceedings of the Third Scientific Conference on Andean Orchids, Quito. Lankesteriana 11(3): 325-336.

Pupulin, F. \& D. Bogarín. 2012. A taxonomic revision of Encyclia (Orchidaceae: Laeliinae) in Costa Rica. Bot. J. Linnean Soc. 168(4): 395-448. doi: 0.1111/j.10958339.2012.01212.x

Pupulin, F. \& R.L. Dressler. 2006. A new species of Ornithocephalus (Orchidaceae) from Panama. Brittonia 58(4): 314-317.

Pupulin, F., A.P. Karremans \& B. Gravendeel. 2012. A reconsideration of the empusellous species of Specklinia (Orchidaceae: Pleurothallidinae) in Costa Rica. Phytotaxa 63: 1-20.

Pupulin, F., D. Bogarín \& M. Fernández. 2010. Illustrations and Studies in Neotropical Orchids.2. A note on Pleurothallis luctuosa (Orchidaceae: Pleurothallidinae), with a new species. Ann. Naturhist. Mus. Wien B112: 239-252.

Ramírez, S.R., B. Gravendeel, R.B. Singer, C.R. Marshall \& N.E. Pierce. 2007. Dating the origin of the Orchidaceae from a fossil orchid with its pollinator. Nature 448: 1042-1045. doi:10.1038/nature06039

Reichenbach, H.G. 1852. Gartenorchideen. Bot. Zeitung (Berlin) 10(44): 761-772.

Reichenbach, H.G. 1854. Orchideae Warscewiczianae recentiores. Bonplandia 2: 96-102.

Reichenbach, H.G. 1866. Beitrage zu einer orchideenkunde Central-Amerika's. Hamburg. T.G. Meissner, p. 97.

Romero-González, G.A. \& C.H. Dodson. 2010. A la tercera se gana: the validation of Benzingia (Orchidaceae:
Zygopetalinae). Lankesteriana 9: 526. doi: ttp://dx.doi. org/10.15517/lank.v0i0.12115

Romero-González, G.A. \& G. Carnevali FernándezConcha. 1999. Notes on the species of Cyrtopodium (Cyrtopodinae, Orchidaceae) from Florida, the Greater Antilles, Mexico, Central America and northern South America. Harvard Pap. Bot. 4: 327-341.

Romowicz, A. \& D.L. Szachetko. 2006. Genera et species orchidalium. 12. Oncidieae. Polish Bot. J. 51: 43-47.

Santiago, E. \& E. Hágsater. 2007. Epidendrum schlechterianum Ames P1. 982 in: E. Hágsater \& L. Sánchez-Saldaña (eds.). The genus Epidendrum. Part 6. Species new \& old in Epidendrum. Icon. Orchid. (Mexico) 9: i-xiii, pl. 901-1000.

Santiago, E. \& E. Hágsater. 2008. Epidendrum piliferum Rchb.f. Pl. 1160 in: E. Hágsater \& L. Sánchez-Saldaña (eds.). 2008. The genus Epidendrum. Part 7. Species new \& old in Epidendrum. Icon. Orchid. (Mexico) 11: i-xxvii, pl. 1101-1200.

Savage, J.M. 1970. On the trail of the golden frog: with Warscewicz and Gabb in Central America. Proc. Calif. Acad. Sci., Fourth Series, 38: 273-288.

Schlechter, F.R.R. 1913a. Orchidaceae novae et criticae. Decas XXXVIII. Repert. Spec. Nov. Regni Veg. Beih. 12 (317-321): 202-206.

Schlechter, F.R.R. 1913b. Orchidaceae novae et criticae. Decas XXXIX. Repert. Spec. Nov. Regni Veg. Beih. 12 (322-324): 212-216.

Schlechter, F.R.R. 1918. Kritische Aufzählung der bisher aus Zentral-Amerika bekanntgewordenen Orchidaceen. Beih. Bot. Centralbl., Abt. 2 36(2): 321-520.

Schlechter, F.R.R. 1920a. Orchidaceae novae et criticae. Decas LXV. Repert. Spec. Nov. Regni Veg. Beih. 16 (462-467): 353-358.

Schlechter, F.R.R. 1920b. Orchidaceae novae et criticae. Decas LXVI-LXVII. Repert. Spec. Nov. Regni Veg. Beih. 16 (468-473): 437-450.

Schlechter, F.R.R. 1921. Orchidaceae novae et criticae. Decas LXIX. . Additamenta ad Orchideologiam Central-Americanam. Repert. Spec. Nov. Regni Veg. Beih. 17(481-485): 138-144.

Schlechter, F.R.R. 1922. Beitrage zur Orchideenkunde von Zentralamerika. I. Orchidaceae Powellianae Panamenses. Repert. Spec. Nov. Regni Veg. Beih. 17: $1-95$.

Schlechter, F.R.R. 1924. Beitrage zur Orchideenkunde von Colombia: Orchidaceae Hoppianae. Repert. Spec. Nov. Regni Veg. Beih. 27: 5-123.

Seemann, C.B. 1852-1857. The botany of the voyage of H.M.S. Herald, under the command of Captain Henry Kellet, R.N., C.B., during the years 1845-51. Lovell Reeve, London.

Seemann, C.B. 1854. Flora of the Isthmus of Panama 
/ [published under the authority of the Lords Commissioners of the Admiralty by Berthold Seemann]. Lovell Reeve, London. 254 pp.

Soto Arenas, M.A. \& R.L. Dressler. 2010. A revision of the Mexican and Central American species of Vanilla Plum. ex Mill. with a characterization of their ITS region of the nuclear ribosomal DNA. Lankesteriana 9(3): 285-354.

Standley, P.C. 1927. The Flora of Barro Colorado Island. Series: Smithsonian Miscellaneous Collections. 78(8): 1-32. http://dx.doi.org/10.5962/bhl.title.46805

Szlachetko, D.L. 2003. Senghasia, eine neue Gattung der Zygopetaleae. J. Orchideenfr. 10: 332-344.

Szlachetko, D.L. 2004a. Matériaux pour la révision des Habenariinae (Orchidaceae, Orchidoideae) - 4. Richardiana 4: 52-65.

Szlachetko, D.L. 2004b. Matériaux pour la révision des Habenariinae (Orchidaceae, Orchidoideae) - 5. Richardiana 4: 103-108.

Szlachetko, D.L. 2006. Genera et species orchidalium. 11. Oncidieae. Polish Bot. J. 51: 39-41.

Szlachetko, D.L. 2007. Notes sur l'alliance Stanhopea (Stanhopeinae, Vandoideae). Richardiana 7: 45-49.

Szlachetko, D.L. \& H.B. Margońska. 2001. Genera et species orchidalium. 3. Polish Bot. J. 46: 113-121.

Szlachetko, D.L. \& H.B. Margońska. 2006. Redefinition of the genera Malaxis Sol. ex Sw. and Microstylis (Nutt.) Eaton (Orchidaceae, Epidendroideae). Acta Soc. Bot. Poloniae 75: 229-231.

Szlachetko, D.L. \& M. Kras. 2006. Notes sur le genre Habenella. Richardiana 6: 33-39.

Szlachetko, D.L.\& M. Kulak. 2006. Nouvelles combinaisons dans le genre Zosterophyllanthos Szlachetko \& Margónska (Orchidaceae, Pleurothallidinae). Richardiana 6: 183-195.

Szlachetko, D.L. \& M. Śmiszek. 2006 [2007]. Nouveaux genres dans le complexe Maxillaria (Orchidaceae). Richardiana 7: 26-32.

Szlachetko, D.L. \& P. Rutkowski. 2008. Classification of Spiranthinae, Stenorrhynchidinae and Cyclopogoninae. Pp. 130-222 in: P. Rutkowski, D. L. Szlachetko \& M. Górniak (eds.), Phylogeny and taxonomy of the subtribes Spiranthinae, Stenorrhynchidinae and Cyclopogoninae (Spirantheae, Orchidaceae) in Central and South America. Wydawnictwo Uniwersytetu Gdańskiego, Gdańsk, Poland.

Szlachetko, D. L. \& Rutkowski, P. 2008. Classification of Spiranthinae, Stenorrhynchidinae and Cyclopogoninae. — In: P. Rutkowski, D.L. Szlachetko \& M. Górniak. (eds.), Phylogeny and taxonomy of the subtribes Spiranthinae, Stenorrhynchidinae and Cyclopogoninae (Spirantheae, Orchidaceae) in Central and South America: 166-167. Gdańsk University Press.
Szlachetko, D.L. \& Sitko, M. 2007. Noveaux genres dans le complexe Maxillaria (Orchidaceae). - Richardiana 7: 26-32.

Szlachetko, D.L., J. Mytnik-Ejsmont \& A. Romowicz. 2006. Genera et species orchidalium. 14. Oncidieae. Polish Bot. J. 51: 53-55.

Szlachetko, D.L., J. Mytnik-Ejsmont, M. Górniak \& M. Śmiszek. 2006. Genera et species orchidalium. 15. Maxillarieae. Polish Bot. J. 51: 57-59.

Szlachetko, D.L., M. Kulak \& A. Romowicz. 2006. Notes sur le genre Senghasia Szlachetko (Orchidaceae, Huntleyinae). Richardiana 6: 180-182.

Szlachetko, D.L., P. Rutkowski \& J. Mytnik. 2005. Contributions to the taxonomic revision of the subtribes Spiranthinae, Stenorrhynchidinae and Cyclopogoninae (Orchidaceae) in Mesoamerica and the Antilles. Polish Bot. Stud. 20: 3-387.

TROPICOS. 2014. Missouri Botanical Garden. Available from: http://www.tropicos.org/ (August 2014).

van den Berg, C., D.H. Goldman, J.V. Freudenstein, A.M. Pridgeon, K.M. Cameron, M.W. Chase. 2005. An overview of the phylogenetic relationships within Epidendroideae inferred from multiple DNA regions and recircumscription of Epidendreae \& Arethuseae (Orchidaceae). Am. J. Bot. 92:613-624. doi: 10.3732/ajb.92.4.613.

van den Berg, C., Higgins, W.E., Dressler, R.L. 2000. A phylogenetic analysis of Laeliinae (Orchidaceae) based on sequence data from internal transcribed spacers (ITS) of nuclear ribosomal DNA. Lindleyana. 15: 96-114.

Wallace, J. 1700-1701. Part of a Journal Kept from Scotland to New Caledonia in Darien, with a Short Account of That Country. Communicated by Dr Wallace, F. R. S. Phil. Trans. 22: 536-543.

Whitten, W.M., N.H. Williams, R.L. Dressler, G. Gerlach \& F. Pupulin. 2005. Generic relationships of Zygopetalinae (Orchidaceae: Cymbideae): combined molecular evidence. Lankesteriana 5(2): 87-107.

Williams, L.O. 1940. Orchidaceae. Pp. s271-286 in: R.E. Jr. Woodson \& R.W. Schery (eds.), Contributions toward a Flora of Panama. IV. Miscellaneous collections, chiefly by Paul H. Allen. Ann. Missouri Bot. Gard. 27. doi: http://dx.doi.org/10.2307/2394356

Williams, L.O. 1946a. Orchidaceae. Pp. 1-140 in: R.E. Jr. Woodson \& R.W. Schery (eds.), Flora of Panama. Part III, Fascicle 2. Ann. Missouri Bot. Gard., 33. doi: http:// dx.doi.org/10.2307/2394519

Williams, L.O. 1946b. Orchidaceae. Pp. 315-404 in: R.E. Jr. Woodson \& R.W. Schery (eds.), Flora of Panama. Part III, Fascicle 3. Ann. Missouri Bot. Gard. 33. doi: http://dx.doi.org/10.2307/2394495

Williams, L.O. 1956. An enumeration of the Orchidaceae of Central America, British Honduras and Panama. Ceiba 5(1): 25 . 
Williams, L.O. \& P.H. Allen. 1980. A facsimile reprint of the Orchidaceae, Flora of Panama. Pp. 107-590 in: W. G. D'Arcy (ed.), Orchids of Panama. Missouri Bot. Garden Press.

Williams, N.H. \& W.M. Whitten. 1988. Stanhopea panamensis, a new species from central Panama (Orchidaceae). Lindleyana 3(1): 9-11.

Williams, N.H., M.W. Chase, T. Fulcher \& W.M. Whitten. 2001. Molecular systematics of the Oncidiinae based on evidence from four DNA sequence regions: expanded circumscriptions of Cyrtochilum, Erycina, Otoglossum, and Trichocentrum and a new genus (Orchidaceae). Lindleyana 16: 113-139.
Williams, N.H., W.M. Whitten \& R. L. Dressler. 2005. Molecular systematics of Telipogon (Orchidaceae: Oncidiinae) and its allies: nuclear and plastid DNA sequence data. Lankesteriana 5: 163-184.

Withner, C.L. \& P.A. Harding. 2004. The Cattleyas and their relatives: the debatable Epidendrums. Timber Press, Portland, OR/Cambridge, UK.

Woodson, R.E., Jr. \& R.W. Shery. 1943-1981. Flora of Panama. Ann. Missouri Bot. Garden USA, Vol. 30-67.

Yañez, E. \& M. Soto. 2003. Osmoglossum egertonii (Lindl.) Schltr. Pl. 634 in: E. Hágsater \& M. Soto (eds.). 2003. Orchids of Mexico. Parts 2 \& 3. Icon. Orchid. 5-6: pls. 501-700. 

